\title{
LYAPUNOV-BASED HYBRID CONTROL FOR ROBUST TRAJECTORY TRACKING OF ROBOTIC MANIPULATORS
}

\author{
bY \\ Shafiqul Islam \\ M. Sc. in Control Engineering, \\ B. Sc. in Electrical and Electronic Engineering
}

A thesis submitted to the Faculty of Graduate and Postdoctoral Affairs in partial fulfillment of the requirements for the degree of Doctor of Philosophy in Electrical and Computer Engineering

Ottawa-Carleton Institute of Electrical and Computer Engineering Department of Systems and Computer Engineering Carleton University, Ottawa, Ontario, Canada, K1S 5B6

August, 2010

(C) Copyright 2010, Shafiqul Islam 
Library and Archives

Canada

Published Heritage

Branch

395 Wellington Street

Ottawa ON K1A ON4

Canada
Bibliotheque et

Archives Canada

Direction du

Patrimoine de l'édition

395, rue Wellington

Ottawa ON K1A ON4

Canada

Your file Votre référence

ISBN: 978-0-494-70524-7

Our file Notre référence

ISBN: $978-0-494-70524-7$

NOTICE:

The author has granted a nonexclusive license allowing Library and Archives Canada to reproduce, publish, archive, preserve, conserve, communicate to the public by telecommunication or on the Internet, loan, distribute and sell theses worldwide, for commercial or noncommercial purposes, in microform, paper, electronic and/or any other formats.

The author retains copyright ownership and moral rights in this thesis. Neither the thesis nor substantial extracts from it may be printed or otherwise reproduced without the author's permission.
AVIS:

L'auteur a accordé une licence non exclusive permettant à la Bibliothèque et Archives Canada de reproduire, publier, archiver, sauvegarder, conserver, transmettre au public par télécommunication ou par l'Internet, prêter, distribuer et vendre des thèses partout dans le monde, à des fins commerciales ou autres, sur support microforme, papier, électronique et/ou autres formats.

L'auteur conserve la propriété du droit d'auteur et des droits moraux qui protège cette thèse. Ni la thèse ni des extraits substantiels de celle-ci ne doivent être imprimés ou autrement reproduits sans son autorisation.
In compliance with the Canadian Privacy Act some supporting forms may have been removed from this thesis.

While these forms may be included in the document page count, their removal does not represent any loss of content from the thesis.
Conformément à la loi canadienne sur la protection de la vie privée, quelques formulaires secondaires ont été enlevés de cette thèse.

Bien que ces formulaires aient inclus dans la pagination, il n'y aura aucun contenu manquant.

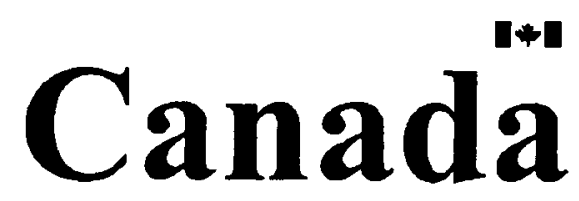




\begin{abstract}
In the face of large-scale modeling errors and uncertainties, the single-model based classical control approach requires high observer-controller gains in order to meet desired tracking objectives. In practice, the sensitivity of the control system to noise, disturbance and uncertainty increases with high gains, causing highfrequency chattering and fast switching, which might make classical design not implementable since available control efforts are usually limited. In addition, high gains could amplify the input and output disturbance in an unusual way, which may excite hidden unmodeled dynamics, resulting in poor tracking performance.

In order to reduce the control gains/efforts as well as to improve the robustness of classical control systems with respect to these non-ideal operating conditions, this thesis introduces a new hybrid adaptive control strategy based on multipleparameter models/controls. The hybrid strategy reduces the observer-controller gains by reducing modeling errors and uncertainties via identifying an appropriate controller from a finite set of candidates that closely estimate the plant at each instant of time. The proposed scheme works as follows: first, the compact set of parameters is split into smaller subsets, and a candidate controller for each of these smaller subsets is designed; then, the stability-guaranteed Lyapunov-based switching mechanism is employed to select a candidate controller that best approximates the plant at any instant of time among the finite set of candidates. Simulation and experimental studies of the proposed methods are conducted on real robot systems to demonstrate the theoretical development for real-life applications.
\end{abstract}




\section{Acknowledgments}

I would like to express my sincere gratitude to my supervisor Professor Peter Xiaoping Liu for his continuous financial support and excellent guidance. Professor Liu has played a significant role on this research, writing, and state of mind ever since our first meeting. His quick response, quick mind, excellent technical skills, and enthusiasm inspired me to pursue my Ph.D. degree. I would also like to express my sincere gratitude to Professor Howard Schwartz for his guidance and financial support in the beginning of the program. Without their support, I would never have reached this point.

I sincerely appreciate for the inputs from Dr. Guangjun Liu from the Department of Aerospace Engineering at Ryerson University, Dr. Jerome Talim from the Department of Systems and Computer Engineering at Carleton University, Dr. Mojtaba Ahmadi from the Department of Mechanical and Aerospace Engineering at Carleton University, and Dr. Abdulmotaleb El Saddik from the School of Information Technology and Engineering at the University of Ottawa. Their constructive comments are very helpful in improving the presentation of this thesis.

I thank Dr. Ilia G. Polouchine from the Faculty of Engineering at the University of Western Ontario for e-mail discussion on the model of the Phantom ${ }^{\mathbf{T M}}$ experimental systems that used in this thesis.

I would like to thank the Faculty of Graduate and Postdoctoral Affairs of Carleton University for Research Excellence Award in Science and Engineering of Carleton University for the year 2009-2010.

Finally, I thank to my wife for her unflagging support and great patient over last four years. I am grateful to my parents for their prayer, guidance and love that brought me who I am today. 


\section{Contents}

$1 \quad$ INTRODUCTION 19

2 PD-Based Linear Control System 33

2.1 System Dynamics and Properties . . . . . . . . . . . . . . 34

2.2 Output Feedback Design . . . . . . . . . . . . . . . . 35

2.2.1 Error Bound Analysis Under PD State Feedback Control De$\operatorname{sign} \ldots \ldots \ldots \ldots \ldots \ldots \ldots \ldots$

2.2.2 Error Bound Under PD Output Feedback Controller . . . . 48

2.3 Design Synthesis and Simulation Results . . . . . . . . . . . 55

2.4 Summary . . . . . . . . . . . . . . . . . . . . . . 69

3 Robust Adaptive Fuzzy Control System 70

3.1 System Dynamics . . . . . . . . . . . . . . . . . . . . . 71

3.1 .1 Fuzzy System . . . . . . . . . . . . . . . . . 72

3.2 Hybrid Adaptive Control System Using Direct Adaptive Fuzzy and Robust Adaptive Control . . . . . . . . . . . . . . . . . 73

$3.2 .1 \quad$ State Feedback Approach _. . . . . . . . . . . 73

3.2 .2 Output Feedback Approach _ . . . . . . . . . . . . . 79

3.2 .3 Design Procedure and Steps . . . . . . . . . . . . . . 92

3.2.4 Design and Simulation Results . . . . . . . . . . . . . . . 92 
3.2.5 Comparison Between Theorem 3.1 and Theorem 3.3 ... 98

3.3 Hybrid Adaptive Control System Using Indirect Adaptive Fuzzy and Robust Adaptive control . . . . . . . . . . . . . . . . . . 102

3.3.1 State Feedback Approach _. . . . . . . . . . . . . 102

3.3 .2 Simulation Results . . . . . . . . . . . . . . . . . . 112

3.3.3 Simulation Results with Theorem 3.6 . . . . . . . . . . 113

3.3.4 Comparison of CAOFB with Hybrid Design . . . . . . 115

3.3.5 Comparison of Theorem 3.4 with Theorem $3.6 \ldots \ldots$. . 116

3.4 Summary . . . . . . . . . . . . . . . . . . . . . 117

4 Distributed Sliding Mode Control 119

4.1 Classical Sliding Mode Control . . . . . . . . . . . . . . . . . 120

4.1.1 Output Feedback Sliding Mode Control (OFBSMC) Design . 123

4.1.2 Adaptive Sliding Mode Control (ASMC) . . . . . . . . 132

4.1.3 Adaptive Output Feedback Sliding Mode Control (AOFBSMC) 133

4.1.4 Design and Implementation Process . . . . . . . . . . 137

4.1 .5 Simulation Results . . . . . . . . . . . . . . . . . 141

4.1.6 Comparison with Output Feedback SMC . . . . . . . . 144

4.2 Distributed Sliding Mode Control: State and Output Feedback Design152

4.2.1 Multiple Lyapunov-functions Based Distributed SMC . . . 162

4.2 .2 Simulation Results . . . . . . . . . . . . . . . . . 167

4.3 Summary . . . . . . . . . . . . . . . . . . . . . 174

5 Multi-model Hybrid Adaptive Control 178

5.1 Single Model Classical Adaptive Control . . . . . . . . . . . . . 179

5.1.1 State Feedback Controller . . . . . . . . . . . . . 180

5.1.2 Disturbance Rejection in Adaptive State Feedback Design 184 
5.1.3 Adaptive Output Feedback Design . . . . . . . . . . . 185

5.1.4 Disturbance Rejection in Adaptive Output Feedback Design 190

5.1.5 Design Steps for CE-based CAOFB . . . . . . . . 190

5.1.6 Motivating Example . . . . . . . . . . . . . . . . 192

5.2 Adaptive Feedback Using Multi-model Control Approach: State and Output Feedback Design . . . . . . . . . . . . . . 196

5.2.1 Robust Adaptive Feedback Using multi-model Control Approach . . . . . . . . . . . . . . . 205

5.2 .2 Simulation Results . . . . . . . . . . . . . . 206

5.2.3 Comparison of Theorem 5.2 and Theorem $5.6 \ldots 208$

5.2.4 Comparison of Theorem 5.3 and Theorem 5.7 . . . . . 209

5.2.5 Comparison of Theorem 5.6 with the CAC Design (State Feedback) . . . . . . . . . . . . . 210

5.2.6 Comparison of Theorem 5.7 and Theorem 5.1 (Output Feedback $\ldots \ldots \ldots \ldots \ldots \ldots \ldots$. . . . . . . . . . . . 211

5.3 Summary . . . . . . . . . . . . . . . . . . 212

6 Experimental Results 214

6.1 Model Dynamics . . . . . . . . . . . . . . . . . . . . 214

6.2 Trajectory Generation . . . . . . . . . . . . 220

6.3 Implementation Results with PD-based Linear Control System Design: State and Output Feedback Case . . . . . . . . . . . 221

6.4 Implementation Results with CE-based Nonlinear Adaptive Control Design: State and Output Feedback Case . . . . . . . . . . . . . 224

6.5 Experimental Results with Multi-model Hybrid Adaptive Design: State Feedback Approach . . . . . . . . . . . . 233 
6.6 Experimental Results with Multi-model Hybrid Adaptive Design: Output Feedback Approach . . . . . . . . . . . . . . . . . . 244

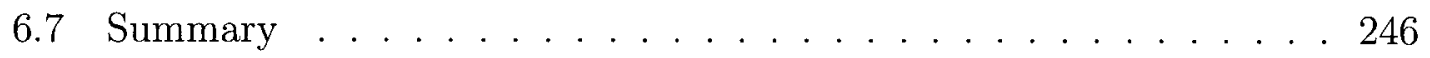

7 Conclusions 248

7.1 Thesis Contributions ................... 248

7.2 Manuscripts Published, Accepted, Under Review and Submitted . . 250

7.3 Future Research . . . . . . . . . . . . . . . . . . . 252 


\section{List of Figures}

2.1 The implementation block diagram representation of observer-controller

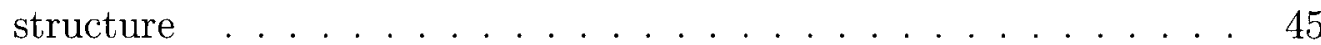

2.2 The desired (dash), output trajectory (radians) (solid) and control inputs (newton-meters) for joints 1 and 2 without using input and output disturbance noise under Theorem 2.1. . . . . . . . . . 51

2.3 The tracking errors for joints 1 and 2 without using input and output disturbance noise under Theorem 2.1. . . . . . . . . . . . 53

2.4 The desired (dash), output trajectory (radians) (solid) and control inputs (newton-meters) for joints 1 and 2 using output feedback design of Theorem 2.1 with initial conditions. . . . . . . . . . 55

2.5 The tracking errors for joints 1 and 2 using with output feedback design of Theorem 2.1 using with initial conditions. . . . . . . . 56

2.6 The disturbance level of $w(t) \ldots \ldots \ldots \ldots \ldots$

2.7 The desired (dash), output trajectory (radians) (solid) and control inputs (newton-meters) for joints 1 and 2 using output feedback design of Theorem 2.1 using with input and output disturbance noise

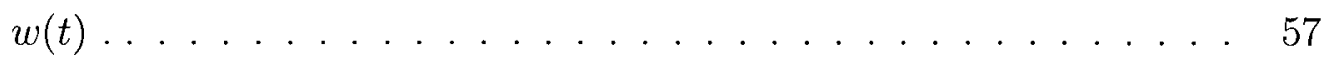

2.8 The tracking errors of joints 1 and 2 under Theorem 2.1 with the disturbance noise $w(t)$. 
2.9 The desired (dash), output tracking (radians) (solid) and control inputs (newton-meters) for the joint 1 and joint 2 with Theorem 2.1 under dynamics changes. . . . . . . . . . . . . . . .

2.10 The tracking errors for joints 1 and 2 with Theorem 2.1 under model

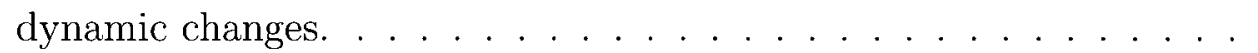

2.11 The desired (dash), output tracking (radians) (solid) and control inputs (newton-meters) for the joint 1 and joint 2 under Theorem 2.2. 65

2.12 The tracking errors for joint 1 and joint 2 with Theorem 2.2. . . . 66

2.13 The desired (dash), output tracking (radians) (solid) and control inputs (newton-meters) for joints 1 and 2 under output feedback design of Theorem 2.3 without using the input and output disturbance noise. . . . . . . . . . . . . . . . . .

2.14 The tracking errors for joints 1 and 2 with the PD output feedback design of Theorem 2.3 without using the input and output disturbance noise.

2.15 The desired (dash), output tracking (radians) (solid) and control inputs (newton-meters) for joints 1 and 2 under output feedback design of Theorem 2.3 using with the input and output disturbance noise $w(t) \ldots \ldots \ldots$. . . . . . . . . . . . . . . . .

2.16 The The tracking errors for joint 1 and joint 2 under PD output feedback design with the input and output disturbance noise measurement $w(t)$. . . . . . . . . . . . . . . . . . .

2.17 The tracking errors for joints 1 and 2 under PD and classical adaptive output feedback design under dynamics changes. . . . . . . . . . . .

3.1 The tracking errors (radians) for joint 1 and joint 2 under CAOFB and hybrid adaptive output feedback design with model dynamic changes. The solid-line is for CAOFB design and the red-dash-line is for Theorem 3.3. . . . . . . . . . . . . . . . . . . 
3.2 The tracking errors (radians), the control input (newton-meters) for joint 1 and joint 2 under Theorem 3.1 and Theorem 3.3. The solidline is for Theorem 3.1 and the red-dash-line is for Theorem 3.3. . . 97

3.3 The disturbance noise level $d(t)=w(t)$ (newton-meters). . . . . 99

3.4 The tracking errors (radians), the control input (newton-meters) for joint 1 and joint 2 under Theorem 3.1 and Theorem 3.3. The sloidline is for Theorem 3.1 and the red-dash-line is for Theorem 3.3. . 100

3.5 The tracking errors (radians), the control input (newton-meters) for joint 1 and joint 2 under Theorem 3.1 and Theorem 3.3. The sloidline is for Theorem 3.1 and the red-dash-line is for Theorem 3.3. . . 101

3.6 The tracking errors (radians), the control input (newton-meters) for joint 1 and joint 2 under Theorem 3.1 and Theorem 3.3. The sloidline is for Theorem 1 and the red-dash-line is for Theorem $3 \ldots 103$

3.7 The time varying disturbance for two joints $d_{1}(t), d_{2}(t)$ of $(3.53) \ldots 113$

3.8 The tracking errors (radians), the control input (newton-meters) for joint 1 and joint 2 under Theorem 3.3. . . . . . . . . . . . 114

3.9 The tracking errors (radians), the control input (newton-meters) for joint 1 and joint 2 under CE-based state and output feedback design. 116

3.10 The tracking errors (radians), the control input (newton-meters) for joint 1 and joint 2 under Theorem 3.4 (state feedback) and Theorem 3.6 (output feedback). . . . . . . . . . . . . . . . . 117

4.1 The desired (dash), output tracking (radians) (solid) and control input (newton-meters) for joints $1 \& 2$ under state feedback based ASMC for $\tilde{\theta}=10$ without using input and output disturbance noise. 136

4.2 The disturbance $w(t) \ldots \ldots \ldots \ldots \ldots \ldots$ 
4.3 The desired (dash), output tracking (radians) (solid) and control input (newton-meters) for joints $1 \& 2$ under state feedback based ASMC for $\tilde{\theta}=8$ with non ideal operating condition. . . . . . . 138

4.4 The desired (black-dash), output tracking (radians) (black-solid) and control input (newton-meters) (n-m) for joints $1 \& 2$ under AOFBSMC design with $\tilde{\theta}=10$ and without using input and output disturbance noise $w(t) \ldots \ldots \ldots \ldots$. . . . . . . . . 140

4.5 The desired (black-dash), output tracking (radians) (black-solid) and control input (newton-meters) (n-m) for joints $1 \& 2$ under AOFBSMC design using with disturbance noise $w(t)$ and the estimation error as $\tilde{\theta}=10 \ldots \ldots \ldots$. . . . . . . . . . . . . . 141

4.6 The simulation results with observers (4.9) and (4.46) based AOFBSMC under ideal condition: Left column is for $\tilde{\theta}_{\text {observer }}=4$ and right column is for $\tilde{\theta}_{\text {observer }}=0$, where $a$ : output tracking errors (radians) (dash-line is for the observer (4.46), solid-line is for the observer (4.9)) for joint 1, $b$ : output tracking errors (radians) (dashline is for the observer (4.46), solid-line is for the observer (4.9)) for joint 2, $c$ : control input for joint 1 (dash-line is for the observer (4.46), solid-line is for the observer (4.9)), $d$ : control input for joint 2 (dash-line is for the observer (4.46), solid-line is for the observer $(4.9))$

4.7 Observer estimation error $\tilde{e}_{11}$ for joint $1 \ldots \ldots \ldots \ldots$

4.8 Observer velocity estimation error $\tilde{q}_{21}$ for joint $1 . \ldots \ldots \ldots$

4.9 Derivative of observer estimation error $\dot{\tilde{e}}_{11}$ for joint $1 \ldots \ldots . . .146$

4.10 Discontinuous nonlinear vector $v_{1}$ for joint $1 . \ldots \ldots \ldots$

4.11 The parameter estimate $\hat{\theta}_{1} \ldots \ldots \ldots \ldots \ldots \ldots$ 
4.12 The simulation results with Theorem 4.3 and Theorem 4.7 under $\tilde{\theta}=4$ : Left column is for Theorem 4.3 and right column is for Theorem 4.7, where $a$ : output tracking errors (radians) for joint $1, b$ : output tracking errors (radians) for joint $2, c$ : control input (newton-meters) for joint $1, d$ : control input (newton-meters) for joint 2. . . . . . . . . . . . . . . . . 169

4.13 The simulation results with Theorem 4.3 and Theorem 4.7 under $\tilde{\theta}=8:$ Left column is for Theorem 4.3 and right column is for Theorem 4.7, where $a$ : output tracking errors (radians) for joint 1, $b$ : output tracking errors (radians) for joint 2, $c$ : control input (newton-meters) for joint $1, d$ : control input (newton-meters) for joint 2. . . . . . . . . . . . . . . . . . . . . 170

4.14 The simulation results with Theorem 4.7 and SM-based ASMC un$\operatorname{der} \tilde{\theta}=8$ : Left column is for ASMC algorithm (4.35), (4.36) and right column is for Theorem 4.7, where $a$ : output tracking errors (radians) for joint $1, b$ : output tracking errors (radians) for joint 2, $c$ : control input (newton-meters) for joint $1, d$ : control input (newton-meters) for joint 2.

4.15 The disturbance level $w(t) \ldots \ldots$. . . . . . . . . . . . . . . 172

4.16 The simulation results with Theorem 4.7 and SM-based ASMC un$\operatorname{der} \tilde{\theta}=8$ with non ideal condition: Left column is for ASMC algorithm (4.35)-(4.36) and right column is for Theorem 4.7, where $a$ : output tracking errors (radians) for joint $1, b$ : output tracking errors (radians) for joint 2, $c$ : control input (newton-meters) for joint $1, d$ : control input (newton-meters) for joint 2. . . . . . . . . . . 173 
4.17 The simulation results with Theorem 4.4 and Theorem 4.8 with $\tilde{\theta}=$ 4: Left column is for Theorem 4.4 and right column is for Theorem 4.8, where $a$ : output tracking errors (radians) for joint $1, b$ : output tracking errors (radians) for joint $2, c$ : control input (newton-meters) for joint $1, d$ : control input (newton-meters) for joint $2 \ldots \ldots$. . 174

4.18 The simulation results with Theorem 4.4 and Theorem 4.8 with $\tilde{\theta}=$ 8: Left column is for theorem 4.4 and right column is for theorem 4.8, where $a$ : output tracking errors (radians) for joint $1, b$ : output tracking errors (radians) for joint $2, c$ : control input (newton-meters) for joint $1, d$ : control input (newton-meters) for joint $2 \ldots \ldots \ldots$

4.19 The simulation results with Theorem 4.4 and Theorem 4.8 with $\tilde{\theta}=$ 4: Left column is for Theorem 4.4 and right column is fo Theorem 4.8 , where $a$ is for output tracking for joint $1, b$ is for the output tracking of joint 2, $c$ is for the control input (newton-meters) for joint $1, d$ is for the control input (newton-meters) of joint $2 . \quad \ldots 176$

4.20 The simulation results with Theorem 4.4 and Theorem 4.8 with $\tilde{\theta}=$ 8: Left column is for Theorem 4.4 and right column is for Theorem 4.8 , where $a$ is for the output tracking (radians) for joint $1, b$ is for the output tracking (radians) for joint $2, c$ : control input (newtonmeters) for joint $1, d$ : control input (newton-meters) for joint $2 . \quad$. 176

4.21 The simulation results with Theorem 4.8 and SM-based AOFBSMC under $\tilde{\theta}=8$ with ideal condition: Left column is for AOFBSMC algorithm (4.41)-(4.42) and right column is for Theorem 4.8, where $a$ : output tracking errors (radians) for joint $1, b$ : output tracking errors (radians) for joint 2, $c$ : control input (newton-meters) for joint $1, d$ : control input (newton-meters) for joint $2 \ldots \ldots \ldots . . \ldots 177$ 
4.22 The simulation results with Theorem 4.8 and SM-based AOFBSMC under $\tilde{\theta}=8$ with non ideal condition: Left column is for AOFBSMC algorithm (4.41)-(4.42) and right column is for Theorem 4.8, where $a$ : output tracking errors (radians) for joint $1, b$ : output tracking errors (radians) for joint 2, $c$ : control input (newton-meters) for joint $1, d$ : control input (newton-meters) for joint $2 \ldots \ldots \ldots$

5.1 First column is for $K_{P}=200 I_{2 \times 2}, K_{D}=250 I_{2 \times 2}, \Gamma=15 I_{2 \times 2}$, $H_{1}=5 I_{2 \times 2}, H_{2}=5 I_{2 \times 2}, \epsilon=0.002$ and $\tilde{\theta}=8$, second column is for $K_{P}=200 I_{2 \times 2}, K_{D}=250 I_{2 \times 2}, \Gamma=15 I_{2 \times 2}, H_{1}=5 I_{2 \times 2}, H_{2}=5 I_{2 \times 2}$, $\epsilon=0.01$ and $\tilde{\theta}=8$, where $a$ : output tracking errors (radians) for joint $1, b$ : output tracking errors (radians) for joint $2, c$ : control input (newton-meters) for joint $1, d$ : control input (newton-meters)

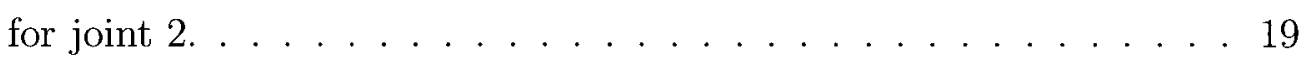

5.2 First column is for $K_{P}=60 I_{2 \times 2}, K_{D}=60 I_{2 \times 2}, \Gamma=15 I_{2 \times 2}, H_{1}=$ $5 I_{2 \times 2}, H_{2}=5 I_{2 \times 2}, \epsilon=0.002$ and $\tilde{\theta}=8$, second column is for $K_{P}=200 I_{2 \times 2}, K_{D}=250 I_{2 \times 2}, \Gamma=15 I_{2 \times 2}, H_{1}=5 I_{2 \times 2}, H_{2}=5 I_{2 \times 2}$, $\epsilon=0.01$ and $\tilde{\theta}=8$, where $a$ : output tracking errors (radians) for joint $1, b$ : output tracking errors (radians) for joint $2, c$ : control input (newton-meters) for joint $1, d$ : control input (newton-meters) for joint 2 . . . . . . . . . . . . . . . . . . . . . . . . . 194

5.3 The simulation results with Theorem 5.2 and Theorem 5.6 under $\tilde{\theta}=4$. First column is for Theorem 5.2 and the second column is for Theorem 5.6, where $a$ : output tracking errors (radians) for joint $1, b$ : output tracking errors (radians) for joint $2, c$ : control input (newton-meters) for joint $1, d$ : control input (newton-meters) for joint $2 \ldots \ldots \ldots \ldots \ldots \ldots \ldots$ 
5.4 The simulation results with Theorem 5.2 and Theorem 5.6 under $\tilde{\theta}=8$. First column is for Theorem 5.2 and the second column is for Theorem 5.6, where a: output tracking errors (radians) for joint $1, b$ : output tracking errors (radians) for joint 2, $c$ : control input (newton-meters) for joint $1, d$ : control input (newton-meters) for joint $2 \ldots \ldots \ldots \ldots \ldots \ldots \ldots \ldots \ldots \ldots$

5.5 The simulation results with Theorem 5.3 and Theorem 5.7 with $\tilde{\theta}=$ 8. First column is for Theorem 5.3 and the second column is for Theorem 5.7, where $a$ : output tracking errors (radians) for joint 1,b: output tracking errors (radians) for joint 2, $c$ : control input (newton-meters) for joint $1, d$ : control input (newton-meters) for

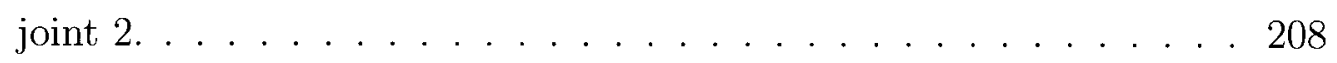

5.6 The simulation results with Theorem 5.6 and SM-based CAC design (5.3), (5.4) with $\tilde{\theta}=4$. First column is for Theorem 5.6 and the second column is for CAC design (5.3), (5.4), where $a$ : output tracking errors (radians) for joint $1, b$ : output tracking errors (radians) for joint 2, $c$ : control input (newton-meters) for joint $1, d$ : control input (newton-meters) for joint $2 . \ldots \ldots \ldots \ldots \ldots$

5.7 The simulation results with Theorem 5.6 and SM-based CAC design (5.3), (5.4) under $\tilde{\theta}=8$. First column is for Theorem 5.6 and the second column is for the single-model CAC (5.3), (5.4), where $a$ : output tracking errors (radians) for joint $1, b$ : output tracking errors (radians) for joint 2, $c$ : control input (newton-meters) for joint $1, d$ : control input (newton-meters) for joint 2. . . . . . . . . . 211 
5.8 The tested results with Theorem 5.7 and SM-based CAC design of Theorem 5.1 under $\tilde{\theta}=8$ with $H_{1}=I_{2 \times 2}, H_{2}=I_{2 \times 2}$ and $\epsilon=$ 0.01 . First column is for Theorem 5.7 and the second column is for single model based CAOFB design of Theorem 5.1, where a: output tracking errors (radians) for joint $1, b$ : output tracking errors (radians) for joint $2, c$ : control input (newton-meters) for joint $1, d$ : control input (newton-meters) for joint 2 . . . . . . . . . . . . 212

5.9 The simulation results with Theorem 5.7 and SM-based CAC design of Theorem 5.1 under $\tilde{\theta}=8$ with $H_{1}=20 I_{2 \times 2}, H_{2}=20 I_{2 \times 2}$ and $\epsilon=0.1$. First column is for Theorem 5.7 and the second column is for CAOFB design of Theorem 5.1, where $a$ : output tracking errors (radians) for joint 1, b: output tracking errors (radians) for joint 2, c: control input (newton-meters) for joint 1, $d$ : control input (newton-meters) for joint 2 .

6.1 Experimental set-up for 3-DOF Phantom 1 TM (left side) and Phantom ${ }_{2}^{\text {TM }}$ (right side) device for real-time application of CAC and hybrid design. 215

6.2 Phantom $_{1}^{\text {TM }}$ robotic system. . . . . . . . . . . . . 215

6.3 Phantom $_{2}^{\mathrm{TM}}$ robotic system. . . . . . . . . . . . 216

6.4 Phantom ${ }_{1}^{\text {TM }}$ robotic manipulator is tracking desired trajectory with an additional $0.85 \mathrm{~kg}$. load attached to its end-effector. . . . . . . 217

6.5 Implementation block diagram for real-time operation of PD-output feedback design with Computer/Simulink/Real-time Workshop/SensAble.217

6.6 Implementation block diagram for real-time operation of hybrid adaptive design with Computer/Simulink/Real-time Workshop/SensAble. 219

6.7 Left column: a, b \& c) The desired (solid-line) and output tracking (dash-line) for joints 1, 2 and 3, Right column: d, e \& f) The tracking errors for joints 1,2 and 3 under PD state feedback design of Theorem 2.2. 
$6.8 \mathrm{a}, \mathrm{b} \& \mathrm{c}$ ) The control inputs for joints 1,2 and 3 under PD state feedback design. . . . . . . . . . . . . . . . . . . 220

6.9 Left column: a, b \& c) The desired (solid-line) and output tracking (dash-line) for joints 1, 2 and 3, Right column: d, e \& f) The tracking errors for joints 1,2 and 3 under PD state feedback design of Theorem 2.2 under dynamic model changes.

$6.10 \mathrm{a}, \mathrm{b} \& \mathrm{c}$ ) The control inputs for joints 1,2 and 3 with Theorem 2.2 under model dynamic changes. . . . . . . . . . . . . . . . . . . . 222

6.11 Left column: a, b \& c) The desired (solid-line) and output tracking (dash-line) for joints 1, 2 and 3, Right column: d, e \& f) The tracking errors for joints 1,2 and 3 with PD output feedback design of Theorem 2.3 with $\epsilon=0.1$.

$6.12 \mathrm{a}, \mathrm{b} \& \mathrm{c}$ ) The control inputs for joints 1,2 and 3 under Theorem 2.3 using with $\epsilon=0.1$.

6.13 Left column: a, b \& c) The desired (solid-line) and output tracking (dash-line) for joints 1, 2 and 3, Right column: d, e \& f) The tracking errors for joints 1,2 and 3 with PD output feedback design of Theorem 2.3 using with $\epsilon=0.01 \ldots \ldots \ldots 226$

$6.14 \mathrm{a}, \mathrm{b} \& \mathrm{c})$ The control inputs for joints 1,2 and 3 using with Theorem

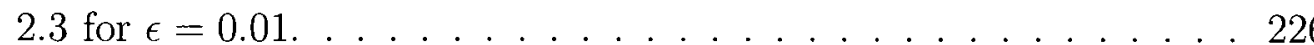

6.15 Left column: a, b \& c) The desired (solid-line) and output tracking (dash-line) for joints 1, 2 and 3, Right column: d, e \& f) The tracking errors for joints 1,2 and 3 with Theorem 2.3 under parameter

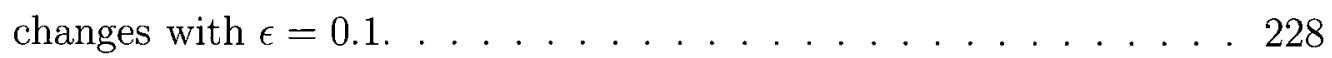

$6.16 \mathrm{a}, \mathrm{b} \& \mathrm{c})$ The control inputs for joints 1,2 and with Theorem 2.3

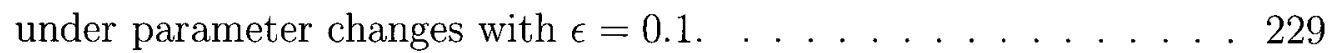


6.17 Left column: a, b \& c) The desired (solid-line) and output tracking (dash-line) for joints 1, 2 and 3, Right column: d, e \& f) The tracking errors for joints 1,2 and 3 with algorithm $(6.3),(6.4) \ldots \ldots . .230$

6.18 a, b \& c) The control inputs for joints 1, 2 and 3 with nonlinear adaptive state feedback control (6.3), (6.4) . . . . . . . . . 230

6.19 Left Column: a, b \& c) The tracking errors for joints 1, 2 and 3, Right Column: d, e \& f) The control inputs for joints 1, 2 and 3 under nonlinear adaptive controller with learning gains $\Gamma=\operatorname{diag}(550,550,550) .231$

6.20 Left Column: a, b \& c) The desired (solid-line) and output tracking (dash-line) for joints 1, 2 and 3, Right Column: d, e \& f) The tracking errors for joints 1,2 and 3 with algorithm (6.3), (6.4). . . 231

$6.21 \mathrm{a}, \mathrm{b} \& \mathrm{c})$ The control inputs for joints 1, 2 and 3 with (6.3), (6.4) under $\Gamma=\operatorname{diag}(700,700,700) \ldots \ldots \ldots \ldots \ldots \ldots \ldots \ldots$

6.22 Left column: a, b \& c) The desired (solid-line) and output tracking (dash-line) for joints 1, 2 and 3, Right column: d, e \& f) The tracking errors for joints 1, 2 and 3 with nonlinear adaptive output design

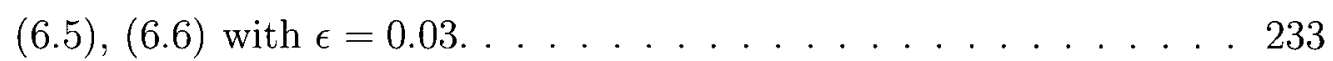

$6.23 \mathrm{a}, \mathrm{b} \& \mathrm{c})$ The control inputs for joints 1,2 and 3 under $(6.5),(6.6)$

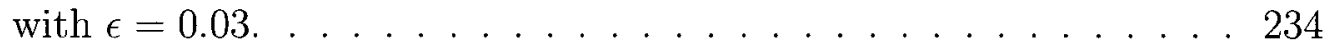

6.24 Left column: a, b \& c) The desired (solid-line) and output tracking (dash-line) for joints 1, 2 and 3, Right column: d, e \& f) The tracking errors for joints 1, 2 and 3 under nonlinear adaptive output design (6.5), (6.6) with $\epsilon=0.02 \ldots \ldots \ldots \ldots \ldots . \ldots . \ldots 234$

$6.25 \mathrm{a}, \mathrm{b} \& \mathrm{c})$ The control inputs for joints 1, 2 and 3 with CAOFB design

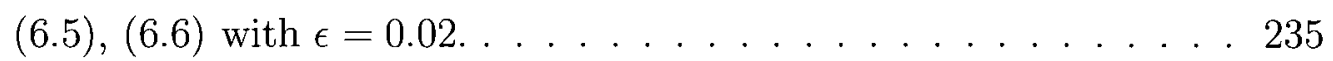


6.26 Left Column: a, b \& c) The tracking error (dash-line is for hybrid adaptive design and solid-line is for CAC algorithm (6.3)) for joints 1, 2 and 3, Right Column: d, e \& f) The control inputs (dash-line is for hybrid adaptive design and solid-line is for CAC algorithm (6.3)) for joints 1,2 and $3 . \ldots \ldots . \ldots . \ldots 236$

$6.27 \mathrm{a}, \mathrm{b}, \mathrm{c} \& \mathrm{~d})$ The parameter estimate $\theta_{1}, \theta_{2}, \theta_{3}$ and $\theta_{4}$ with hybrid adaptive design where the dash-line is for hybrid adaptive design and the solid-line is for the estimate defined by (6.3) . . . . . . 236

$6.28 \mathrm{a}, \mathrm{b}, \mathrm{c} \& \mathrm{~d})$ The parameter estimate $\theta_{5}, \theta_{6}, \theta_{7}$ and $\theta_{8}$ under (6.3) (solid-line) and hybrid adaptive design (dash-line). . . . . . . . . 238

6.29 Left Column: a, b \& c) The tracking errors for joints 1,2 and 3 , Right Column: d, e \& f) The control inputs for joints 1, 2 and 3. . . 238

$6.30 \mathrm{a}, \mathrm{b}, \mathrm{c} \& \mathrm{~d})$ The parameter estimate $\theta_{1}, \theta_{2}, \theta_{3}$ and $\theta_{4}$ with (6.3) and hybrid adaptive design of Theorem 5.6. The dash-line is for hybrid adaptive design and the solid-line is for algorithm (6.3) . . . . . . . 239

$6.31 \mathrm{a}, \mathrm{b}, \mathrm{c} \& \mathrm{~d})$ The parameter $\theta_{5}, \theta_{6}, \theta_{7}$ and $\theta_{8}$ under classical estimate (6.3) (solid-line) and hybrid adaptive design of Theorem 5.6 (dash-

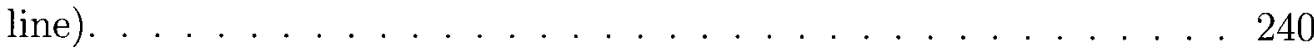

6.32 Left Column: a, b \& c) The tracking errors for joints 1, 2 and 3, Right Column: d, e \& f) The control inputs for joints 1,2 and 3 with additional load attached to the end-effector. . . . . . . . . . . 240

$6.33 \mathrm{a}, \mathrm{b}, \mathrm{c} \& \mathrm{~d})$ The parameter $\theta_{1}, \theta_{2}, \theta_{3}$ and $\theta_{4}$ with classical estimate and hybrid adaptive design. The dash-line is for hybrid adaptive design and the solid-line is for algorithm (6.3) with additional load attached to the end-effector. . . . . . . . . . . . . . . . . . 241

$6.34 \mathrm{a}, \mathrm{b}, \mathrm{c} \& \mathrm{~d})$ The parameter $\theta_{5}, \theta_{6}, \theta_{7}$ and $\theta_{8}$ under CAC estimate (solid-line) and hybrid adaptive design (dash-line) with additional load attached to the end-effector. . . . . . . . . . . . . . . . 242 
6.35 Left Column: $\mathrm{a}, \mathrm{b} \& \mathrm{c}$ ) The tracking errors (dash-line is for hybrid adaptive design of Theorem 5.6 and solid-line is for CAC) for joints 1, 2 and 3, Right Column: d, e \& f) The control inputs (dash-line is for Theorem 5.6 and solid-line is for CAC) for joints 1, 2 and 3. . 243

$6.36 \mathrm{a}, \mathrm{b}, \mathrm{c} \& \mathrm{~d})$ The parameter estimate $\theta_{1}, \theta_{2}, \theta_{3}$ and $\theta_{4}$ with algorithm (6.3) (solid-line) and hybrid adaptive design (dash-line) under dynamic model parameter changes. . . . . . . . . . . . . . 243

$6.37 \mathrm{a}, \mathrm{b}, \mathrm{c} \& \mathrm{~d})$ The parameter estimate $\theta_{5}, \theta_{6}, \theta_{7}$ and $\theta_{8}$ under CE-based CAC design (6.3) (solid-line) and hybrid adaptive design (dash-line) with dynamic model parameter changes. . . . . . . . . . . . . 244

6.38 Left Column: a, b \& c) The tracking errors (dash-line is for hybrid adaptive output feedback design and solid-line is for CAOFB design (6.5)) for joints 1, 2 and 3, Right Column: d, e \& f) The control inputs (dash-line is for hybrid design of Theorem 5.7 and solid line is for $(6.5))$ for joints 1,2 and $3 \ldots \ldots \ldots . \ldots 245$

$6.39 \mathrm{a}, \mathrm{b}, \mathrm{c} \& \mathrm{~d})$ The parameter estimate $\theta_{1}, \theta_{2}, \theta_{3}$ and $\theta_{4}$ with estimation algorithm (6.5) (solid-line) and hybrid adaptive output feedback design of Theorem 5.7 (dash-line). . . . . . . . . . . . . . 246

$6.40 \mathrm{e}, \mathrm{f}, \mathrm{g} \& \mathrm{~h})$ The parameter estimate $\theta_{5}, \theta_{6}, \theta_{7}$ and $\theta_{8}$ under classical estimator (6.5) (solid-line) and hybrid adaptive output feedback design of Theorem 5.7 (dash-line) . . . . . . . . . . . . . . . 247 


\section{Chapter 1}

\section{INTRODUCTION}

OVER past decades, there has been tremendous progress in the development of advanced control systems for the trajectory tracking control problem for a class of uncertain nonlinear systems, such as, robotic systems, chemical and nuclear reactors, air-craft systems, electrical network systems, hydraulic systems, unmanned airborne vehicles systems, turbojet engine systems, renewable energy control systems, etc. The existing control systems can be divided into five categories as proportionalderivative (PD) and proportional-integral-derivative (PID) control $[23,29,45,49$, $50,66,67,71,72,73,74,75,78,79,81]$, feed forward and computed torque control $[23,29,42,49,50]$, adaptive control $[10,11,16,22,23,25,26,27,28,29,30,34$, $38,39,40,41,49,50,51,56,62,65,68,70,82,83,102,103,104,105,110]$, fuzzy and neural network control $[2,8,9,14,19,33,47,48,54,57,60,61,63,80,107$, $111,112,113,114,115,116,117,118,119]$, and variable structure control $[12,13$, $17,18,21,23,24,26,27,28,29,38,42,43,44,46,52,53,76,84,108,118]$. In this thesis, robotic systems are considered as a class of multi-input multi-output (MIMO) nonlinear mechanical systems. Therefore, all designs, developments and implementation results reported in the thesis are based on using MIMO robotic systems.

Most of today's advanced industrial robot systems are controlled by using only proportional-derivative (PD) or proportional-integral-derivative (PID) based lin- 
ear controllers $[4,5,6$, and 49]. Such PD type industrial control system can be traced back to the literature $[67,80]$. These designs comprise PD control plus gravity compensation and the PD control plus desired gravity compensation term. Authors in those papers prove that a PD-type robot controller can be used to asymptotically stabilize the joint positions of rigid robot manipulators. However, these control algorithms requires the knowledge of the gravitational loading vector of the robot dynamics.

Adaptive forms of these controllers can be traced back to the reported results in $[73,74]$. Also, the parameters of these controllers require to meet complex design inequalities that are very difficult to realize in real-time operation. As a matter of fact, in the presence of the gravitational loading vector, PD design may cause a steady-state tracking error, which can be reduced via tuning the proportional and derivative gains or via adding an integral control action.

In $[45,75,79]$, authors show local stability proof for a model-free PID control law for industrial robot systems. The control design does not require knowledge of the robot dynamics. It shows that the control design parameters depending on the robot manipulator dynamics can be selected to ensure desired tracking objective. Motivated by the controller proposed in [45, 75, 79], a stable positioncontroller called $P I^{2} D$ controller without using gravitational loading vector of the robot dynamics was proposed in [78].

A new type of control strategy was introduced in $[74,81]$ to remove the requirement of the gravitational loading vector from the controller design. The control structure combined an integral term developed by using a bounded nonlinear function of the position error with a PD controller term. The controller was developed via combining a saturated, proportional, and differential feedback term with a proportional-integral term formulated by a linear sum of velocity and saturated position errors. The basic idea is to use the energy-shaping technique as well as the passivity theory. The method can achieve desired tracking if the designer can choose suitable design parameters via using Lyapunov second method. How- 
ever, the controller parameters of these controllers are required to satisfy complex inequalities that may not attract industrials for the real-world applications.

In [71], Rocco proposed Lyapunov-based PID controller where the local stability property of the closed-loop system was established. Authors in [71] showed that the integral gain in PID controller required very small values making PID control structure to a simple PD controller.

The implementation of PD or PID controller is, however, difficult since it requires velocity signals in addition to joint position signals. The practical problem is that most advanced robotic systems do not provide velocity sensors due to the constraints of weight and cost. In order to obtain velocity signals, the common practical approach is to differentiate the position measurements obtained from encoders or resolvers, which often leads to severe noise. As a consequence, the performance of the PD or PID controller is limited as, in practice, the noise is amplified with the increase of the values of controller gains.

To attenuate the noise amplification, the cut-off frequency of the filter may be chosen via a trial-and-error method on the basis of the derivative control gains, the amount of measurement noise associated with the sensor and the filtered derivative action of the velocity signals $[4,5$, and 6$]$. While it may work for some applications, such a simple approximation is often inadequate for cases where velocities are very low and/or very high. Moreover, the quantization effect of the noisy velocity signals usually produces undesirable oscillations in the joint which renders the controlled system unstable. In addition, there is no theoretical proof for such an ad hoc solution that has been used in existing industrial robot controllers.

In this work, we propose a linear estimator to estimate the unknown velocity signal for PD-based industrial robot controllers [85, 94]. We are mainly interested in using an observer that is free from the nonlinear robot dynamics and uncertain parameters, which makes the design very robust. Based on using this linear observer, we first propose a PD-like output-feedback control law. The design comprises a PD term with nominal robot model dynamics where the unknown ve- 
locity signals are estimated by the output of the linear estimator. The Lyapunov method is utilized to establish necessary conditions that guarantee the asymptotic stability of the closed-loop error model dynamics. This condition sets the bound on the tracking error trajectory of the closed-loop system. In the second design, we remove the nominal model dynamics from the control design to formulate a model-independent PD-type output feedback approach.

The convergence analysis of the proposed method is given in two steps. We first design a PD controller as a state feedback approach. Based on using Lyapunov method, we obtain the bound on the tracking error signals for the closed-loop error model. It is shown in our analysis that the tracking error bound can be made arbitrarily small by increasing the minimal eigen-value of the control gains. Second, for a given set of initial conditions, we estimate the region of interest of the PD based state feedback design. Then we saturate the control outside the estimated region to obtain bounded control input. Afterwards, we use the linear observer to estimate unknown velocity signals to develop a PD based output feedback control law. The idea of introducing saturated control is to protect the controlled plant from the effect of exponentially fast observer dynamics in the output feedback design. The bounded control allows the designer to increase the speed of the observer dynamics without sacrificing the tracking performance. Using asymptotic analysis of the singularly perturbed closed-loop model, we prove that the observer and tracking error variables are ultimately bounded and their bounds can be made to a small neighborhood of the origin by using observer-controller gains. The introduced control strategy can be applied in industrial robot manipulators that are working under a filtered version of the PD based state feedback control law just by replacing the velocity signal with the output of the linear estimator.

The proposed design is simulated on a 2-DOF robot manipulator. The method is also experimentally tested on 3-DOF Phantom ${ }^{\mathbf{T M}}$ mechatronic systems to demonstrate the effectiveness of the theoretical developments for real-time applications. 
In the last two decades, there has been growing interest in designing fuzzy and neural networks based control systems for nonlinear systems $[12,13,17,18,21$, $22,23,24,26,27,28,29,38,42,43,44,46,52,53,76,84,108,117,118$, and references therein]. The idea of using fuzzy and neural networks systems is to remove the demand of a priori knowledge of the system dynamics. Most of these reported results are based on using the requirement of the known bound of fuzzy approximation errors, external disturbance and other modeling error uncertainties. On the other hand, there is not a well-recognized fuzzy and neural network based adaptive output feedback methodology for developing a smooth controller-observer algorithm for highly uncertain multi-input multi-output nonlinear mechanical systems with modeling error and disturbance uncertainties. Therefore, the field of fuzzy and neural network based adaptive output feedback control also remains a difficult theoretical and practical problem.

In this work, we propose novel hybrid control schemes for a class of nonlinear mechanical systems $[88,90,96$, and 97]. In the first scheme, we combine direct adaptive fuzzy system with robust adaptive control law. The direct adaptive fuzzy system is used to approximate the certainty equivalence (CE) principle-based classical nonlinear adaptive controller.

In the second scheme, an indirect adaptive fuzzy system is employed to estimate nonlinear system dynamics. The robust adaptive controller in both schemes is deployed to cope with uncertainty due to the presence of the fuzzy approximation errors, external disturbance and other unmodeled dynamics. The convergence analysis of the proposed method uses simple theoretical arguments that allow a designer to formulate an output feedback form of any state feedback based fuzzy and neural network based design reported in the literature.

In contrast with the structures of existing fuzzy and neural network control mechanisms, the hybrid design in this work is more efficient in terms of cost and hardware implementation since the design uses only one fuzzy system to approximate the CE-based nonlinear adaptive controller. This is possible as the observer 
dynamics is free from uncertain parameters and nonlinear system dynamics. Unlike the existing fuzzy approach, the design introduced in this work does not require the known bound of fuzzy approximation errors, external disturbance and any other modeling error uncertainties.

The proposed design can be applied for both state (position-velocity) and output feedback cases. In the state feedback case, we develop a hybrid adaptive control system with the assumption that the system states are available for feedback design. The design comprises two components, i.e., adaptive fuzzy and robust adaptive component. The adaptive fuzzy component is employed to estimate nonlinear system dynamics and CE-based classical nonlinear adaptive control law. A robustfying adaptive control component together with an adaptation mechanism is used to learn bounded uncertainties representing fuzzy modeling errors, external disturbance and other modeling error uncertainties. Using Lyapunov-like energy function, we show that the tracking error signals under state feedback based closed loop system are bounded and their bounds meet desired tracking objectives. In the output feedback case, for the given set of initial conditions, we estimate the region of interest of the state feedback based hybrid design and saturate the controller outside the estimated region of interest. Then, we replace the velocity signals in the saturated control law by the output of the linear estimator to formulate hybrid adaptive output feedback control system. We then prove that the tracking error bounds achieved under state feedback based design can be recovered asymptotically.

The design is more effective with respect to cost and real-time implementation as the proposed design does not require an additional fuzzy system for observer dynamics. This is mainly because the observer dynamics is free from uncertain parameters and nonlinearities. In addition, the design consists of a robust adaptive control term for estimating unknown modeling error and disturbance uncertainty on-line, which avoids the problem associated with the existing fuzzy approach. The controller design and implementation procedure are simpler as there are no need 
of the filtering or error augmentation techniques. The controller does not require a priori knowledge of the system dynamics, which makes the design simple such that it is easy to implement in a real-time environment. The proposed algorithms are evaluated on a 2-DOF serial robot manipulators to demonstrate the usefulness of theoretical developments for practical applications.

The sliding mode control (SMC) technique has been extensively studied by many researchers $[12,13,17,18,21,23,24,26,27,28,29,38,42,43,44,46,52,53$, $76,84,108,118$, and many other references reported in the literature]. Most SMC (details definition can be found in [108]) designs reported in the literature, however, assume that velocity signals are available for feedback. But, in practice, most systems only provide position sensors in order to reduce the weight and cost. So, the required joint velocity signals have to be derived by differentiating the position measurements that are often contaminated by severe noise, limiting the tracking performance as the noise is amplified with the increase of the discontinuous control gains. Specifically, the control system under the SMC design may exhibit excessive chattering and infinitely fast switching causing poor tracking performance. To deal with the above problem, we propose to estimate the velocity signals in the SMC design. Results in this topic can be found in $[12,17,18,21$, and 38].

The nonlinear dynamic observer was proposed in [18] for a planar flexible manipulator. The observer dynamics is based on the use of system dynamics, uncertain model parameters and nonlinear control inputs. This method guarantees local stability of the closed-loop system. Moreover, the design is not adaptive, which requires large control efforts to cope with the modeling error uncertainty.

Recently, authors in [38] proposed a sliding mode observer (SMO) based output feedback SMC for a certain class of nonlinear dynamics. The design and stability analysis of their technique resemble the output feedback method earlier reported in [83] for a class of nonlinear systems. The theoretical concepts and critical assumptions of such SMO can be found in [83]. Further difficulties associated with the SMO design synthesis are clearly illustrated via using simulation 
examples.

The SMO structure [38], however, requires system dynamics, nonlinear control input, switching control input terms and undesirable switching function sign(). The narrow stability range of initial conditions with the assumption of the zero initial position estimation error and the difficulties associated with the chattering activity of the switching controls as well as the switching function in the SMO structure are also discussed. In the face of large-scale parametric uncertainty, such a non adaptive SMC [38] demands very large control effort as nonlinear control terms and the high-frequency switching function intensify the control chattering activity in the sliding surface. The method, however, imposes global growth conditions on the system nonlinearities. In addition, the observer-controller ensure only local stability of the closed loop design.

More recently, dynamic observer based output feedback full-order sliding mode scheme is proposed for a certain class of nonlinear systems [17]. The idea of this approach is in two fold. The first one is to introduce an auxiliary dynamics whose input is the system output, and the other one is to transform the augmented system into an observable linear system with an injection term which contains the system output as well as the state of the auxiliary dynamics. The parameters of the controllers-observer are chosen via using complex matrix-vector transformations. As a matter of fact, such a large number of tuning parameters may not be realistic for highly uncertain MIMO nonlinear mechanical systems. Moreover, the designed controller is not robust as it cannot cope with parametric uncertainty. This is mainly because the design uses fixed system dynamics which may cause poor tracking performance in the face of large uncertainty.

In this thesis, we propose linear observer based output feedback SMC design for robotic systems $[89,93]$. The observer structure is simple in the sense that it does not require system dynamics, control inputs as well as undesirable switching terms. The proof of stability of this method is established as follows. We first derive the SMC as a state feedback approach with an assumption that all the state 
variables are available for feedback. The Lyapunov method is utilized to show that the state feedback based SMC design ensures the global stability property for all the signals in the closed-loop system. This property is established by assuming that the parameters are unknown but belongs to a known compact set which is relatively large. In the second step, we replace the unknown velocity signals with the output of the linear estimator. For the given set of initial conditions, we first define the estimated region of the interest for the state feedback based SMC design. Then, we saturate the controller outside the estimated region of interest ensuring that the output feedback controller remains bounded over the estimated region. The idea of using the saturation mechanism is to deal with large-scale uncertainty and disturbance. The saturation mechanism allows the speed of the observer to be increased without sacrificing the transient tracking performance. In convergence analysis, we show that the stability region under the position-velocity based SMC design can be obtained by the output feedback sliding mode control (OFBSMC) design.

In comparison, the method introduced in this thesis is adaptive while the output feedback methods introduced in $[12,17,18,21$, and 38] are not in adaptive control framework. In addition, the output feedback design proposed in $[12,17,18$, 21 , and 38], ensures only local stability while our approach guarantees semi-global stability of the closed-loop system. The proposed design can be applied to design an output feedback form of any state feedback based classical SMC design reported in the literature.

If we consider that the uncertain parameters and initial conditions belong to a relatively large compact set, then the existing single model (SM)-based SMC design either for state or output feedback will exhibit poor tracking performance. To improve overall tracking response, one can deploy high observer, discontinuous and adaptation gains in order to increase the convergence of the state and parameter estimates. More specifically, the state feedback based classical SMC approach requires high learning gains as well as high discontinuous control gains to obtain 
fast convergence of the parameter estimator. In the output feedback case, high observer gains are essential for robust reconstruction of the state estimates, and high learning and discontinuous control gains are required to obtain fast parameter learning to achieve desired tracking performance. The requirement of high observer speed makes the output feedback based SMC design even more complex in realtime application as high observer speed amplifies the input and output disturbance causing high-frequency chattering and infinitely fast switching control phenomenon. As a consequence, the existing SMC design, either for the state or output feedback, might not be practically implementable or might be very expensive as, in practice, the control efforts in most practical systems are limited. To improve overall tracking performance with smaller observer-controller gains, we introduce a distributed SMC technique for a class of nonlinear mechanical systems. Results in this direction for single-input single-output (SISO) systems can be found [55, 58]. Theses methods assume that the position-velocity signals are available for feedback. In addition, the design reported in the literature $[55,58]$ exhibits large transient tracking error due to the pre-routed nature of the switching scheme.

In this thesis, we first propose a pre-ordered control switching based distributed SMC technique for MIMO nonlinear mechanical systems. An inequality for the derivative of the Lyapunov-function candidate is used as a switching criterion to identify an appropriate SMC from the distributed SMC. It shows that the pre-ordered switching nature may cause undesirable transient tracking in the presence of a large number of the distributed candidate sliding mode controllers.

To improve the transient tracking performance from the pre-ordered control switching mechanism, we allow classical sliding mode controllers to be switched instantaneously among the finite set of distributed candidates so that overall tracking performance can be improved. This idea can be described as follows. First, we distribute the compact set of uncertain parameters into a smaller number of compact subsets. Second, we construct a family of candidate of distributed sliding mode controllers corresponding to these smaller parameter compact subsets. Then, at 
each instant of time, we compare a family of distributed candidates to see which candidate generates the largest decrease in the value of the Lyapunov inequality. If the controller that currently acting in the loop satisfies the Lyapunov inequalities, then we keep it. Otherwise, we switch to the distributed SMC candidate that best approximates the plant. The design is applicable for both full state and partial state measurement cases. The proposed method is evaluated on a 2-DOF robot manipulator to demonstrate the effectiveness of the theoretical development for real-world applications.

The single model (SM) certainty equivalence (CE) principle-based classical adaptive control technique has been attracted the automatic control community for several years $[10,11,16,22,23,25,26,27,28,29,30,34,38,39,40,41,49,50,51,56$, $62,65,68,70,82,83,102,103,104,105,110$, and many other references]. These designs can be classified into two categories as state and output feedback based designs.

When operating conditions change in smaller magnitude, such a single model CE principle-based adaptive control design either for the state or output feedback approach can adapt to the new environment asymptotically. The problem is when environments change in large magnitude, very high observer and learning control gains are essential to improve the transient tracking performance. This means that if uncertain parameters and initial conditions become large, then the CE-based nonlinear adaptive design provides poor transient tracking performance.

Another reason for the current approaches showing poor transient tracking performance is the well-known assumption that uncertain model parameters are required to be linear with respect to nonlinear functions. For example, the performance of the CE principle-based nonlinear adaptive robot control system design relies on strict linearized dynamical properties of the systems $[3,5,6,15$, $21,22,23,25,26,27,28,29,30,35$, and many others reported in the literature], where uncertain model parameters are assumed to appear linearly with respect to nonlinear robot dynamics, such as, the inertial matrix $M(q) \in R^{n \times n}$ is 
symmetric, bounded, and positive definite and satisfies the following inequalities: $\|M(q)\| \leq M_{M}$ and $\left\|M^{-1}(q)\right\| \leq M_{M I}$, where $M_{M}$ and $M_{M I}$ are bounded positive constants, the matrix $\dot{M}(q)-2 C(q, \dot{q})$ is skew symmetric and the norm of the gravity and centrifugal-coriolis forces are upper bounded as $\|C(q, \dot{q})\| \leq C_{M}\|\dot{q}\|$ and $\left\|C\left(q, \dot{q}_{d}\right)\right\| \leq C_{M}\left\|\dot{q}_{d}\right\| \leq \alpha_{c}$, where $C_{M}$ and $\alpha_{c}$ are bounded positive constants. Therefore, when unknown parameters $\left(M_{M}, M_{M I}, C_{M}, \alpha_{c}\right)$ are large, then the SM-based CE designs, either for state or output feedback, exhibit poor transient tracking performance due to the presence of large modeling error uncertainty. To improve transient tracking response, one may assign high observer-adaptation gains to speed up the convergence rate of the state and parameter estimates via reducing the modeling error uncertainties of the closed loop system. This implies that the position-velocity based classical adaptive design requires high adaptation gains to obtain fast convergence rate of the parameter estimates. In output feedback design, high observer-learning gains are required to achieve robust reconstruction of the true velocity signals and fast parameter learning to ensure good transient tracking performance. However, the demand of high gains makes the CE-based adaptive design unrealistic in practical applications as high observer-adaptation gains excites high frequency dynamics as well as the input and output unmodeled dynamics, which in turn causes poor transient tracking performance.

To deal with the problem associated with high observer-adaptation gains in classical adaptive control (CAC) framework, we propose a hybrid adaptive control strategy for a class of MIMO nonlinear systems [86, 95, 101]. This method extends the classical adaptive design by allowing its parameter estimate to be changed into a family of candidate models. In other words, the method switches the CAC design into a finite set of candidate controller that best approximates the plant. For this purpose, we uniformly split the compact set of unknown parameters into a finite number of smaller compact subsets $[1,20]$. Then, we design a candidate controller corresponding to each of these smaller compact subsets. The Lyapunov-function inequality is utilized as a resetting criterion to select an appropriate candidate 
model that closely estimates the plant at any instant of time. The design can be applied for both the state and output feedback designs. The proposed design is

simulated on a 2-DOF serial robot and experimented on 3-DOF Phantom ${ }^{\text {TM }}$ robot manipulators to demonstrate the effectiveness of the theoretical development for possible practical applications.

\section{Thesis Overview}

The thesis has the following structure.

In Chapter 2, linear controller-observer schemes are developed for a class of nonlinear mechanical systems. The asymptotic stability property of the closed loop signals is presented via using Lyapunov-like energy function. Then, we remove the nominal model dynamics from the controller design to formulate a modelindependent PD-type output feedback approach. Through asymptotic analysis, we also show that all the signals under model-free PD type output feedback design are bounded and their bounds can be made arbitrarily small by using observercontroller gains. This asymptotic analysis is our main technical tool that allows us to investigate the stability of different output feedback control systems presented in the subsequent chapters. Implementation results demonstrate the potential application of the proposed method on real systems.

Chapter 3 develops novel hybrid adaptive control systems where adaptive fuzzy system combines with adaptive sliding mode control algorithm. These designs can be applied for both state and output feedback case. In convergence analysis, it is proven that the tracking of the output feedback design can converge asymptotically to the performance achieved under the state feedback control design. The algorithms are evaluated on a robot manipulator to demonstrate the theoretical development.

Chapter 4 introduces variable structure control technique. The contributions of this chapter can be divided into two parts. First part proposes an output feed- 
back variable structure control strategy in order to deal with the problem associated with the state feedback based design. Then, distributed variable control structure technique is developed to obtain desired tracking objectives with relatively smaller observer-controller gains in the presence of large-scale parametric uncertainty. The control algorithms are evaluated on a 2-DOF robot manipulator to demonstrate the effectiveness of the theoretical development.

In chapter 5 , a multi-model based adaptive control method in certainty equivalent principle framework is proposed for large scale nonlinear systems. This method extends classical adaptive design by allowing its parameter estimate to be reset into a finite set of candidate parameter models. The proposed method is simulated on a serial link robot manipulator to illustrate the efficacy of theoretical developments for real-time applications.

Chapter 6 presents experimental results of some classical and multi-model based adaptive control algorithms on 3-DOF Phantom ${ }^{\text {TM }}$ robot systems. The aim of this chapter is to experimentally compare the tracking performance of multimodel based hybrid adaptive design with the single model based classical control design.

Finally, future research directions as well as thesis contributions are briefly discussed in Chapter 7. 


\section{Chapter 2}

\section{PD-BASED Linear CONTROL}

\section{SYSTEM}

This chapter presents PD-type output feedback linear control schemes for the trajectory tracking control problem for industrial robotic systems [85, 94].

In the first part of this chapter, we design PD-like output-feedback control laws. The design comprises a PD term with nominal robot dynamics where the unknown velocity signals are estimated by the output of the linear estimator. Using Lyapunov analysis, we characterize the asymptotic property of all the signals in the closed-loop error model dynamics. This property sets the bound on the tracking error trajectory of the closed loop system.

In the second part, we remove the nominal model dynamics from the control design to formulate model-independent PD-type linear feedback controller. In convergence analysis, we prove that all the signals under PD feedback design are bounded and their bounds can be made arbitrarily small by using observercontroller design parameters. Implementation results on a 2-DOF robot manipulator demonstrate the potential applications of the proposed method on real systems.

The rest of the chapter is organized as follows: Section II describes the system model and its dynamical properties. In section III, we propose a PD-like output- 
feedback control algorithm. We then remove the nominal model dynamics from the control law to formulate the model-free feedback control approach. This section also provides the error bound analysis of the proposed method via using Lyapunovlike energy function. To validate our theoretical arguments, these methods are implemented and evaluated on a 2-DOF robot manipulator in section IV. Section V summarize this chapter.

\subsection{System Dynamics and Properties}

Let us first consider the equation of motion for an $n$-link rigid robot systems [82101] given by

$$
M(q) \ddot{q}+C(q, \dot{q}) \dot{q}+G(q)=\tau
$$

where $q \in \Re^{n}$ is the joint position vector, $\dot{q} \in \Re^{n}$ is the joint velocity vector,, $\ddot{q} \in \Re^{n}$ is the joint acceleration vector, $\tau \in \Re^{n}$ is the input torque, $M(q) \in \Re^{n \times n}$ is the symmetric and uniformly positive definite inertia matrix, $C(q, \dot{q}) \dot{q} \in \Re^{n}$ is the Coriolis and centrifugal loading vector, and $G(q) \in \Re^{n}$ is the gravitational loading vector. We now define the joint position and velocity error vector as $e=\left(q-q_{d}\right)$ and $\dot{e}=\left(\dot{q}-\dot{q}_{d}\right)$. Then, we represent the robot model (2.1) in state space form as follows

$$
\begin{aligned}
& \dot{e}_{1}=e_{2} \\
& \dot{e}_{2}=\phi_{1}(e)+\phi_{2}\left(e_{1}\right) \tau-\ddot{q}_{d}
\end{aligned}
$$

where $e_{1}=e, e_{2}=\dot{e}, \phi_{1}(e)=-M\left(e_{1}+q_{d}\right)^{-1}\left[C\left(e_{1}+q_{d}, e_{2}+\dot{q}_{d}\right)\left(e_{2}+\dot{q}_{d}\right)+\right.$ $\left.G\left(e_{1}+q_{d}\right)\right]$ and $\phi_{2}\left(e_{1}\right)=M\left(e_{1}+q_{d}\right)^{-1}$.

The objective of this chapter is to design a PD-based output feedback controller which ensures the boundedness of all signals in the closed-loop model such that the output of the system tracks a given desired trajectory $q_{d}(t)$ which satisfies the following assumption: 
$\left.A_{1}\right)$ The reference trajectory, its first and second derivatives are bounded as $Q_{d} \in$ $\Re^{3 n}=\left[q_{d}, \dot{q}_{d}, \ddot{q}_{d}\right]^{T} \in \Omega_{d}$ with compact set $\Omega_{d}$.

The following dynamic properties will also be employed in convergence analysis $[3,5,6,15,21,22$, and many others]:

1) $M(q) \in R^{n \times n}$ is a symmetric, bounded, and positive definite matrix that satisfies $\|M(q)\| \leq M_{M}$ and $\left\|M^{-1}(q)\right\| \leq M_{M I}$, where $M_{M}$ and $M_{M I}$ are bounded positive constant. 2) The matrix $\dot{M}(q)-2 C(q, \dot{q})$ is skew-symmetric. 3) The norm of the gravity and centrifugal-coriolis forces are upper bounded as $\|C(q, \dot{q})\| \leq$ $C_{M}\|\dot{q}\|$ and $\left\|C\left(q, \dot{q}_{d}\right)\right\| \leq k_{c d}\left\|\dot{q}_{d}\right\| \leq k_{c}$, where $C_{M}, k_{c}$ and $k_{c d}$ are bounded positive constant.

The contributions of this chapter can be divided into two parts. In the first part, we show that PD-like output feedback design can be used to achieve asymptotic stability for industrial robot control systems. Explicit bounds on the observer gains are given by using Lyapunov method. In the second part, we remove the nominal dynamics from the design and achieve semi-global stability property. It is shown that, for the given set of initial conditions of interest, the designer can obtain suitable bound on the observer-controller parameter that ensures the desired tracking objectives. The initial sets can be defined to cover any given initial conditions of interest, but once they are chosen the controller will be designed based on them.

\subsection{Output Feedback Design}

In this section, we first introduce PD-like output-feedback control algorithm for the system (2.2). The control law combines PD term with nominal robot model dynamics where unknown velocity signals is estimated by using linear observer. The error bound analysis of the observer-controller closed-loop system established using singularly perturbed theorem. Later, the nominal model dynamics will be removed to formulate a model-free PD type output feedback design for robotic 
systems.

Let us first state the main results for PD-like output-feedback method in the following Theorem.

Theorem 2.1: Consider the closed-loop system composed of the system (2.2) with the following observer-controller algorithm

$$
\begin{aligned}
\tau\left(e_{1}, \hat{e}_{2}, Q_{d}\right) & =-K_{p} e_{1}-K_{d} \hat{e}_{2}+M\left(e_{1}+q_{d}\right) \ddot{q}_{d}+C\left(e_{1}+q_{d}, \dot{q}_{d}\right) \dot{q}_{d}+G(q) \\
\dot{\hat{e}}_{1} & =\hat{e}_{2}+\frac{H_{1}}{\epsilon} \tilde{e}_{1}, \dot{\hat{e}}_{2}=\frac{H_{2}}{\epsilon^{2}} \tilde{e}_{1}
\end{aligned}
$$

where $\tilde{e}_{1}=\left(e_{1}-\hat{e}_{1}\right), \tilde{e}_{2}=\left(e_{2}-\hat{e}_{2}\right), \hat{e}_{1}$ and $\hat{e}_{2}$ denote the estimated values of $e_{1}$ and $e_{2}, H_{1}$ and $H_{2}$ are positive definite matrices, $\epsilon$ is chosen as a small positive parameter, and $K_{p} \in \Re^{n \times n}, K_{d} \in \Re^{n \times n}$ are constant positive matrices. If $d$ be the positive number such that $d \in(0,1)$, and if there exists a continuous interval $\epsilon \in\left(0, \epsilon^{*}\right)$ such that $\epsilon^{*}$ satisfies

$$
\epsilon^{*}(d)=\frac{\alpha_{1} \alpha_{2}}{\alpha_{1} \gamma+\frac{1}{4 d(1-d)}\left[(1-d) \beta_{1}+d \beta_{2}\right]}
$$

and the bound on $\epsilon^{*}(d)$ is given by

$$
\epsilon^{*}=\frac{\alpha_{1} \alpha_{2}}{\beta_{1} \beta_{2}+\alpha_{1} \gamma}
$$

where $\alpha_{1}, \alpha_{2}, \gamma, \beta_{1}$ and $\beta_{2}$ are non-negative constants, then the origin of the closedloop system is asymptotically stable for all $0<\epsilon<\epsilon^{*}$.

Proof: To prove Theorem 2.1, let us first define the closed loop tracking error model as

$$
\begin{aligned}
& \dot{e}_{1}=e_{2} \\
& \dot{e}_{2}=\phi_{1}(e)+\phi_{2}\left(e_{1}\right) \tau\left(e_{1}, \hat{e}_{2}, Q_{d}\right)-\ddot{q}_{d}
\end{aligned}
$$

Then, we formulate the observer error model dynamics as standard singularly perturbed system as

$$
\begin{aligned}
\epsilon \dot{\eta}_{1} & =\eta_{2}-H_{1} \eta_{1} \\
\epsilon \dot{\eta}_{2} & =\epsilon\left[-\ddot{q}_{d}+\phi_{1}(e)+\phi_{2}\left(e_{1}\right) \tau\left(e_{1}, \hat{e}_{2}, Q_{d}\right)\right]-H_{2} \eta_{1}
\end{aligned}
$$


with $\epsilon \eta_{1}=\tilde{e}_{1}$ and $\eta_{2}=\tilde{e}_{2}$. We can see that the model (2.4)is in a standard nonlinear singularly perturbed form as

$$
\begin{aligned}
\dot{e} & =f(e, \eta, 0) \\
\epsilon \dot{\eta} & =f(e, \eta, \epsilon)
\end{aligned}
$$

Our aim is to analyze the property of the singularly perturbed system from those of the reduced and the boundary layer models. To begin with this analysis, we first find the reduced and boundary layer model by setting $\epsilon=0$ in (2.4) as

$$
\begin{aligned}
& \dot{\eta}_{1} * 0=\tilde{\eta}_{2}-H_{1} \tilde{\eta}_{1} \\
& \dot{\eta}_{2} * 0=0 *\left[-\ddot{q}_{d}+\phi_{1}(e)+\phi_{2}\left(e_{1}\right) \tau\left(e_{1}, \hat{e}_{2}, Q_{d}\right)\right]-H_{2} \tilde{\eta}_{1}
\end{aligned}
$$

which implies $0=\tilde{\eta}_{2}-H_{1} \tilde{\eta}_{1}, 0=-H_{2} \tilde{\eta}_{1}$. That is, $\eta_{1}=0$ and $\eta_{2}=0$. This also says that $\tilde{e}_{1}=0$ and $\tilde{e}_{2}=0$. This means that $e_{1}=\hat{e}_{1}$ and $e_{2}=\hat{e}_{2}$ are the equilibrium points of (2.4). We now use the control law $\tau\left(e_{1}, \hat{e}_{2}, Q_{d}\right)$ and then add and subtract $C\left(e_{1}+q_{d}, e_{2}\right) \dot{q}_{d}$ to simplify the tracking error model as

$$
\dot{e}_{2}=M^{-1}\left[-C\left(e_{1}+q_{d}, e_{2}\right) e_{2}-K_{d} e_{2}+K_{d} \tilde{e}_{2}-2 C\left(e_{1}+q_{d}, \dot{q}_{d}\right) e_{2}-K_{p} e_{1}\right]
$$

Applying $\tilde{e}_{2}=0$ in $\dot{e}_{2}$, one obtains the reduced model as

$$
\begin{aligned}
& \dot{e}_{1}=e_{2} \\
& \dot{e}_{2}=M^{-1}\left[-C\left(e_{1}+q_{d}, e_{2}\right) e_{2}-K_{d} e_{2}-2 C\left(e_{1}+q_{d}, \dot{q}_{d}\right) e_{2}-K_{p} e_{1}\right]
\end{aligned}
$$

which has an equilibrium at $e=0,\left(e_{1}, e_{2}\right)=(0,0)$. The boundary layer system can be defined in faster time scale $\zeta$ as

$$
\begin{gathered}
\frac{d \eta_{1}(\zeta)}{d \zeta}=\tilde{\eta}_{2}(\zeta)-H_{1} \tilde{\eta}_{1}(\zeta) \\
\frac{d \eta_{2}(\zeta)}{d \zeta}=-H_{2} \tilde{\eta}_{1}(\zeta)
\end{gathered}
$$

where $\zeta=\frac{t}{\epsilon}$. The boundary-layer model has the following compact form

$$
\frac{d \eta(\zeta)}{d \zeta}=A_{0} \tilde{\eta}
$$


with $A_{0}=\left[\begin{array}{cc}-H_{1} & I_{n \times n} \\ -H_{2} & 0_{n \times n}\end{array}\right]$ and $\eta(\zeta)=\left[\eta_{1}^{T}(\zeta), \eta_{2}^{T}(\zeta)\right]^{T}$. This implies that boundary or fast model has an equilibrium at $\eta=0,\left(\eta_{1}, \eta_{2}\right)=(0,0)$.

At this point, we claim the following Lemma.

Lemma 2.1: The equilibrium point of the reduced or slow model and boundary or fast model are asymptotically stable.

Proof: To prove the Lemma 2.1, we use the following controller Lyapunov function $V_{c}$ for the reduced model and observer Lyapunov function $V_{0}$ for the boundary-layer system

$$
V_{c}=\frac{1}{2} e_{1}^{T} K_{p} e_{1}+\frac{1}{2} e_{2}^{T} M e_{2}, V_{0}=\frac{1}{2} \eta^{T} P_{0} \eta
$$

First, we take the derivative of $V_{c}$ with respect to time along with the reduced model as

$$
\dot{V}_{c}=\dot{e}_{1}^{T} K_{p} e_{1}+\frac{1}{2} e_{2}^{T} \dot{M} e_{2}+e_{2}^{T} M \dot{e}_{2}
$$

Using the property 2 and $\dot{e}_{2}$, one can simplify the derivative of $V_{c}$ as

$$
\dot{V}_{c}=e_{2}^{T}\left[-K_{d} e_{2}-2 C\left(e_{1}+q_{d}, \dot{q}_{d}\right) e_{2}\right]
$$

In view of the property 3 , we can further simplify $\dot{V}_{c}$ as

$$
\dot{V}_{c} \leq-e_{2}^{T} K_{d c} e_{2}<0
$$

where $K_{d c}=2 k c+k_{d}$ with a positive constant derivative control gain $k_{d}$. By invoking the LaSalles invariance principle, the only solution of $\dot{e}_{1}=e_{2}$ and $\dot{e}_{2}=$ $M^{-1}\left[-C\left(e_{1}+q_{d}, e_{2}\right) e_{2}-K_{d} e_{2}-2 C\left(e_{1}+q_{d}, \dot{q}_{d}\right) e_{2}-K_{p} e_{1}\right]$ evolving in the set $\left\{\left(e_{1}, e_{2}\right) \mid e_{2}=0\right\}$ are $\dot{e}_{1}=0$ and $0=-\left[M^{-1} K_{p}\right] e_{1}$. Then, we have $e_{1}=0$.

Let us now take derivative $V_{0}$ along with the fast or boundary-layer model as

$$
\dot{V}_{0}=2 \eta^{T} P_{0} \dot{\eta}
$$

As the matrix $A_{0}=\left[\begin{array}{cc}-H_{1} & I_{n \times n} \\ -H_{2} & 0_{n \times n}\end{array}\right]$ is Hurwitz, then there exists a positive definite matrix $P_{0}$ such that $A_{0}^{T} P_{0}+P_{0} A_{0}=-Q$ with the given positive definite design 
matrix $Q$. Then we can write $\dot{V}_{0}$ as

$$
\dot{V}_{0} \leq-\eta^{T} Q \eta \leq-\lambda_{\min }(Q)\|\eta\|^{2}<0
$$

which guarantees that the equilibrium point $\eta=0$ of the fast model is asymptotically stable. That concludes the proof of Lemma 2.1.

Note that the Lemma 2.1 is established for the reduced and boundary layer model by assuming that $\epsilon=0$. However, in practice, it may not be possible to apply $\epsilon=0$ in observer dynamics. Therefore, our aim is to establish the stability property of singularly perturbed model (2.3) and (2.4) such that there exists a bound on $\epsilon$ for which the derivative of the Lyapunov function is negative. To explore that, let us consider the following composite quadratic-Lyapunov function candidate $V_{c o}$ for the singularly perturbed model (2.3) and (2.4)

$$
V_{c o}=\frac{(1-d)}{2}\left[e_{2}^{T} M e_{2}+e_{1}^{T} K_{p} e_{1}\right]+\frac{1}{2} \eta^{T} P_{0} \eta
$$

We take derivative along with perturbed model, one has

$$
\dot{V}_{c o}=(1-d)\left[\dot{e}_{1}^{T} K_{p} e_{1}+\frac{1}{2} e_{2}^{T} \dot{M} e_{2}+e_{2}^{T} M \dot{e}_{2}\right]+d \eta^{T} P_{0} \dot{\eta}
$$

Now, we use the control law $\tau\left(e_{1}, \hat{e}_{2}, Q_{d}\right)$ and then add and subtract $C\left(e_{1}+q_{d}, e_{2}\right) \dot{q}_{d}$ to simplify $\dot{V}_{c o}$ as as

$$
\begin{aligned}
\dot{V}_{c o}= & \frac{(1-d)}{2} e_{2}^{T} \dot{M} e_{2}+(1-d) e_{2}^{T}[f(e, 0,0)+f(0, \eta, \epsilon)] \\
& +\frac{d}{\epsilon} \eta^{T} P_{0} \eta+d \eta^{T} P_{0}[g(0, \eta, \epsilon)+g(e, 0,0)]
\end{aligned}
$$

where $e_{2}=\dot{e}_{1}, \eta_{2}=\tilde{e}_{2}, g(0, \eta, \epsilon)=K_{d} M^{-1} \eta_{2}, f(e, 0,0)=\left[-C\left(e_{1}+q_{d}, e_{2}\right) e_{2}-\right.$ $\left.K_{d} e_{2}-2 C\left(e_{1}+q_{d}, \dot{q}_{d}\right) e_{2}\right], f(0, \eta, \epsilon)=K_{d} \eta_{2}$ and $g(e, 0,0)=M^{-1}\left[-C\left(e_{1}+q_{d}, e_{2}\right) e_{2}-\right.$ $\left.K_{d} e_{2}-K_{p} e_{1}-2 C\left(e_{1}+q_{d}, \dot{q}_{d}\right) e_{2}\right]$. Applying $A_{0}^{T} P_{0}+P_{0} A_{0}=-Q$ together with the property of 2 and 3 , equation (2.8) becomes

$$
\begin{aligned}
\dot{V}_{c o} \leq & -(1-d) e^{T}\left[\begin{array}{cc}
0 & 0 \\
0 & K_{d c}
\end{array}\right] e+(1-d) e^{T}\left[\begin{array}{cc}
0 & 0 \\
0 & K_{d}
\end{array}\right] \eta \\
& -\frac{d}{\epsilon} \eta^{T} Q \eta+d \eta^{T} P_{0}[g(0, \eta, \epsilon)+g(e, 0,0)]
\end{aligned}
$$


This implies that, for a small value of $\epsilon$, the closed-loop trajectory enters into a positively invariant set over a finite time and remains there for all future time. The time and size of this set can be made very small by using small values of $\epsilon$. Let us now find the bound on $\epsilon$. In view of the property 1 and the property 3 , the bound on the modeling error terms $g(0, \eta, \epsilon)$ and $g(e, 0,0)$ can be obtained as $\|g(0, \eta, \epsilon)\| \leq g_{\epsilon}\|\eta\|$ and $\|g(e, 0,0)\| \leq g_{c}\|e\|$ with positive constants $g_{\epsilon}$ and $g_{c}$. Applying $\|g(0, \eta, \epsilon)\| \leq g_{\epsilon}\|\eta\|$ and $\|g(e, 0,0)\| \leq g_{c}\|e\|$, equation (2.9) can be simplified as

$$
\begin{aligned}
\dot{V}_{c o} \leq & -(1-d) \alpha_{1} \psi^{2}(e)+(1-d) \beta_{1} \psi(e) \psi(\eta)-\frac{d}{\epsilon} \alpha_{2} \psi^{2}(\eta) \\
& +d \gamma \psi^{2}(e)+d \beta_{2} \psi(e) \psi(\eta)
\end{aligned}
$$

where $\psi(e)=\|e\|, \psi(\eta)=\|\eta\|, \alpha_{1}=\left\|\left[\begin{array}{cc}0 & 0 \\ 0 & K_{d c}\end{array}\right]\right\|, \beta_{1}=\left\|\left[\begin{array}{cc}0 & 0 \\ 0 & K_{d}\end{array}\right]\right\|, \alpha_{2}=$ $\|Q\|, \beta_{2}=g_{c}\left\|P_{0}\right\|$ and $\gamma=g_{\epsilon}\left\|P_{0}\right\|$. Then, equation (2.10) can be rewritten in the following compact form

$$
\dot{V}_{c o} \leq-\left[\begin{array}{c}
\Psi_{1}(e) \\
\Psi_{2}(\eta)
\end{array}\right]^{T} T_{p d}\left[\begin{array}{l}
\Psi_{1}(e) \\
\Psi_{2}(\eta)
\end{array}\right]
$$

with

$$
T_{p d}=\left[\begin{array}{cc}
(1-d) \alpha_{1} & -\frac{1}{2}(1-d) \beta_{1}-\frac{1}{2} d \beta_{2} \\
-\frac{1}{2}(1-d) \beta_{1}-\frac{1}{2} d \beta_{2} & d\left(\left(\frac{\alpha_{2}}{\epsilon}\right)-\gamma\right)
\end{array}\right]
$$

This implies that $\dot{V}_{c o}<0$ if there exists a positive constant $\epsilon^{*}(d)$ given by $\epsilon^{*}(d)=$ $\frac{\alpha_{1} \alpha_{2}}{\alpha_{1} \gamma+\frac{1}{4 d(1-d)}\left[(1-d) \beta_{1}+d \beta_{2}\right]}$. For maximum value of $d=\frac{\beta_{1}}{\left(\beta_{1}+\beta_{2}\right)}$, one can find the bound on $\epsilon^{*}(d)$ as $\epsilon^{*}=\frac{\alpha_{1} \alpha_{2}}{\beta_{1} \beta_{2}+\alpha_{1} \gamma}$, where $\alpha_{1}, \alpha_{2}, \gamma, \beta_{1}$ and $\beta_{2}$ are non-negative constants. Then $\dot{V}_{c o}$ can be written as

$$
\dot{V}_{c o} \leq-\lambda_{\min }\left(T_{p d}\right)\|\tilde{\zeta}\|^{2}
$$

where $\tilde{\zeta}=\left[\begin{array}{ll}\Psi_{1}(e) & \Psi_{2}(\eta)\end{array}\right]^{T}$ and $\lambda_{\min }($.$) is the minimum eigen value of the positive$ definite matrix $T_{p d}$. This implies that, for $\epsilon<\epsilon^{*}$, the origin of the singularly perturbed system (2.3) and (2.4) is asymptotically stable. That concludes the proof of Theorem 2.1 . 
Remark 2.1: In view of the observer and the closed loop robot dynamics, we can notice that the speed of the observer dynamics is much higher than the closedloop robot dynamics. Then, we can say that the observer has a faster dynamics than the robotic system and the PD controller. As the observer dynamics is faster and free from robot dynamics, then the perturbation in the model parameters will be negligible.

It is worth noting to mention that if the initial error estimates and inertial parameters become very large then the output feedback control input given by Theorem 2.1 may also become very large value during transient phase. This is mainly because the observer speed requires to increase with increase of the error state estimates and the modeling error uncertainties. The demand of high-speed observer, that is small value of $\epsilon$, can be illustrated by analyzing the observer error model dynamics (2.4). From the error model (2.4)

$$
\epsilon \dot{\eta}=A_{0} \eta+B \epsilon\left[-\ddot{q}_{d}+\phi_{1}\left(e, q_{d}, \dot{q}_{d}\right)+\phi_{2}\left(e_{1}, q_{d}\right) \tau\left(e_{1}, \hat{e}_{2}, Q_{d}\right)\right]
$$

with $A_{0}=\left[\begin{array}{cc}-H_{1} & I_{n \times n} \\ -H_{2} & 0_{n \times n}\end{array}\right]$ and $B=\left[\begin{array}{c}0_{n \times n} \\ I_{n \times n}\end{array}\right]$, we can see that if the uncertainty level increase, then the second term of the bracket will also be increased. To reduce the influence of the modeling error uncertainties from the observer dynamics, that is to minimize the effect of the second term, we have to use very small $\epsilon$ to make the term close to zero which may cause large transient control input. The practical problem is that the large control efforts during transient phase may excite unmodeled high-frequency dynamics resulting undesirable chattering activity. To protect the plant from the large transient control effect, let us introduce saturated control technique, where the input is bounded over the domain of interest provided by the state feedback controller. We consider that $\Omega_{c 1}$ is an estimate of the region of attraction of the state feedback controller $\tau\left(e_{1}, e_{2}, Q_{d}\right)$ such that the controller is bounded over the set $\Omega_{c_{r 1}}$ via using a smooth saturated function as $\tau^{s}\left(e_{1}, e_{2}, Q_{d}\right)=$ $\tau_{\text {max }} \operatorname{Sat}\left(\frac{\tau\left(e_{1}, e_{2}, Q_{d}\right)}{\tau_{\max }}\right)=\tau\left(e_{1}, e_{2}, Q_{d}\right), \forall e(0) \in \Omega_{c_{o 1}}, \forall e \in \Omega_{c_{1}}$ and $\tau_{\text {max }}$ is taken over the set $\Omega_{c_{r 1}}$, where $\Omega_{c_{o 1}}, \Omega_{c r 1}$ and $\Omega_{c 1}$ are the compact sets with $c_{r 1}>c_{1} \geq$ 
$c_{o 1}, Q_{d} \in \Omega_{d}, \tau_{\max }=\max \left|\tau\left(e_{1}, e_{2}, Q_{d}\right)\right|$ and $\operatorname{Sat}($.$) is bounded smooth saturation$ function defined as follows

$$
\operatorname{Sat}(y)=\left\{\begin{array}{cc}
-1 & \text { if } y<-1 \\
y & \text { if }|y| \leq 1 \\
1 & \text { if } y>1
\end{array}\right.
$$

Then, $\forall e \in \Omega_{c 1}$, one has $\left|\tau\left(e_{1}, e_{2}, Q_{d}\right)\right| \leq \tau_{\max } \forall t \geq 0$ and therefore $\tau^{s}\left(e_{1}, e_{2}, Q_{d}\right)=$ $\tau\left(e_{1}, e_{2}, Q_{d}\right)$. We now replace the state vectors $e$ in the control law by the output of the linear estimator $\hat{e}$. The bounded output feedback controller can also be achieved by saturating outside the region of interest $\Omega_{c 1}$ as follows: $\tau^{s}\left(e_{1}, \hat{e}_{2}, Q_{d}\right)=$ $\tau_{\max } \operatorname{Sat}\left(\frac{\tau\left(e_{1}, \hat{e}_{2}, Q_{d}\right)}{\tau_{\max }}\right)=\tau\left(e_{1}, \hat{e}_{2}, Q_{d}\right), \forall \hat{e} \in \Omega_{c 1}, \forall \hat{e}(0) \in \Omega_{c o 1}, Q_{d} \in \Omega_{d}$. Then one has $\left|\tau\left(e_{1}, \hat{e}_{2}, Q_{d}\right)\right| \leq \tau_{\max } \forall t \geq 0$ which implies $\tau\left(e_{1}, \hat{e}_{2}, Q_{d}\right)=\tau^{s}\left(e_{1}, \hat{e}_{2}, Q_{d}\right)$. In practice, the maximum bounds on the control input is given by the manufacturer. It is important to note that the saturation mechanism will only be used when the initial conditions of interest becomes very large. Then, we can write tracking error model as

$$
\dot{e}_{1}=e_{2} \dot{e}_{2}=\phi_{1}(e)+\phi_{2}\left(e_{1}\right) \tau^{s}\left(e_{1}, \hat{e}_{2}, Q_{d}\right)-\ddot{q}_{d}
$$

The observer-error dynamics has the following form

$$
\begin{aligned}
& \epsilon \dot{\eta}_{1}=\tilde{e}_{2}-\frac{H_{1}}{\epsilon} \tilde{e}_{1} \\
& \epsilon \dot{\eta}_{2}=\epsilon\left[-\ddot{q}_{d}+\phi_{1}(e)+\phi_{2}\left(e_{1}\right) \tau^{s}\left(e_{1}, \hat{e}_{2}, Q_{d}\right)\right]-H_{2} \eta_{1}
\end{aligned}
$$

We now analyze the property of the closed-loop observer-controller error model dynamics. First we add and subtract $\tau^{s}\left(e, Q_{d}, 0,0\right)$ with the third term of $\epsilon \dot{\eta}_{2}$ and then use $\tau^{s}\left(e, Q_{d}, 0,0\right)=\tau\left(e, Q_{d}, 0,0\right)$ to simplify the derivative of $V_{c o}$ as

$$
\begin{aligned}
\dot{V}_{c o}= & (1-d) \frac{1}{2} e_{2}^{T} \dot{M} e_{2}+\frac{d}{\epsilon} \eta^{T} P_{0} \eta+(1-d) e_{2}^{T}\left[\tau^{s}\left(e, Q_{d}, \eta, \epsilon\right)-\tau^{s}\left(e, Q_{d}, 0,0\right)+\right. \\
& \left.M\left(-C\left(e_{1}+q_{d}, e_{2}\right) e_{2}-K_{d} e_{2}-2 C\left(e_{1}+q_{d}, \dot{q}_{d}\right) e_{2}\right)\right]+d \eta^{T} P_{0}[\xi(0, \eta, \epsilon) \\
& +\xi(e, 0,0)]
\end{aligned}
$$


where

$$
\begin{aligned}
& \xi(e, 0,0)=M^{-1}\left[-2 C\left(e_{1}+q_{d}, \dot{q}_{d}\right) e_{2}-K_{p} e_{1}-K_{d} e_{2}-C\left(e_{1}+\dot{q}_{d}, e_{2}\right) e_{2}\right] \\
& \xi(0, \eta, \epsilon)=K_{d} M^{-1} \eta_{2}
\end{aligned}
$$

Applying $A_{0}^{T} P_{0}+P_{0} A_{0}=-Q$ and the property 1 and the property $3, \dot{V}_{c o}$ can be further simplified as

$$
\begin{aligned}
\dot{V}_{c o} \leq & -(1-d) e^{T}\left[\begin{array}{cc}
0 & 0 \\
0 & K_{d c}
\end{array}\right] e+(1-d) e_{2}^{T}\left[\tau^{s}\left(e, Q_{d}, \eta, \epsilon\right)-\tau^{s}\left(e, Q_{d}, 0,0\right)\right] \\
& -\frac{d}{\epsilon} \eta^{T} Q \eta+d \eta^{T} P_{0}[\xi(0, \eta, \epsilon)+\xi(e, 0,0)]
\end{aligned}
$$

Since $\tau^{s}\left(e_{1}, e_{2}, Q_{d}, 0,0\right)$ and $\tau^{s}\left(e_{1}, e_{2}, Q_{d}, \eta, \epsilon\right)$ are continuous function with respect to their arguments, then, using properties 1 and 3 , one has

$$
\begin{gathered}
\left\|\tau^{s}\left(e, Q_{d}, \eta, \epsilon\right)-\tau^{s}\left(e, Q_{d}, 0,0\right)\right\| \leq \beta_{1}\|\eta\| \\
\|\xi(0, \eta, \epsilon)\| \leq \delta_{1}\|\eta\| \\
\|\xi(e, 0,0)\| \leq \delta_{2}\|e\|
\end{gathered}
$$

where $\beta_{1}, \delta_{1}$ and $\delta_{2}$ are bounded positive constants. With these above inequalities, $\dot{V}_{c o}$ has the following simplified form as

$$
\begin{aligned}
\dot{V}_{c o} \leq & -(1-d) \alpha_{1} \psi^{2}(e)+(1-d) \beta_{1} \psi(e) \psi(\eta)-\frac{d}{\epsilon} \alpha_{2} \psi^{2}(\eta)+d \gamma\left\|P_{0}\right\| \psi^{2}(e) \\
& +d \beta_{2} \psi(e) \psi(\eta)
\end{aligned}
$$

where $\psi(e)=\|e\|, \psi(\eta)=\|\eta\|, \alpha_{1}=\left\|\left[\begin{array}{cc}0 & 0 \\ 0 & K_{d c}\end{array}\right]\right\|, \beta_{2}=\delta_{2}\left\|P_{0}\right\|$ and $\gamma=\delta_{1}\left\|P_{0}\right\|$. Then $\dot{V}_{c o}$ can be re-written in the following compact form

$$
\dot{V}_{c o} \leq-\left[\begin{array}{c}
\Psi_{1}(e) \\
\Psi_{2}(\eta)
\end{array}\right]^{T} U_{p d}\left[\begin{array}{l}
\Psi_{1}(e) \\
\Psi_{2}(\eta)
\end{array}\right]
$$

with $U_{p d}=\left[\begin{array}{cc}(1-d) \alpha_{1} & -\frac{1}{2}(1-d) \beta_{1}-\frac{1}{2} d \beta_{2} \\ -\frac{1}{2}(1-d) \beta_{1}-\frac{1}{2} d \beta_{2} & d\left(\left(\frac{\alpha_{2}}{\epsilon}\right)-\gamma\right)\end{array}\right]$. In view of $\dot{V}_{c o}$, we can conclude that $\dot{V}_{c o}<0$ if there exists a positive constant $\epsilon^{*}(d)$ such that

$$
\epsilon^{*}(d)=\frac{\alpha_{1} \alpha_{2}}{\alpha_{1} \gamma+\frac{1}{4 d(1-d)}\left[(1-d) \beta_{1}+d \beta_{2}\right]}
$$


and, for the maximum value of $d=\frac{\beta_{1}}{\left(\beta_{1}+\beta_{2}\right)}$, the upper bound on $\epsilon^{*}(d)$ can be calculated as follows $\epsilon^{*}=\frac{\alpha_{1} \alpha_{2}}{\beta_{1} \beta_{2}+\alpha_{1} \gamma}$, where $\alpha_{1}, \alpha_{2}, \gamma, \beta_{1}$ and $\beta_{2}$ are positive constants. Then, for every $\epsilon$, the bound on $\dot{V}_{c o}$ becomes $\dot{V}_{c o} \leq-\lambda_{\text {min }}\left(U_{p d}\right)\|\tilde{\zeta}\|^{2}$ where $\tilde{\zeta}=$ $\left[\begin{array}{ll}\Psi_{1}(e), \quad \Psi_{2}(\eta)\end{array}\right]^{T}$ and $\lambda_{\min }($.$) is the minimum eigen-value of the positive definite$ matrix $U_{p d}$. This implies that the origin of the singularly perturbed system (2.3) and (2.4) is asymptotically stable. That concludes the proof of Theorem 2.1 when the control input is bounded by a saturated function.

It is important to point out that the output feedback algorithm developed in Theorem 1 depends on the nominal robot dynamics. We now remove the robot dynamics from the controller to develop the following model-free controller-observer algorithm

$$
\tau\left(e_{1}, \hat{e}_{2}\right)=-K\left(\hat{e}_{2}+\lambda e_{1}\right)
$$

with $\lambda>0, K \in \Re^{n \times n}$ and $\hat{e}$ designed as $\dot{\hat{e}}_{1}=\hat{e}_{2}+\frac{H_{1}}{\epsilon} \tilde{e}_{1}$ and $\dot{\hat{e}}_{2}=\frac{H_{2}}{\epsilon^{2}} \tilde{e}_{1}$. The implementation block diagram of this controller-observer is shown in Figure 4.1. In view of the controller and observer structure, we can observe that the design is independent of the system dynamics and parameters. Then the control input with linear observer can be written as

$$
\tau\left(e_{1}, \hat{e}_{2}\right)=-K \lambda e_{1}-K e_{2}+K \tilde{e}_{2}
$$

Now substitute this input $\tau\left(e_{1}, \hat{e}_{2}\right)$ to formulate the closed-loop tracking error model as $\dot{e}_{1}=e_{2}$ and $\dot{e}_{2}=M^{-1}\left[-C\left(e_{1}+q_{d}, e_{2}+\dot{q}_{d}\right) e_{2}-C\left(e_{1}+q_{d}, e_{2}+\dot{q}_{d}\right) \dot{q}_{d}-K \lambda e_{1}-\right.$ $\left.K e_{2}+K \tilde{e}_{2}\right]-\ddot{q}_{d}$. Then construct observer error dynamics as

$$
\dot{\tilde{e}}=A_{0} \tilde{e}+B f_{o}\left(Q_{d}, e, \tilde{e}\right)
$$

where

$$
\begin{gathered}
f_{o}\left(Q_{d}, e, \tilde{e}\right)=\left[M ^ { - 1 } \left(-C\left(e_{1}+q_{d}, e_{2}+\dot{q}_{d}\right) e_{2}-C\left(e_{1}+q_{d}, e_{2}+\dot{q}_{d}\right) \dot{q}_{d}-K \lambda e_{1}-K e_{2}\right.\right. \\
\left.\left.+K \tilde{e}_{2}\right)-\ddot{q}_{d}\right] \\
A_{0}=\left[\begin{array}{cc}
-H_{1} & I_{n \times n} \\
-H_{2} & 0_{n \times n}
\end{array}\right] \text { and } B=\left[\begin{array}{c}
0_{n \times n} \\
I_{n \times n}
\end{array}\right] . \text { Then the observer-controller error }
\end{gathered}
$$




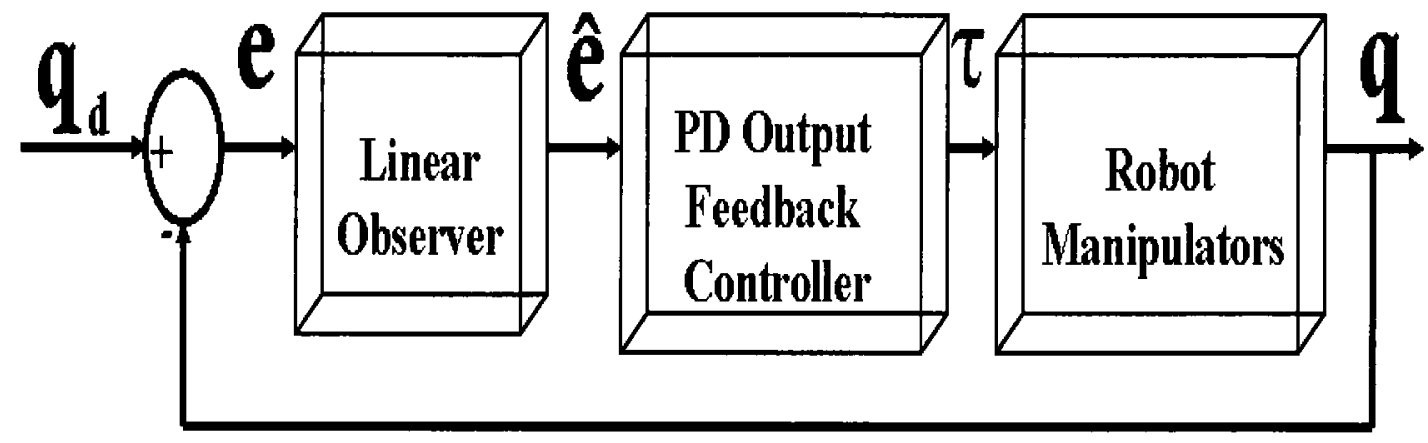

Figure 2.1: The implementation block diagram representation of observer-controller structure

model dynamics can be written in the newly defined variables $\eta$ as

$$
\begin{aligned}
\dot{e} & =f_{s}\left(Q_{d}, e, \epsilon, \eta\right) \\
\epsilon \dot{\eta} & =A_{0} \eta+B \epsilon f_{o}\left(Q_{d}, e, \epsilon, \eta\right)
\end{aligned}
$$

where $f_{s}\left(Q_{d}, e, \epsilon, \eta\right)$ is defined as

$\left[e_{2} ; M^{-1}\left(-C\left(e_{1}+q_{d}, e_{2}+\dot{q}_{d}\right) e_{2}-C\left(e_{1}+q_{d}, e_{2}+\dot{q}_{d}\right) \dot{q}_{d}-K \lambda e_{1}-K e_{2}+K \eta_{2}\right)-\ddot{q}_{d}\right]$

and $f_{o}\left(Q_{d}, e, \epsilon, \eta\right)$ is defined as

$$
M^{-1}\left(-C\left(e_{1}+q_{d}, e_{2}+\dot{q}_{d}\right) e_{2}-C\left(e_{1}+q_{d}, e_{2}+\dot{q}_{d}\right) \dot{q}_{d}-K \lambda e_{1}-K e_{2}+K \eta_{2}\right)-\ddot{q}_{d}
$$

with $\epsilon \eta_{1}=\tilde{e}_{1}, \eta_{2}=\tilde{e}_{2}$ and a small positive parameter $\epsilon$. The terms $f_{s}\left(Q_{d}, e, \epsilon, \eta\right)$ and $f_{o}\left(Q_{d}, e, \epsilon, \eta\right)$ represent the effects of the nonlinear dynamics of the manipulator and the effects due to the observer errors. The above model is in a standard nonlinear singularly perturbed form which can be defined as $\dot{e}=f(e, 0,0)$ and $\epsilon \dot{\eta}=f(e, \eta, \epsilon)$. Notice that if $\epsilon=0$ in (2.15) then we have $\eta=0$. Using $\epsilon=0$ and $\eta=0$, one has the following closed-loop tracking error dynamics under state 
feedback control law defined as

$$
\dot{e}=f_{s}\left(Q_{d}, e, 0,0\right)=\left[\begin{array}{l}
f_{s 1} \\
f_{s 2}
\end{array}\right]
$$

where $f_{s 1}=e_{2}$ and $f_{s 2}=\left[M^{-1}\left(-C\left(e_{1}+q_{d}, e_{2}+\dot{q}_{d}\right) e_{2}-C\left(e_{1}+q_{d}, e_{2}+\dot{q}_{d}\right) \dot{q}_{d}-\right.\right.$ $\left.\left.K \lambda e_{1}-K e_{2}\right)-\ddot{q}_{d}\right]$. Equation (4.36) represents the trajectory tracking error dynamics driven by the nonlinear terms defining the dynamics of the robot manipulator.

\subsubsection{Error Bound Analysis Under PD State Feedback Con- trol Design}

Let us now derive the property of the closed-loop error signals under the position and velocity based PD feedback controller given by

$$
\tau\left(e_{1}, e_{2}\right)=-K \lambda e_{1}-K e_{2}
$$

The filtered version of this PD controller usually used in most advanced industrial and practical robot control algorithm, see for example [4], [5], [6]. In our subsequent development, we remove this assumption via replacing the derivative signals by the output of the linear observer. Then we derive a condition under which all the signals in the PD output feedback control law will be bounded. We begin with the analysis by finding an error boundedness property of the closed loop system (2.16) under above PD based state feedback system. To find the tracking error bound of the PD control law, let us consider the following Lyapunov function candidate

$$
V_{r}=\frac{1}{2} S^{T} M S
$$

with $S=e_{2}+\lambda e_{1}$ and $\lambda=\operatorname{diag}\left[\lambda_{1}, \lambda_{2}, \ldots ., \lambda_{n}\right]$ with positive constants $\lambda_{n}$. Take the derivative of (2.17) along the solution of closed-loop system (2.16). Then, using assumption $A_{1}$ as well as the property $2, \dot{V}$ becomes

$$
\dot{V}_{r} \leq-\lambda_{\min .}(K)\|S\|^{2}+\left\|S^{T}\right\| k_{o}
$$


where $\|\left[\lambda C\left(e_{1}+q_{d}, e_{1}\right) \dot{q}_{d}-C\left(e_{1}+q_{d}, \dot{q}_{d}\right) \dot{q}_{d}\right]+M\left(-\ddot{q}_{d}+\lambda e_{2}\right)+\left[\lambda C\left(e_{1}+q_{d}, e_{1}\right)-C\left(e_{1}+\right.\right.$ $\left.\left.q_{d}, \dot{q}_{d}\right)\right] e_{2} \| \leq k_{o} \forall\left(e, Q_{d}\right) \in \Omega_{c} \times \Omega_{d}$ with $k_{o}>0$. Now, applying $\|S\|^{2} \geq \frac{V_{r}}{\lambda_{\max .\left(\alpha_{o}\right)}}$ and $\|S\| \leq \sqrt{\frac{V_{r}}{\lambda_{\min .}\left(\alpha_{o}\right)}}$, equation (2.18) can be written as

$$
\dot{V}_{r} \leq-\psi_{o} V_{r}+v_{o} \sqrt{V_{r}}
$$

with $\alpha_{o}=0.5\left[\begin{array}{ll}M_{11} & M_{12} \\ M_{21} & M_{22}\end{array}\right], \psi_{o}=\frac{\Xi}{\lambda_{\max }\left(\alpha_{o}\right)}, \Xi=\lambda_{\min }(K)$ and $v_{o}=\frac{k_{o}}{\sqrt{\lambda_{\min .\left(\alpha_{o}\right)}}}$. Then, we can find the ultimate bound on the error trajectory as follows

$$
V_{r}(t) \leq V_{r}(0) e^{-\gamma_{o} t}+\frac{\beta_{o}}{\gamma_{o}}\left(1-e^{-\gamma_{o} t}\right)
$$

for any $\alpha_{o}>0, \psi_{o}>\frac{\alpha_{o}}{2}, \gamma_{o}=\left(\psi_{o}-\frac{\alpha_{o}}{2}\right)$, and $\beta_{o}=\frac{v_{o}^{2}}{2 \alpha_{o}}$. Using $V_{r} \geq \frac{4 k_{o}^{2} \lambda_{\max }^{2}\left(\alpha_{o}\right)}{\lambda_{\min .}\left(\alpha_{o}\right) \Xi^{2}}$, the bound on $\dot{V}_{r}$ can be simplified further as

$$
\dot{V}_{r} \leq-\kappa V_{r}
$$

where $\kappa=\frac{\psi_{o}}{2}$. Thus, the solution of (2.19) can be written as

$$
V_{r}(t) \leq V_{r}(0) e^{-\kappa t}
$$

This implies that the trajectory starting in a region where $V_{r}(0)>c$ will continue to decrease until the trajectory enters into the set $\Omega_{c}$ where $\Omega_{c}=\left\{e \mid V_{r} \leq c\right\}$. Now using $V_{r}=\frac{4 k_{o}^{2} \lambda_{\max .}^{2}\left(\alpha_{o}\right)}{\lambda_{\min .}\left(\alpha_{o}\right) \Xi^{2}}$, we have $\dot{V}_{r} \leq 0$, where $c=\frac{2 k_{o}^{2} \lambda_{\max .}^{2}\left(\alpha_{o}\right)}{\lambda_{\min .}\left(\alpha_{o}\right) \Xi^{2}}$ which implies that the solution of (2.16) starting in the set $\Omega_{c}=\left\{e \mid V_{r} \leq c\right\}$ will remain there $\forall t \geq 0$ as $\dot{V}_{r}$ is negative on the boundary $V_{r}=c$.

Remark 2.2: The boundary of the set $\Omega_{c}$ defines the maximum errors that one can expect from the PD state feedback controller. Notice from the relationship $c=\frac{2 k_{o}^{2} \lambda_{\max }^{2}\left(\alpha_{o}\right)}{\lambda_{\min .\left(\alpha_{o}\right) \Xi}}$ that the set $\Omega_{c}$ can be made arbitrarily small by increasing the minimal eigenvalue of the control gain $K$.

Based on our above analysis, let us now state the following Theorem 2.2.

Theorem 2.2: Consider that the closed loop control system (2.16) composed of the nonlinear robot dynamics (2.2) and the control law $\tau\left(e_{1}, e_{2}\right)=-K \lambda e_{1}-K e_{2}$. 
Then, for any given initial error states $e(0)$, there exists a controller gain $K$ such that the tracking error signals are ultimately bounded by a bound that can be made arbitrarily small closed to origin by increasing the minimal eigenvalue of the control gains $K$.

\subsubsection{Error Bound Under PD Output Feedback Controller}

In this part of the chapter, we aim to show that the state variables of the closed loop system under PD output feedback controller are bounded by a bound that can be made arbitrarily small by using observer-controller design parameter $\epsilon$ and $K$. More precisely, we will show in our subsequent analysis that, for the given set of initial conditions of interest, there exists controller-observer control gains, $K$ and $\epsilon_{1}^{*}$, such that for every $0<\epsilon<\epsilon_{1}^{*}$ all the state variables of the closed-loop system under PD output feedback are bounded by a bound that can be made very small via using small value of $\epsilon$. In other words, the performance under PD state feedback design can be recovered asymptotically by the PD output feedback design. To proceed with that we consider that all the initial error states are bounded that belongs to the compact set $\Omega_{b}=\left\{e(0) \mid V_{r}(0) \leq b\right\}$, where $e_{1}(0) \in \Omega_{1 o}$ and $e_{2}(0) \in \Omega_{2 o}$. The initial sets $\Omega_{1 o}$ and $\Omega_{2 o}$ can be chosen arbitrarily large enough to cover any given bounded initial errors. We also consider that the bounded initial state estimates $\hat{e}(0)=\hat{e}_{0}$ belongs to the compact set $\Omega_{b}$. Now, for the given initial conditions of interest, we define the compact set $\Omega_{r}$ such that $\Omega_{r}=\left\{e \mid V_{r} \leq c_{r}\right\}$ with $c_{r} \geq b$ is the domain of interest for the PD state feedback controller.

To ensure the bounded PD-output feedback control, we saturate the input outside the domain of interest $\Omega_{r}$. The bounded control requires to prevent the system being affected from the large transient control phenomenon from high speed observer error variable in the face of the large inertia parameters and initial error states. Once the short transient period is over, the observer error variable becomes small and saturation function becomes idle.

Let us now state our main results for PD-output feedback design where linear 
observer incorporates to estimate the unknown velocity signals.

Theorem 2.3: $\quad$ Consider the observer-controller closed-loop control system defined by (2.14) and (2.15). Then, for any given compact set $(e(0), \hat{e}(0)) \in \Omega_{b}$, there exists observer-controller gains $\epsilon_{1}^{*}$ and $K$ such that for every $0<\epsilon<\epsilon_{1}^{*}$ the state variables $(e, \eta)$ of the closed loop system are bounded by a bound that can be made arbitrarily small closed to the origin by choosing observer-controller design parameters.

Proof: To prove above Theorem 2.3 [85, 94], we first show that there exists a transient time period $T_{1}(\epsilon) \in\left[0, T_{2}\right]$ during which the fast variable $\eta$ approaches a function of the order $O(\epsilon)$, while the slow variables remain in the subset $\Omega_{r}$ of the domain of attraction $\Omega_{c}$. Second, we ensure the boundedness property of the signal $e(t)$ for all $t \in\left[T_{1}(\epsilon), T_{3}\right]$, where $T_{1}(\epsilon) \in\left(0, \frac{T_{2}}{2}\right]$ and $T_{3} \geq T_{2}$ is the first time $e(t)$ exists from the set $\Omega_{r 1}$ where $\Omega_{r 1}=\left\{e \mid V_{r} \leq c_{r 1}\right\}$ with $c_{r 1} \geq b$ and $c_{r 1}<c_{r}$. In this part, $T_{3}$ is shown to tend to infinity, which implies that the state variables $e(t)$ remain bounded for $t \geq 0$. This means that the state trajectory $(e, \eta)$ is trapped inside the set which can be made very small by using small value of observer design constant $\epsilon$.

We first prove that there is a short transient period under which the observer error variables $\eta$, namely fast variables, decay to $O(\epsilon)$ for small value of $\epsilon>0$. During this transient period, the state variables $e$, namely slow variables, remain within a bounded domain of interest $\Omega_{r}$. In this period, the fast variables converge faster than the exponential mode of the form $\frac{1}{\epsilon} \exp ^{\frac{-a t}{\epsilon}}$ for any $a>0$. We start by proving that there exist a finite time $T_{2}$ independent of $\epsilon$ such that every trajectory $e$ starting in $\Omega_{b}$ will be remained in $\Omega_{r 1}$ for all $t \in\left[0, T_{2}\right]$. To prove this argument, let us take the following Lyapunov function candidate $V_{r}=\frac{1}{2} S^{T} M S$. Then we take the derivative along the solution of the trajectory (2.14) to simplify the derivative as one has

$$
\begin{aligned}
\dot{V}_{r}= & -S^{T} K S-S^{T}\left[-C\left(e_{1}+q_{d}, \dot{q}_{d}\right) e_{2}-C\left(e_{1}+q_{d}, \dot{q}_{d}\right) \dot{q}_{d}\right. \\
& \left.+\lambda C\left(e_{1}+q_{d}, e_{1}\right) \dot{q}_{d}+\lambda C\left(e_{1}+q_{d}, e_{1}\right) e_{2}-M\left(\ddot{q}_{d}-\lambda e_{2}\right)+K \eta_{2}\right]
\end{aligned}
$$


$\forall(e(0), \hat{e}(0)) \in \Omega_{b}, \forall e \in \Omega_{r}$ and $\forall \hat{e} \in \Omega_{r}$, the second term of (2.20) is satisfied the inequality

$$
\begin{aligned}
& \|\left[\lambda C\left(e_{1}+q_{d}, e_{1}\right) \dot{q}_{d}-C\left(e_{1}+q_{d}, \dot{q}_{d}\right) \dot{q}_{d}\right]+M\left(-\ddot{q}_{d}+\lambda e_{2}\right)+K \eta_{2}+ \\
& {\left[\lambda C\left(e_{1}+q_{d}, e_{1}\right)-C\left(e_{1}+q_{d}, \dot{q}_{d}\right)\right] e_{2} \| \leq v_{1 o}}
\end{aligned}
$$

$\forall\left(e, \eta, Q_{d}\right) \in \Omega_{r} \times \Omega_{d}$ and $\forall \eta \in \Omega_{r}$ with $v_{1 o}>0$. Applying $\|S\|^{2} \geq \frac{V_{r}}{\lambda_{\max .\left(\alpha_{o}\right)}}$ and $\|S\| \leq \sqrt{\frac{V_{r}}{\lambda_{\min .}\left(\alpha_{o}\right)}}$, equation (2.20) then can be simplified as

$$
\dot{V}_{r} \leq-\psi_{o} V_{r}+v_{1 o} \sqrt{V_{r}}
$$

where $\Xi=\lambda_{\min }(K), \psi_{o}=\frac{\Xi}{\lambda_{\max }\left(\alpha_{o}\right)}$ and $v_{1 o}=\frac{k_{10}}{\sqrt{\lambda_{\min }\left(\alpha_{o}\right)}}$. Using Young's inequality, the second term can be written as

$$
v_{1 o} \sqrt{V_{r}} \leq \frac{\alpha_{o}}{2} V_{r}+\frac{1}{2 \alpha_{o}} v_{1 o}^{2}
$$

with $\alpha_{0}>0$. Then, $\dot{V}_{r}$ can be further simplified as

$$
\dot{V}_{r} \leq-\gamma_{o 1} V_{r}+\beta_{1}
$$

with $\psi_{o}>\frac{\alpha_{o}}{2}, \beta_{1}=\frac{v_{1 o}^{2}}{2 \alpha_{o}}$ and $\gamma_{o 1}=\left(\psi_{o}-\frac{\alpha_{o}}{2}\right)$. Now, the solution of the differential equation can be derived as follows

$$
V_{r}(t) \leq V_{r}(0) e^{-\gamma_{o 1} t}+\frac{\beta_{1}}{\gamma_{o 1}}\left(1-e^{-\gamma_{o 1} t}\right)
$$

Since $V_{r}(0) \leq c_{r 1}<c_{r}$ with $c_{r 1}>b, c_{r 1}<c_{r}$ and $\Omega_{r 1}=\left\{e \mid V_{r} \leq c_{r 1}\right\}$, then there always exists a finite time $T_{2}$, independent of $\epsilon$, such that $V_{r}(t)<c_{r}$ for all $t \in\left[0, T_{2}\right]$.

We now turn our attention to the fast equation and analyze its solution over the interval $t \in\left[0, T_{2}\right]$. We show that there exists a very short time interval $\left[0, T_{1}(\epsilon)\right]$ for $\lim _{\epsilon \rightarrow 0} T_{1}(\epsilon)=0$ such that the observer error variables $\eta$ approach to the order of $O(\epsilon)$. To show that, let us take the following Lyapunov function candidate for the observer error model (2.15)

$$
V_{b}=\eta^{T} P_{o} \eta
$$



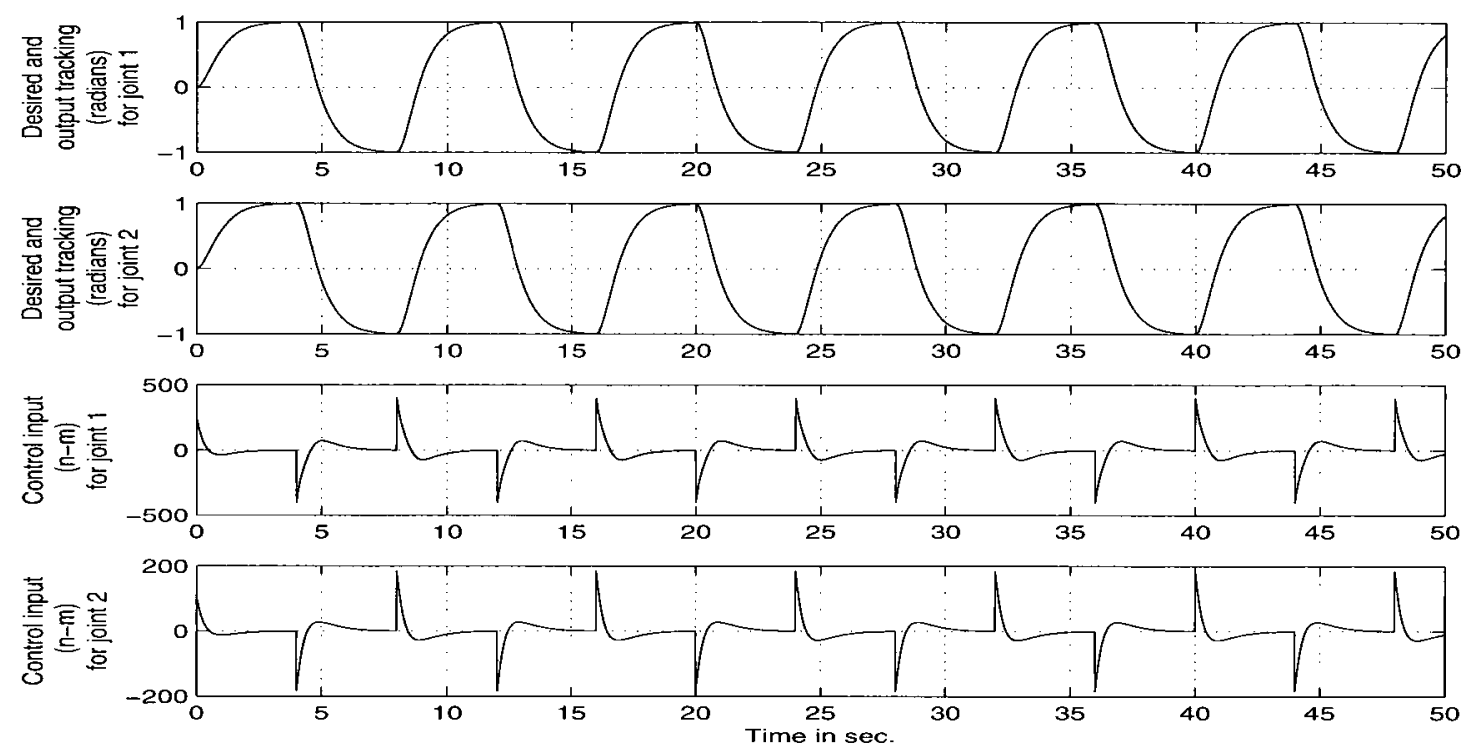

Figure 2.2: The desired (dash), output trajectory (radians) (solid) and control inputs (newton-meters) for joints 1 and 2 without using input and output disturbance noise under Theorem 2.1.

where $P_{o}=P_{o}^{T}>0$ is the solution of the Lyapunov equation $P_{o} A_{0}+A_{0}^{T} P_{o}=-I$. Take derivative (2.21) along the trajectory (2.15), one has

$$
\dot{V}_{b}=\frac{2}{\epsilon} \eta^{T} P_{o} A_{0} \eta+\frac{2}{\epsilon} \epsilon \eta^{T} P_{0} B f_{o}\left(Q_{d}, e, \epsilon, \eta\right)
$$

Now, we can use the following boundedness property of the function $f_{o}\left(Q_{d}, e, \epsilon, \eta\right)$

$$
\left\|f_{o}\left(Q_{d}, e, \epsilon, \eta\right)\right\| \leq k_{3}
$$

$\forall e \in \Omega_{r}$, where $k_{3}$ is a positive constant. Applying $P_{o} A_{0}+A_{0}^{T} P_{o}=-I$ and $(2.23)$, one has

$$
\dot{V}_{b} \leq-\frac{1}{\epsilon}\|\eta\|^{2}+2 k_{3}\|P\|\|\eta\|
$$

Using $\|\eta\|^{2} \geq \frac{V_{b}}{\lambda_{\max .\left(P_{o}\right)}}$ and $\|\eta\|^{2} \leq \frac{V_{b}}{\lambda_{\min .\left(P_{o}\right)}}$, equation (2.23) can be simplified as

$$
\dot{V}_{b} \leq-\frac{V_{b}}{\epsilon \lambda_{\max .}\left(P_{o}\right)}+2 k_{3}\|P\| \sqrt{\frac{V_{b}}{\lambda_{\min .}\left(P_{o}\right)}}
$$

where $\|P\|=\lambda_{\max }\left(P_{o}\right)$. From (2.24), we now analyze the property of the fast variable $\eta$ over the interval $t \in\left[0, T_{1}(\epsilon)\right]$ for three cases when $V_{b}>\epsilon^{2} \beta, V_{b}=\epsilon^{2} \beta$ 
and $V_{b}<\epsilon^{2} \beta$. We first consider if $V_{b}>\epsilon^{2} \beta$, then (2.24) can be simplified as

$$
\dot{V}_{b} \leq-\frac{\gamma}{\epsilon} V_{b}
$$

for $\gamma=\frac{1}{2 \lambda_{\max }\left(P_{o}\right)}$ and the inequality $V_{b}>\epsilon^{2} \beta$ exists if $\frac{V_{b}}{2 \epsilon \lambda_{\max }\left(P_{o}\right)}>2 k_{3}\|P\| \sqrt{\frac{V_{b}}{\lambda_{\min }\left(P_{o}\right)}}$ holds, that is $\frac{1}{2 \epsilon \lambda_{\max }\left(P_{o}\right)} V_{b}>2 \frac{\|P\| k_{3} \sqrt{V_{b}}}{\sqrt{\lambda_{\min }\left(P_{o}\right)}}$ and $V_{b}>\epsilon^{2} \beta$ where $\beta=16\|P\|^{2} k_{3}^{2} \lambda_{\max }\left(P_{o}\right)=$ $16\|P\|^{3} k_{3}^{2}$. Now from (2.25), one gets the solution for $V_{b}$ as

$$
V_{b}<V_{b}(0) \exp ^{\left(-\frac{\gamma t}{\varepsilon}\right)}
$$

for $V_{b}>\epsilon^{2} \beta$.

Let us now choose a bounded initial condition for the initial estimates as $\hat{e}(0) \in$ $\Omega_{b}$ then the corresponding scaled initial state estimation error is also bounded by $\eta(0)=\frac{e(0)-\hat{e}(0)}{\epsilon} \leq \frac{k_{a}}{\epsilon}$ with positive constant $k_{a}$ where $k_{a}$ depends on the size of the set $\Omega_{b}$. Then $V_{b}$ can be further simplified as

$$
V_{b}(\eta(t, \epsilon)) \leq V_{b}(0) \exp ^{\left(-\frac{\gamma t}{\epsilon}\right)} \leq \frac{k_{o}}{\epsilon^{2}} \exp ^{\left(-\frac{\gamma t}{\epsilon}\right)}
$$

where $k_{o}=k_{a}^{2} \lambda_{\max }\left(P_{o}\right)=\frac{k_{a}^{2}}{2 \gamma}$ with $\gamma=\frac{1}{2 \lambda_{\max }\left(P_{o}\right)}$. We now calculate the transient peaking time $T_{1}(\epsilon)$ when $V_{b}=\epsilon^{2} \beta$. Let $\epsilon_{1}^{*}>0$ be small so that for all $0<\epsilon<\epsilon_{1}^{*}$, the time $T_{1}(\epsilon)$ is calculated when $V_{b}=\epsilon^{2} \beta$ as $T_{1}(\epsilon)=\frac{\epsilon}{\gamma} \ln \left(\frac{k_{o}}{\beta \epsilon^{4}}\right)$. Then, at time $T_{1}(\epsilon), V_{b}=\epsilon^{2} \beta$ and from equation (2.25) we have $\dot{V}_{b} \leq-\gamma \epsilon \beta$. Therefore, $V_{b}(\eta(t))$ will continue to decrease and for the time $t>T_{1}(\epsilon)$ the inequality $V_{b} \leq \epsilon^{2} \beta$ holds. We now examine the property of $\eta$ when $V_{b}<\epsilon^{2} \beta$. Notice that the time $T_{1}$ is a function of the observer gain $\epsilon$ and $T_{1}(\epsilon)$ tends to zero when $\epsilon \rightarrow 0$. This implies that we can choose $T_{1}(\epsilon)$ small enough such that, during the short transient time $T_{1}(\epsilon)$, the estimated state variables approach to the true state. This also says that we can choose $T_{1}(\epsilon)$ small enough such that $T_{1}(\epsilon) \in\left(0, T_{2}\right]$. That is, there is a time $T_{1}(\epsilon) \in\left(0, T_{2}\right]$ such that for all $t \in\left[T_{1}(\epsilon), T_{3}\right], V_{b}<\epsilon^{2} \beta$, where $T_{3} \geq T_{2}$ is the first time $e(t)$ exists from the set $\Omega_{r}$ and $T_{3}$ may be equal to infinity. Since the transient time $T_{1}(\epsilon)$ is a function of $\epsilon$ then one can make $T_{1}(\epsilon) \rightarrow 0$ as $\epsilon \rightarrow 0$. Using Lyapunov equation (2.21), we can say that as $V_{b}<\epsilon^{2} \beta \forall t \in\left[T_{1}(\epsilon), T_{3}\right]$. This implies that $\|\eta\|$ is of the order $O(\epsilon)$ in the same interval. 

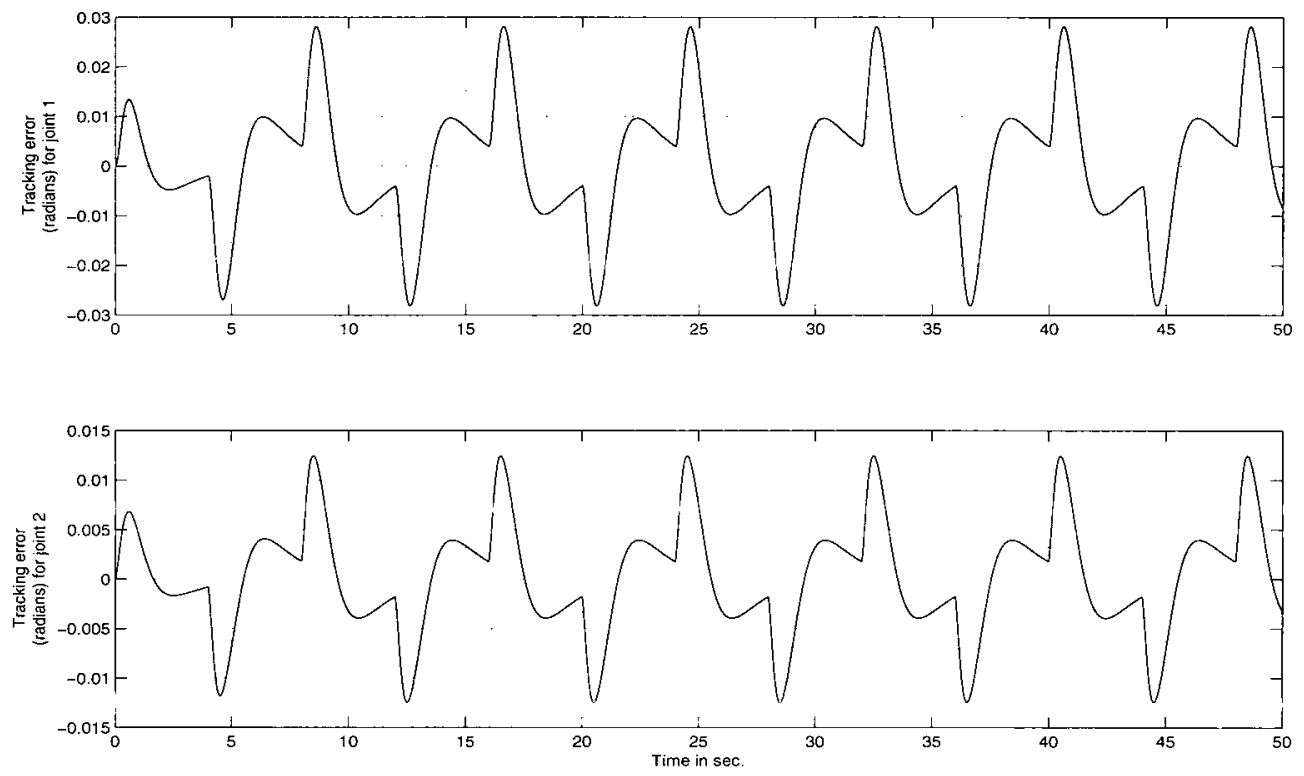

Figure 2.3: The tracking errors for joints 1 and 2 without using input and output disturbance noise under Theorem 2.1.

We now study slow model over the time interval $t \in\left[T_{1}(\epsilon), T_{3}\right]$. The system can be viewed as a perturbation of the closed loop system under state feedback control, with the perturbation term of the order $\|\eta\|$. It is shown in our previous analysis that the fast observer error variables $\eta$ decay to $O(\epsilon)$, that is, $V_{b} \leq \epsilon^{2} \beta$ $\forall t \in\left[T_{1}(\epsilon), T_{3}\right]$. Using this fact, we now show that there exists a positive constant such that for all $0<\epsilon<\epsilon_{1}^{*}$ the error signals $(e, \eta)$ are bounded $\forall t \geq T_{1}(\epsilon)$. To proceed with that, we consider the Lyapunov function $V_{r}$ defined in (2.17). First take the derivative of (2.17) along the trajectory of (2.14), one has

$$
\dot{V}_{r}=\frac{\partial V_{r}}{\partial e} f_{s}\left(Q_{d}, e, \epsilon, \eta\right)
$$

By adding and subtracting $\frac{\partial V_{r}}{\partial e} f_{s}\left(Q_{d}, e, 0,0\right)$, we have

$$
\dot{V}_{r}=\frac{\partial V_{r}}{\partial e}\left[f_{s}\left(Q_{d}, e, \epsilon, \eta\right)-f_{s}\left(Q_{d}, e, 0,0\right)\right]+\frac{\partial V_{r}}{\partial e} f_{s}\left(Q_{d}, e, 0,0\right)
$$

The second term of (2.26) represents the time derivative of $\dot{V}_{r}$ along the solution of the closed-loop system (2.16) under PD state feedback control. Then, we can 
simplify $\dot{V}_{r}$ as

$$
\dot{V}_{r} \leq-\kappa V_{r}+\frac{\partial V_{r}}{\partial e}\left[f_{s}\left(Q_{d}, e, \epsilon, \eta\right)-f_{s}\left(Q_{d}, e, 0,0\right)\right]
$$

Now, the second part of (2.27) has the following upper bound as

$$
\left\|\frac{\partial V_{r}}{\partial e}\left[f_{s}\left(Q_{d}, e, \epsilon, \eta\right)-f_{s}\left(Q_{d}, e, 0,0\right)\right]\right\| \leq k_{2}\|\eta\|
$$

$\forall e \in \Omega_{r}$ with $k_{2}>0$. With this inequality, (2.27) leads to

$$
\dot{V}_{r} \leq-\kappa V_{r}+k_{2}\|\eta\|
$$

Applying $\|\eta\| \leq \sqrt{\frac{V_{b}}{\lambda_{\min }\left(P_{o}\right)}}, \dot{V}_{r}$ becomes

$$
\dot{V}_{r} \leq-\kappa V_{r}+k_{2} \sqrt{\frac{V_{b}}{\lambda_{\min }\left(P_{o}\right)}}
$$

Using Lyapunov function $V_{r}$ of (2.17) and $V_{b} \leq \epsilon^{2} \beta$, we have

$$
\dot{V}_{r} \leq-\kappa e^{T} Q_{L} e+k_{2 o} \epsilon
$$

where $k_{2 o}=k_{2} \sqrt{\frac{\beta}{\lambda_{\min \left(P_{o}\right)}}}$ and $Q_{L}=\left[\begin{array}{cc}0.5 \lambda^{2} M & 0.5 \lambda M \\ 0.5 I & 0.5 M\end{array}\right]$. From $\dot{V}_{r}$, we can see that on the boundary of $V_{r}=c_{r}$, we have $\dot{V}_{r}<0$ when $c_{r}>\frac{\epsilon k_{20}}{\kappa}$. Since $\epsilon$ is small and $c_{r}$ is chosen strictly greater than $b$ and $\frac{\epsilon k_{20}}{\kappa}$ then we can conclude that the set $\Omega_{r}=\left\{e \mid V_{r} \leq c_{r}\right\}$ is a positively invariant set. Note that for the given set of initial conditions of interest, we can calculate the bound on $\epsilon$ as $\epsilon=\frac{\Lambda}{k_{2 o}}$ with $\Lambda=\kappa \lambda_{\min }\left(Q_{L}\right)\|e\|^{2}$. Thus the state trajectory $e$ is trapped inside the set $\Omega_{c r} \times \Omega_{\epsilon}$ with $\Omega_{\epsilon}=\left\{\eta \mid W(\eta) \leq \epsilon^{2} \beta\right\}$. The boundary of the set $\Omega_{r}$ defines the maximum error that we can expect from the PD output feedback controller. Then, we conclude that all the state variables of (2.14) and (2.15) are bounded $\forall t>0$ and $\eta$ is of $O(\epsilon) \forall t \geq T_{1}(\epsilon)$. Therefore, if $\epsilon \rightarrow 0$ then the error bounded set $\Omega_{r}$ under output feedback converge to the bounded set under the state feedback based PD control law. To show that, let us integrate $\dot{V}_{r}$ from $t=0$ to $t=T$ yields,

$$
V_{r}(T)-V_{r}(0) \leq-\int_{0}^{T} \kappa \lambda_{\min }\left(Q_{L}\right)\|e\|^{2} d t+\gamma_{1} \epsilon
$$



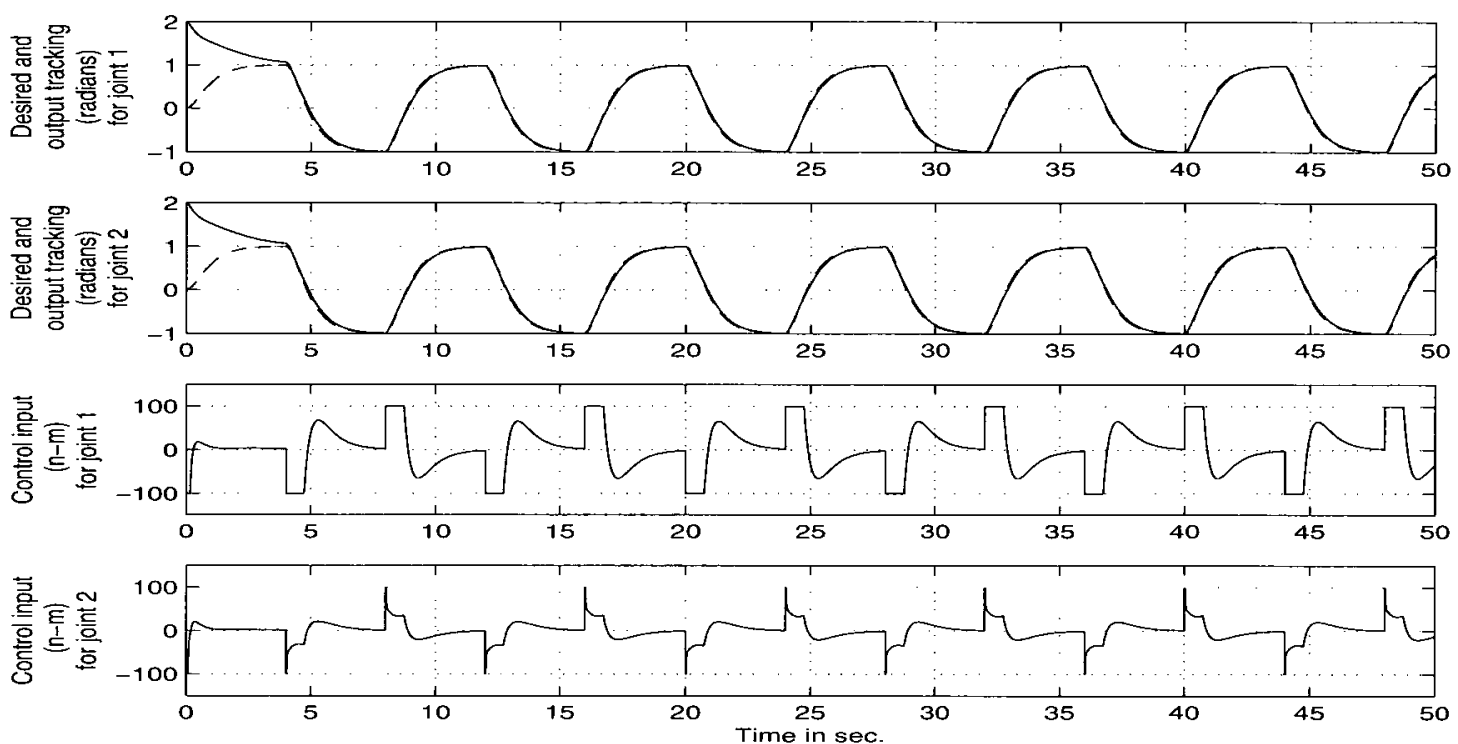

Figure 2.4: The desired (dash), output trajectory (radians) (solid) and control inputs (newton-meters) for joints 1 and 2 using output feedback design of Theorem 2.1 with initial conditions.

where $\gamma_{1}=T k_{2 o}$. Using the Lyapunov equation (2.17), we can write

$$
\int_{0}^{T} \kappa \lambda_{\min }\left(Q_{L}\right)\|e\|^{2} d t \leq \frac{1}{2} e(0)^{T} Q_{L} e(0)-V(T)+\gamma_{1} \epsilon
$$

Using $T=\infty$ and $V(\infty) \geq 0$, then the tracking error bound can be calculated as

$$
\int_{0}^{T} \kappa \lambda_{\min }\left(Q_{L}\right)\|e\|^{2} d t \leq \frac{1}{2} e(0)^{T} Q_{L} e(0)+\gamma_{1} \epsilon
$$

If $\epsilon \rightarrow 0$ then the error bound under output feedback converge to the error bound under state feedback as defined by (2.19).

\subsection{Design Synthesis and Simulation Results}

We now show the design process of the proposed output-feedback control algorithms on a 2-DOF serial link manipulator [82-101]. The equation of motion for this robot system is defined as 

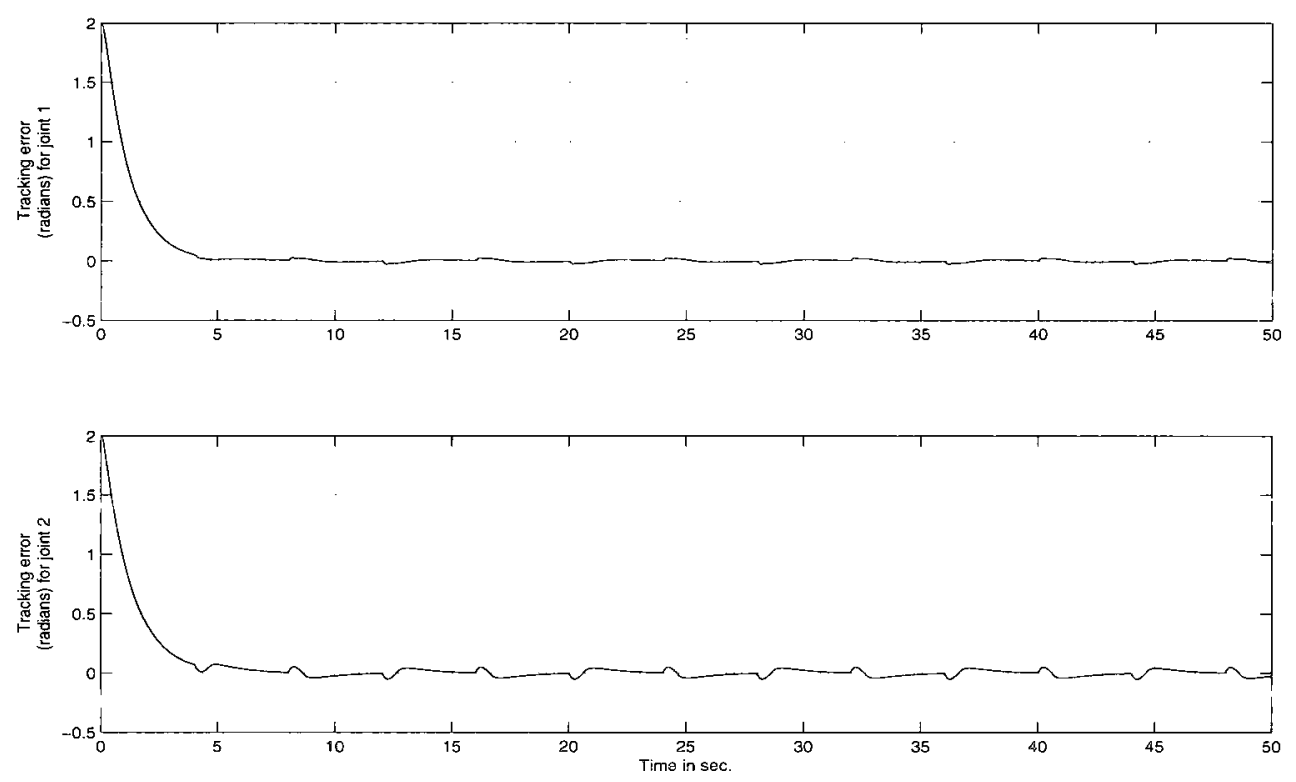

Figure 2.5: The tracking errors for joints 1 and 2 using with output feedback design of Theorem 2. $\mathrm{I}$ using with initiall comcitions.

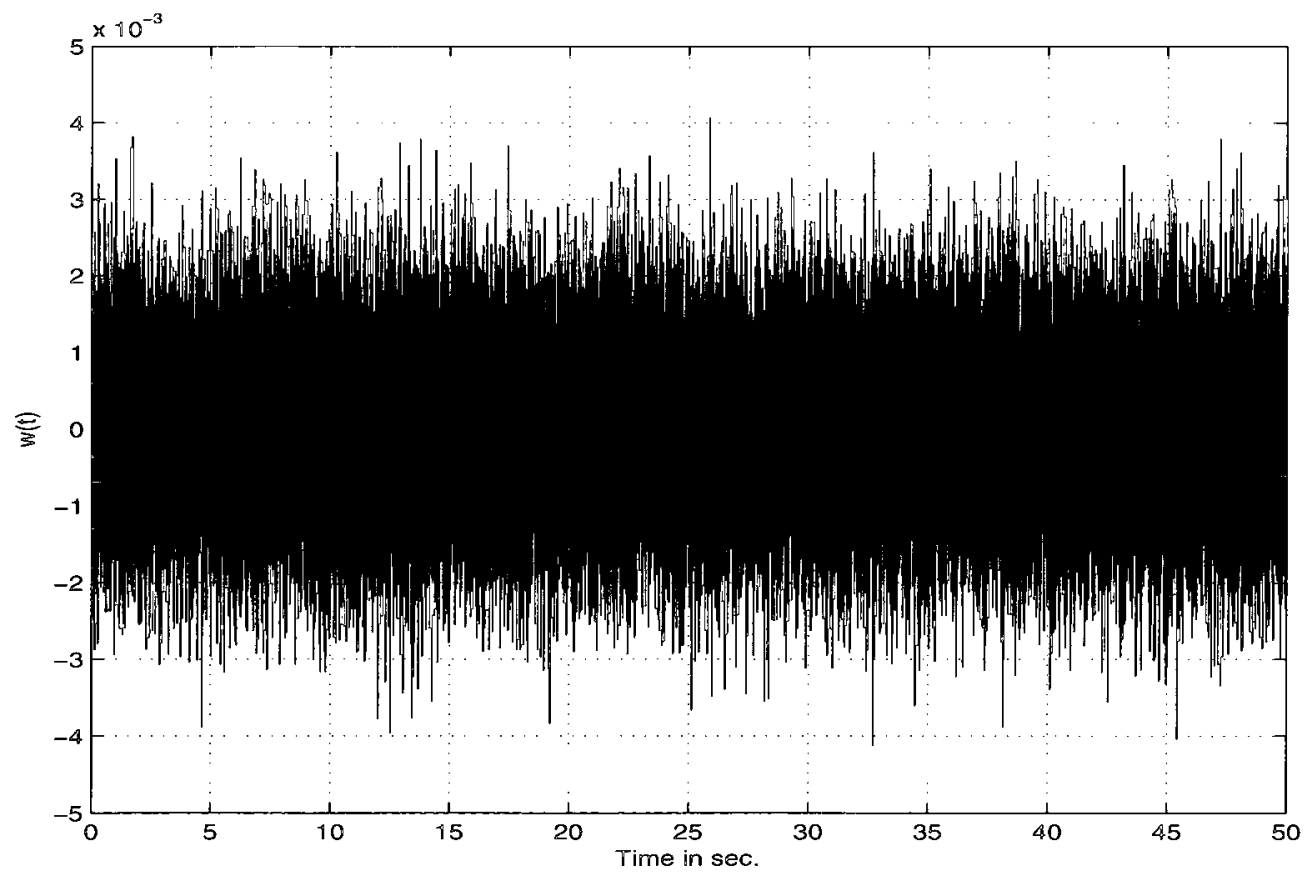

Figure 2.6: The disturbance level of $w(t)$. 

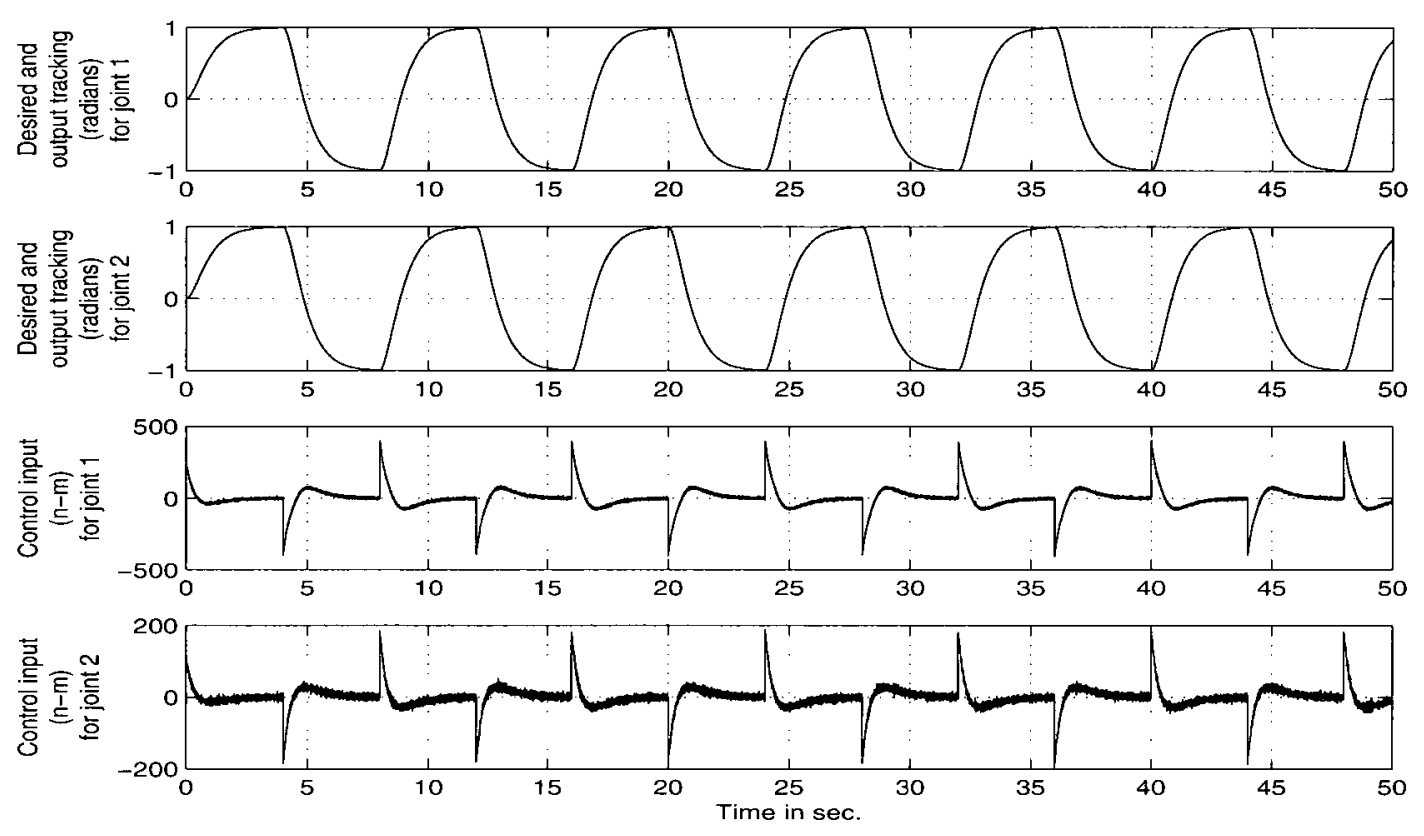

Figure 2.7: The desired (dash), output trajectory (radians) (solid) and control inputs (newton-meters) for joints 1 and 2 using output feedback design of Theorem 2.1 using with input and output disturbance noise $w(t)$.

$$
\left[\begin{array}{ll}
m_{11} & m_{12} \\
m_{21} & m_{22}
\end{array}\right]\left[\begin{array}{c}
\ddot{q}_{1} \\
\ddot{q}_{2}
\end{array}\right]+\left[\begin{array}{ll}
c_{11} & c_{12} \\
c_{21} & c_{22}
\end{array}\right]\left[\begin{array}{l}
\dot{q}_{1} \\
\dot{q}_{2}
\end{array}\right]=\left[\begin{array}{l}
\tau_{1} \\
\tau_{2}
\end{array}\right]
$$

with $m_{11}=\left(\theta_{1}+2 \theta_{2}+2 \theta_{2} \cos q_{2}\right), m_{12}=\left(\theta_{2}+\theta_{2} \cos q_{2}\right), m_{21}=\left(\theta_{2}+\theta_{2} \cos q_{2}\right)$, $m_{22}=\theta_{2}, c_{11}=-2 \dot{q}_{2} \theta_{2} \sin q_{2}, c_{12}=-\dot{q}_{2} \theta_{2} \sin q_{2}, c_{21}=\dot{q}_{1} \theta_{2} \sin q_{2}$ and $c_{22}=0$, where $\theta_{1}=m_{1} l^{2}=8, \theta_{2}=m_{2} l^{2}=9, l$ is the link lengths, $m_{1}$ and $m_{2}$ are the masses of links 1 and link 2, respectively. The robot operates in the horizontal plane so the gravitational force vector is $G=0$.

To define the reference trajectory for the robot to follow, a square wave with a period of 8 seconds and an amplitude of \pm 1 radians is pre filtered with a critically damped 2nd-order linear filter using a bandwidth of $\omega_{n}=2.0 \mathrm{rad} / \mathrm{sec}$.

To obtain desired control signal to follow the desired path, let us take the coefficients of the PD controller arbitrarily as: $K_{p}=200 I_{2 \times 2}$ and $K_{d}=250 I_{2 \times 2}$. These parameters are chosen in such a way that meets the transient tracking objectives. 
This implies to select the values of $K_{p}$ and $K_{d}$ such that the given closed-loop system would be critically damped and the response would be fast enough. The initial position and velocity errors are chosen as $e(0)=2$. With these design parameters, our first aim is to show the design and implementation process of Theorem 2.1 on the given system.

Note that one may choose different design parameters, and then follow the following design steps to calculate the bound on $\epsilon$. The following design steps are also applicable for any robot systems. To begin with the design process, we first calculate $\beta_{1}$ using the following inequality

$$
\left\|\tau^{s}\left(e, Q_{d}, \eta, \epsilon\right)-\tau^{s}\left(e, Q_{d}, 0,0\right)\right\| \leq \beta_{1}\|\eta\|
$$

with $\beta_{1}=\left\|\left[\begin{array}{cc}0 & 0 \\ 0 & K_{d}\end{array}\right]\right\|=250$. Applying the property 3 , we calculate the maximum bound on $C\left(q, \dot{q}_{d}\right)$ as $k_{c}=39.6117$, where $k_{c}=k_{c d}\left\|\dot{q}_{d}\right\|$ with $\left\|\dot{q}_{d}\right\|=1.4671$, $\sin q_{2}=1$ and $\cos q_{2}=1$. Using $\left\|\dot{q}_{d}\right\|$ and $k_{c}, \alpha_{1}$ is calculated as $\alpha_{1}=2 k_{c}+k_{d}=$ 329.2234 with $k_{d}=250$. Now, find the constant $\beta_{2}$ and $\gamma$. To calculate these constants, one requires to choose $\alpha_{2}=\|Q\|=I$ and $H_{i}=5 I_{2 \times 2}$ with $i=1,2$. Then, we use Lyapunov equation to determine the value of $P$ as $P=1.1101$. Using the property 1 and property 3 along with given initial condition of interest, we then calculate the value of $\delta_{1}$ and $\delta_{2}$ as,

$$
\delta_{1}=\left\|M^{-1}\right\|\left\|\left[\begin{array}{cc}
0 & 0 \\
0 & -K_{d}
\end{array}\right]\right\|=215.2778
$$

and

$$
\delta_{2}=\left\|M^{-1}\right\|\left\|\left[\begin{array}{cc}
-K_{P} & 0 \\
0 & -\left[2 * C\left(q, \dot{q}_{d}\right)+C\left(q, e_{2}\right)+K_{d}\right]
\end{array}\right]\right\|=329.9937
$$

By knowing $\|P\|, \delta_{1}$ and $\delta_{2}$, we now determine the values of $\beta_{2}$ and $\gamma$ as $\beta_{2}=$ 366.3260 and $\gamma=238.9799$. Using constants $\beta_{1}, \beta_{2}, \alpha_{1}$ and $\alpha_{2}$, the value of $d^{*}$ is obtained as $d^{*}=\frac{\beta_{1}}{\beta_{1}+\beta_{2}}=0.4056$. The choice of $d^{*}$ results in bound on $\epsilon^{*}=\frac{\alpha_{1} \alpha_{2}}{\beta_{1} \beta_{2}+\alpha_{1} \gamma}=0.0019 \approx 0.002$. Applying the above defined observer-controller 

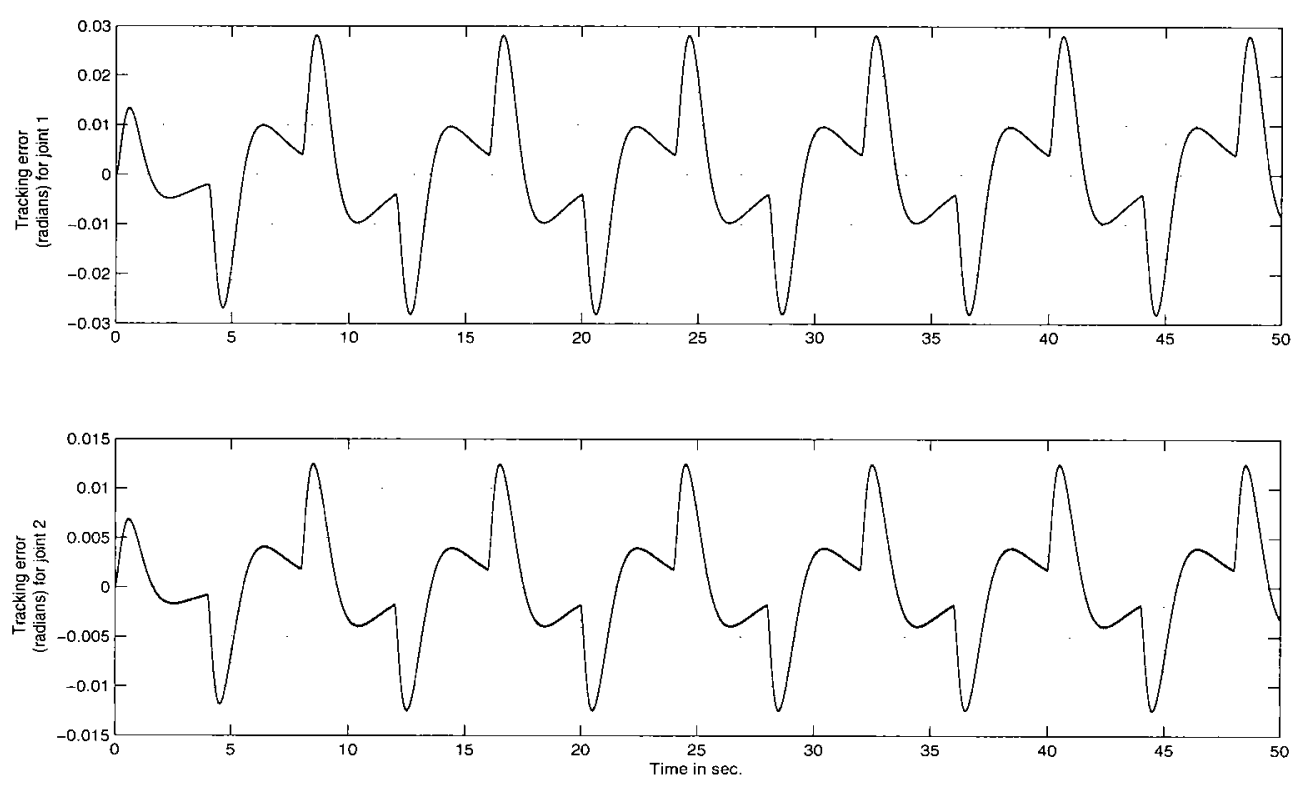

Figure 2.8: The tracking errors of joints 1 and 2 under Theorem 2.1 with the disturbance noise $w(t)$.

design parameters, we implement Theorem 2.1 on the given robotic system. The results are given in Figures 2.2 to 2.3. From these results, we can observe that the output trajectory tracks the desired one with small tracking errors.

We now increase the initial conditions from zero to $e(0)=2$, but we keep all other design parameters same as used for our last evaluation. Due to the presence of initial error estimates, the design may exhibit large transient control input during transient phase. To protect the plant from such transient control action, we saturate the control input over the domain of interest for the given initial conditions. In practice, one requires to use the pre-defined saturation level provided by the manufacturer. Therefore, we consider that the saturation levels for the control inputs are pre-defined by manufacturer as $\tau_{1 \max }=100$ newton-meters and $\tau_{2 \max }=100$ newton-meters. For the given set of initial interest, this level can also be determined using simulation as well as Lyapunov-function estimation 
technique introduced in this paper as,

$\tau_{1 \max }=\left|m_{11}(q)\right|\left|\ddot{q}_{d}\right|+\left|m_{12}(q)\right|\left|\ddot{q}_{d}\right|+\left|c_{11}\left(q, \dot{q}_{d}\right)\right|\left|\dot{q}_{d}\right|+\left|c_{12}\left(q, \dot{q}_{d}\right)\right|\left|\dot{q}_{d}\right|+K_{p 1}\left|e_{1}\right|+K_{d 1}\left|e_{2}\right|$

and

$\tau_{2 \max }=\left|m_{21}(q)\right|\left|\ddot{q}_{d}\right|+\left|m_{22}(q)\right|\left|\ddot{q}_{d}\right|+\left|c_{21}\left(q, \dot{q}_{d}\right)\right|\left|\dot{q}_{d}\right|+\left|c_{22}\left(q, \dot{q}_{d}\right)\right|\left|\dot{q}_{d}\right|+K_{p 2}\left|e_{3}\right|+K_{d 2}\left|e_{4}\right|$

with $q_{d 1}=q_{d 2}=q_{d}$. We then apply Theorem 2.1 on the same system with the same set of design parameters that used in our last evaluation. The tested results are shown in Figures 2.4 to 2.5. Due to the presence of the initial error estimates, the output trajectory peaks before convergence to the desired one.

The presented results above are obtained by assuming that the control system is operating under ideal operating condition. Let us now examine the tracking performance of Theorem 2.1 on the given system under non ideal operating conditions. To do that, we add a band limited white noise, $w(t)$, into the output measurement $q(t)$ (signal obtained from encoders also corrupted by noise, see for example [5] and input $\tau(t)$ in the model. For our evaluation, we use the level for $w(t)$ as depicted in Figure 2.6. Then we apply the output feedback design stated by Theorem 2.1 under the same set of design parameters as used for our last evaluation. The implemented results are shown in Figures 2.7 to 2.8. Even with the input and output disturbance, the performance under output feedback design meets the desired tracking objectives.

To examine the robustness property of Theorem 2.1, we now introduce a more complicated situation. In this test, we aim to change the plant parameter of the joint 1 and joint 2 on-line when the manipulator tracks the desired task with various loads. We consider that the plant is initially operating under the parameters $\theta_{1}=8$ and $\theta_{2}=8$. Then, at 12 sec., the parameters are changed from $\theta_{1}=8$ and $\theta_{2}=8$ to $\theta_{1}=4$ and $\theta_{2}=4$. Again, at 31 sec., the parameters $\theta$ are changed from $\theta_{1}=4$ and $\theta_{2}=4$ to $\theta_{1}=2$ and $\theta_{2}=2$. Therefore, in the whole process, there are three dynamics changes with relatively large modeling error uncertainty. Then, we implement Theorem 2.1 on the given system with the same design constants 

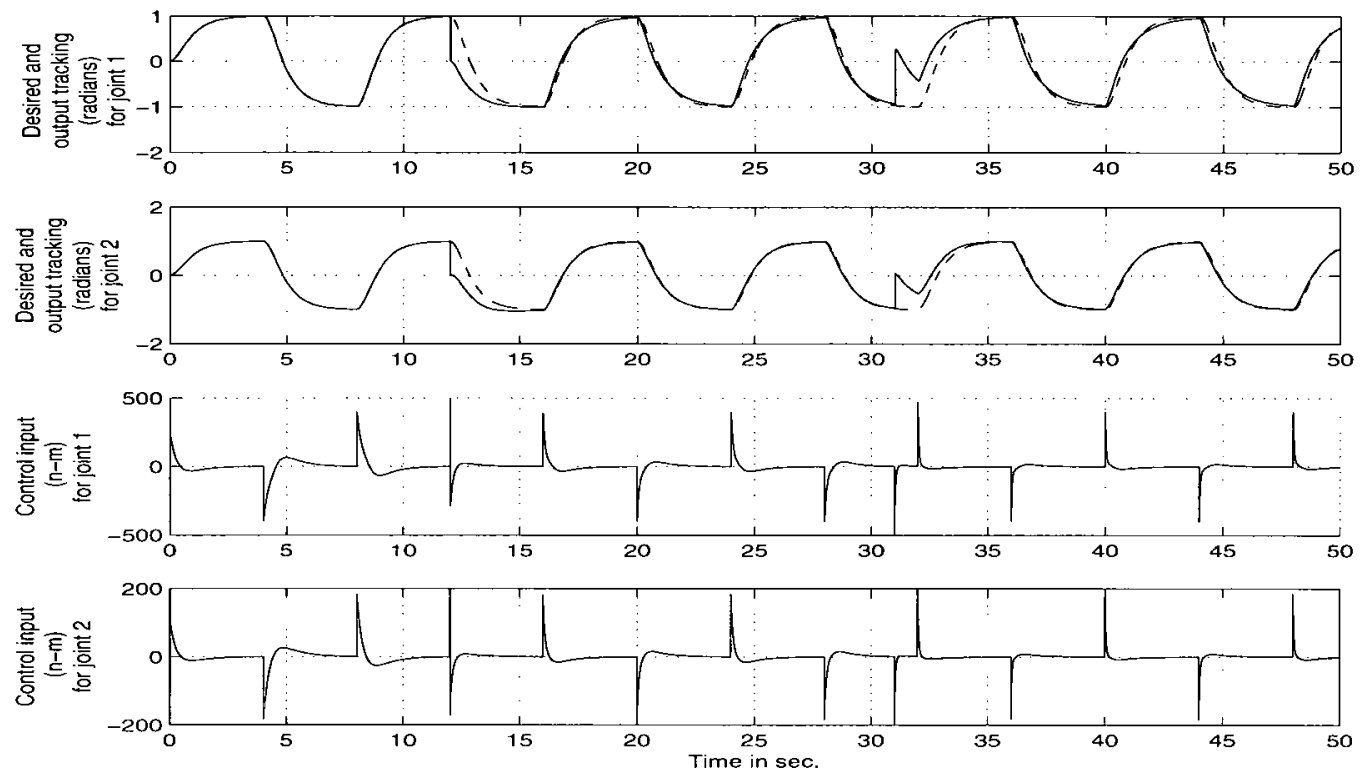

Figure 2.9: The desired (dash), output tracking (radians) (solid) and control inputs (newton-meters) for the joint 1 and joint 2 with Theorem 2.1 under dynamics changes.

as used for our last implementation. The tested results are depicted in Figures 2.9 to 2.10 with the given saturation level for two control inputs as $\tau_{1 \max }=500$ newton-meters and $\tau_{2 m a x}=200$ newton-meters. The saturation mechanism under dynamics changes is introduced to protect the plant from large control action as the control becomes large values at the time of dynamics changes. We notice from our implementation results that the transient tracking errors increase at the time of the dynamics changes. However, the transient tracking errors can be reduced by increasing the controller gains.

Now, our aim is to show the design and implementation process of Theorems 2.2 and 2.3 on the system (2.28). To do that, we first consider $e(0)=2$. The parameters $\theta_{1}$ and $\theta_{2}$ are chosen as $\theta_{1}=1$ and $\theta_{2}=2$. For the given initial conditions of interest, let us define the region of interest as follows. Using property 1 and property 3 , we calculate the upper bound for $k_{o}=67.245104$ and $\Omega_{b}=$ 

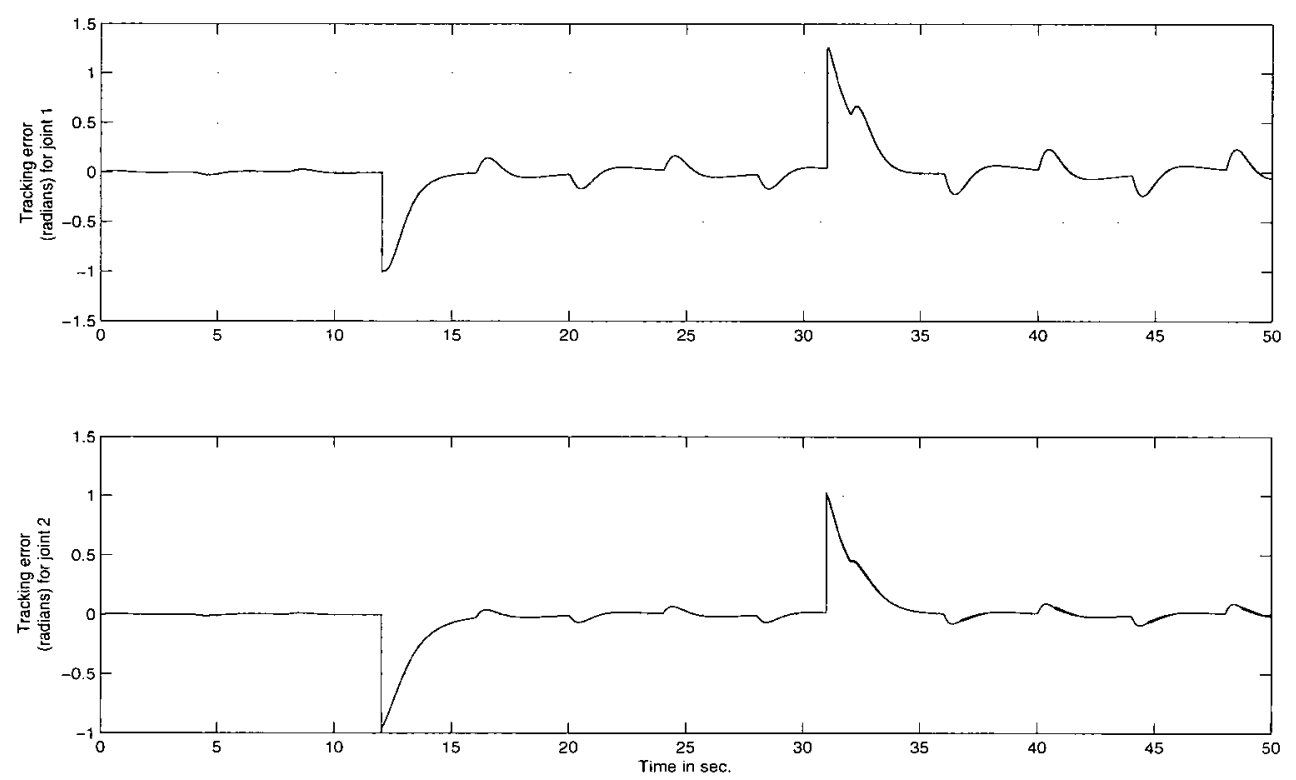

Figure 2.10: The tracking errors for joints 1 and 2 with Theorem 2.1 under model dynamic changes.

$\left\{e(0) \mid V_{r}(0) \leq 104\right\}$. Then, we define $c_{r}=105$ as the region of interest for $e$ as $\Omega_{c r}=\left\{e \mid V_{r} \leq 105\right\}$. Applying $c=\frac{4 k_{o}^{2} \lambda_{\max .}^{2}\left(Q_{L}\right)}{\lambda_{\min \left(Q_{L}\right) \Xi^{2}}}$, we can calculate the bound on the error trajectory under PD state feedback design as $\|e(t)\| \leq \sqrt{\frac{c}{\lambda_{\min }\left(Q_{L}\right)}} \leq \frac{237.72573}{\lambda_{\min }(K)}$. Notice that by increasing the minimal eigenvalue of the control gain $K$, one can make $\|e(t)\|$ under PD state feedback control be very small. For the given estimated region of interest, we now find the bound on the observer design parameter $\epsilon$.

We start by considering that, during the transient period, the fast variable $\eta$ approaches to the domain of interest $\Omega_{r 1}$ before converging to a very small value. The, define $H_{1}=I_{2 \times 2}$ and $H_{2}=I_{2 \times 2}$ to solve the Lyapunov equation for $\|P\|=$ 1.8090. Using $C_{M}, M_{M I}$ and $\eta \in \Omega_{r 1}$, one calculates the maximum bound on $f_{o}\left(Q_{d}, e, \epsilon, \eta\right)$ as $k_{3}=3.891317 \times 10^{4}$. By knowing $k_{3}$ and $\|P\|, \beta$ is calculated as $\beta=1.4342577 \times 10^{11}$. Using $K=60 I_{2 \times 2}$ and $\lambda_{\min }\left(P_{o}\right)=0.6910$, we now calculate $k_{2 o}=k_{2} \sqrt{\frac{\beta}{\lambda_{\min }\left(P_{o}\right)}}=27.335359 \times 10^{6}$. Then, we determine the maximum bound on the error trajectory as $\|e\|^{2} \leq \frac{c_{r 1}}{\lambda_{\min }(Q)}$. For the given controller gains, 
we now calculate $c_{r 1}=75.6533396 \times 10^{4} \epsilon$. This implies that $c_{r}$ can be made very small by using small value of $\epsilon$. For our implementation, let us use the value of $\epsilon$ as $\epsilon=0.002$. Using with these design parameters, we then apply the PD-based output feedback control law (2.12) on the given model (2.28). The conducted results are depicted in Figures 2.11 to 2.14. Figures 2.11 to 2.12 are pictured the tracking convergence under PD based state feedback approach of Theorem 2.2. The control performance under PD based output feedback approach of Theorem 2.3 is shown in Figures 2.13 to 2.14. In view of Figures 2.11 to 2.14, we can see that the control performance under PD output feedback recovers the performance achieve under PD state feedback approach.

We now examine the convergence property of Theorem 2.3 for the case when the control system is operating under non ideal condition. For this purpose, we add a band limited white noise $w(t)$, as given in Figure 2.6, to the control input $\tau(t)$ and output measurement $q(t)$. Then we apply PD-based output feedback approach on the given system. The conducted results are depicted in Figures 2.15 to 2.16. This result confirm the desired control objective as the output tracking for joints 1 and 2 converges closed to zero.

Let us inspect the transient tracking performance of Theorem 2.3 with respect to dynamics changes. To induce such dynamics changes, we first operate the system with the parameters $\theta_{1}=2$ and $\theta_{2}=2$. Then, at $12 \mathrm{sec}$, the parameters $\theta$ are changed from $\theta_{1}=2$ and $\theta_{2}=2$ to $\theta_{1}=4$ and $\theta_{2}=4$. Again, at 31 sec., the parameters $\theta$ are changed from $\theta_{1}=4$ and $\theta_{2}=4$ to $\theta_{1}=2$ and $\theta_{2}=2$. In view of our whole dynamic set up, we can notice that there exists modeling error uncertainty at $12 \mathrm{sec}$. as well as $31 \mathrm{sec}$. This means that there are three dynamic changes in the whole process dynamics. Then, we implement Theorem 2.3 on the given system with the same design constants as used for our last implementation. The tested results are depicted in Figure 2.17 (solid-line) with the given saturation level for two control inputs as $\tau_{1 \max }=50$ newton-meters and $\tau_{2 \max }=50$ newtonmeters. By comparing Figure 2.14 and the solid-line of Figure 2.17, we can see that 
the control performance under dynamic changes exhibits relatively large transient tracking errors (see after at $12 \mathrm{sec}$, and $31 \mathrm{sec}$. ) than the tracking performance achieved under the control system designed for the given initial conditions and initial dynamics of interest. This is, in fact, the common feature in the semiglobal stable control system. As the output feedback method is semiglobal, then the designer can tune the controller parameters to cover for large initial conditions and initial dynamics of interest that ensures good transient tracking performance.

We now compare the tracking performance of Theorem 2.3 with the classical adaptive output feedback (CAOFB) law [82] under dynamics changes. This controller-observer algorithm can be designed as,

$$
\tau\left(\hat{e}, Q_{d}, \hat{\theta}\right)=Y\left(\hat{e}, \dot{q}_{d}, \ddot{q}_{d}\right) \hat{\theta}-K_{P} e_{1}-K_{D} \hat{e}_{2}
$$

with $\dot{\hat{\theta}}=-\Gamma Y^{T}\left(\hat{e}, \dot{q}_{d}, \ddot{q}_{d}\right) \hat{S}$, where $Y\left(\hat{e}, \dot{q}_{d}, \ddot{q}_{d}\right) \hat{\theta}=\hat{M}(q) \ddot{q}_{d}+\hat{C}\left(q, \dot{\hat{q}}_{r}\right) \dot{q}_{d}+\hat{G}(q), K_{P} \in$ $\Re^{n \times n}, K_{D} \in \Re^{n \times n}, \hat{S}=\hat{e}_{2}+\lambda e_{1}, \dot{\hat{q}}_{r}=\left(\dot{\hat{q}}_{2}-\lambda e_{1}\right), \lambda=\frac{\lambda_{0}}{1+\left\|e_{1}\right\|}, \lambda_{0}>0$ and $\hat{M}, \hat{C}($.$) and$ $\hat{G}($.$) define the estimates of the M(),. C($.$) and G($.$) , respectively. To construct the$ nonlinear regressor model $Y\left(\hat{e}, \dot{q}_{d}, \ddot{q}_{d}\right)$ [82], one requires a priori knowledge of the system dynamics. The unknown velocity signal in equation (2.29) is now replaced by the output of the linear observer as

$$
\dot{\hat{e}}_{1}=\hat{e}_{2}+\frac{H_{1}}{\epsilon} \tilde{e}_{1}, \dot{\hat{e}}_{2}=\frac{H_{2}}{\epsilon^{2}} \tilde{e}_{1}
$$

For comparison, we consider the same set of observer-controller design parameters that used for PD-based output design of Theorem 2.3 as, $K_{P}=60 I_{2 \times 2}, K_{D}=$ $60 I_{2 \times 2}, \lambda_{0}=2, H_{1}=I_{2 \times 2}, H_{2}=I_{2 \times 2}$ and $\epsilon=0.002$. The learning gains $\Gamma$ are chosen as $\Gamma=15 I_{2 \times 2}$. The adaptation gains are tuned to achieve faster parameter learning via using trial and error search technique. Then, we apply the controllerobserver (6.5) on the system (6.6). The tested results are depicted in Figure 2.17 (blue line).

In view of blue and black solid line of Figure 2.17, we notice that the tracking errors under $\mathrm{CAOFB}$ are larger than the tracking errors obtained with PD-output feedback design. One may choose higher values of the controller gains to reduce 

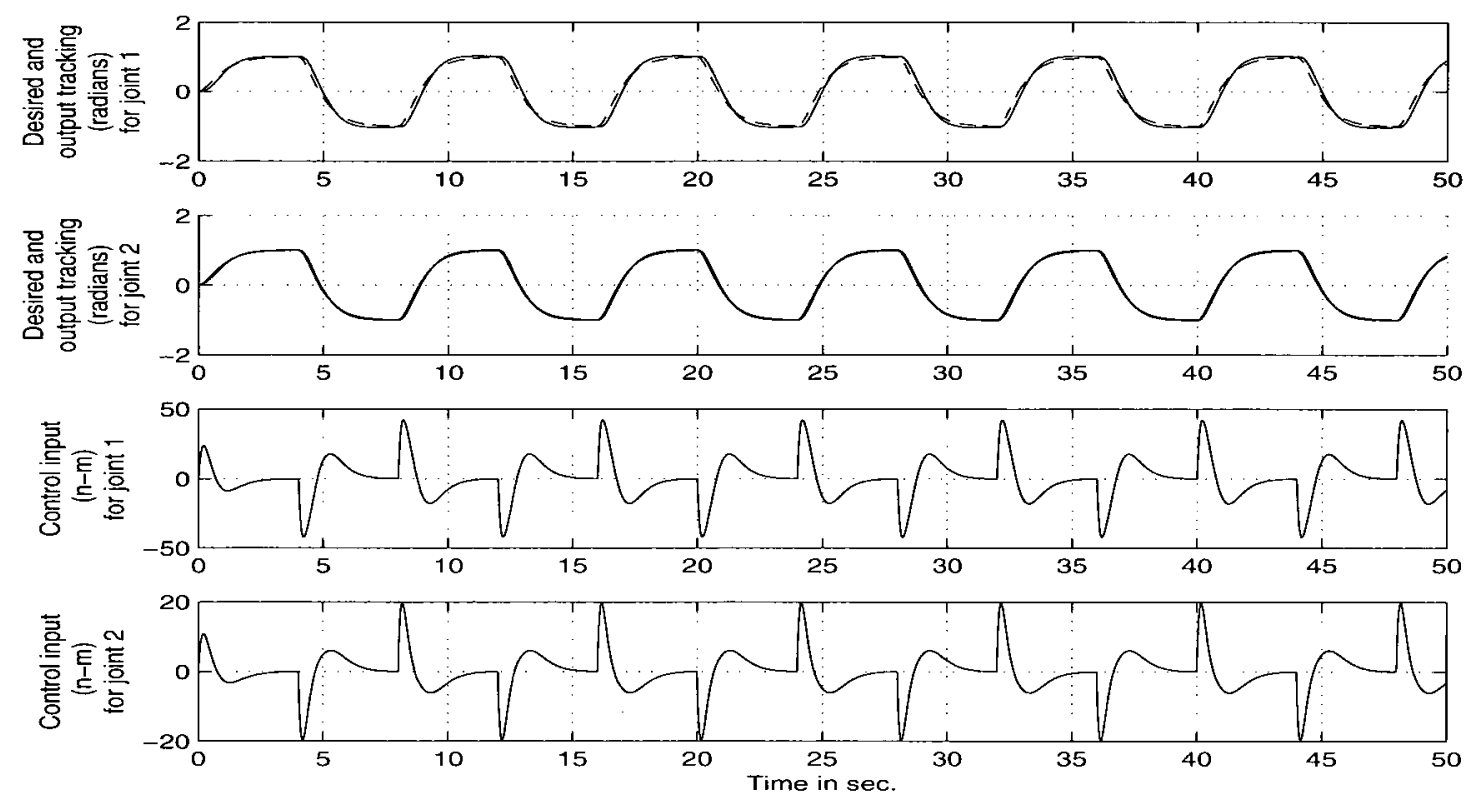

Figure 2.11: The desired (dash), output tracking (radians) (solid) and control inputs (newton-meters) for the joint 1 and joint 2 under Theorem 2.2.

transient tracking errors via using trail and error methods. The reason for showing poor tracking performance of CAOFB design with respect to large scale of initial conditions and parameter errors because of the assumption that the nonlinear functions are assumed to be appeared linearly with respect to unknown parameters.

Remark 2.3: In the face of large scale modeling error uncertainties, the single model-based design, either PD or classical adaptive control, demands large control gain and control saturation level in order to achieve good tracking performance. The well known problem of having high gains based control system design is that it may amplify the input and output disturbance as well as excite hidden unmodeled dynamics causing poor tracking performance in the real-time operation.

Remark 2.4: In all theorems reported in this chapter, one can show that the tracking error and its time derivative can be made arbitrarily small by increasing the minimal eigenvalues of the control gains. 

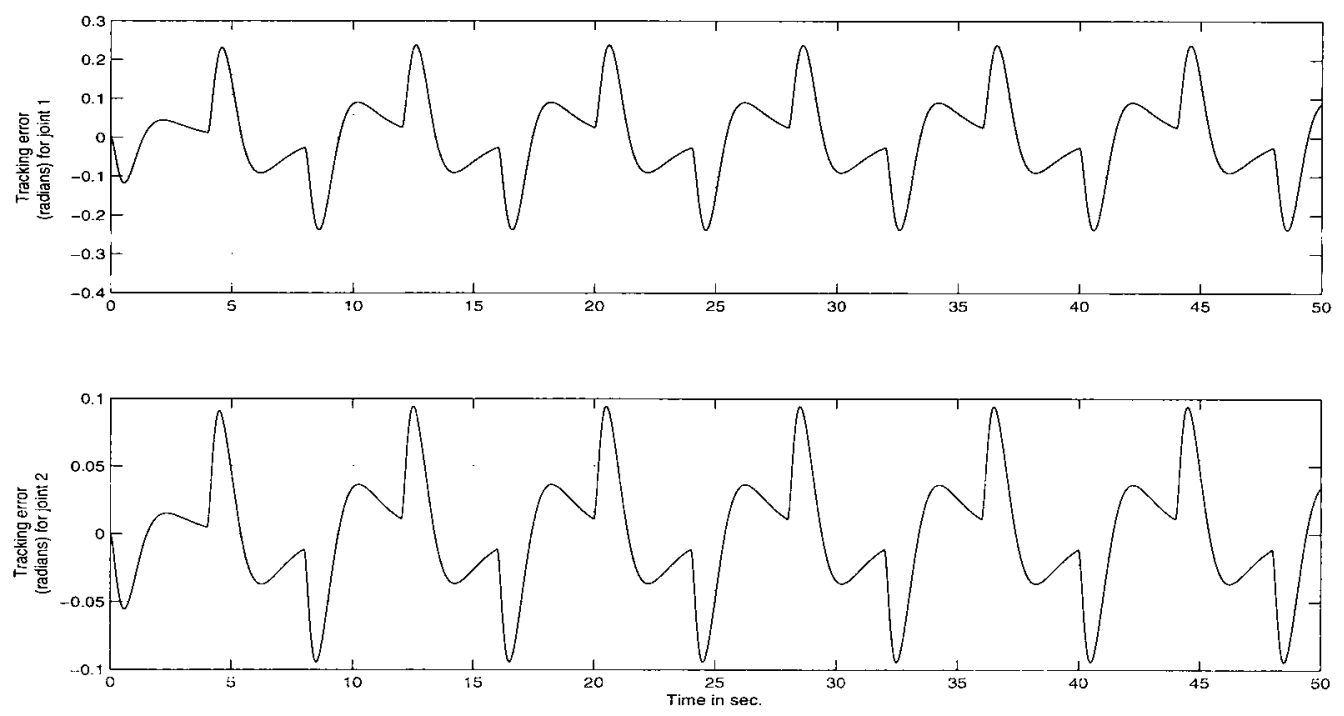

Figure 2.12: The tracking errors for joint 1 and joint 2 with Theorem 2.2.
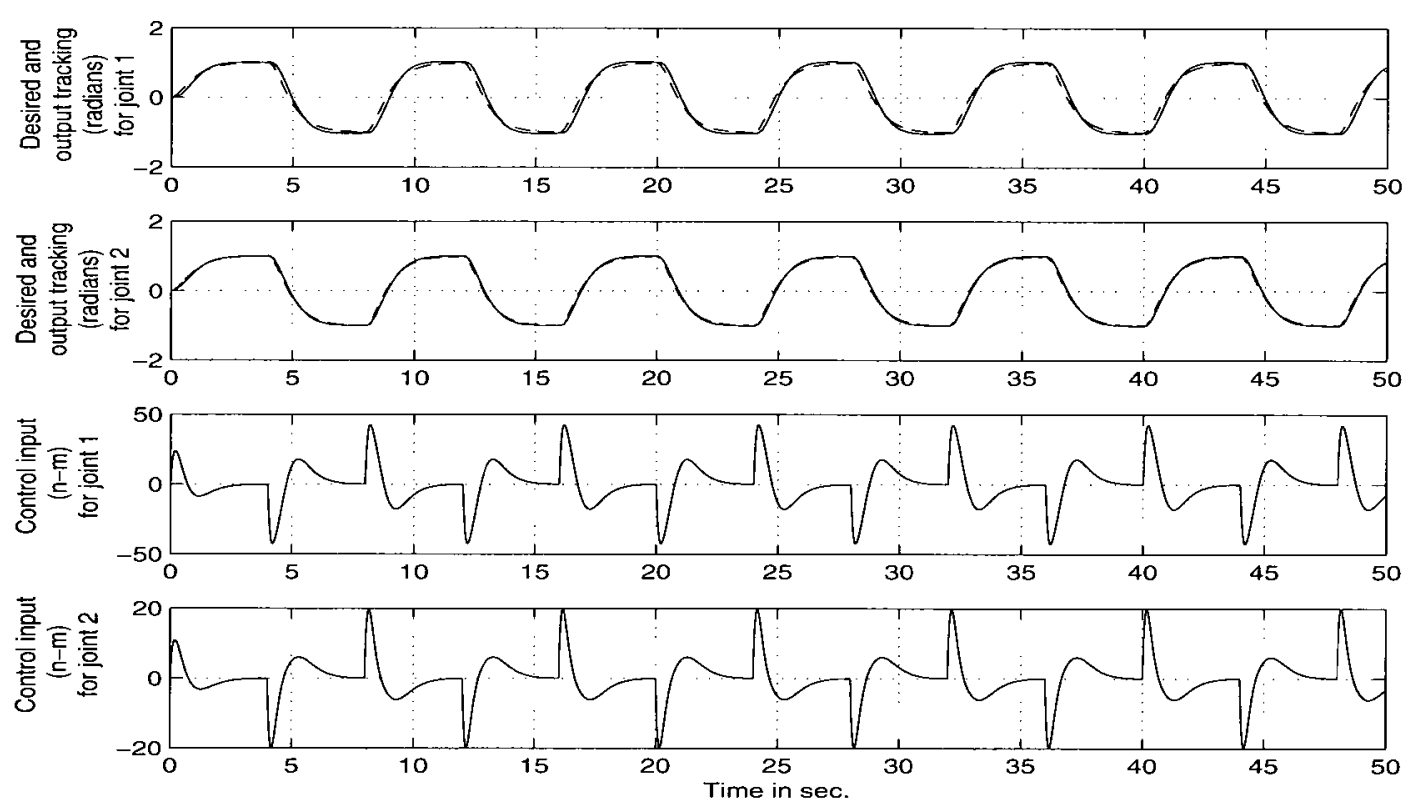

Figure 2.13: The desired (dash), output tracking (radians) (solid) and control inputs (newton-meters) for joints 1 and 2 under output feedback design of Theorem 2.3 without using the input and output disturbance noise. 

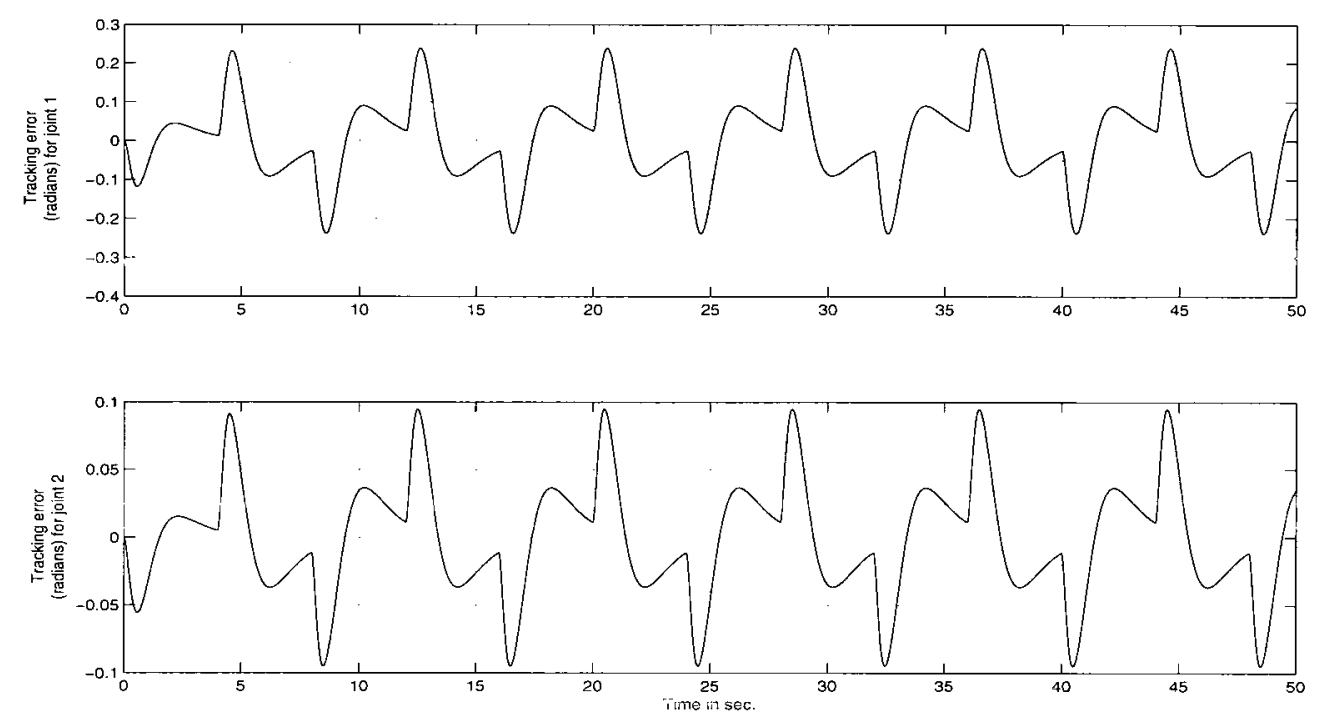

Figure 2.14: The tracking errors for joints 1 and 2 with the PD output feedback design of Theorem 2.3 without using the input and output disturbance noise.
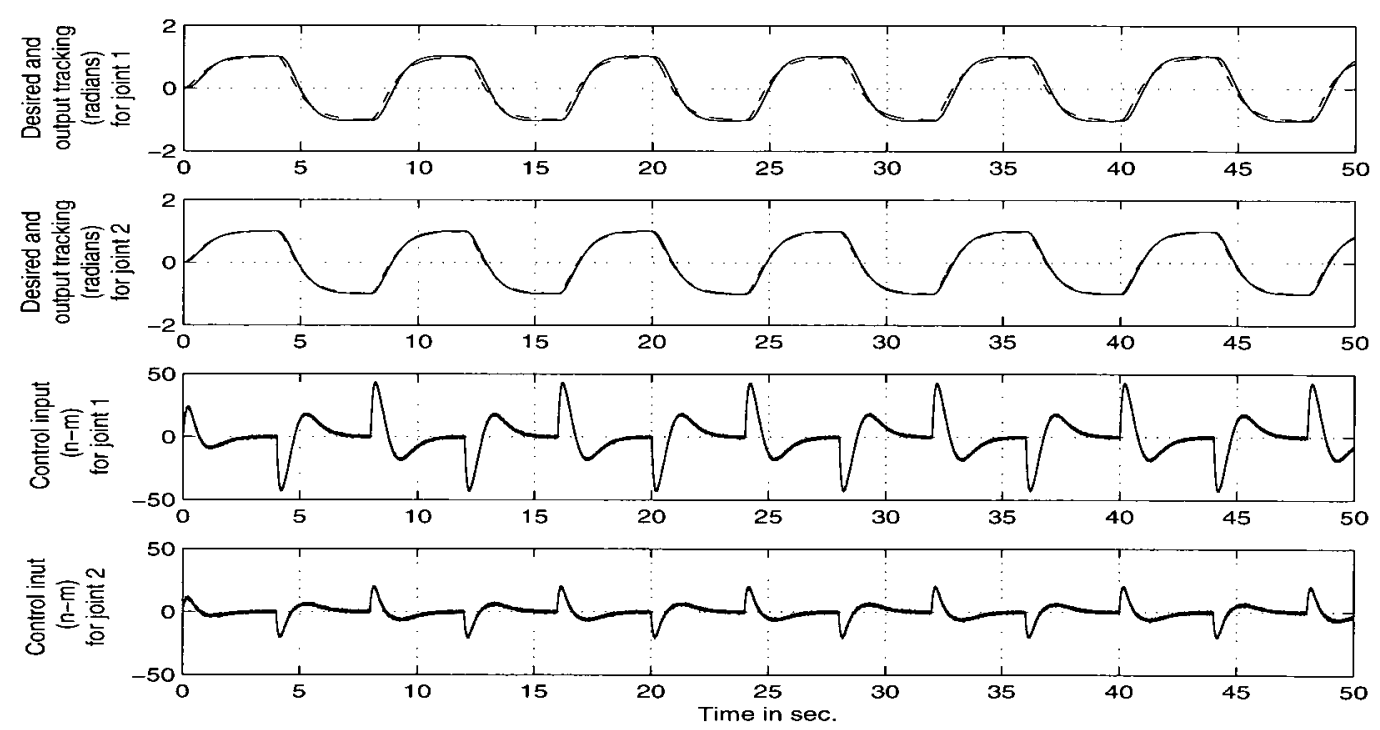

Figure 2.15: The desired (dash), output tracking (radians) (solid) and control inputs (newton-meters) for joints 1 and 2 under output feedback design of Theorem 2.3 using with the input and output disturbance noise $w(t)$. 

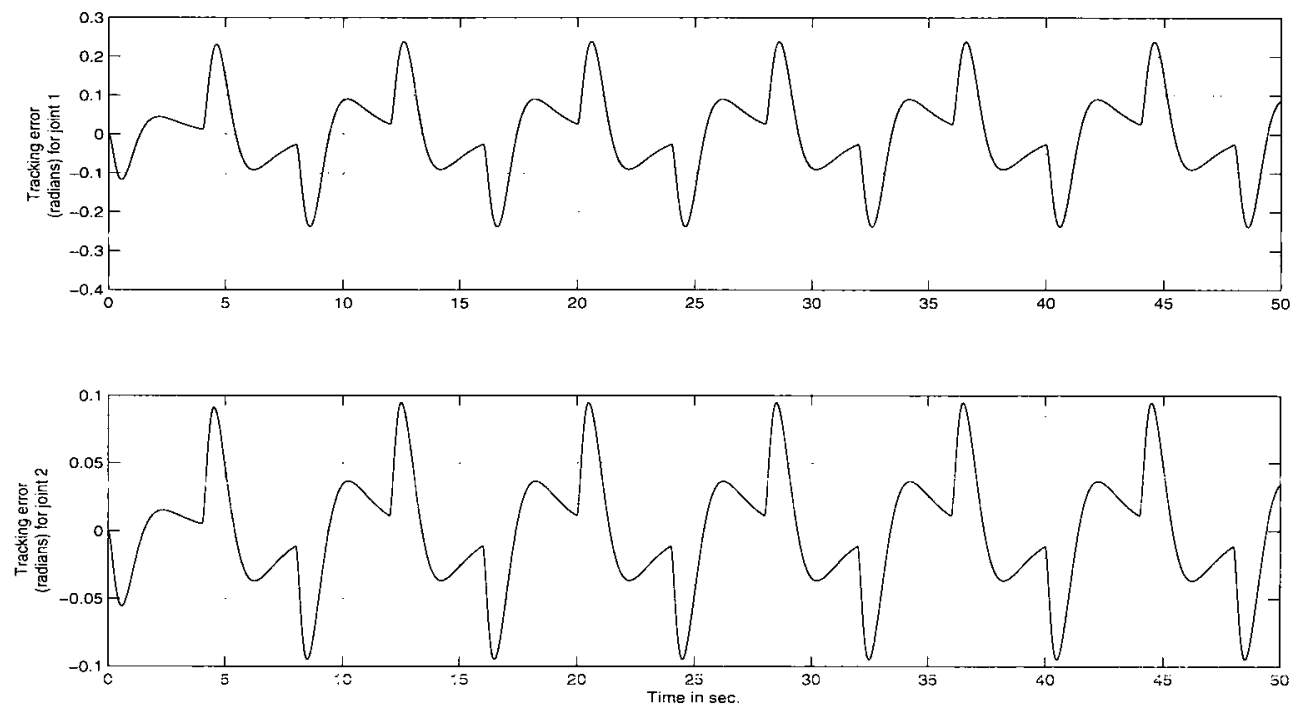

Figure 2.16: The The tracking errors for joint 1 and joint 2 under PD output feedback design with the input and output disturbance noise measurement $w(t)$.
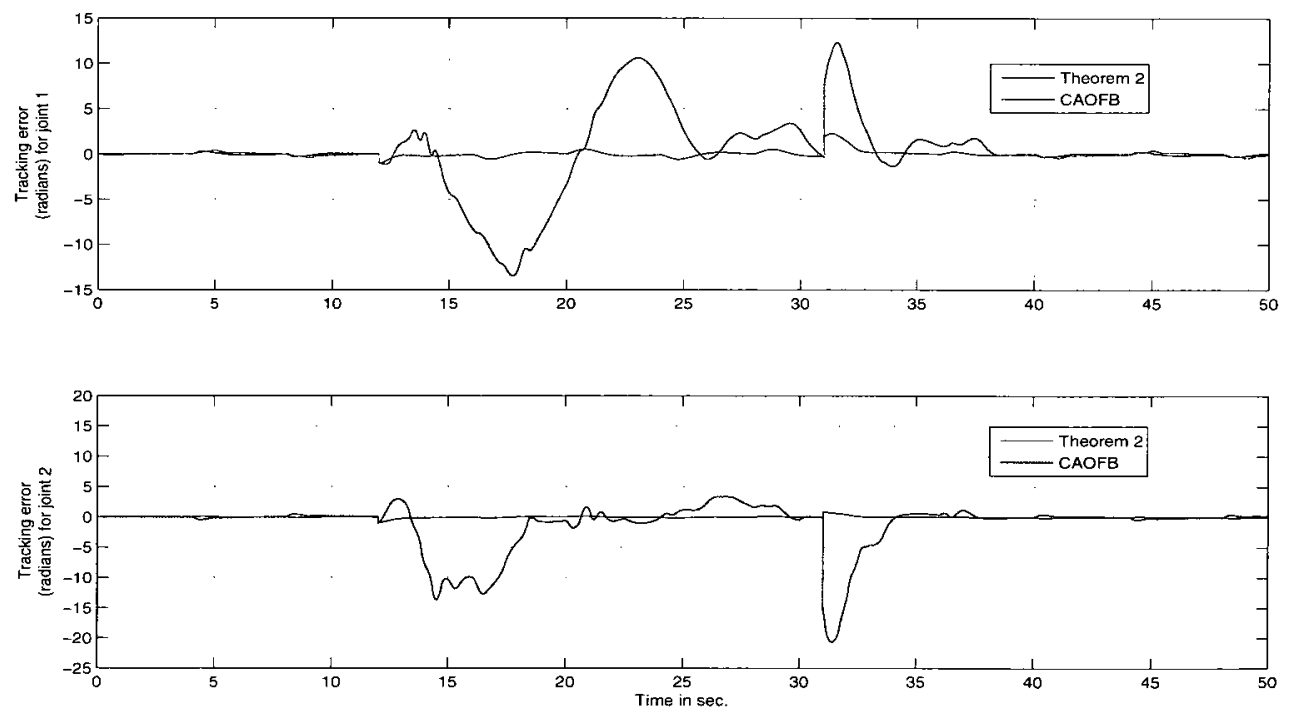

Figure 2.17: The tracking errors for joints 1 and 2 under PD and classical adaptive output feedback design under dynamics changes. 


\subsection{Summary}

In this chapter, we have shown that the model-free linear-observer-based PD outputfeedback control design can be used to solve the output feedback stabilization and tracking control problem for industrial robotic systems. The Lyapunov method has been utilized to characterize the observer-controller error bounds, where a detailed analysis has been given to show that all the signals in the closed loop system are bounded. In our first design, the stability condition sets the lower bound on the observer speed which reflects the uncertainties in initial conditions and initial parameters. In the second design, we have shown that the error bounded set can be made arbitrarily small by using observer-controller design parameters. Simulations on a 2-DOF robot has been used to demonstrate the theoretical developments for real-world operation. In the face of large scale modeling error uncertainties, the design may demand high controller gain to achieve good transient tracking performance. To deal with the problem associated with high controller gains, hybrid adaptive control approach using multi-model/control technique will be developed in chapter 5 . 


\section{Chapter 3}

\section{Robust Adaptive Fuzzy Control}

\section{SYSTEM}

In this chapter, we develop model-free hybrid adaptive control schemes for robust trajectory tracking control problem for a class of nonlinear systems. The design combines adaptive fuzzy system with robust adaptive control algorithm [88, 90, and 96].

In the first design, the direct adaptive fuzzy system is used to approximate the CE-based optimal controller. In second design, we introduce indirect adaptive fuzzy system to estimate nonlinear system dynamics. The robust adaptive control term in both designs is employed to learn and compensate uncertainties as a result of the external disturbance, fuzzy approximation errors and any other modeling errors. In two designs, we first develop a stable hybrid control law by assuming that the system output and its derivatives are available for feedback control design. Using Lyapunov second method, we explore asymptotic stability property of the closed loop system. Then, an output feedback form of the position-velocity (state feedback) controller is proposed, where unknown velocity signal is replaced by the output of the model-free linear estimator. It is proven that the tracking of the output feedback design can converge asymptotically to the performance achieved 
under the state feedback control design in the face of modeling error uncertainty and disturbances. The proposed design does not require a priori knowledge of the system dynamics. Finally, the design is evaluated on a 2-DOF robot to demonstrate the theoretical development of this chapter.

The rest of this chapter is organized as follows; In section II, we describe MIMO nonlinear mechanical systems and structure of the fuzzy systems. Section III presents direct adaptive fuzzy based hybrid control system. A detail convergence analysis of this method is given in Section III. Section III also shows the simulation results of the proposed method on a robotic system. Section IV develops indirect adaptive fuzzy based hybrid control system. This section provides the convergence property of the closed loop signals under indirect adaptive fuzzy based hybrid control system. The design and implementation procedure of this design is also illustrated in section IV. Finally, section V concludes the chapter.

\subsection{System Dynamics}

Let us consider a class of MIMO nonlinear mechanical systems having the following form

$$
\ddot{q}_{1}=\phi_{f}\left(q_{1}, \dot{q}_{1}\right)+\phi_{g}\left(q_{1}\right) \tau+\phi_{g}\left(q_{1}\right) d
$$

where $q_{1} \in \Re^{n}$ is the position vector, $\dot{q}_{1} \in \Re^{n}$ is the velocity vector, $\ddot{q}_{1} \in \Re^{n}$ is the acceleration vector, $\tau \in \Re^{n}$ is the control input vector, $d$ is the vector representing bounded external disturbance and any other unmodeled dynamical effects, $\phi_{f}\left(q_{1}, \dot{q}_{1}\right)$ and $\phi_{g}\left(q_{1}\right)$ are smooth continuous nonlinear functions. Note also that the vector $d$ is independent of the position and velocity state vetors $q_{1}$ and $q_{2}$.

Remark 3.1: The system (1.1) represents the equation of motion for an $n$-link robot manipulators [67, 68, 82-101, 110 and many others] as

$$
M\left(q_{1}\right) \ddot{q}_{1}+C\left(q_{1}, \dot{q}_{1}\right) \dot{q}_{1}+G\left(q_{1}\right)=\tau+d
$$


Then, we can write

$$
\ddot{q}_{1}=-M\left(q_{1}\right)^{-1}\left[C\left(q_{1}, \dot{q}_{1}\right) \dot{q}_{1}+G\left(q_{1}\right)\right]+M\left(q_{1}\right)^{-1} M\left(q_{1}\right)^{-1}
$$

Then, we can use $\phi_{f}\left(q_{1}, \dot{q}_{1}\right)=-M\left(q_{1}\right)^{-1}\left[C\left(q_{1}, \dot{q}_{1}\right) \dot{q}_{1}+G\left(q_{1}\right)\right]$ and $\phi_{g}\left(q_{1}\right)=M\left(q_{1}\right)^{-1}$ to obtain the model (1.1). In view of the dynamical properties of this system $[3$, $5,6,15$, and many others], we consider that $\phi_{f}\left(q_{1}, q_{2}\right)$ and $\phi_{g}\left(q_{1}\right)$ are smooth continuous nonlinear functions as $M\left(q_{1}\right) \in \Re^{n \times n}, C\left(q_{1}, \dot{q}_{1}\right) \dot{q}_{1} \in \Re^{n}$ and $G\left(q_{1}\right) \in \Re^{n}$ are continuous over the given compact sets.

\subsubsection{Fuzzy System}

Before starting to derive hybrid adaptive control system, let us briefly introduce basic structure of fuzzy logic system (FLS) which can approximate any well-defined nonlinear function over a given compact set with any degree of accuracy. A FLS consists of four parts as the fuzzy rule base (knowledge base), the fuzzifier, the fuzzy inference engine working on fuzzy rules, and the defuzzifier $[54,112]$. The fuzzy rule base for the FLS comprises a collection of fuzzy if-then rules. The fuzzy inference engine utilizes the fuzzy if-then rules to perform a mapping from an input vector $x=\left[x_{1}, x_{2}, x_{3}, x_{4}, \ldots \ldots, x_{n}\right]^{T} \in \Omega_{U} \subset \Re^{n}$ to an output vector $y=\left[y_{1}, y_{2}, y_{3}, y_{4}, \ldots \ldots, y_{m}\right]^{T} \in \Omega_{V} \subset \Re^{m}$. Then the if-then fuzzy rules are of the following form $R^{l}$ : If $x_{1}$ is $A_{1}^{l}$ and.... and $x_{n}$ is $A_{n}^{l}$, then $y$ is $y^{l}$ where $A_{1}^{l}, A_{2}^{l}, \ldots \ldots, A_{n}^{l}$ are fuzzy variables and $y^{l}$ is a Singleton vector. Now, using product inference, center-average and singleton fuzzifier, we obtain the output of the fuzzy systems as

$$
y(x)=\frac{\sum_{l=1}^{M} \bar{y}^{l}\left(\prod_{i=1}^{n} \mu_{A_{i}^{l}}\left(x_{i}\right)\right)}{\sum_{l=1}^{M}\left(\prod_{i=1}^{n} \mu_{A_{i}^{l}}\left(x_{i}\right)\right)}=\Theta \xi(x)
$$

where $\mu_{A_{i}^{l}}\left(x_{i}\right)$ is the membership function of the fuzzy variables $x_{i}$ and $\Theta$ is the adjustable parameter vector composed of consequent parameters. 


\subsection{Hybrid Adaptive Control System Using Di- rect Adaptive Fuzzy and Robust Adaptive Control}

\subsubsection{State Feedback Approach}

The objective is now to design a state feedback based control systems such that the manipulator joint position $q(t)$ asymptotically tracks the bounded desired joint position $q_{d}(t)$ in the presence of the modeling error uncertainty.

If the nonlinear functions are known and the plant dynamics of equation (3.3) is free from external disturbance, then one can use the following CE-based nonlinear controller to achieve desired tracking objective

$$
\tau_{c}=\phi_{g}^{-1}\left(q_{1}\right)\left[-\phi_{f}\left(q_{1}, \dot{q}_{1}\right)+\ddot{q}_{d}+K_{D} \dot{e}_{1}+K_{P} e_{1}\right]
$$

$e_{1}=\left(q_{d}-q_{1}\right), \dot{e}_{1}=\left(\dot{q}_{d}-\dot{q}_{1}\right), K_{P} \in \Re^{n \times n}$ and $K_{D} \in \Re^{n \times n}$ are symmetric, positive definite, constant diagonal matrices. Then using equation (3.5) in (3.3), we have the following error model

$$
\ddot{e}_{1}+K_{D} \dot{e}_{1}+K_{P} e_{1}=0
$$

Then, the choice of the control design parameter $K_{D}$ and $K_{P}$ ensures that $\lim _{t \rightarrow \infty} e(t)=$ 0 as $t \rightarrow \infty$. This performance can only be obtained when nonlinear functions and parameter uncertainty in the plant dynamics are known. In addition, the disturbance free system dynamics is also required to obtain such a globally asymptotically stable property of the tracking error signals. However, in practice, the plant dynamics are highly uncertain and nonlinear that associated with many structured and unstructured uncertainties, so obtaining a control law defined in equation (3.6) that ensures $\lim _{t \rightarrow \infty} e_{1}(t)=0$ and $\lim _{t \rightarrow \infty} \dot{e}_{1}(t)=0$ are not possible.

In this work, our goal is to derive hybrid system by combining robust adaptive control with direct adaptive fuzzy systems. The overall control law is then defined 
as follows

$$
\tau=\tau_{d}+\tau_{s}
$$

where $\tau_{d}$ is the direct adaptive fuzzy controller to estimate CE controller $\tau_{c}(3.5)$ and $\tau_{s}$ is an additional robustifying adaptive control term designed through Lyapunov analysis. The control term $\tau_{s}$ is used to update the bounded fuzzy approximation errors, external disturbances and other modeling error uncertainties that entered into the closed-loop system. Then, the closed-loop error model dynamics becomes

$$
\ddot{e}_{1}=-K_{D} \dot{e}_{1}-K_{P} e_{1}+\phi_{g}\left(e_{1}\right)\left[\tau-\tau_{d}-d\right]-\phi_{g}\left(e_{1}\right) \tau_{s}
$$

At this point, we consider the following assumption.

Assumption 3.1: The fuzzy approximation errors, external disturbances and other unmodeled dynamics are bounded as $\left\|w_{e}\right\| \leq \theta_{s}^{*}$.

Remark 3.1 Note that the parameter defined above is unknown which will be estimated by using robust adaptive control law.

As the mass matrix $M\left(e_{1}\right)$ is a symmetric, bounded and positive definite, then the bound on $\phi_{g}\left(e_{1}\right)$ satisfies the inequality $\left\|\phi_{g}\left(e_{1}\right)\right\| \leq \phi_{g o}\left(e_{1}\right)[3,5,6,15,21$, $22,23,25$, and many others]. We now introduce state variable as $e=\left[e_{1}^{T}, e_{2}^{T}\right]^{T}$, where $e_{1}=\left(q_{d}-q_{1}\right)$ and $e_{2}=\left(\dot{q}_{d}-\dot{q}_{1}\right)$. The error model equation under hybrid controller can be re-defined in the following error state-space form

$$
\dot{e}=A e+B \phi_{g o}\left(e_{1}\right)\left[\tau-\tau_{d}\right]-B \phi_{g o}\left(e_{1}\right) d-B \phi_{g o}\left(e_{1}\right) \tau_{s}
$$

where $A=\left[\begin{array}{cc}0_{n \times n} & I_{n \times n} \\ -K_{P} & -K_{D}\end{array}\right]$ and $B=\left[\begin{array}{c}0_{n \times n} \\ I_{n \times n}\end{array}\right]$. Let us consider that there exists a unique positive definite matrix $\mathrm{P}$ which satisfies the following Lyapunov equation $A^{T} P+P A=-Q$ where $Q$ is a positive definite design matrix. Now our main focus is to develop an adaptive controller $\tau_{d}$. To do that, define the optimal parameter vector

$$
\theta_{d}^{*}=\arg \min _{\theta_{d} \in \Omega_{d}}\left[\sup _{e \in \Omega_{c}}\left(\tau_{d}\left(e \mid \theta_{d}\right)-\tau\right)\right]
$$


where $\Omega_{d}$ and $\Omega_{c}$ are the compact set. The optimal parameters $\theta_{d}^{*}$ is used to minimize the fuzzy approximation errors which is defined as follows

$$
w_{e}=\left[\tau_{d}\left(e \mid \theta_{d}^{*}\right)-\tau\right]+d
$$

To adjust the parameter vector $\theta_{d}$, we use adaptive fuzzy systems given in equation (3.4) as $\tau_{d}\left(e \mid \theta_{d}\right)=\theta_{d} \xi(e)$. The optimal parameter controller is then defined by $\tau_{d}\left(e \mid \theta_{d}^{*}\right)=\theta_{d}^{*} \xi(e)$ Then the error model has the following form

$$
\dot{e}=A e+B \phi_{g o}\left(e_{1}\right)\left[\tilde{\theta}_{d} \xi(e)-w_{e}-\tau_{s}\right]
$$

with $\tilde{\theta}_{d}=\left(\theta_{d}^{*}-\theta_{d}\right)$. We now use Lyapunov second method to obtain stability condition for the closed-loop system (3.10) as well as design robust adaptive control term $\tau_{s w}$. To do that, let us consider the following Lyapunov function candidate

$$
V=\frac{1}{2} e^{T} P e+\frac{1}{2 \Gamma_{d}} \operatorname{tr}\left(\tilde{\theta}_{d}^{T} \tilde{\theta}_{d}\right)+\frac{1}{2 \Gamma_{s}} \tilde{\theta}_{s}^{2}
$$

where $\operatorname{tr}($.$) denotes the trace of matrix, \tilde{\theta}_{s}=\left(\theta_{s}^{*}-\theta_{s}\right), \Gamma_{d}>0$ and $\Gamma_{s}>0$. The parameter $\theta_{s}$ is a constant defined in assumption 3.1. Now we differentiate equation (3.11) with respect to time along with the tracking trajectory of the closed-loop equation (3.10). Then, to cope with uncertainty that entered into the closed-loop system as a result of the fuzzy approximation errors, external disturbances and any other modeling uncertainties, we introduce the following robust adaptive control law

$$
\tau_{s}=-\theta_{s} \operatorname{sgn}\left(e^{T} P B \phi_{g o}\left(e_{1}\right)\right)
$$

Then $\dot{V}$ can be written in the following simplified form

$$
\begin{aligned}
\dot{V}= & \operatorname{tr}\left\{\tilde{\theta}_{d}^{T}\left(\dot{\tilde{\theta}}_{d}+\Gamma_{d} \phi_{g o}^{T}\left(e_{1}\right) B^{T} P e \xi^{T}(e)\right)\right\}-\frac{1}{2} e^{T} Q e \\
& +\left[\tilde{\theta}_{s}\left(\dot{\tilde{\theta}}_{s}-\Gamma_{s}\left(e^{T} P B \phi_{g o}\left(e_{1}\right)\right) \operatorname{sgn}\left(e^{T} P B \phi_{g o}\left(e_{1}\right)\right)\right)\right]
\end{aligned}
$$

To obtain $\dot{V} \leq 0$, one needs to consider the following parameter adaptation laws

$$
\dot{\theta}_{d}=\Gamma_{d} \phi_{g o}^{T}\left(e_{1}\right) B^{T} P e \xi^{T}(e)
$$


or, equivalently $\dot{\tilde{\theta}}_{d}=-\Gamma_{d} \phi_{g o}^{T}\left(e_{1}\right) B^{T} P e \xi^{T}(e)$ and using the fuzzy rule tuning method as

$$
\dot{\theta}_{s}=-\Gamma_{s}\left(e^{T} P B \phi_{g o}\left(e_{1}\right)\right) \operatorname{sgn}\left(e^{T} P B \phi_{g o}\left(e_{1}\right)\right)
$$

or, equivalently $\dot{\tilde{\theta}}_{s}=\Gamma_{s}\left(e^{T} P B \phi_{g o}\left(e_{1}\right)\right) \operatorname{sgn}\left(e^{T} P B \phi_{g o}\left(e_{1}\right)\right)$. In our earlier work [83], we show that adaptation law given in equations (3.14) and (3.15) may exhibit discontinuous property even after the learning estimate converges to the actual one results in unbounded parameter estimate. To ensure that the parameter $\theta_{d}$ and $\theta_{s}$ remains bounded over the compact sets as $\theta_{d} \in \Omega_{d}$ and $\theta_{s} \in \Omega_{s}$, let us introduce a projection algorithm to limit the parameter estimates. To proceed with that, let us now define the compact sets $\hat{\Omega}_{d}$ and $\hat{\Omega}_{s}$ such that $\hat{\Omega}_{d} \subset \Omega_{d}$ and $\hat{\Omega}_{s} \subset \Omega_{s}$. The convex hypercube $\Omega_{d}$ is defined as $\Omega_{d}=\left\{x_{i} \leq \theta_{d i} \leq y_{i}\right\}$. Let $\Omega_{\delta d}$ as $\Omega_{\delta d}=\left\{x_{i}-\delta \leq \theta_{d i} \leq y_{i}-\delta\right\}$ where $\delta$ is chosen such that $\hat{\Omega}_{d} \subset \Omega_{\delta d}$ and adaptation gain $\Gamma_{d}$ to be positive constant. Then, the projection of the parameter adaptation rule can be written as $\dot{\theta}_{d}=\operatorname{Proj}\left(\theta_{d}, \Phi_{d}\right)$ with [37]

$$
\left[\operatorname{Proj}\left(\theta_{d}, \Phi_{d}\right)\right]_{i}=\left\{\begin{array}{cc}
\Gamma_{d} \Phi_{d i} & \text { if } x_{i} \leq \theta_{d i} \leq y_{i} \text { or } \\
& \text { if } \theta_{d i}>y_{i} \text { and } \Phi_{d i} \leq 0 \text { or } \\
& \text { if } \theta_{d i}<x_{i} \text { and } \Phi_{d i} \geq 0 \\
\Gamma_{d} \overline{\Phi_{d i}} & \text { if } \theta_{d i}>y_{i} \text { and } \Phi_{d i}>0 \\
\Gamma_{d} \breve{\Phi_{d i}} & \text { if } \theta_{d i}<x_{i} \text { and } \Phi_{d i}<0
\end{array}\right.
$$

where $\bar{\Phi}_{d i}=\left[1+\frac{y_{i}-\theta_{d i}}{\delta}\right] \Phi_{d i}, \breve{\Phi_{d i}}=\left[1+\frac{\theta_{d i}-x_{i}}{\delta}\right] \Phi_{d i}$ and $\Phi_{d i}$ is the $i$-th element of $\phi_{g o}^{T}\left(e_{1}\right) B^{T} P e \xi^{T}(e)$. Similarly, we can limit the parameter $\dot{\theta}_{s}$ using projection mechanism. To show that $\theta_{d}(t) \in \Omega_{d \delta}$ and $\theta_{s}(t) \in \Omega_{s \delta} \forall t>0$, we now apply above projection based adaptation mechanism to update $\theta_{d}$ and $\theta_{s}$ as

$$
\dot{\theta}_{d}=\operatorname{Proj}\left(\theta_{d}, \Gamma_{d} \phi_{g o}^{T}\left(e_{1}\right) B^{T} P e \xi^{T}(e)\right)
$$

and

$$
\dot{\theta}_{s}=\operatorname{Proj}\left(\theta_{s},-\Gamma_{s}\left(e^{T} P B \phi_{g o}\left(e_{1}\right)\right) \operatorname{sgn}\left(e^{T} P B \phi_{g o}\left(e_{1}\right)\right)\right)
$$


This impels that if the parameter estimate starts in the set $\theta_{d}(0) \in \Omega_{d}$ and $\theta_{s}(0) \in$ $\Omega_{s}$ will remain in $\theta_{d}(t) \in \Omega_{\delta d}$ and $\theta_{s}(t) \in \Omega_{\delta s} \forall t \geq 0$.

Based on our above analysis, we now state our main results for the positionvelocity based robust hybrid adaptive fuzzy control design as a state feedback approach.

Theorem 3.1: Let assumption 3.1 holds. Then, all the signals in the closedloop system (3.10) composed of (3.3), (3.7), (3.12), (3.14), (3.15) along with parameter projection mechanism are bounded and the tracking errors converge to zero globally.

Proof: Using (3.7), (3.12), (3.14), (3.15) along with parameter projection mechanism, one can write the derivative $V$ of (3.13) as

$$
\dot{V} \leq-\frac{1}{2} e^{T} Q e \leq 0
$$

If $e \neq 0$, then we conclude that $\dot{V}$ is negative in $e$ space. This implies that $\left(V, e, \theta_{d}, \theta_{s}\right) \in L_{\infty}$. Since all the variables in the right hand side of equation (3.10) are bounded then we can also conclude that $\dot{e} \in L_{\infty}$. Hence, $e$ is uniformly continuous and bounded. Now take integral (3.16) from 0 to $T$, we have

$$
V(T)-V(0) \leq-\int_{0}^{T} \frac{\lambda_{\min .}(Q)}{2}\|e\|^{2}
$$

Using Lyapunov equation (3.11), we can write

$$
\int_{0}^{T} \frac{\lambda_{\text {min. }}(Q)}{2}\|e\|^{2} \leq \frac{1}{2} e(0)^{T} Q e(0)+\frac{1}{2 \Gamma_{d}} \operatorname{tr}\left\{\tilde{\theta}_{d}(0)^{T} \tilde{\theta}_{d}(0)\right\}+\frac{1}{2 \Gamma_{s}} \tilde{\theta}_{s}^{T}(0) \tilde{\theta}_{s}(0)
$$

which implies that $e \in L_{2}$. Since $e$ is uniformly continuous over the interval $[0, \infty)$ with $T=\infty$ then, using Barbalat's lemma [106], we can conclude that $\lim _{t \rightarrow \infty} \dot{V}=$ 0 and $\lim _{t \rightarrow \infty} e=0$ provided that the parameter estimation errors are bounded.

Notice that the $\operatorname{sgn}($.$) function in (3.12), (3.15) may cause discontinuous con-$ trol law $\tau_{s}$. To smooth out $\tau_{s}$, we can estimate $\operatorname{sgn}($.$) by using following bounded$ 
control input as

$$
\begin{aligned}
& \tau_{s}=-\theta_{s} \tanh \left(\frac{e^{T} P B \phi_{g o}\left(e_{1}\right)}{\epsilon_{o}}\right) \\
& \tau_{s}=-\Gamma_{s}\left(e^{T} P B \phi_{g o}\left(e_{1}\right)\right) \tanh \left(\frac{e^{T} P B \phi_{g o}\left(e_{1}\right)}{\epsilon_{o}}\right)
\end{aligned}
$$

where

$$
\dot{\theta}_{s}=\operatorname{Proj}\left(\theta_{s},-\Gamma_{s}\left(e^{T} P B \phi_{g o}\left(e_{1}\right)\right) \tanh \left(\frac{e^{T} P B \phi_{g o}\left(e_{1}\right)}{\epsilon_{o}}\right)\right)
$$

with the small value of $\epsilon_{o}>0$ and $\tanh ($.$) is a smooth bounded saturation function,$ where

$$
\begin{aligned}
& \tanh \left(\frac{e^{T} P B \phi_{g o}\left(e_{1}\right)}{\epsilon_{o}}\right)= \\
& {\left[\tanh \left(\left(\frac{e^{T} P B \phi_{g o}\left(e_{1}\right)}{\epsilon_{o}}\right)_{1}\right), \ldots \ldots ., \tanh \left(\left(\frac{e^{T} P B \phi_{g o}\left(e_{1}\right)}{\epsilon_{o}}\right)_{n}\right)\right]^{T}}
\end{aligned}
$$

and $\left(\frac{e^{T} P B \phi_{g o}\left(e_{1}\right)}{\epsilon_{o}}\right)_{i}$ defines the $i$-th column of $\left(\frac{e^{T} P B \phi_{g o}\left(e_{1}\right)}{\epsilon_{o}}\right)$. With this modification, Theorem 3.1 has the following modified form.

Theorem 3.2: Let assumption 3.1 holds. Then, all the signals in the closedloop system (3.10) formulated by (3.3), (3.7), (3.14), (3.17) with parameter projection mechanism are bounded as $\left(e, \theta_{d}, \theta_{s}\right) \in L_{\infty}$.

Proof: The proof of Theorem 3.2 can be shown along the line of the proof of Theorem 3.1. We first replace the $\operatorname{sgn}($.) function in controller and adaptation law (3.12) and (3.15). Then, we follow the steps that used for Theorem 3.1. After some manipulations, we can write $\dot{V}$ as

$$
\dot{V} \leq-\frac{1}{2} e^{T} Q e+\left\|e^{T}\right\|\|P B\|\left\|\phi_{g o}\left(e_{1}\right)\right\| \zeta_{o}
$$

where

$$
\begin{gathered}
\left\|\tilde{\theta}_{s} \Gamma_{s} \tanh \left(\frac{e^{T} P B \phi_{g o}\left(e_{1}\right)}{\epsilon_{o}}\right)-\tilde{\theta}_{s} \Gamma_{s} \tanh \left(\frac{e^{T} P B \phi_{g o}\left(e_{1}\right)}{\epsilon_{o}}\right)\right\| \leq \zeta_{o} \\
\forall\left(e, Q_{d}, \theta_{d}, \theta_{s}\right) \in\left(\Omega_{c} \times \Omega_{D} \times \Omega_{\delta d} \times \Omega_{\delta s}\right) \text { and } e \in \Omega_{c}, Q_{d} \in \Omega_{D}, \theta_{d}(t) \in \Omega_{\delta d}, \theta_{s}(t) \in
\end{gathered}
$$


$\Omega_{\delta s}$ for all $t>0$. We now simplify $\dot{V}$ as

$$
\begin{aligned}
\dot{V} \leq & -\frac{1}{2} \lambda_{\min }(Q)\|e\|^{2}+\left\|e^{T}\right\|\|P B\|\left\|\phi_{g o}\left(e_{1}\right)\right\| \zeta_{o} \\
\leq & {\left[\frac{\lambda_{\max }(P)}{\lambda_{\min }(Q)} \zeta_{o} B^{T} P B \zeta_{o}+\frac{\lambda_{\min }(Q)}{4 \lambda_{\max }(P)} e^{T} P e\right]-} \\
& {\left[\frac{\lambda_{\max }(P)}{\lambda_{\min }(Q)} \zeta_{o} B^{T} P B \zeta_{o}+\frac{\lambda_{\min }(Q)}{4 \lambda_{\max }(P)} e^{T} P e\right]+} \\
& \left\|e^{T}\right\|\|P B\|\left\|\phi_{g o}\left(e_{1}\right)\right\| \zeta_{o}-\frac{1}{2} \lambda_{\min }(Q)\|e\|^{2}
\end{aligned}
$$

which can be further simplified as

$$
\begin{aligned}
\dot{V} \leq & {\left[\frac{\lambda_{\min }(Q)}{4 \lambda_{\max }(P)} e^{T} P e+\frac{\lambda_{\max }(P)}{\lambda_{\min }(Q)} \zeta_{o} B^{T} P B \zeta_{o}\right] } \\
& -\frac{1}{2} \lambda_{\min }(Q)\|e\|^{2} \\
\leq & -\frac{1}{4} \lambda_{\min }(Q)\|e\|^{2}+\frac{\lambda_{\max }(P)}{\lambda_{\min }(Q)}\left\|B^{T}\right\|\|P\|\|B\| \zeta_{o}^{2} \\
\leq & -\frac{1}{4} \lambda_{\min }(Q)\|e\|^{2}+\frac{\lambda_{\max }^{2}(P)}{\lambda_{\min }(Q)}\|B\|^{2} \zeta_{o}^{2}
\end{aligned}
$$

This implies that $\dot{V}$ is negative outside the compact set as

$$
\Omega_{u} \equiv\left\{e \mid\|e\| \leq\left(\frac{2 \lambda_{\max }(P)}{\lambda_{\min }(Q)}\right)\|B\| \zeta_{o}\right\}
$$

This means that the tracking error signals are uniformly bounded as the boundedness property of the parameter estimates $\theta_{d}, \theta_{s}, \tilde{\theta}_{d}$ and, $\tilde{\theta}_{s}$ are guaranteed by using projection feature of their learning estimates.

\subsubsection{Output Feedback Approach}

The robust hybrid adaptive control algorithm developed in previous section is based on using the strict assumption that the output and its derivatives are available for feedback. This assumption may become an important issue in the real-time operation. This is because advanced mechanical systems only equipped with encoder that provides only position measurement while the velocity sensors are removed to reduce the weight and cost of the overall systems $[4,5,6,36]$. We now remove this 
assumption from state feedback based control algorithm proposed in previous section via incorporating a linear observer to estimate unknown velocity error states as

$$
\dot{\hat{e}}_{1}=\hat{e}_{2}+\frac{H_{1}}{\epsilon} \tilde{e}_{1}, \dot{\hat{e}}_{2}=\frac{H_{2}}{\epsilon^{2}} \tilde{e}_{1}
$$

where $\tilde{e}_{1}=\left(e_{1}-\hat{e}_{1}\right) \epsilon$ is a design constant needs to be specified and $\hat{e}_{1}$ is the estimate of $e_{1}$. The dynamics (3.22) is independent of the model uncertainty, nonlinear dynamics and any other auxiliary dynamical nonlinearities. The observer dynamics can be re-written in the following compact form as

$$
\dot{\hat{e}}=A_{c} \hat{e}+L D(\epsilon) \tilde{e}_{1}
$$

where $A_{c}=\left[\begin{array}{cc}0 & I \\ 0 & 0\end{array}\right], L=\left[\begin{array}{cc}H_{1} & 0 \\ 0 & H_{2}\end{array}\right]$ and $D(\epsilon)=\left[\begin{array}{c}\frac{1}{\epsilon} I_{n \times n} \\ \frac{1}{\epsilon^{2}} I_{n \times n}\end{array}\right]$ with zero and identity matrix of 0 and $I$ and $H_{1}$ and $H_{2}$ are positive definite design matrices. We now only replace $e$ in the state feedback control law by $\hat{e}$ while all other signals/terms in the closed loop system under output feedback case remain same as the closed-loop system under state feedback design. Then, we show that the performance achieved under state feedback design (3.7) can be recovered asymptotically by the output feedback controller in which velocity signals are replaced by the output of the linear observer (3.22). This means that the tracking error bound obtained under state feedback based design can be recovered asymptotically by using output feedback design. In other words, with small value of $\epsilon$, a certain degree of separation between perturbed observer and controller closed-loop system can be easily achieved ensuring certain disturbance rejection properties that allows asymptotic recovery of the performance achieved under state feedback. More specifically, we aim to show that the stability domain obtained under state feedback can asymptotically recover by the output feedback control system design as the observer design parameter $\epsilon \rightarrow 0$. The asymptotic here means that the tracking behavior of the closed-loop dynamics with respect to the value of the observer design constant $\epsilon$. To make $\epsilon$ very small (to increase the speed of the observer dynamics), we introduce saturation mechanism where we saturate the state feedback control input outside 
the estimated region to obtain bounded output feedback control input.

The performance recovery analysis under output feedback design is shown via using perturbation theory with a small perturbed design parameter $\epsilon$. To show that, let us first develop singularly perturbed observer-controller closed loop system as

$$
\dot{\tilde{e}}=A_{o} \tilde{e}+B\left[\phi_{g o}\left(e_{1}\right) \tilde{\theta}_{d} \xi(e)-\phi_{g o}\left(e_{1}\right) \tau_{s}-\phi_{g o}\left(e_{1}\right) w_{e}-K e\right]
$$

with $A_{o}=\left[A_{c}-\bar{L} C\right], \bar{L}=\left[\begin{array}{l}H_{1} \\ H_{2}\end{array}\right], K=\left[K_{P}, K_{D}\right], \tilde{e}=\left[\tilde{e}_{1}^{T}, e_{2}^{T}\right]^{T}$ and $C=$ $\left[\begin{array}{ll}I_{n \times n} & 0_{n \times n}\end{array}\right]$. Now we re-define the observer error dynamics by scaled estimation error to construct singularly perturbed system. For this purpose, we introduce new variables as $\epsilon \eta_{1}=\tilde{e}_{1}, \eta_{2}=\tilde{e}_{2}$ with a small positive parameter $\epsilon$. Using this scaled estimation error, we construct the standard singularly perturbed systems as follows

$$
\begin{aligned}
\epsilon \dot{\eta} & =A_{o} \eta+\epsilon B\left[\phi_{g o}\left(e_{1}\right)\left(\tilde{\theta}_{d} \xi(e)-w_{e}-\tau_{s}\right)-K e\right] \\
\dot{e} & =A e+B \phi_{g o}\left(e_{1}\right)\left[\tilde{\theta}_{d} \xi(e)-\tau_{s}-w_{e}\right]
\end{aligned}
$$

with $\tilde{\eta}=\left[\tilde{\eta}_{1}^{T}, \eta_{2}^{T}\right]^{T}$. It is important to notice from above observer error model that if the uncertainty level increase, then the second term will also be increased. To reduce the influence of the large scale uncertainties that is the last term of (3.24) $B\left[\phi_{g o}\left(e_{1}\right)\left(\tilde{\theta}_{d} \xi(e)-w_{e}-\tau_{s}\right)-K e\right]$, we have to use small $\epsilon$ to make the term close to zero.

The advantage of using perturbation analysis is that we can easily split the stability problem into two subsystems as the slow subsystem and the fast subsystem. Then, it is possible to analyze both subsystems independently using relatively higher speed of the subsystem (3.24) (faster subsystem) with respect to the slower speed of the system (3.25) (slower subsystem). The difference speed between two subsystem can be made by choosing smaller value of $\epsilon$. Now, in view of closedloop model (3.24) and (3.25), we can see that the perturbed system has stable boundary-layer model. Also, the system has a reduced model which has the same 
dynamics as the closed-loop system under state feedback (3.10). This means that if $\epsilon \rightarrow 0$ then the second term of (3.24) closed to zero. On other hand, if $\epsilon \rightarrow 0$ then one can show that the model (3.25) has the same form of the closed-loop system under state feedback design (3.10). However, in the face of large scale uncertainties, small value of $\epsilon$ may cause large control input during state transient recovery phase. Note that, for the given set of initial condition of interest, the designer can find the minimum bound on $\epsilon$ and state transient recovery time under which the fast variable $\eta$ converges closed to zero. To attenuate large transient control efforts, we need to use bounded output feedback controller where the control action is globally bounded over the domain of interest provided by the state feedback based hybrid design. To do that, we use the Lyapunov estimation technique as follows. We first consider that all the initial conditions are bounded by the compact set $\Omega_{b}$. Then we choose the initial sets as $\theta_{d}^{*}(0) \in \Omega_{d}, \theta_{s}^{*}(0) \in \Omega_{s}$ and $e(0) \in \Omega_{e}$.

Using the universal approximation theorem, we also consider that the optimal parameter controller is bounded $\forall e \in \Omega_{c}$, that is, $\theta_{d}^{*} \in \Omega_{d}$. As we know from our state feedback design that if $\theta_{d}(0) \in \Omega_{d}$ and $\theta_{s}(0) \in \Omega_{s}$, then projection adaptive law ensures $\theta_{s}(t) \in \Omega_{\delta s}$ and $\theta_{d}(t) \in \Omega_{\delta d} \forall t \geq 0$. Then, we can choose $c_{1 d}=\max _{e \in \Omega_{e}} \frac{1}{2} e^{T} P e, c_{2 d}=\max _{\theta_{d}^{*} \in \Omega_{d}, \theta_{d} \in \Omega_{\delta d}} \frac{1}{2 \Gamma_{d}} \operatorname{tr}\left[\left(\theta_{d}^{*}-\theta_{d}\right)^{T}\left(\theta_{d}^{*}-\theta_{d}\right)\right]$ and $c_{3 d}=\max _{\theta_{s}^{*} \in \Omega_{s}, \theta_{s} \in \Omega_{\delta s}} \frac{1}{2 \Gamma_{s}}\left(\theta_{s}^{*}-\theta_{s}\right)^{T}\left(\theta_{s}^{*}-\theta_{s}\right)$ where $c_{1 d}>0, c_{2 d}>0$ and $c_{3 d}>0$. Then we choose a positive constant $c$ such that $c>\left(b=c_{1 d}+c_{2 d}+c_{3 d}\right)$. Now we define the compact set $\Omega_{c}$ such that $e(t) \in \Omega_{c}=\left\{e \mid e^{T} P e \leq c\right\} \forall t \geq 0$ as the domain of interest for the state feedback based control design as given in equation (3.7). Since the input $\tau_{d}$ and $\tau_{s}$ are continuous function with respect to $e, Q_{d}, \theta_{d}$ and $\theta_{s}$ then they are bounded on the compact set of these variables as $\Omega_{c} \times \Omega_{D} \times \Omega_{\delta d} \times \Omega_{\delta s}$ with $Q_{d} \in \Omega_{D} \subset \Re^{3 n}=\left[q_{d}, \dot{q}_{d}, \ddot{q}_{d}\right]^{T}$. Now we define the maximum bound for the control input $\tau$ as $S=S_{1}+S_{2}$ provided that $\tau_{d}$ and $\tau_{s}$ have maximum bound as $S_{1}=\max \left|\tau_{d}\left(e, Q_{d}, \theta_{d}\right)\right|$ and $S_{2}=\max \left|\tau_{s}\left(e, \theta_{s}\right)\right|$. Then, during transient peaking period, the control input saturate outside the set $\Omega_{c r}$ with $c_{r}>c$ and we have 
$\tau^{S}=\tau$ where

$$
\tau=\theta^{T} \xi(e)+\theta_{s} \operatorname{sgn}\left(e^{T} P B \phi_{g o}\left(e_{1}\right)\right)
$$

for all $e(0) \in \Omega_{e}, \theta_{s}(0) \in \Omega_{s}, \theta_{d}(0) \in \Omega_{d}, e(t) \in \Omega_{c}, \theta_{s}(t) \in \Omega_{s \delta}$ and $\theta_{d}(t) \in \Omega_{d \delta}$. If we now replace the state vectors $e$ in the control law by the output of the state estimator $\hat{e}$ of equation (3.22) then the bounded adaptive output feedback controller can also be achieved via saturated function. Then the closed-loop system in state space form can be re-written in the following singular perturbed form ${ }^{1}$

$$
\begin{aligned}
\epsilon \dot{\eta} & =A_{o} \eta+\epsilon B\left[\phi_{g o}\left(\hat{e}_{1}\right)\left(\tilde{\theta}_{d} \xi(e)-\tau_{s}-w_{e}\right)-K e\right] \\
\dot{e} & =A e+B \phi_{g o}\left(e_{1}\right)\left[\tilde{\theta}_{d} \xi(e)-\tau_{s}-w_{e}\right]
\end{aligned}
$$

with

$$
\begin{gathered}
\dot{\theta}_{d}=\operatorname{Proj}\left(\theta_{d}, \phi_{d}\left((e-\zeta(\epsilon)) \eta, Q_{d}, \theta_{d}\right)\right) \\
\dot{\theta}_{s}=\operatorname{Proj}\left(\theta_{s}, \phi_{s}\left((e-\zeta(\epsilon) \eta), \theta_{s}\right)\right)
\end{gathered}
$$

and $\tau^{S}=\tau$ that is $\tau_{s}=\tau_{s}^{S}$ and $\tau_{d}=\tau_{d}^{S}$. We now state the main results for the robust hybrid adaptive output feedback design.

Theorem 3.3: Consider the closed-loop control system defined by equation (3.26), (3.27) composed of the plant equation (3.3) and the output feedback control laws given by equations (3.7), (3.12), (3.14), (3.15) along with the observer (3.22) and the projection parameter mechanism. If we take the initial conditions e $(0) \in$ $\Omega_{e} \subseteq \Omega_{c}, \theta_{d}(0) \in \Omega$ and $\theta_{s}(0) \in \Omega$ then there exists $\epsilon^{*}>0$ such that $\forall \epsilon \in\left[0, \epsilon^{*}\right]$, all the state variables of the closed-loop system are bounded by a bound that can be made airbitrarily small with the small value of $\epsilon$.

Proof: The proof of Theorem 3.3 can be shown as follows $[88,96]$. We first prove that there exists a short transient period $T_{1}(\epsilon) \in\left[0, T_{2}\right]$ during which the fast variable $\eta$ approaches a function of the order $O(\epsilon)$, while the slow variables

\footnotetext{
${ }^{1}$ We may also represent singular perturbed closed loop model $(3.26),(3.27)$ in the following form $\epsilon \dot{\eta}=A_{o} \eta+\epsilon B\left[\phi_{g o}\left(e_{1}\right)\left(\left(\theta_{d}^{*} \xi(e)-\theta_{d} \xi(\hat{e})\right)-\left(\tau_{s}+w_{e}\right)\right)-K \hat{e}\right]$ and $\dot{e}=A e+B \phi_{g o}\left(e_{1}\right)\left[\left(\theta_{d}^{*} \xi(e)-\right.\right.$ $\left.\left.\theta_{d} \xi(\hat{e})\right)-\left(\tau_{s}+w_{e}\right)\right]$.
} 
$\left(e, \theta_{d}, \theta_{s}\right)$ remain in the subset $\Omega_{r}$ of the domain of attraction $\Omega_{c}$. To prove that, we consider the following Lyapunov function candidate

$$
V=\frac{1}{2} e^{T} P e+\frac{1}{2 \Gamma_{d}} \operatorname{trace}\left\{\tilde{\theta}_{d}^{T} \tilde{\theta}_{d}\right\}+\frac{1}{2 \Gamma_{s}} \tilde{\theta}_{s}^{2}
$$

From (3.28), we can see that the second and third part of the Lyapunov function $V$ is bounded by $c_{2 d}$ and $c_{3 d}$. So, we can define $c_{d}=c_{2 d}+c_{3 d}$. Notice that even if it increases in no time it will be remained bounded as $\theta_{d}(t) \in \Omega_{d \delta}$ and $\theta_{s}(t) \in \Omega_{s \delta}$ for any time greater than zero. This means that we only have to show that the first part of the Lyapunov-function (3.28), that is, $\frac{1}{2} e^{T} P e$ does not reach the constant value $c_{1 d}$ in any time.

To illustrate that, we can differentiate $V_{T_{2}}=\frac{1}{2} e^{T} P e$ along the slow trajectory (3.27) to establish the ultimate boundedness property of the slow subsystem over the time interval $\left[0, T_{2}\right]$. Now we take the derivative (3.28) along the solution of the slow closed-loop trajectories and then use projection based adaptation mechanism that ensures $\theta_{d}(t) \in \Omega_{\delta d}$ and $\theta_{s}(t) \in \Omega_{\delta s} \forall t \geq 0$ whenever $\theta_{d}(0) \in \Omega_{d}$ and $\theta_{s}(0) \in \Omega_{s}$. Then the derivative of $V$ becomes

$$
\begin{aligned}
\dot{V}= & -\frac{1}{2} e^{T} Q e+\left[\tilde{\theta}_{s}\left(\hat{e}^{T} P B \phi_{g o}\left(\hat{e}_{1}\right)\right) \operatorname{sgn}\left(\hat{e}^{T} P B \phi_{g o}\left(\hat{e}_{1}\right)\right)-\tilde{\theta}_{s}\left(e^{T} P B \phi_{g o}\left(e_{1}\right)\right) s g n\right. \\
& \left.\left(e^{T} P B \phi_{g o}\left(e_{1}\right)\right)\right]+\operatorname{tr}\left\{\tilde{\theta}_{d}^{T}\left(\Gamma_{d} \phi_{g o}^{T}\left(e_{1}\right) B^{T} P e \xi^{T}(e)-\Gamma_{d} \phi_{g o}^{T}\left(\hat{e}_{1}\right) B^{T} P \hat{e} \xi^{T}(\hat{e})\right)\right\}
\end{aligned}
$$

Since the input $\tau=\tau_{s}+\tau_{d}$ is bounded over the set $\Omega_{c} \times \Omega_{D} \times \Omega_{\delta d} \times \Omega_{\delta s}$ for all $e \in \Omega_{c}, \hat{e} \in \Omega_{c}, Q_{d} \in \Omega_{D}, \theta_{d}(t) \in \Omega_{\delta d}, \theta_{s}(t) \in \Omega_{\delta s}$ for all $t>0$. Then we have the following upper bound on the second, third and fourth terms of equation (3.29)

$$
\begin{aligned}
& \left\|\operatorname{tr}\left\{\tilde{\theta}_{d}^{T}\left(\Gamma_{d} \phi_{g o}^{T}\left(e_{1}\right) B^{T} P e \xi^{T}(e)-\Gamma_{d} \phi_{g o}^{T}\left(\hat{e}_{1}\right) B^{T} P \hat{e} \xi^{T}(\hat{e})\right)\right\}\right\| \leq k_{1} \\
& \left\|\tilde{\theta}_{s}\left(\hat{e}^{T} P B \phi_{g o}\left(\hat{e}_{1}\right)\right) \operatorname{sgn}\left(\hat{e}^{T} P B \phi_{g o}\left(\hat{e}_{1}\right)\right)-\tilde{\theta}_{s}\left(e^{T} P B \phi_{g o}\left(e_{1}\right)\right) \operatorname{sgn}\left(e^{T} P B \phi_{g o}\left(e_{1}\right)\right)\right\| \\
& \leq k_{2}
\end{aligned}
$$

$\forall\left(e, Q_{d}, \theta_{d}, \theta_{s}\right) \in \Omega_{c} \times \Omega_{D} \times \Omega_{\delta d} \times \Omega_{\delta s}$ with $k_{1}>0$ and $k_{2}>0$. Applying (3.30), equation $(3.29)$ can be simplified as

$$
\dot{V} \leq-\frac{1}{2} e^{T} Q e+k_{s}
$$


where $k_{s}=k_{1}+k_{2}$. In view of Lyapunov function candidate (3.28), the bound on error trajectory can be written as follows

$$
\dot{V} \leq-\frac{1}{2} \frac{\lambda_{\min }(Q)}{\lambda_{\max }(P)} e^{T} P e+k_{s}
$$

Equation (3.32) can be simplified further as

$$
\dot{V} \leq-\gamma_{1 s} V+k_{s v}
$$

where $\gamma_{1 s}=\frac{\lambda_{\min }(Q)}{\lambda_{\max }(P)}$ and $k_{s v}=\chi_{s} c_{2 d}+\chi_{s} c_{3 d}+k_{s}$ with $\chi_{s}=\frac{\lambda_{\min }(Q)}{\lambda_{\max }(P)}, \frac{1}{2 \Gamma_{s}} \tilde{\theta}_{s}^{2} \leq c_{3 d}$ and $\frac{1}{2 \Gamma_{d}} \operatorname{tr}\left\{\tilde{\theta}_{d}^{T} \tilde{\theta}_{d}\right\} \leq c_{2 d}$. Then the solution of the differential equation (3.33) can be written as

$$
V(t) \leq V(0) e^{-\gamma_{1 s} t}+\frac{k_{s v}}{\gamma_{1 s}}\left(1-e^{-\gamma_{1 s} t}\right)
$$

Since $V(0) \leq b<c$, then there is a finite time $T_{2}>0$, which is independent of $\epsilon$, such that $V(t)<c$ for $\forall t \in\left[0, T_{2}\right)$. This implies that the slow variables $\left(e(t), \theta_{d}(t), \theta_{s}(t)\right)$ remain inside the set $\Omega_{c}$. Note that we can also replace $e$ by $\hat{e}$ in the closed loop model

$$
\dot{e}=A e+B\left[\phi_{g o}\left(e_{1}\right)\left(\theta_{d}^{*} \xi(e)-\theta_{d} \xi(\hat{e})-\left(w_{e}+\tau_{s}\right)\right)\right]
$$

and then we can prove that the slow variables $\left(e, \theta_{d}, \theta_{s}\right)$ remain bounded by the compact subset of the region of attraction $\Omega_{c}$ over the short transient time $\left[0, T_{2}\right]$. To show that the slow variables remain bounded, let us take derivative of (3.28) along the defined trajectory of the closed-loop system and use the controller and adaptation law to simplify $\dot{V}$ as

$$
\begin{aligned}
\dot{V}= & -\frac{1}{2} e^{T} Q e+\frac{1}{\Gamma_{d}} \operatorname{tr}\left\{\tilde{\theta}_{d}^{T} \dot{\tilde{\theta}}_{d}\right\}+\frac{1}{\Gamma_{s}} \tilde{\theta}_{s} \dot{\tilde{\theta}}_{s}+e^{T} P B \phi_{g o}\left(e_{1}\right)\left[\theta_{d}^{*} \xi(e)-\theta_{d} \xi(\hat{e})\right] \\
& -e^{T} P B \phi_{g o}\left(e_{1}\right)\left[\tau_{s}+w_{e}\right]
\end{aligned}
$$

In view of assumption 3.1, control law and projection based adaptation mechanism law, we can further simplified $\dot{V}$ as follows,

$$
\begin{aligned}
\dot{V}= & -\frac{1}{2} e^{T} Q e-\operatorname{tr}\left\{\tilde{\theta}_{d}^{T} \phi_{g o}^{T}\left(\hat{e}_{1}\right) B^{T} P \hat{e} \xi^{T}(\hat{e})\right\}+\tilde{\theta}_{s}\left(\hat{e}^{T} P B \phi_{g o}\left(\hat{e}_{1}\right)\right) \operatorname{sgn}\left(\hat{e}^{T} P B \phi_{g o}\left(\hat{e}_{1}\right)\right) \\
& +e^{T} P B \phi_{g o}\left(e_{1}\right)\left[\theta_{d}^{*} \xi(e)-\theta_{d} \xi(\hat{e})\right]+\theta_{s}^{*}\left(e^{T} P B \phi_{g o}\left(e_{1}\right)\right) \operatorname{sgn}\left(e^{T} P B \phi_{g o}\left(e_{1}\right)\right)- \\
& \theta_{s}\left(e^{T} P B \phi_{g o}\left(e_{1}\right)\right) \operatorname{sgn}\left(\hat{e}^{T} P B \phi_{g o}\left(\hat{e}_{1}\right)\right)
\end{aligned}
$$


Then $\forall\left(e, Q_{d}, \theta_{s}, \theta_{d}, \hat{e}\right) \in \Omega_{c} \times \Omega_{D} \times \Omega_{\delta s} \times \Omega_{\delta d} \times \Omega_{c}$, we have the following bounds

$$
\left\|-\operatorname{tr}\left\{\tilde{\theta}_{d}^{T} \phi_{g o}^{T}\left(\hat{e}_{1}\right) B^{T} P \hat{e} \xi^{T}(\hat{e})\right\}+e^{T} P B \phi_{g o}\left(e_{1}\right)\left[\theta_{d}^{*} \xi(e)-\theta_{d} \xi(\hat{e})\right]\right\| \leq k_{1_{T_{2}}}
$$

and

$$
\begin{aligned}
& \| \tilde{\theta}_{s}\left(\hat{e}^{T} P B \phi_{g o}\left(\hat{e}_{1}\right)\right) \operatorname{sgn}\left(\hat{e}^{T} P B \phi_{g o}\left(\hat{e}_{1}\right)\right)+\theta_{s}^{*}\left(e^{T} P B \phi_{g o}\left(e_{1}\right)\right) \operatorname{sgn}\left(e^{T} P B \phi_{g o}\left(e_{1}\right)\right)- \\
& \theta_{s}\left(e^{T} P B \phi_{g o}\left(e_{1}\right)\right) \operatorname{sgn}\left(\hat{e}^{T} P B \phi_{g o}\left(\hat{e}_{1}\right)\right) \| \leq k_{T_{T_{2}}}
\end{aligned}
$$

where $k_{1_{T 2}}>0$ and $k_{2_{T 2}}>0$. Using these inequalities, $\dot{V}$ becomes

$$
\dot{V} \leq-\frac{1}{2} e^{T} Q e+k_{T}
$$

where $k_{T}=k_{1_{T 1}}+k_{2_{T 2}}$. From $\dot{V}$ we can also deduce that there is a finite time $T_{2}$ which is independent of $\epsilon$, such that for all $t \in\left[0, T_{2}\right] V(t) \leq b_{T 2}<c$ with $b<b_{T_{2}}<c$ and $e(0) \in \Omega_{b_{T_{2}}}=\left\{e \mid e^{T} P e \leq b_{T_{2}}\right\}$.

Let us now study the fast equation (3.26) over the time interval $\forall t \in\left[0, T_{2}\right)$. To do that, we take the following Lyapunov function candidate

$$
W(\eta)=\eta^{T} P_{o} \eta
$$

where $P_{o}$ is the solution of the Lyapunov equation $A_{o}^{T} P_{o}+A_{o} P_{o}=-Q_{o}$ and $Q_{0}$ is a positive definite design matrix. Then we differentiate (3.36) with respect to time along the solution of fast equation (3.26) and apply adaptation algorithms to obtain $\dot{W}(\eta)$ as ${ }^{2}$

$$
\begin{aligned}
\dot{W}(\eta)= & -\frac{1}{\epsilon} \eta^{T} Q_{o} \eta+2 \eta^{T} P_{o} B \phi_{g o}\left(e_{1}\right) \tilde{\theta}_{d} \xi(e)-2 \eta^{T} P_{o} B K e \\
& +2 \eta^{T} P_{o} B \phi_{g o}\left(e_{1}\right) \tilde{\theta}_{s} \operatorname{sgn}\left(\hat{e}^{T} P B \phi_{g}\left(\hat{e}_{1}\right)\right)
\end{aligned}
$$

\footnotetext{
${ }^{2}$ Using $\epsilon \dot{\eta}=A_{o} \eta+\epsilon B\left[\phi_{g o}\left(e_{1}\right)\left(\left(\theta_{d}^{*} \xi(e)-\theta_{d} \xi(\hat{e})\right)-\left(\theta_{s}^{*} \operatorname{sgn}\left(e^{T} P B \phi_{g o}\left(e_{1}\right)\right)-\right.\right.\right.$ $\left.\left.\left.\theta_{s} \operatorname{sgn}\left(\hat{e}^{T} P B \phi_{g o}\left(\hat{e}_{1}\right)\right)\right)\right)-K \hat{e}\right]$, one can also follow the same steps as derived from (3.36)(3.44) to show that $\|\eta\|$ converges to a small value namely order of $O(\epsilon)$. In this case, the bound on $\left\|-K \hat{e}+\phi_{g o}\left(e_{1}\right)\left(\theta_{d}^{*} \xi(e)-\theta_{d} \xi(\hat{e})\right)+\phi_{g o}\left(e_{1}\right)\left(\theta_{s}^{*} \operatorname{sgn}\left(e^{T} P B \phi_{g o}\left(e_{1}\right)\right)-\theta_{s} \operatorname{sgn}\left(\hat{e}^{T} P B \phi_{g o}\left(\hat{e}_{1}\right)\right)\right)\right\| \leq k_{2 s}$ exists $\forall\left(e, Q_{d}, \theta_{d}, \theta_{s}, \hat{e}\right) \in \Omega_{c} \times \Omega_{D} \times \Omega_{\delta d} \times \Omega_{\delta s} \times \Omega_{c}$.
} 
Since the fast variable $\eta$ enters into the slow model via bounded function $\tau((e-$ $\left.\zeta(\epsilon) \eta), Q_{d}, \theta\right)$ then the slow variables $\left(e, \theta_{d}, \theta_{s}\right)$ remain bounded on the set $\Omega_{c} \times$ $\Omega_{D} \times \Omega_{\delta d} \times \Omega_{\delta s}$. Then, we consider the following bound

$$
\left\|-K e+\phi_{g o}\left(e_{1}\right) \tilde{\theta}_{d} \xi(e)+\phi_{g o}\left(e_{1}\right) \tilde{\theta}_{s} \operatorname{sgn}\left(\hat{e}^{T} P B \phi_{g o}\left(e_{1}\right)\right)\right\| \leq k_{2 s}
$$

where $k_{2 s}>0 \forall\left(e, Q_{d}, \theta, \hat{e}\right) \in \Omega_{c} \times \Omega_{D} \times \Omega_{\delta d} \times \Omega_{\delta s} \times \Omega_{c}$. Using the above inequality, equation (3.37) can be written as

$$
\dot{W}(\eta) \leq-\frac{1}{\epsilon} \lambda_{\min }\left(Q_{o}\right)\|\eta\|^{2}+2\left\|\eta^{T}\right\|\left\|P_{o}\right\|\|B\| k_{2 s}
$$

Applying equation (3.36), we now simplify equation (3.39) to find the bound on $W(\eta)$

$$
\dot{W}(\eta) \leq-\frac{\Psi}{\epsilon} W+\Upsilon \sqrt{W}
$$

with $\Psi=\frac{\lambda_{\min }\left(Q_{o}\right)}{2 \lambda_{\max }\left(P_{o}\right)}$ and $\Upsilon=\frac{2\left\|P_{o}\right\|\|B\| k_{2 s}}{\sqrt{\lambda_{\min }\left(P_{o}\right)}}$. Using $W(\eta) \geq \epsilon^{2} \beta$ with $\beta=\frac{16\left\|P_{o}\right\|^{4} k_{2 s}^{2}}{\lambda_{\min }^{2}\left(Q_{o}\right) \lambda_{\min }\left(P_{o}\right)}$ and $\left\|P_{o}\right\|=\lambda_{\max }\left(P_{o}\right)$, equation (3.40) can be simplified as

$$
\dot{W}(\eta) \leq-\frac{\psi}{\epsilon} W
$$

where $\psi=\frac{\Psi}{2}$. Now for $\left\{\left(e, \theta_{d}, \theta_{s}\right) \mid V \leq c\right\}$ and $\left\{\eta \mid W(\eta) \geq \epsilon^{2} \beta\right\}$, one has the solution of (3.41) as

$$
W(\eta(t)) \leq W(0) \exp ^{\left(-\frac{\psi t}{\epsilon}\right)}
$$

Let us now define a bounded initial sets for the estimates $\hat{e}(0) \in \Omega_{e}$, where $\Omega_{e}$ is the compact set, then corresponding scaled initial state estimation errors are also bounded by $\eta(0)=\frac{e(0)-\hat{e}(0)}{\epsilon} \leq \frac{k}{\epsilon}$ for a non-negative constant $k$ that depends on the initial set $\Omega_{e}$. Then equation (3.42) can be written as

$$
W(\eta(t)) \leq W(0) \exp ^{\left(-\frac{\psi t}{\epsilon}\right)} \leq \frac{k_{o}}{\epsilon^{2}} \exp ^{\left(-\frac{\psi t}{\epsilon}\right)}
$$

where $k_{o}=k^{2} \lambda_{\max }(P)$. To calculate the transient peaking time $T_{1}(\epsilon)$ when $W(\eta(t))=\epsilon^{2} \beta$, let us consider $\epsilon_{1}^{*}>0$ be small enough so that for all $0<\epsilon<\epsilon_{1}^{*}$, the time $T_{1}(\epsilon)$ is

$$
T_{1}(\epsilon)=\frac{\epsilon}{\gamma} \ln \left(\frac{k_{o}}{\beta \epsilon^{4}}\right) \leq T_{2}
$$


Then at time $T_{1}(\epsilon), W(\eta(t))=\epsilon^{2} \beta$ and from equation (3.36) we have $\dot{W}(\eta) \leq-\gamma \epsilon \beta$. Therefore, $W(\eta)$ will continue to decrease and for the time $t>T_{1}(\epsilon)$ the inequality $W(\eta(t)) \leq \epsilon^{2} \beta$ holds

Let us now examine the property of $\eta$ as $W(\eta(t)) \leq \epsilon^{2} \beta$. Notice from equation (3.44) that $T_{1}(\epsilon)$ tends to zero as $\epsilon \rightarrow 0$ that is the right-hand side of the above inequality tends to zero when $\epsilon \rightarrow 0$. This implies that one can choose $\epsilon$-dependent time $T_{1}(\epsilon)$ small enough such that $T_{1}(\epsilon) \in\left[0, T_{2}\right]$. Hence, we deduce that for $t \in\left[0, T_{2}\right]$, there exists a time $T_{1}(\epsilon) \in\left[0, T_{2}\right]$ such that for all $t \in\left[T_{1}(\epsilon), T_{3}\right]$ $W(\eta(t)) \leq \epsilon^{2} \beta$, where $T_{3} \geq T_{2}$ is the first time $(e(t), \theta(t))$ exists from the set $\Omega_{E}=\Omega_{c} \times \Omega_{D} \times \Omega_{\delta}$, where $T_{3}=\infty$. Since the time $T_{1}(\epsilon)$ is a function of $\epsilon$ then one can make $T_{1}(\epsilon) \rightarrow 0$ as $\epsilon \rightarrow 0$. From Lyapunov equation (3.36) we conclude that as $W(\eta(t)) \leq \epsilon^{2} \beta \forall t \in\left[T_{1}(\epsilon), T_{3}\right]$ then $\|\eta\| \leq k_{\epsilon} \epsilon$ where $k_{\epsilon}>0$ and $\epsilon>0$, that is, $\eta$ is of order $O(\epsilon)$ in the same interval.

We now analyze the stability property of slow variables $\left(e(t), \theta_{d}(t), \theta_{s}(t)\right)$ during time interval $t \in\left[T_{1}, T_{3}\right]$, where $T_{3}$ is the first time when $\left(e(t), \theta_{d}(t), \theta_{s}(t)\right)$ exist from the set $\Omega_{E}$. During this time interval, fast variable $\eta$ approaches to the order $O(\epsilon)$ as $W(\eta(t)) \leq \epsilon^{2} \beta, \forall e(0) \in \Omega_{e}, \forall \hat{e}(0) \in \Omega_{e}$ and $\forall \theta(0) \in \Omega \forall t \in\left[T_{1}, T_{3}\right]$. Using this fact, we now prove that $\forall \epsilon \in\left(0, \epsilon_{1}^{*}\right)$ the output feedback controller guarantees the stability of the singularly perturbed closed-loop system $\forall t \in\left[T_{1}, T_{3}\right]$. To establish this argument, we present the perturbed error dynamics as

$$
\dot{e}=A e+B \phi_{g o}\left(e_{1}\right)\left[\theta_{d}^{*} \xi(e)-\theta_{d} \xi(\hat{e})\right]-B \phi_{g o}\left(e_{1}\right)\left[\tau_{s}+w_{e}\right]
$$

Now our goal is to prove that the signals $\left(e(t), \theta_{d}(t), \theta_{s}(t)\right)$ in equation (3.45) remains bounded for all $t \geq T_{1}(\epsilon)$. To explore that, let us consider the Lyapunov function candidate defined by equation (3.28). Then, take derivative of (3.28) along the trajectory of the closed-loop system equation (3.45) and use the controller and adaptation law to simplify $\dot{V}$ as

$$
\begin{aligned}
V= & -\frac{1}{2} e^{T} Q e+\frac{1}{\Gamma_{d}} \operatorname{tr}\left\{\tilde{\theta}_{d}^{T} \dot{\tilde{\theta}}_{d}\right\}+\frac{1}{\Gamma_{s}} \tilde{\theta}_{s} \dot{\tilde{\theta}}_{s}+e^{T} P B \phi_{g o}\left(e_{1}\right)\left[\theta_{d}^{*} \xi(e)-\theta_{d} \xi(\hat{e})\right]- \\
& e^{T} P B \phi_{g o}\left(e_{1}\right)\left[\tau_{s}+w_{e}\right]
\end{aligned}
$$


Then, applying assumption 3.1, control law (3.7), (3.12), (3.14), (3.15) along with estimator (3.22) and projection based adaptation mechanism law, we have

$$
\begin{aligned}
& \dot{V}=-\frac{1}{2} e^{T} Q e-\operatorname{tr}\left\{\tilde{\theta}_{d}^{T} \phi_{g o}^{T}\left(\hat{e}_{1}\right) B^{T} P \hat{e} \xi^{T}(\hat{e})\right\}+\tilde{\theta}_{s}\left(\hat{e}^{T} P B \phi_{g o}\left(\hat{e}_{1}\right)\right) \operatorname{sgn} \\
& \left(\hat{e}^{T} P B \phi_{g o}\left(\hat{e}_{1}\right)\right)+e^{T} P B \phi_{g o}\left(e_{1}\right)\left[\theta_{d}^{*} \xi(e)-\theta_{d} \xi(\hat{e})\right]+\theta_{s}^{*}\left(e^{T} P B \phi_{g o}\left(e_{1}\right)\right) \\
& \operatorname{sgn}\left(e^{T} P B \phi_{g o}\left(e_{1}\right)\right)-\theta_{s}\left(e^{T} P B \phi_{g o}\left(e_{1}\right)\right) \operatorname{sgn}\left(\hat{e}^{T} P B \phi_{g o}\left(\hat{e}_{1}\right)\right)
\end{aligned}
$$

Since $\eta$ is bounded by $\|\eta\| \leq k_{\epsilon} \epsilon \forall t \in\left[T_{1}, T_{3}\right]$, then we consider the following bounds

$$
\left\|-\operatorname{tr}\left\{\tilde{\theta}_{d}^{T} \phi_{g o}^{T}\left(\hat{e}_{1}\right) B^{T} P \hat{e} \xi^{T}(\hat{e})\right\}+e^{T} P B \phi_{g o}\left(e_{1}\right)\left[\theta_{d}^{*} \xi(e)-\theta_{d} \xi(\hat{e})\right]\right\| \leq k_{1 o}\|\eta\| \leq k_{1 o} \epsilon
$$

and

$$
\begin{aligned}
& \| \tilde{\theta}_{s}\left(\hat{e}^{T} P B \phi_{g o}\left(\hat{e}_{1}\right)\right) \operatorname{sgn}\left(\hat{e}^{T} P B \phi_{g o}\left(\hat{e}_{1}\right)\right)+\theta_{s}^{*}\left(e^{T} P B \phi_{g o}\left(e_{1}\right)\right) \operatorname{sgn}\left(e^{T} P B \phi_{g o}\left(e_{1}\right)\right) \\
& -\theta_{s}\left(e^{T} P B \phi_{g o}\left(e_{1}\right)\right) \operatorname{sgn}\left(\hat{e}^{T} P B \phi_{g o}\left(\hat{e}_{1}\right)\right)\left\|\leq k_{2 o}\right\| \eta \| \leq k_{2 o} \epsilon
\end{aligned}
$$

$\forall\left(e, Q_{d}, \theta_{s}, \theta_{d}, \eta\right) \in \Omega_{c} \times \Omega_{D} \times \Omega_{\delta s} \times \Omega_{\delta d} \times \Omega_{\epsilon}$, where $\left.\Omega_{\epsilon}=\left\{\eta \mid W(\eta) \leq \epsilon^{2} \beta\right)\right\}$, $k_{1 o}=k_{\epsilon} k_{1 s}, k_{2 o}=k_{\epsilon} k_{2 s}$ with $k_{1 s}>0, k_{2 s}>0, k_{1 o}>0$ and $k_{2 o}>0$. Now using (3.47) and (3.48), equation (3.46) becomes

$$
\dot{V} \leq-\frac{1}{2} e^{T} Q e+k_{\mathcal{O}} \epsilon
$$

where $k_{\mathcal{O}}=\left(k_{1 o}+k_{2 o}\right)$. In view of equation (3.28), we can calculate the bound on $V$ as follows

$$
\dot{V} \leq-\chi V+\frac{\chi}{2 \Gamma_{d}} \operatorname{tr}\left\{\tilde{\theta}_{d}^{T} \tilde{\theta}_{d}\right\}+\frac{\chi}{2 \Gamma_{s}} \tilde{\theta}_{s}^{T} \tilde{\theta}_{s}+k_{\mathcal{O}} \epsilon
$$

where $\chi=\frac{\lambda_{\min }(Q)}{\lambda_{\max }(P)}$. As $\theta_{d}(t) \in \Omega_{\delta d}$ and $\theta_{s}(t) \in \Omega_{\delta s}, \forall \theta_{d}(0) \in \Omega_{d}$ and $\forall \theta_{s}(0) \in \Omega_{s}$ then we have $\frac{1}{2 \Gamma_{d}} \operatorname{tr}\left\{\tilde{\theta}_{d}^{T} \tilde{\theta}_{d}\right\} \leq c_{2 d}$ and $\frac{1}{2 \Gamma_{s}} \tilde{\theta}_{s}^{T} \tilde{\theta}_{s} \leq c_{3 d}$. Then equation (3.50) becomes

$$
\dot{V} \leq-\chi V+\chi c_{o}+k_{\mathcal{O}} \epsilon
$$


with $c_{o}=\left(c_{2 d}+c_{3 d}\right)$. Now on the boundary $V=c$, we have $\dot{V}<0$ when $c>$ $\left[c_{o}+\frac{k_{\mathcal{O}} \epsilon}{\chi}\right]$. Since $c>\left(c_{1 d}+c_{2 d}+c_{3 d}\right)$ then, for small $\epsilon$, we conclude that the set $\left\{e \in \Omega_{c}\right\} \cap\left\{\theta \in \Omega_{\delta}\right\}$ is a positively invariant set. This implies that all the state trajectory $\left(e, \theta_{d}, \theta_{s}, \eta\right)$ is trapped inside $\left\{e \in \Omega_{c}\right\} \times\left\{\theta_{d} \in \Omega_{\delta d}\right\} \times\left\{\theta_{s} \in \Omega_{\delta s}\right\} \times\{\eta \in$ $\left.\Omega_{\epsilon}\right\}$ and remains there for all time. Therefore, we can conclude that all the signal in the closed-loop model equations (3.26) and (3.27) are bounded for $\forall t>0$ and $\eta$ is bounded $\forall t \in\left[T_{1}, T_{3}\right]$. Now integrating (3.49) from $t=0$ to $t=T$ yields

$$
V(T)-V(0) \leq-\int_{0}^{T} \frac{\lambda_{\min }(Q)}{2}\|e\|^{2}+T k_{\mathcal{O}} \epsilon
$$

Using Lyapunov function (3.28) together with the fact that $V(T) \geq 0$, we can simplify the bound for the error trajectory as

$$
\begin{gathered}
\int_{0}^{T} \frac{\lambda_{\min }(Q)}{2}\|e\|^{2} d t=\frac{1}{2} e(0)^{T} P e(0)+\frac{1}{2 \Gamma_{d}} \operatorname{tr}\left\{\tilde{\theta}_{d}(0)^{T} \tilde{\theta}_{d}(0)\right\}+\frac{1}{2 \Gamma_{s}} \tilde{\theta}_{s}(0)^{T} \tilde{\theta}_{s}(0) \\
+T k_{\mathcal{O}} \epsilon
\end{gathered}
$$

This implies that if $\epsilon \rightarrow 0$ then the hybrid adaptive output feedback controller can recover the performance achieved under hybrid adaptive state feedback controller.

Now, we can replace $\operatorname{sgn}\left(\hat{e}^{T} P B \phi_{g o}\left(\hat{e}_{1}\right)\right)$ in the controller-adaptation law (3.12), (3.15) by using smooth bounded function $\tanh \left(\frac{\hat{e}^{T} P B \phi_{g o}\left(\hat{e}_{1}\right)}{\epsilon_{o}}\right)$ along with linear observer (3.22). With this modification, we can follow the above design steps to show that the tracking error bound under state feedback design stated in Theorem 3.2 $\left(\Omega_{u}\right)$ can asymptotically $(\epsilon \rightarrow 0)$ recover by the output feedback design. The details performance recovery analysis is omitted for brevity.

Remark 3.2: The main ingredient in the semi-global results is the existence of small observer design parameter $\epsilon>0$ where $\epsilon$ represents the bandwidth of the observer dynamics. Notice from our analysis that if the speed of the observer dynamics is relatively faster than the closed-loop robot dynamics then the output feedback can recover the performance achieved under the state feedback deign. As the observer dynamics is free from model dynamics then, for any given set of initial interests, it is not hard to obtain the minimum bound on $\epsilon$ from the combined 
Lyapunov function candidate $V_{Q}=\frac{(1-d)}{2} e^{T} P e+\frac{d}{2} \eta^{T} P \eta$ with the design constant $d>0$.

Remark 3.3: Notice from our design is that the adaptation law depends on the value of $\phi_{g o}\left(e_{1}\right)$. Since, the inertial matrix $M\left(e_{1}\right)$ is a symmetric, bounded and positive definite then we can obtain the bound on $\phi_{g}\left(e_{1}\right)$ by using the nominal model parameters. This is not a restrictive assumption as the bound depends on known position error measurements $e_{1}$ that are available from encoders. On the other hand, we can choose $\left\|\phi_{g o}\left(e_{1}\right)\right\|=1$ to make the proposed design free from plant information accept the known sign of $\phi_{g o}\left(e_{1}\right)$.

Remark 3.4: Unlike the existing fuzzy control approach, the design uses only one fuzzy system, which is an important feature for practical application as it reduces the cost and computational efforts by saving the memory space.

Remark 3.5: The Lyapunov stability analysis in most fuzzy control system reported in the literature relies on the fact that the fuzzy approximation errors, external disturbance as well as modeling error uncertainties are bounded by a small positive constant. In view of universal fuzzy approximation theorem, one may find a fuzzy system using with large number of fuzzy membership function to estimate any given real continuous function with a small fuzzy approximation errors. However, in real-time application, the designer can only develop a fuzzy system that uses finite number of fuzzy rules and fuzzy membership functions as memory space is limited in most practical application. As a result, large fuzzy approximation errors may cause unstable closed-loop control system. To deal with that, we introduce robust adaptive control term to learn and compensate bounded uncertainties of the fuzzy approximation errors, unmodeled dynamics and other unknown external disturbance.

Remark 3.6: The proposed algorithm does not use robot dynamics as well as uncertain model parameters while they should be known in CE-based nonlinear adaptive feedback design. In comparison with the model-based method, the design that we introduced in this work does not require nonlinear function to be linearly 
parameterized with uncertain model parameters. This feature makes the proposed design insensitive to the model parameter variations which helps to reduce the control gains as well as the control efforts. Unlike model based approach, the proposed design does not need to compute tedious nonlinear-regressor model dynamics.

\subsubsection{Design Procedure and Steps}

The following is the design steps of the proposed method:

a. First define the control gains $K_{P}$ and $K_{D}$ such that $A$ is Hurwitz.

b. Then, choose observer design parameters $H_{1}, H_{2}$ and small value of $\epsilon$ such that $A_{o}$ is exponentially fast observer dynamics.

c. We then specify the matrix $Q$ to solve the Lyapunov equation for the positive definite matrix $P$.

d. Using estimate $\hat{e}$ along with observer dynamics, we obtain state estimate $\hat{q}$.

e. For the given initial conditions of interest $\Omega_{e}$ and $\Omega$ define the region of interest $\Omega_{c}$ as well as the saturation level $\Omega_{c r}$.

f. Then, specify the membership functions $\mu_{A_{j}^{l}}$ and calculate the fuzzy basis function $\xi(\hat{e})$.

g. Finally, we design and implement the control law to compute adaptive law in order to learn the parameter vector $\theta_{d}$ and $\theta_{s}$.

\subsubsection{Design and Simulation Results}

In this subsection, we present the design and implementation process of the hybrid scheme based on using with the direct adaptive fuzzy and robust adaptive control on a 2-DOF robotic system. The dynamic equations for this robot system can be 
defined as [82-101]

$$
\left[\begin{array}{ll}
m_{11} & m_{12} \\
m_{21} & m_{22}
\end{array}\right]\left[\begin{array}{l}
\ddot{q}_{1} \\
\ddot{q}_{2}
\end{array}\right]+\left[\begin{array}{ll}
c_{11} & c_{12} \\
c_{21} & c_{22}
\end{array}\right]\left[\begin{array}{l}
\dot{q}_{1} \\
\dot{q}_{2}
\end{array}\right]=\left[\begin{array}{l}
\tau_{1} \\
\tau_{2}
\end{array}\right]
$$

with $m_{11}=\left(\theta_{1}+2 \theta_{2}+2 \theta_{2} \cos q_{2}\right), m_{12}=\left(\theta_{2}+\theta_{2} \cos q_{2}\right), m_{21}=\left(\theta_{2}+\theta_{2} \cos q_{2}\right)$, $m_{22}=\theta_{2}, c_{11}=-2 \dot{q}_{2} \theta_{2} \sin q_{2}, c_{12}=-\dot{q}_{2} \theta_{2} \sin q_{2}, c_{21}=\dot{q}_{1} \theta_{2} \sin q_{2}$ and $c_{22}=0$ where $\theta_{1}=m_{1} l^{2}$ and $\theta_{2}=m_{2} l^{2}$ and $l$ is the link lengths and $m_{1}$ and $m_{2}$ are the masses of link 1 and link 2, respectively. The mass of the link 1 and link 2 assumed to be changed when the manipulator tracks the desired task with various loads. To generate the reference trajectory for the given robot model to follow, a square wave with a period of 8 seconds and an amplitude of \pm 1 radians is pre filtered with a critically damped 2nd-order linear filter using a bandwidth of $\omega_{n}=2.0 \mathrm{rad} / \mathrm{sec}$. We then define the initial values of the position and velocity error vectors for two joints as $e_{1}(0)=2, e_{2}(0)=2, e_{3}(0)=2$ and $e_{4}(0)=2$.

We first implement and evaluate the CE-principle based nonlinear classical adaptive output feedback design (CAOFB) on the given system. The objective is to design a CAOFB control law such that the manipulator position $q(t)$ asymptotically tracks the desired position $q_{d}(t)$. We assume that the plant parameter belong to the known interval $\theta \in[-10,10]$ with $\theta \in \Re^{2}$. In our evaluation, we aim to analyze the dynamic performance of the given system using CAOFB design with respect to model parameter changes. This means that the model dynamics will be changed over the known interval while tracking the desired task. For this purpose, we consider that the plant is initially operating under the parameters $\theta_{1}=8$ and $\theta_{2}=8$. Then, at $12 \mathrm{sec}$, the parameter $\theta$ is changed from $\theta_{1}=8$ and $\theta_{2}=8$ to $\theta_{1}=4$ and $\theta_{2}=4$. Therefore, there are total two dynamics changes in the whole process. To meet the control objective, let us design the CAOFB law [82] as

$$
\tau\left(\hat{e}, Q_{d}, \hat{\theta}\right)=Y\left(\hat{e}, \dot{q}_{d}, \ddot{q}_{d}\right) \hat{\theta}-K_{P} e_{1}-K_{D} \hat{e}_{2}
$$

with $\dot{\hat{\theta}}=-\Gamma Y^{T}\left(\hat{e}, \dot{q}_{d}, \ddot{q}_{d}\right) \hat{S}$, where $Y\left(\hat{e}, \dot{q}_{d}, \ddot{q}_{d}\right) \hat{\theta}=\hat{M}(q) \ddot{q}_{d}+\hat{C}\left(q, \dot{\hat{q}}_{r}\right) \dot{q}_{d}+\hat{G}(q), K_{P} \in$ $\Re^{n \times n}, K_{D} \in \Re^{n \times n}, \hat{S}=\hat{e}_{2}+\lambda e_{1}, \dot{\hat{q}}_{r}=\left(\dot{\hat{q}}_{2}-\lambda e_{1}\right), \lambda=\frac{\lambda_{0}}{1+\left\|e_{1}\right\|}, \lambda_{0}>0$ and $\hat{M}, \hat{C}($.$) and$ 

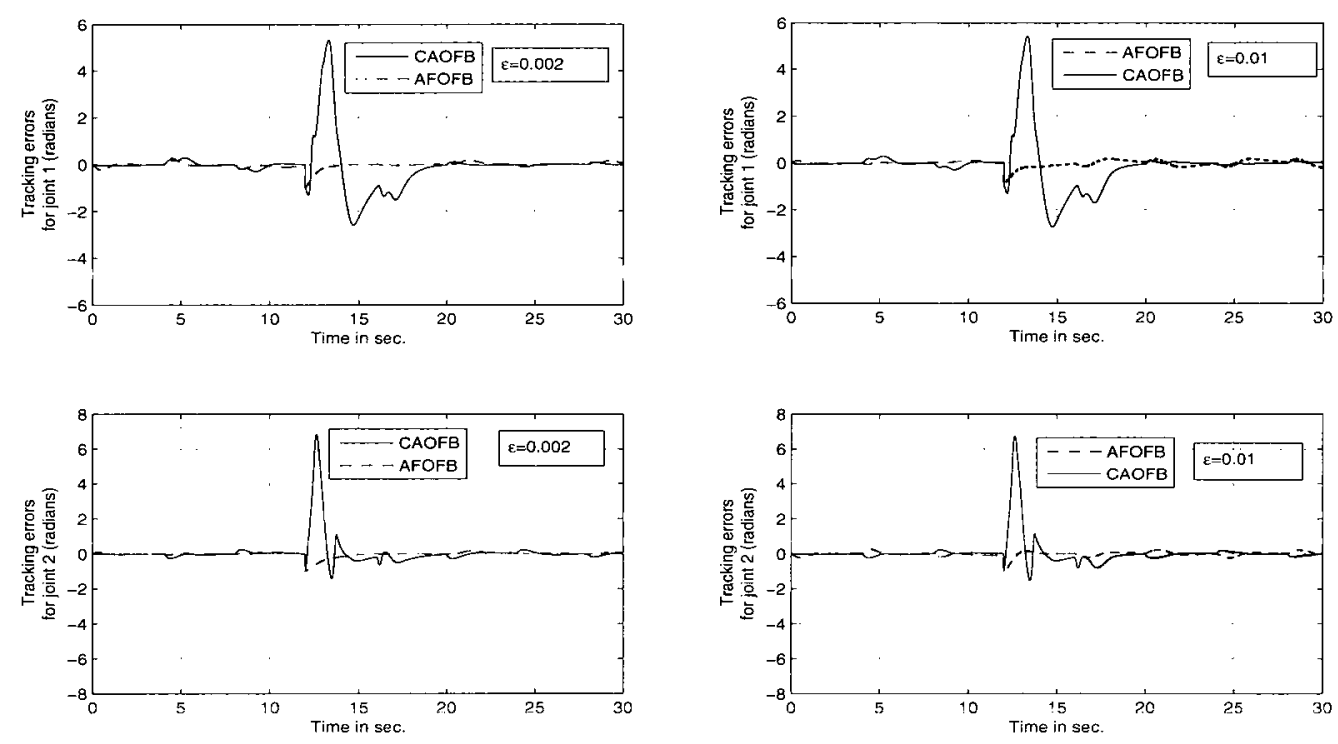

Figure 3.1: The tracking errors (radians) for joint 1 and joint 2 under CAOFB and hybrid adaptive output feedback design with model dynamic changes. The solid-line is for CAOFB design and the red-dash-line is for Theorem 3.3. .

$\hat{G}($.$) define the estimates of the M(),. C($.$) and G($.$) , respectively. We now select$ controller design parameters $K_{P}, K_{D}$ and $\lambda_{o}$ as $K_{P}=150 I_{2 \times 2}, K_{D}=150 I_{2 \times 2}$ and $\lambda_{o}=2$ to ensure an acceptable transient and steady state tracking performance of the given closed loop system. The learning gains $\Gamma$ is chosen to ensure fast parameter learning. For our evaluation, the value of $\Gamma$ is selected as $\Gamma=15 I_{2 \times 2}$ by assuming that $\theta \in[-10,10]$. As our results are semi-global, then the designer can select the control gains to guarantee exponential convergence of the closed-loop trajectory that starts inside a given region of interest.

Then, the unknown velocity signal in equation (3.54) is now replaced by the output of the linear observer as

$$
\dot{\hat{e}}_{1}=\hat{e}_{2}+\frac{H_{1}}{\epsilon} \tilde{e}_{1}, \dot{\hat{e}}_{2}=\frac{H_{2}}{\epsilon^{2}} \tilde{e}_{1}
$$

For our evaluation, we consider two sets of observer design constants as $H_{1}=I_{2 \times 2}$, $H_{2}=I_{2 \times 2}, \epsilon=0.002$ and $H_{1}=I_{2 \times 2}, H_{2}=I_{2 \times 2}, \epsilon=0.01$. With these design 
constants, we apply CAOFB design (3.54) on the given system (3.53). We notice that the control action become very large values at the time of dynamics changes. To protect the plant from large control action, we saturate the input outside the given domain of interest. The saturation level for two control inputs is considered as $\tau_{1 \max }=500$ newton-meters and $\tau_{2 \max }=500$ newton-meters. This level is obtained by using Lyapunov-function estimation technique. In practice, one requires to use the pre-defined saturation level provided by manufacturer. The implementation results are depicted in Figures 3.1 and 3.2. The solid-line of these Figures is pictured the tracking convergence of CAOFB design. The left colum of Figures 3.1 and 3.2 depicts the control performance under the following observer design constants: $H_{1}=I_{2 \times 2}, H_{2}=I_{2 \times 2}$ and $\epsilon=0.002$. The right colum of these Figures shows the control performance when the observer design constants are chosen as $H_{1}=I_{2 \times 2}$, $H_{2}=I_{2 \times 2}$ and $\epsilon=0.01$.

We now compare the tracking performance of model-free hybrid adaptive output feedback Theorem 3.3 with the CAOFB design. To construct Theorem 3.3, we assume that the compact set as $\Omega_{d}=\left\{-10 \leq \theta_{d} \leq 10\right\}, \Omega_{s}=\left\{-20 \leq \theta_{s} \leq 20\right\}$ and take $\delta=0.1$. For comparison, we keep the same model parameter changes as applied for CAOFB design. We also use the same PD controller gains of CAOFB design as $K_{P}=\operatorname{diag}(150,150)$ and $K_{D}=\operatorname{diag}(150,150)$. For the given error set, we now develop the fuzzy system by defining the input variables as $\left\{q_{1}, q_{2}, \dot{q}_{1}, \dot{q}_{2}\right\}$. To formulate the fuzzy basis function, we choose five fuzzy sets for each input variables. Then membership functions for each inputs $q_{j}(j=1,2)$ are chosen arbitrarily as

$$
\begin{gathered}
\mu_{A_{j}^{1}}\left(q_{j}\right)=\frac{1}{1+\left[\exp \left(\frac{\left(q_{j}+0.75\right)}{0.1}\right)\right]^{2}} \\
\mu_{A_{j}^{2}}\left(q_{j}\right)=\exp \left(-\left(\frac{\left(q_{j}+0.5\right)}{0.3}\right)^{2}\right) \\
\mu_{A_{j}^{3}}\left(q_{j}\right)=\exp \left(-\left(\frac{q_{j}}{0.3}\right)^{2}\right)
\end{gathered}
$$




$$
\mu_{A_{j}^{4}}\left(q_{j}\right)=\exp \left(-\left(\frac{\left(q_{j}-0.5\right)}{0.3}\right)^{2}\right)
$$

and

$$
\mu_{A_{j}^{5}}\left(q_{j}\right)=\frac{1}{1+\left[\exp \left(-\left(\frac{\left(q_{j}-0.75\right)}{0.1}\right)\right)\right]^{2}}
$$

The membership functions for each inputs $\dot{q}_{j}(j=1,2)$ are also defined as

$$
\begin{gathered}
\mu_{A_{j}^{1}}\left(\dot{q}_{j}\right)=\frac{1}{1+\left[\exp \left(\frac{\left(\dot{q}_{j}+1.13\right)}{0.2}\right)\right]^{2}} \\
\mu_{A_{j}^{2}}\left(\dot{q}_{j}\right)=\exp \left(-\left(\frac{\left(\dot{q}_{j}+0.75\right)}{0.45}\right)^{2}\right) \\
\mu_{A_{j}^{3}}\left(\dot{q}_{j}\right)=\exp \left(-\left(\frac{\dot{q}_{j}}{0.45}\right)^{2}\right) \\
\mu_{A_{j}^{4}}\left(\dot{q}_{j}\right)=\exp \left(-\left(\frac{\left(\dot{q}_{j}-0.75\right)}{0.45}\right)^{2}\right)
\end{gathered}
$$

and

$$
\mu_{A_{j}^{5}}\left(\dot{q}_{j}\right)=\frac{1}{1+\left[\exp \left(-\left(\frac{\left(\dot{q}_{j}-1.13\right)}{0.2}\right)\right)\right]^{2}}
$$

The membership functions are selected such that they covers the bound on the joint position and velocity error vectors. It is important to note that, to determine the membership function efficiently in fuzzy control, one requires to know the bound on the input vector of the fuzzy system a priori. Now, we choose identity matrix of $Q$ to solve the Lyapunov-equation $A^{T} P+P^{T} A=-Q$. For the given set of the initial conditions of interest, we choose the learning gains $\Gamma_{d}=500$ and $\Gamma_{s}=500$. To make the design free from system dynamics, we use $\phi_{g o}=1$. We then choose the small value of controller design constant $\epsilon_{o}=1$. Finally, we use two sets of observer design parameters as $H_{1}=I_{2 \times 2}, H_{2}=I_{2 \times 2}, \epsilon=0.002$ and $H_{1}=I_{2 \times 2}$, $H_{2}=I_{2 \times 2}, \epsilon=0.01$. Note that the control inputs under hybrid design of Theorem 3.3 are saturated with the same bounds as used for CAOFB design. With these design constants, we then implement Theorem 3.3 on the same system with the 

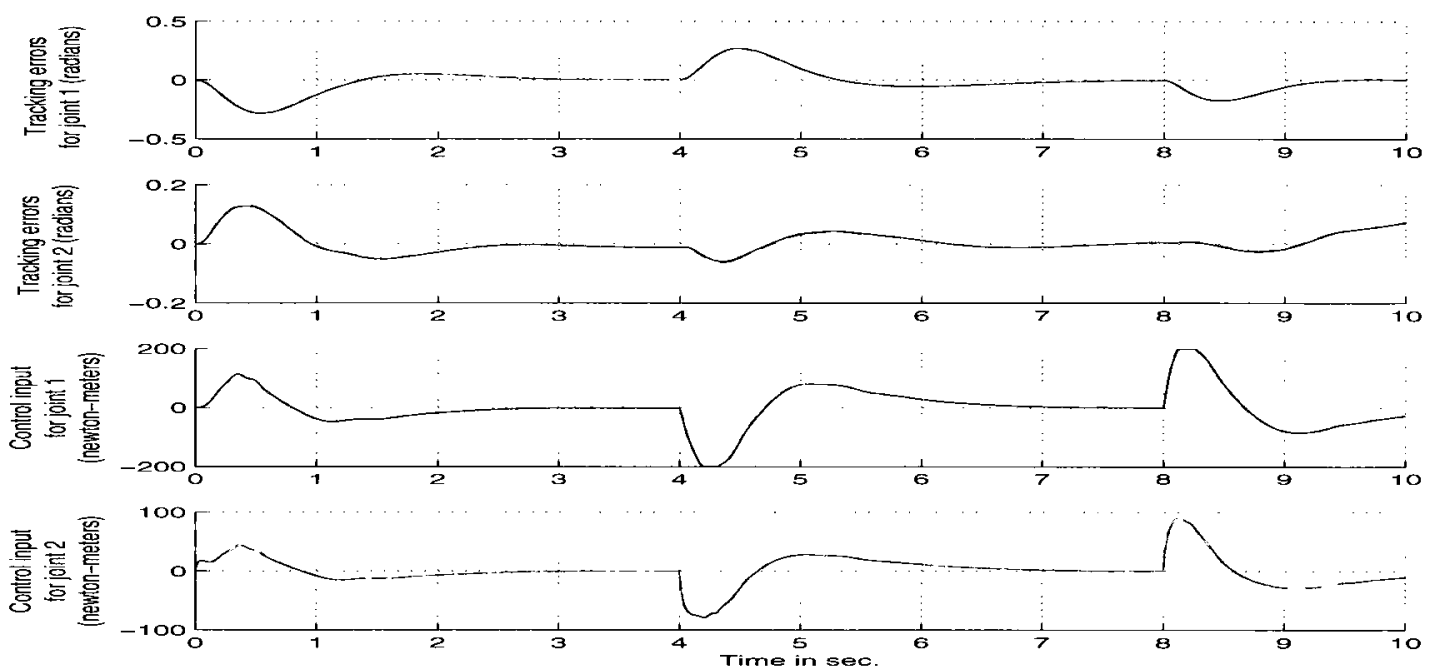

Figure 3.2: The tracking errors (radians), the control input (newton-meters) for joint 1 and joint 2 under Theorem 3.1 and Theorem 3.3. The solid-line is for Theorem 3.1 and the red-dash-line is for Theorem 3.3.

same dynamic sets up that used for CAOFB design. The tested results under Theorem 3.3 are given in Figures 3.1 and 3.2. The red-dash-line of Figures 3.1 and 3.2 (titled as AFOFB) is pictured the tracking convergence under Theorem 3.3. The left colum of these Figures depicts the control performance under the following observer design constants: $H_{1}=I_{2 \times 2}, H_{2}=I_{2 \times 2}$ and $\epsilon=0.002$. The right colum of these Figures shows the implementation results when the observer design constants are chosen as $H_{1}=I_{2 \times 2}, H_{2}=I_{2 \times 2}$ and $\epsilon=0.01$.

By comparing solid-line and red-dash-line of Figures 3.1 and 3.2, we notice that the control performance under Theorem 3.3 is much better than the performance achieved with CAOFB design. More specfically, at the time of dynamic changes, the tracking errors and control inputs under CAOFB design are larger than the tracking errors and control inputs obtained with the hybrid design of Theorem 3.3. This is mainly because the tracking performance of CAOFB design is sensitive to the model parameter changes, while the hybrid design of Theorem 3.3 is insensitive to the model parameter variations. 
Remark 3.7: We notice from our evaluations that, in the face of large scale parametric uncertainties, the CE-based CAOFB appoach exhibits poor transient tracking performance while the control tracking performance under hybrid adaptive output feedback design is relatively small as the proposed scheme is insensitive to the model parameters.

\subsubsection{Comparison Between Theorem 3.1 and Theorem 3.3}

In this part of this chapter, our aim is to compare the performance achieved under Theorem 3.1 (state feedback) with Theorem 3.3 (output feedback). In theorem 3.1, we assume that position-velocity signals are vailable for feedback design. Most fuzzy based control designs reported in the literature requires full state state measurements of the system. This is, in fact, a restrictive assumption as advanced nonlinear systems provide only measurements of joint positions through sensors such as encoders or resolvers $[4,5,6,36]$. In contrast, joint velocities are usually obtained by means of tachometers or by differentiating the position measurements. This means that the obtained velocity signals are often severely contaminated by measurement noise. Therefore, the performance of the position-velocity based hybrid design of Theorem 3.1 is restricted as, in practice, the measurement noise associated with the estimation of the velocity signals is amplified with the increase of the values of the controller gains.

To illustrate that arguement, let us implement Theorem 3.1 and Theorem 3.3 on the given system (3.53). The controller design constants are kept similar to our last test. The observer design parameters are chosen as $H_{1}=I_{2 \times 2}, H_{2}=I_{2 \times 2}$ and $\epsilon=0.002$. The implemented results are depicted in Figure 3.2. The red-dash-line of Figure 3.2 presents the tracking convergence of Theorem 3.3. The solid-line of this Figure pictures the control performance of Theorem 3.1. In view of Figure 3.2 , we can see that the control performance under output feedback (Theorem 3.3) recovers the performance achieved under state feedback approach (Theorem 3.1).

The presented results is assumed that the control system is operating under 


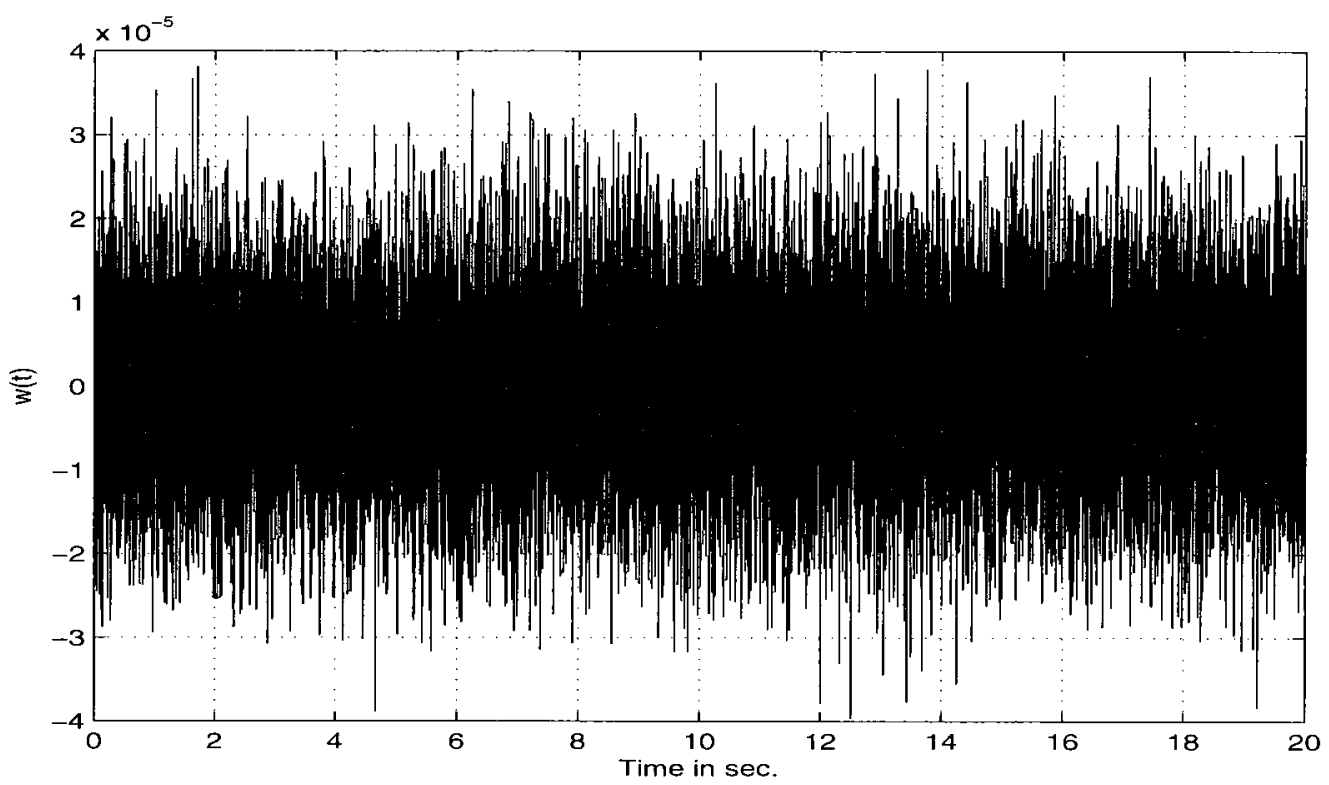

Figure 3.3: The disturbance noise level $d(t)=w(t)$ (newton-meters).

ideal situation. We now investigate the tracking performance of Theorem 3.1 and Theorem 3.3 with respect to non ideal operating conditions. To create such a practical situation, let us add an arbitrarily chosen band limited white noise $d(t)=$ $w(t)$ into the output $q(t)$ and input $\tau(t)$ of the given system (5.23). The level $w(t)$ that used in our test depicted in Figure 3.3. Then, we apply Theorem 3.1 and Theorem 3.3 with the same controller design parameters that used for the previous evaluation under ideal operating condition. The observer design parameters for this evaluation is chosen as $H_{1}=I_{2 \times 2}, H_{2}=I_{2 \times 2}$ and $\epsilon=0.0018$. The results are depicted in Figure 3.4. The red-dash-line of this Figure presents the tracking convergence of Theorem 3.3. The solid-line of the Figure 3.4 pictures the control performance obtained under Theorem 3.1.

By comparing solid and red-dash-line of the Figure 3.4, we can see that the control performance with output feedback based design of Theorem 3.3 exhibits better performance than the performance achieved under state feedback design. In view of our results, we notice that the control input and the output tracking error 

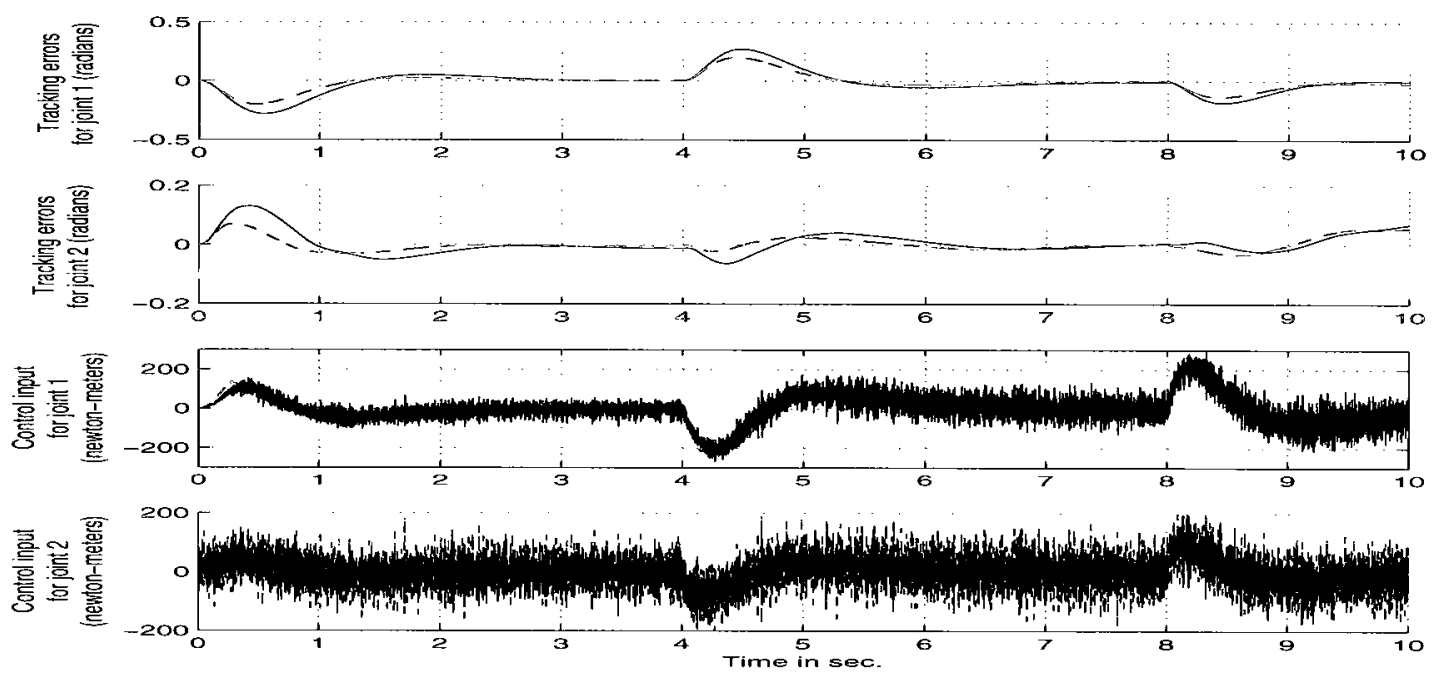

Figure 3.4: The tracking errors (radians), the control input (newton-meters) for joint 1 and joint 2 under Theorem 3.1 and Theorem 3.3. The sloid-line is for Theorem 3.1 and the red-dash-line is for Theorem 3.3.

under Theorem 3.1 are greater than the control input as well as the output tracking error of Theorem 3.3 .

Let us now increase the initial error states $e(0)$ from $e(0)=0$ to $e(0)=$ 1. The saturation level for two control inputs is assumed to be pre-defined by manufacturer as $\tau_{1 \max }=500$ newton-meters and $\tau_{2 \max }=500$ newton-meters. The control saturation mechanism is introduced to protect the plant from large transient control action that appeared from using initial error estimates. Then, we repeat the last experiment of Theorems 3.1 and 3.3 on the same system (3.53). The results are given in Figure 3.5. The blue-dash-line and solid-line of the Figure 3.5 depicts the control performance under Theorem 3.1 and Theorem 3.3 with the presence of the initial error estimates. The red-dash-line of the Figure 3.5 presents the tracking convergence of Theorem 3.3. The solid-line of this Figure pictures the control performance of Theorem 3.1. We notice from Figure 3.5 is that the output trajectory for both joints peaks before converge to the desired one due to the presence of initial error estimates. 

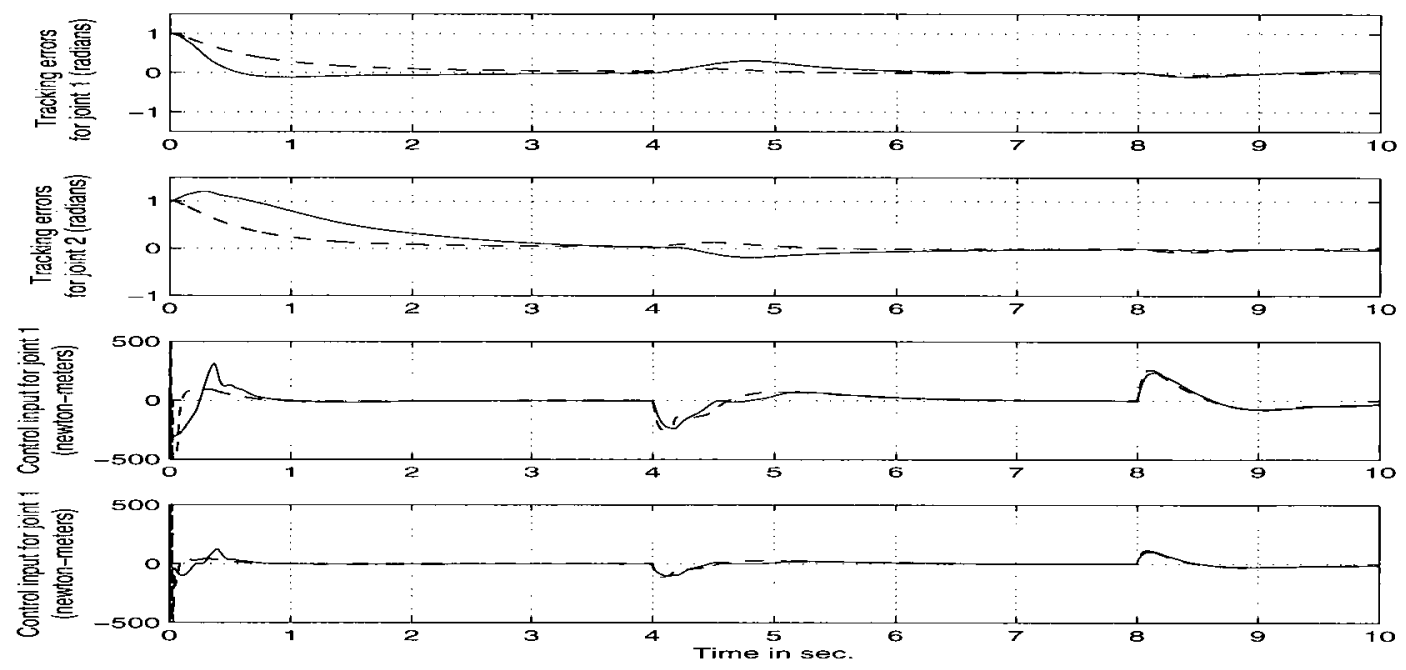

Figure 3.5: The tracking errors (radians), the control input (newton-meters) for joint 1 and joint 2 under Theorem 3.1 and Theorem 3.3. The sloid-line is for Theorem 3.1 and the red-dash-line is for Theorem 3.3.

We now compare the tracking performance of state and output feedback based design with respect to non ideal operating conditions. For this purpose, the system model (3.53) is corrupted by disturbance noise via adding an arbitrarily chosen band limited white noise $w(t)$ into the output $q(t)$ and input $\tau(t)$ as given in Figure 3.3. Then, we apply Theorems 3.1 and 3.3 with the same design parameters that used for the previous experimentation under ideal operating condition. The conducted results are depicted in Figure 3.6. The red-dash-line of Figure 3.6 is given for the tracking convergence of Theorem 3.3. The solid-line of Figure 3.6 depicts the control performance of Theorem 3.1.

In view of solid and red-dash-line of Figure 3.6, we can again observe that the control chattering and tracking errors under Theorem 3.3 are relatively smaller than the control input and tracking error under CAOFB design of Thoerem 3.1. The sampling time is set to $1 \mathrm{~ms}$. for all of our results presented in this section.

Remark 3.8: The tracking convergence under state feedback based hybrid 
design deteriorates as the disturbance noise associated with the velocity signals (derivative action of the noisy position measurement $q(t)+w(t)$ ) as well as with the input signals $(\tau(t)+w(t))$ amplifies with the control gains (see Figure 3.5 and Figure 3.6). To deal with this practical problem, one can deploy the proposed hybrid adaptive output feedback based control system where the velocity signals are replaced by the output of the observer.

Remark 3.9: From Figures 3.5 and 3.6, we can see that the output trajectory for both joints peaks before converge to the desired one due to the presence of initial error estimates. Note that this transient tracking error can be reduced by increasing the speed of the observer dynamics.

\subsection{Hybrid Adaptive Control System Using In- direct Adaptive Fuzzy and Robust Adaptive control}

\subsubsection{State Feedback Approach}

In this section, we propose hybrid adaptive control system by combing indirect adaptive fuzzy system with robust adaptive control technique. The fuzzy control structure in this design is used to approximate uncertain nonlinear system dynamics. The robust adaptive control term is employed to learn and compensate uncertainties as a result of disturbance and any other modeling errors. Then, overall hybrid adaptive strategy can be written as [97]

$$
\begin{aligned}
\tau_{c}\left(e_{1}, \dot{e}_{1}, Q_{d}, \theta_{f}, \theta_{g}, \theta_{s w}\right)= & \hat{\phi}_{g}^{-1}\left(e_{1} \mid \theta_{g}\right)\left[-\hat{\phi}_{f}\left(\left(e_{1}, \dot{e}_{1}\right) \mid \theta_{f}\right)+\ddot{q}_{d}+K_{D} \dot{e}_{1}+\right. \\
& \left.K_{P} e_{1}+\tau_{s w}\right]
\end{aligned}
$$

where fuzzy system is used to estimate nonlinear functions $\hat{\phi}_{g}^{-1}\left(e_{1} \mid \theta_{g}\right)$ and $\hat{\phi}_{f}\left(e \mid \theta_{f}\right)$ and the robust adaptive control term $\tau_{s w}$ will be designed via using Lyapunov 

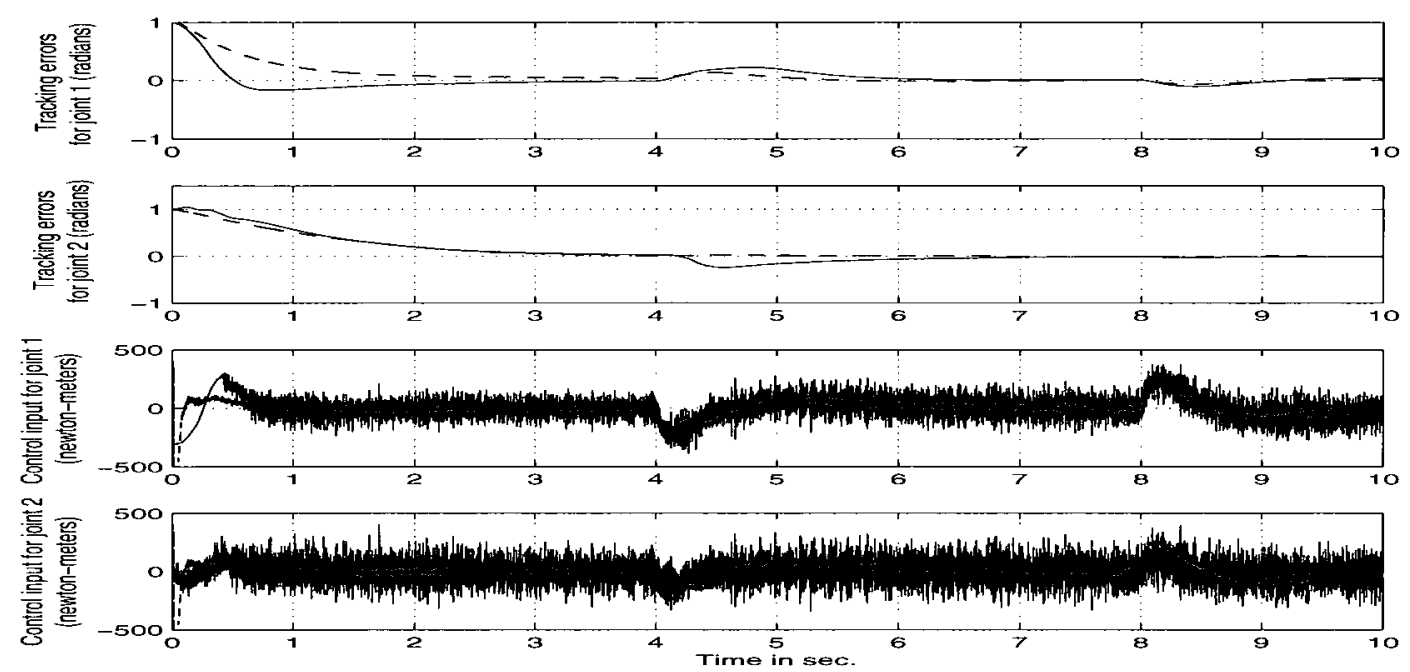

Figure 3.6: The tracking errors (radians), the control input (newton-meters) for joint 1 and joint 2 under Theorem 3.1 and Theorem 3.3. The sloid-line is for Theorem 1 and the red-dash-line is for Theorem 3.

function analysis. Using (3.56), one obtains the following error model

$$
\begin{aligned}
\ddot{e}_{1}= & -K_{D} \dot{e}_{1}-K_{P} e_{1}-\left[\phi_{f}\left(e_{1}, \dot{e}_{1}\right)-\hat{\phi}_{f}\left(\left(e_{1}, \dot{e}_{1}\right) \mid \theta_{f}\right)\right]+ \\
& {\left[\hat{\phi}_{g}\left(e_{1} \mid \theta_{g}\right)-\phi_{g}\left(e_{1}\right)\right] \tau_{c}-\tau_{s w}-d }
\end{aligned}
$$

Let us define new state variable as $e_{1}=\left(q_{d}-q_{1}\right)$ and $e_{2}=\left(\dot{q}_{d}-\dot{q}_{1}\right)$. The closedloop tracking error model (3.57) can then be defined in the following state-space form as ${ }^{3}$

$$
\begin{aligned}
\dot{e}= & B\left(\left[\phi_{f}(e)-\hat{\phi}_{f}\left(e \mid \theta_{f}\right)\right]+\left[\phi_{g}\left(e_{1}\right)-\hat{\phi}_{g}\left(e_{1} \mid \theta_{g}\right)\right] \tau_{c}\right) \\
& -B \tau_{s w}-B d+A e
\end{aligned}
$$

where $A=\left[\begin{array}{cc}0_{n \times n} & I_{n \times n} \\ -K_{P} & -K_{D}\end{array}\right], B=\left[\begin{array}{c}0_{n \times n} \\ I_{n \times n}\end{array}\right]$ and $e=\left[e_{1}^{T}, e_{2}^{T}\right]^{T}$. To update the unknown parameters $\theta_{f}, \theta_{g}$ and $\theta_{s w}$, we will use Lyapunov technique and show

\footnotetext{
${ }^{3}$ For brevity, we represent control term $\tau_{c}\left(e_{1}, \dot{e}_{1}, Q_{d}, \theta_{f}, \theta_{g}, \theta_{s w}\right)$ as $\tau_{c}$, where we remove its arguments.
} 
boundedness property for all the signals in the closed-loop error model dynamics (3.58). We consider that the error vectors $e$ and the parameter vectors $\theta_{f}$ and $\theta_{g}$ belong to the compact sets as $\Omega_{e}, \Omega_{f}$ and $\Omega_{g}$, respectively. Then the optimal parameter vectors $\theta_{f}^{*}$ and $\theta_{g}^{*}$ of $\hat{\phi}_{f}($.$) and \hat{\phi}_{g}($.$) can be defined as$

$$
\begin{aligned}
\theta_{f}^{*} & =\arg \min _{\theta_{f} \in \Omega_{f}}\left[\sup _{e \in \Omega_{c}}\left\|\hat{\phi}_{f}\left(e \mid \theta_{f}\right)-\phi_{f}(e)\right\|\right] \\
\theta_{g}^{*} & \left.=\arg \min _{\theta_{g} \in \Omega_{g}}\left[\sup _{e \in \Omega_{c}} \| \hat{\phi}_{g}\left(e_{1} \mid \theta_{g}\right)-\phi_{g}\left(e_{1}\right)\right) \|\right]
\end{aligned}
$$

The optimal vectors $\theta_{f}^{*}$ and $\theta_{g}^{*}$ are chosen as the values of $\theta_{f}$ and $\theta_{g}$ that minimizes the fuzzy approximation error over the given set $\Omega_{f}$ and $\Omega_{g}$, respectively. The minimum approximation error can be defined as follows

$$
w_{e}=\left[\hat{\phi}_{f}\left(e \mid \theta_{f}^{*}\right)-\phi_{f}(e)\right]+\left[\hat{\phi}_{g}\left(e_{1} \mid \theta_{g}^{*}\right)-\phi_{g}\left(e_{1}\right)\right] \tau_{c}
$$

Applying (3.60), the error system under hybrid control leads to

$$
\begin{aligned}
\dot{e}= & A e+B\left[\phi_{f}\left(e \mid \theta_{f}^{*}\right)-\hat{\phi}_{f}\left(e \mid \theta_{f}\right)\right]+B\left(w_{e}-d\right)-B \tau_{s w} \\
& +B\left[\phi_{g}\left(e_{1} \mid \theta_{g}^{*}\right)-\hat{\phi}_{g}\left(e_{1} \mid \theta_{g}\right)\right] \tau_{c}
\end{aligned}
$$

The above error model dynamics can be simplified as

$$
\dot{e}=A e+B \tilde{\theta}_{f} \xi(e)+B \tilde{\theta}_{g} \xi(e) \tau_{c}+B w-B \tau_{s w}
$$

where $w=\left(w_{e}-d\right), \tilde{\theta}_{f}=\left(\theta_{f}^{*}-\theta_{f}\right)$ and $\tilde{\theta}_{g}=\left(\theta_{g}^{*}-\theta_{g}\right)$. We now consider the following assumption.

Assumption 3.2: The modeling errors, external disturbances and any other unmodeled dynamical effects are bounded as $\|w\| \leq \theta_{s w}^{*}$ with $\theta_{s w}^{*}>0$.

Like the assumption 3.1, the parameter defined above $\theta_{s w}^{*}$ is unknown that will be estimated by using robust adaptive control law. We now establish stability preserving adaptation law to design and update robust adaptive control as well as update the unknown parameter vectors of the fuzzy systems defined in the closedloop error model (3.62). To do that, we consider the following Lyapunov function 
candidate

$$
V=\frac{1}{2} e^{T} P e+\frac{1}{2 \Gamma_{1}} \tilde{\theta}_{f}^{T} \tilde{\theta}_{f}+\frac{1}{2 \Gamma_{2}} \operatorname{tr}\left\{\tilde{\theta}_{g}^{T} \tilde{\theta}_{g}\right\}+\frac{1}{2 \Gamma_{3}} \tilde{\theta}_{s w}^{T} \tilde{\theta}_{s w}
$$

where, $\Gamma_{1}>0, \Gamma_{2}>0$ and $\Gamma_{3}>0$. Taking derivative (3.63) along the trajectory of (3.62), we simplify $\dot{V}$ as

$$
\begin{aligned}
\dot{V}= & -\frac{1}{2} e^{T} Q e+\tilde{\theta}_{f}^{T}\left(\Gamma_{1}^{-1} \dot{\tilde{\theta}}_{f}+B^{T} P e \xi^{T}(e)\right)+e^{T} P B \\
& \left(\theta_{s w}^{*}-\tau_{s w}\right)+\operatorname{tr}\left\{\tilde{\theta}_{g}^{T} \Gamma_{2}^{-1} \dot{\tilde{\theta}}_{g}\right\}+\operatorname{tr}\left\{\tau_{c}^{T} \xi_{g}^{T}(e) \tilde{\theta}_{g}^{T} B^{T} P e\right\}+\frac{1}{\Gamma_{3}} \tilde{\theta}_{s w}^{T} \dot{\tilde{\theta}}_{s w}(3.6)
\end{aligned}
$$

From (3.64), it is not hard to design $\tau_{s w}$ which can cope with uncertainties that enters into the closed-loop system due to the presence of the external disturbances and any other modeling errors as

$$
\tau_{s w}=\theta_{s w} \operatorname{sgn}\left(e^{T} P B\right)
$$

Applying (3.65), one then simplifies (3.64) as

$$
\begin{aligned}
\dot{V} \leq & -\frac{1}{2} e^{T} Q e+\tilde{\theta}_{f}^{T}\left(\Gamma_{1}^{-1} \dot{\tilde{\theta}}_{f}+B^{T} P e \xi^{T}(e)\right)+ \\
& \tilde{\theta}_{s w}^{T}\left[\Gamma_{3}^{-1} \dot{\tilde{\theta}}_{s w}+e^{T} P B \operatorname{sgn}\left(e^{T} P B\right)\right]+\tilde{\theta}_{g}^{T}\left(\Gamma_{2}^{-1} \dot{\tilde{\theta}}_{g}+B^{T} P e \tau_{c}^{T} \xi_{g}^{T}(e)\right)
\end{aligned}
$$

It is clear from (3.66) that one needs the following adaptation law to make $\dot{V} \leq 0$

$$
\begin{aligned}
\dot{\tilde{\theta}}_{f} & =-\Gamma_{1} B^{T} P e \xi^{T}(e) \\
\dot{\tilde{\theta}}_{g} & =-\Gamma_{2} B^{T} P e \tau_{c}^{T} \xi_{g}^{T}(e) \\
\dot{\tilde{\theta}}_{s w} & =-\Gamma_{3}\left(e^{T} P B\right) \operatorname{sgn}\left(e^{T} P B\right)
\end{aligned}
$$

Then use projection based parameter adaptation mechanism to limit the parameter estimate to guarantee that $\theta_{f}, \theta_{g}$ and $\theta_{s w}$ are bounded over the compact sets $\Omega_{f}$, $\Omega_{g}$ and $\Omega_{s w}$. To show that, we now define the compact sets $\hat{\Omega}_{f}, \hat{\Omega}_{g}$ and $\hat{\Omega}_{s w}$ such that $\hat{\Omega}_{f} \subset \Omega_{f}, \hat{\Omega}_{g} \subset \Omega_{g}$ and $\hat{\Omega}_{s w} \subset \Omega_{s w}$. The convex hypercube $\Omega_{f}$ is defined as $\Omega_{f}=\left\{x_{i} \leq \theta_{f i} \leq y_{i}\right\}$. Let $\Omega_{\delta f}$ as $\Omega_{\delta f}=\left\{x_{i}-\delta \leq \theta_{f i} \leq y_{i}-\delta\right\}$ where $\delta$ is chosen such that $\hat{\Omega}_{f} \subset \Omega_{\delta f}$ and adaptation gain $\Gamma_{1}$ to be positive constant. Then, the projection of the parameter adaptation rule can be written as $\dot{\theta}_{f}=\operatorname{Proj}\left(\theta_{f}, \Phi_{f}\right)$ 
[37] with $\bar{\Phi}_{f i}=\left[1+\frac{y_{i}-\theta_{f i}}{\delta}\right] \Phi_{f i}, \breve{\Phi}_{f i}=\left[1+\frac{\theta_{f i}-x_{i}}{\delta}\right] \Phi_{f i}$ and $\Phi_{f i}$ is the $i$-th element of $B^{T} P e \xi^{T}(e)$. To show $\theta_{f}(t) \in \Omega_{\delta f}, \theta_{g}(t) \in \Omega_{\delta g}$ and $\theta_{s w}(t) \in \Omega_{\delta_{s w}} \forall t>0$, we modify (3.67) to update $\theta_{f}, \theta_{g}$ and $\theta_{s}$ as

$$
\begin{gathered}
\dot{\theta}_{f}=\operatorname{Proj}\left(\theta_{f}, \Gamma_{1} B^{T} P e \xi^{T}(e)\right) \\
\dot{\theta}_{g}=\operatorname{Proj}\left(\theta_{g}, \Gamma_{2} B^{T} P e \tau_{c}^{T} \xi_{g}^{T}(e)\right) \\
\dot{\theta}_{s w}=\operatorname{Proj}\left(\theta_{s w}, \Gamma_{3}\left(e^{T} P B\right) \operatorname{sgn}\left(e^{T} P B\right)\right)
\end{gathered}
$$

The idea of using projection scheme is that if the parameters estimate start in the set $\theta_{f}(0) \in \Omega_{f}, \theta_{g}(0) \in \Omega_{g}$ and $\theta_{s w}(0) \in \Omega_{s w}$, then their estimate will be remained in $\theta_{f}(t) \in \Omega_{\delta f}, \theta_{g}(t) \in \Omega_{\delta g}$ and $\theta_{s w}(t) \in \Omega_{\delta_{s w}} \forall t \geq 0$.

Based on our above analysis, we now state our main results in Theorem 3.4 for the position-velocity hybrid control system.

Theorem 3.4: Let assumption 3.2 holds. Then the closed-loop system (3.62) composed of (3.56), (3.65), (3.67) along with parameter projection mechanism is bounded and the tracking errors converge to zero globally.

Proof: Applying hybrid controller with parameter projection mechanism, the derivative of $V(3.66)$ can be written as

$$
\dot{V} \leq-\frac{1}{2} e^{T} Q e \leq 0
$$

Then, we can conclude that $\left(V, e, \theta_{f}, \theta_{g}, \theta_{s w}\right) \in L_{\infty}$. In view of the right hand side of equation (3.62), we can see that all the variables are bounded. This implies that $\dot{e} \in L_{\infty}$. Taking integral (3.68) from 0 to $T$, we have

$$
V(T)-V(0) \leq-\int_{0}^{T} \frac{\lambda_{\min .}(Q)}{2}\|e\|^{2}
$$

Then, in view of (3.63), we can write

$$
\begin{array}{r}
\int_{0}^{T} \frac{\lambda_{m i n .}(Q)}{2}\|e\|^{2} \leq \frac{1}{2} e(0)^{T} Q e(0)+\frac{1}{2 \Gamma_{1}} \tilde{\theta}_{f}(0)^{T} \tilde{\theta}_{f}(0) \\
+\frac{1}{2 \Gamma_{2}} \operatorname{tr}\left\{\tilde{\theta}_{g}(0)^{T} \tilde{\theta}_{g}(0)\right\}+\frac{1}{2 \Gamma_{3}} \tilde{\theta}_{s w}(0)^{T} \tilde{\theta}_{s w}(0)
\end{array}
$$


This means that $e \in L_{2}$. Thus, $\lim _{t \rightarrow \infty} \dot{V}=0$ and $\lim _{t \rightarrow \infty} e=0$ provided that the parameter estimation errors are bounded.

It is important to note that the $\operatorname{sgn}($.$) function in (3.65) and \dot{\theta}_{s w}$ may cause discontinuous control action. To smooth out $\tau_{s w}$ and $\dot{\theta}_{s w}$, we can estimate $\operatorname{sgn}($.) by using following bounded input as

$$
\tau_{s w}=\theta_{s w} \tanh \left(\frac{e^{T} P B}{\epsilon_{o}}\right)
$$

with

$$
\dot{\theta}_{s w}=\operatorname{Proj}\left(\theta_{s w}, \Gamma_{3}\left(e^{T} P B\right) \tanh \left(\frac{e^{T} P B}{\epsilon_{o}}\right)\right)
$$

where $\epsilon_{o}>0$ and $\tanh ($.$) is a smooth bounded saturation function$

With this modification, Theorem 3.4 has the following form.

Theorem 3.5: Let assumption 3.2 holds. Then, all the signals in the closedloop system (3.62) composed of (3.56), (3.67), (3.70), (3.71) with the parameter projection mechanism are bounded that is the tracking errors and parameter errors are bounded as $\left(e, \theta_{f}, \theta_{g}, \theta_{s w}\right) \in L_{\infty}$.

Proof: The proof of Theorem 3.5 can be shown along the line of the proof of Theorem 3.4 [97]. So, we removed the details proof for brevity.

The hybrid control system developed in Theorem 3.4 and Theorem 3.5 is based on using strict assumption that the output and its derivatives are available for feedback. We now remove this assumption by replacing the unknown velocity signals $e$ in (3.56) by the output of the linear observer $\hat{e}$ (3.22). We then prove that the stability condition under state feedback based design of Theorem 3.4 and Theorem 3.5 can be obtained asymptotically by the output feedback form of Theorem 3.4 and Theorem 3.5. This recovery analysis can be shown via using perturbation theory provided that there exists a small perturbed observer design parameter $\epsilon$. To show that, let us develop singularly perturbed observer-controller 
closed-loop system ${ }^{4}$

$$
\begin{aligned}
\epsilon \dot{\eta}= & B \epsilon\left[-K e+\tilde{\theta}_{f} \xi(e)+\tilde{\theta}_{g} \xi_{g}(e) \tau_{c}+\tilde{\theta}_{s w} \operatorname{sgn}\left(\hat{e}^{T} P B\right)\right] \\
& +A_{o} \eta \\
\dot{e}= & A e+B\left[\tilde{\theta}_{f} \xi(e)+\tilde{\theta}_{g} \xi_{g}(e) \tau_{c}+\tilde{\theta}_{s w} \operatorname{sgn}\left(\hat{e}^{T} P B\right)\right]
\end{aligned}
$$

with $A_{o}=\left[A_{c}-\bar{L} C\right], \bar{L}=\left[\begin{array}{c}H_{1} \\ H_{2}\end{array}\right], K=\left[K_{P} K_{D}\right], \tilde{e}=\left[\tilde{e}_{1}^{T}, e_{2}^{T}\right]^{T}, \tilde{\eta}=\left[\tilde{\eta}_{1}^{T}, \eta_{2}^{T}\right]^{T}$, $C=\left[\begin{array}{ll}I_{n \times n} & 0_{n \times n}\end{array}\right]$ and adaptation laws are given by

$$
\begin{gathered}
\dot{\theta}_{f}=\operatorname{Proj}\left(\theta_{f}, \Gamma_{1} B^{T} P \hat{e} \xi^{T}(\hat{e})\right) \\
\dot{\theta}_{g}=\operatorname{Proj}\left(\theta_{g}, \Gamma_{2} B^{T} P \hat{e} \tau_{c}^{T} \xi_{g}^{T}(\hat{e})\right) \\
\dot{\theta}_{s w}=\operatorname{Proj}\left(\theta_{s w}, \Gamma_{3}\left(\hat{e}^{T} P B\right) \operatorname{sgn}\left(\hat{e}^{T} P B\right)\right)
\end{gathered}
$$

Now, it is not hard to show from (3.72), (3.73) that the performance achieved under state feedback design can be recovered asymptotically by the output feedback controller. Then we can state the main results for hybrid adaptive output feedback control in the following Theorem 3.6.

Theorem 3.6: Consider the closed-loop system (3.72), (3.73) formulated by using (3.3), (3.56), (3.65), (3.67) with the parameter projection mechanism and linear observer (3.22). Then, for any given $e(0) \in \Omega_{o}, \theta_{f}(0) \in \Omega, \theta_{g}(0) \in \Omega$ and $\theta_{s w}(0) \in \Omega_{s w}$, there exists $\epsilon^{*}$ such that $\forall \epsilon \in\left[0, \epsilon^{*}\right]$ all signals in closed loop dynamics are bounded and their bound can be made very small for small value of $\epsilon$.

Proof: The proof of Theorem 3.6 can be shown along the line of [97]. It is proven that there exists a short transient period $T_{1}(\epsilon) \in\left[0, T_{2}\right]$ during which the fast variable $\eta$ approaches to a function of the order $O(\epsilon)$, while the slow variables $\left(e, \theta_{f}, \theta_{g}, \theta_{s w}\right)$ remain in the subset of the domain of attraction $\Omega_{c}$. Then, show that

\footnotetext{
${ }^{4}$ One may use the observer-controller closed-loop model as $\epsilon \dot{\eta}=A_{o} \eta+B \epsilon\left[-K \hat{e}+\theta_{f}^{*} \xi(e)-\right.$ $\left.\theta_{f} \xi(\hat{e})+\theta_{g}^{*} \xi_{g}(e) \tau_{c}-\theta_{g} \xi_{g}(\hat{e}) \tau_{c}+\theta_{s w}^{*} \operatorname{sgn}\left(e^{T} P B\right)-\theta_{s w} \operatorname{sgn}\left(\hat{e}^{T} P B\right)\right], \dot{e}=A e+B\left[\theta_{f}^{*} \xi(e)-\theta_{f} \xi(\hat{e})+\right.$ $\left.\theta_{g}^{*} \xi_{g}(e) \tau_{c}-\theta_{g} \xi_{g}(\hat{e}) \tau_{c}+\theta_{s w}^{*} \operatorname{sgn}\left(e^{T} P B\right)-\theta_{s w} \operatorname{sgn}\left(\hat{e}^{T} P B\right)\right]$. For brevity, we represent the control term $\tau_{c}\left(\hat{e}, Q_{d}, \theta_{f}, \theta_{g}, \theta_{s w}\right)$ as $\tau_{c}$.
} 
the boundedness property of the signal $e(t)$ for all $t \in\left[T_{1}(\epsilon), T_{3}\right]$, where $T_{1}(\epsilon) \in$ $\left(0, T_{2}\right]$ and $T_{3} \geq T_{2}$ is the first time $e(t)$ exists from the subset of the domain of attraction $\Omega_{c}$ of the state feedback design (3.56), (3.65), (3.67) along with the parameter projection mechanism. This implies that the state trajectories $(e, \eta)$ are locked into a set that can be made very small by using small value of observer design parameter $\epsilon$.

We begin our analysis by showing that there exists a short transient period during which the fast variable $\eta$ decays to a small level as $O(\epsilon)$ for a small value of $\epsilon>0$. To prove that, we consider the positive definite Lyapunov-function candidate as defined in equation (3.63). From (3.63), we can see that the second, third and fourth part of the Lyapunov function $V$ is bounded by $c_{2}, c_{3}$ and $c_{4}$. First assume that all the initial conditions are bounded on the given compact subsets of $\Omega_{c}$ as $\theta_{f}(0) \in \Omega_{f}, \theta_{g}(0) \in \Omega_{g}, \theta_{s w}(0) \in \Omega_{s w}$ and $e(0) \in \Omega_{o}$. Then, we use universal approximation theorem and assumption 3.2 to show that the optimal parameter $\theta_{f}^{*}$ and $\theta_{g}^{*}$ are bounded by the compact set as $\theta_{f}^{*} \in \Omega_{f}$ and $\theta_{g}^{*} \in \Omega_{g}$, respectively. In view of the projection mechanism, we also know that the parameter estimate is bounded as $\theta_{f}(t) \in \Omega_{\delta f}, \theta_{g}(t) \in \Omega_{\delta g}$ and $\theta_{s w}(t) \in \Omega_{\delta s w}$. Let

$$
\begin{gathered}
c_{1}=\max _{e \in \Omega_{o}} \frac{1}{2} e^{T} P e \\
c_{2}=\max _{\theta_{f}^{*} \in \Omega_{f}, \theta_{f} \in \Omega_{\delta f}} \frac{1}{2 \Gamma_{1}}\left(\theta_{f}^{*}-\theta_{f}\right)^{T}\left(\theta_{f}^{*}-\theta_{f}\right) \\
c_{3}=\max _{\theta_{g}^{*} \in \Omega_{g}, \theta_{g} \in \Omega_{\delta g}} \frac{1}{2 \Gamma_{2}} \operatorname{tr}\left\{\left(\theta_{g}^{*}-\theta_{g}\right)^{T}\left(\theta_{g}^{*}-\theta_{g}\right)\right\} \\
c_{4}=\max _{\theta_{s w}^{*} \in \Omega_{s w}, \theta_{s w} \in \Omega_{\delta s w}} \frac{1}{2 \Gamma_{3}}\left(\theta_{s w}^{*}-\theta_{s w}\right)^{T}\left(\theta_{s w}^{*}-\theta_{s w}\right)
\end{gathered}
$$

where $c_{1}>0, c_{2}>0, c_{3}>0$ and $c_{4}>0$. Then define positive constant $c$ with $c>\left(c_{o}=c_{1}+c_{2}+c_{3}+c_{4}\right)$ such that $\Omega_{c}$ be the domain of attraction as $e(t) \in \Omega_{c}=\left\{e \mid e^{T} P e \leq c\right\} \forall t \geq 0$. Since the input $\tau_{c}$ is continuous function with respect to their arguments, then they are bounded on the compact set of these variables as $\forall\left(e, Q_{d}, \theta_{f}, \theta_{g}, \theta_{s w}\right) \in \Omega_{c} \times \Omega_{D} \times \Omega_{\delta f} \times \Omega_{\delta g} \times \Omega_{\delta s w}$. We then define the maximum bound on $\tau_{\max } \geq \tau_{c} \forall e \in \Omega_{c r}$ with $e \in \Omega_{c r}=\left\{e \mid e^{T} P e \leq c r\right\}$ and 
$c r>c$. The bounded control input can then be defined as $\tau_{c}^{s}=\tau_{\max } \operatorname{sat}\left(\frac{\tau_{c}}{\tau_{\text {max }}}\right)=\tau_{c}$ with a smooth bounded saturation function $\operatorname{sat}($.). Therefore, $V$ will be remained bounded by a constant $c_{T_{2}}$ which is less than $c$ and greater than $c_{o}$ that is $c>c_{T_{2}}$ and $c_{T_{2}}>c_{o}$ with $c_{T_{2}}=c_{2}+c_{3}+c_{4}$ as $\theta_{f}(t) \in \Omega_{\delta f}, \theta_{g}(t) \in \Omega_{\delta g}$ and $\theta_{s w}(t) \in \Omega_{\delta_{s w}}$. To illustrate that, we differentiate (3.63) along the slow trajectory (3.73) to simplify the derivative of $V$ as

$$
\begin{aligned}
\dot{V}= & -\tilde{\theta}_{s w}\left(\hat{e}^{T} P B\right) \operatorname{sgn}\left(\hat{e}^{T} P B\right)+\tilde{\theta}_{s w}\left(e^{T} P B\right) \operatorname{sgn}\left(e^{T} P B\right) \\
& +\operatorname{tr}\left\{\tilde{\theta}_{g}^{T}\left(B^{T} P e \tau_{c}^{s^{T}} \xi_{g}^{T}(e)-B^{T} P \hat{e} \tau_{c}^{s^{T}} \xi^{T}(\hat{e})\right)\right\} \\
& -\frac{1}{2} e^{T} Q e+\tilde{\theta}_{f}^{T}\left(-B^{T} P \hat{e} \xi^{T}(\hat{e})+B^{T} P e \xi^{T}(e)\right)
\end{aligned}
$$

Since the input and adaptation law are bounded over the set $\Omega_{c} \times \Omega_{D} \times \Omega_{\delta f} \times \theta_{\delta g} \times$ $\Omega_{\delta s w}$ for all $e \in \Omega_{c}, \hat{e} \in \Omega_{c}, Q_{d} \in \Omega_{D}, \theta_{f}(t) \in \Omega_{\delta f}, \theta_{g}(t) \in \Omega_{\delta g}, \theta_{s w}(t) \in \Omega_{\delta s w}$, then we have the following bound on the first, second and fourth terms of equation (3.74)

$$
\begin{gathered}
\left\|\operatorname{tr}\left\{\tilde{\theta}_{g}^{T}\left(B^{T} P e \tau_{c}^{s^{T}} \xi_{g}^{T}(e)-B^{T} P \hat{e} \tau_{c}^{s^{T}} \xi_{g}^{T}(\hat{e})\right)\right\}\right\| \leq k_{1 s v} \\
\left\|-\tilde{\theta}_{s w}\left(\hat{e}^{T} P B\right) \operatorname{sgn}\left(\hat{e}^{T} P B\right)+\tilde{\theta}_{s w}\left(e^{T} P B\right) \operatorname{sgn}\left(e^{T} P B\right)\right\| \leq k_{2 s v} \\
\left\|\tilde{\theta}_{f}^{T}\left(-B^{T} P \hat{e} \xi^{T}(\hat{e})+B^{T} P e \xi^{T}(e)\right)\right\| \leq k_{3 s v}
\end{gathered}
$$

with $k_{1 s v}>0, k_{2 s v}>0$ and $k_{3 s v}>0$. Applying above inequalities, equation (3.74) can be simplified as

$$
\dot{V} \leq-\frac{1}{2} e^{T} Q e+k_{s}
$$

where $k_{s}=k_{1 s v}+k_{2 s v}+k_{3 s v}$. In view of Lyapunov function candidate (3.63), the bound on error trajectory can be written as follows

$$
\dot{V} \leq-\frac{1}{2} \frac{\lambda_{\min }(Q)}{\lambda_{\max }(P)} e^{T} P e+k_{s}
$$


Equation (3.76) can be simplified further as

$$
\dot{V} \leq-\gamma_{1 s} V+k_{s v}
$$

where $\gamma_{1 s}=\frac{\lambda_{\min }(Q)}{\lambda_{\max }(P)}$ and $k_{s v}=\chi_{s} c_{2}+\chi_{s} c_{3}+\chi_{s} c_{4}+k_{s}$ with $\chi_{s}=\frac{\lambda_{\min }(Q)}{\lambda_{\max }(P)}, \frac{1}{2 \Gamma_{f}} \tilde{\theta}_{f}^{T} \tilde{\theta}_{f} \leq$ $c_{2}, \frac{1}{2 \Gamma_{s w}} \tilde{\theta}_{s w}^{T} \tilde{\theta}_{s w} \leq c_{4}$ and $\frac{1}{2 \Gamma_{g}} \operatorname{tr}\left\{\tilde{\theta}_{g}^{T} \tilde{\theta}_{g}\right\} \leq c_{3}$. Then, the solution of the differential equation (3.77) can be written as

$$
V(t) \leq V(0) e^{-\gamma_{1 s} t}+\frac{k_{s v}}{\gamma_{1 s}}\left(1-e^{-\gamma_{1 s} t}\right)
$$

Since $V(0) \leq c_{T 2}<c$ with $c_{T 2}>c_{o}$, then there is a finite time $T_{2}>0$, which is independent of $\epsilon$, such that $V(t)<c$ for $\forall t \in\left[0, T_{2}\right)$. This implies that the slow variables remain inside the set $\Omega_{c}$. One can also replace $e$ by $\hat{e}$ in the closed loop model

$\dot{e}=A e+B\left[\left(\theta_{f}^{*} \xi(e)-\theta_{f} \xi(\hat{e})\right)+\left(\theta_{g}^{*} \xi_{g}(e) \tau_{c}^{s}-\theta_{g} \xi_{g}(\hat{e}) \tau_{c}^{s}\right)+\theta_{s w}^{*} \operatorname{sgn}\left(e^{T} P B\right)-\theta_{s w} \operatorname{sgn}\left(\hat{e}^{T} P B\right)\right]$

Then, we can prove that the slow variables remain bounded by the compact subset of the region of attraction $\Omega_{c}$ over the short transient time $\left[0, T_{2}\right]$. To show that, let us take derivative of (3.63) along the trajectory of the closed-loop system defined above to simplify $\dot{V}$ as

$$
\begin{aligned}
\dot{V}= & -\frac{1}{2} e^{T} Q e+\frac{1}{\Gamma_{1}} \tilde{\theta}_{f}^{T} \dot{\tilde{\theta}}_{f}+\frac{1}{\Gamma_{2}} \operatorname{tr}\left\{\tilde{\theta}_{g}^{T} \dot{\tilde{\theta}}_{g}\right\}+\frac{1}{\Gamma_{3}} \tilde{\theta}_{s w} \dot{\tilde{\theta}}_{s w} \\
& +e^{T} P B\left[\theta_{f}^{*} \xi(e)-\theta_{f} \xi(\hat{e})\right]+e^{T} P B\left[\theta_{g}^{*} \xi_{g}(e) \tau_{c}^{s}-\theta_{g} \times\right. \\
& \left.\xi_{g}(\hat{e}) \tau_{c}^{s}\right]+e^{T} P B\left[\theta_{s w}^{*} \operatorname{sgn}\left(e^{T} P B\right)-\theta_{s w} \operatorname{sgn}\left(\hat{e}^{T} P B\right)\right]
\end{aligned}
$$

Using the control along with the projection based adaptation mechanism, we can simplify $\dot{V}$ as follows

$$
\begin{aligned}
\dot{V}= & -\frac{1}{2} e^{T} Q e-\operatorname{tr}\left\{\tilde{\theta}_{g}^{T} B^{T} P \hat{e} \tau_{c}^{s^{T}} \xi_{g}^{T}(\hat{e})\right\}-\tilde{\theta}_{f}^{T} B^{T} P \hat{e} \xi^{T}(\hat{e})-\tilde{\theta}_{s w}\left(\hat{e}^{T} P B\right) s g n \\
& \left(\hat{e}^{T} P B\right)+e^{T} P B\left[\theta_{f}^{*}-\xi(e) \theta_{f} \xi(\hat{e})\right]+e^{T} P B\left[\theta_{s w}^{*} \operatorname{sgn}\left(e^{T} P B\right)-\theta_{s w} \times\right. \\
& \left.\operatorname{sgn}\left(\hat{e}^{T} P B\right)\right]+e^{T} P B\left[\theta_{g}^{*} \xi_{g}(e) \tau_{c}^{s}-\theta_{g} \xi_{g}(\hat{e}) \tau_{c}^{s}\right]
\end{aligned}
$$


Then, $\forall\left(e, Q_{d}, \theta_{f}, \theta_{g}, \theta_{s w}, \hat{e}\right) \in \Omega_{c} \times \Omega_{D} \times \Omega_{\delta f} \times \Omega_{\delta g} \times \Omega_{s w} \times \Omega_{c}$, we have the following bounds

$$
\begin{aligned}
& \left\|-\operatorname{tr}\left\{\tilde{\theta}_{g}^{T} B^{T} P \hat{e} \tau_{c}^{s^{T}} \xi_{g}^{T}(\hat{e})\right\}+e^{T} P B\left[\theta_{g}^{*} \xi_{g}(e) \tau_{c}^{s}-\theta_{g} x i_{g}(\hat{e}) \tau_{c}^{s}\right]\right\| \leq k_{1_{T_{2} s}} \\
& \left\|-\tilde{\theta}_{s w}\left(\hat{e}^{T} P B\right) \operatorname{sgn}\left(\hat{e}^{T} P B\right)+\theta_{s w}^{*}\left(e^{T} P B\right) \operatorname{sgn}\left(e^{T} P B\right)-\theta_{s w}\left(e^{T} P B\right) \operatorname{sgn}\left(\hat{e}^{T} P B \hat{e}\right)\right\| \\
& \leq k_{2_{T_{2}}} \\
& \left\|-\tilde{\theta}_{f}^{T} B^{T} P \hat{e} \xi^{T}(\hat{e})+e^{T} P B\left[\theta_{f}^{*} \xi(e)-\theta_{f} \xi(\hat{e})\right]\right\| \leq k_{3_{T 3 s}}
\end{aligned}
$$

where $k_{1_{T 2 s}}>0, k_{2_{T 2 s}}>0$ and $k_{3_{T 3 s}}>0$. With these inequalities, $\dot{V}$ becomes $\dot{V} \leq-\frac{1}{2} e^{T} Q e+k_{T s}$ where $k_{T s}=k_{1_{T 1 s}}+k_{2_{T 2 s}}+k_{3_{T 3 s}}$. This implies that there is a finite time $T_{2}$ which is independent of $\epsilon$, such that for all $t \in\left[0, T_{2}\right] V(t) \leq c_{T 2}<c$ with $c_{o}<c_{T_{2}}<c$ and $e \in \Omega_{c_{T_{2}}}=\left\{e \mid e^{T} P e \leq c_{T_{2}}\right\}$. The remaining proof is omitted and can be found in [97].

Note that the switching function $\operatorname{sgn}($.$) in robust adaptive output feedback$ control design requires to estimate by using saturation function $\tanh ($.$) to reduce$ the chattering phenomenon $[5,6]$. This means that we need to replace $\operatorname{sgn}\left(\hat{e}^{T} P B\right)$ in the output feedback controller by using bounded function $\tanh \left(\frac{\hat{e}^{T} P B}{\epsilon_{o}}\right)$. Applying this modification, we then follow the above design steps to show that the tracking error bound under state feedback design stated in Theorem 3.5 can asymptotically $(\epsilon \rightarrow 0)$ recover by the output feedback design. The details performance recovery is omitted for brevity and can be found in [97] .

\subsubsection{Simulation Results}

To demonstrate the theoretical development, we now implement hybrid schemes that introduced in previous section on a 2-DOF robotic system (3.53). Our prime target is to track the same desired task as used in subsection 3.2.3. In our evaluation, the disturbance for two joints $d_{1}(t), d_{2}(t)$ in (3.53) are depicted in Figure 3.7 . 


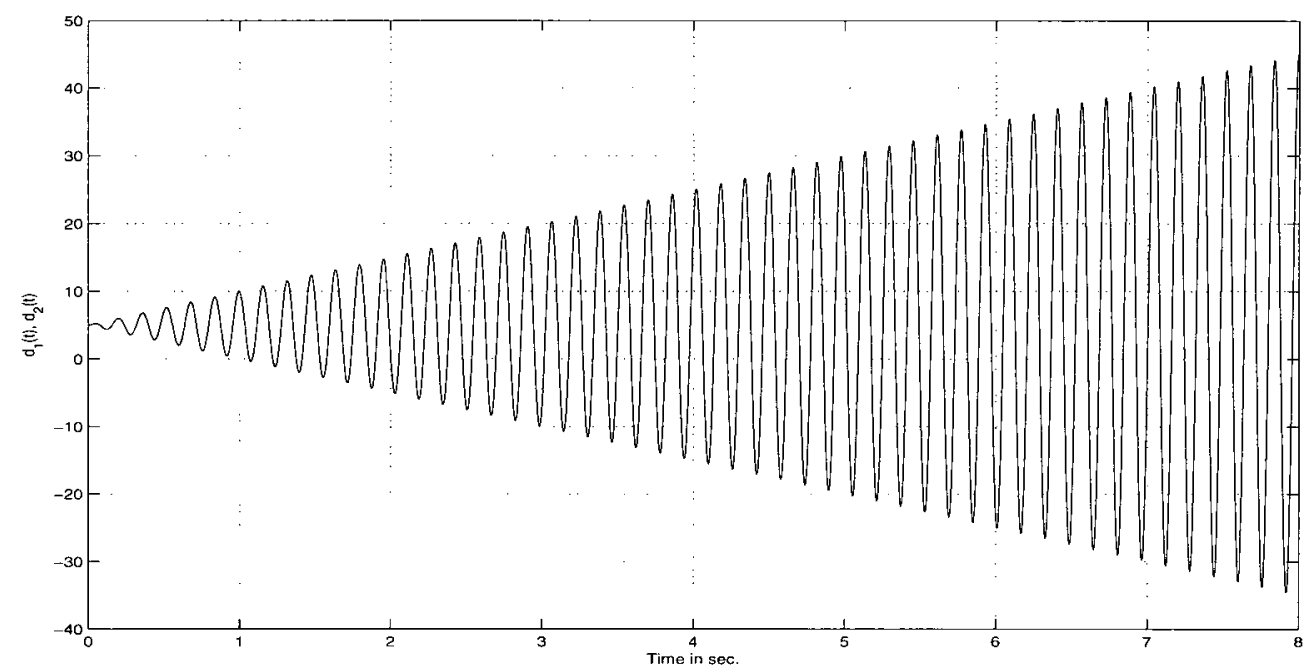

Figure 3.7: The time varying disturbance for two joints $d_{1}(t), d_{2}(t)$ of $(3.53)$

\subsubsection{Simulation Results with Theorem 3.6}

Let us implement and evaluate output feedback based hybrid design of Theorem 3.6 on the given system. The design assume that the velocity signals $q_{2}$ is not available for feedback design, but the position signals $q_{1}$ is available. To construct Theorem 3.6, we first formulate the fuzzy basis function for indirect fuzzy systems via choosing five fuzzy sets for each input variables. The membership functions for each inputs $q_{j}(j=1,2)$ are chosen as

$$
\begin{gathered}
\mu_{A_{j}^{1}}\left(q_{j}\right)=\frac{1}{1+\left[\exp \left(\frac{\left(q_{j}+0.75\right)}{0.1}\right)\right]^{2}} \\
\mu_{A_{j}^{2}}\left(q_{j}\right)=\exp \left(-\left(\frac{\left(q_{j}+0.5\right)}{0.3}\right)^{2}\right) \\
\mu_{A_{j}^{3}}\left(q_{j}\right)=\exp \left(-\left(\frac{q_{j}}{0.3}\right)^{2}\right) \\
\mu_{A_{j}^{4}}\left(q_{j}\right)=\exp \left(-\left(\frac{\left(q_{j}-0.5\right)}{0.3}\right)^{2}\right)
\end{gathered}
$$



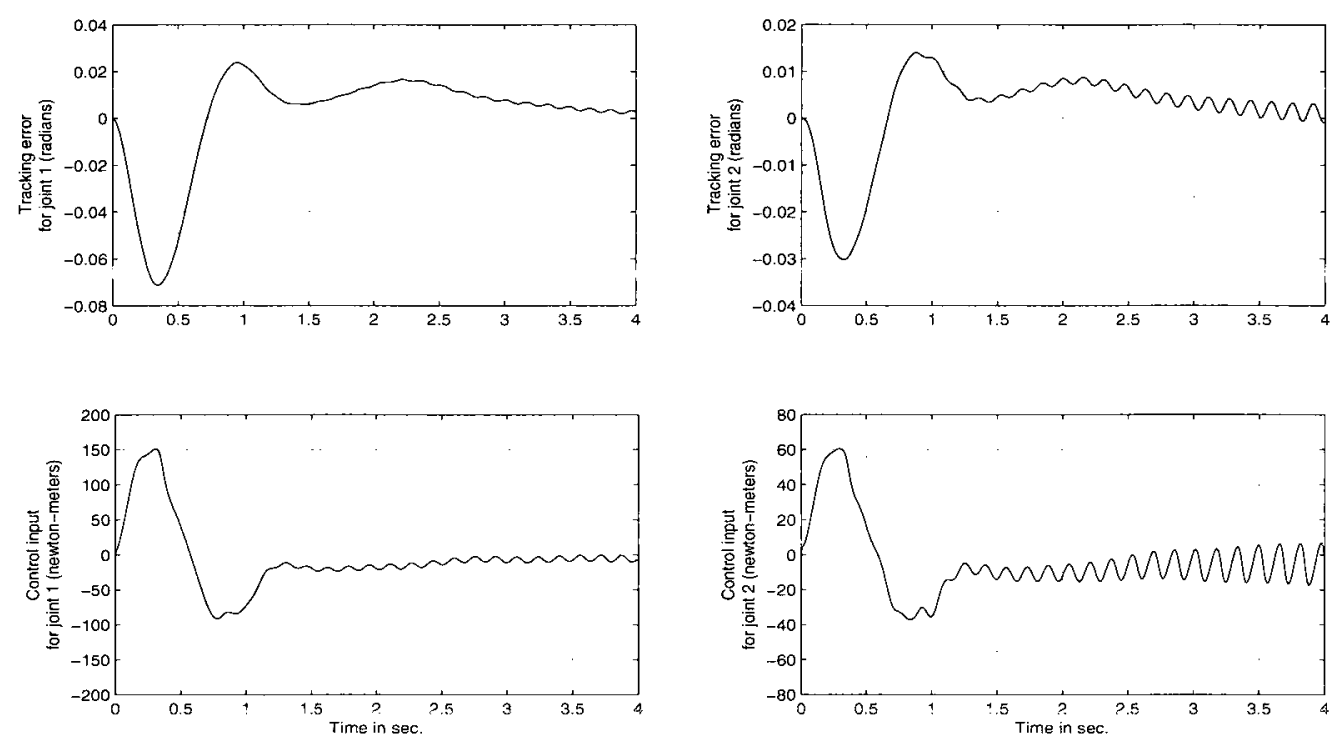

Figure 3.8: The tracking errors (radians), the control input (newton-meters) for joint 1 and joint 2 under Theorem 3.3.

and

$$
\mu_{A_{j}^{5}}\left(q_{j}\right)=\frac{1}{1+\left[\exp \left(-\left(\frac{\left(q_{j}-0.75\right)}{0.1}\right)\right)\right]^{2}}
$$

We then select PD-controller design parameters as $K_{P}=150 I_{2 \times 2}, K_{D}=150 I_{2 \times 2}$ and $\lambda_{o}=2$. Define the compact set as: $\Omega_{f}=\Omega_{g}=\left\{-10 \leq \theta_{d} \leq 10\right\}$, $\Omega_{s w}=\left\{-20 \leq \theta_{s} \leq 20\right\}$ and take $\delta=0.1$. We choose identity matrix of $Q$ to solve the Lyapunov-equation $A^{T} P+P^{T} A=-Q$ for $P$. We then define the learning gains $\Gamma_{1}=0.005, \Gamma_{2}=0.005$ and $\Gamma_{3}=0.005$. Finally, we define linear observer design parameters (3.22) $H_{1}=I_{2 \times 2}, H_{2}=I_{2 \times 2}$ and $\epsilon=0.005$. With these design constants, we implement Theorem 3.6 on the given system (5.53). The tested results are given in Figure 3.8. In view of Figure 3.8, we can see that the control performance under output feedback achieves desired tracking objective as the tracking errors for both joints almost closed to zero. 


\subsubsection{Comparison of CAOFB with Hybrid Design}

We now compare the tracking convergence of Theorem 3.4 and Theorem 3.6 with the CE-based nonlinear adaptive feedback design [82]. To do that, we consider that the plant is initially operating under the parameters $\theta_{1}=8$ and $\theta_{2}=8$. Then, at 5 sec., the parameter $\theta$ is changed from $\theta_{1}=8$ and $\theta_{2}=8$ to $\theta_{1}=4$ and $\theta_{2}=4$. This means that there are total two dynamics changes in the whole process dynamics. For comparison, we first design CE-based state feedback law [82] with the same PD-controller design parameters that applied in Theorem 3.6 as $K_{P}=150 I_{2 \times 2}$, $K_{D}=150 I_{2 \times 2}$ and $\lambda_{o}=2$. For our evaluation, the value of $\Gamma$ is selected as $\Gamma=15 I_{2 \times 2}$ such that the learning gains provides faster parameter learning. Note that the designer can select the control gains to guarantee exponential convergence of the closed-loop trajectory that starts inside a given region of interest. With these design constants, we then apply CE-based state feedback design [82]

$$
\tau\left(e, Q_{d}, \hat{\theta}\right)=Y\left(e, \dot{q}_{d}, \ddot{q}_{d}\right) \hat{\theta}-K_{P} e_{1}-K_{D} e_{2}
$$

on the given system (3.53). However, we notice that the control action become very large values at the time of dynamics changes. To protect the plant from large control action, we then saturate the input signals outside the given domain of interest. The saturation level for two control inputs is considered as $\tau_{1 \max }=500$ newton-meters and $\tau_{2 \max }=500$ newton-meters. Using with the above saturation level, we again implement above CE-based state feedback design on the system (5.53). The tested results are depicted in Figure 3.9. With this saturation level, we again apply state feedback based CE design on the system [82]. The tested results are given in Figure 3.8 .

We now assume that the velocity signals $q_{2}$ is not available but estimated by the output of the observer (3.22). The observer design constants are chosen as $H_{1}=I_{2 \times 2}, H_{2}=I_{2 \times 2}$ and $\epsilon=0.005$. Then, we apply (3.54) with (3.22) on the robot system (5.53). The tested results under output feedback designs (3.54) are depicted in the Figure 3.9. 

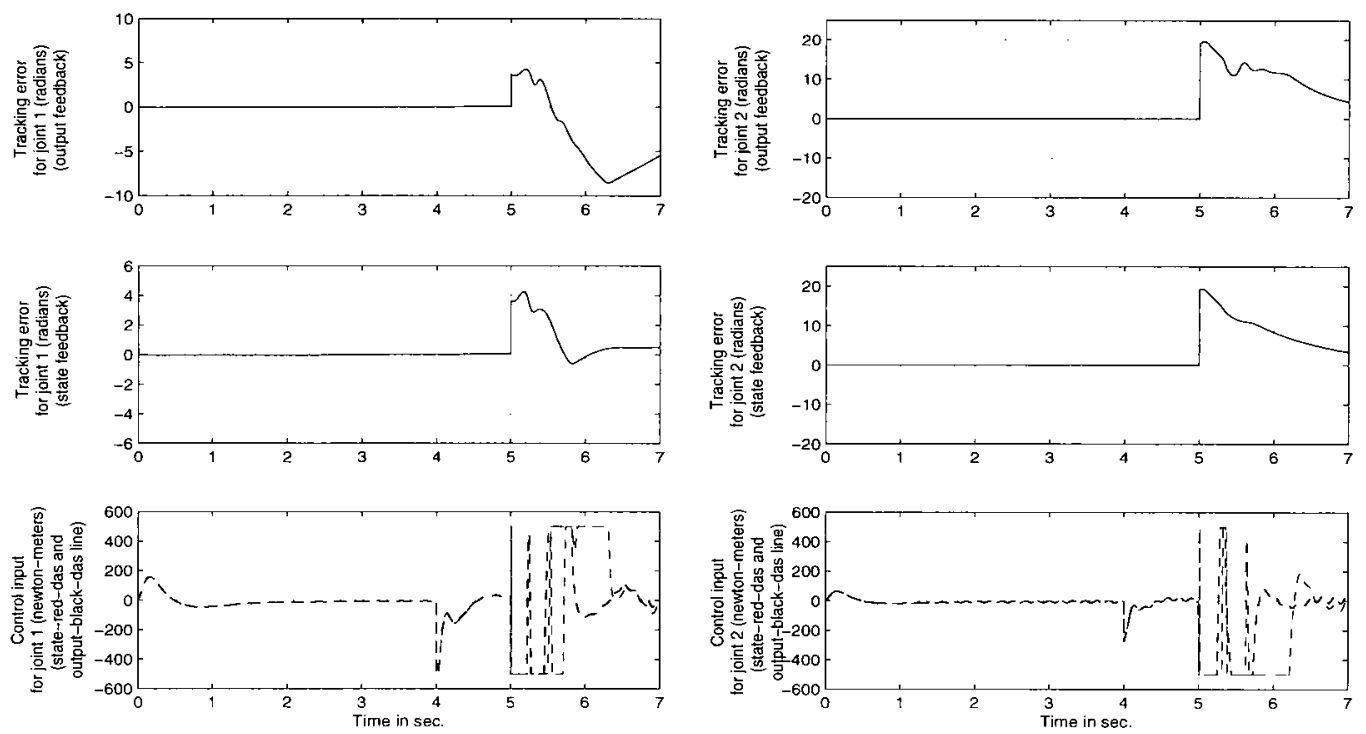

Figure 3.9: The tracking errors (radians), the control input (newton-meters) for joint 1 and joint 2 under CE-based state and output feedback design.

\subsubsection{Comparison of Theorem 3.4 with Theorem 3.6}

For comparison, we now implement Theorem 3.4 and Theorem 3.6 under the same parameter sets up as used in our last evaluation for the CE-based design. The conducted results under Theorem 3.4 (state feedback) and Theorem 3.6 (output feedback) designs are presented in Figure 3.10. The design parameters for this evaluation are kept similar to our last evaluation for Theorem 3.6 in subsection 3.2.3.

In view of Figures 3.9 and 3.10, we can observe that the control performance under Theorem 3.4 and Theorem 3.6 is much better than the performance achieved under CE-based nonlinear adaptive design. Specifically, at the time of dynamic changes, the tracking errors and control inputs under classical approach are larger than the tracking errors and control inputs under hybrid control system design. This is mainly because the control design of CE-based design is sensitive to the model parameter changes while the performance under proposed design is insensi- 

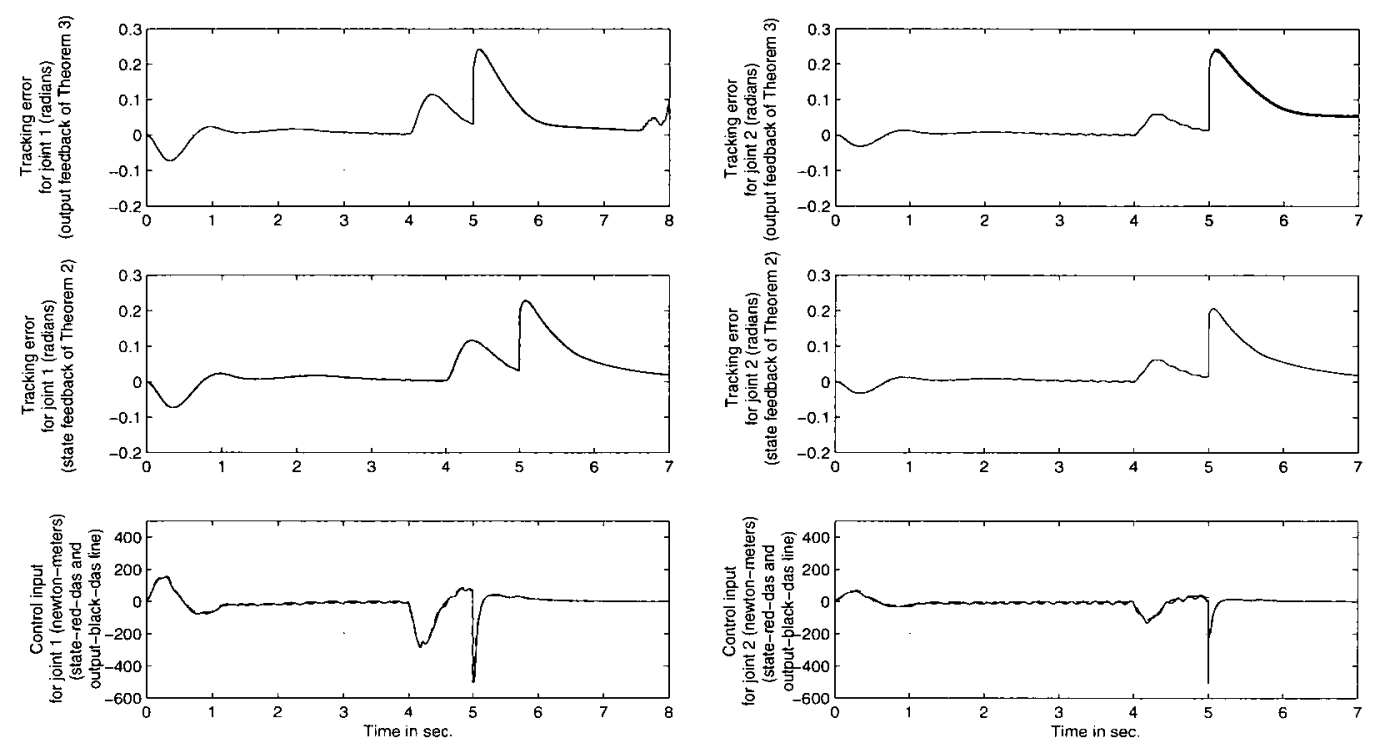

Figure 3.10: The tracking errors (radians), the control input (newton-meters) for joint 1 and joint 2 under Theorem 3.4 (state feedback) and Theorem 3.6 (output feedback).

tive to the model parameter variation.

\subsection{Summary}

In this chapter, we have presented hybrid adaptive feedback control system for robust trajectory tracking for a class of nonlinear systems. The proposed design combined robust adaptive control with direct and indirect adaptive fuzzy control in order to deal with the external disturbances and modeling error uncertainties appearing from fuzzy approximation errors. A detailed convergence analysis has been given to show the boundedness of all the signals in the observer-controller closed-loop system. In stability analysis, we have introduced parameter projection and control saturation techniques to cope with the large scale uncertainties. Unlike the existing classical and fuzzy control system, the introduced method does 
not require known bound of the uncertainty that appeared from modeling errors and disturbances. We have shown that controller-observer design parameters can be tuned in order to achieve semi-global stability property of all signals under output feedback. The evaluation on a 2-DOF robotic system verified the theoretical development of this chapter for possible real-time applications. 


\section{Chapter 4}

\section{Distributed Sliding Mode}

\section{Control}

In this chapter, we propose distributed sliding mode control algorithms for robust trajectory tracking control problem for a class of nonlinear systems.

The first part of this chapter derives single model classical sliding mode control as a state and output feedback control approach [89, 93]. In the face of large scale parametric uncertainties, the SM-based SMC approach, however, demands high observer, adaptation and discontinuous control gains in order to achieve good tracking performance.

A distributed hybrid SMC technique is then proposed to reduce the level of the parametric uncertainty so as to reduce the observer, adaptation and discontinuous control gains from single model classical SMC [84, 87, 92, 98, and 100]. The design begins by uniformly distributing the compact set of parameters into a finite number of smaller compact subsets provided that the model parameter belongs to a large compact set. Then a family of distributed candidate SMC for these smaller compact subsets is developed. The derivative of the Lyapunov-function candidate is assigned to supervise distributed models/controls and to identify the closest possible candidate model/control that closely represents the plant at each instant 
of time. The method is evaluated on a 2-DOF robot manipulator to demonstrate the effectiveness of the theoretical development for practical applications.

The rest of the chapter is organized as follows. In section II, we formulate the problem associated with the SM-based SMC approach that motivates this works. In section III, distributed models/controls based sliding mode design is developed to improve overall tracking response as well as to reduce the control chattering phenomenon from classical robust control approach. This section also presents implementation results to demonstrate the theoretical development for the realworld applications. Finally, section IV concludes the chapter.

\subsection{Classical Sliding Mode Control}

In this section, we first formulate the stability criterion for the SMC as a state feedback (position-velocity) control approach. Then, an output feedback version of this SMC is presented which incorporates a linear observer in order to remove the demand of the velocity signals from SMC design. To begin with these SMC algorithm designs, let us first model the equation of the motion for MIMO nonlinear robotic systems [82-101] as

$$
M(q) \ddot{q}+C(q, \dot{q}) \dot{q}+G(q)=\tau
$$

where $q \in \Re^{n}$ is the joint position vector, $\dot{q} \in \Re^{n}$ is the joint velocity vector, $\ddot{q} \in \Re^{n}$ is the joint acceleration vector, $\tau \in \Re^{n}$ is the input torque vector, $M(q) \in \Re^{n \times n}$ is the symmetric positive definite inertia matrix, $C(q, \dot{q}) \dot{q} \in \Re^{n}$ is the coriolis and centrifugal loading vector, and $G(q) \in \Re^{n}$ is the gravitational loading vector. We now represent the robot model (4.1) in error-state space form as follows

$$
\dot{e}_{1}=e_{2}, \dot{e}_{2}=\phi_{1}\left(e, q_{d}, \dot{q}_{d}\right)+\phi_{2}\left(e_{1}, q_{d}\right) \tau-\ddot{q}_{d}
$$

where $e_{1}=\left(q_{1}-q_{d}\right) \in \Re^{n}$ is the vector of joint position tracking error with $q_{1}=q$, $e_{2}=\left(q_{2}-\dot{q}_{d}\right) \in \Re^{n}$ is the vector of joint velocity tracking error with $q_{2}=\dot{q}$, 
$e=\left[e_{1}^{T}, e_{2}^{T}\right]^{T}, \phi_{1}\left(e, q_{d}, \dot{q}_{d}\right)=-M^{-1}(q)[C(q, \dot{q}) \dot{q}+G(q)]$ and $\phi_{2}\left(e_{1}, q_{d}\right)=M^{-1}(q)$. We assume that the desired trajectory $q_{d}(t)$ and its first and second derivatives are bounded as $Q_{d} \in \Omega_{d}=\left[q_{d}, \dot{q}_{d}, \ddot{q}_{d}\right]^{T} \subset \Re^{3 n}$ with compact set $\Omega_{d}$. Then, we consider the following well-know properties of the robot dynamics $[3,5,6,15,21$, $22,23,25,26,27,28,29,30,35,38,39,40$, to name a few] [1)] $M(q) \in R^{n \times n}$ is a symmetric, bounded, and positive definite matrix that satisfies the following inequalities as $\|M(q)\| \leq M_{M}$ and $\left\|M^{-1}(q)\right\| \leq M_{M I}$, where $M_{M}$ and $M_{M I}$ are bounded positive constants. [2)] The matrix $\dot{M}(q)-2 C(q, \dot{q})$ is skew-symmetric. [3)] The norm of the gravity and centrifugal-coriolis forces are bounded and can be represented as, $\|C(q, \dot{q})\| \leq C_{M}\|\dot{q}\|$ and $\left\|C\left(q, \dot{q}_{d}\right)\right\| \leq k_{c d}\left\|\dot{q}_{d}\right\| \leq k_{c}$, where $C_{M}, k_{c d}$ and $k_{c}$ are bounded positive constants.

Let us define the reference state as $\dot{q}_{r}=\left(\dot{q}_{d}-\lambda e_{1}\right)$ where $\lambda=\operatorname{diag}\left[\lambda_{1}, \lambda_{2}, \ldots \ldots, \lambda_{n}\right]$ with $\lambda_{1}>0, \lambda_{2}>0, \lambda_{n}>0$. Then we define the sliding surface as $S=e_{2}+\lambda e_{1}$. The control objective is to drive the position signals $q(t)$ to the desired position $q_{d}(t)$. To obtain such control objective, we consider the following robust control law

$$
\tau\left(e, Q_{d}, \hat{\theta}\right)=\hat{M}(q) \ddot{q}_{r}+\hat{C}(q, \dot{q}) \dot{q}_{r}+\hat{G}(q)-\mathcal{K} S-K \operatorname{sgn}(S)
$$

where $\ddot{q}_{r}=\left(\ddot{q}_{d}-\lambda e_{2}\right), \hat{M}$ and $\hat{C}$ are the estimates of $M(q)$ and $C(q, \dot{q})$, respectively, $\mathcal{K}=\operatorname{diag}\left[\mathcal{K}_{1}, \mathcal{K}_{2}, \ldots \ldots, \mathcal{K}_{n},\right], K=\operatorname{diag}\left[K_{1}, K_{2}, \ldots \ldots, K_{n}\right]$ with $\mathcal{K}_{1}>0, \mathcal{K}_{2}>0, \mathcal{K}_{n}>$ 0 and $K_{1}>0, K_{2}>0, K_{n}>0$. Using (4.3), we simplify the closed loop dynamics as follows,

$$
\begin{gathered}
M \dot{S}+(C+\mathcal{K}) S=\triangle \beta-K \operatorname{sgn}(S) \\
\triangle \beta=(\hat{M}-M) \ddot{q}_{r}+(\hat{C}-C) \dot{q}_{r}+(\hat{G}-G)=\triangle M \ddot{q}_{r}+\triangle C \dot{q}_{r}, \text { where } \triangle M=\hat{M}-M, \\
\triangle C=\hat{C}-C \text { and } \triangle G=\hat{G}-G .
\end{gathered}
$$

We now explore the convergence condition of the closed loop system (4.4) in the Lyapunov-sense. To do that, we define the following positive definite Lyapunovlike candidate function

$$
V=\frac{1}{2} S^{T} M S
$$


where $M$ is symmetric and positive definite matrix. As $M$ is symmetric and positive definite then $V>0$ for $S \neq 0$. The function $V$ can be considered as an indicator of the energy for $\mathrm{S}$. Let us show that the energy $V$ decays as long as $S \neq 0$. To show that, we take the derivative along the closed-loop trajectory (4.4) and then use the property 2 to obtain $\dot{V}$ as,

$$
\begin{aligned}
\dot{V} & =\frac{1}{2} S^{T} \dot{M} S+S^{T} M \dot{S} \\
& =-S^{T} \mathcal{K} S-\sum_{i=1}^{n}\left(S_{i}\left[K_{i} \operatorname{sgn}\left(\left|S_{i}\right|\right)-\triangle \beta_{i}\right]\right)
\end{aligned}
$$

From (4.6), we can see that $\dot{V} \leq 0$ holds if

$$
\sum_{i=1}^{n}\left(S_{i}\left[K_{i} \operatorname{sgn}\left(\left|S_{i}\right|\right)-\left|\triangle \beta_{i}\right| \mid\right) \geq 0\right.
$$

This can be shown when $K_{i} \geq\left|\triangle \beta_{i}\right|_{\max }$ with upper bound $\left|\triangle \beta_{i}\right|_{\max }$ that satisfies $\left|\triangle \beta_{i}\right|_{\max }>\left|\triangle \beta_{i}\right|$. If $S_{i}>0$ and $K_{i} \geq\left|\triangle \beta_{i}\right|_{\max }$, then we have

$$
\triangle \beta_{i}-K_{i} \operatorname{sgn}\left(\left|S_{i}\right|\right)=\triangle \beta_{i}+K_{i} \leq 0
$$

This implies that

$$
S_{i}\left[\triangle \beta_{i}-K_{i} \operatorname{sgn}\left(\left|S_{i}\right|\right)\right] \leq 0
$$

Similarly, if $S_{i}<0$ and $K_{i} \geq\left|\triangle \beta_{i}\right|_{\max }$, then we can write $\triangle \beta_{i}-K_{i} \operatorname{sgn}\left(\left|S_{i}\right|\right)=$ $\triangle \beta_{i}-K_{i} \geq 0$ such that

$$
S_{i}\left[\triangle \beta_{i}-K_{i} \operatorname{sgn}\left(\left|S_{i}\right|\right)\right] \leq 0
$$

Hence $\sum_{i=1}^{n}\left[S_{i}\left(\triangle \beta_{i}-K_{i} \operatorname{sgn}\left(\left|S_{i}\right|\right)\right)\right] \leq 0$. As $\mathcal{K}$ is a positive definite matrix, then first term of (4.6) can be written as

$$
\dot{V} \leq-S^{T} \mathcal{K} S \leq 0
$$

Based on our above analysis, we can write (4.6) as

$$
\dot{V}=\sum_{i=1}^{n}\left(S_{i}\left[\triangle \beta_{i}-K_{i} \operatorname{sgn}\left(\left|S_{i}\right|\right)\right]\right)-S^{T} \mathcal{K} S \leq 0
$$


Then equation (4.5) can be viewed as an energy indicator for $S$. This implies the decay of the energy of $S$ as long as $S \neq 0$. Thus, the sufficient condition $\frac{1}{2} \frac{d}{d t} S_{i}^{2} \leq-\eta_{i}\left|S_{i}\right|$, where $\eta_{i}$ is a positive constant, is satisfied [43]. To reduce the control chattering activity, we can estimate the switching function $\operatorname{sgn}($.$) by using$ a smooth bounded saturation function sat(.) $[5,6]$.

\subsubsection{Output Feedback Sliding Mode Control (OFBSMC) Design}

The analysis presented above is based on the strict assumption that all the state variables are available for feedback. However, the algorithm cannot be applied directly as most advanced system removes the velocity sensors to reduce the weight and cost of the system. To obtain the velocity signals, the common practical approach is to differentiate the position measurements obtained from encoders or resolvers which are often contaminated by noise, see for example [4], [5], [36]. The performance under state feedback based SMC approach is limited as, in practice, the measurement noise associated with velocity signals is amplified with the increase of the values of the discontinuous controller gains $K$. In the face of the large parametric uncertainty $\Delta \beta$, the demand of large discontinuous control gains makes the design even more complex as high control gains intensifies high-frequency control chattering activity.

To deal with above mentioned practical control problem, we propose to estimate the velocity signals by the output of the observer [93]. We consider a model-free linear observer to formulate an output feedback form of classical SMC approach. The observer-controller algorithm is given as,

$$
\begin{aligned}
\tau\left(\hat{e}, Q_{d}, \hat{\theta}\right)= & \hat{M}(q) \ddot{\hat{q}}_{r}+\hat{C}\left(q, \hat{e}_{2}+\dot{q}_{d}\right) \dot{\hat{q}}_{r}+\hat{G}(q)- \\
& \mathcal{K}\left(\hat{e}_{2}+\lambda \hat{e}_{1}\right)-K \operatorname{sat}\left(\frac{\hat{S}}{\phi}\right)
\end{aligned}
$$

where $\operatorname{sat}($.$) is a bounded smooth saturation function, \dot{\hat{q}}_{r}=\left(\dot{q}_{d}-\lambda \hat{e}_{1}\right), \ddot{\hat{q}}_{r}=$ 
$\left(\ddot{q}_{d}-\lambda \hat{e}_{2}\right), \hat{S}=\left(\hat{e}_{2}+\lambda \hat{e}_{1}\right)$ and the unknown velocity signals $\hat{e}_{2}$ is replaced by the output of the following linear estimator designed as follows,

$$
\dot{\hat{e}}_{1}=\hat{e}_{2}+\frac{H_{1}}{\epsilon} \tilde{e}_{1}, \dot{\hat{e}}_{2}=\frac{H_{2}}{\epsilon^{2}} \tilde{e}_{1}
$$

where $\tilde{e}_{1}=\left(e_{1}-\hat{e}_{1}\right), \tilde{e}_{2}=\left(e_{2}-\hat{e}_{2}\right), \hat{e}_{1}$ and $\hat{e}_{2}$ are the estimates of $e_{1}$ and $e_{2}, \epsilon$ is a small positive constant needs to be specified and $H_{1}$ and $H_{2}$ are the positive constant matrices. The observer dynamics for SMC approach can be viewed as a simple chain of integrator plus correction terms injected by the output error term. Since the observer is linear, then its dynamics can be made exponentially fast of the form $\frac{1}{\epsilon} \exp ^{\frac{-a t}{\epsilon}}$ with $a>0$. The observer structure is independent of the system dynamics, uncertain model parameters, nonlinear switching control input and nonlinear control inputs. In comparison, the performance with nonlinear observer based non adaptive SMC design $[12,17,18,38]$ relies on the fact that there exists a known system dynamics. For a robotic manipulator, however, it is very difficult to obtain an exact system dynamics which ensures robust reconstruction of unknown states in order to guarantee asymptotic tracking error convergence. This is mainly because nonlinear system dynamics is associated with many structured and unstructured uncertainties that cannot be exactly modeled.

To analyze the convergence rate for the proposed OFBSMC design, we use singularly perturbation method [93]. To begin with that, we first define an observer error dynamics. Using (4.8), one obtains $\dot{e}_{2}$

$$
\dot{e}_{2}=\phi_{1}\left(e, q_{d}, \dot{q}_{d}\right)+\phi_{2}\left(e_{1}, q_{d}\right) \tau\left(\hat{e}, Q_{d}, \hat{\theta}\right)-\ddot{q}_{d}
$$

The observer error becomes

$$
\begin{aligned}
& \dot{\tilde{e}}_{1}=\tilde{e}_{2}-\frac{H_{1}}{\epsilon} \tilde{e}_{1} \\
& \dot{\tilde{e}}_{2}=-\ddot{q}_{d}+\phi_{1}\left(e, q_{d}, \dot{q}_{d}\right)+\phi_{2}\left(e_{1}, q_{d}\right) \tau\left(\hat{e}, Q_{d}, \hat{\theta}\right)-\frac{H_{2}}{\epsilon^{2}} \tilde{e}_{1}
\end{aligned}
$$

Now replace the observer errors by the scaled estimation errors $\epsilon \eta_{1}=\tilde{e}_{1}$ and $\eta_{2}=\tilde{e}_{2}$ with a small positive parameter $\epsilon$ to form a singularly perturbed system. Using 
the scaled estimation errors, one gets

$$
\begin{aligned}
\epsilon \dot{\eta}= & B \epsilon\left[-\ddot{q}_{d}+\phi_{1}\left(e, q_{d}, \dot{q}_{d}\right)+\phi_{2}\left(e_{1}, q_{d}\right) \tau\left(e-\zeta(\epsilon) \eta, Q_{d}, \hat{\theta}\right)\right] \\
& +A_{o} \eta
\end{aligned}
$$

where $A_{o}=\left[\begin{array}{cc}-H_{1} & I \\ -H_{2} & 0_{n \times n}\end{array}\right], \zeta(\epsilon)=\left[\begin{array}{cc}\epsilon I_{n \times n} & 0_{n \times n} \\ 0 & I_{n \times n}\end{array}\right]$ and $H_{1}$ and $H_{2}$ are positive constant matrices such that the matrix $A_{o}$ is Hurwitz. In output feedback design, we show that the performance achieved under state feedback based SMC (4.3) can be recovered asymptotically by the OFBSMC design (4.8). To show this performance recovery, we first construct the closed loop system in standard singularly perturbed form

$$
\begin{aligned}
\dot{e} & =B\left[\phi_{1}\left(e, q_{d}, \dot{q}_{d}\right)+\phi_{2}\left(e_{1}, q_{d}\right) \tau\left((e-\zeta(\epsilon) \eta), Q_{d}, \hat{\theta}\right)-\ddot{q}_{d}\right]+A e \\
\epsilon \dot{\eta} & =B \epsilon\left[-\ddot{q}_{d}+\phi_{1}\left(e, q_{d}, \dot{q}_{d}\right)+\phi_{2}\left(e_{1}, q_{d}\right) \tau\left(e-\zeta(\epsilon) \eta, Q_{d}, \hat{\theta}\right)\right]+A_{o} \eta
\end{aligned}
$$

where $A=\left[\begin{array}{cc}0 & I_{n \times n} \\ 0_{n \times n} & 0\end{array}\right]$. We now consider that the system output and its derivatives are available. Then design SMC as a state feedback control law (4.3) such that the design meets the desired tracking objectives. We then replace the unknown velocity state vectors in SMC by the output of the observer. If we consider for the situation where the initial state estimates as well as the initial parameter estimates become large then the observer speed will require to increase for robust reconstruction of unknown velocity states. The problem with high speed observer is that it may cause large control efforts during transient phase which may enter into closed-loop system resulting unstable control system. To protect the plant from large transient control effect, we saturate the control input within the estimated region of interest. This saturation function will only be active during the transient period. To obtain such a bounded control, let us consider that $\Omega_{c}=\left\{e \mid e^{T} Q_{s m} e \leq\right.$ $c\}, c>0$, be an estimate of the region of attraction of the state feedback based SMC design $\tau\left(e, Q_{d}, \hat{\theta}\right)$ with $Q_{s m}=0.5\left[\begin{array}{cc}\lambda^{2} M & \lambda M \\ I & M\end{array}\right]$. Since $\tau\left(e, Q_{d}, \hat{\theta}\right)$ is a continuous 
function over $e, Q_{d}$ and $\hat{\theta}$, then there always exists a maximum control $\tau_{i}$ max $=$ $\max \left|\tau_{i}\left(e, Q_{d}, \hat{\theta}\right)\right|, 1 \leq i \leq n$, such that the input can be saturated over the compact set $\Omega_{c r}$ that satisfies $\tau_{i}^{s}\left(e, Q_{d}, \hat{\theta}\right)=\tau_{i} \max \operatorname{sat}\left(\frac{\tau_{i}\left(e, Q_{d}, \hat{\theta}\right)}{\tau_{i} \max }\right)=\tau_{i}\left(e, Q_{d}, \hat{\theta}\right), \forall e(0) \in$ $\Omega_{c o}=\left\{e(0) \mid e(0)^{T} Q_{s m} e(0) \leq c_{4}\right\}$ where $c_{4}<c, \theta \in \Omega, \hat{\theta} \in \Omega, Q_{d} \in \Omega_{d}$ and $\tau_{i}$ max is taken over for all $e \in \Omega_{c_{r}}$ with $c_{r}>c$ and $\operatorname{sat}($.$) is the smooth bounded$ saturation function. Then, $\forall e(t) \in \Omega_{c}$ and $\hat{\theta}(t) \in \Omega$, one has $\left|\tau_{i}\left(e, Q_{d}, \hat{\theta}\right)\right| \leq \tau_{i} \max$ $\forall t \geq 0$ and $\tau_{i}^{s}\left(e, Q_{d}, \hat{\theta}\right)=\tau_{i}\left(e, Q_{d}, \hat{\theta}\right)$. Hence, the saturation function will not be effective when short transient time period is over.

We now replace the state vectors $e$ in the control law by state estimator $\hat{e}$. Then, the bounded output feedback SMC can also be achieved via saturating outside of the region of interest $\Omega_{c}$ as follows: $\tau_{i}^{s}\left(\hat{e}, Q_{d}, \hat{\theta}\right)=\tau_{i}$ max sat $\left(\frac{\tau_{i}\left(\hat{e}, Q_{d}, \hat{\theta}\right)}{\tau_{i} m a x}\right)=$ $\tau_{i}\left(\hat{e}, Q_{d}, \hat{\theta}\right) \forall \hat{e}(0) \in \Omega_{c o}, \forall \hat{e} \in \Omega_{c}, \forall e(0) \in \Omega_{c o}, \forall e \in \Omega_{c}, \forall \hat{\theta} \in \Omega, \forall \theta \in \Omega$ and $Q_{d} \in \Omega_{d}$. Let us now summerize our main results for the OFBSMC design.

Theorem 4.1: Consider the closed-loop system (4.12) and (4.13). Then, for any given compact set of $e(0) \in \Omega_{c o}, \hat{e}(0) \in \Omega_{c o}$ and $\hat{\theta}(0) \in \Omega$ there exists a small $\epsilon_{1}^{*}$ such that for all $0<\epsilon<\epsilon_{1}^{*}$, all the state variables of the closed-loop systems are bounded by a bound that can be made arbitrary small by making $\epsilon_{1}^{*}$ small.

Proof: The proof of Theorem 4.1 can be shown along the line of the method proposed in [93]. We first show that there exists a short transient period $T_{1}(\epsilon) \epsilon$ $\left[0, T_{2}\right]$ during which the fast variable $\eta$ approaches a function of the order $O(\epsilon)$, while the slow variables $(e, \hat{\theta})$ remain in a subset of the domain of attraction. To show that, let us define the following positive definite Lyapunov-like function candidate

$$
V=\frac{1}{2} S^{T} M S
$$

We consider that all initial conditions are bounded. Thus, we choose $e(0) \in \Omega_{c o} \subseteq$ $\Omega_{c}$, that includes $e_{1}(0) \in \Omega_{c o_{1}}$ and $e_{2}(0) \in \Omega_{c o_{2}}, \theta(0) \in \Omega$, where $\Omega_{c}$ is the domain of attraction, $\Omega_{c o}$ is the compact set chosen to cover any bounded initial condition. Then, for the given initial set of $e_{1}$ and $e_{2}$, we have $c_{4}=\max _{e_{1} \in \Omega_{c o 1}, e_{2} \in \Omega_{c o 2}} \frac{1}{2} S^{T} M S$ 
where $c_{4}>0$. Then define the compact set as $e(t) \in \Omega_{c}$ with $c>c_{4}$. Now, our aim is to prove that the energy function $V$ remains bounded by a constant $c$. To verify that we first take the derivative of the Lyapunov function (6.5). Then we use the property 2 and the bounded output-feedback law on the set $\Omega_{c r}$ as

$$
\tau^{s}\left(\hat{e}, Q_{d}, \hat{\theta}\right)=\left[\tau^{s}\left(e, Q_{d}, \hat{\theta}\right)-\tau^{s}\left(\zeta(\epsilon) \eta, Q_{d}, \hat{\theta}\right)\right]
$$

with $\tau\left(e, Q_{d}, \hat{\theta}\right)=\tau^{s}\left(e, Q_{d}, \hat{\theta}\right)$ to simplify the derivative of $V$ as

$$
\begin{aligned}
\dot{V}= & -S^{T} \mathcal{K} S-\sum_{i=1}^{n}\left(S_{i}\left[K_{i} \operatorname{sat}\left(\frac{\left|S_{i}\right|}{\phi_{i}}\right)-\triangle \hat{\beta}_{i}\right]\right) \\
& -S^{T} \tau^{s}\left(\zeta(\epsilon) \eta, Q_{d}, \hat{\theta}\right)
\end{aligned}
$$

where $\hat{\triangle} \beta$ is defined as

$$
\triangle \hat{\beta}=\triangle \beta+\left[(\hat{M}-M) \lambda \eta_{2}+(\hat{C}-C) \lambda \epsilon \eta_{1}\right]
$$

with $\hat{S}=S-\left(\eta_{2}+\lambda \epsilon \eta_{1}\right), \ddot{\hat{q}}_{r}=\ddot{q}_{r}+\lambda \eta_{2}$ and $\dot{\hat{q}}_{r}=\dot{q}_{r}+\lambda \epsilon \eta_{1}$. Since $\epsilon$ is a small positive constant and the fast variable $\eta$ enters into the slow dynamics via bounded function $\tau^{s}\left((e-\zeta(\epsilon) \eta), Q_{d}, \hat{\theta}\right), \forall e \in \Omega_{c}, \forall Q_{d} \in \Omega_{d}, \forall \theta \in \Omega$ and $\forall \hat{\theta} \in \Omega$, then, in view of the dynamical property of 1 and 3 , the second part of (4.16) satisfies the following inequality,

$$
\left\|(\hat{M}-M) \lambda \eta_{2}+(\hat{C}-C) \lambda \epsilon \eta_{1}\right\| \leq k_{s m}
$$

for $k_{s m}>0$. We then simplify the derivative of (6.6) as,

$$
\begin{aligned}
\dot{V} \leq & -\sum_{i=1}^{n}\left(S_{i}\left[K_{i} \operatorname{sat}\left(\frac{\left|S_{i}\right|}{\phi_{i}}\right)-\triangle \beta_{i}-k_{s m_{i}}\right]\right) \\
& -S^{T} \mathcal{K} S-S^{T} \tau^{s}\left(\zeta(\epsilon) \eta, Q_{d}, \hat{\theta}\right)
\end{aligned}
$$

Now, for $\left|K_{i}\right| \geq\left|\triangle \beta_{i}\right|$, we have $\left[K_{i}\right.$ sat $\left.\left(\frac{\left|S_{i}\right|}{\phi}\right)-\triangle \beta_{i}\right] \geq 0$. Then equation (4.18) can be written in a simplified form as,

$$
\dot{V} \leq-S^{T} \mathcal{K} S+\|S\| k_{s m}-S^{T} \tau^{s}\left(\zeta(\epsilon) \eta, Q_{d}, \hat{\theta}\right)
$$


As $\eta$ enters into slow subsystem via bounded function then the above equation can be simplified as,

$$
\dot{V} \leq-\varpi_{o} V+\alpha_{o}
$$

$\forall e \in \Omega_{c}, \forall \hat{\theta} \in \Omega$ and $\forall \theta \in \Omega$, where $\alpha_{o}=\gamma_{s} k_{s m}+\gamma_{s} \alpha_{1}, \varpi_{o}=\frac{\lambda_{\min }(\mathcal{K})}{\lambda_{\max }\left(Q_{s m}\right)}, \gamma_{s}$ and $\alpha_{1}$ are the bound for $\|S\|$ and $\|\eta\|$ over the set $\Omega_{c}$, respectively. Then, the solution of the differential equation (4.20) can be derived as follows,

$$
V(t) \leq V(0) e^{-\varpi_{o} t}+\frac{\alpha_{o}}{\varpi_{o}}\left(1-e^{-\varpi_{o} t}\right)
$$

As $V(0) \leq c_{4}<c$, then we conclude that there always exists a finite time $T_{2}$, independent of $\epsilon$, such that for all $t \in\left[0, T_{2}\right] V(t) \leq c$. Note that, during $t \in\left[0, T_{2}\right]$, we can alternatively show the ultimately boundedness property of the closed-loop trajectories as follows

$$
\dot{V}=-S^{T} \mathcal{K} S-\sum_{i=1}^{n}\left(S_{i}\left[K_{i} s a t\left(\frac{\left|S_{i}\right|}{\phi_{i}}\right)-\triangle \beta_{i}\right]\right)-S^{T} \tau^{s}\left(\zeta(\epsilon) \eta, Q_{d}, \hat{\theta}\right)
$$

Using $\left[K_{i} \operatorname{sat}\left(\frac{\left|S_{i}\right|}{\phi_{i}}\right)-\triangle \beta_{i}\right] \geq 0$, we have

$$
\dot{V} \leq-S^{T} \mathcal{K} S+S^{T} \tau^{s}\left(\zeta(\epsilon) \eta, Q_{d}, \hat{\theta}\right)
$$

Since $\epsilon$ is a bounded by a positive constant and the fast variable $\eta$ enters into the slow dynamics via bounded function $\tau^{s}\left((e-\zeta(\epsilon) \eta), Q_{d}, \hat{\theta}\right), \forall e \in \Omega_{c}, Q_{d} \in \Omega_{d}$, $\forall \theta \in \Omega$ and $\forall \hat{\theta} \in \Omega$, then we have

$$
\dot{V} \leq-\varpi_{o} V+\alpha_{o}
$$

$\forall e \in \Omega_{c}, \forall \hat{\theta} \in \Omega$ and $\forall \theta \in \Omega$, where $\alpha_{o}=\gamma_{s} \alpha_{1}$, and $\gamma_{s}$ and $\alpha_{1}$ are the bound for $\|S\|$ and $\|\eta\|$ over the set $\Omega_{c}$, respectively.

We now prove that over the time interval $\left[0, T_{2}\right]$ the fast variable $\eta$ converge to a very small value. Based on the choice of the observer design parameter $\epsilon$, one can make the bound on $\eta$ very small. To show that, let us consider the following Lyapunov function candidate for the fast observer-error model (4.13)

$$
W(\eta)=\eta^{T} P \eta
$$


where $P=P^{T}>0$ is the solution of the Lyapunov equation $P A_{0}+A_{0}^{T} P=-I$. Applying $P A_{0}+A_{0}^{T} P=-I$, one can simplify the derivative of (4.22) along the trajectory (4.13),

$$
\begin{gathered}
\dot{W}(\eta) \leq-\frac{1}{\epsilon}\|\eta\|^{2}+2 \eta^{T} P B\left[-\ddot{q}_{d}+\phi_{1}\left(e, q_{d}, \dot{q}_{d}\right)+\right. \\
\left.\phi_{2}\left(e_{1}, q_{d}\right) \tau^{s}\left((e-\zeta(\epsilon) \eta), Q_{d}, \hat{\theta}\right)\right]
\end{gathered}
$$

Now, for any given $e(0) \in \Omega_{c o}, \hat{e}(0) \in \Omega_{c o}, \theta(0) \in \Omega$ and $\hat{\theta}(0) \in \Omega$, the following inequality holds

$$
\left\|\left[-\ddot{q}_{d}+\phi_{1}\left(e, q_{d}, \dot{q}_{d}\right)+\phi_{2}\left(e_{1}, q_{d}\right) \tau^{s}\left((e-\zeta(\epsilon) \eta), Q_{d}, \hat{\theta}\right)\right]\right\| \leq k_{1}
$$

Substituting this inequality, we obtain $\dot{W}(\eta)$ as,

$$
\dot{W}(\eta) \leq-\frac{1}{\epsilon}\|\eta\|^{2}+2 k_{1}\|P\|\|\eta\|
$$

with $\|B\|=I$. Using the Lyapunov equation (4.22), equation (4.24) can be simplified as,

$$
\dot{W}(\eta) \leq-\frac{W(\eta)}{\epsilon \lambda_{\max .}(P)}+2 k_{1}\|P\| \sqrt{\frac{W(\eta)}{\lambda_{\min .}(P)}}
$$

We now investigate the property of $\eta$ for three different situations as $W(\eta) \geq \epsilon^{2} \beta$, $W(\eta)=\epsilon^{2} \beta$ and $W(\eta) \leq \epsilon^{2} \beta$. If $W(\eta) \geq \epsilon^{2} \beta$, then we can write (4.25) in the following form,

$$
\dot{W}(\eta) \leq-\frac{\gamma}{\epsilon} W(\eta)
$$

with $W(\eta) \geq \epsilon^{2} \beta$ where $\beta=16\|P\|^{2} k_{1}^{2} \lambda_{\max } .(P)=16\|P\|^{3} k_{1}^{2}$ and $\|P\|=\lambda_{\max }(P)$. Since $\{V \leq c\}$ and $\left\{W \geq \epsilon^{2} \beta\right\}$, then we have the solution of the differential equation (4.26) as,

$$
W(\eta(t)) \leq W(0) \exp ^{\left(-\frac{\gamma t}{\epsilon}\right)}
$$

As we know that initial conditions are bounded for the estimates $\hat{e}(0) \in \Omega_{c o}$ then corresponding scaled initial state estimation error also bounded by $\eta(0)=$ 
$\frac{e(0)-\hat{e}(0)}{\epsilon} \leq \frac{k}{\epsilon}$ for some positive constant $k$ that depends on the initial set $\Omega_{c o}$. Then, equation (4.27) can be further simplified,

$$
W(\eta(t)) \leq W(0) \exp ^{\left(-\frac{\gamma t}{\epsilon}\right)} \leq \frac{k_{o}}{\epsilon^{2}} \exp ^{\left(-\frac{\gamma t}{\epsilon}\right)}
$$

where $k_{o}=k^{2} \lambda_{\max .}(P)=\frac{k^{2}}{2 \gamma}$ with $\gamma=\frac{1}{2 \lambda_{\max .(P)}}$. We now calculate the transient peaking time $T_{1}(\epsilon)$ when $W(\eta(t))=\epsilon^{2} \beta$. Let $\epsilon_{1}^{*}>0$ be small enough so that for all $0<\epsilon<\epsilon_{1}^{*}$, the time $T_{1}(\epsilon)$ is calculated when $W(\eta(t))=\epsilon^{2} \beta$ as

$$
T_{1}(\epsilon)=\frac{\epsilon}{\gamma} \ln \left(\frac{k_{o}}{\beta \epsilon^{4}}\right) \leq \frac{1}{2} T_{2}
$$

This implies that at time $T_{1}(\epsilon), W(\eta(t))=\epsilon^{2} \beta$ and $\dot{W}(\eta) \leq-\gamma \epsilon \beta$. Hence, $W(\eta)$ will continue to decrease and for the time $t>T_{1}(\epsilon)$ the inequality $W(\eta(t)) \leq \epsilon^{2} \beta$ holds. We now investigate the property of $\eta$ for the case when $W(\eta(t)) \leq \epsilon^{2} \beta$. One can see from (4.29) that $T_{1}(\epsilon)$ tends to zero as $\epsilon \rightarrow 0$. This means that right-hand side of the above inequality tends to zero as $\epsilon \rightarrow 0$. This implies that we can choose $T_{1}(\epsilon)$ small enough such that $T_{1}(\epsilon) \in\left(0, \frac{1}{2} T_{2}\right]$. That is for $t \in\left[0, T_{2}\right]$, there always exists a short transient period $T_{1}(\epsilon) \in\left(0, \frac{1}{2} T_{2}\right]$ such that for all $t \in\left[T_{1}(\epsilon), T_{3}\right]$ $W(\eta(t)) \leq \epsilon^{2} \beta$. The time $T_{3} \geq T_{2}$ is the first time $(e(t), \hat{\theta}(t))$ exists from the set $\Omega_{c}$ and time $T_{3}$ may be equal to infinity. Since the time $T_{1}(\epsilon)$ in (4.29) is a function of $\epsilon$ then one can make $T_{1}(\epsilon) \rightarrow 0$ as $\epsilon \rightarrow 0$. In view of Lyapunov equation (4.22), we can conclude that as $W(\eta(t)) \leq \epsilon^{2} \beta \forall t \in\left[T_{1}(\epsilon), T_{3}\right]\|\eta\| \leq K_{o} \epsilon, K_{o}>0$. This means that $\eta$ is of order $O(\epsilon)$ over the time interval $\forall t \in\left[T_{1}(\epsilon), T_{3}\right]$.

Let us now study the slow subsystems (4.12) over the time interval $\left[T_{1}(\epsilon), T_{3}\right]$. That is to study the property of the slow variable $(e, \hat{\theta})$ when $\|\eta\|$ converges closed to the origin. For the time interval $\left[T_{1}(\epsilon), T_{3}\right]$, we choose $e(0) \in \Omega_{c o}$ and $\hat{\theta}(0) \in \Omega$. Then we can write the tracking error model as,

$$
\begin{aligned}
\dot{e}_{2}= & {\left[\phi_{1}\left(e, q_{d}, \dot{q}_{d}\right)+\phi_{2}\left(e_{1}, q_{d}\right) \tau^{s}\left(e, Q_{d}, \hat{\theta}\right)-\ddot{q}_{d}\right]+} \\
& {\left[\phi_{2}\left(e_{1}, q_{d}\right) \tau^{s}\left(\hat{e}, Q_{d}, \hat{\theta}\right)-\phi_{2}\left(e_{1}, q_{d}\right) \tau^{s}\left(e, Q_{d}, \hat{\theta}\right)\right] }
\end{aligned}
$$

This can be viewed as a perturbed closed-loop model under state feedback over the time interval $\left[T_{1}(\epsilon), T_{3}\right]$. From the first part, we already know that the perturbation term $\|\eta\|$ decays exponentially fast to a level where $\|\eta\|$ is of order $O(\epsilon)$ 
and $W(\eta(t)) \leq \epsilon^{2} \beta$. Now $\forall(e, \hat{\theta}, \eta) \in\left\{e \in \Omega_{c}\right\} \times\{\hat{\theta} \in \Omega\} \times\left\{\eta \in \Omega_{\epsilon}\right\}$ with $\Omega_{\epsilon}=\left\{\eta \mid W(\eta(t)) \leq \epsilon^{2} \beta\right\}$ for all $t \in\left[T_{1}(\epsilon), T_{3}\right]$, we have

$$
\begin{aligned}
\left\|\tau^{s}\left(\hat{e}, Q_{d}, \hat{\theta}\right)-\tau^{s}\left(e, Q_{d}, \hat{\theta}\right)\right\| & \leq k_{\epsilon 1}\|\eta\| \\
\left\|(\hat{M}-M) \lambda \eta_{2}+(\hat{C}-C) \lambda \epsilon \eta_{1}\right\| & \leq k_{s m_{1}}\|\eta\|
\end{aligned}
$$

with $k_{\epsilon 1}>0$ and $k_{s m_{1}}>0$. Now using perturbed error model (4.30) as well as the inequality (4.31), the derivative of the Lyapunov function (6.5) has the following simplified form,

$$
\dot{V} \leq-S^{T} \mathcal{K} S+\|S\| k_{s m_{1}}\|\eta\|+k_{\epsilon 1}\|\eta\|\|S\|
$$

As we have shown in part 1 that $\eta$ is bounded by a constant $K_{o} \epsilon$ with $K_{o}>0$ and $\epsilon>0$, then the term $\|\eta\|$ also bounded. Then, equation (4.32) can be simplified as

$$
\dot{V} \leq-\lambda_{\min }(\mathcal{K})\|S\|^{2}+K_{o} \epsilon k_{\epsilon 1}\|S\|+\|S\| k_{s m_{1}} K_{o} \epsilon
$$

Now, $\forall e \in \Omega_{c}, \forall \hat{\theta} \in \Omega$ and $\forall \theta \in \Omega$, we can further simplify $\dot{V}$ as,

$$
\dot{V} \leq-\varpi_{o} V+\chi \epsilon
$$

$\forall e \in \Omega_{c}, \forall \hat{\theta} \in \Omega$ and $\forall \theta \in \Omega$, where $\chi=K_{o} \gamma_{p} k_{\epsilon 1}+\gamma_{p} k_{s m_{1}} K_{o}, \varpi_{o}=\frac{\lambda_{\min .}(\mathcal{K})}{\lambda_{\max .}\left(Q_{s m}\right)}$ and $\gamma_{p}$ is the bound for the error state $\|S\|$ over the set $\Omega_{c}$. From (4.34), we can see that on the boundary $V=c, \dot{V}<0$ when $c>\frac{\chi \epsilon}{\varpi_{0}}$. Since $\epsilon$ is a small bounded positive constant and $c$ is chosen strictly greater than $c_{4}$ and $\frac{\chi \epsilon}{\varpi_{0}}$, then we can conclude that the set $\Omega_{c} \times \Omega \times \Omega_{\epsilon}$ is a positively invariant set and inside this set $e \in \Omega_{c}$. This implies that the state trajectory $(e, \hat{\theta}, \eta)$ is confined inside the set $\Omega_{c} \times \Omega \times \Omega_{\epsilon}$. By making $\epsilon$ small, one can make this invariant set very small closed to the origin. Hence, we conclude that $T_{3}=\infty$ and all the signals in (4.12) and (4.13) are bounded $\forall t \geq 0$ and $\eta$ is $O(\epsilon) \forall t \in\left[T_{1}, T_{3}\right]$. Now integrating (4.34) from $t=0$ to $t=T$ yields

$$
V(t)-V(0) \leq-\int_{0}^{T} \alpha_{1}\|e\|^{2} d t+T \chi \epsilon
$$


Since $V(0) \geq 0$ and also from (6.5) we can write the above equation as

$$
\int_{0}^{T} \alpha_{1}\|e\|^{2} d t \leq \frac{1}{2} S(0)^{T} M S(0)+k_{6} \epsilon
$$

with $k_{6}=T \chi$. This implies that if $\epsilon \rightarrow 0$ then the OFBSMC (Lyapunov stability condition defined by (4.34)) can recover the performance (the bound on error trajectories) achieved by the state feedback-based SMC controller (Lyapunov stability condition defined by $(4.7)) \cdot \bullet$

\subsubsection{Adaptive Sliding Mode Control (ASMC)}

The level of uncertainty in classical SMC design (4.3) and (4.8) can be reduced by adding an adaptation term. For this purpose, we introduce an estimation algorithm to develop an adaptive SMC algorithm as a state feedback as

$$
\begin{aligned}
\tau\left(e, Q_{d}, \hat{\theta}\right) & =Y\left(e, \dot{q}_{r}, \ddot{q}_{r}\right) \hat{\theta}-\mathcal{K} S-K s a t\left(\frac{S}{\phi}\right) \\
\dot{\hat{\theta}} & =\Gamma Y^{T}\left(e, \dot{q}_{r}, \ddot{q}_{r}\right) S
\end{aligned}
$$

where $\Gamma=\operatorname{diag}\left[\Gamma_{1}, \Gamma_{2}, \ldots \ldots, \Gamma_{n}\right]$ with constant diagonal elements $\Gamma_{n}>0, Y\left(e, \dot{q}_{r}, \ddot{q}_{r}\right)$ is the regressor matrix and $\hat{\theta}$ denotes the estimation of the manipulator parameters and the masses of the working loads. To construct $Y\left(e, \dot{q}_{r}, \ddot{q}_{r}\right)$, one requires the model structure of the system. Such a parameter adaptation law may exhibit discontinuous property even after learning estimate converge to actual parameter [82]. Therefore, the parameter estimates $\hat{\theta}$ are required to adjust with the smooth parameter projection scheme [37] as

$$
\dot{\hat{\theta}}_{i}=[\operatorname{Proj}(\hat{\theta}, \Psi)]_{i}
$$

for $\left.\theta \in \Omega=\left\{\theta \mid a_{i} \leq \theta_{i} \leq b_{i}\right\}, 1 \leq i \leq p\right\}$ where, $\Psi_{i}$ is the $i$-the element of the column vector $Y^{T}\left(e, \dot{q}_{r}, \ddot{q}_{r}\right) S, \gamma_{i i}$ is the $i$ the element of $\Gamma$ and $\delta>0$ is chosen such that $\Omega \subset \Omega_{\delta}$ with $\left.\Omega_{\delta}=\left\{\theta \mid a_{i}-\delta \leq \theta_{i} \leq b_{i}+\delta\right\}, 1 \leq i \leq p\right\}$. Then the closed-loop error model can be written as

$$
M \dot{S}=Y\left(e, \dot{q}_{r}, \ddot{q}_{r}\right) \tilde{\theta}-(C+\mathcal{K}) S-K s a t\left(\frac{S}{\phi}\right)
$$


where $\tilde{\theta}=(\theta-\hat{\theta})$ and $\theta$ denotes the actual manipulator parameters and the masses of the working loads. The proposed adaptive control law is designed by using the following control Lyapunov function

$$
V=\frac{1}{2} S^{T} M S+\frac{1}{2} \tilde{\theta} \Gamma^{-1} \tilde{\theta}
$$

Using the property 2 , the time derivative of (4.39) along the closed-loop error trajectories (4.38) can be simplified as

$$
\dot{V}(e, \tilde{\theta}) \leq-\lambda_{\min }(\mathcal{K})\|S\|^{2}-K\|S\| \leq 0
$$

$\forall e \in \Omega_{c}, \forall \hat{\theta}(0) \in \Omega, \forall \theta(0) \in \Omega$ and $\hat{\theta}(t) \in \Omega_{\delta}$.

\subsubsection{Adaptive Output Feedback Sliding Mode Control (AOF- $\mathrm{BSMC)}$}

The above design is implementable only when all the process states are measurable. To relax this strict assumption, we now replace the unknown state vectors $e$ in (4.35)-(4.37) by the output of the estimator as

$$
\begin{aligned}
\tau\left(\hat{e}, Q_{d}, \hat{\theta}\right) & =Y\left(\hat{e}, \dot{\hat{q}}_{r}, \ddot{\hat{q}}_{r}\right) \hat{\theta}-\mathcal{K} \hat{S}-K \operatorname{sat}\left(\frac{\hat{S}}{\phi}\right) \\
\dot{\hat{\theta}} & =\Gamma Y^{T}\left(\hat{e}, \dot{\hat{q}}_{r}, \ddot{\hat{q}}_{r}\right) S
\end{aligned}
$$

To smooth the parameter estimates, we may use parameter projection scheme (4.37), where the state estimates replaced by the output of the observer. Then the closed-loop model under AOFBSMC scheme has the following form,

$$
\begin{aligned}
\dot{e} & =B\left[\phi_{1}\left(e, q_{d}, \dot{q}_{d}\right)+\phi_{2}\left(e_{1}, q_{d}\right) \tau\left((e-\zeta(\epsilon) \eta), Q_{d}, \hat{\theta}\right)-\ddot{q}_{d}\right]+A e \\
\dot{\hat{\theta}} & \left.=\operatorname{Proj}\left(\hat{\theta}, \Psi(e-\zeta(\epsilon)) \eta, Q_{d}, \hat{\theta}\right)\right) \\
\epsilon \dot{\eta} & =B \epsilon\left[-\ddot{q}_{d}+\phi_{1}\left(e, q_{d}, \dot{q}_{d}\right)+\phi_{2}\left(e_{1}, q_{d}\right) \tau\left(e-\zeta(\epsilon) \eta, Q_{d}, \hat{\theta}\right)\right]+A_{o} \eta
\end{aligned}
$$

For any given $e(0) \in \Omega_{c o}, \hat{e}(0) \in \Omega_{c o}, \theta(0) \in \Omega$ and $\hat{\theta}(0) \in \Omega$ then we have the following inequality

$$
\left\|\left[-\ddot{q}_{d}+\phi_{1}\left(e, q_{d}, \dot{q}_{d}\right)+\phi_{2}\left(e_{1}, q_{d}\right) \tau^{s}\left((e-\zeta(\epsilon) \eta), Q_{d}, \hat{\theta}\right)\right]\right\| \leq k_{2 o}
$$


for $k_{2 o}>0, \forall e(t) \in \Omega_{c}, \forall \hat{\theta}(t) \in \Omega_{\delta}$.

We now state the main results for the AOFBSMC design in the following Theorem 4.2 .

Theorem 4.2 : $\quad$ Let us consider the closed-loop system (4.43), (4.44) with the adaptive output feedback control law (4.41), (4.42), (4.37) along with linear observer. Then, for any given $\hat{\theta}(0) \in \Omega$ and $(e(0), \hat{e}(0)) \in \Omega_{c o} \subseteq \Omega_{c}$, there exists $\epsilon_{1}^{*}$ such that for all $0<\epsilon<\epsilon_{1}^{*}$, all the signals in the closed-loop system will be bounded by a bound that can be made arbitrarily small for small value of the observer design constant $\epsilon$.

Proof: The proof of stability of Theorem 4.2 is similar to the Theorem 4.1. So, we removed the details stability analysis for brevity.

Remark 4.1: It is worth noting that the bounded inequalities

$$
\left\|\left[-\ddot{q}_{d}+\phi_{1}\left(e, q_{d}, \dot{q}_{d}\right)+\phi_{2}\left(e_{1}, q_{d}\right) \tau^{s}\left((e-\zeta(\epsilon) \eta), Q_{d}, \hat{\theta}\right)\right]\right\| \leq k_{1}
$$

and

$$
\left\|\left[-\ddot{q}_{d}+\phi_{1}\left(e, q_{d}, \dot{q}_{d}\right)+\phi_{2}\left(e_{1}, q_{d}\right) \tau^{s}\left((e-\zeta(\epsilon) \eta), Q_{d}, \hat{\theta}\right)\right]\right\| \leq k_{2 o}
$$

used in observer error dynamics analysis do not impose any restriction either in theory or in practice. Note also that these inequalities do not enforce growth condition on systems nonlinearities. More specifically, the constants $k_{1}$ and $k_{2 o}$ are used to establish the semi-global stability property of Theorems 4.1 and 4.2. For the given set of initial conditions of interest, the designer can calculate the values of $k_{1}$ and $k_{2 o}$ over the domain of attraction $\Omega_{c}$. Their values can be obtained as follows. For the given $e(0) \in \Omega_{c o}$ and $\theta(0) \in \Omega$, one calculates the terms $\phi_{1}\left(e, q_{d}, \dot{q}_{d}\right)$ and $\phi_{2}\left(e_{1}, q_{d}\right)$. Then, for the given $\ddot{q}_{d}$ and initial conditions of interest, we define the saturation level $\tau_{\max }$ (maximum bound on $\tau\left(e, Q_{d}, \hat{\theta}\right)$ ). For a small value of $\epsilon$ and initial error estimates $\hat{e}(0) \in \Omega_{c o}$, we then calculate the bound on the output feedback controller $\tau^{s}\left((e-\zeta(\epsilon) \eta), Q_{d}, \hat{\theta}\right)$ as well as the bound on the term $-\ddot{q}_{d}+\phi_{1}\left(e, q_{d}, \dot{q}_{d}\right)+\phi_{2}\left(e_{1}, q_{d}\right) \tau^{s}\left((e-\zeta(\epsilon) \eta), Q_{d}, \hat{\theta}\right)$. However, in practice, the designer does not require to calculate the maximum bound on $\tau_{\max }$ as it predefines 
by a manufacturer.

Remark 4.2: The design parameter $\epsilon$ represents the bandwidth of the observer dynamics. For the given set of initial interests, it is not hard to obtain the minimum bound on $\epsilon$ from the following combined Lyapunov-function candidate as

$$
V_{C}=\frac{(1-d)}{2} S^{T} M S+\frac{d}{2} \eta^{T} P \eta
$$

with the design constant $d>0$. To calculate the bound on $\epsilon$, let us differentiate $V_{C}$ with respect to time along the perturbed closed-loop trajectory to simplify $\dot{V}_{C}$ ass

$$
\dot{V}_{C} \leq-(1-d)\left[\alpha_{1}\|e\|^{2}+\zeta_{1}\|e\|\|\eta\|\right]-d \frac{\alpha_{2}}{\epsilon}\|\eta\|^{2}+2 d \eta^{T} P B f_{o}\left(e, Q_{d}, \theta, \eta, \epsilon\right)
$$

where $\zeta_{1}=\varsigma_{o}\left(k_{\epsilon 1}+k_{s m_{1}}\right)$ with $\varsigma_{o}=\left\|\lambda^{2} \lambda ; \lambda I\right\|, \alpha_{1}=\lambda_{\min }(\mathcal{V})$ with $\mathcal{V}=\varsigma_{o} \mathcal{K}$ and $\alpha_{2}=\left\|Q_{o}\right\|$ where $Q_{o}$ is a positive definite matrix for solving Lyapunov equation $A_{0}^{T} P+P A_{0}=-Q_{0}$ and

$$
f_{o}\left(e, Q_{d}, \theta, \eta, \epsilon\right)=\left[-\ddot{q}_{d}+\phi_{1}\left(e, q_{d}, \dot{q}_{d}\right)+\phi_{2}\left(e_{1}, q_{d}\right) \tau^{s}\left(\hat{e}, Q_{d}, \theta\right)\right]
$$

By knowing the upper bound on the inertial parameter $\theta \in \Omega$ as well as the bound on the desired trajectories $Q_{d} \in \Omega_{d}$, we can simplify the modeling error term $f_{o}\left(e, Q_{d}, \theta, \eta, \epsilon\right)=[\psi(0, \eta, \epsilon)+\psi(e, 0,0)]$ as $\|\psi(0, \eta, \epsilon)\| \leq \gamma_{s p}\|\eta\|$ and $\|\psi(e, 0,0)\| \leq$ $\gamma_{s}\|e\|$. Then, $\dot{V}_{C}$ can be written as

$\dot{V}_{C} \leq-(1-d)\left[\alpha_{1}\|e\|^{2}+\zeta_{1}\|e\|\|\eta\|\right]-d \frac{\alpha_{2}}{\epsilon}\|\eta\|^{2}+2 d\|\eta\|\|P\| \gamma_{s p}\|\eta\|+2 d\|\eta\|\|P\| \gamma_{s}\|e\|$ with $\|B\|=I$. We now define $\Psi_{1}(e)=\|e\|$ and $\Psi_{2}(\eta)=\|\eta\|$. Then $\dot{V}_{C}$ can be further simplified as

$\dot{V}_{C} \leq-(1-d) \alpha_{1} \Psi_{1}^{2}(e)-d \frac{\alpha_{2}}{\epsilon} \Psi_{2}^{2}(\eta)+(1-d) \zeta_{1} \Psi_{1}(e) \Psi_{2}(\eta)+d \zeta_{2} \Psi_{1}(e) \Psi_{2}(\eta)+d \gamma \Psi_{2}^{2}(\eta)$ where $\zeta_{2}=2\|P\| \gamma_{s}$ and $\gamma=2\|P\| \gamma_{s p}$. Now, $\dot{V}_{C}$ can be expressed in compact matrix form as

$$
\dot{V}_{C} \leq-\left[\begin{array}{c}
\Psi_{1}(e) \\
\Psi_{2}(\eta)
\end{array}\right]^{T} U\left[\begin{array}{l}
\Psi_{1}(e) \\
\Psi_{2}(\eta)
\end{array}\right]
$$



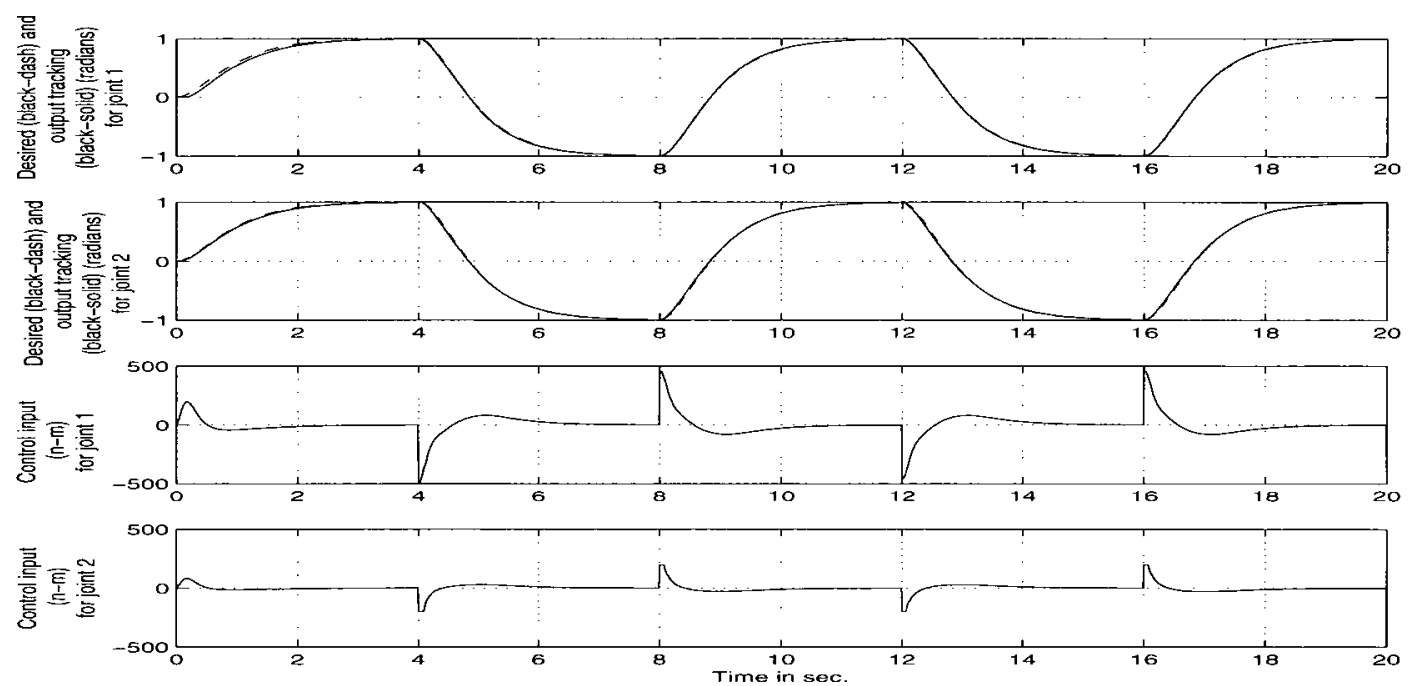

Figure 4.1: The desired (dash), output tracking (radians) (solid) and control input (newton-meters) for joints $1 \& 2$ under state feedback based ASMC for $\tilde{\theta}=10$ without using input and output disturbance noise.

with $U=\left[\begin{array}{cc}(1-d) \alpha_{1} & -\frac{1}{2}(1-d) \zeta_{1}-\frac{1}{2} d \zeta_{2} \\ -\frac{1}{2}(1-d) \zeta_{1}-\frac{1}{2} d \zeta_{2} & d\left(\left(\frac{\alpha_{2}}{\epsilon}\right)-\gamma\right)\end{array}\right]$. This implies that $\dot{V}_{C}$ is negative definite if the matrix $U$ is positive definite and satisfies the inequality $\left[d(1-d) \alpha_{1}\left(\frac{\alpha_{2}}{\epsilon}-\gamma\right)\right]>\frac{1}{4}\left[(1-d) \zeta_{1}+d \zeta_{2}\right]$. This means that for a given $d \in(0,1)$ the matrix $U$ will be positive definite and there exists a continuous interval $\left(0, \epsilon^{*}\right)$ such that $\forall \epsilon \in\left(0, \epsilon^{*}\right), \epsilon^{*}$ satisfies $\epsilon^{*}(d)=\frac{\alpha_{1} \alpha_{2}}{\alpha_{1} \gamma+\frac{1}{4 d(1-d)}\left[(1-d) \zeta_{1}+d \zeta_{2}\right]}$ for the maximum value of $d^{*}=\frac{\zeta_{1}}{\zeta_{1}+\zeta_{2}}$, one gets the bound on $\epsilon^{*}$ as $\epsilon^{*}=\frac{\alpha_{1} \alpha_{2}}{\zeta_{1} \zeta_{2}+\alpha_{1} \gamma}$. Then $\dot{V}_{c}$ becomes $\dot{V}_{c} \leq-\lambda_{\min }(U)\|\tilde{\eta}\|^{2}$, where $\tilde{\eta}=\left[\begin{array}{ll}\Psi_{1}^{T}(e), & \Psi_{2}^{T}(\eta)\end{array}\right]^{T}$ and $\lambda_{\min }(U)$ is the minimum eigen-value of the positive definite matrix $U$. This implies that all the signals in the closed loop system are bounded and trajectories converge closed to zero within a finite time. 


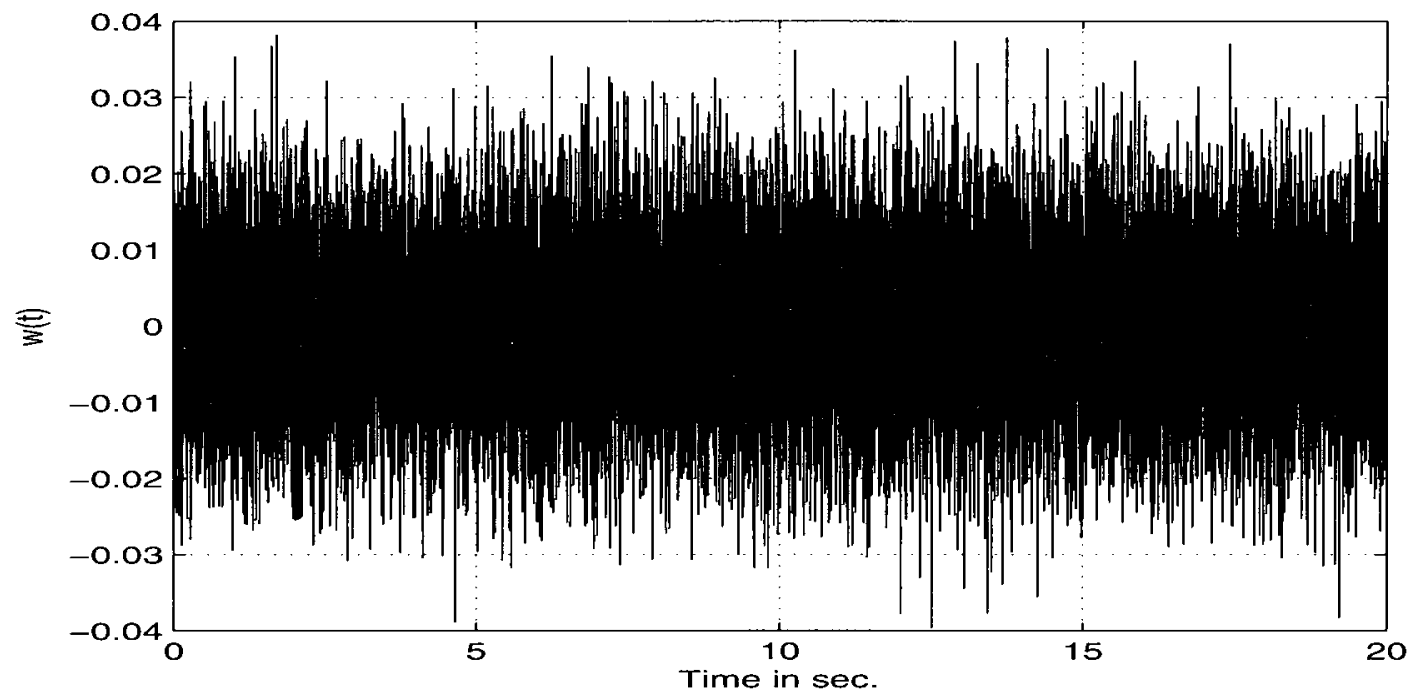

Figure 4.2: The disturbance $w(t)$.

\subsubsection{Design and Implementation Process}

To illustrate the design procedure of the proposed scheme, let us now consider a 2-link robotic system [82-101] which has the following motion equation

$$
\left[\begin{array}{ll}
m_{11} & m_{12} \\
m_{21} & m_{22}
\end{array}\right]\left[\begin{array}{l}
\ddot{q}_{1} \\
\ddot{q}_{2}
\end{array}\right]+\left[\begin{array}{ll}
c_{11} & c_{12} \\
c_{21} & c_{22}
\end{array}\right]\left[\begin{array}{l}
\dot{q}_{1} \\
\dot{q}_{2}
\end{array}\right]=\left[\begin{array}{l}
\tau_{1} \\
\tau_{2}
\end{array}\right]
$$

with $m_{11}=\left(\theta_{1}+2 \theta_{2}+2 \theta_{2} \cos q_{2}\right), m_{12}=\left(\theta_{2}+\theta_{2} \cos q_{2}\right), m_{21}=\left(\theta_{2}+\theta_{2} \cos q_{2}\right)$, $m_{22}=\theta_{2}, c_{11}=-2 \dot{q}_{2} \theta_{2} \sin q_{2}, c_{12}=-\dot{q}_{2} \theta_{2} \sin q_{2}, c_{21}=\dot{q}_{1} \theta_{2} \sin q_{2}, c_{22}=0, \theta_{1}=$ $m_{1} l^{2}, \theta_{2}=m_{2} l^{2}$ and $l$ is the link lengths and $m_{1}$ and $m_{2}$ are the masses of link 1 and link 2 , respectively. The robot operates in the horizontal plane so the gravitational force vector is zero, that is, $G=0$. We consider that the parameters $\theta_{1}$ and $\theta_{2}$ of the above robot dynamics are unknown but belong to comparatively large compact sets as $\theta \in \Omega=[-10,10]$. We also consider that the initial states belong to the set $e(0) \in \Omega_{c o}=[-2,2]$. We now generate the reference trajectory, $q_{d}(t)$, for the given robot model to follow, a square wave with a period of 8 seconds and an amplitude of \pm 1 radians is pre filtered with a critically damped 2 nd-order linear filter using a bandwidth of $\omega_{n}=2.0 \mathrm{rad} / \mathrm{sec}$. 

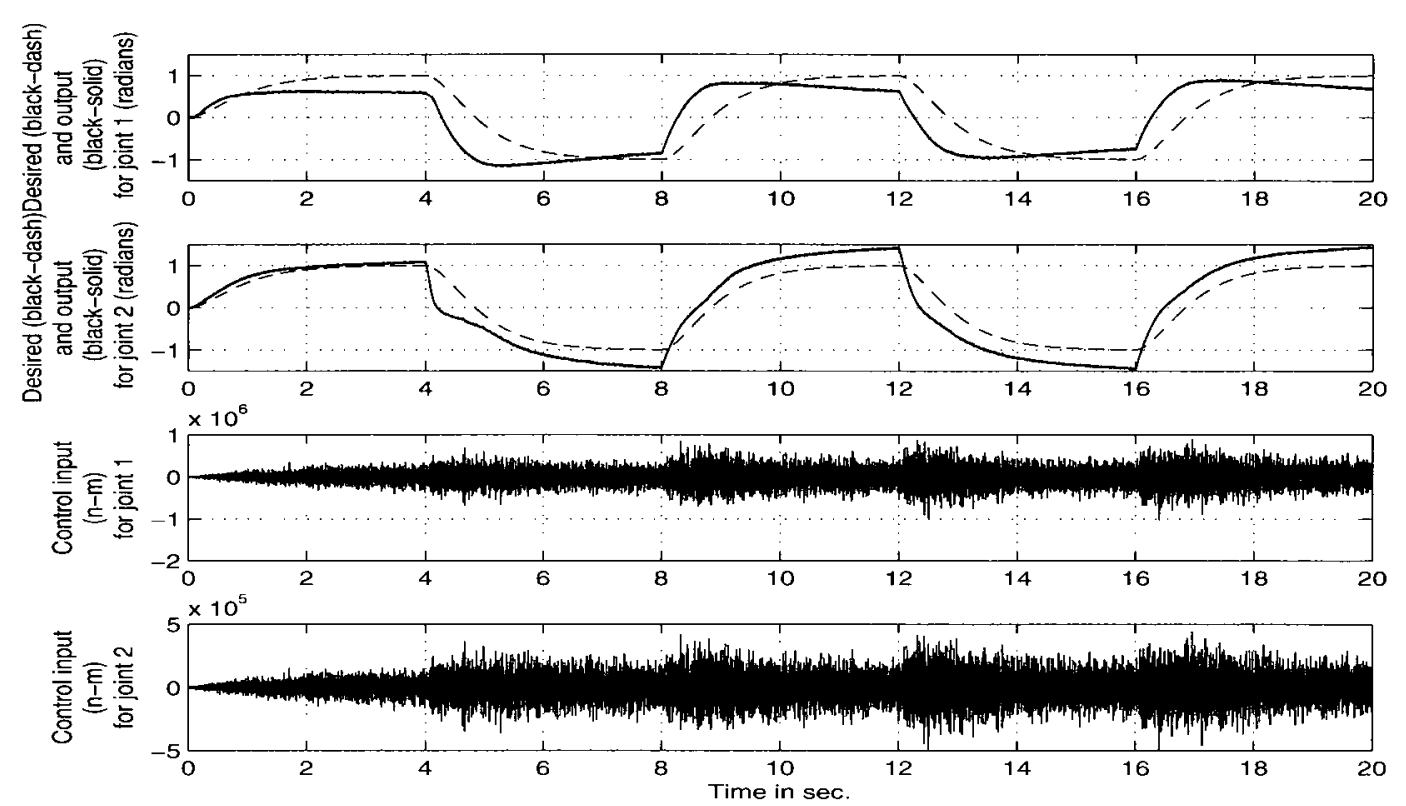

Figure 4.3: The desired (dash), output tracking (radians) (solid) and control input (newton-meters) for joints $1 \& 2$ under state feedback based ASMC for $\tilde{\theta}=8$ with non ideal operating condition.

If we know the uncertain parameter $\theta$ of the given system, then the controller is a simple feedback-linearizing regulator as

$$
\tau\left(e, Q_{d}, \theta\right)=M(q) \ddot{q}_{r}+C(q, \dot{q}) \dot{q}_{r}+G(q)-\mathcal{K} S-K\left(\frac{S}{\phi}\right)
$$

with positive constant $K$ and $\phi$. Then the origin of the closed-loop system under the above control law can be shown globally exponentially stable provided that the value of positive constant $K$ and $\phi$ are chosen via using well known pole-placement technique. Now, if we consider that parameter $\theta$ is unknown then we can estimate the parameter $\hat{\theta}$ of $\theta$. Then, the continuous sliding mode control has the following form

$$
\tau\left(e, Q_{d}, \hat{\theta}\right)=\hat{M}(q) \ddot{q}_{r}+\hat{C}(q, \dot{q}) \dot{q}_{r}+\hat{G}(q)-\mathcal{K} S-K s a t\left(\frac{S}{\phi}\right)
$$

with $\phi$ is a small positive constant and sat(.) is a bounded saturation function that 
satisfies

$$
\operatorname{sat}(y)=\left\{\begin{array}{cc}
-1 & \text { if } y<-1 \\
y & \text { if }|y| \leq 1 \\
1 & \text { if } y>1
\end{array}\right.
$$

We first show how to design the values of $K$ and $\phi$ for the given initial conditions of interest. The value of $K$ and $\phi$ can be chosen to ensure that the closed-loop trajectories converge to an invariant set where $|S| \leq \phi$. This requires to guarantee $K \geq|\triangle \beta|_{\max }$ with $\triangle G=0$ as given system operating in the horizontal plane. our aim is now to show an exponential convergence property of the closed loop trajectory that satisfies the Lyapunov-stability property as $\dot{V}+\sigma V \leq 0$ with $\sigma=\frac{\lambda_{\min } \mathcal{K}}{\lambda_{\max }(M)}$. To show $\dot{V}+\sigma V \leq 0$, the value of $K$ and $\phi$ are required to satisfy the inequality as $\frac{K}{\phi} \geq 771.1321$ with $\Omega_{c o} \subset\left\{S(0)^{T} M(0) S(0) \leq 3357.2\right\}$. If $|S|>\phi$ then the control gain $K$ requires to satisfy the inequality as $K \geq 771.1321$. Note that, for the given set, the designer can increase the value of $\phi$ and decrease the value of $K$ to reduce the chattering phenomenon.

We now show how to calculate the feedback controller gain $\mathcal{K}$ for the given initial conditions and bandwidth of the controller. This implies to find the value of the feedback controller gains $\mathcal{K}$ such that they ensures an acceptable transient performance of the closed-loop system. To show that, let us consider positive definite Lyapunov-like energy function as $V_{r}=\frac{1}{2} S^{T} M S$ with $S=\left[S_{1}^{T}, S_{2}^{T}\right]^{T}, M=$ $\left[\begin{array}{ll}m_{11} & m_{12} \\ m_{21} & m_{22}\end{array}\right]$ and $C=\left[\begin{array}{ll}c_{11} & c_{12} \\ c_{21} & c_{22}\end{array}\right]$. First take the derivative and then use the control input $\tau\left(e_{1}, e_{2}\right)=-\mathcal{K} S$ and the property 2 to obtain $\dot{V}_{r}$ as

$$
\dot{V}_{r} \leq-\lambda_{\min } .(\mathcal{K})\|S\|^{2}+\left\|S^{T}\right\| k_{o s}
$$

where $\|\left[\lambda C\left(e_{1}+q_{d}, e_{1}\right) \dot{q}_{d}-C\left(e_{1}+q_{d}, \dot{q}_{d}\right) \dot{q}_{d}\right]+M\left(-\ddot{q}_{d}+\lambda e_{2}\right)+\left[\lambda C\left(e_{1}+q_{d}, e_{1}\right)-C\left(e_{1}+\right.\right.$ $\left.\left.q_{d}, \dot{q}_{d}\right)\right] e_{2} \| \leq k_{o s} \forall\left(e, Q_{d}\right) \in \Omega_{c_{1}} \times \Omega_{d}$ with $k_{o s}>0$. Now, applying $\|S\|^{2} \geq \frac{V_{r}}{\lambda_{\max .\left(\alpha_{o s}\right)}}$ and $\|S\| \leq \sqrt{\frac{V_{r}}{\lambda_{\min .}\left(\alpha_{o s}\right)}}, \dot{V}_{r}$ can be written as $\dot{V}_{r} \leq-\psi_{o s} V_{r}+v_{o s} \sqrt{V_{r}}$ with $\alpha_{o s}=$ $0.5\left[\begin{array}{ll}m_{11} & m_{12} \\ m_{21} & m_{22}\end{array}\right], \psi_{o s}=\frac{\Xi}{\lambda_{\max }\left(\alpha_{o s}\right)}, \Xi=\lambda_{\min }(\mathcal{K})$ and $v_{o s}=\frac{k_{o s}}{\sqrt{\lambda_{\min }\left(\alpha_{o s}\right)}}$. Then, we 

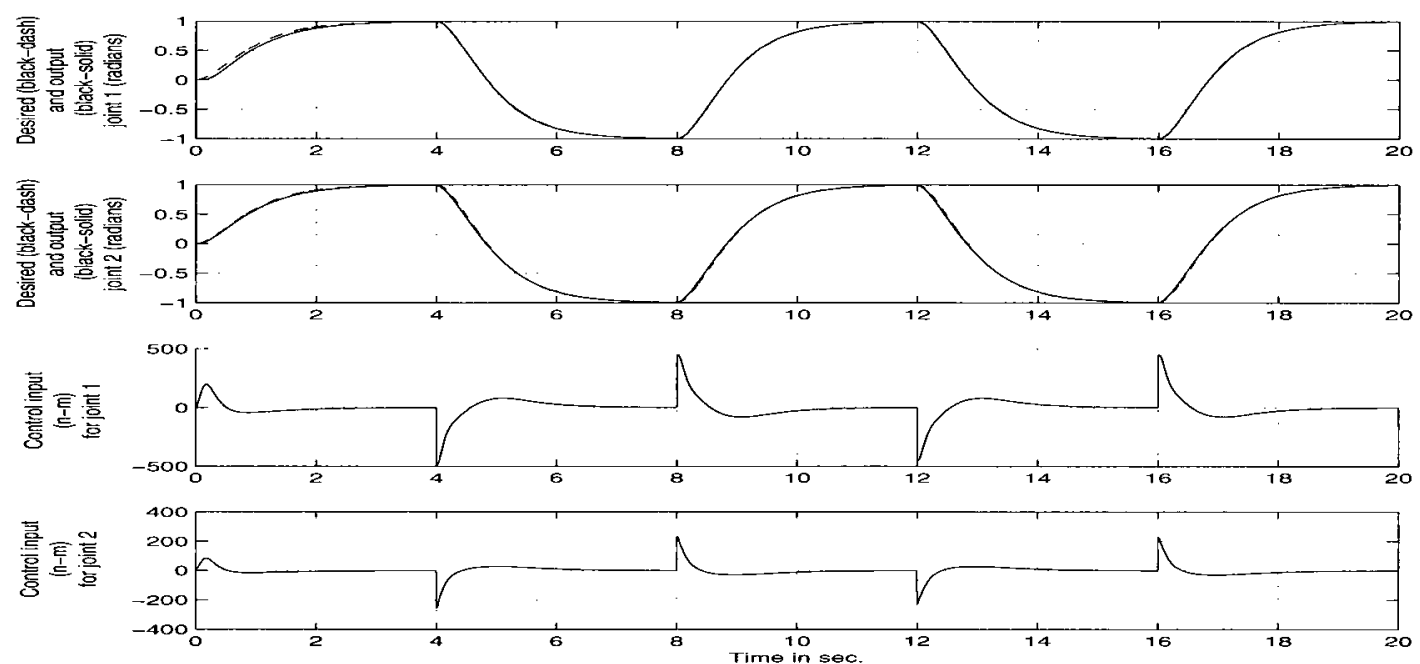

Figure 4.4: The desired (black-dash), output tracking (radians) (black-solid) and control input (newton-meters) (n-m) for joints $1 \& 2$ under AOFBSMC design with $\tilde{\theta}=10$ and without using input and output disturbance noise $w(t)$.

can find the bound on the error trajectory as $V_{r}(t) \leq V_{r}(0) e^{-\gamma_{o s} t}+\frac{\beta_{o s}}{\gamma_{o s}}\left(1-e^{-\gamma_{o s} t}\right)$ for any $\alpha_{o s}>0$ with $\psi_{o s}>\frac{\alpha_{o s}}{2}, \gamma_{o s}=\left(\psi_{o s}-\frac{\alpha_{o s}}{2}\right)$ and $\beta_{o s}=\frac{v_{o s}^{2}}{2 \alpha_{o s}}$. Using $V_{r} \geq$ $\frac{4 k_{o s}^{2} \lambda_{\max }^{2}\left(\alpha_{o s}\right)}{\lambda_{\min .}\left(\alpha_{o s}\right) \Xi^{2}}$, the bound on $\dot{V}_{r}$ can be written as $\dot{V}_{r} \leq-\kappa V_{r}$ where $\kappa=\frac{\psi_{o s}}{2}$. This means that the tracking error bound can be obtained as $V_{r}(t) \leq V_{r}(0) e^{-\kappa t}$ which implies that the trajectory starting in a region where $V_{r}(0)>c_{1 s}$ will continue to decrease until the trajectory enters into the set $\Omega_{c_{1 s}}$ with $\Omega_{c_{1 s}}=\left\{e \mid V_{r} \leq c_{1 s}\right\}$. Now using $V_{r}=\frac{4 k_{o s}^{2} \lambda_{\max .}^{2}\left(\alpha_{o s}\right)}{\lambda_{\min .}\left(\alpha_{o s}\right) \Xi^{2}}$, we have $\dot{V}_{r} \leq 0$, where $c_{1 s}=\frac{2 k_{o s}^{2} \lambda_{\max }^{2}\left(\alpha_{o s}\right)}{\lambda_{\min .}\left(\alpha_{o s}\right) \Xi^{2}}$ which implies that the trajectory starting in the set $\Omega_{c_{1 s}}=\left\{e \mid V_{r} \leq c_{1 s}\right\}$ will remain there $\forall t \geq 0$ as $\dot{V}_{r}$ is negative on $V_{r}=c_{1 s}$ that defines the maximum errors under $\tau=-\mathcal{K} S$. In view of $c_{1 s}=\frac{2 k_{o}^{2} \lambda_{\max }^{2}\left(\alpha_{o}\right)}{\lambda_{\min .}\left(\alpha_{o}\right) \Xi}$, we can observe that the tracking error bound can be made arbitrarily small by increasing the minimal eigenvalue of the control gain $\mathcal{K}$. Then, we can say that, for any given initial conditions of interest and $\lambda$, there exists a controller gain $\mathcal{K}$ such that the tracking error signals are bounded by a bound that can be made arbitrarily small closed to origin by increasing the minimal eigenvalue of the control gains $\mathcal{K}$. 

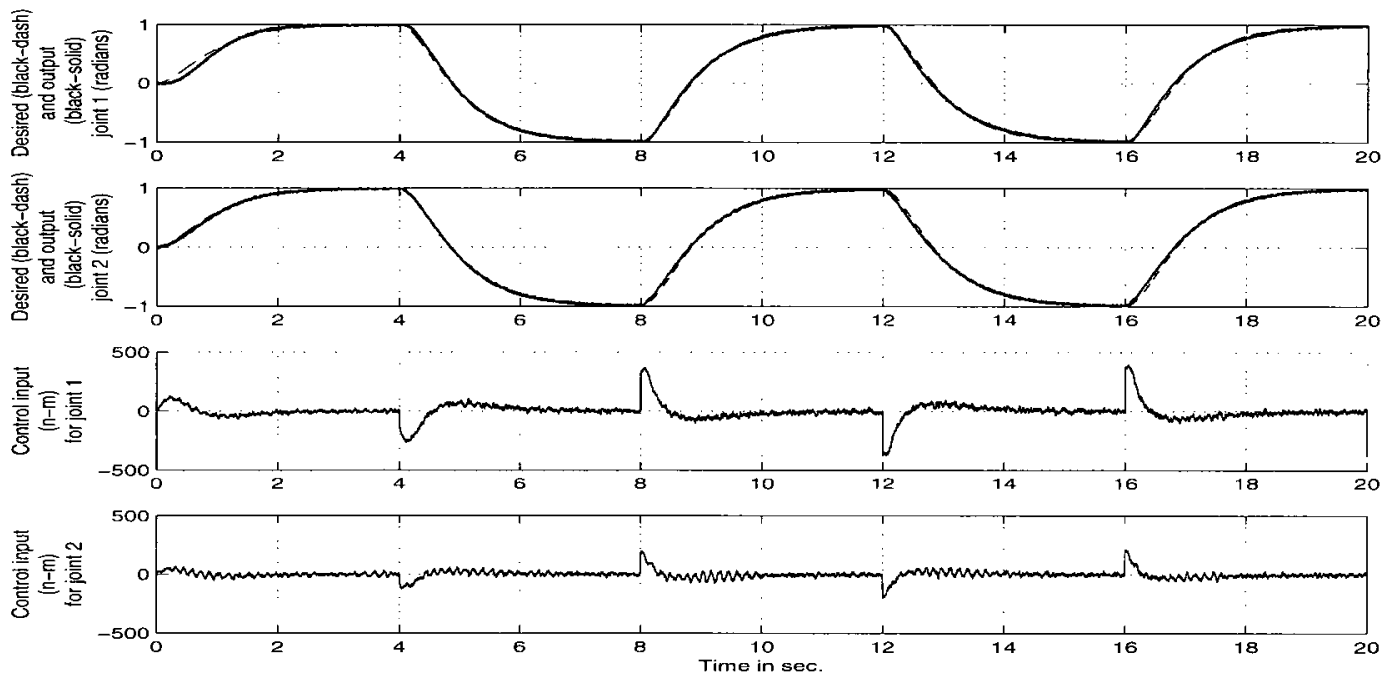

Figure 4.5: The desired (black-dash), output tracking (radians) (black-solid) and control input (newton-meters) (n-m) for joints $1 \& 2$ under AOFBSMC design using with disturbance noise $w(t)$ and the estimation error as $\tilde{\theta}=10$.

Finally, we design observer gain matrices $H_{1}$ and $H_{2}$ such that the dynamics represented by $A_{o}$ is Hurwitz. Then, for the given $e(0) \in \Omega_{c o}, \theta(0) \in \Omega, \mathcal{K}, H_{1}$, $H_{2}$ and $\lambda$, we select the small value of $\epsilon$ that makes observer dynamics relatively faster than the closed-loop robot dynamics to guarantee robust reconstruction of the unknown velocity state. Note that, for the given $e(0) \in \Omega_{c o}, \theta(0) \in \Omega, \mathcal{K}, H_{1}$, $H_{2}$ and $\lambda$, one can calculate the minimum bound of $\epsilon$ a priori to ensure robust reconstruction of the unknown velocity signals.

\subsubsection{Simulation Results}

In this subsection, we follow the above design steps to implement and evaluate the property of the proposed method on the given system (4.45). In our evaluation, we will examine the tracking convergence property of ASMC and AOFBSMC approach with respect to estimation errors as $\tilde{\theta}=10$ and $\tilde{\theta}=8$. For brevity, we have removed the evaluation of Theorem 4.1 . 
The observer-controller design parameters are calculated along the line of the design steps given in previous section for the chosen initial conditions. For given initial sets of interest, the design parameters for ASMC approach are obtained as, $\mathcal{K}=125 I_{2 \times 2}, K=15 I_{2 \times 2}, \Gamma=10 I_{2 \times 2}, \phi_{1}=0.7$ and $\phi_{2}=0.7$. With these control parameters, we first apply ASMC design on the given system. The tested results are given in Figure 4.1. The Figure 4.1 shows the tracking performance with the chosen parameter estimator errors as $\tilde{\theta}=10$.

We now examine the tracking performance of ASMC algorithms under non ideal operating situation. For this purpose, we add a band limited white noise, $w(t)$, into the output $q(t)$ (as position measurement obtained from encoders is contaminated by disturbance noise [4], [5]) and input $\tau(t)$ to the system. For our evaluation, the level $w(t)$ for the output $q(t)$ and input $\tau(t)$ are given in Figure 4.2. Then, we apply ASMC on the given system by using the same design parameters as used in our last evaluation. The implemented results are given in Figure 4.3. Figure 4.3 pictures the tracking performance under non ideal operating condition. The estimation errors for this test are set to $\tilde{\theta}=8$. The design parameters are used for this experimentation as $\mathcal{K}=125 I_{2 \times 2}, K=15 I_{2 \times 2}, \Gamma=I_{2 \times 2}, \phi_{1}=0.7$ and $\phi_{2}=0.7$. Notice from Figures 4.1 and 4.3 is that the tracking errors increase with the increase of the disturbance noise associated with input and output measurement. More specifically, one can notice that the control effort become very large that may not be realistic for the real time operation as available control inputs are restricted in most nonlinear control system design. Such a large control efforts is mainly because of the derivative action of the noisy position signals that used in position-velocity based ASMC.

Now our aim is to show that control chattering phenomenon under positionvelocity based ASMC design can be reduced by using AOFBSMC design. To explore that, we keep the same controller design parameters as applied for the evaluation of the position-velocity based ASMC algorithm. Then, we define observer design constants as follows: $H_{1}=I_{2 \times 2}, H_{2}=I_{2 \times 2}$ and $\epsilon=0.05$ such that 

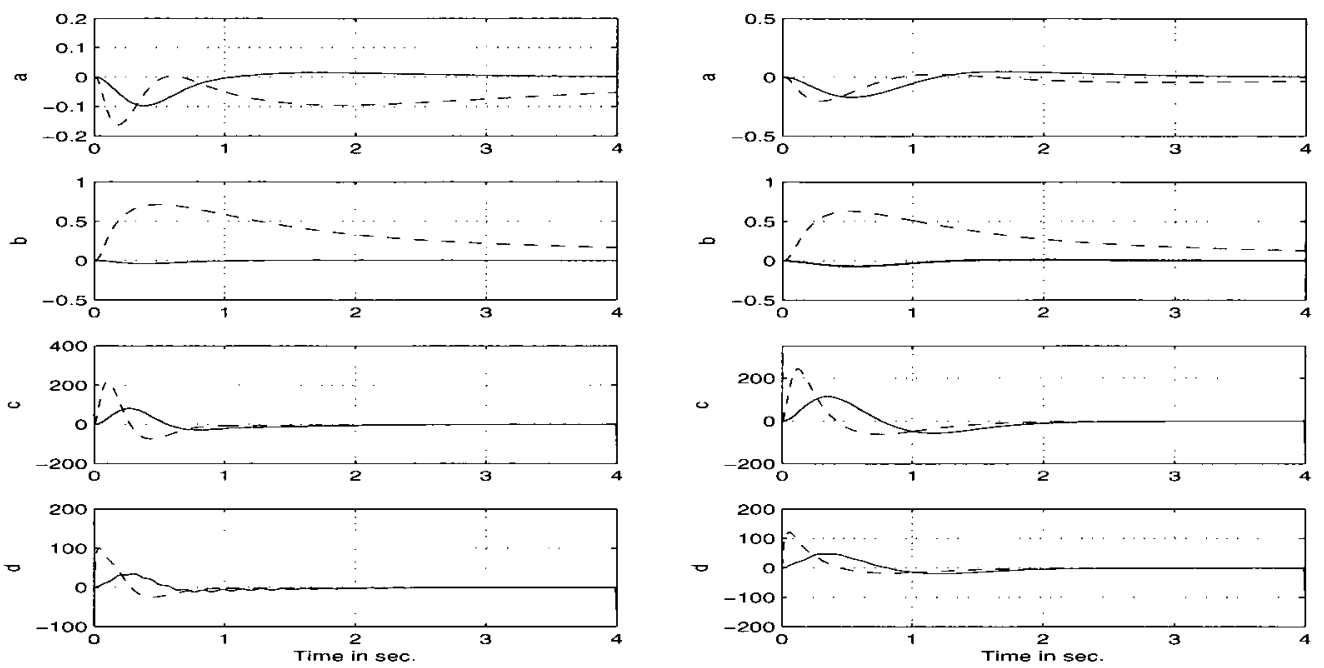

Figure 4.6: The simulation results with observers (4.9) and (4.46) based AOFBSMC under ideal condition: Left column is for $\tilde{\theta}_{\text {observer }}=4$ and right column is for $\tilde{\theta}_{\text {observer }}=0$, where $a$ : output tracking errors (radians) (dash-line is for the observer (4.46), solid-line is for the observer (4.9)) for joint 1, b: output tracking errors (radians) (dash-line is for the observer (4.46), solid-line is for the observer (4.9)) for joint 2, $c$ : control input for joint 1 (dash-line is for the observer (4.46), solid-line is for the observer (4.9)), $d$ : control input for joint 2 (dash-line is for the observer (4.46), solid-line is for the observer (4.9)).

the observer dynamics is faster than the closed loop robot dynamics. With these sets up, we now apply the AOFBSMC algorithm on the system (4.45). The implementation results are given in Figure 4.4. By observing Figures 4.1 and 4.4, we can see that the performance under AOFBSMC design is similar to the performance under ASMC design.

Let us examine the robustness property of AOFBSMC design in the presence of input and output disturbances noise. For this purpose, we add a band limited white noise, $w(t)$, into the output measurement $q(t)$ and input $\tau(t)$ of the system. For fair comparison of the performance of AOFBSMC with ASMC design, we use the 
same level of $w(t)$ as used for the evaluation of ASMC design as depicted in Figure 4.2. Then, using the following observer-controller design parameters $\mathcal{K}=125 I_{2 \times 2}$, $\Gamma=10 I_{2 \times 2}, K=15 I_{2 \times 2}, \phi_{1}=0.7, \phi_{2}=0.7, \epsilon=0.05, H_{1}=I_{2 \times 2}$ and $H_{2}=I_{2 \times 2}$, we then implement AOFBSMC on the system. The tested results are presented in Figure 4.5 with $\tilde{\theta}=10$. By comparing Figures 4.3 (ASMC) and 4.5 (AOFBSMC), we can clearly observe that the tracking performance under AOFBSMC is almost closed to zero while large tracking errors can be seen under ASMC design even with smaller uncertainty. Notice also from these results that the required control effort under AOFBSMC is much smaller than the control effort demanded under ASMC design.

\subsubsection{Comparison with Output Feedback SMC}

Let us now compare our approach with the method proposed in $[18,38]$. In comparison, the robot dynamics, uncertain model parameters as well as nonlinear control inputs are not required in the proposed observer while they should be known in $[18,38]$. The method introduced in [18] only provides local stability. In addition, we propose adaptive SMC approach but the method [18] is not in adaptive control framework. For comparison, let us design nonlinear observer [18] for rigid robot manipulators as,

$$
\begin{aligned}
& \dot{\hat{e}}_{1}=\hat{e}_{2}+\frac{H_{1}}{\epsilon} \tilde{e}_{1} \\
& \dot{\hat{e}}_{2}=\frac{H_{2}}{\epsilon^{2}} \tilde{e}_{1}-\ddot{q}_{d}+\phi_{1}\left(\hat{e}, q_{d}, \dot{q}_{d}\right)+\phi_{2}\left(\hat{e}_{1}, q_{d}\right) \tau\left(\hat{e}, Q_{d}, \hat{\theta}\right)
\end{aligned}
$$

Then, we replace the unknown velocity signals by the output of the nonlinear observer (4.46) to formulate nonlinear observer based AOFBSMC. We use the observer (4.46) in ASMC that introduced in this work to formulate AOFBSMC. We examine the tracking performance under linear observer and observer (4.46) based adaptive sliding mode control approach. It is important to note that, for fair comparison, we use adaptive controller instead of non adaptive SMC proposed in [18]. 


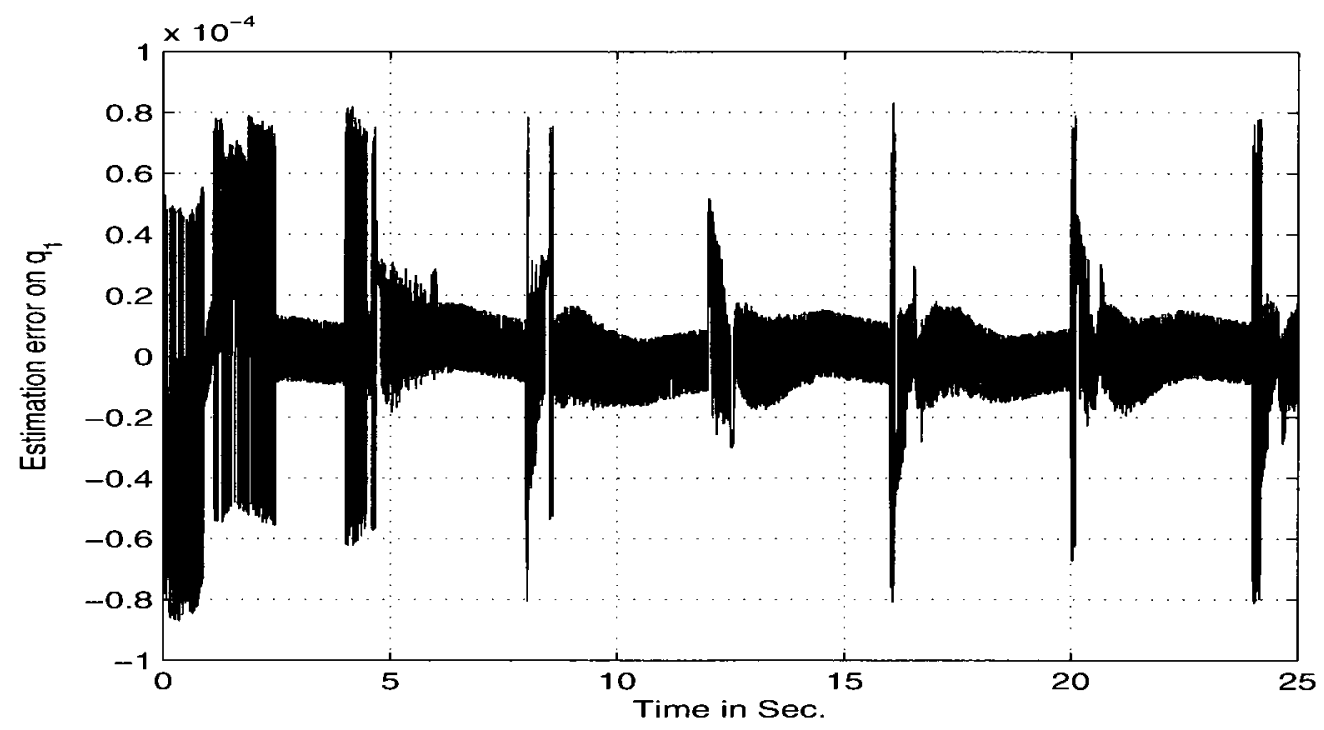

Figure 4.7: Observer estimation error $\tilde{e}_{11}$ for joint 1.

As the observer is based on model parameters, we consider two different estimation error uncertainties for the observer error dynamics as, $\tilde{\theta}_{\text {observer }}=(\theta-$ $\left.\hat{\theta}_{\text {observer }}\right)=4$ and $\tilde{\theta}_{\text {observer }}=\left(\theta-\hat{\theta}_{\text {observer }}\right)=0$, that is, the model parameters that used in the observer dynamics are exactly known. Here, $\hat{\theta}_{\text {observer }}$ denotes the parameter estimates in nonlinear observer dynamics. Then we implement both model free and model based observer based AOFBSMC design on the given robotic system.

The results are given in Figure 4.6 with the following observer design constants; $H_{1}=20 I_{2 \times 2}, H_{2}=20 I_{2 \times 2}$ and $\epsilon=0.1$, but we keep the same set of controller design parameters that applied for the evaluation of ASMC. The left column of the Figure 4.6 presents the control performance when the estimation error for the nonlinear observer is set to $\tilde{\theta}_{\text {observer }}=4$. The right column of the Figure 4.6 depicts the tracking convergence when the estimation error for the nonlinear observer is chosen to $\tilde{\theta}=0$. The dash-line of the Figure 4.6 is for the nonlinear observer-based AOFBSMC while solid-line is for the linear observer-based AOFBSMC. By comparing solid and dash line of the Figure 4.6, we can notice that the tracking error and control efforts under nonlinear observer-based AOFBSMC are larger than the tracking error and control efforts under model-free linear observer-based AOFBSMC. 


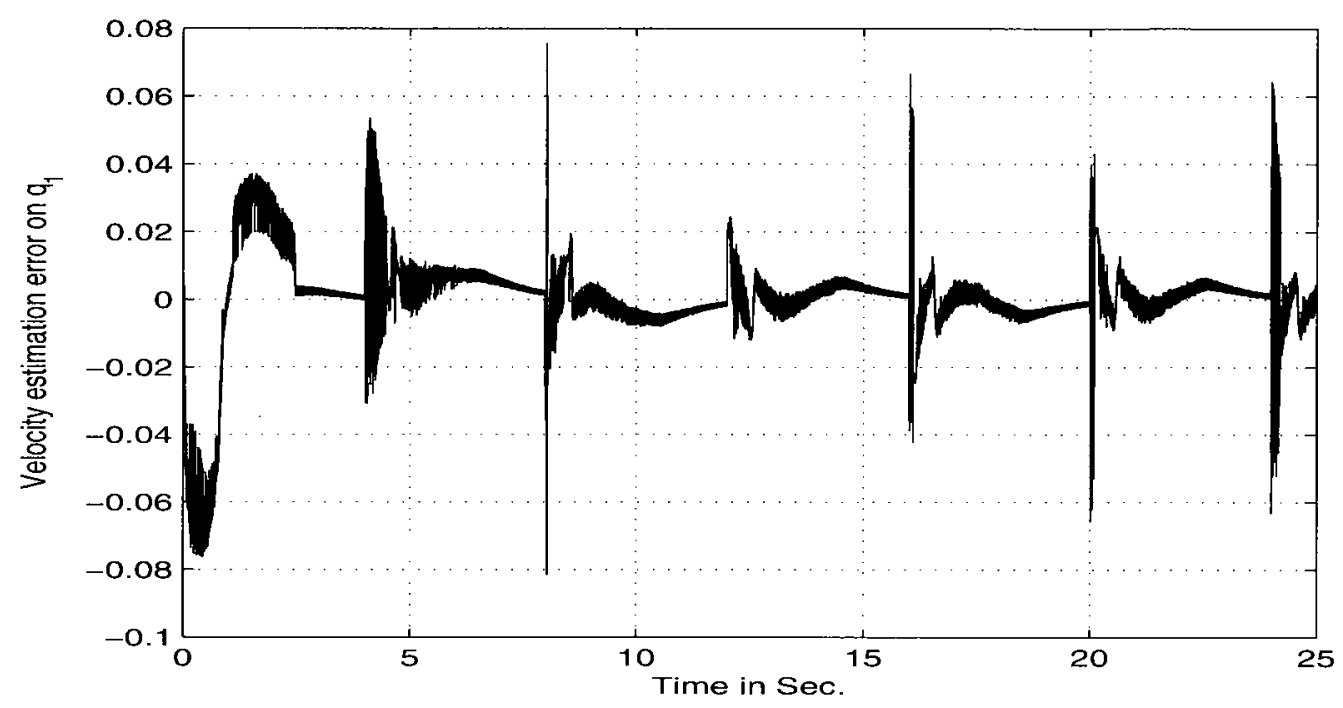

Figure 4.8: Observer velocity estimation error $\tilde{q}_{21}$ for joint 1.

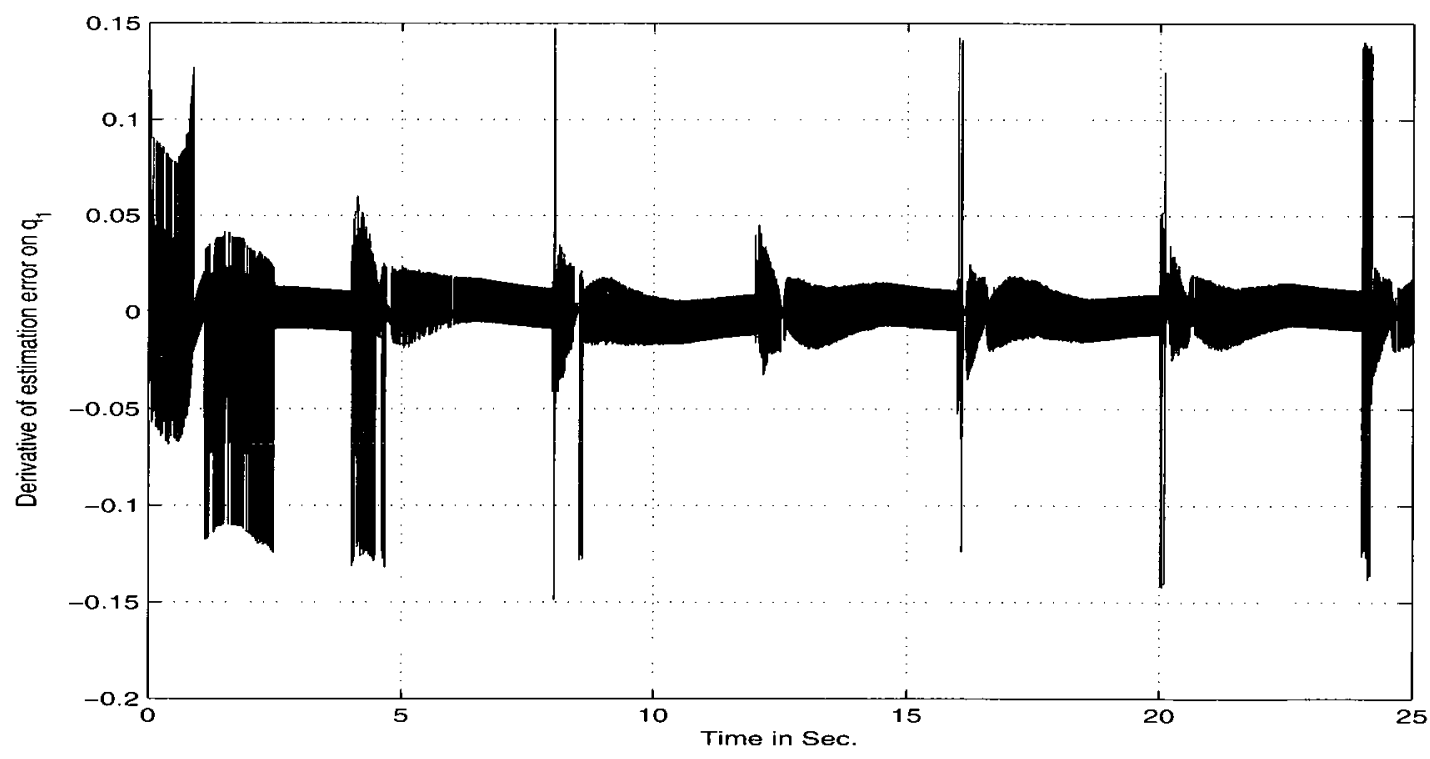

Figure 4.9: Derivative of observer estimation error $\dot{\tilde{e}}_{11}$ for joint 1 . 


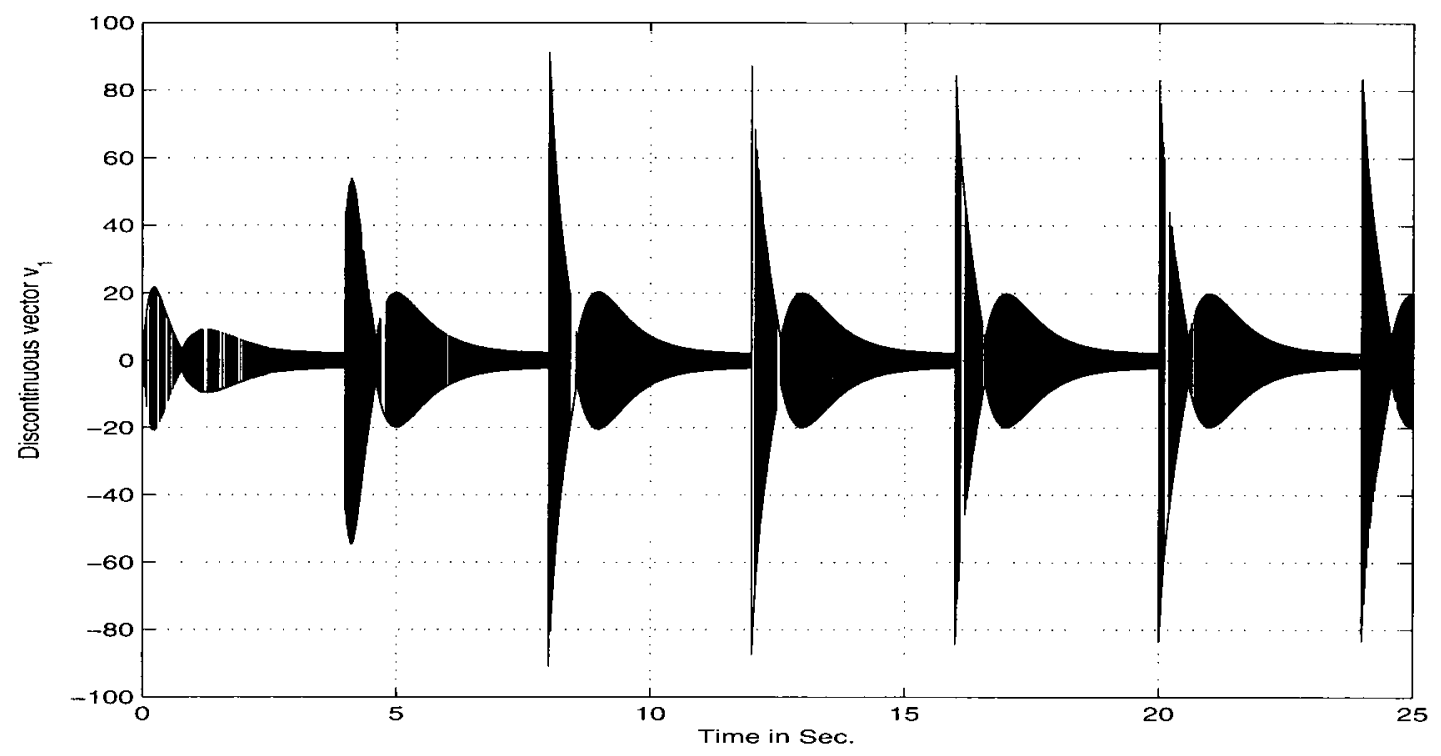

Figure 4.10: Discontinuous nonlinear vector $v_{1}$ for joint 1 .

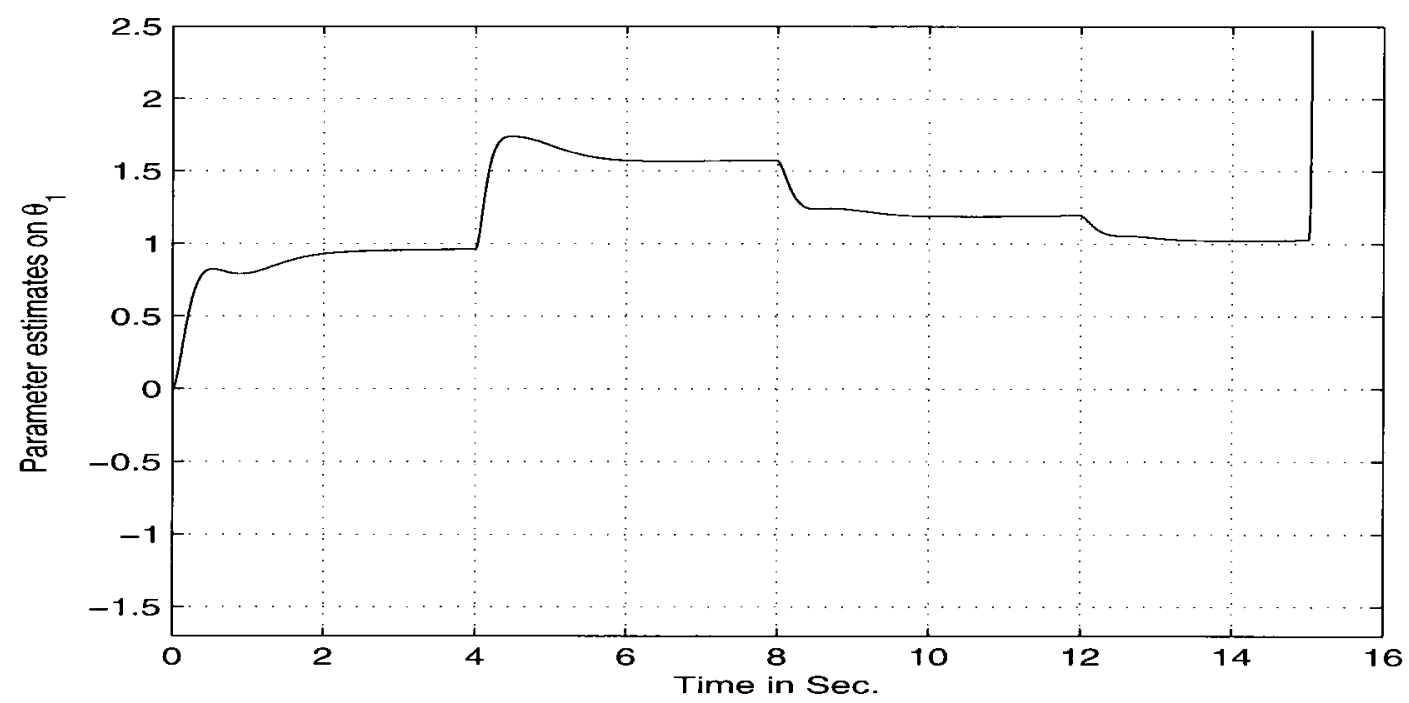

Figure 4.11: The parameter estimate $\hat{\theta}_{1}$. 
Let us now show the difficulties associated with the SMO based output feedback design [38]. Like the method reported in $[12,17]$, the SMO based OFBSMC reported in [38] only ensures local stability. The main assumption in SMO based design is the requirement of the zero initial conditions $\tilde{e}_{1}=0$ and the growth condition on unknown velocity signals (see, equations (4.10) and (4.40) of [38] for a certain class of system).

Let us examine the SMO based adaptive output feedback design for robotic systems. The following design and implementation for robotic system can be viewed as an extension of the work reported in [38] for a class of nonlinear systems. To derive SMO based adaptive approach, let us construct SMO for robotic systems (4.1)

$$
\begin{aligned}
& \dot{\hat{q}}_{1}=\hat{q}_{2}-\Lambda_{1 o} \tilde{e}_{1}-\delta_{1 o} \operatorname{sgn}\left(\tilde{e}_{1}\right) \\
& \dot{\hat{q}}_{2}=-\delta_{2 o} \operatorname{sgn}\left(\tilde{e}_{1}\right)-W\left(q, \dot{q}_{r}, \hat{\theta}\right) \hat{s}+W\left(q, \dot{q}_{r}, \hat{\theta}\right) \delta_{1 o} \operatorname{sgn}\left(\tilde{e}_{1}\right)+v
\end{aligned}
$$

where, $\hat{q}_{1}$ is the observer estimate of the joint positions, $\hat{q}_{2}$ is the estimate of the joint velocities and $\hat{s}=\left(\hat{q}_{2}-\dot{q}_{d}\right)+\Lambda\left(q-q_{d}\right)$. The constants $\Lambda_{1 o}, \delta_{1 o}$ and $\delta_{2 o}$ are $n \times n$ positive definite diagonal design matrices. The design elements are $\Lambda_{1 o}=$ $\operatorname{diag}\left(\gamma_{1 o}, \gamma_{1 o}, \cdots \gamma_{1 o}\right), \delta_{1 o}=\operatorname{diag}\left(\lambda_{1 o}, \lambda_{1 o}, \cdots \lambda_{1 o}\right), \delta_{2 o}=\operatorname{diag}\left(\lambda_{2 o}, \lambda_{2 o}, \cdots \lambda_{2 o}\right)$. Notice from the SMO design (4.47) that the observer consists of undesirable highfrequency switching terms such as $\operatorname{sgn}(),. W\left(q, \dot{q}_{r}, \hat{\theta}\right)$ and $v$. These switching terms are designed in such away that they meets the eventual Lyapunov-stability condition.

To construct observer dynamics for the system (4.45), we first define the matrix $W\left(q, \dot{q}_{r}, \hat{\theta}\right)$ as,

$$
W\left(q, \dot{q}_{r}, \hat{\theta}\right)=\left[\begin{array}{ll}
W_{11} & W_{12} \\
W_{21} & W_{22}
\end{array}\right]
$$

and the diagonal matrix $\Lambda=\left[\begin{array}{ll}\Lambda_{s 1} & \Lambda_{s 2}\end{array}\right]$. The sliding mode observer for link 1 and 
link 2 is given by equation (4.47) as

$$
\begin{aligned}
& \dot{\hat{q}}_{11}=\hat{q}_{21}-\gamma_{1 o} \tilde{e}_{11}-\lambda_{1 o} \operatorname{sgn}\left(\tilde{e}_{11}\right) \\
& \dot{\hat{q}}_{21}=-\lambda_{2} \operatorname{sgn}\left(\tilde{e}_{11}\right)-W_{11} \hat{s}_{1}-W_{12} \hat{s}_{2}+v_{1} \\
& \dot{\hat{q}}_{12}=\hat{q}_{22}-\gamma_{10} \tilde{e}_{12}-\lambda_{1 o} \operatorname{sgn}\left(\tilde{e}_{12}\right) \\
& \dot{\hat{q}}_{22}=-\lambda_{2 o} \operatorname{sgn}\left(\tilde{e}_{12}\right)-W_{21} \hat{s}_{1}-W_{22} \hat{s}_{2}+v_{2}
\end{aligned}
$$

where $\tilde{e}_{11}=\left(\hat{q}_{1}-q_{1}\right)$ is the known position estimation error for joint $1, \tilde{e}_{12}=$ $\left(\hat{q}_{2}-q_{2}\right)$ is the known position estimation error for joint $2, \hat{q}_{11}$ is the observer estimate of the position for joint $1, \hat{q}_{21}$ is the estimate of the velocity for joint 1 , $\hat{q}_{12}$ is the observer estimate of the position for joint $2, \hat{q}_{22}$ is the estimate of the velocity for joint 2

$$
\begin{aligned}
& W_{11}=\left[-2 \cos \left(q_{2}\right) \hat{\theta}_{2} \Lambda_{s 1}-2 \hat{\theta}_{2} \Lambda_{s 1}-\hat{\theta}_{1} \Lambda_{s 1}-\mathcal{K}_{1}\right] \\
& W_{12}=\left[-\cos \left(q_{2}\right) \hat{\theta}_{2} \Lambda_{s 2}-\hat{\theta}_{2} \Lambda_{s 2}-2 \sin \left(q_{2}\right) \dot{q}_{r 1} \hat{\theta}_{2}-\sin \left(q_{2}\right) \dot{q}_{r 2} \hat{\theta}_{2}\right] \\
& W_{21}=\left[-\cos \left(q_{2}\right) \hat{\theta}_{2} \Lambda_{s 1}-\hat{\theta}_{2} \Lambda_{s 1}-\hat{\theta}_{2} \sin \left(q_{2}\right) \dot{q}_{r 1}\right] \\
& W_{22}=\left[-\hat{\theta}_{2} \Lambda_{12}-\mathcal{K}_{2}\right]
\end{aligned}
$$

with $\dot{q}_{r 1}=\dot{q}_{d 1}-\Lambda_{s 1}\left(q_{1}-q_{d 1}\right)$ and $\dot{q}_{r 2}=\dot{q}_{d 2}-\Lambda_{s 2}\left(q_{2}-q_{d 2}\right)$. The nonlinear compensation law $v_{1}$ and $v_{2}$ for joint 1 and joint 2 can be designed as follows

$$
v_{1}=\left\{\begin{array}{cl}
\psi\left(\hat{q}_{2}, \tau\right) \operatorname{sgn}\left(\tilde{e}_{11}\right) & \text { if }\left|\operatorname{sgn}\left(\tilde{e}_{11}\right)\right| \neq 0 \\
0 & \text { if }\left|\operatorname{sgn}\left(\tilde{e}_{11}\right)\right|=0
\end{array}\right.
$$

and

$$
v_{2}=\left\{\begin{array}{cl}
\psi\left(\hat{q}_{2}, \tau\right) \operatorname{sgn}\left(\tilde{e}_{12}\right) & \text { if } \mid \operatorname{sgn}\left(\tilde{e}_{12} \mid \neq 0\right. \\
0 & \text { if }\left|\operatorname{sgn}\left(\tilde{e}_{12}\right)\right|=0
\end{array}\right.
$$

where $\psi\left(\hat{q}_{2}, \tau\right)$ is given as $\psi\left(\hat{q}_{2}, \tau\right)=\alpha_{1}\left|\hat{q}_{2}\right|^{2}+2 \alpha_{1}\left|\hat{q}_{2}\right| \lambda_{1 o}+\lambda_{1 o}^{2}+\alpha_{2}+\alpha_{3}|\tau|$. Then, the adaptive controller with SMO observer can be designed as,

$$
\begin{aligned}
\tau & =\hat{M}(q) \ddot{\hat{q}}_{r}+\hat{C}\left(q, \hat{q}_{2}\right) \dot{q}_{r}+\hat{G}(q)-\mathcal{K} \hat{s} \\
& =Y^{\tau}\left(q, \hat{q}_{2}, \dot{q}_{r}, \ddot{\hat{q}}\right) \hat{\theta}-\mathcal{K} \hat{s}
\end{aligned}
$$


with the positive definite matrix $\mathcal{K} \in \Re^{n \times n}$ and the regressor model as

$$
Y^{\tau}\left(q, \hat{q}_{2}, \dot{q}_{r}, \ddot{\hat{q}}_{r}\right)=\left[\begin{array}{cc}
Y_{11}^{\tau} & Y_{12}^{\tau} \\
Y_{21}^{\tau} & Y_{22}^{\tau}
\end{array}\right]
$$

where, $Y_{11}^{\tau}=\ddot{\hat{q}}_{r 1}, Y_{12}^{\tau}=2 \ddot{\hat{q}}_{r 1}+2 \cos \left(q_{2}\right) \ddot{\hat{q}}_{r 1}+\ddot{\hat{q}}_{r 2}+\cos \left(q_{2}\right) \ddot{\hat{q}}_{r 2}-2 \hat{q}_{22} \sin \left(q_{2}\right) \dot{q}_{r 1}-$ $\hat{q}_{22} \sin \left(q_{2}\right) \dot{q}_{r 2}, Y_{21}^{\tau}=0$ and $Y_{22}^{\tau}=\ddot{\hat{q}}_{r 1}+\cos \left(q_{2}\right) \ddot{\hat{q}}_{r 1}+\ddot{\hat{q}}_{r 2}+\sin \left(q_{2}\right) \dot{q}_{r 1} \hat{q}_{21}$ where, $\ddot{\hat{q}}_{r 1}=$ $\ddot{q}_{d 1}-\Lambda_{s 1}\left(\hat{q}_{21}-\dot{q}_{d 1}\right)$ and $\ddot{\hat{q}}_{r 2}=\ddot{q}_{d 2}-\Lambda_{s 2}\left(\hat{q}_{22}-\dot{q}_{d 2}\right)$ with $q_{d 1}=q_{d 2}=q_{d}$. Let us now formulate projection-free parameter adaptation law. For this purpose, we define the adaptation gain matrix as $\Gamma^{-1}=\operatorname{diag}\left[\eta_{1} \eta_{2}\right]$, the standard adaptation law takes the following form

$$
\dot{\hat{\theta}}=-\Gamma^{-1} Y^{T}\left(q, \dot{q}, \dot{q}_{r}, \ddot{q}_{r}\right) s
$$

where, $Y_{11}=\ddot{q}_{r 1}, Y_{12}=2 \ddot{q}_{r 1}+2 \cos \left(q_{2}\right) \ddot{q}_{r 1}+\ddot{q}_{r 2}+\cos \left(q_{2}\right) \ddot{q}_{r 2}-2 \dot{q}_{2} \sin \left(q_{2}\right) \dot{q}_{r 1}-$ $\dot{q}_{2} \sin \left(q_{2}\right) \dot{q}_{r 2}, Y_{21}=0$ and $Y_{22}=\ddot{q}_{r 1}+\cos \left(q_{2}\right) \ddot{q}_{r 1}+\ddot{q}_{r 2}+\sin \left(q_{2}\right) \dot{q}_{r 1} \dot{q}_{1}$. The parameter adaptation law can be written as

$$
\begin{aligned}
& \dot{\hat{\theta}}_{1}=-\eta_{1}\left[Y_{11} s_{1}+Y_{21} s_{2}\right] \\
& \dot{\hat{\theta}}_{2}=-\eta_{2}\left[Y_{12} s_{1}+Y_{22} s_{2}\right.
\end{aligned}
$$

The unknown terms in adaptation mechanism can be defined as follows, $s_{1}=$ $\hat{s}_{1}-\lambda_{1 o} \operatorname{sgn}\left(\tilde{e}_{11}\right), s_{2}=\hat{s}_{2}-\lambda_{1 o} \operatorname{sgn}\left(\tilde{e}_{12}\right), \ddot{q}_{r 1}=\ddot{\hat{q}}_{r 1}+\lambda_{1 o} \Lambda_{s 1} \operatorname{sgn}\left(\tilde{e}_{11}\right)$ and $\ddot{q}_{r 2}=$ $\ddot{\hat{q}}_{r 2}+\lambda_{10} \Lambda_{s 2} \operatorname{sgn}\left(\tilde{e}_{12}\right)$. The observer design starts by choosing a particular observer estimation error sliding regime such that $\lambda_{10} \geq\left|\tilde{e}_{2}\right|$ (the bound on the velocity error signals, see equations (4.10) and (4.40) of [38]).

Now, question is how to choose such a priori bound on unknown velocity signals. Another important assumption in SMO design is the requirement of strict condition of $\tilde{e}_{11}=0$ and $\tilde{e}_{12}=0$. For our evaluation, we choose $\tilde{e}_{11}=0$ and $\tilde{e}_{12}=0$ and find an appropriate value of $\lambda_{1 o}$ via trial and error search technique without injecting discontinuous control term. We notice that once we inject the discontinuous control term in (4.48), the closed-loop control system immediately exhibit finite escape time phenomenon as the observer-controller system generates 
excessive chattering from four different control switching operation. The design parameters $\alpha_{1}$ to $\alpha_{3}, \gamma_{1 o}, \lambda_{1 o}, \lambda_{2 o}, \Lambda_{s 1}, \Lambda_{s 2}, \eta_{1}$ and $\eta_{2}$ are determined by trial and error. The observer estimation error sliding regime is arbitrarily selected as $\lambda_{1 o}=0.1$. One then uses a trial and error procedure to find appropriate parameters such that $\left|\tilde{q}_{2}\right| \leq 0.1$.

The controller and observer parameters are selected as follows: $\alpha_{1}=2, \alpha_{2}=2$, $\alpha_{3}=1, \mathcal{K}_{1}=25, \mathcal{K}_{2}=30, \lambda_{1 o}=0.1, \lambda_{2 o}=5, \gamma_{1 o}=2, \Lambda_{s 1}=0.5, \Lambda_{s 2}=0.5$ and $\eta_{1}=\eta_{2}=3$. The true values of the parameters are set at $\theta_{1}=1$ and $\theta_{2}=2$. Then, the parameter estimation are initialized to $\tilde{\theta}_{1}=1$ and $\tilde{\theta}_{2}=2$. With these design sets, we now implement SMO based output feedback design (4.47)-(4.48) on the given system (4.45). The results are depicted in Figure 4.7. Figure 4.7 shows the observer estimation error in the position of joint 1 . The switching behavior is clearly visible. It is clear from Figure 4.7, that the assumption in SMO design that is estimation error of link 1 position is not zero, $\tilde{e}_{11} \neq 0$. Consequently, Figure 4.9 demonstrates the assumption on the derivative of the observer estimation error of joint $1 \dot{\tilde{e}}_{11}$ is also not zero. Figure 4.8 also illustrates the observer velocity estimation error. In view of Figures 4.7 and 4.9, we notice that the assumptions that used in SMO design, i.e., the zero initial conditions on $\tilde{e}_{11}$ and $\dot{\tilde{e}}_{11}$ are not meet even when the control system operating under ideal condition with small estimation error uncertainty. Figure 4.10 depicts the effect of the switching term $v$ in SMO dynamics for joint 1. Finally, we show the drifting effect of the projection free adaptation mechanism in Figure 4.11. Notice from Figure 4.11 is that the parameter estimates of $\theta_{1}$ approach the true value of $\theta_{1}=1.0$ and then drifts away from the correct value. For brevity, we remove the discontinuous property of the estimates $\theta_{2}$. The sampling period is chosen as $1 \mathrm{~ms}$. for all the results reported in this paper.

Remark 4.3: It is assumed in our evaluation that, with the chosen design parameters, the sliding regime is established that satisfies the bounded inequality $\lambda_{1 o} \geq\left|\tilde{e}_{2}\right|$. This condition, however, makes the SMO design is unrealistic as it 
is very hard for the designer to select the bound that provides required sliding regime. On the other hand, due to the presence of control switching terms in SMO design, it is also very difficult for this method to be robust with respect to large parametric uncertainty and disturbance noise with $q(t)$ and $\tau(t)$. Therefore, in the face of large scale uncertainty, the SMO based output feedback SMC design cannot achieve desired tracking objectives as the designer cannot increase the observercontroller gain due to excessive control chattering phenomenon.

\subsection{Distributed Sliding Mode Control: State and Output Feedback Design}

In previous section, we have analyzed classical sliding mode control design on robotic systems. It has been noticed from our theoretical and practical evaluations that the classical SMC design exhibits poor tracking response in the presence of large scale parametric uncertainty $\Delta \beta$. This means that when the initial conditions and parameter errors become large then the tracking errors will also become large.

Our evaluation shows that designer may use high values of observer, discontinuous and adaptation gains in order to achieve desired tracking performance via minimizing the modeling error uncertainty $\Delta \beta$. In particular, when the level of uncertainty is large, four parameters of the observer-controller $\left(\frac{1}{\epsilon}, \Gamma, K\right.$ and $\left.\frac{1}{\phi}\right)$ require to be very high to ensure good tracking performance. To increase the domain of interest (stability domain), the control saturation level (maximum bound on the state feedback control input) requires to increase resulting unacceptable transient peaking phenomenon. The practical problem, however, is that the observer, adaptation and discontinuous gains needs to be increased with the increase of the parametric uncertainty resulting very large control efforts. As a matter of fact, the use of high-gain is not a practical solution as high gains causes the control system deteriorate significantly. This is because high gains increase the steady-state noise 
sensitivity causing control chattering activity $[5,6]$. In addition, in practice, such a large control efforts based design may not be realizable as available control efforts in most practical system designs are restricted.

To deal with the above mentioned practical problems from classical SMC technique, we propose to use distributed SMC technique that allows to keep smaller values of $\Gamma$ and $K$ and higher values of $\phi$ and $\epsilon[84,87,92,98,100]$. The idea is to reduce the level of parametric uncertainty via switching the classical SMC into a distributed SMC which best approximates the plant at each instant of time. This implies to identify a control vector $\tau\left(e, Q_{d}, \theta\right)$ corresponding to a distributed candidate model that closely estimates the parameters of the manipulator and its loads that operating in the workspace.

The design steps for the proposed method can be described as follows. First, we consider that the unknown plant parameter $\theta$ belongs to a known but comparatively large compact set $\Omega$. $\theta$ denotes the inertial parameters of the robot arms and its load operating in the work space. Then, we equally distribute the parameter set $\Omega$ into a finite number of smaller compact subsets such that $\theta_{i} \in \Omega_{i}$ with $\Omega=\bigcup_{i=1}^{N} \Omega_{i}$ and $\theta \in \Omega_{i}$. For a given compact set of the initial conditions of interest $e(0) \in \Omega_{c o}$, we construct a family of candidate SMC (5.49), bounded in $e$ via saturating outside the region of interest, correspond to smaller parameter compact subsets as

$$
\tau^{i}\left(e, Q_{d}, \theta_{i}\right)=Y\left(e, \dot{q}_{r}, \ddot{q}_{r}\right) \theta_{i}-\mathcal{K} S-K_{i} \operatorname{sat}\left(\frac{S}{\phi_{i}}\right)
$$

with $\left(\theta, \theta_{i}\right) \in \Omega_{i}$, such that for every $\theta \in \Omega_{i}$ all the signals in the closed loop system under (4.49) started inside the sets $\Omega_{c o}$ are bounded and the output tracking error trajectories converge to zero within short time. The control term $\mathcal{K} S$ is common to all candidate SMC. The regressors model $Y\left(e, \dot{q}_{r}, \ddot{q}_{r}\right)$ is also common to all the candidate controllers. To identify best possible candidate controller from a family of candidates (4.49), we introduce on-line estimation of the derivative of the Lyapunov-function candidate [84, 87, 92, and 98].

Remark 4.4: The important question is how to choose distributed parameter 
model sets. Naturally, the model selection is based on a priori known bound of the robot dynamics and its operating environments. If the manipulator parameters and the masses of the working loads are known to be within a specified range then the model sets can be distributed within the given specified range around with the nominal parameter values. To simplify the control design, the compact parameter set $\Omega$ is partitioned into a finite number of smaller compact subsets $\Omega_{i}$. For the simplicity of the design, we consider nominal parameter for each compact set $\Omega_{i}$. Since the partition of the parameter sets $\Omega_{i}$ allows for overlapping then the tuned controller to the system for $\theta \in \Omega_{i}$ may not be unique. The parameter sets can also be split into non-uniform and non-overlapping regions as long as it covers the entire parameter space. Note that for non-overlapping and non-uniform case, one requires to use strict assumption such that there exists a single model that guarantees asymptotic tracking property.

Our next issue is how to identify a suitable $\operatorname{SMC} \tau\left(e, Q_{d}, \theta\right)$ from a finite set of distributed SMC $\tau^{i}\left(e, Q_{d}, \theta_{i}\right)$. To identify which candidate SMC will be used to generate the final control vector, a switching-logic has to be selected in such a way that guarantees that all the signals in the closed-loop systems are bounded, and the error trajectories converge to zero within the finite time period. To ensure that, we assume that there exist a small dwell time constant $t_{d}$ such that the solution of the closed loop system does not have a finite-escape time property. This assumption implies that the switching inequality can guarantee the boundedness property of the tracking error signals or the finiteness property of the Lyapunovfunction. The finiteness property of the Lyapunov-function candidate prevents the occurrence of finite-escape time phenomenon, in the sequel guarantees the boundedness of tracking errors. This also implies that the supervisor can ensure finite time identification of unstable loops through on-line checking of the Lyapunov inequality. For each switching operating condition, we consider a candidate SMC exists which satisfies the Lyapunov stability as

$$
V_{i}\left(t_{i}\right) \leq V_{i}\left(t_{i-1}\right)-\int_{t_{i-1}}^{t_{i}} \mathcal{V}\left\|e\left(\tau_{o}\right)\right\|^{2} d \tau_{o}
$$


with $t_{i}:=t_{o}+t_{d}$ and $t_{o}=t_{i}$. This property holds as the manipulator parameters and the masses of the working loads belong to the compact subsets $\Omega_{i}$ and the model selection is based on using known bound of the system dynamics and its operating environments. This is not unusual as, in practice, one has the maximum bound on $\theta$. Note also that the parameters $\theta=\left[\theta_{1}, \theta_{2}, \theta_{3}, \ldots ., \theta_{p}\right]^{T}$ are unknown but the vector $\theta$ belongs to the known set $\Omega$. This means that, for each operating condition, a candidate controller exists such that it satisfies the Lyapunov stability condition.

Let us consider $i\left(t_{i}\right): \mathcal{M} \rightarrow\{1,2,3,4, \ldots, N\}$ be the controller index acting in the loop at time instant $t_{i}$. If there exist a controller $\tau^{i}\left(e, Q_{d}, \theta_{i}\right)\left(t_{i}\right)$ such that it does not guarantee the convergence trajectory then we have

$$
V_{i}\left(t_{i}\right)>V_{i}\left(t_{i-1}\right)-\int_{t_{i-1}}^{t_{i}} \mathcal{V}\left\|e\left(\tau_{o}\right)\right\|^{2} d \tau_{o}
$$

and then switching takes place. The next candidate controller will be selected by using the logic introduced later in Algorithms 1,2 and 3 via checking the derivative of the Lyapunov function inequality. If the controller does not satisfies the inequality then the supervisor replaces the actually acting controller to the next candidate. As the parameter belongs to the given compact subsets then there exists finite switching steps to find a candidate controller such that

$$
V_{i}\left(t_{i}\right) \leq V_{i}\left(t_{i-1}\right)-\int_{t_{i-1}}^{t_{i}} \mathcal{V}\left\|e\left(\tau_{o}\right)\right\|^{2} d \tau_{o}
$$

If the resetting criterion is satisfied then there will not be any more switching and we can write $V_{i}\left(t_{i}\right) \leq V_{i}(0) \forall t_{i} \geq 0$. Thus the tracking error is uniformly continuous and bounded as a result of the radial unboundedness property of $V_{i}$. On the other hand, $V_{i}\left(t_{i}\right)$ is lower-bounded and nonincreasing sequence then we have $\lim _{t \rightarrow \infty} V_{i}\left(t_{i}\right) \geq 0$. Therefore, one can write

$$
\int_{t_{i-1}}^{t_{i}} \mathcal{V}\left\|e\left(\tau_{o}\right)\right\|^{2} d \tau_{o} \leq V_{i}\left(t_{i-1}\right)-V_{i}\left(t_{i}\right)
$$

If the bound on $\lim _{t \rightarrow \infty} V_{i}\left(t_{i}\right)=V_{M} \geq 0$, then we have

$$
\int_{t_{i-1}}^{t_{i}} \mathcal{V}\left\|e\left(\tau_{o}\right)\right\|^{2} d \tau_{o} \leq V_{i}\left(t_{i-1}\right)-V_{M}
$$


This means that $\|e(t)\|^{2} \rightarrow 0$. This also implies that the boundedness property of $e(t)$. The continuity property of Lyapunov-function implies that the finite escape time property does not exist. The finiteness property of the Lyapunov-function under output feedback case can also be shown along the line of the above analysis provided that there exists a small value of observer design constant $\epsilon$.

Now, our aim is to develop a switching strategy such that it improves tracking response while guaranteeing the closed loop stability of overall control systems. How to choose the next candidate model is an important decision which directly affects the overall control tracking performance.

First, we introduce a pre-ordered supervisory technique for on-line identification of candidate SMC corresponding to the parameter model $\theta_{i} *$, with $i=i *$ and $i * \in \mathcal{M}(i *)$, from a finite set of candidate parameters using on-line estimation of the Lyapunov-inequality. A similar switching-logic is proposed in [55, 59 and references therein] for a class of SISO systems. In [55, 59], a quadratic performance index using model identification errors is used as a switching condition to select an appropriate model from a set of distributed candidate model. The switching supervisory condition that we introduced in Algorithm 4.1 is based on using the inequality for the derivative of the Lyapunov-function candidate [84, 87]. Since the parameter sets $\Omega_{i}$ are allowed to overlap then the model/control that satisfies the switching condition does not require to be unique. The switching-logic derived in $[55,59]$ for a class of SISO systems is assumed that the position-velocity signals are available for feedback. The proposed method can be applied for both state and output feedback sliding mode control design for a class of MIMO nonlinear systems.

Algorithm 4.1: Suppose that the controller index $i \in \mathcal{M}$ is acting in the loop at time $t$. Then, we follow the following switching scheme to identify candidate SMC that satisfies the pre-specified Lyapunov inequality

- A. Assuming that the initial time $t_{o}=0$, control index $i \in \mathcal{M}=\{1,2,3, . ., N\}$ and a dwell time constant $t_{d}>t_{o}$. 
- B. We then put the classical $S M C \tau\left(e, Q_{d}, \hat{\theta}\right)$ with $\hat{\theta}$ is provided by a standard adaptation law (for state feedback case (4.36) and output feedback case (4.42)), in the loop and dwell it for a short period of time $t \in\left[t_{o}, t_{o}+t_{d}\right]$.

- C. For $t \geq t_{o}+t_{d}$, we check the pre-specified suitching inequality using with the derivative of the Lyapunov-function candidate $\dot{V}(t) \leq 0$. If the inequality satisfies then we apply the classical ASMC to the system. If not then we put the first candidate $S M C \tau^{i}\left(e, Q_{d}, \theta_{i}\right)$ with $i=1$.

- D. We again dwell this controller for small time $t_{d}$ and monitor the inequality for the derivative of the Lyapunov function to see whether or not the function decreasing sufficiently fast to switch to the next candidate SMC. If the controller does not satisfy the switching inequality then the supervisor switches again to the next candidate $S M C \tau^{i}\left(e, Q_{d}, \theta_{i}\right)$ with $i=2$. The logic repeats the search until the supervisor identifies a candidate that satisfies the derivative of the Lyapunov inequality.

Based on our above analysis, we now state the main results in the following Theorem 4.3 by assuming that the position and velocity signals are available to design candidate SMCs (4.49).

Theorem 4.3: Consider the closed loop system formulated by (4.2) and (4.49) under the switching-logic defined in Algorithm 4.1. Then there exists a time such that the controller according to the logic stated in Algorithm 4.1 is tuned to the plant that ensures $\dot{V}(t) \leq 0$.

Remark 4.5: Note that the plant parameters are assumed to belong to one of the candidate subsets $\Omega_{i}$ then there exists a finite number of search such that at least one of the candidate SMC satisfies the Lyapunov inequality. Therefore, the number of switching search as well as the switching time period is finite. In addition, the tuned controller does not need to be unique as the parameter sets are allowed to be overlapping.

In theorem 4.3, we assume that all the state vectors are available for feedback 
to construct distributed sliding mode controllers (4.49). Let us consider that the velocity signals are unavailable then the distributed control laws (4.49) cannot be directly employed as, in practice, the velocity signals are not available for feedback design. Therefore, to estimate unknown velocity signal, we replace $e$ by the output of the linear estimator. Then we modify the algorithm (4.49) to formulate adaptive output feedback based distributed SMCs as

$$
\tau^{i}\left(\hat{e}, Q_{d}, \theta_{i}\right)=Y\left(\hat{e}, \dot{\hat{q}}_{r}, \ddot{\hat{q}}_{r}\right) \theta_{i}-\mathcal{K} \hat{S}-K_{i} \operatorname{sat}\left(\frac{\hat{S}}{\phi_{i}}\right)
$$

We also require estimating the Lyapunov inequality that modifies the switching criterion for the distributed AOFBSMCs design. To do that, we need to ensure the robust reconstruction of unknown velocity state vectors. As we have already seen from SM-based SMC design that we cannot make state estimation errors to zero as $\epsilon \neq 0$. So, we have to find the bound on the non-vanishing estimation errors term in the switching inequality provided by the derivative of the Lyapunov-function candidate. This implies that, for small positive observer design constant $\epsilon$, there exists a short transient period such that the state estimates $\hat{e}$ decay exponential fast to a small set $\Omega_{\epsilon}$. For the given $\epsilon$ as well as the initial state estimates, the short transient peaking time $T_{1}(\epsilon)$ can be determined from (4.29) as $T_{1}(\epsilon)=\frac{\epsilon}{\gamma} \ln \left(\frac{k_{0}}{\beta \epsilon^{4}}\right)$ where $k_{o}=k^{2} \lambda_{\max }(P)=\frac{k^{2}}{2 \gamma}, \gamma=\frac{1}{2 \lambda_{\max .(P)}}$ and $e(0)-\hat{e}(0) \leq k$ with $k \geq 0$. After this transient peaking time, the estimation error converge to a small value, namely $O(\epsilon)$, that closed to zero. To ensure the estimation error converge to a small value, the dwell time $t_{d}$ requires to chose such that $T_{1}(\epsilon)<t_{d}$. Using a small value of the observer design constant $\epsilon$, the peaking time period can be made very small so that the designer can use smaller dwell time constant to guarantee the solution of the closed-loop system does not have a finite-escape time property. Then, we propose to use the following modified switching inequality for the derivative of the Lyapunov function for the distributed AOFBSMCs

$$
\dot{V}+\lambda_{\min }(\mathcal{K})\|S\|^{2} \leq k_{f}
$$

where $k_{f}=\chi \epsilon$ with $\chi>0$. The constant $k_{f}$ has appeared due to the presence 
of the non-vanishing estimation error term $\eta$. The value of $k_{f}$ can be made very small by making $\epsilon$ smaller. Then, the value of the dwell time constant $t_{d}$ will also be smaller.

Based on the above analysis, let us now state our main results for the output feedback based distributed SMC scheme in the following Theorem.

Theorem 4.4 : Consider the closed loop control system designed by using (4.2) and (4.50) with the switching-logic defined in Algorithm 4.1. Then, for any given $(e(0), \hat{e}(0)) \in \Omega_{c o}, \theta \in \Omega_{i}$ and $\theta_{i} \in \Omega_{i}$ with $i \in \mathcal{M}$, there exists a small $\epsilon>0$ and dwell time $t_{d}>T_{1}(\epsilon)$ such that the candidate AOFBSMC, corresponding to an appropriate model, is tuned to the plant which ensures that all the state variables of the closed loop systems are bounded by a bound that can be made arbitrarily small for small value of $\epsilon$ and $k_{f}$.

Proof: The proof of Theorem 4.3 and Theorem 4.4 can be shown along the line of the switching-logic defined in Algorithm 4.1 [84, 85]. We first assume that the all the error sate vectors $e$ are available for feedback to construct the candidate controllers (4.49). Then we proceed with the following steps to prove Theorem 4.3. Case 1: Let us first put the classical ASMC $\tau\left(e, Q_{d}, \hat{\theta}\right)$ along with an adaptation law (4.42) into the loop for a short period $t \in\left[t_{o}, t_{o}+t_{d}\right]$. If the parameter estimates, $\hat{\theta}$, provided by standard adaptation mechanism is the closest model to the plant then the derivative of the Lyapunov inequality will be satisfied. This implies that the error trajectories will be decreasing sequence, and there will not be any more switching. Thus all the signals in the closed-loop systems under (4.49) are bounded and converge to zero within a finite time.

Case 2: If the parameter estimates, $\hat{\theta}$, provided by standard adaptation mechanism is not the right model that is $\hat{\theta} \notin \Omega_{i}$, then the classical ASMC will not satisfy the Lyapunov inequality. This implies that the error trajectory will be increasing sequence and the model obtained from classical ASMC will be excluded. Then, at $t=t_{i}$ with $t_{i} \geq t_{o}+t_{d}$, a candidate AMC $\tau^{i}\left(e, Q_{d}, \theta_{i}\right)$ with the model $\theta_{i}$ and $i=1$ will be put into the loop. Once again, we dwell it for a short period of time 
$t_{o}+t_{d}$ to see whether or not the switching inequality decreasing sufficiently fast to switch to the next candidate controller. If the controller does not satisfy the Lyapunov inequality then we switch again to the next candidate $\operatorname{ASMC} \tau^{i}\left(e, Q_{d}, \theta_{i}\right)$ with $i=2$. We repeat the search until we find a candidate ASMC that satisfies the inequality $\dot{V}(t) \leq 0$. Since $\theta \in \Omega_{i}$ there exists a candidate ASMC with $\theta_{i *}$ where $i=i *$ and $i \in \mathcal{M}(i *)$ that best approximates the plant satisfying the resetting inequality.

Case 3: Once the logic identify candidate ASMC that guarantees the switching inequality, no more switching will be allowed. Then we can conclude that the tracking trajectories converge to zero within a finite time. The proof of Theorem 4.3 is complete.

Let us now replace unknown velocity signals by the output of the linear observer. To ensure robust state estimates, we choose a small value of $\epsilon$ and $t_{d}>T_{1}(\epsilon)$. Using the linear observer, it is shown that the closed-loop error trajectories satisfy semi-global stability property as $\dot{V}(t) \leq k_{f}$. This implies that for a given $k_{f}$, using a small value of $\epsilon>0$, the trajectory will be bounded and the error bound $|e(t)|$ can be made small for a small value of $\epsilon$ and $k_{f}$. Therefore, we use $\dot{V}(t) \leq k_{f}$ as an inequality for the switching condition, and we follow the same steps that used for proving the Theorem 4.3. The proof of Theorem 4.4 is complete.

The problem of the pre-ordered switching Algorithm 4.1 is that when the number of the distributed ASMC becomes large then the long switching search (number of search) may produce unacceptable transient tracking performance. This is mainly because, in the presence of large number of candidate controllers, the switching has to travel through a large number of candidate controllers before converging to the one that satisfies the Lyapunov inequality. On the other hand, if the parameter changes after switching events (if any due to fault) then the logic stated in Algorithm 4.1 will be insensitive to the parameter change which may cause large transient tracking performance.

To avoid unacceptable transient tracking from pre-ordered switching-logic, we 
allow ASMCs to be switched instantaneously using with the following switching Algorithm 4.2.

Algorithm 4.2: Suppose that the controllers $i \in \mathcal{M}=\{1,2,3, \ldots ., N\}$ and the switching inequality are available at any time $t$. Then we follow the following steps to identify a candidate ASMC that best approximate the plant at any instant of time.

- A. Assuming that the initial time $t_{0}=0$, the controller index $i \in \mathcal{M}=$ $\{1,2,3, \ldots \ldots ., N\}$ and the small positive dwell time constant $t_{d}>0$.

- B. We first put classical $A S M C \tau\left(e, Q_{d}, \hat{\theta}\right)$ along with standard adaptation law $\hat{\theta}$ and dwell it for some time, $t \in\left[t_{o}, t_{o}+t_{d}\right]$.

- C. At $t \geq t_{o}+t_{d}$, we check the derivative of the fixed Lyapunov inequality $\dot{V}(t) \leq 0$ to see which candidate controller satisfies the switching condition. If the classical ASMC guarantees the inequality then we stay with that controller until the moment of time the switching inequality is violated. If the classical $A S M C$ does not satisfy $\dot{V}(t) \leq 0$ then at $t \geq t_{o}+t_{d}$ we switch to the candidate ASMC that ensures the switching condition.

- D. If the inequality $\dot{V}(t) \leq 0$ never violated then there will not be any switching. Then the output trajectory tracks the desired one that is $q(t) \rightarrow q_{d}(t)$. If at some time, say $t_{i} \geq t_{o}+t_{d}$ with $t_{o}=t_{i}$, tuned ASMC does not satisfy the switching inequality then another candidate that guarantees $\dot{V}(t) \leq 0$ will be put into the system.

We now summarize our main results in the following Theorem when all the process states are measurable.

Theorem 4.5: Consider the closed-loop system formulated by using (4.2) and control input (4.49) under the switching Algorithm 4.2. Then, there exists a time $t$ such that the classical ASMC (4.35), where the parameter estimate provided by the standard adaptation law (4.36), is switched into a family of distributed ASMC 
$\tau^{i}\left(e, Q_{d}, \theta_{i}\right)$ with $i \in \mathcal{M}$ that satisfies the inequality $\dot{V}(t) \leq 0$. Then we conclude that the adaptive sliding mode control system with the given switching condition ensures that all the signals in the closed-loop system are bounded and the tracking errors converge to zero within a short time.

The following theorem is given for the distributed adaptive output feedback sliding mode control algorithms (4.50) where the unknown velocity state vectors are estimated by the output of the linear observer.

Theorem 4.6: Consider the closed-loop system formulated by using (4.2), and (4.50) under the switching logic defined in Algorithm 4.2. Then, for any given $(e(0), \hat{e}(0)) \in \Omega_{c o}$ and $\theta \in \Omega_{i}$ with $i \in \mathcal{M}$, there exists a small $\epsilon$ and $t_{d}>T_{1}(\epsilon)$ such that classical AOFBSMC with standard adaptation law (4.42) is switched into a candidate controller corresponding to distributed candidate models $\theta_{i}$ with $i \in \mathcal{M}$

that ensures the derivative of the Lyapunov inequality $\dot{V}(t) \leq k_{f}$. Then the adaptive output feedback sliding mode control system guarantees that the closed loop error trajectories under (4.50) are bounded by a bound that can be made arbitrarily small for small value of $\epsilon$ and $k_{f}$.

Proof: The proof of Theorem 4.5 and Theorem 4.6 can be shown along the line of Algorithm 4.2. So, we omitted the details proof for brevity.

\subsubsection{Multiple Lyapunov-functions Based Distributed SMC}

The switching-logic proposed in previous section is based on using a fixed inequality for the derivative of the Lyapunov-function. In this section, we introduce a switching-logic that depends on the derivative of multiple Lyapunov-function inequalities. To begin with this development, we first consider that, for a given compact set of initial conditions of interest $e(0) \in \Omega_{c o}$ and for every $\theta \in \Omega_{i}$, with $i \in \mathcal{M}=\{1,2,3, \ldots, N\}$, we design a family of distributed candidate ASMC $\tau^{i}\left(e, Q_{d}, \theta_{i}\right)$ bounded in $e$ by saturating them outside the domain of interest such that for every $\theta \in \Omega_{i}$ all the signals in the closed-loop system started inside $\Omega_{c o}$ are bounded such that the error trajectory converge to zero within a short time. 
We also consider that a family of Lyapunov-function candidate corresponding to candidate ASMCs as $\alpha_{2}^{i}\|e\|^{2} \leq V_{i}\left(e, \tilde{\theta}_{i}\right) \leq \alpha_{3}^{i}\|e\|^{2} \forall e \in \Omega_{c}^{i}=\left\{\left(e, \tilde{\theta}_{i}\right) \mid V_{i}\left(e, \tilde{\theta}_{i}\right) \leq c\right\}$ and $\forall\left(\theta, \theta_{i}\right) \in \Omega_{i}$, where $c>0, \tilde{\theta}_{i}=\left(\theta_{i}-\theta\right)$ and $\alpha_{2}^{i}$ and $\alpha_{3}^{i}$ are bounded positive constants. Then we follow the following logic based switching supervisory control technique to identify a candidate SMC which best approximates the plant at each instant of time such that all the trajectories converge to zero within a very short time.

Algorithm 4.3: Suppose that the candidate ASMCs $\tau^{i}\left(e, Q_{d}, \hat{\theta}_{i}\right)$ as well as Lyapunov-function candidates $V_{i}\left(e, \tilde{\theta}_{i}\right)$ with $i \in \mathcal{M}=\{1,2,3, \ldots . ., N\}$ are available at any time $t$.

- A. Define the initial time $t_{o}=0$, the switching index $i \in \mathcal{M}=\{1,2,3, \ldots ., N\}$ and a small positive dwell time constant $t_{d}>0$.

- B. Put the classical ASMC $\tau\left(e, Q_{d}, \hat{\theta}\right)$ with standard adaptation mechanism for short period of time $t \in\left[t_{o}, t_{o}+t_{d}\right]$.

- C. For $t \geq t_{o}+t_{d}$, we continuously check the inequality for the multiple Lyapunov-function candidates to see which candidate ASMC generates guaranteed decrease in the value of $\triangle W_{i}(t)=V_{i}\left(t_{s}\right)-V_{0}(t) \leq 0$ where $t_{s}>t_{o}+t_{d}$ is the switching time and $V_{0}(t)$ is the Lyapunov function for the classical ASMC (4.36). We keep this classical ASMC in the loop until the moment of time $t_{i} \geq t_{o}+t_{d}$ when the switching inequality is violated. If classical ASMC does not satisfy then at $t=t_{i}$ switch it to the candidate ASMC that generates largest guaranteed decrease in the value of $\Delta W_{i}(t) \leq 0$. Note that if the classical ASMC satisfies the switching inequality then we stay with that controller.

- D. If the inequality $\Delta W_{i}(t) \leq 0$ is never violated then there will not be any more switching. Then we conclude that the plant output tracks the desired one, i.e. $q(t) \rightarrow q_{d}(t)$. If at some time, say $t_{i}$ with $t_{i} \geq t_{o}+t_{d}$ and $t_{i}=t_{o}$, the candidate ASMC that acting in the loop does not satisfy $\triangle W_{i}(t) \leq 0$ then 
another candidate ASMC will be put in the system as there always exists a guaranteed minimum value of $\Delta W(t) \leq 0$ at that instant of time.

We now summarize the results for the multiple Lyapunov-functions based ASMC as a state feedback control design in the following Theorem.

Theorem 4.7 : Consider the closed-loop system formulated by using (4.2) with the candidate ASMCs (4.49) under the switching control logic defined in Algorithm 4.3. Then, there exists a time $t$ such that, according to Algorithm 4.3, the ASMC corresponding to the guaranteed decrease in the value of $\triangle W_{i}(t) \leq 0$ is tuned to the plant which ensures that all the signals in the closed-loop model are bounded and $e(t) \rightarrow 0$ within a very short time.

Theorem 4.7 can be applied when all the state vectors are available for feedback to design candidate ASMCs (4.49). Now, we assume that the velocity signals are not available and replace the unknown velocity signals in (4.49) by the output of the linear estimator to formulate distributed candidate AOFBSMCs (4.50). Then, we present the main results for the distributed AOFBSMC in the following Theorem 4.8.

Theorem 4.8: Consider the closed-loop system (4.2) and (4.50) under the switching-logic stated in Algorithm 4.3 [92, 98]. Then, for any given $(e(0), \hat{e}(0)) \in$ $\Omega_{c o}$ and $\theta_{i} \in \Omega_{i}$ with $i \in \mathcal{M}$, there exists a small value of $\epsilon>0$ and $t_{d}>T_{1}(\epsilon)$ such that the controller corresponding to a guaranteed decrease in the value of $\triangle W_{i}(t) \leq$ 0 is tuned to the plant. Then the output feedback control system ensures that all the state variables of the closed-loop system are bounded by a bound that can be made arbitrarily small for small value of $\epsilon$.

Proof: The proof of Theorem 4.7 and Theorem 4.8 can be shown along the line of the switching scheme defined in Algorithm 4.3. The main idea in Algorithm 4.3 is to compare the distributed $\operatorname{ASMCs} \tau^{i}\left(e, Q_{d}, \theta_{i}\right)$ with $i \in \mathcal{M}$, at each instant of time to see which distributed candidate provides the highest decrease in the 
value of the Lyapunov-function that is

$$
\triangle W_{i}(t)=V_{i}\left(t_{s}\right)-V_{0}(t)
$$

where

$$
V_{0}(t)=\frac{1}{2} e^{T}(t) Q_{s m} e(t)+\frac{1}{2} \tilde{\theta}^{T}(t) \Gamma^{-1} \tilde{\theta}(t)
$$

and

$$
V_{i}\left(t_{s}\right)=\frac{1}{2} e^{T}\left(t_{s}\right) Q_{s m} e\left(t_{s}\right)+\frac{1}{2} \tilde{\theta}_{i}^{T}\left(t_{s}\right) \Gamma^{-1} \tilde{\theta}_{i}\left(t_{s}\right)
$$

with $\tilde{\theta}(t)=(\hat{\theta}(t)-\theta), \tilde{\theta}_{i}\left(t_{s}\right)=\left(\theta_{i}\left(t_{s}\right)-\theta\right)$ and $t_{s}>t_{o}+t_{d}$ is the time when the parameter estimate $\hat{\theta}(t)$ provided by standard adaptation mechanism is switched into a model among distributed model sets $\theta_{i}\left(t_{s}\right)$ which best approximates the plant $\theta$. This means that the switching will be occurred if $V_{i}\left(t_{s}\right)$ is a non-increasing sequence with respect to $i$ that is $\Delta W_{i}(t) \leq 0$. Here, $V_{0}$ is the Lyapunov-function for classical ASMC controller (4.39) and $V_{i}$ is the multiple Lyapunov-functions for distributed ASMCs (4.49). To identify an appropriate distributed controller, let us simplify $\triangle W_{i}(t)$ as

$\Delta W_{i}(t)=\frac{1}{2} e^{T}\left(t_{s}\right) Q_{s m} e\left(t_{s}\right)-\frac{1}{2} e^{T}(t) Q_{s m} e(t)-\frac{1}{2}\left[\Theta_{i}^{T} \Gamma^{-1} \Theta_{i}-2 \Theta_{i}^{T} \Gamma^{-1}\left(\theta_{i}\left(t_{s}\right)-\theta\right)\right]$ where $\Theta_{i}=\left(\theta_{i}\left(t_{s}\right)-\hat{\theta}(t)\right)$. For simplicity, we define $V_{i}\left(t_{s}\right)$ and $V_{0}(t)$ instead of $V_{i}\left(e\left(t_{s}\right), \tilde{\theta}_{i}\left(t_{s}\right)\right)$ and $V_{0}(e(t), \tilde{\theta}(t))$, respectively.

To show $\triangle W_{i}(t) \leq 0$, we need to calculate the value of $\left(\theta_{i}\left(t_{s}\right)-\theta\right)$. To do that, let us define the torque prediction error models as

$$
E_{i}(t)=Y\left(e, \dot{q}_{r}, \ddot{q}_{r}\right)\left(\theta_{i}-\theta\right)
$$

where $Y\left(e, \dot{q}_{r}, \ddot{q}_{r}\right)$ is the regressor matrix common to all candidate models. For the sake of simplicity of the analysis, we consider that $\theta$ is time-invariant, but the proposed control logic can be used for situation when uncertain parameter $\theta$ is time-varying. Then, we can write after some manipulations as

$$
\left(\theta-\theta_{i}\right)=\xi^{-1}(t) \omega_{i}(t)
$$


where $\xi(t)>0$,

$$
\xi(t)=\int_{o}^{t} Y\left(e, \dot{q}_{r}, \ddot{q}_{r}\right) Y^{T}\left(e, \dot{q}_{r}, \ddot{q}_{r}\right) d \tau
$$

and

$$
\omega_{i}(t)=\int_{o}^{t} Y\left(e, \dot{q}_{r}, \ddot{q}_{r}\right) E_{i} d \tau
$$

Since the control input is bounded outside the domain of interest via using saturation mechanism then $Y\left(e, \dot{q}_{r}, \ddot{q}_{r}\right)$ is also bounded. Then, the switching condition can be obtained as

$$
\triangle W_{i}(t)=-\frac{1}{2} \Theta_{i}^{T} \Gamma^{-1}\left[\Theta_{i}+2 \xi^{-1}(t) \omega_{i}(t)\right]+\frac{1}{2} e^{T}\left(t_{s}\right) Q_{s m} e\left(t_{s}\right)-\frac{1}{2} e^{T}(t) Q_{s m} e(t) \leq 0
$$

This implies that the parameter estimate $\hat{\theta}(t)$ will be switched to the distributed candidate models $\theta_{i}\left(t_{s}\right)$ only if the data has a strong evidence of the parameters.

If $\xi(t)=0$ then there will not be any switching. Thats is the case when the system operates in steady state condition.

But, if there is sudden change in the parameters then the switching condition will be satisfied. When the system is poorly excited, that is $\xi(t) \approx 0$, then the error $E_{i}$ requires to be very small to switch the parameter estimate of (4.36).

During transient phase, the data contains strong evidence of the parameters satisfying $\triangle W_{i}(t) \leq 0$ which will improve the transient tracking performance. Thus, $V_{i}$ is a non-increasing sequence as $V_{0}$ is bounded and the switching will be occurred during transient phase.

Based on the above analysis, we now proceed the proof of Theorem 4.7 along the line of Algorithm 4.3.

Case 1: We first first put the classical ASMC into the loop for the time period $t \in\left[t_{o}, t_{o}+t_{d}\right]$. At $t \geq t_{o}+t_{d}$, we check the inequality $\triangle W_{i}(t) \leq 0$. If it is satisfied, then we stay with that controller as long as $\Delta W_{i}(t) \leq 0$ holds. Then we can conclude that the error trajectories converge to zero, $e(t) \rightarrow 0$ within a very short time.

Case 2: If the classical ASMC does not satisfy switching criterion then new candidate ASMC will be put in the system based upon a guaranteed decrease in the 
value of $\triangle W_{i}(t)$.

Case 3 If the inequality, $\triangle W_{i}(t) \leq 0$, never violated then there will not be any switching. This implies that the plant output tracks the desired one, i.e., $q(t) \rightarrow q_{d}(t)$.

Case 4: If there exists a time $t_{i} \geq t_{i}+t_{d}$ with $t_{o}+t_{d}=t_{i}$ such that the controller that acting in the loop does not satisfy $\triangle W_{i}(t) \leq 0$, then another candidate ASMC will be put in the loop via comparing a family of the candidate ASMC that provides largest guaranteed minimum values of $\triangle W_{i}(t)$ (largest negative jump in the Lyapunov-function with respect to $i$ ) at that instant of time. The proof of Theorem 4.7 is complete.

Let us now replace unknown velocity signals by the output of the linear observer to construct distributed AOFBSMCs (4.50). To ensure robust state estimates, one requires to select small value of $\epsilon$ such that $\|\eta\|$ is small and $t_{d}>$ $T_{1}(\epsilon)$. Then, we replace $Y\left(\hat{e}, \dot{\hat{q}}_{r}, \ddot{\hat{q}}_{r}\right)$ by $Y\left(e, \epsilon \eta, \dot{q}_{r}, \ddot{q}_{r}\right)$ and we follow the same steps that used for proving Theorem 4.7 to obtain the reseting inequality $\triangle W_{i}(t)=$ $-\frac{1}{2} \Theta_{i}^{T} \Gamma^{-1}\left[\Theta_{i}+2\left(\theta-\theta_{i}\left(t_{s}\right)\right)\right]+\frac{1}{2} e^{T}\left(t_{s}\right) Q_{s m} e\left(t_{s}\right)-\frac{1}{2} e^{T}(t) Q_{s m} e(t) \leq 0$ with $\Theta_{i}=$ $\left(\theta_{i}\left(t_{s}\right)-\hat{\theta}(t)\right)$. The proof of Theorem 4.8 is complete.

\subsubsection{Simulation Results}

This subsection presents the design and implementation process of the distributed SMC strategy to demonstarte the theoretical development for the real-time applications. For our evaluations, we consider a 2-DOF serial link manipulator as defined in (4.45). We first consider that the plant parameters $\theta \in \Re^{2}$ in (4.45) belong to a known but comparatively large compact set as $\Omega \in[-10,10]$. We define the initial states as $|e(0)| \leq 2,|\hat{e}(0)| \leq 2$ and $\hat{\theta}(0)=0$. Then, we distribute the parameter set $\Omega$ equally into a finite number of smaller compact subsets as $\theta_{i} \in \Omega_{i}$ with $\Omega=$ $\bigcup_{i=1}^{41}\left\{\Omega_{i}\right\}$, that is, $\Omega=\bigcup_{i=1}^{41}\left\{\theta_{i}\right\}=\{-10,-9.5, ., ., . ., . ., ., . ., .9 .5,10\} \times\{-10,10\}$. The control design parameters $\lambda_{1}, \lambda_{2}, \mathcal{K}_{1}$ and $\mathcal{K}_{2}$ are chosen as $\lambda_{1}=2, \lambda_{2}=2$, $\mathcal{K}_{1}=15$ and $\mathcal{K}_{2}=15$. Note that the control gains $\lambda_{1}, \lambda_{2}, \mathcal{K}_{1}$ and $\mathcal{K}_{2}$ are common 
to all $i=41$ candidate controllers.

The value of $\lambda_{1}, \lambda_{2}, \mathcal{K}_{1}$ and $\mathcal{K}_{2}$ are required to choose in such a way that they ensures acceptable transient tracking performance. From the practical point of view, $\lambda_{1}, \lambda_{2}, \mathcal{K}_{1}$ and $\mathcal{K}_{2}$ can be designed according to well established design rules for PD control, for example, such that the closed loop would be critically damped and the response would be fast enough. The discontinuous control gains $K$ and $\frac{1}{\phi}$ as well as the learning gains $\Gamma$ for adaptive SMC controller are chosen such that $\left(\theta, \theta_{i}\right) \in \Omega_{i}$. For our evaluation, these design constants are chosen as $K_{i 1}=15$, $K_{i 2}=15, \phi_{i 1}=0.7, \phi_{i 2}=0.7$ and $\Gamma=\left[\begin{array}{llll}\Gamma_{1} & 0 ; 0 & \Gamma_{2}\end{array}\right]$ with $\Gamma_{1}=10$ and as $\Gamma_{2}=10$. Note that for the given initial conditions of interest, we can increase the value of $\phi$ and reduce the value of $K$. On the other hand, one may use boundary layer technique to avoid control chattering activity. For the given set of initial interests, we now define $t_{d}=0.003$. Applying with the above design constants, let us now construct a family of candidate controllers as a state feedback

$$
\tau^{i}\left(e, Q_{d}, \theta_{i}\right)=Y\left(e, \dot{q}_{r}, \ddot{q}_{r}\right) \theta_{i}-\mathcal{K} S-K_{i} \operatorname{sat}\left(\frac{S}{\phi_{i}}\right)
$$

with $i=41$. Then we implement Theorem 4.3 and Theorem 4.7 on the given model (4.45) to examine the tracking performance of the switching logic defined in Algorithm 4.1 and Algorithm 4.3. The implemented results are given in Figures 4.12 and 4.13 (state feedback case) with $\tilde{\theta}=4$ and $\tilde{\theta}=8$, that is, $i *=29$ and $i *=37$, respectively. The left column of each Figure is for Theorem 4.3 and the right column of each figure is for Theorem 4.7.

In view of Figures 4.12-4.13, one can observe that the tracking errors under Theorem 4.3 of switching Algorithm 4.1 are larger than the errors obtained under Theorem 4.7 of switching Algorithm 4.3. Notice also from Figure 4.13 is that the transient tracking errors under pre-ordered switching control of Theorem 4.3 increase with the increase of $i^{*}$. This is mainly because the switching has to scan through a number of candidate controllers before converging to the one that satisfies Lyapunov inequality. As a result, relatively large transient tracking errors can be seen during transient phase. Such undesirable transient behavior of pre-ordered 

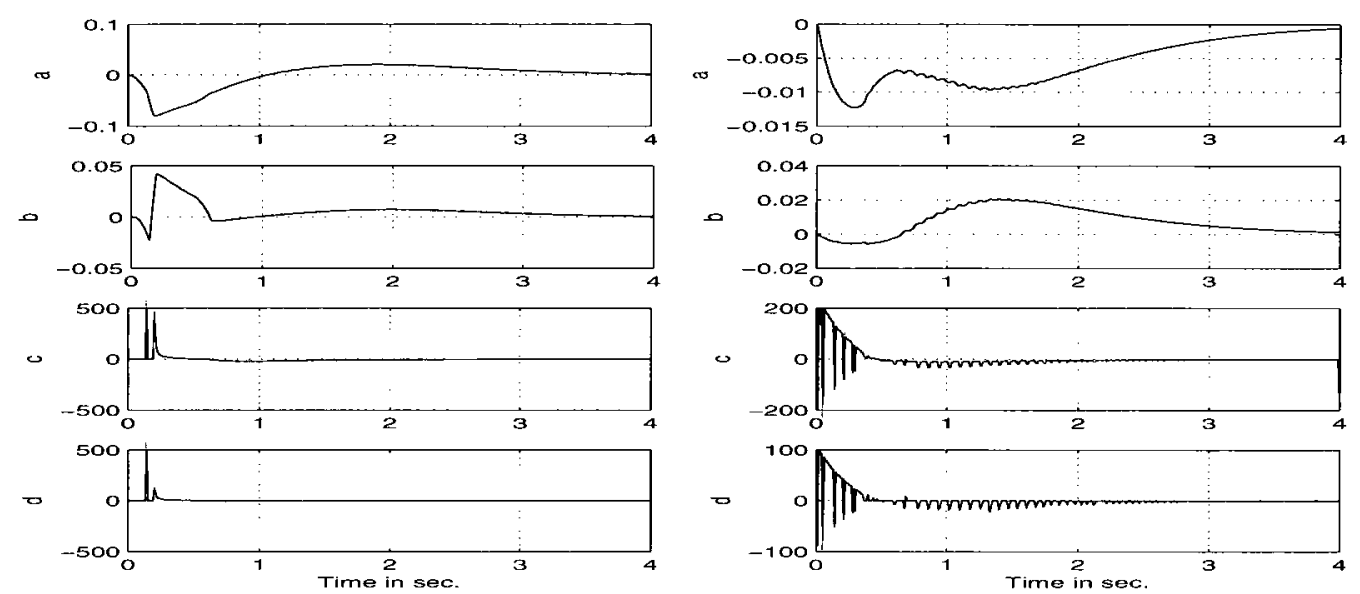

Figure 4.12: The simulation results with Theorem 4.3 and Theorem 4.7 under $\tilde{\theta}=4$ : Left column is for Theorem 4.3 and right column is for Theorem 4.7, where $a$ : output tracking errors (radians) for joint $1, b$ : output tracking errors (radians) for joint $2, c$ : control input (newton-meters) for joint $1, d$ : control input (newtonmeters) for joint 2 .

switching can be reduced by using instantaneous switching technique proposed in Algorithm 4.2 of Theorem 4.5 and switching Algorithm 4.3 of Theorem 4.7. The idea in switching Algorithms 4.2 and 4.3 is to monitor continuously the switching condition (fixed Lyapunov-function inequality in Algorithm 4.2 and multiple Lyapunov-function inequalities in Algorithm 4.3) to see which candidate ASMC among a family of candidate ASMCs satisfy the inequality, and then switch to the candidate controller that satisfies the derivative of the Lyapunov-inequality.

Let us now compare the tracking performance of Theorem 4.7 with the SMbased adaptive SMC algorithm (4.35), (4.36). The tested results are depicted in Figure 4.14 with $\tilde{\theta}=8$ (right column is for Theorem 4.7 and left column is for the SM-based ASMC (4.35)-(4.36)). The tests are conducted under the same set of the controller design parameters as considered for our last evaluation.

By comparing left and right column of the Figure 4.14, we can observe that the tracking errors under multiple Lyapunov-functions based distributed SMC control 

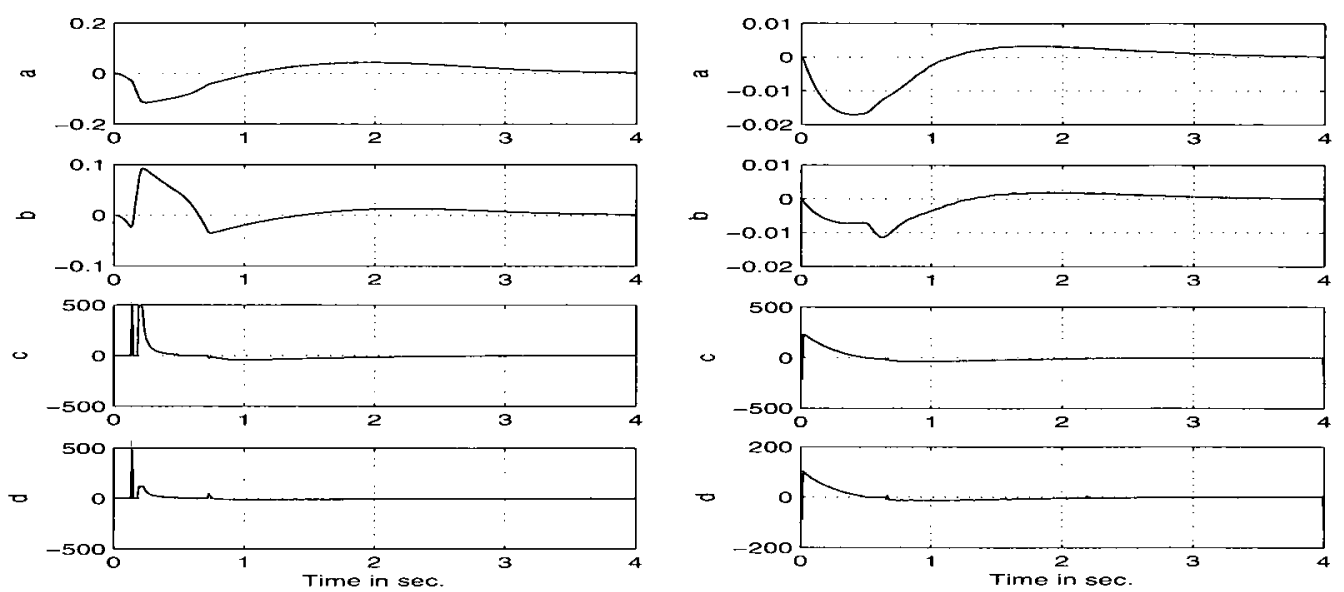

Figure 4.13: The simulation results with Theorem 4.3 and Theorem 4.7 under $\tilde{\theta}=8$ : Left column is for Theorem 4.3 and right column is for Theorem 4.7, where $a$ : output tracking errors (radians) for joint $1, b$ : output tracking errors (radians) for joint 2, $c$ : control input (newton-meters) for joint $1, d$ : control input (newtonmeters) for joint 2 .

technique almost closed to zero, but relatively large tracking errors can be seen under single model based classical ASMC algorithm.

We now investigate the robustness of Theorem 4.7 with respect to non ideal (practical) operating conditions. To create such environment, let us add a band limited white noise, $w(t)$, into the output $q(t)$ and input $\tau(t)$ of the given system (4.45). For our evaluation, the disturbance level $w(t)$ for the input and output is considered as shown in Figure 4.15. We then repeat the last evaluation with the same set of the design parameters. The conducted results are pictured in Figure 4.16 (right column is for Theorem 4.7 and left column is for SM-based ASMC). This Figure shows the control performance of Theorem 4.7 and the SM-based ASMC algorithm under $\tilde{\theta}=8$. In view of these figures, we can see that the control performance of Theorem 4.7 under ideal operating condition is almost similar to the performance achieved under non ideal situation. However, comparatively large tracking errors and control efforts (see, left column of the Figure 4.16), can be seen 

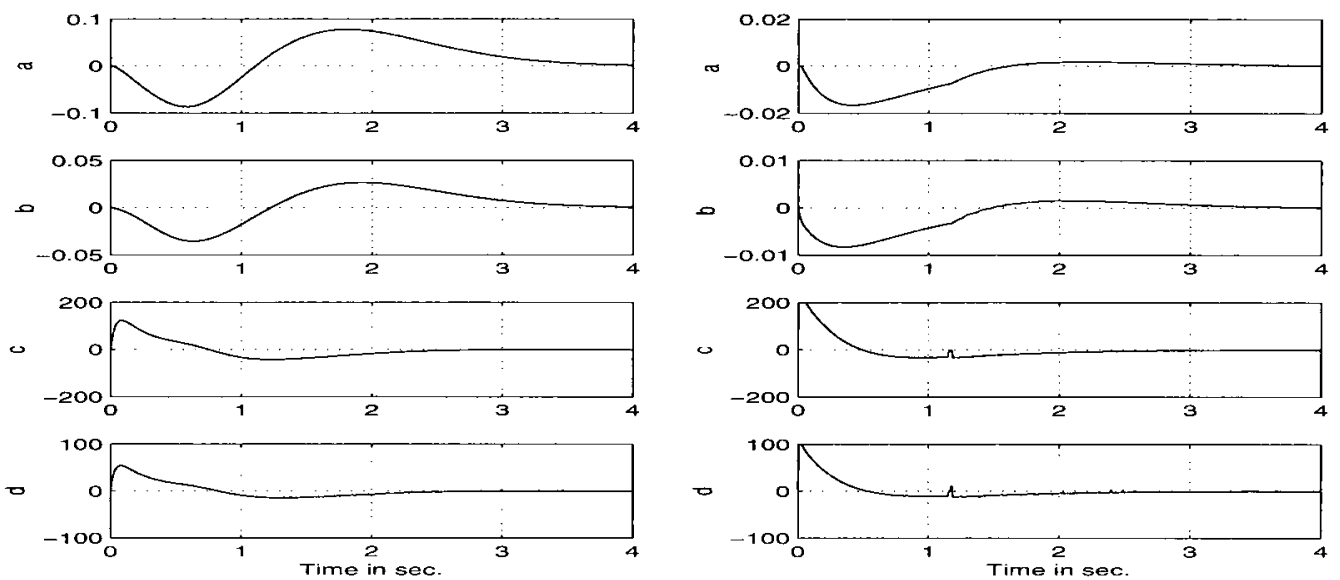

Figure 4.14: The simulation results with Theorem 4.7 and SM-based ASMC under $\tilde{\theta}=8$ : Left column is for ASMC algorithm (4.35), (4.36) and right column is for Theorem 4.7, where $a$ : output tracking errors (radians) for joint $1, b$ : output tracking errors (radians) for joint 2, $c$ : control input (newton-meters) for joint 1 , $d$ : control input (newton-meters) for joint 2 .

under SM-based ASMC approach.

Now we show that the performance obtained under state feedback based design (Theorem 4.3 and Theorem 4.7) can be recovered by using output feedback design (Theorem 4.4 and Theorem 4.8). To show that, our first task is to ensure robust reconstruction of the unknown states via using exponentially first observer dynamics. We compare the control performance of Theorem 4.4 with Theorem 4.8 for two value of $\epsilon$ as 0.005 (very high speed observer) and 0.1 (slow speed observer). Note that we keep the controller design parameters same as used for the evaluation of the state feedback based design (Theorem 4.3 and Theorem 4.7). The two sets of the observer design parameters are considered for the evaluation as $H_{1}=I_{2 \times 2}$, $H_{2}=I_{2 \times 2}, \epsilon=0.005$ and $H_{1}=20 I_{2 \times 2}, H_{2}=20 I_{2 \times 2}, \epsilon=0.1$. For the given set of the design parameters and the initial conditions of interest, we consider $t_{d}$ as $t_{d}=0.005$. Then, we choose the value of $k_{f 1}=0.05$ and $k_{f 2}=0.05$. With these design constants, we apply control laws (4.50) under the switching-logic defined 


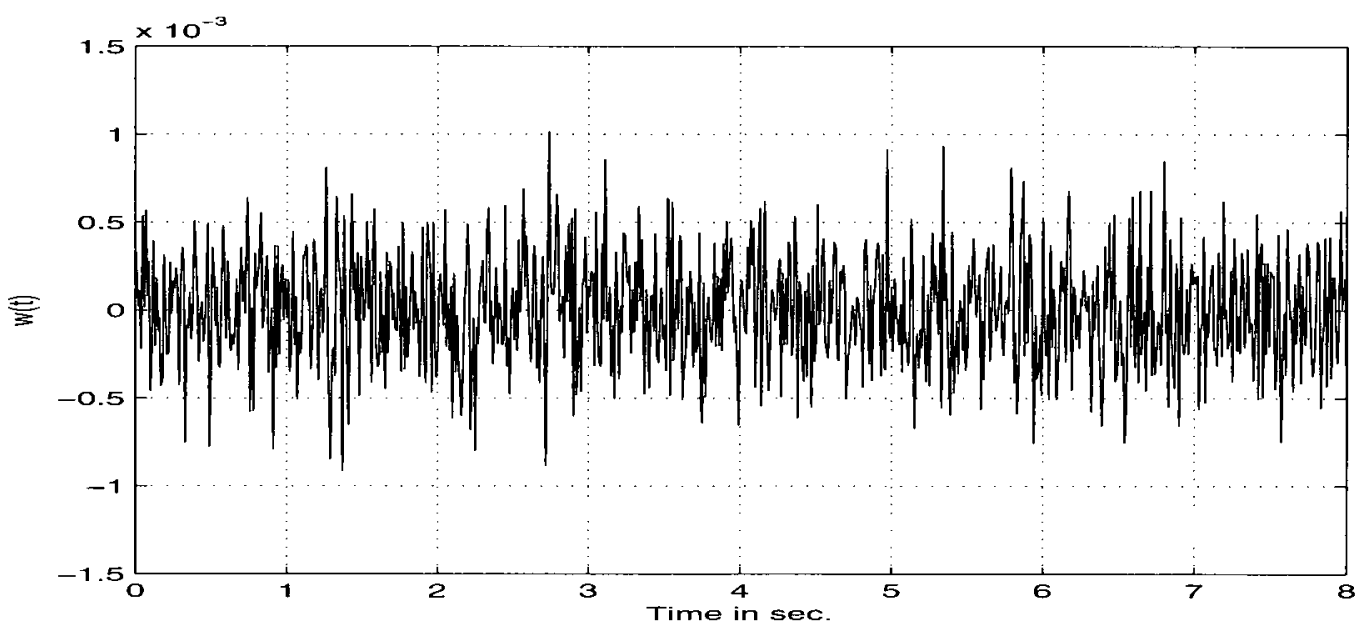

Figure 4.15: The disturbance level $w(t)$.

by Algorithm 4.1 (Theorem 4.4) and Algorithm 4.3 (Theorem 4.8) on the given system (4.45). The tested results are given in Figures 4.17-4.20. Figures 4.17 and 4.19 show the tracking performance when $\tilde{\theta}=4$. Figures 4.18 and 4.20 present the implementation results under $\tilde{\theta}=8$. Figures 4.17-4.18 depict the control performance when observer design constants are chosen as $H_{1}=I_{2 \times 2}, H_{2}=I_{2 \times 2}$, and $\epsilon=0.005$. Figures 4.19-4.20 picture the convergence with the following observer design constants $H_{1}=20 I_{2 \times 2}, H_{2}=20 I_{2 \times 2}$ and $\epsilon=0.1$. The left column of each Figure is for Theorem 4.4 and the right column of each figure is for Theorem 4.8. In view of Figures 4.12-4.13 and Figures 4.17-4.20 (see right column of each Figure), one can see that results under Theorem 4.8 (output feedback case) recover the performance achieved under Theorem 4.7 (state feedback case). However, undesirable transient tracking and control oscillation with output feedback based pre-ordered switching logic of Theorem 4.4 are clearly observed (see left column of Figures 4.17-4.20). Like Theorem 4.7, Theorem 4.8 can be used to reduce the transient tracking behavior that appeared in pre-routed switching-logic of Theorem 4.4.

We now compare the tracking performance of Theorem 4.8 with the single model based AOFBSMC algorithm (4.41)-(4.42). The conducted results are depicted in Figure 4.21 with $\tilde{\theta}=8$ and $H_{1}=20 I_{2 \times 2}, H_{2}=20 I_{2 \times 2}$ and $\epsilon=0.1$. For 

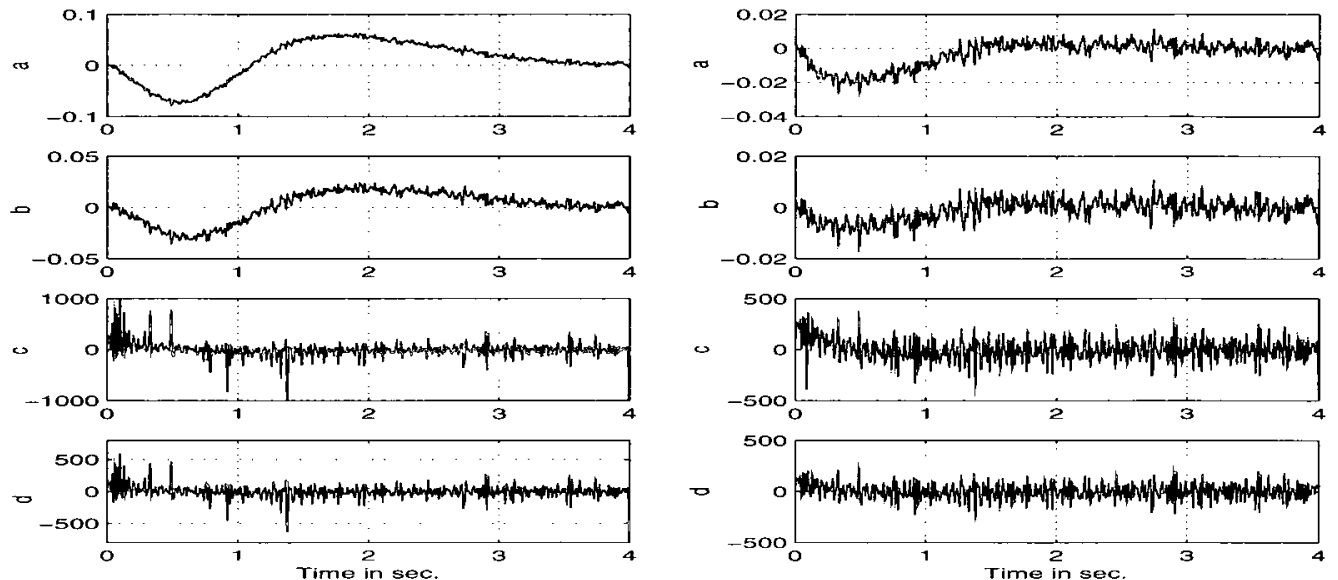

Figure 4.16: The simulation results with Theorem 4.7 and SM-based ASMC under $\tilde{\theta}=8$ with non ideal condition: Left column is for ASMC algorithm (4.35)-(4.36) and right column is for Theorem 4.7, where $a$ : output tracking errors (radians) for joint $1, b$ : output tracking errors (radians) for joint 2, $c$ : control input (newtonmeters) for joint $1, d$ : control input (newton-meters) for joint 2 .

fair comparison, we keep the same set of controller design parameters that applied for the evaluation of the state feedback adaptive SMC.

By comparing left (SM-based ASMC) and right column of the Figure 4.21 (Theorem 4.8), we can observe that the output tracking under theorem 8 converge to the desired one while, quite a large tracking errors can be seen under single model based AOFBSMC approach.

To examine the robustness of Theorem 4.8 with respect to non ideal operating conditions, we add a band limited white noise, $w(t)$, into the output $q(t)$ and input $\tau(t)$ of the given system. The disturbance level $w(t)$ for the input and output signals is chosen as depicted in Figure 4.14. Then, we repeat the last test with the same set of controller-observer design parameters. The tested results are depicted in Figure 4.22. The Figure 4.22 presents the control performance of Theorem 4.8 (right column of the Figure 2.2 is for Theorem 4.8) and SM-based AOFBSMC (left column of the Figure 4.22 is for algorithm ((4.41)-(4.42)). Notice from Figure 4.22 

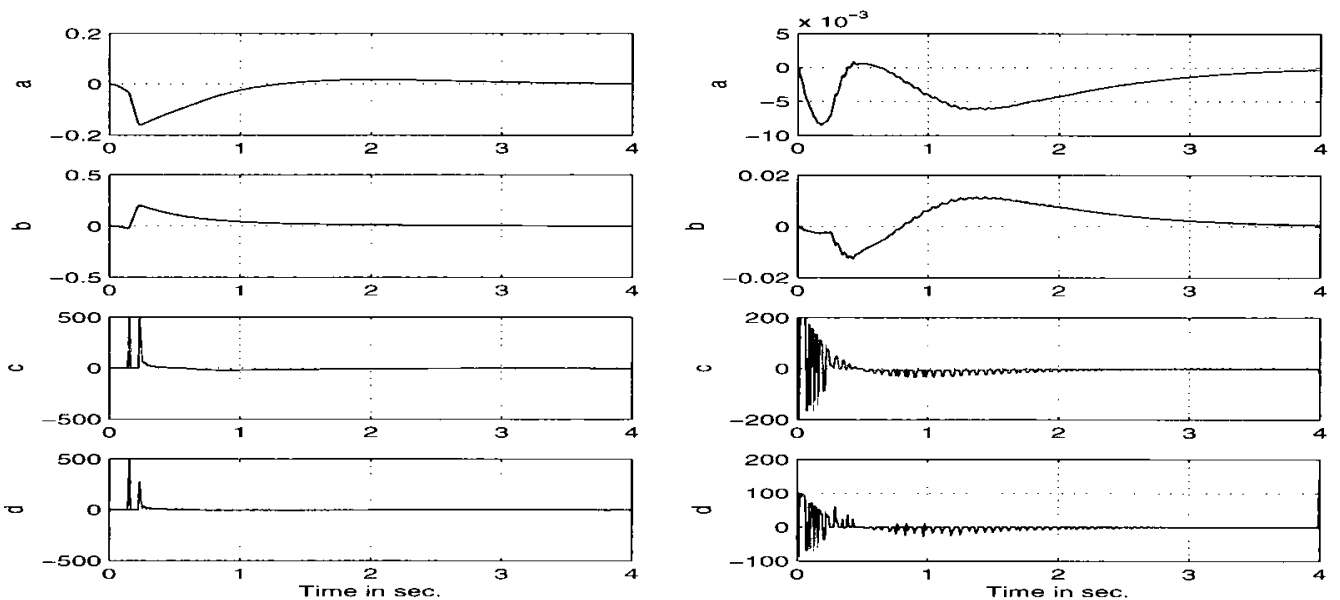

Figure 4.17: The simulation results with Theorem 4.4 and Theorem 4.8 with $\tilde{\theta}=4$ : Left column is for Theorem 4.4 and right column is for Theorem 4.8, where $a$ : output tracking errors (radians) for joint 1, $b$ : output tracking errors (radians) for joint 2, c: control input (newton-meters) for joint $1, d$ : control input (newtonmeters) for joint 2 .

is that control performance of Theorem 4.8 under non ideal operating condition is closed to the performance achieved under ideal situation (see right column of the Figure 4.21). The sampling time was set to $0.01 \mathrm{sec}$. for all the results reported in this chapter.

\subsection{Summary}

The contributions of this chapter can be divided into two parts.

First, we have introduced single model-based output feedback sliding mode control technique for a certain class of nonlinear systems. The design can be employed to remove the problem associated with state-feedback based robust control design reported in the literature. It has been shown that controller parameters can be tuned to achieve semi-global stability property of all the closed loop signals. 

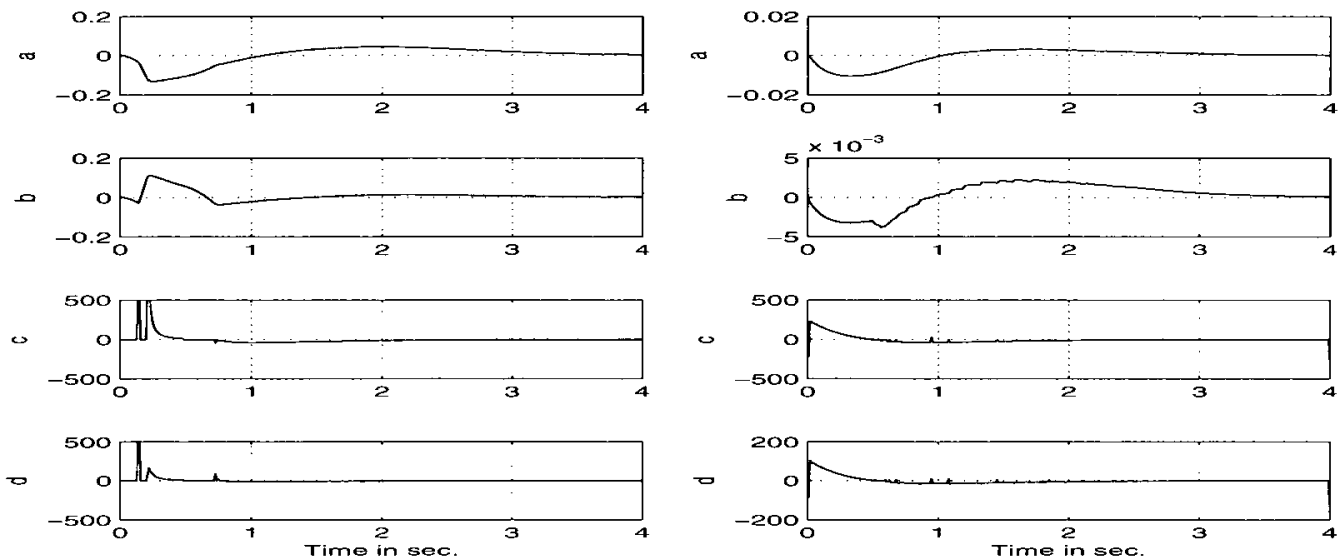

Figure 4.18: The simulation results with Theorem 4.4 and Theorem 4.8 with $\tilde{\theta}=8$ : Left column is for theorem 4.4 and right column is for theorem 4.8 , where $a$ : output tracking errors (radians) for joint $1, b$ : output tracking errors (radians) for joint 2 , $c$ : control input (newton-meters) for joint $1, d$ : control input (newton-meters) for joint 2 .

In the face of large scale modeling/parametric uncertainty, the SM-based SMC design, however, required very high discontinuous, learning and observer gains to achieve desired tracking objectives. In the second part, distributed controller/parameter based sliding mode technique has been proposed to improve the tracking performance with smaller values of the observer, discontinuous, learning control design parameters. The method switches among distributed SMC controllers through on-line estimation of the Lyapunov-function inequality. The evaluation on a 2-DOF robotic system has been used to demonstrate the theoretical development for real-world applications. These evaluations clearly showed the demand of the distributed SMC strategy over classical SMC approach. 

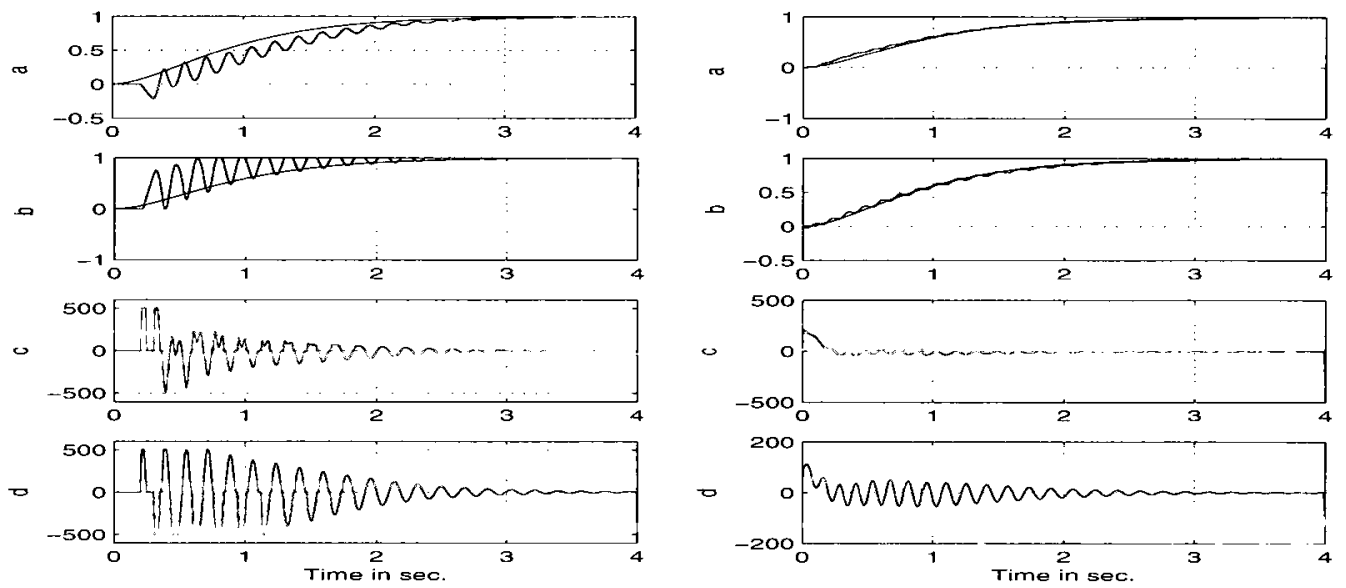

Figure 4.19: The simulation results with Theorem 4.4 and Theorem 4.8 with $\tilde{\theta}=4$ : Left column is for Theorem 4.4 and right column is fo Theorem 4.8, where $a$ is for output tracking for joint $1, b$ is for the output tracking of joint $2, c$ is for the control input (newton-meters) for joint $1, d$ is for the control input (newton-meters) of joint 2 .
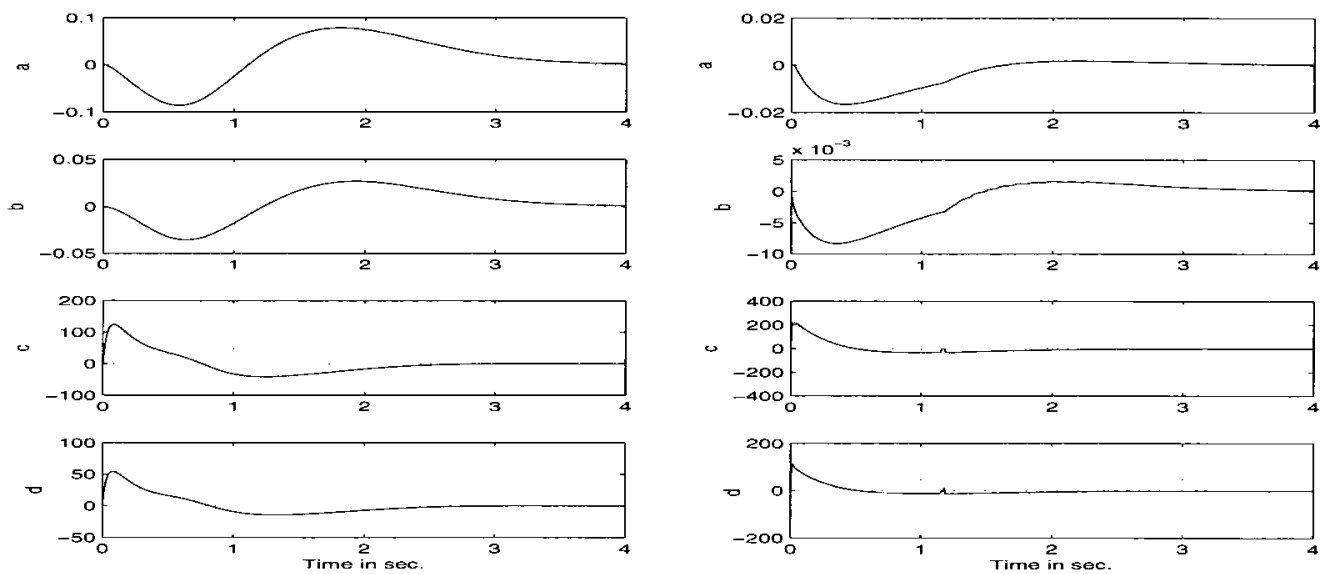

Figure 4.20: The simulation results with Theorem 4.4 and Theorem 4.8 with $\tilde{\theta}=8$ : Left column is for Theorem 4.4 and right column is for Theorem 4.8, where $a$ is for the output tracking (radians) for joint $1, b$ is for the output tracking (radians) for joint 2, $c$ : control input (newton-meters) for joint $1, d$ : control input (newtonmeters) for joint 2 . 

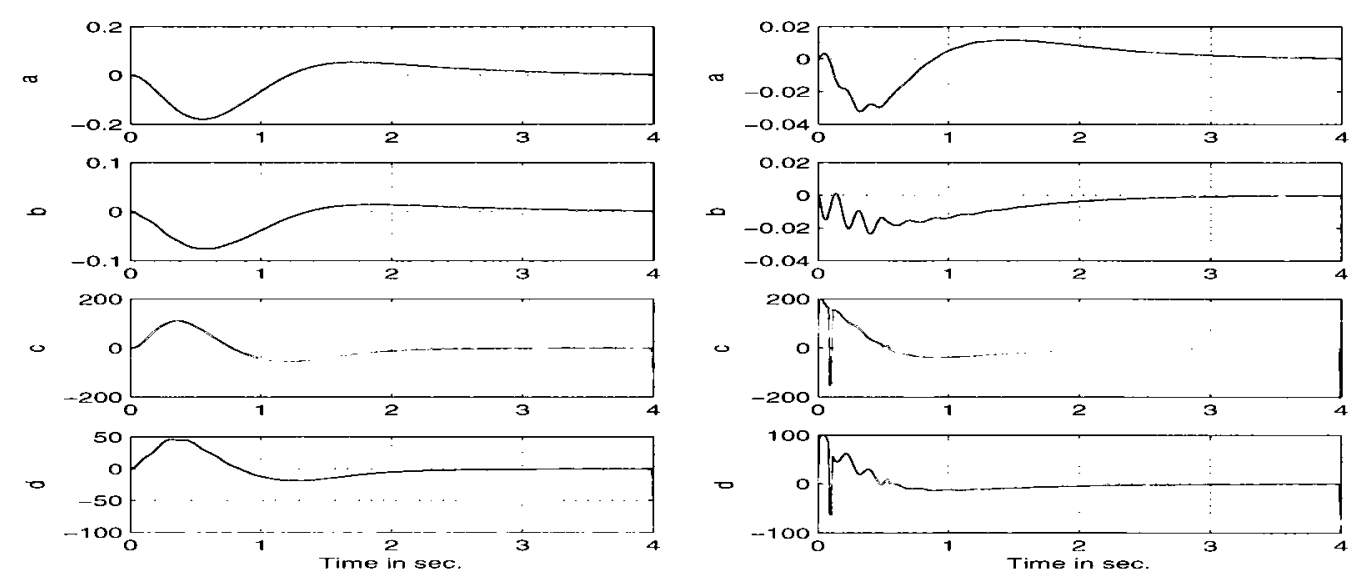

Figure 4.21: The simulation results with Theorem 4.8 and SM-based AOFBSMC under $\tilde{\theta}=8$ with ideal condition: Left column is for AOFBSMC algorithm (4.41)(4.42) and right column is for Theorem 4.8, where a: output tracking errors (radians) for joint $1, b$ : output tracking errors (radians) for joint $2, c$ : control input (newton-meters) for joint $1, d$ : control input (newton-meters) for joint 2.
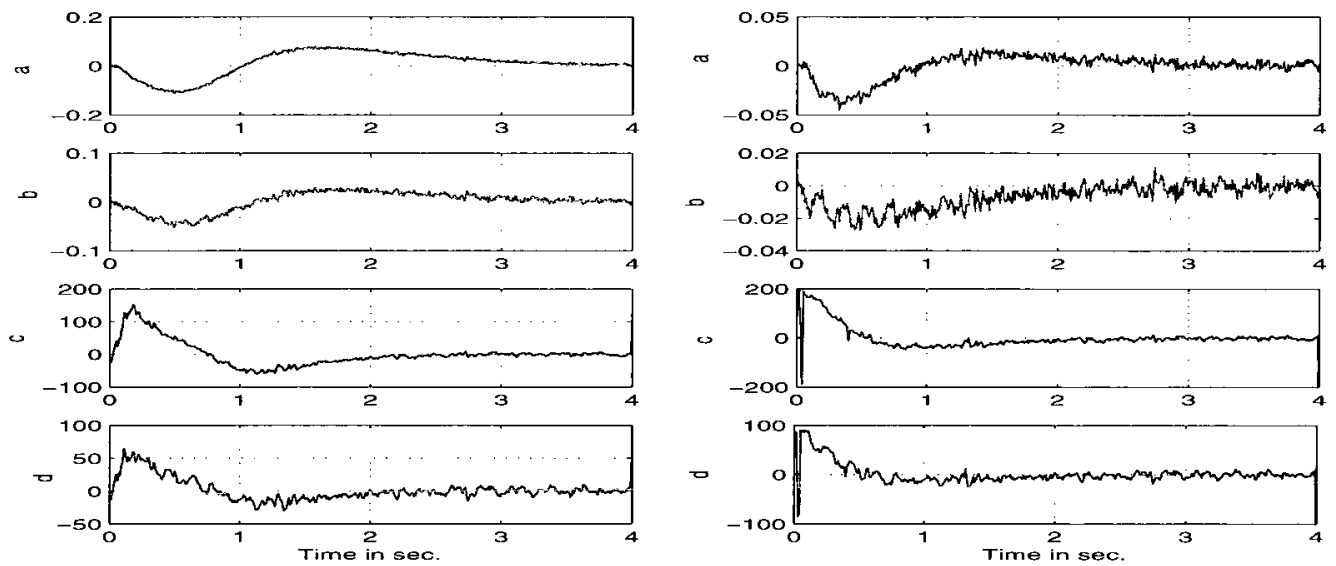

Figure 4.22: The simulation results with Theorem 4.8 and SM-based AOFBSMC under $\tilde{\theta}=8$ with non ideal condition: Left column is for AOFBSMC algorithm (4.41)-(4.42) and right column is for Theorem 4.8, where $a$ : output tracking errors (radians) for joint $1, b$ : output tracking errors (radians) for joint $2, c$ : control input (newton-meters) for joint $1, d$ : control input (newton-meters) for joint 2. 


\section{Chapter 5}

\section{Multi-model Hybrid Adaptive}

\section{Control}

In this chapter, we develop multi-model based hybrid adaptive strategy in classical adaptive control framework for trajectory tracking control problem for robotic systems $[86,95,101]$. The method extends CE-based classical adaptive controller by allowing its parameter estimate to be reset into a candidate model among finite set of candidates that best estimates the plant. The idea of introducing hybrid design is to reduce the observer-controller gains so as to reduce control efforts from $\mathrm{CE}$ principle based classical adaptive control technique.

The hybrid adaptive control system can be derived as follows. We first uniformly divide the compact set of unknown parameters into a finite number of smaller compact subsets. Then construct candidate controller that corresponds to each of these smaller compact subsets. The stability guaranteed Lyapunov-function candidate is utilized for on-line identification of an appropriate candidate controller that closely approximates the plant at each instant of time. This hybrid control approach is developed in classical adaptive control framework in order to achieve good transient tracking performance with smaller values of controller gains in the presence of large scale parametric uncertainties. The proposed method is imple- 
mented and evaluated on a 2-DOF robot model to demonstrate the effectiveness of the theoretical development for real-world applications.

The rest of the chapter is organized as follows: In section II, we derive the CE-based classical adaptive control (CAC) approach for robotic systems. In section III, multi-model based hybrid adaptive control design is proposed to deal with the problem associated with high gains in CE-based CAC approach. Section III presents simulation results to demonstrate the theoretical development of this chapter. Finally, section IV concludes this chapter.

\subsection{Single Model Classical Adaptive Control}

In this section, we first derive CE principle based classical adaptive controller for robotic systems as a state feedback by assuming that the position and velocity signals are available for feedback. Then, we replace the unknown velocity signals by the output of the linear estimator to develop an adaptive output feedback method [82]. Finally, we illustrate the problem associated with classical adaptive control approach via simulation example. Let us first consider the error model equation for MIMO robotic systems [82-101 and references therein] as

$$
\begin{aligned}
& \dot{e}_{1}=e_{2} \\
& \dot{e}_{2}=\phi_{1}(e)+\phi_{2}\left(e_{1}\right) \tau-\ddot{q}_{d}
\end{aligned}
$$

where $e_{1}=\left(q-q_{d}\right), e_{2}=\left(\dot{q}-\dot{q}_{d}\right), e=\left[e_{1}^{T} e_{2}^{T}\right]^{T}, \phi_{2}\left(e_{1}\right)=M^{-1}\left(e_{1}+q_{d}\right)$ and $\phi_{1}(e)=-M^{-1}\left(e_{1}+q_{d}\right)\left[C\left(e_{1}+q_{d}, e_{2}+\dot{q}_{d}\right)\left(e_{2}+\dot{q}_{d}\right)+G\left(e_{1}+q_{d}\right)\right]$

\section{Objectives}

The objective of this chapter can be divided into two parts.

First, we develop single model CE principle based CAC design for the tracking error model (5.1), (5.2). The stability property of the CAC approach is based on using the linearized properties of the robot dynamics $[3,5,6,15,21,22,23,25,26$, 
27, to name a few]; [1)] $M(q) \in R^{n \times n}$ is symmetric, bounded, and positive definite and satisfies the following inequalities $\|M(q)\| \leq M_{M}$ and $\left\|M^{-1}(q)\right\| \leq M_{M I}$ where $M_{M}$ and $M_{M I}$ are bounded positive constant. [2)] The matrix $\dot{M}(q)-2 C(q, \dot{q})$ is skew symmetric. [3)] The norm of the gravity and centrifugal-coriolis forces are upper bounded as $\|C(q, \dot{q})\| \leq C_{M}\|\dot{q}\|$ and $\left\|C\left(q, \dot{q}_{d}\right)\right\| \leq C_{M}\left\|\dot{q}_{d}\right\| \leq \alpha_{c}$, where $C_{M}$ and $\alpha_{c}$ are bounded positive constant. Second, we introduce multiple model based hybrid adaptive control technique for the system (5.1), (5.2). This method extends classical design derived in the first part of the chapter.

\subsubsection{State Feedback Controller}

Our aim is now to design an adaptive controller as a state feedback such that the position $q(t)$ asymptotically tracks the desired position $q_{d}(t)$. To obtain this objective, we assume that $q_{d}(t)$, its first and second derivatives are bounded. Then define $Q_{d}=\left[\begin{array}{lll}q_{d} & \dot{q}_{d} & \ddot{q}_{d}\end{array}\right]^{T}$ with $Q_{d}$ be given any compact set $\Omega_{d} \subset \Re^{3 n}$. To meet the control objective, we consider the following adaptive control law [82 and references therein] as

$$
\begin{aligned}
\tau\left(e, Q_{d}, \hat{\theta}\right) & =Y\left(e, \dot{q}_{d}, \ddot{q}_{d}\right) \hat{\theta}-K_{P} e_{1}-K_{D} e_{2} \\
\dot{\hat{\theta}} & =-\Gamma Y^{T}\left(e, \dot{q}_{d}, \ddot{q}_{d}\right) S
\end{aligned}
$$

where $Y\left(e, \dot{q}_{d}, \ddot{q}_{d}\right) \hat{\theta}=\hat{M}(q) \ddot{q}_{d}+\hat{C}\left(q, \dot{q}_{r}\right) \dot{q}_{d}+\hat{G}(q), \hat{\theta}$ is an estimate of uncertain parameters representing the inertial parameters of robot arms and its load, $K_{P} \in$ $\Re^{n \times n}, K_{D} \in \Re^{n \times n}, e_{1}=q_{1}-q_{d}, S=e_{2}+\lambda e_{1}, \dot{q}_{r}=\left(\dot{q}_{2}-\lambda e_{1}\right), \lambda=\frac{\lambda_{0}}{1+\left\|e_{1}\right\|}, \lambda_{0}>0$ and $\hat{M}, \hat{C}($.$) and \hat{G}($.$) define the estimates of the M(),. C($.$) and G($.$) .$

In our earlier work [83] we show that such adaptation mechanism may cause discontinuous property in the parameter estimates even after learning estimate converge to the actual parameter. To remove the discontinuous property from learning estimates, $\hat{\theta}$ can be adjusted with the smooth parameter projection scheme 
$[37]$

$$
\dot{\hat{\theta}}_{i}=[\operatorname{Proj}(\hat{\theta}, \Phi)]_{i}=\left\{\begin{array}{cc}
\gamma_{i i} \Phi_{i} & \text { if } a_{i} \leq \hat{\theta}_{i} \leq b_{i} \text { or } \\
& \text { if } \hat{\theta}_{i}>b_{i} \text { and } \Phi_{i} \leq 0 \text { or } \\
& \text { if } \hat{\theta}_{i}<a_{i} \text { and } \Phi_{i} \geq 0 \\
\gamma_{i i} \bar{\Phi}_{i} & \text { if } \hat{\theta}_{i}>b_{i} \text { and } \Phi_{i}>0 \\
\gamma_{i i} \breve{\Phi}_{i} & \text { if } \hat{\theta}_{i}<a_{i} \text { and } \Phi_{i}<0
\end{array}\right.
$$

for $\left.\theta \in \Omega=\left\{\theta \mid a_{i} \leq \theta_{i} \leq b_{i}\right\}, 1 \leq i \leq p\right\}$ where, $\tilde{\Phi}_{i}=\left[1+\frac{b_{i}-\hat{\theta}_{i}}{\delta}\right] \Phi_{i}, \breve{\Phi}_{i}=$ $\left[1+\frac{\hat{\theta_{i}}-a_{i}}{\delta}\right] \Phi_{i}, \Phi_{i}$ is the $i$ the element of the column vector, $\Phi=-Y^{T}\left(e, \dot{q}_{d}, \ddot{q}_{d}\right) S$ and $\delta>0$ is chosen such that $\Omega \subset \Omega_{\delta}$ with $\left.\Omega_{\delta}=\left\{\theta \mid a_{i}-\delta \leq \theta_{i} \leq b_{i}+\delta\right\}, 1 \leq i \leq p\right\}$. Notice from the adaptive controller design (5.3), (5.4) is that the nonlinear system dynamics that is regressor model $Y\left(e, \dot{q}_{d}, \ddot{q}_{d}\right)$ is appeared linearly with respect to uncertain parameter $\theta$. In fact, such a linearity assumption can be seen in most $\mathrm{CAC}$ design reported in the literature. Then, the closed loop system can be defined as

$$
\begin{aligned}
\dot{e} & =A e+B\left[\phi_{1}\left(e, q_{d}, \dot{q}_{d}\right)+\phi_{2}\left(e_{1}, q_{d}\right) \tau\left(e, Q_{d}, \hat{\theta}\right)-\ddot{q}_{d}\right] \\
\dot{\hat{\theta}} & =\operatorname{Proj}\left(\hat{\theta}, \phi\left(e, Q_{d}, \hat{\theta}\right)\right)
\end{aligned}
$$

where $A=\left[\begin{array}{cc}0 & I_{n \times n} \\ 0_{n \times n} & 0\end{array}\right]$ and $B=\left[\begin{array}{c}0_{n \times n} \\ I_{n \times n}\end{array}\right]$. To explore the stability condition, we can consider the following Lyapunov-function candidate [826]

$$
V(e, \tilde{\theta})=\frac{1}{2} S^{T} M S+\frac{1}{2} e^{T} K_{P} e+\frac{1}{2} \tilde{\theta} \Gamma^{-1} \tilde{\theta}
$$

with $\tilde{\theta}=(\hat{\theta}-\theta)$. We first take time derivative, $\dot{V}(e, \tilde{\theta})$, along with the closed loop trajectories, formulated by using the error system (5.6) and the control law (5.3)(5.4) along with parameter projection mechanism (5.5). Let us take the derivative along the solution of (5.6)

$$
\dot{V}(e, \tilde{\theta})=\frac{1}{2} S^{T} \dot{M} S+S^{T} M \ddot{e}+S^{T} \dot{\lambda} M e+S^{T} \lambda M \dot{e}+\dot{e}^{T} K_{P} e+\tilde{\theta} \Gamma^{-1} \dot{\tilde{\theta}}
$$

Then, the term $M \ddot{e}$ of the above equation can be simplified as

$$
M(q) \ddot{q}+C(q, \dot{q}) \dot{q}+G(q)=\hat{M}(q) \ddot{q}_{d}+\hat{C}\left(q, \dot{q}_{r}\right) \dot{q}_{d}+\hat{G}(q)-K_{p} e-k_{d} \dot{e}
$$


Substituting $\ddot{e}=\left(\ddot{q}-\ddot{q}_{d}\right)$ and $\dot{e}=\left(\dot{q}-\dot{q}_{d}\right)$, one obtains

$M(q) \ddot{e}+M(q) \ddot{q}_{d}+C(q, \dot{q})\left(\dot{e}+\dot{q}_{d}\right)+G(q)=\hat{M}(q) \ddot{q}_{d}+\hat{C}\left(q, \dot{q}_{r}\right) \dot{q}_{d}+\hat{G}(q)-K_{p} e-K_{d} \dot{e}$

Now add and subtract the term $\lambda C(q, \dot{e}) \dot{q}_{d}$ to obtain $M(q) \ddot{e}$ as

$$
\begin{aligned}
& M(q) \ddot{e}+M(q) \ddot{q}_{d}+C(q, \dot{q})\left(\dot{e}+\dot{q}_{d}\right)-\lambda C(q, \dot{e}) \dot{q}_{d}+\lambda C(q, \dot{e}) \dot{q}_{d}+G(q)=\hat{M}(q) \ddot{q}_{d} \\
& +\hat{C}\left(q, \dot{q}_{r}\right) \dot{q}_{d}+\hat{G}(q)-K_{p} e-K_{d} \dot{e}
\end{aligned}
$$

Which implies

$$
M(q) \ddot{e}=-C(q, \dot{q}) \dot{e}-\lambda C(q, \dot{e}) \dot{q}_{d}+Y\left(q, \dot{q}_{r}, \dot{q}_{d}, \ddot{q}_{d}\right) \tilde{\theta}-K_{p} e-K_{d} e
$$

where $Y\left(q, \dot{q}_{r}, \dot{q}_{d}, \ddot{q}_{d}\right) \tilde{\theta}=\hat{M}(q) \ddot{q}_{d}-M(q) \ddot{q}_{d}+\hat{C}\left(q, \dot{q}_{r}\right) \dot{q}_{d}-C(q, \dot{q}-\lambda e) \dot{q}_{d}+\hat{G}(q)-G(q)$ and $\tilde{\theta}=(\hat{\theta}-\theta)$. The above simplified formed is obtained by applying the wellknown property of $C(e, x+\delta y)=C(e, x)+C(e, \delta y)$. Now, using the skew symmetric property of $\dot{M}\left(e_{1}\right)-2 C\left(e_{1}, e_{2}\right)$, the derivative of the Lyapunov function has the following form

$$
\begin{aligned}
\dot{V}(e, \tilde{\theta})= & S^{T} Y\left(q, \dot{q}_{r}, \dot{q}_{d}, \ddot{q}_{d}\right) \tilde{\theta}+\tilde{\theta} \Gamma^{-1} \dot{\tilde{\theta}}+\dot{\lambda} S^{T} M e+\dot{e}^{T} K_{P} e-S^{T}\left[K_{d}-\lambda M\right] \dot{e}- \\
& S^{T} K_{p} e+\lambda S^{T} C(q, \dot{e}) e
\end{aligned}
$$

Using $\dot{e}=S-\lambda e, \dot{V}(e, \tilde{\theta})$ can be written as follows

$$
\begin{aligned}
\dot{V}(e, \tilde{\theta})= & \tilde{\theta}^{T}\left[Y^{T}\left(q, \dot{q}_{r}, \dot{q}_{d}, \ddot{q}_{d}\right) S+\Gamma^{-1} \dot{\tilde{\theta}}\right]+\dot{\lambda} S^{T} M e-S^{T}\left[K_{d}-\lambda M\right] \dot{e}-\lambda e^{T} K_{P} e \\
& +\lambda S^{T} C(q, \dot{e}) e
\end{aligned}
$$

To simplify the above equation, we define new variables $S_{1}$ as $S_{1}=\dot{e}+\frac{\lambda}{2} e$. Then using $S=\dot{e}+\frac{\lambda}{2} e+\frac{\lambda}{2} e=S_{1}+\frac{\lambda}{2} e$ and $\dot{e}=\left(S_{1}-\frac{\lambda}{2} e\right), \dot{V}(e, \tilde{\theta})$ can be further simplified as

$$
\begin{aligned}
\dot{V}(e, \tilde{\theta})= & \tilde{\theta}^{T}\left[Y^{T}\left(q, \dot{q}_{r}, \dot{q}_{d}, \ddot{q}_{d}\right) S+\Gamma^{-1} \dot{\tilde{\theta}}\right]+\dot{\lambda} S^{T} M e+\lambda S^{T} C(q, \dot{e}) e- \\
& S_{1}^{T}\left[K_{d}-\lambda M\right] S_{1}-\left(\frac{\lambda}{2} e\right)^{T}\left[\frac{4}{\lambda} K_{p}-\left(K_{d}-\lambda M\right)\right] \frac{\lambda}{2} e
\end{aligned}
$$


The term $\dot{\lambda} S^{T} M e$ and $\lambda S^{T} C(q, \dot{e}) e$ in the above equation can be simplified as follows:

$$
\dot{\lambda} S^{T} M e \leq 2 \lambda_{0} M_{m}\left[\left\|S_{1}\right\|^{2}+\left\|\frac{\lambda}{2} e\right\|^{2}\right]
$$

and

$$
\lambda S^{T} C(q, \dot{e}) e \leq 2 \lambda_{0} C_{M}\left[\left\|S_{1}\right\|^{2}+\left\|\frac{\lambda}{2} e\right\|^{2}\right]
$$

where we used

$$
\begin{gathered}
\dot{\lambda}=\frac{d}{d t}\left[\frac{\lambda_{0}}{1+\|e\|}\right]=-\frac{\lambda \dot{e}}{(1+\|e\|)} \\
{\left[\left\|S_{1}\right\|^{2}+2\left\|S_{1}\right\|\left\|\frac{\lambda}{2} e\right\|+\left\|\frac{\lambda}{2} e\right\|^{2}\right] \leq 2\left[\left\|S_{1}\right\|^{2}+\left\|\frac{\lambda}{2} e\right\|^{2}\right]}
\end{gathered}
$$

$\dot{e}=S_{1}-\frac{\lambda}{2} e, S=S_{1}+\frac{\lambda}{2} e,\|M\| \leq M_{M}$ and $\|C(q, e)\| \leq C_{M}\|e\|$. Using these inequalities, $\dot{V}(e, \tilde{\theta})$ becomes

$$
\dot{V}(e, \dot{e}, \tilde{\theta}) \leq \tilde{\theta}^{T}\left[Y^{T}\left(e, \dot{q}_{d}, \ddot{q}_{d}\right) S+\Gamma^{-1} \dot{\tilde{\theta}}\right]-K_{1}\left\|S_{1}\right\|^{2}-K_{2}\left\|\frac{\lambda}{2} e\right\|^{2}
$$

where $Y^{T}\left(q, \dot{q}_{r}, \dot{q}_{d}, \ddot{q}_{d}\right)=Y^{T}\left(e, \dot{q}_{d}, \ddot{q}_{d}\right), K_{1}=\left[\lambda_{\text {min. }}\left(K_{d}\right)-3 \lambda_{0} M_{M}-2 \lambda_{0} C_{M}\right], K_{2}=$ $\left[\frac{4 \lambda_{\min } . K_{P}}{\lambda_{0}}-\lambda_{\max } .\left(K_{d}\right)-2 \lambda_{0} M_{M}-2 \lambda_{0} C_{M}\right]$. Using (5.4) along with (5.5) and $\hat{\theta}(0) \epsilon$ $\Omega$ and $\hat{\theta}(t) \in \Omega_{\delta} \forall t \geq 0$, we have

$$
\begin{aligned}
\dot{V}(e, \tilde{\theta}) & \leq-[e \dot{e}]\left[\begin{array}{cc}
\frac{\lambda I}{2} & \frac{\lambda I}{2} \\
I & 0
\end{array}\right]\left[\begin{array}{cc}
K_{1} I & 0 \\
0 & K_{2} I
\end{array}\right]\left[\begin{array}{cc}
\frac{\lambda I}{2} & I \\
\frac{\lambda I}{2} & 0
\end{array}\right]\left[\begin{array}{l}
e \\
\dot{e}
\end{array}\right] \\
& \leq-\lambda_{\min .}(\Pi)\|e\|^{2} \leq 0
\end{aligned}
$$

$\forall \hat{\theta}(0) \in \Omega, \forall \theta(0) \in \Omega, \forall e(0) \in \Omega_{c o}, \forall \hat{\theta} \in \Omega_{\delta}, \Omega_{c}=\left\{e \mid e^{T} Q_{s m} e \leq c\right\}, c>0$ and $Q_{s m}=\left[\begin{array}{cc}0.5 M & 0.5 M \lambda \\ 0.5 M \lambda & 0.5\left(\lambda^{2}+K_{P}\right)\end{array}\right], \Pi=\Theta^{T} \Delta \Theta$ with $\Delta$ and $\Theta$ defined as $\Delta=$ $\left[\begin{array}{cc}K_{1} I & 0 \\ 0 & K_{2} I\end{array}\right]$ and $\Theta=\left[\begin{array}{cc}\frac{\lambda I}{2} & I \\ \frac{\lambda I}{2} & 0\end{array}\right]$ with $K_{1}=\left[\lambda_{\text {min. }}\left(K_{D}\right)-3 \lambda_{0} M_{M}-2 \lambda_{0} C_{M}\right]$ and $K_{2}=\left[\frac{4 \lambda_{\text {min }} K_{P}}{\lambda_{0}}-\lambda_{\max .}\left(K_{D}\right)-2 \lambda_{0} M_{M}-2 \lambda_{0} C_{M}\right]$. 


\subsubsection{Disturbance Rejection in Adaptive State Feedback Design}

The design derived in previous section is assumed that system model is free from disturbances. We now remove this assumption by adding unmodeled dynamics and external disturbances $\tau_{d}$ with the error model (5.1), (5.2) as

$$
\dot{e}_{1}=e_{2}, \quad \dot{e}_{2}=\phi_{1}(e)+\phi_{2}\left(e_{1}\right)\left(\tau+\tau_{d}\right)-\ddot{q}_{d}
$$

where $\tau_{d}$ is the vector containing unmodeled dynamics and any other unknown disturbances $\tau_{d}$. We then derive robust adaptive control law for robotic systems as

$$
\begin{aligned}
\tau\left(e, Q_{d}, \hat{\theta}\right) & =\Upsilon\left(e, \dot{q}_{r}, \dot{q}_{d}, \ddot{q}_{d}\right) \hat{\theta}-K_{P} e_{1}-K_{D} e_{2} \\
\dot{\hat{\theta}} & =-\Gamma \Upsilon^{T}\left(e, \dot{q}_{r}, \dot{q}_{d}, \ddot{q}_{d}\right) S
\end{aligned}
$$

where the matrix $\Upsilon\left(e, \dot{q}_{r}, \dot{q}_{d}, \ddot{q}_{d}\right)[91,99]$ is defined as

$$
\Upsilon\left(e, \dot{q}_{r}, \dot{q}_{d}, \ddot{q}_{d}\right)=\left[\zeta\left(e, \dot{q}_{r}, \dot{q}_{d}, \ddot{q}_{d}\right) \operatorname{sgn}(S)\right]
$$

$\hat{\theta}(t)=\left[\hat{\theta}_{l}^{T}(t) \hat{\theta}_{g d}\right]^{T}$ is an estimate of uncertain parameters $\theta(t)=\left[\begin{array}{ll}\theta_{l}^{T}(t) & \theta_{g d}\end{array}\right]^{T}$, $\theta_{l}(t)$ represent the parameters associated with inertial matrix $M$ and centrifugalcoriolis matrix $C,\|G(q)-d\| \leq \theta_{g d}, \operatorname{sgn}(S)$ is the vector obtained by applying the signum function to all elements of $S$ and $\zeta\left(e, \dot{q}_{r}, \dot{q}_{d}, \ddot{q}_{d}\right) \hat{\theta}_{l}$ is defined as

$$
\zeta\left(e, \dot{q}_{r}, \dot{q}_{d}, \ddot{q}_{d}\right) \hat{\theta}_{l}=\hat{M}(q) \ddot{q}_{d}+\hat{C}\left(q, \dot{q}_{r}\right) \dot{q}_{d}
$$

To show that all the signals in the closed loop model are bounded, we will follow the same steps that used to establish the stability condition for the control law (5.3), (5.4). To begin with that, we first develop the closed-loop error model under robust adaptive controller as follows:

$$
\begin{aligned}
& M(q) \ddot{e}+M(q) \ddot{q}_{d}+C(q, \dot{q})\left(\dot{e}+\dot{q}_{d}\right)-\lambda C(q, \dot{e}) \dot{q}_{d}+\lambda \\
& C(q, \dot{e}) \dot{q}_{d}+\beta=\hat{M}(q) \ddot{q}_{d}+\hat{C}\left(q, \dot{q}_{r}\right) \dot{q}_{d}+\hat{\beta}-K_{p} e-K_{d} \dot{e}
\end{aligned}
$$


Which implies

$$
\begin{aligned}
M(q) \ddot{e}= & -C(q, \dot{q}) \dot{e}-\lambda C(q, \dot{e}) \dot{q}_{d}+\zeta\left(e, \dot{q}_{r}, \dot{q}_{d}, \ddot{q}_{d}\right) \tilde{\theta}_{l}+\tilde{\beta} \\
& -K_{p} e-K_{d} \dot{e}
\end{aligned}
$$

where $\tilde{\beta}=(\hat{\beta}-\beta)$ and $\zeta\left(e, \dot{q}_{r}, \dot{q}_{d}, \ddot{q}_{d}\right) \tilde{\theta}_{l}$ is defined as

$$
\left[\hat{M}(q) \ddot{q}_{d}-M(q) \ddot{q}_{d}+\hat{C}\left(q, \dot{q}_{r}\right) \dot{q}_{d}-C(q, \dot{q}-\lambda e) \dot{q}_{d}\right]
$$

We then follow from equation (5.7) to (5.8) to derive the convergence condition for the above closed loop system. To reduce the chattering phenomenon, the switching function $\operatorname{sgn}($.$) in robust adaptive control design requires to estimate by using$ saturation function sat(.) $[5,6]$.

\subsubsection{Adaptive Output Feedback Design}

To implement the state feedback controller (5.3), (5.4), we assume that system output and its derivatives are available for feedback. We now consider the practical situation where velocity sensors are not available. This means that the state vector $e$ in the above control law (5.3), (5.4) is not available for measurement and is required to be estimated by a suitable estimator $\hat{e}$. If $e$ is replaced by an estimate $\hat{e}$ in $\tau\left(\hat{e}, Q_{d}, \hat{\theta}\right)$, then one obtains output feedback form of $(5.3),(5.4)$ as [82]

$$
\tau\left(\hat{e}, Q_{d}, \hat{\theta}\right)=Y\left(\hat{e}, \dot{q}_{d}, \ddot{q}_{d}\right) \hat{\theta}-K_{P} \hat{e}_{1}-K_{D} \hat{e}_{2}
$$

where $\dot{\hat{q}}_{r}=\left(\hat{e}_{2}+\dot{q}_{d}-\lambda \hat{e}_{1}\right)$ and $\hat{\theta}$ is similar to define in controller (5.3) accept $e$ is replaced by the following linear estimator, $\hat{e}$, as

$$
\dot{\hat{e}}_{1}=\hat{e}_{2}+\frac{H_{1}}{\epsilon} \tilde{e}_{1}, \dot{\hat{e}}_{2}=\frac{H_{2}}{\epsilon^{2}} \tilde{e}_{1}
$$

where $\tilde{e}_{1}=e_{1}-\hat{e}_{1}, \epsilon$ is a small constant design parameters needs to be specified, $H_{1}=\operatorname{diag}\left\{\omega_{1 i}\right\}$ and $H_{2}=\operatorname{diag}\left\{\omega_{2 i}\right\}$ with $i=1,2, \ldots, n$. The value of $\omega_{i j}$ is chosen such that the matrix $\left[\begin{array}{cc}-H_{1} & I \\ -H_{2} & 0_{n \times n}\end{array}\right]$ is Hurwitz. Then the observer error model 
can be written as

$$
\begin{aligned}
& \dot{\tilde{e}}_{1}=\tilde{e}_{2}-\frac{H_{1}}{\epsilon} \tilde{e}_{1} \\
& \dot{\tilde{e}}_{2}=-\ddot{q}_{d}+\phi_{1}(e)+\phi_{2}\left(e_{1}\right) \tau\left(\hat{e}, Q_{d}, \hat{\theta}\right)-\frac{H_{2}}{\epsilon^{2}} \tilde{e}_{1}
\end{aligned}
$$

We now analyze the convergence property of this CE-based adaptive output feedback design by using singularly perturbation method. To begin with that, we first define singularly perturbed closed loop observer error model as

$$
\epsilon \dot{\eta}=A_{\circ} \eta+B \epsilon\left[-\ddot{q}_{d}+\phi_{1}(e)+\phi_{2}\left(e_{1}\right) \tau\left(e-\zeta(\epsilon) \eta, Q_{d}, \hat{\theta}\right)\right]
$$

where $\eta=\left[\begin{array}{c}\eta_{1} \\ \eta_{2}\end{array}\right], \epsilon \dot{\eta}_{1}=\eta_{2}-H_{1} \eta_{1}, \epsilon \dot{\eta}_{2}=\epsilon\left[-\ddot{q}_{d}+\phi_{1}(e)+\phi_{2}\left(e_{1}\right) \tau\left(\hat{e}, Q_{d}, \hat{\theta}\right)\right]-$ $H_{2} \eta_{1}, \hat{e}=e-\zeta(\epsilon) \eta, \epsilon \eta_{1}=\tilde{e}_{1}, \eta_{2}=\tilde{e}_{2}$ with a small positive parameter $\epsilon, \zeta(\epsilon)=$ $\left[\begin{array}{cc}\epsilon I_{n \times n} & 0_{n \times n} \\ 0 & I_{n \times n}\end{array}\right], A_{0}=\left[\begin{array}{cc}-H_{1} & I \\ -H_{2} & 0_{n \times n}\end{array}\right], H=\left[\begin{array}{c}H_{1} \\ H_{2}\end{array}\right], B=\left[\begin{array}{c}0_{n \times n} \\ I_{n \times n}\end{array}\right]$ and $C=$ $\left[\begin{array}{ll}I_{n \times n} & 0_{n \times n}\end{array}\right]$. Then we show that the performance achieved under adaptive state feedback $\tau\left(e, Q_{d}, \hat{\theta}\right)$ can be recovered by using an adaptive output feedback control design $\tau\left(\hat{e}, Q_{d}, \hat{\theta}\right)$. The resulting adaptive output feedback system has the following form

$$
\begin{aligned}
\dot{e} & =B\left[\phi_{1}(e)+\phi_{2}\left(e_{1}\right) \tau\left((e-\zeta(\epsilon) \eta), Q_{d}, \hat{\theta}\right)-\ddot{q}_{d}\right]+A e \\
\dot{\hat{\theta}} & =\operatorname{Proj}\left(\hat{\theta}, \phi\left(e-\zeta(\epsilon) \eta, Q_{d}, \hat{\theta}\right)\right)
\end{aligned}
$$

where $A=\left[\begin{array}{cc}0 & I_{n \times n} \\ 0_{n \times n} & 0\end{array}\right]$.

We then state our main results for the SM-based classical adaptive output feedback (CAOFB) design.

Theorem 5.1 : Consider the closed-loop system (5.12), (5.13) and (5.14). Then, for any given $\forall e(0) \in \Omega_{c o} \subseteq \Omega_{c}, \forall \hat{e}(0) \in \Omega_{c o}, \forall \hat{\theta}(0) \in \Omega$ and $\forall \theta(0) \in \Omega$, there exists a small $\epsilon_{1}^{*}$ such that for all $0<\epsilon<\epsilon_{1}^{*}$, all the state variables of the closed loop system are bounded and their bound can be made very small using small value of $\epsilon$. 
Proof: The proof of theorem 5.1 is similar to the proof of Theorem 1 of [82]. First, it is proven that there exists a short transient period $T_{1}(\epsilon) \in\left[0, T_{2}\right]$ during which the fast variable $\eta$ approaches a function of the order $O(\epsilon)$, while the slow variables $(e, \theta)$ remain in a subset of the domain of attraction. Second, the boundedness of the signal $e(t)$ is given for all $t \in\left[T_{1}(\epsilon), T_{3}\right]$, where $T_{1}(\epsilon) \in\left(0, \frac{T_{2}}{2}\right]$ and $T_{3} \geq T_{2}$. This means that the state variables $(e(t), \theta(t))$ remain bounded for $t \geq T_{1}(\epsilon)$ provided that the fast variable $\eta$ is of the order $O(\epsilon)$ and there exists a Lyapunov function for the fast model $W(\eta)=\eta^{T} P \eta$ such that the fast variables converge to the set $\Omega_{\epsilon}=\left\{\eta \mid W(\eta) \leq \epsilon^{2} \beta\right\}$ where $\beta=16\|P\|^{2} k_{1}^{2} \lambda_{\max }(P)=$ $16\|P\|^{3} k_{1}^{2}$ with

$$
\left\|\left[-\ddot{q}_{d}+\phi_{1}(e)+\phi_{2}\left(e_{1}\right) \tau^{s}\left((e-\zeta(\epsilon) \eta), Q_{d}, \hat{\theta}\right)\right]\right\| \leq k_{1}
$$

for some $k_{1}>0,\|P\|=\lambda_{\max }(P)$ and $P$ is the solution of the Lyapunov-equation as $P A_{o}+A_{o}^{T} P=-I$, for all $t \in\left[T_{1}(\epsilon), T_{3}\right]$. This implies that the state trajectory $(e, \theta)$ is trapped inside the set which can be made very small by using small value of observer design constant $\epsilon$ as

$$
\dot{V} \leq-\lambda_{m i n}(\Pi)\|e\|^{2}+\chi \epsilon
$$

with $\chi>0$. This implies that for small value of $\epsilon$ the closed-loop trajectory can be made arbitrarily small closed neighborhood of the origin over a finite time.

The main ingredient in theorem 5.1 is the existence of small observer design parameter $\epsilon>0$ which represents the bandwidth of the observer dynamics. However, Theorem 5.1 does not provide the minimum bound on the value of $\epsilon$. The design depends on trial and error method to obtain an appropriate value of $\epsilon$ for which the estimation error converges to zero exponentially fast so that the closedloop trajectories asymptotically convergence to zero. However, such a trail and error method may not be realizable in practice as in-appropriate choice of $\epsilon$ may cause an unstable closed loop system.

Let us now find the minimum bound of $\epsilon$ which ensures robust reconstruction of the unknown velocity signals. Specifically, for the given set of inertial parameters 
and effect of payloads, our aim is to calculate the value of observer speed that ensures exponentially fast reconstruction of the unknown velocity signals. To show that, we consider the following combined Lyapunov function candidate for the singularly perturbed system (5.12), (5.14)

$$
V_{Q}=(1-d)\left[\frac{1}{2} S^{T} M S+\frac{1}{2} e^{T} K_{P} e\right]+d \frac{1}{2} \eta^{T} P \eta
$$

Now take derivative to simplify $\dot{V}_{Q}$ as

$$
\begin{aligned}
\dot{V}_{Q} \leq & -(1-d)\left[\alpha_{1}\|e\|^{2}+\beta_{1}\|e\|\|\eta\|\right]-d \frac{\alpha_{2}}{\epsilon}\|\eta\|^{2} \\
& +2 d \eta^{T} P B \xi\left(e, Q_{d}, \theta, \eta, \epsilon\right)
\end{aligned}
$$

$\alpha_{1}=\lambda_{\text {min. }}(\Pi), \beta_{1}=\lambda k_{\epsilon 1}$ where

$$
\left\|\tau^{s}\left(\hat{e}, Q_{d}, \theta\right)-\tau^{s}\left(e, Q_{d}, \theta\right)\right\| \leq k_{\epsilon 1}\|\eta\|
$$

with $k_{\epsilon 1}>0, \alpha_{2}=\left\|Q_{o}\right\|$ where $Q_{o}$ is positive definite matrix for solving Lyapunov equation $A_{0}^{T} P+P A_{0}=-Q_{o}$

$$
\xi\left(e, Q_{d}, \theta, \eta, \epsilon\right)=\left[-\ddot{q}_{d}+\phi_{1}(e)+\phi_{2}\left(e_{1}\right) \tau^{s}\left(\hat{e}, Q_{d}, \theta\right)\right]
$$

By knowing the upper bound on the inertial parameter $\theta \in \Omega$ as well as the bound of the desired trajectories $Q_{d} \in \Omega_{d}$, we can simplify the term $\xi\left(e, Q_{d}, \theta, \eta, \epsilon\right)$ as $\xi\left(e, Q_{d}, \theta, \eta, \epsilon\right)=[\psi(0, \eta, \epsilon)+\psi(e, 0,0)]$. Then equation (5.18) has the following simplified form

$$
\begin{gathered}
\dot{V}_{Q} \leq-(1-d)\left[\alpha_{1}\|e\|^{2}+\beta_{1}\|e\|\|\eta\|\right]-d \frac{\alpha_{2}}{\epsilon}\|\eta\|^{2}+ \\
2 d \eta^{T} P B[\psi(0, \eta, \epsilon)+\psi(e, 0,0)]
\end{gathered}
$$

This implies that, for a small value of $\epsilon$, the closed-loop trajectory enters into a positively invariant set over a finite time and remains there for all future time. The time and size of this set can be made very small by using small values of $\epsilon$. Our aim is now to find the bound on $\epsilon$. For the given $\theta \in \Omega$ and $Q_{d} \in \Omega_{d}$, we calculate the upper bound on the modeling error terms $\psi(0, \eta, \epsilon)$ and $\psi(e, 0,0)$ of $(5.19)$ 
as $\|\psi(0, \eta, \epsilon)\| \leq \gamma_{s p}\|\eta\|$ and $\|\psi(e, 0,0)\| \leq \gamma_{s}\|e\|$ where $\gamma_{s p}$ and $\gamma_{s}$ are bounded positive constants. With these inequalities, (5.19) leads to

$$
\begin{gathered}
\dot{V}_{Q} \leq-(1-d) \alpha_{1}\|e\|^{2}+(1-d) \beta_{1}\|e\|\|\eta\|-d \frac{\alpha_{2}}{\epsilon}\|\eta\|^{2}+ \\
2 d\|\eta\|\|P\| \gamma_{s p}\|\eta\|+2 d\|\eta\|\|P\| \gamma_{s}\|e\|
\end{gathered}
$$

Let us define $\Psi_{1}(e)=\|e\|$ and $\Psi_{2}(\eta)=\|\eta\|$. Then, (5.19) can be re-written as follows:

$$
\begin{aligned}
& \dot{V}_{Q} \leq-(1-d) \alpha_{1} \Psi_{1}^{2}(e)-d \frac{\alpha_{2}}{\epsilon} \Psi_{1}^{2}(\eta)+(1-d) \beta_{1} \Psi_{1}(e) \Psi_{2}(\eta)+ \\
& d \beta_{2} \Psi_{1}(e) \Psi_{2}(\eta)+d \gamma \Psi_{2}^{2}(\eta)
\end{aligned}
$$

where $\beta_{2}=2\|P\| \gamma_{s}$ and $\gamma=2\|P\| \gamma_{s p}$. Now, we can write (5.21) in a matrix form as follows:

$$
\dot{V}_{Q} \leq-\left[\begin{array}{c}
\Psi_{1}(e) \\
\Psi_{2}(\eta)
\end{array}\right]^{T} U\left[\begin{array}{c}
\Psi_{1}(e) \\
\Psi_{2}(\eta)
\end{array}\right]
$$

with $U=\left[\begin{array}{cc}(1-d) \alpha_{1} & -\frac{1}{2}(1-d) \beta_{1}-\frac{1}{2} d \beta_{2} \\ -\frac{1}{2}(1-d) \beta_{1}-\frac{1}{2} d \beta_{2} & d\left(\left(\frac{\alpha_{2}}{\epsilon}\right)-\gamma\right)\end{array}\right]$. Equation (5.22) is negative definite if the matrix $U$ is positive definite and satisfies the inequality

$$
\left[d(1-d) \alpha_{1}\left(\frac{\alpha_{2}}{\epsilon}-\gamma\right)\right]>\frac{1}{4}\left[(1-d) \beta_{1}+d \beta_{2}\right]
$$

This implies that for a given $d \in(0,1)$ the matrix $U$ will be positive definite and there exists a continuous interval $\left(0, \epsilon^{*}\right)$ such that $\forall \epsilon \in\left(0, \epsilon^{*}\right), \epsilon^{*}$ satisfies $\epsilon^{*}(d)=\frac{\alpha_{1} \alpha_{2}}{\alpha_{1} \gamma+\frac{1}{4 d(1-d)}\left[(1-d) \beta_{1}+d \beta_{2}\right]}$ for the maximum value of $d^{*}=\frac{\beta_{1}}{\beta_{1}+\beta_{2}}$, one gets bounds on $\epsilon^{*}$ as follows $\epsilon^{*}=\frac{\alpha_{1} \alpha_{2}}{\beta_{1} \beta_{2}+\alpha_{1} \gamma}$. Equation (5.22) can be further simplified as

$$
\dot{V}_{Q} \leq-\lambda_{\min }(U)\|\tilde{\eta}\|^{2}
$$

where $\tilde{\eta}=\left[\begin{array}{ll}\Psi_{1}^{T}(e), & \Psi_{2}^{T}(\eta)\end{array}\right]^{T}$ and $\lambda_{\min }(U)$ is the minimum eigen-value of the positive definite matrix $U$. Then, we can conclude that for a small value of $\epsilon$ all the signals in the singularly perturbed system (5.12)-(5.14) are bounded and their bounds can be made closed to zero via using small value of $\epsilon$. 


\subsubsection{Disturbance Rejection in Adaptive Output Feedback Design}

We now include disturbances in the error model (5.1), (5.2) to derive robust adaptive output feedback law as follows

$$
\begin{aligned}
\tau\left(\hat{e}, Q_{d}, \hat{\theta}\right) & =\Upsilon\left(\hat{e}, \dot{\hat{q}}_{r}, \dot{q}_{d}, \ddot{q}_{d}\right) \hat{\theta}-K_{P} \hat{e}_{1}-K_{D} \hat{e}_{2} \\
\dot{\hat{\theta}} & =-\Gamma \Upsilon^{T}\left(\hat{e}, \dot{\hat{q}}_{r}, \dot{q}_{d}, \ddot{q}_{d}\right) \hat{S}
\end{aligned}
$$

where matrix $\Upsilon\left(\hat{e}, \dot{\hat{q}}_{r}, \dot{q}_{d}, \ddot{q}_{d}\right)$ is defined as $\Upsilon\left(\hat{e}, \dot{\hat{q}}_{r}, \dot{q}_{d}, \ddot{q}_{d}\right)=\left[\zeta\left(\hat{e}, \dot{\hat{q}}_{r}, \dot{q}_{d}, \ddot{q}_{d}\right) \operatorname{sgn}(\hat{S})\right]$, $\hat{\theta}(t)=\left[\begin{array}{ll}\hat{\theta}_{l}^{T}(t) & \hat{\theta}_{g d}\end{array}\right]^{T}$ is an estimate of uncertain parameters $\theta(t)=\left[\begin{array}{ll}\theta_{l}^{T}(t) & \theta_{g d}\end{array}\right]^{T}$ $\theta_{l}(t)$ define the model parameters associated with $M$ and $C,\|G(\hat{q})-d\| \leq \theta_{g d}$ and $\zeta\left(\hat{e}, \dot{\hat{q}}_{r}, \dot{q}_{d}, \ddot{q}_{d}\right) \hat{\theta}_{l}$ is defined as

$$
\zeta\left(\hat{e}, \dot{\hat{q}}_{r}, \dot{q}_{d}, \ddot{q}_{d}\right) \hat{\theta}_{l}=\hat{M}(q) \ddot{q}_{d}+\hat{C}\left(q, \dot{\hat{q}}_{r}\right) \dot{q}_{d}
$$

To show that all the signals in the closed loop model with the above robust adaptive output control law are bounded, we can follow the same steps that used in the stability analysis for the control law (5.9), (5.10) of Theorem 5.1. Therefore, we remove the detail proof for brevity.

\subsubsection{Design Steps for CE-based CAOFB}

The design steps for the CAOFB design are as follows. First, we choose the feedback controller gains $K_{P}, K_{D}$ and $\lambda_{o}$ such that they ensures an acceptable transient performance for the closed loop system. This means that the values of $K_{P}, K_{D}$ and $\lambda_{o}$ can be selected according to design rules for PD control such that the closed loop would be critically damped and the response would be fast enough.

The observer design constants matrices $H_{1}$ and $H_{2}$ are selected such that $A_{0}$ is stable. Then, we solve the Lyapunov-equation for $P$. For the given initial conditions of interest, one then calculate the value of $\epsilon$ to ensure robust reconstruction of 

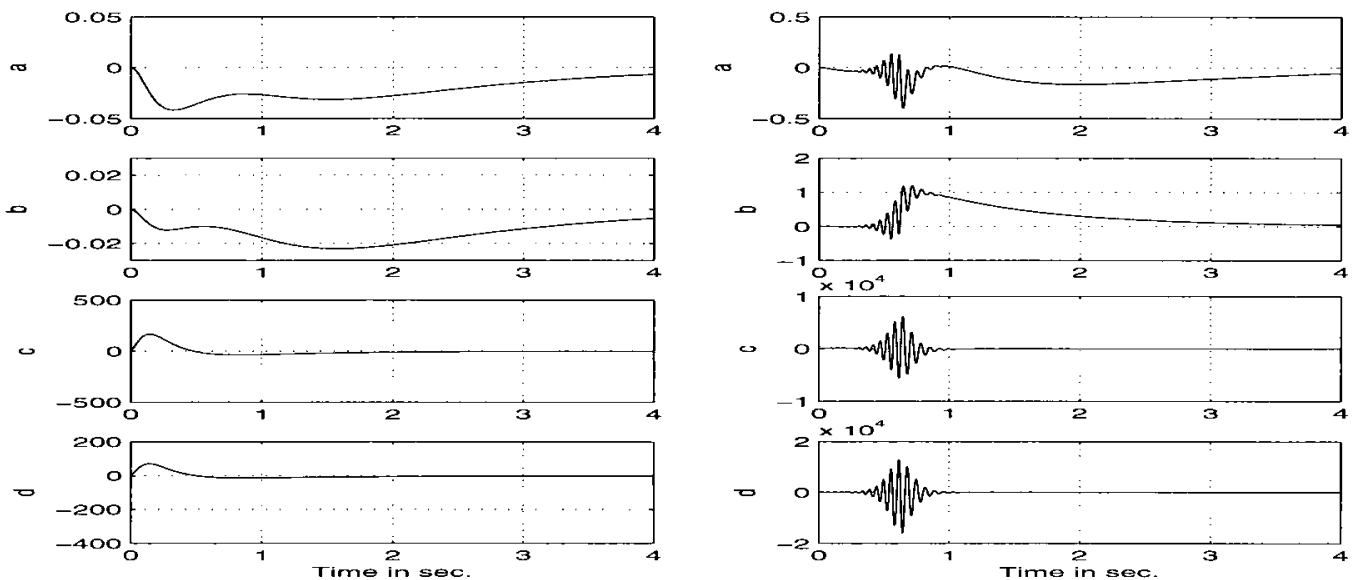

Figure 5.1: First column is for $K_{P}=200 I_{2 \times 2}, K_{D}=250 I_{2 \times 2}, \Gamma=15 I_{2 \times 2}, H_{1}=$ $5 I_{2 \times 2}, H_{2}=5 I_{2 \times 2}, \epsilon=0.002$ and $\tilde{\theta}=8$, second column is for $K_{P}=200 I_{2 \times 2}$, $K_{D}=250 I_{2 \times 2}, \Gamma=15 I_{2 \times 2}, H_{1}=5 I_{2 \times 2}, H_{2}=5 I_{2 \times 2}, \epsilon=0.01$ and $\tilde{\theta}=8$, where $a$ : output tracking errors (radians) for joint $1, b$ : output tracking errors (radians) for joint $2, c$ : control input (newton-meters) for joint $1, d$ : control input (newtonmeters) for joint 2 .

unknown velocity signal which guarantees asymptotic tracking trajectories starting inside a given compact set of interest.

Remark 5.1: Notice that the value of $k_{1}$ will increase with the increase of the domain of attraction that is with the initial set $\Omega$ and $\Omega_{c o}$. This simply means that the value of $\Gamma$ and $\frac{1}{\epsilon}$ will also increase with the increase of the bounded parameters and initial conditions. The demand of small value of $\epsilon$ can also be illustrated by analyzing the observer error model dynamics $\epsilon \dot{\eta}=A_{o} \eta+B \epsilon\left[\tilde{\phi}_{1}\left(\zeta(\epsilon) \eta, q_{d}, \dot{q}_{d}\right)+\right.$ $\left.\tilde{\phi}_{2}\left(\epsilon \eta_{1}, q_{d}\right) \tau\left((e-\zeta(\epsilon) \eta), Q_{d}, \hat{\theta}\right)\right]$ with $\tilde{\phi}_{1}()=.\phi_{1}($.$) and \tilde{\phi}_{2}()=.\phi_{2}($.$) . From \epsilon \dot{\eta}$, we can see that if the uncertainty level increase, then the second term of the bracket $\epsilon \dot{\eta}$ will also increase. To reduce the influence of the uncertainties from observer error dynamics, one has to use very large value of $\frac{1}{\epsilon}$ to make the term close to zero. On the other hand, the increase of the observer speed demands to increase the value of the saturation level, $\tau_{\max }$, to protect the plant from peaking phenomenon. 


\subsubsection{Motivating Example}

In this subsection, we show the design and implementation process of CE-based CAOFB design on robotic systems. In our evaluation, our prime focus is to examine the effect of observer-controller gains on overall control tracking performance. For this purpose, we consider 2-DOF serial link robot manipulator [82-101] which has the following dynamics

$$
\left[\begin{array}{ll}
m_{11} & m_{12} \\
m_{21} & m_{22}
\end{array}\right]\left[\begin{array}{l}
\ddot{q}_{1} \\
\ddot{q}_{2}
\end{array}\right]+\left[\begin{array}{ll}
c_{11} & c_{12} \\
c_{21} & c_{22}
\end{array}\right]\left[\begin{array}{c}
\dot{q}_{1} \\
\dot{q}_{2}
\end{array}\right]=\left[\begin{array}{c}
\tau_{1} \\
\tau_{2}
\end{array}\right]
$$

with $m_{11}=\left(\theta_{1}+2 \theta_{2}+2 \theta_{2} \cos q_{2}\right), m_{12}=\left(\theta_{2}+\theta_{2} \cos q_{2}\right), m_{21}=\left(\theta_{2}+\theta_{2} \cos q_{2}\right)$, $m_{22}=\theta_{2}, c_{11}=-2 \dot{q}_{2} \theta_{2} \sin q_{2}, c_{12}=-\dot{q}_{2} \theta_{2} \sin q_{2}, c_{21}=\dot{q}_{1} \theta_{2} \sin q_{2}$ and $c_{22}=0$, where $\theta_{1}=m_{1} l^{2}$ and $\theta_{2}=m_{2} l^{2}$ and $l$ is the link lengths and $m_{1}$ and $m_{2}$ are the masses of link 1 and link 2, respectively. As the manipulator is operating in horizontal plane, the gravitational loading vectors $G(q)$ are zero. Let us now define given desired trajectory for the robot (5.23) to follow. To generate that, a square wave with a period of 8 seconds and an amplitude of \pm 1 radians is pre-filtered with a critically damped second-order filter using a bandwidth of $\omega_{n}=2.0 \mathrm{rad} / \mathrm{sec}$. The output of this filter is our desired trajectory for the above robot model to follow. Our main target is to use smooth desired trajectory that usually uses in industrial robotic systems $[5,6]$.

In our evaluation, we consider for the case when the parameter $\theta$ belongs to a comparatively large compact set as defined as follows: $\Omega=\left\{\theta \in \Re^{2} \mid-8<\theta_{1}<\right.$ $\left.8,-9<\theta_{2}<9\right\}$ and take $\delta=0.1$ so that the parameter $\hat{\theta}$ belongs to the set $\Omega_{\delta}$. We now define the initial values of position and velocity error as $e(0)=2$. The initial parameter estimates are chosen as $\hat{\theta}(0)=0$.

Let us choose the controller design parameters as $K_{P}=200 I_{2 \times 2}, K_{D}=$ $250 I_{2 \times 2}, \lambda_{o}=2$ and $\Gamma=15 I_{2 \times 2}$. In our example, the value of $\Gamma=15 I_{2 \times 2}$ is chosen by assuming that $\theta \in \Omega$. We also note that the learning gain requires to increase with the increase of the parameter set to achieve fast parameter conver- 
gence. With these design parameters, our interest is to calculate the minimum bound of $\epsilon$. Note that one may choose different controller design parameters, and then follow the following design steps to calculate $\epsilon$ for the implementation.

Our first task is to calculate the values of $K_{1}$ and $K_{2}$ defined in equation (5.8). Applying the property 1 , we calculate $K_{1}$ and $K_{2}$ as follows:

$$
K_{1}=\left[\lambda_{\text {min. }}\left(K_{D}\right)-3 \lambda_{0} M_{M}-2 \lambda_{0} C_{M}\right]=41.1856
$$

and

$$
\begin{aligned}
K_{2}= & {\left[\frac{4 \lambda_{\min .}\left(K_{P}\right)}{\lambda_{0}}-\lambda_{\max .}\left(K_{D}\right)-2 \lambda_{0} M_{M}-2 \lambda_{0} C_{M}\right] } \\
& =392.7904
\end{aligned}
$$

Now, recall equation $\Pi=\Theta^{T} \Delta \Theta$ which is used to determine the value of $\alpha_{1}$ as

$$
\alpha_{1}=\lambda_{\min }\left(\left[\begin{array}{cc}
\frac{\lambda I}{2} & I \\
\frac{\lambda I}{2} & 0
\end{array}\right]^{T}\left[\begin{array}{cc}
K_{1} I & 0 \\
0 & K_{2} I
\end{array}\right]\left[\begin{array}{cc}
\frac{\lambda I}{2} & I \\
\frac{\lambda I}{2} & 0
\end{array}\right]\right)=35.3852
$$

We now calculate $k_{\epsilon 1}$ as

$$
\tau^{s}\left(\hat{e}, Q_{d}, \hat{\theta}\right)-\tau^{s}\left(e, Q_{d}, \hat{\theta}\right)=\left[\begin{array}{cc}
0 & 0 \\
0 & -\hat{C}\left(q, \dot{q}_{d}\right)+K_{D}
\end{array}\right]\left[\begin{array}{c}
\eta_{1} \\
\eta_{2}
\end{array}\right]
$$

with $\dot{\hat{q}}_{r}=\left(e_{2}-\tilde{e}_{2}+\dot{q}_{d}-\lambda e_{1}\right)$ and $\tilde{e}_{2}=\eta_{2}$, which can be written as

$$
\left\|\tau^{s}\left(\hat{e}, Q_{d}, \hat{\theta}\right)-\tau^{s}\left(e, Q_{d}, \hat{\theta}\right)\right\| \leq\left\|\left[\begin{array}{cc}
0 & 0 \\
0 & -\hat{C}\left(q, \dot{q}_{d}\right)+K_{D}
\end{array}\right]\right\|\left\|\left[\begin{array}{l}
\eta_{1} \\
\eta_{2}
\end{array}\right]\right\|=k_{\epsilon 1}\|\eta\|
$$

where $k_{\epsilon 1}$ is

$$
k_{\epsilon 1}=\left\|\left[\begin{array}{cc}
0 & 0 \\
0 & -\hat{C}\left(q, \dot{q}_{d}\right)+K_{d}
\end{array}\right]\right\|
$$

Let us now calculate the maximum bound on $\hat{C}\left(q, \dot{q}_{d}\right)$ as $\alpha_{c}=39.6117$ where $\alpha_{c}=C_{M}\left\|\dot{q}_{d}\right\|$ with the maximum bound on $\left\|\dot{q}_{d 1}\right\|=\left\|\dot{q}_{d 2}\right\|=\left\|\dot{q}_{d}\right\|=1.4671$. By knowing $\alpha_{c}$ and $K_{D}$, we now determine the value of $k_{\epsilon 1}=210.3883$. Using $\lambda_{0}=2$ and $k_{\epsilon 1}$, the value of $\beta_{1}$ is determined as $\beta_{1}=210.3883$. Then we find the constant 

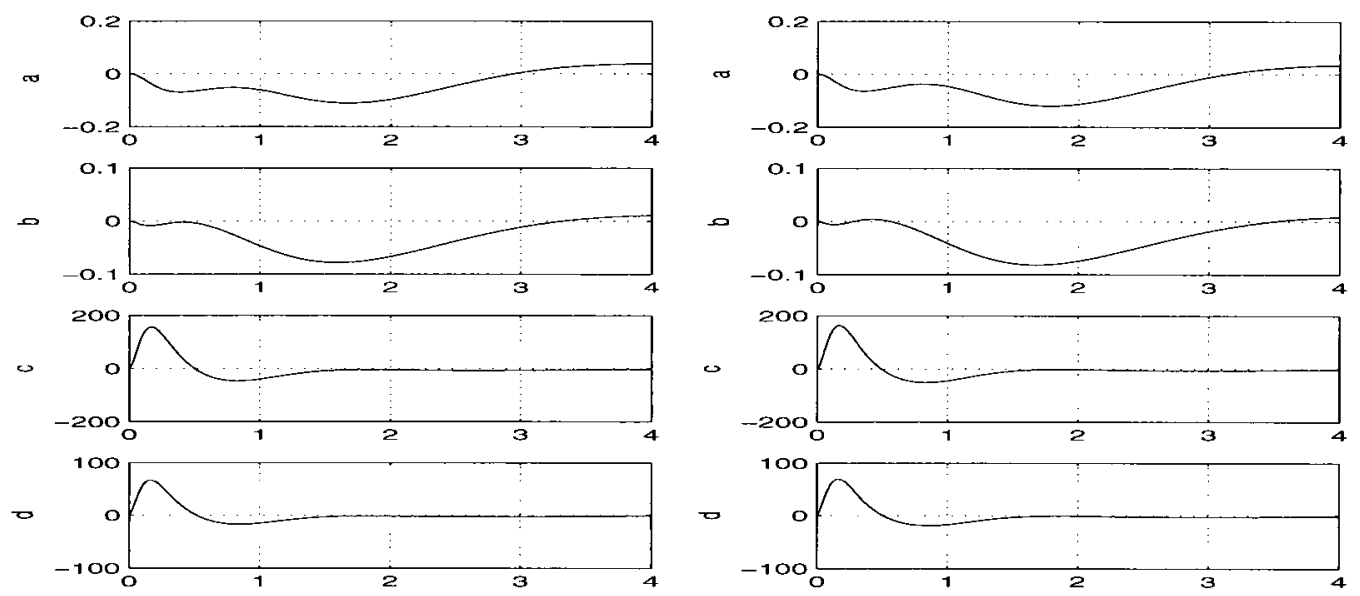

Figure 5.2: First column is for $K_{P}=60 I_{2 \times 2}, K_{D}=60 I_{2 \times 2}, \Gamma=15 I_{2 \times 2}, H_{1}=$ $5 I_{2 \times 2}, H_{2}=5 I_{2 \times 2}, \epsilon=0.002$ and $\tilde{\theta}=8$, second column is for $K_{P}=200 I_{2 \times 2}$, $K_{D}=250 I_{2 \times 2}, \Gamma=15 I_{2 \times 2}, H_{1}=5 I_{2 \times 2}, H_{2}=5 I_{2 \times 2}, \epsilon=0.01$ and $\tilde{\theta}=8$, where $a$ : output tracking errors (radians) for joint $1, b$ : output tracking errors (radians) for joint 2, $c$ : control input (newton-meters) for joint $1, d$ : control input (newtonmeters) for joint 2 .

$\gamma_{s p}$ and $\gamma_{s}$. To calculate the value of $\gamma_{s p}$ and $\gamma_{s}$, we choose $\alpha_{2}=\left\|Q_{o}\right\|=1$. The value of $H_{i}=5 I_{2 \times 2}$ with $i=1,2$ are chosen such that $A_{o}$ is stable. Then, use the Lyapunov equation to find the value of $P$ as $P=1.1101$. In view of the property 1 and 3, we then calculate the value of $\gamma_{s p}$ and $\gamma_{s}$ as $\gamma_{s p}=150.7853$ and $\gamma_{s}=379.2944$. By knowing $\|P\|, \gamma_{s p}$ and $\gamma_{s}$, we now determine the values of $\beta_{2}$ and $\gamma$ as $\beta_{2}=842.1094$ and $\gamma=334.7691$. Using constants $\beta_{1}, \beta_{2}, \alpha_{1}$ and $\alpha_{2}$, the value of $d^{*}$ is obtained as $d^{*}=\frac{\beta_{1}}{\beta_{1}+\beta_{2}}=0.1999$. The choice of $d^{*}=0.1999$ results in the bounds on $\epsilon^{*}=\frac{\alpha_{1} \alpha_{2}}{\beta_{1} \beta_{2}+\alpha_{1} \gamma}=0.00018721 \approx 0.0002$. Note that we obtained the value of $\epsilon$ for the given design parameters. It is clear from our evaluation that $\epsilon$ will decrease (increase the observer speed) with the increase of the initial conditions and the inertial parameters. We now investigate the tracking convergence of CAOFB scheme with respect to different observer-controller design constants. 
Case 1: Using $K_{P}=60 I_{2 \times 2}, K_{D}=60 I_{2 \times 2}, \Gamma=15 I_{2 \times 2}, H_{1}=5 I_{2 \times 2}, H_{2}=$ $5 I_{2 \times 2}, \epsilon=0.002$ and $\tilde{\theta}=8$, we apply CAOFB controller on the given system (5.23). The tested results for SM-based classical adaptive output feedback (5.9), (5.10) are depicted in the left column of Figure 5.1. Notice that the design provides good tracking at the expense of high controller-observer gains.

To investigate the influence of $\epsilon$ on the tracking performance, let us reduce the observer speed by increasing the value of $\epsilon$ from 0.002 to 0.01 , and keep all other design constants similar to our last evaluation. With these design sets, we implement CAOFB scheme (5.9), (5.10) on the given model (5.23). The implementation results are given in the right column of Figure 1. By comparing left and right column of Figure 5.1, we notice that, for the slow observer speed, that is higher value of $\epsilon$, the tracking performance is not impressive due to the presence of the large estimation error uncertainty. Notice also that the design demands very large control efforts (see right column of Figure 5.1) with higher values of observer design constant $\epsilon$.

Case 2: We now repeat the last two tests with smaller values of proportional and derivative gains as $K_{P}=60 I_{2 \times 2}$ and $K_{D}=60 I_{2 \times 2}$. Note that the learning gains $\Gamma$ are kept similar to our last two tests as $\Gamma=15 I_{2 \times 2}$. Using $H_{1}=5 I_{2 \times 2}$, $H_{2}=5 I_{2 \times 2}, \epsilon=0.002$ and $\tilde{\theta}=8$, we then implement classical adaptive output feedback controller on the system (5.23). The implemented results are depicted in the left column of Figure 5.2.

To examine the influence of $\epsilon$ on the tracking performance, we now reduce the observer speed by increasing the value of $\epsilon$ from 0.002 to 0.01 , and keep all other design constants similar to our last evaluation. The implemented results are shown in the right column of Figure 5.2. In view of left and right column of Figure 5.2 , we can observe that the tracking performance under single model classical adaptive output feedback design with smaller controller-observer gains does not provide good tracking performance.

Remark 5.2: In CE-based CAC design, the dynamical properties defined in 
section 5.1 are frequently employed for obtaining Lyapunov stability condition for robotic systems. These properties make the classical design overly conservative as the unknown nonlinear functions (linearized bound defined in property 1,2 and 3) are required to appear linearly with respect to unknown model parameters of the robot dynamics. Such a strict linearity assumption is very difficult to meet for uncertain nonlinear systems as nonlinear systems associated with many structured and unstructured uncertainties resulting large modeling error uncertainty. As a matter of the fact, for a general class of nonlinear systems, unknown nonlinear functions are also assumed to be appeared linearly with respect to unknown parameters to establish Lyapunov stability condition.

\subsection{Adaptive Feedback Using Multi-model Con- trol Approach: State and Output Feedback Design}

In previous section, we have examined the complexity of the CE-based single model $\mathrm{CAC}$ approach for state and output feedback design. The main drawback of the classical adaptive control design is its poor transient tracking response. Specifically, when the initial conditions and parameter errors become large then the transient tracking performance will also become unacceptably large values. This is mainly because of the well-known assumption that the nonlinear functions are assumed to be appeared linearly with respect to uncertain parameters. Most importantly, the design only provide asymptotic convergence property while transient tracking property is not considered in the stability analysis.

Our evaluation shows that the designer may use high values of observeradaptation gains as well as the control saturation level to achieve desired transient tracking performance via reducing initial parameter error uncertainties. However, the use of high gains and large saturation levels makes the design unimplementable 
as, in practice, high gains increase the control chattering activity limiting tracking performance.

In this work, we propose to use multiple models based hybrid adaptive control technique to reduce the observer-controller gains from CE-based CAC design [86, $95,101]$. This technique permits the designer to keep smaller value of learning gains $\Gamma$ and higher value of observer design constants $\epsilon$. The idea behind this approach is to reduce the level of uncertainty via resetting the parameter estimate of CAC design into a family of candidate at any instant of time. To identify best possible model from a family of candidate, we use on-line estimation of the the Lyapunov-function candidate.

We begin with the hybrid design by considering that the unknown plant parameter $\theta$ belongs to a known but comparatively large compact set $\Omega$. Then, we equally split the parameter set $\Omega$ into a finite number of smaller compact subsets such that $\theta_{i} \in \Omega_{i}$ with $\Omega=\bigcup_{i=1}^{N} \Omega_{i}$ and $\theta \in \Omega_{i}$. We then design a family of candidate controller bounded in $e$ via saturating outside the region of interest $\Omega_{c}$ correspond to each of these smaller parameter subsets as

$$
\tau^{i}\left(e, Q_{d}, \theta_{i}\right)=Y\left(e, \dot{q}_{d}, \ddot{q}_{d}\right) \theta_{i}-K_{P} e_{1}-K_{D} e_{2}
$$

with $\left(\theta, \theta_{i}\right) \in \Omega_{i}$, such that for every $\theta \in \Omega_{i}$ all the signals in the closed-loop system started inside the sets $\Omega_{c o}$ with $e(0) \in \Omega_{c o}$ are bounded, and the output tracking error trajectories converge to zero, $e(t) \rightarrow 0$ as the time goes to infinity. The constant diagonal elements of the positive definite matrices $K_{P}$ and $K_{D}$ are chosen such that they ensures an acceptable transient performance of the closedloop system. The control gains $K_{P}$ and $K_{D}$ are common to all the candidate controllers N. We also notice from (5.24) that the regressors model $Y\left(e, \dot{q}_{d}, \ddot{q}_{d}\right)$ is also common to all candidate controllers (5.24). The model selection is based upon a prior $i$ known bound of the system dynamics and its operating environments. This means that if the system parameter belongs to a known specified range then the model sets can be split within the specified range.

We now develop resetting-logic to identify a suitable candidate $\tau\left(e, Q_{d}, \theta\right)$ 
from a finite set of candidate controller $\tau^{i}\left(e, Q_{d}, \theta_{i}\right)$. Specifically, logic needs to be selected in such a way that guarantees all the signals in the closed-loop systems are bounded, and the error trajectories converge to zero as time goes to zero. We consider that there exists a small time constant such that the solution of the closed loop system with the switching-logic is well defined. For the given set of initial condition of interest, the candidate controllers are saturated outside of the region of interest in order to ensure that the closed loop trajectories under switching remain bounded by the domain of interest.

To develop model/control resetting strategy such that it improves overall tracking response while guaranteeing the closed loop stability for overall control systems, we first introduce a pre-routed resetting logic. The idea is to identify a controller corresponding to the parameter model $\theta_{i}$, with $i=i *$ and $i * \in \mathcal{M}(i *)$, from a finite set of candidate models via using on-line estimation of the Lyapunovinequality $[86,101]$.

Algorithm 5.1: Suppose that the controller index $i \in \mathcal{M}$ is acting in the loop at time $t$. Then, we follow the following pre-routed switching-logic to identify a controller that satisfies the pre-specified Lyapunov inequality

- A. Set the initial time $t_{o}=0$, controller index $i \in \mathcal{M}=\{1,2,3, \ldots ., N\}$ and a dwell time constant $t_{d}>0$.

- B. We first place the CAC algorithm (5.3) with $\hat{\theta}$ is provided by standard adaptation law (the output of $\dot{\hat{\theta}}$ defined in (5.4)) in the loop and dwell it for $t \in\left[t_{o}, t_{o}+t_{d}\right]$.

- C. For $t \geq t_{o}+t_{d}$, we check the pre-specified resetting inequality (5.8) as $\dot{V}(t) \leq 0$. If this inequality satisfies then we keep the classical control in the loop.

- D. If the $C A C$ does not satisfy $\dot{V}(t) \leq 0$ then we put the first candidate controller $\tau^{1}\left(e, Q_{d}, \theta_{1}\right)$ in the loop. We dwell the controller $\tau^{1}\left(e, Q_{d}, \theta_{1}\right)$ for 
small time $t_{d}$ and then monitor the inequality $\dot{V}(t) \leq 0$ to see whether or not the Lyapunov-function decreasing sufficiently fast to switch to the next candidate controller.

- $\mathbf{E}$ If the controller $\tau^{1}\left(e, Q_{d}, \theta_{1}\right)$ satisfy the inequality then we apply it the system. If not then we switch again to the next candidate $\tau^{2}\left(e, Q_{d}, \theta_{2}\right)$. We repeat the search until we find a candidate controller that satisfies the stability guaranteed Lyapunov inequality.

Using our above analysis, let us state the main results in the following Theorem 5.2 provided that all the states (the position and velocity signals) are available for candidate controllers design (5.24).

Theorem 5.2 : Consider the closed loop system formulated by (5.1), (5.2) and (5.24) under the switching-logic defined by Algorithm 5.1. Then there exists a time such that the controller according to the logic stated in Algorithm 5.1 is tuned to the plant that ensures $\dot{V}(t) \leq 0$.

Since we consider that the plant parameter $\theta$ belongs to one of the compact subsets then there exists a candidate controller that guarantees the inequality $\dot{V}(t) \leq 0$.

In Theorem 5.2, we assume that all the state vectors are available for feedback to construct multi-model control law stated in (5.24). We now consider the velocity signals $e$ are unavailable in (5.24). To reproduce unknown velocity signals, we replace $e$ by the output of the linear estimator (5.10). Then, we can modify the algorithm (5.24) to formulate multi-model based adaptive output feedback (AOFB) as

$$
\tau^{i}\left(\hat{e}, Q_{d}, \theta_{i}\right)=Y\left(\hat{e}, \dot{q}_{d}, \ddot{q}_{d}\right) \theta_{i}-K_{P} \hat{e}_{1}-K_{D} \hat{e}_{2}
$$

Then, we use the Lyapunov inequality $\dot{V}+\lambda_{\min }(\Pi)\|e\|^{2} \leq \chi \epsilon(5.16)$ as a resetting criterion for the multi-models based AOFB design. Notice from this Lyapunov inequality that one cannot make state estimation error to zero as $\epsilon \neq 0$. This implies to find the bound on the estimation error term $\|\eta\|$ in $\dot{V}+\lambda_{\min }(\Pi)\|e\|^{2} \leq \chi \epsilon$. 
For any given $\epsilon$ there exists a short transient period such that the state estimates $\hat{e}$ decay exponential fast to a small compact set $\Omega_{\epsilon}$. The short transient peaking time $T_{1}(\epsilon)$ can be determined as $T_{1}(\epsilon)=\frac{\epsilon}{\gamma} \ln \left(\frac{k_{o}}{\beta \epsilon^{4}}\right)$ where $\epsilon>0, k_{o}=k^{2} \lambda_{\max }(P)=\frac{k^{2}}{2 \gamma}$, $\gamma=\frac{1}{2 \lambda_{\max }(P)}$ and $e(0)-\hat{e}(0) \leq k$ with $k \geq 0$ [82]. After this transient peaking time, the estimation error converge to a small value. To ensure that the estimation error converge to a small value, the small time $t_{d}$ needs to select such that $T_{1}(\epsilon)<t_{d}$. The control input is saturated outside the region of interest to guarantee the closed-loop trajectory remains inside the domain of attraction during transient phase. Then we state our main results for the multi-model based AOFB design in the following Theorem $5.3[86,101]$.

Theorem 5.3 : Consider the closed loop control system designed by using (5.1), (5.2), (5.10) and (5.25) under the switching-logic defined in Algorithm 5.1 with the resetting inequality for the derivative of the Lyapunov-function $\dot{V}(t) \leq \chi \epsilon$. Then, for any given $(e(0), \hat{e}(0)) \in \Omega_{c o}, \theta \in \Omega_{i}$ and $\theta_{i} \in \Omega_{i}$ with $i \in \mathcal{M}$, there exists $\epsilon>0$ and $t_{d}>T_{1}(\epsilon)$ such that the candidate controller, corresponding to an appropriate model, according to the Algorithm 5.1, is tuned to the plant which ensures that all the state variables of the closed loop system are bounded.

Proof: The proof of Theorem 5.2 and Theorem 5.3 is similar to the proof of Theorem 4.3 and Theorem 4.4, respectively. So, we removed the details proof for brevity.

The problem with the pre-routed switching Algorithm 5.1 is that if the number of candidates becomes large then the design may produce unacceptable transient tracking errors and high-frequency control oscillation. This is due to the fact in the presence of large number of candidate controllers, the logic has to scan through a number of candidate controllers before identifying an appropriate candidate that satisfies the Lyapunov inequality.

Let us now introduce continuous switching mechanism to remove such transient tracking and control oscillation phenomenon from pre-routed switching-logic. This means that we allow the parameters to be reset continuously among family 
of the candidate models $\theta_{i}$.

Algorithm 5.2: We assume that the multi-model controllers $\tau^{i}\left(\hat{e}, Q_{d}, \hat{\theta}_{i}\right)$ with $i \in \mathcal{M}=\{1,2,3, \ldots ., N\}$ and the switching inequality $\dot{V}(t) \leq 0$ are available at any time $t$, then we follow the following steps to select a candidate controller which best estimates the plant at any instant of time.

- A. Suppose that the initial time $t_{0}=0$, the controller index $i \in \mathcal{M}=$ $\{1,2,3, \ldots \ldots ., N\}$ and the small time constant $t_{d}>0$.

- B. Apply first $C A C \tau\left(\hat{e}, Q_{d}, \hat{\theta}\right)$ (5.3) along with standard adaptation law (5.4) and dwell it for some time, $t \in\left[t_{o}, t_{o}+t_{d}\right]$. Then, at $t \geq t_{o}+t_{d}$, we check the inequality $\dot{V}(t) \leq 0$ to see which candidate controller satisfies the switching condition.

- D. If the CAC satisfies the pre-defined inequality then we stay with that controller until the moment of time the Lyapunov inequality violated. If the CAC does not guarantee $\dot{V}(t) \leq 0$ then we reset to the candidate that satisfies the switching condition. If the pre-specified inequality $\dot{V}(t) \leq 0$ never violated then there will not be any switching. Thus, the output trajectory tracks the desired one asymptotically that is $q(t) \rightarrow q_{d}(t)$ as the time goes to infinity.

- E. If there exists a time, say $t_{i} \geq t_{o}+t_{d}$ with $t_{o}=t_{i}$, tuned controller does not satisfy the switching criterion then another candidate will be put into the system that guarantees the pre-specified inequality $\dot{V}(t) \leq 0$.

We now summarize our main results in the following Theorem 5.4 when all the process states are measurable.

Theorem 5.4 : Consider the closed-loop system formulated by using (5.1), (5.2), (5.24) under the switching-logic defined in Algorithm 5.2. Then, there exists a time such that the classical control law (5.3) (5.4) is switched into a set of candidate controllers $\tau^{i}\left(e, Q_{d}, \theta_{i}\right)$ with $i \in \mathcal{M}$ satisfying the Lyapunov stability condition 
$\dot{V}(t) \leq 0$. Then, hybrid adaptive control system guarantees that all the signals in the closed-loop system are asymptotically stable.

Let us now replace unknown velocity signals $e$ by the output of the linear observer (5.10) to formulate hybrid adaptive output feedback (HAOFB) design (5.25) given in the following Theorem 5.5.

Theorem 5.5: Consider the closed-loop system formulated by using (5.1), (5.2), (5.10), (5.25) with the resetting Algorithm 5.2. Then, for any given $(e(0), \hat{e}(0)) \in$ $\Omega_{c o}$ and $\theta \in \Omega_{i}$ with $i \in \mathcal{M}$, there exists a small $\epsilon$ and $t_{d}>T_{1}(\epsilon)$ such that CAOFB algorithm (5.9) is tuned into a candidate controller corresponding to candidate model $\theta_{i *}$, with $i * \in \mathcal{M}(i *)$, that guarantees the derivative of the Lyapunov inequality $\dot{V}(t) \leq \chi \epsilon$. Then, output feedback based hybrid control scheme with the given control switching condition guarantees that the closed-loop error trajectories are bounded by a bound that can be made arbitrarily small for small value of $\epsilon$ and $k_{f}$.

Remark 5.3: The control switching-logic proposed so far is based on using a fixed inequality for the derivative of the Lyapunov-function. These logics tuned the controller according to the pre-specified fixed rate of the decay of the Lyapunovfunction candidate. Notice that the candidate controller that selected by using Algorithms 5.1 and 5.2 are not unique. This means that the model/control identified by the logic derived in Algorithms 5.1 and 5.2 with the fixed inequality for the derivative of the Lyapunov-function is not the actual one. Another important shortcoming of this Algorithms is that the control logic cannot identify the model that already tested by the switching supervisor.

Let us now develop a control switching-logic that depends on the derivative of multiple Lyapunov-function inequalities [86, 95, 101]. To begin with this development, we first consider that, for a given compact set of initial conditions of interest $e(0) \in \Omega_{c o}$ and for every $\theta \in \Omega_{i}$ with $i \in \mathcal{M}=\{1,2,3, \ldots, N\}$, we construct a set of candidate controller $\tau^{i}\left(e, Q_{d}, \theta_{i}\right)$ bounded in $e$ via saturating outside the domain of interest such that for every $\theta \in \Omega_{i}$ all the signals in the closed-loop system started 
inside $\Omega_{c o}$ are bounded such that the error trajectory converges to zero as the time goes to infinity. We also consider Lyapunov-function candidate corresponding to each of these candidate controllers as

$$
\alpha_{2}^{i}\|e\|^{2} \leq V_{i}\left(e, \tilde{\theta}_{i}\right) \leq \alpha_{3}^{i}\|e\|^{2}
$$

$\forall e \in \Omega_{c}^{i}=\left\{\left(e, \tilde{\theta}_{i}\right) \mid V_{i}\left(e, \tilde{\theta}_{i}\right) \leq c\right\}$ and $\forall\left(\theta, \theta_{i}\right) \in \Omega_{i}$, where $c>0, \tilde{\theta}_{i}=\left(\theta_{i}-\theta\right)$ and $\alpha_{2}^{i}$ and $\alpha_{3}^{i}$ are bounded positive constant. Then, we apply the following resetting strategy to identify a candidate controller $\tau^{i *}\left(e, Q_{d}, \theta_{i *}\right)$ with $i=i^{*}, i^{*} \in \mathcal{M}(i *)$ and $\theta \in \Omega(i *)$ which best approximates the plant at each instant of time such that all the trajectories asymptotically converge to zero as the time goes to infinity.

\section{Algorithm 5.3:}

- A. Suppose that the initial time $t_{o}=0$, the controller index $i \in \mathcal{M}=$ $\{1,2,3, \ldots ., N\}$ and a small positive time constant $t_{d}>t_{o}$.

- B. Apply first CAC $\tau\left(e, Q_{d}, \hat{\theta}\right)$ (5.3) in the loop for a short period of time $t \in\left[t_{o}, t_{o}+t_{d}\right]$. For $t \geq t_{o}+t_{d}$, we continuously monitor the inequality $\triangle W_{i}(t)=V_{i}\left(t_{s}\right)-V_{0}(t) \leq 0$, where $t_{s} \geq t_{o}+t_{d}$ is the resetting time and $V_{0}(t)$ is defined by (5.7), to see which candidate generates guaranteed decrease in the value of $\triangle W_{i}(t) \leq 0$.

- C. We keep the CAC law in the loop until the moment of time $t_{i} \geq t_{o}+t_{d}$ when the resetting inequality is violated. If CAC does not satisfy the Lyapunov inequality then we reset to the candidate control law that generates largest guaranteed decrease in the value of $\triangle W_{i}(t) \leq 0$.

- D. If the inequality $\Delta W_{i}(t) \leq 0$ is never violated then there will not be any switching. This implies that the plant output tracks the desired trajectory that is $q(t) \rightarrow q_{d}(t)$ as the time goes to infinity. If there exist a time such that the candidate controller that acting in the loop does not satisfy $\triangle W_{i}(t) \leq 0$ then another candidate will be put in the system as there exists a candidate 
that provides guaranteed minimum value of $\triangle W_{i}(t) \leq 0$ at that instant of time.

We now present the main results for the multiple Lyapunov-functions based adaptive control by assuming that the position and velocity state vectors are measurable $[86,95,101]$.

Theorem 5.6: Consider the closed loop system composed of (5.1), (5.2) and (5.24) under the switching-logic defined in Algorithm 5.3. Then, there exists a time such that the control law corresponding to the guaranteed decrease in the value of $\triangle W_{i}(t) \leq 0$ is tuned to the plant which ensures that all the signals in the closed-loop model are bounded and $e(t) \rightarrow 0$ when $t \rightarrow \infty$. Note that Theorem 5.6 can only be applied when all the states are available for feedback to construct multi-model controllers (5.24). We now consider that the velocity signals is not available and estimated by the output of the linear estimator (5.10) to formulate hybrid adaptive output feedback based hybrid control design (5.25). We then state our main results in the following Theorem $5.7[96,95,101]$.

Theorem 5.7: Consider the closed-loop system formulated by using (5.1), (5.2), (5.25) and (5.9) with control logic Algorithm 5.3. Then, for any given $(e(0), \hat{e}(0)) \in \Omega_{c o}$ and $\theta_{i} \in \Omega_{i}$ with $i \in \mathcal{M}$, there exists a small value of $\epsilon>0$ and $t_{d}>T_{1}(\epsilon)$ such that the candidate controller corresponding to a guaranteed decrease in the value of $\triangle W_{i}(t) \leq 0$ is applied to the plant. Then, the HAOFB control system ensures that all the state variables of the closed-loop system are bounded.

Proof: The proof of Theorem 5.6 and Theorem 5.7 can be derived along the line of the logic introduced in Algorithm 5.3. So, we omitted the proof for brevity. 

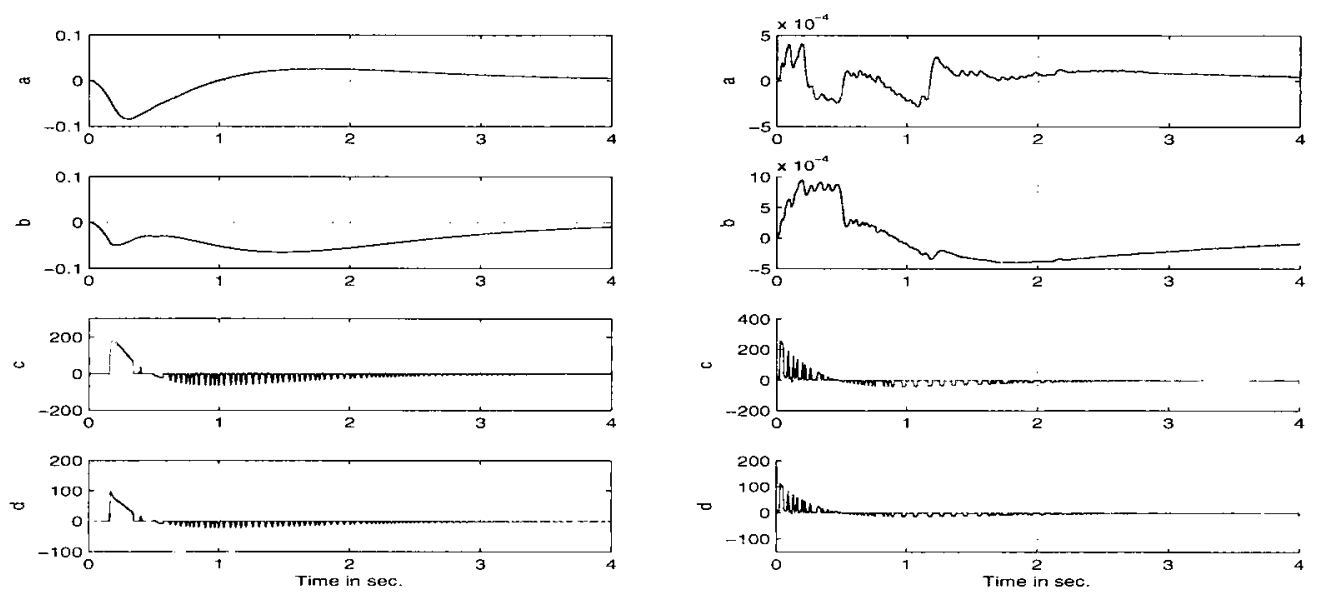

Figure 5.3: The simulation results with Theorem 5.2 and Theorem 5.6 under $\tilde{\theta}=4$. First column is for Theorem 5.2 and the second column is for Theorem 5.6, where $a$ : output tracking errors (radians) for joint $1, b$ : output tracking errors (radians) for joint $2, c$ : control input (newton-meters) for joint $1, d$ : control input (newtonmeters) for joint 2 .

\subsubsection{Robust Adaptive Feedback Using multi-model Con- trol Approach}

1 Due to the presence of uncertainty, such as input and output disturbance, unmodeled actuator dynamics, estimator transients and noisy supervisory signals, the Lyapunov-function inequality may increase leading to erroneous reset.

To avoid such an undesirable switching in practice, one requires to modify Algorithm 5.1 to Algorithm 5.3. For Algorithm 5.1, we need to replace the state

\footnotetext{
${ }^{1}$ We remove the convergence analysis for the modified Algorithm 5.1 to Algorithm 5.3 with modified hybrid adaptive controller design $\tau^{i}\left(e, Q_{d}, \theta_{i}\right)=\Upsilon\left(e, \dot{q}_{d}, \ddot{q}_{d}\right) \theta_{i}-K_{P} e_{1}-K_{D} e_{2}$ and $\tau^{i}\left(\hat{e}, Q_{d}, \theta_{i}\right)=\Upsilon\left(\hat{e}, \dot{q}_{d}, \ddot{q}_{d}\right) \theta_{i}-K_{P} \hat{e}_{1}-K_{D} \hat{e}_{2}$ as they can be shown along the line of the convergence analysis shown for Algorithm 5.1 to Algorithm 5.3 under $\tau^{i}\left(e, Q_{d}, \theta_{i}\right)=Y\left(e, \dot{q}_{d}, \ddot{q}_{d}\right) \theta_{i}-$ $\left.K_{P} e_{1}-K_{D} e_{2}\right)$ and $\tau^{i}\left(\hat{e}, Q_{d}, \theta_{i}\right)=Y\left(\hat{e}, \dot{q}_{d}, \ddot{q}_{d}\right) \theta_{i}-K_{P} \hat{e}_{1}-K_{D} \hat{e}_{2}$.
} 
feedback candidate controllers (5.24)

$$
\tau^{i}\left(e, Q_{d}, \theta_{i}\right)=Y\left(e, \dot{q}_{d}, \ddot{q}_{d}\right) \theta_{i}-K_{P} e_{1}-K_{D} e_{2}
$$

by

$$
\tau^{i}\left(e, Q_{d}, \theta_{i}\right)=\Upsilon\left(e, \dot{q}_{d}, \ddot{q}_{d}\right) \theta_{i}-K_{P} e_{1}-K_{D} e_{2}
$$

In Algorithm 5.2, hybrid adaptive output feedback controller (5.25)

$$
\tau^{i}\left(\hat{e}, Q_{d}, \theta_{i}\right)=Y\left(\hat{e}, \dot{q}_{d}, \ddot{q}_{d}\right) \theta_{i}-K_{P} \hat{e}_{1}-K_{D} \hat{e}_{2}
$$

replace by

$$
\tau^{i}\left(\hat{e}, Q_{d}, \theta_{i}\right)=\Upsilon\left(\hat{e}, \dot{q}_{d}, \ddot{q}_{d}\right) \theta_{i}-K_{P} \hat{e}_{1}-K_{D} \hat{e}_{2}
$$

The proof of stability of Theorems 5.2 to Theorem 5.7 under Algorithm 5.1 to Algorithm 5.3 with the modified hybrid adaptive controller design can be shown along the line of the convergence analysis shown for Theorems 5.2 to Theorems 5.7 with Algorithm 5.1 to Algorithm 5.3.

Remark 5.3: Notice from our analysis that the tracking convergence will increase with the increase of the number of candidate controllers that is with the increase of the smaller number compact subsets $\Omega_{i}$. However, the number of candidate controllers $N$ can be reduced arbitrarily by distributing the candidates around with the bound of the parameter vector $\theta$ that belongs to a known compact set. We also note that the design requires smaller time constant $t_{d}$ to ensure the continuity of the Lyapunov function candidate. In our experimental evaluation later in chapter 6 , we will examine the tracking performance of the hybrid design using with smaller number of candidate controllers with large estimation error uncertainty provided that the parameter does not belong to distributed sets.

\subsubsection{Simulation Results}

In this subsection, we show the design and implementation process of the multimodel/control based adaptive control strategy on robotic systems. For this purpose, we consider a 2-link manipulator system (5.23) that used for the evaluation 

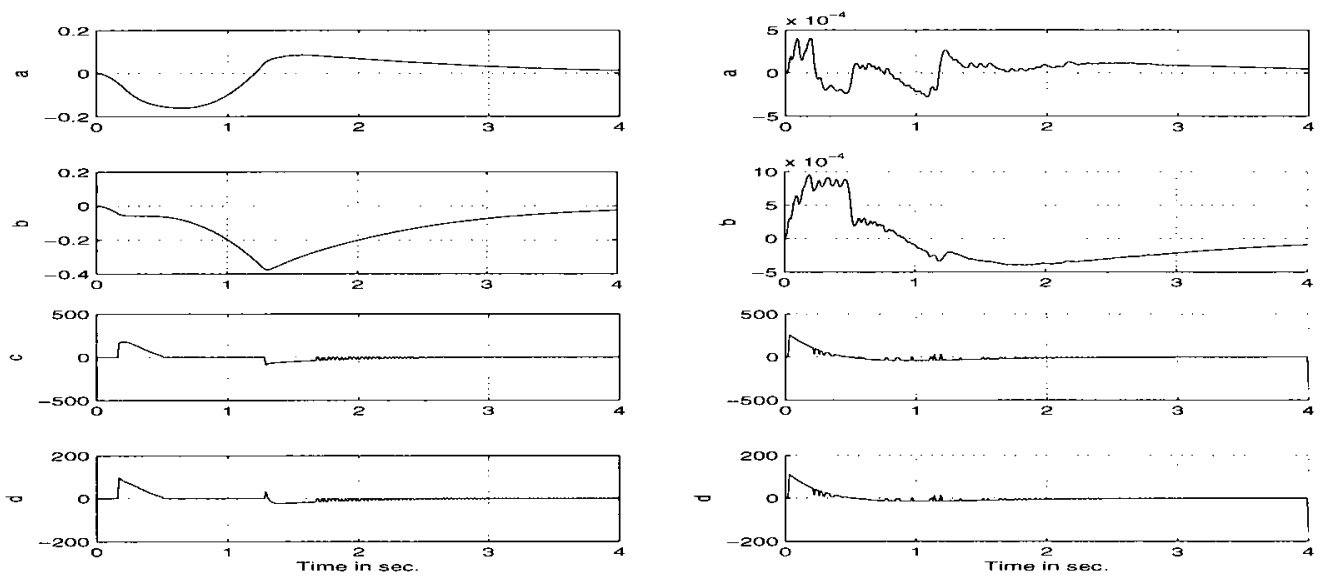

Figure 5.4: The simulation results with Theorem 5.2 and Theorem 5.6 under $\tilde{\theta}=8$. First column is for Theorem 5.2 and the second column is for Theorem 5.6, where $a$ : output tracking errors (radians) for joint $1, b$ : output tracking errors (radians) for joint 2, $c$ : control input (newton-meters) for joint $1, d$ : control input (newtonmeters) for joint 2.

of the SM-based CAC design in previous subsection. We consider that the plant parameters $\theta \in \Re^{2}$ are assumed to be unknown but belong to known compact set as $\Omega \in[-10,10]$. We define the initial conditions of interest as $|e(0)|=2$, $|\hat{e}(0)|=2$ and $\hat{\theta}(0)=0$. Then we split the parameter set $\Omega$ equally into a finite number of smaller compact subsets as $\theta_{i} \in \Omega_{i}$ with $\Omega=\bigcup_{i=1}^{41}\left\{\Omega_{i}\right\}$, that is, $\Omega=\bigcup_{i=1}^{41}\left\{\theta_{i}\right\}=\{-10,-9.5, ., ., ., ., ., ., ., ., 9.5,10\} \times\{-10,10\}$. The control design parameters $\lambda_{0}, K_{P}$ and $K_{D}$ are common to all $i=41$ candidate controllers. The value of $\lambda_{0}, K_{P}$ and $K_{D}$ are chosen in such a way that they ensures an acceptable transient tracking performance. The learning gains $\Gamma$ are chosen such that $\left(\theta, \theta_{i}\right) \in \Omega_{i}$. For our evaluation, the control design parameters are chosen as $\lambda_{0}=2, K_{P 1}=60, K_{P 2}=60, K_{D 1}=60, K_{D 2}=60$ and $\Gamma=10 I_{2 \times 2}$. 

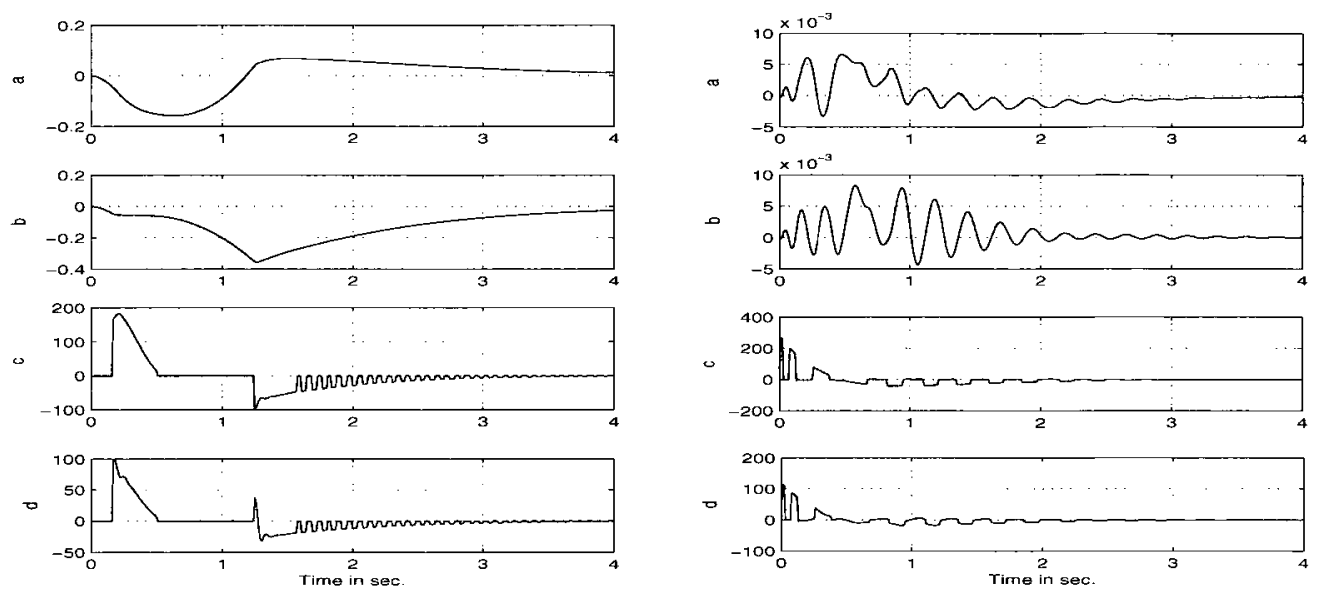

Figure 5.5: The simulation results with Theorem 5.3 and Theorem 5.7 with $\tilde{\theta}=8$. First column is for Theorem 5.3 and the second column is for Theorem 5.7, where $a$ : output tracking errors (radians) for joint $1, b$ : output tracking errors (radians) for joint 2, $c$ : control input (newton-meters) for joint $1, d$ : control input (newtonmeters) for joint 2 .

\subsubsection{Comparison of Theorem 5.2 and Theorem 5.6}

We first compare the tracking performance of the Theorem 5.2 (pre-routed with fixed Lyapunov-function inequality) and Theorem 5.6 (continuous with multiple Lyapunov-function inequalities) on the system (5.23). We then choose small value of $t_{d}$ as $t_{d}=0.03$ and construct candidates (5.24) with $i=41$ as a state feedback controller. The implemented results are given in Figures 5.3 and 5.6 (state feedback case). The Figure 5.3 shows the tracking convergence with $\tilde{\theta}=4$, that is, $i *=29$. The Figure 5.4 depicts the conducted results under $\tilde{\theta}=8$, that is, $i *=37$. The first column of each Figure is for Theorem 5.2 and the second column of each Figure is for Theorem 5.6.

By comparing left and right columns of two Figures, we see that comparatively large transient tracking errors under pre-routed switching-logic of Algorithm 5.1 than Algorithm 5.3. We also notice from this results that the tracking errors with 
Theorem 5.2 increase with the increase of the number $i^{*}$ (see, the left column of Figures 5.3 and 5.4). This is mainly because of the fact that the logic defined in Algorithm 1 has to scan through a number of model-controllers before converging to the one that satisfies the resetting criterion.

\subsubsection{Comparison of Theorem 5.3 and Theorem 5.7}

Let us now compare the performance obtained under state feedback based design (Theorem 5.2 and Theorem 5.6) can be recovered by using output feedback design (Theorem 5.3 and Theorem 5.7). To illustrate that, our first task is to ensure robust reconstruction of the unknown states via using exponentially fast observer dynamics (5.10). We then choose the observer design constants as $H_{1}=I_{2 \times 2}, H_{2}=I_{2 \times 2}$ and $\epsilon=0.01$. For fair comparison, we keep the same controller design parameters that used for the evaluation of the state feedback based design of Theorem 5.2 and Theorem 5.6. Then we define small time $t_{d}$ as $t_{d}=0.005$ and construct multimodel HAOFB (5.25) with $i=41$ such that $t_{d}>T_{1}(\epsilon)$. Then, we choose the value of $k_{f 1}=0.05$ and $k_{f 2}=0.05$. With these set up, we follow the logic defined by Algorithm 5.1 (Theorem 5.3) and Algorithm 5.3 (Theorem 5.7) on the given system (5.23). The tested results are given in Figure 5.5. The Figure 5.5 presents the tracking convergence with the chosen parameter $\tilde{\theta}$ as $\tilde{\theta}=8$. The left column of this Figure shows the control performance under Theorem 5.3. The right column of Figure 5.5 depicts tracking convergence with Theorem 5.7.

In view of the left and right column of Figure 5.5, one can see that results under multi-model based output feedback approach recover the performance achieved under state feedback design. However, like Theorem 5.2, undesirable transient tracking under pre-routed switching-logic of Theorem 5.3 are clearly observed. 

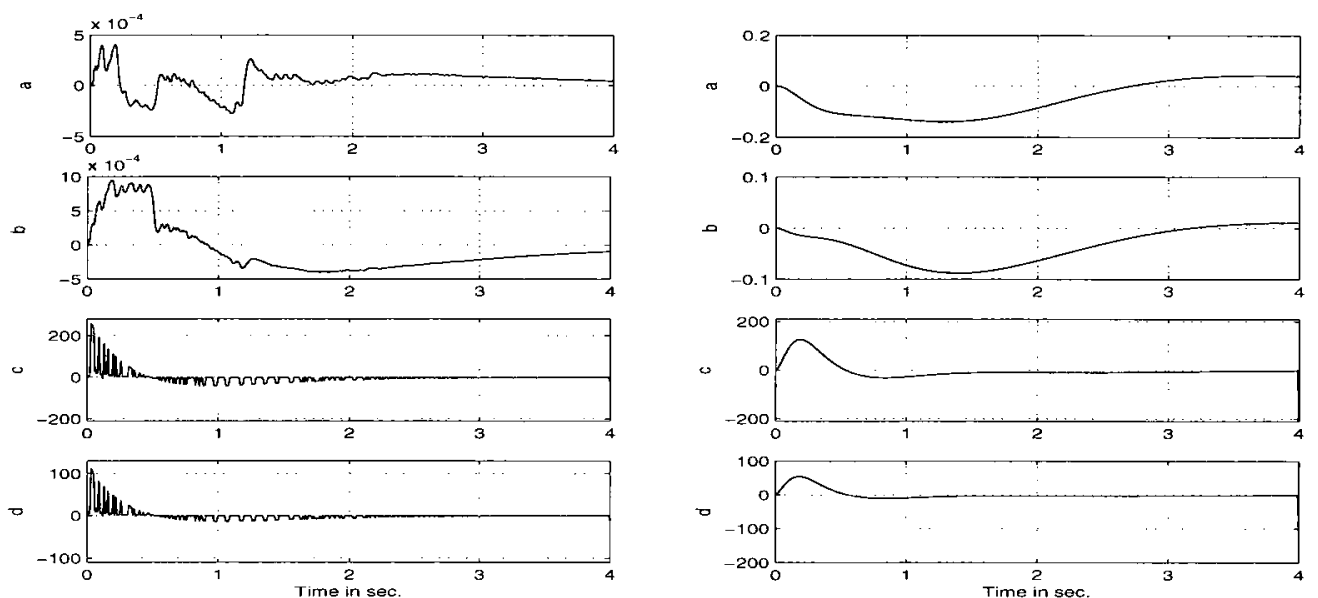

Figure 5.6: The simulation results with Theorem 5.6 and SM-based CAC design (5.3), (5.4) with $\tilde{\theta}=4$. First column is for Theorem 5.6 and the second column is for CAC design (5.3), (5.4), where $a$ : output tracking errors (radians) for joint $1, b$ : output tracking errors (radians) for joint 2, $c$ : control input (newton-meters) for joint $1, d$ : control input (newton-meters) for joint 2 .

\subsubsection{Comparison of Theorem 5.6 with the CAC Design (State Feedback)}

In this subsection, we compare the tracking convergence property of Theorem 5.6 with the SM-based CAC design. For this purpose, we apply Theorem 5.6 and the SM-based CAC algorithm (5.3), (5.4) on the simulated system (5.23). The design parameters are kept similar to our previous evaluation of Theorem 5.6. The conducted results are depicted in Figure 5.6 and Figure 5.7. The Figure 5.6 is for $\tilde{\theta}=4$ and the Figure 5.7 is $\tilde{\theta}=8$. The left colum of each Figure is for Theorem 5.6 and the right column of each Figure is for CAC law. By comparing left and right column of Figures 5.5 and 5.6, we can observe that the output tracking under multi-model based adaptive design converge to the desired one as tracking errors almost converge to zero. But, quite a large tracking errors under single model based CAC design can be seen. Note that the learning gains under CAC design is 

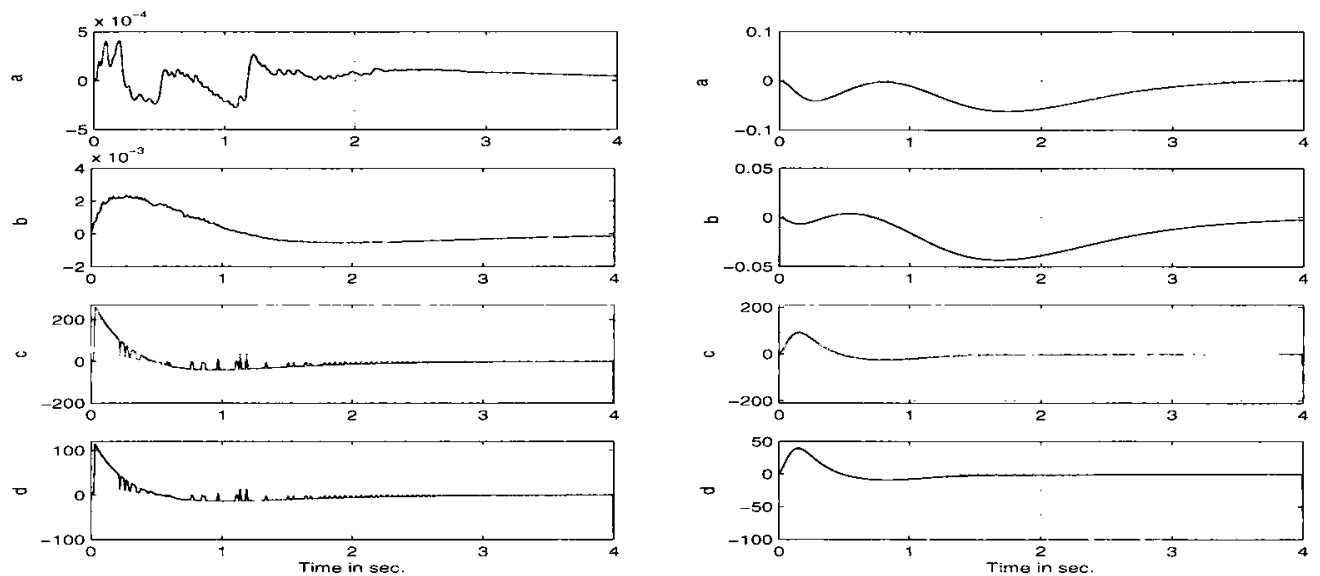

Figure 5.7: The simulation results with Theorem 5.6 and SM-based CAC design (5.3), (5.4) under $\tilde{\theta}=8$. First column is for Theorem 5.6 and the second column is for the single-model CAC (5.3), (5.4), where $a$ : output tracking errors (radians) for joint $1, b$ : output tracking errors (radians) for joint 2, $c$ : control input (newtonmeters) for joint $1, d$ : control input (newton-meters) for joint 2.

ten times higher than multi-model based adaptive control design.

\subsubsection{Comparison of Theorem 5.7 and Theorem 5.1 (Out- put Feedback)}

Our aim is now to compare the tracking performance of Theorem 5.7 with Theorem 5.1. To do that, we consider two sets of observer design constants as $H_{1}=I_{2 \times 2}$, $H_{2}=I_{2 \times 2}, \epsilon=0.01$ and $H_{1}=20 I_{2 \times 2}, H_{2}=20 I_{2 \times 2}, \epsilon=0.1$. For these evaluations, we keep the same set of controller design parameters that applied for the evaluation of Theorem 5.6 and CAC algorithm (5.9), (5.10) of Theorem 5.1. The tested results are given in Figures 5.8 and 5.9. Figures 5.8 and 5.9 show the tracking convergence when the parameter $\theta$ is chosen as $\tilde{\theta}=8$. Figure 5.8 depicts the control performance under the following observer design constants: $H_{1}=I_{2 \times 2}$, $H_{2}=I_{2 \times 2}$ and $\epsilon=0.01$. Figure 5.9 is pictured the tracking convergence with the 

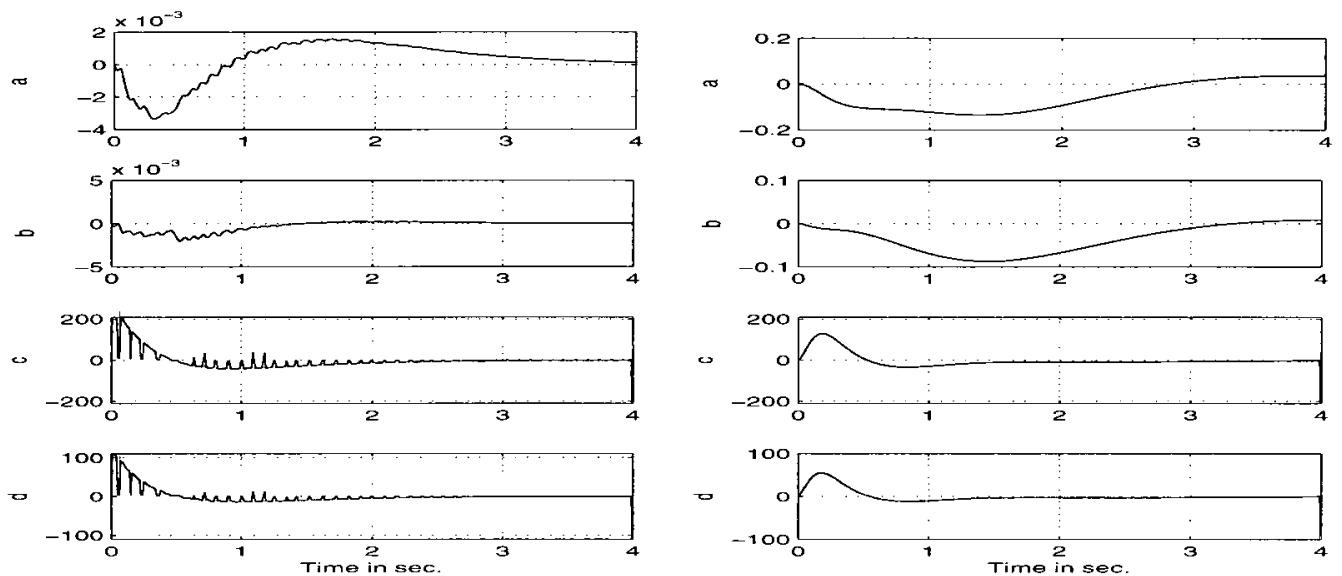

Figure 5.8: The tested results with Theorem 5.7 and SM-based CAC design of Theorem 5.1 under $\tilde{\theta}=8$ with $H_{1}=I_{2 \times 2}, H_{2}=I_{2 \times 2}$ and $\epsilon=0.01$. First column is for Theorem 5.7 and the second column is for single model based CAOFB design of Theorem 5.1, where $a$ : output tracking errors (radians) for joint $1, b$ : output tracking errors (radians) for joint 2, $c$ : control input (newton-meters) for joint 1 , $d$ : control input (newton-meters) for joint 2.

following observer design constants: $H_{1}=20 I_{2 \times 2}, H_{2}=20 I_{2 \times 2}$ and $\epsilon=0.1$. The left column of each Figure is for Theorem 5.7 and the right column of each Figure is for Theorem 5.1. By comparing left and right column of Figures 5.8 to 5.9, we can observe the superiority of the multi-model based HAOFB design of Theorem 5.7 over CAC design of Theorem 5.1. It is important to note that the learning gains under CAOFB design of Theorem 5.1 is ten times higher than multi-model based HAOFB design. The sampling time was set to $0.01 \mathrm{sec}$. for all the results reported in this paper.

\subsection{Summary}

The novelty of this chapter existed in two facts. First, we have considered linear observer based classical adaptive output feedback design for a class of non- 

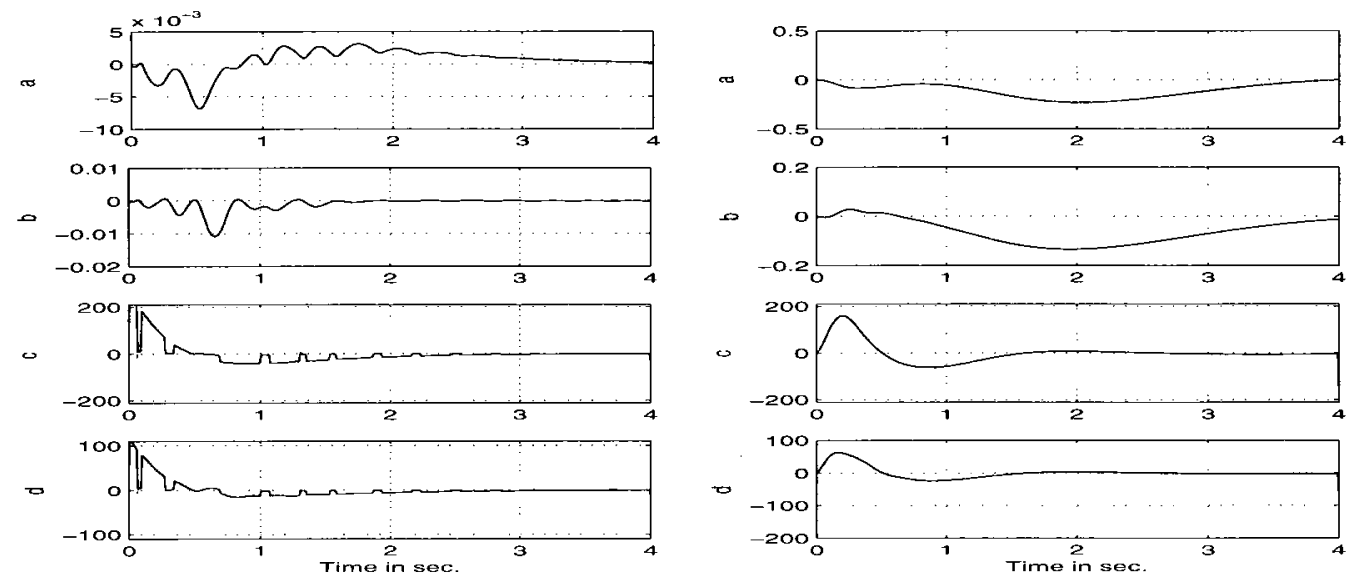

Figure 5.9: The simulation results with Theorem 5.7 and SM-based CAC design of Theorem 5.1 under $\tilde{\theta}=8$ with $H_{1}=20 I_{2 \times 2}, H_{2}=20 I_{2 \times 2}$ and $\epsilon=0.1$. First column is for Theorem 5.7 and the second column is for CAOFB design of Theorem 5.1 , where $a$ : output tracking errors (radians) for joint $1, b$ : output tracking errors (radians) for joint 2, $c$ : control input (newton-meters) for joint $1, d$ : control input (newton-meters) for joint 2.

linear systems. Second, hybrid adaptive control strategy has been introduced to achieve robust tracking with the presence of large scale parametric uncertainty. The method reduced the observer-controller gains from CE-based classical adaptive control scheme via reducing the level of parametric uncertainty through on-line estimation of the Lyapunov-function inequality. The design increased the convergence speed of standard adaptation mechanism by resetting the parameter estimate of CAC design into a family of candidate model that closely approximates the plant at each instant of time. The proposed design can be employed to reduce the sensitivity of the system to noise, disturbances and any other model uncertainties that usually appeared from using high controller-observer gains in single-model based classical design. The evaluation results on a 2-DOF robot manipulator demonstrated the effectiveness of theoretical development for the real-time applications. 


\section{Chapter 6}

\section{EXPERIMENTAL RESULTS}

In this chapter, we implement and evaluate some multi-model based hybrid adaptive and single model-based classical control algorithms on real robotic systems. Our interest in these experimentations is to study the tracking performance of hybrid [86, 95, and 101] and classical control approach [82, 85, and 94]. For experimental evaluations, we use two Phantom ${ }^{\text {TM }}$ (Phantom $_{1}^{\text {TM }}$ and Phantom ${ }_{2}^{\text {TM}}$ ) Premium 1.5A medical mechatronic systems as shown in Fig. 1 provided by SensAble Technologies Inc. The two systems are equipped with standard gimbal endeffectors. Each joint is attached with encoder for joint position measurement. The joint velocity signals are obtained by differentiating the joint position measurement as the system does not have velocity sensors. Such robotic systems are used in bilateral teleoperation systems for biomedical engineering applications [36].

\subsection{Model Dynamics}

To implement the model based hybrid and classical control schemes, let us first model Phantom $^{\mathbf{T M}}$ (Phantom $_{1}^{\mathbf{T M}}$ and Phantom ${ }_{2}^{\text {TM }}$ ) systems as given in Fig. 2 and Fig. 3, respectively. The equation of motion for this two robot systems is defined 


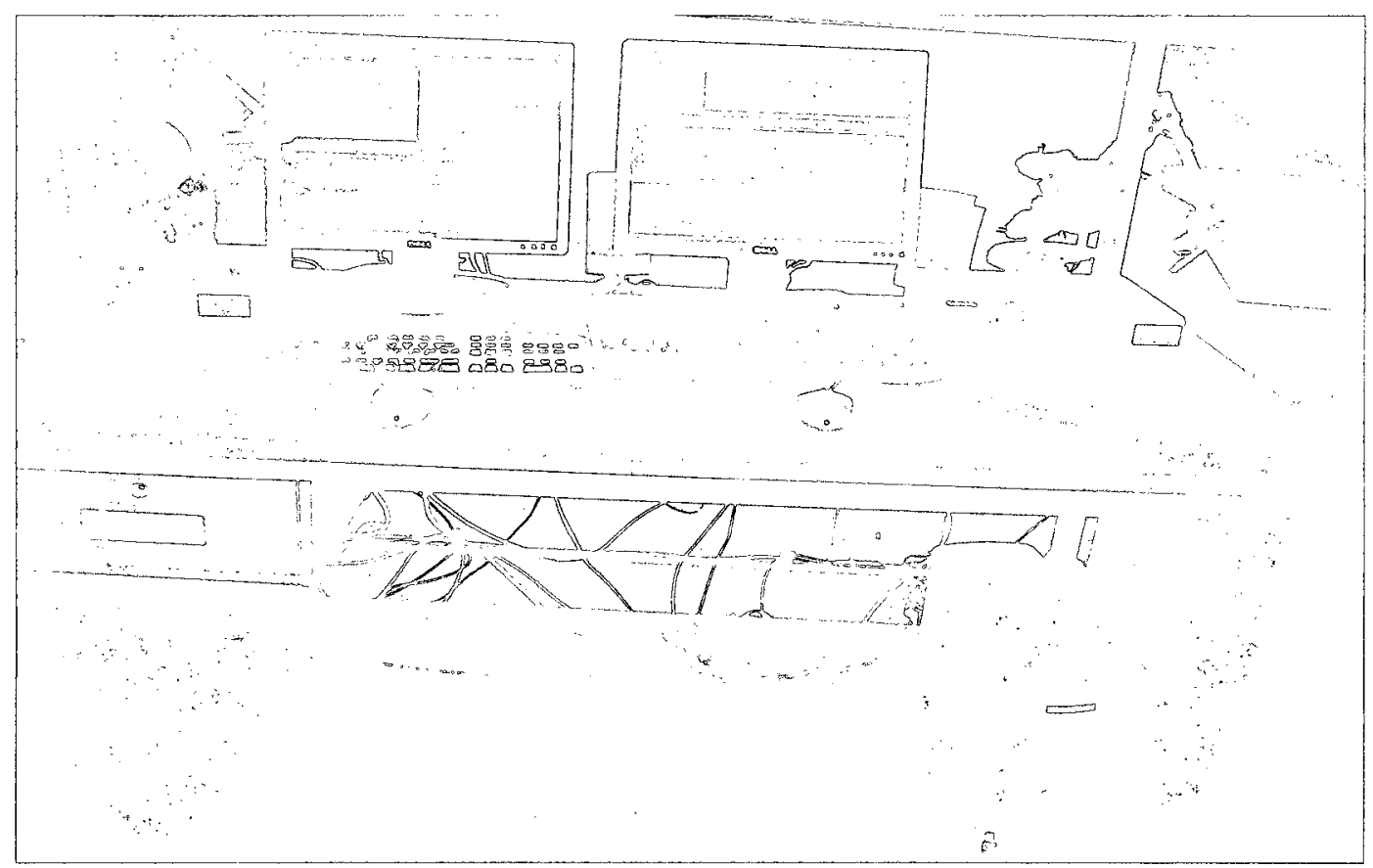

Figure 6.1: Experimental set-up for 3-DOF Phantom ${ }_{1}^{\mathbb{T M}}$ (left side) and Phantom ${ }_{2}^{\text {TM }}$ (right side) device for real-time application of CAC and hybrid design.

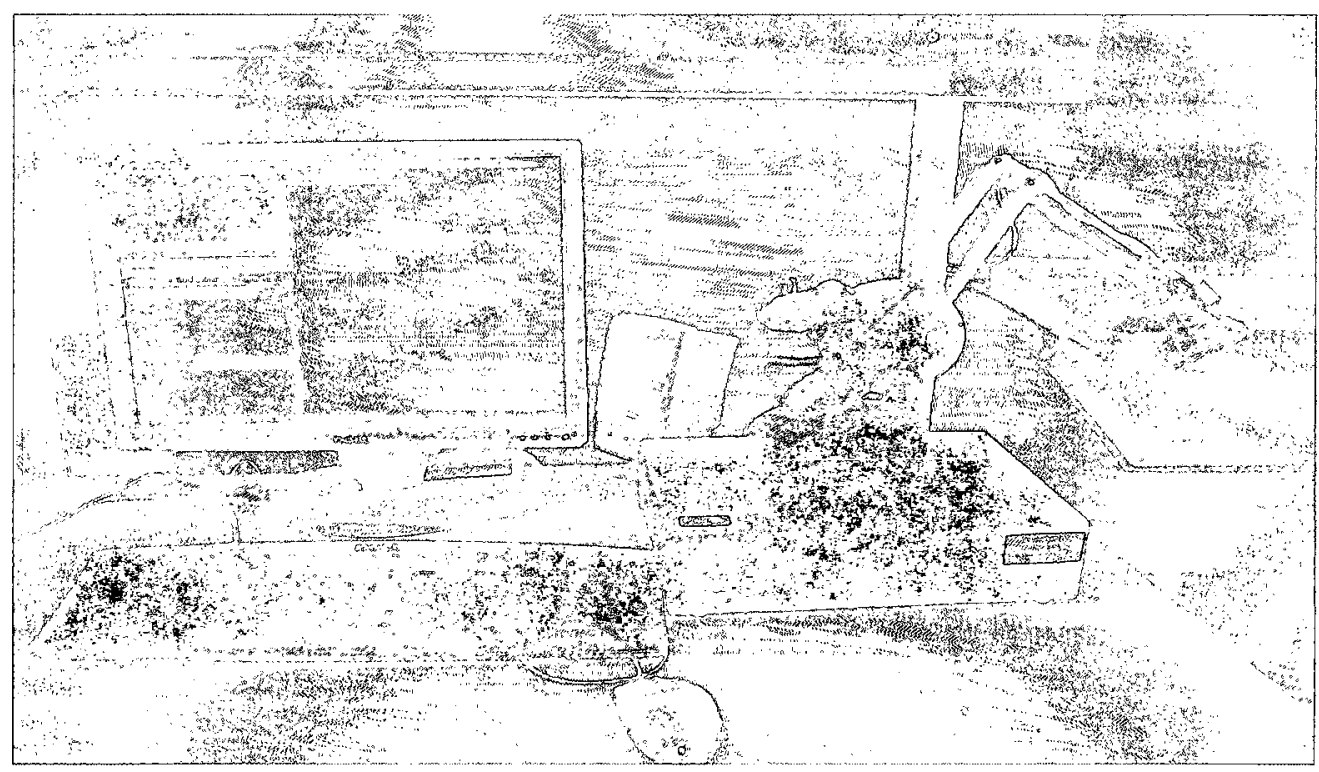

Figure 6.2: $\mathbb{P h a n t o m}{ }_{1}^{\mathrm{TM}}$ robotic system. 


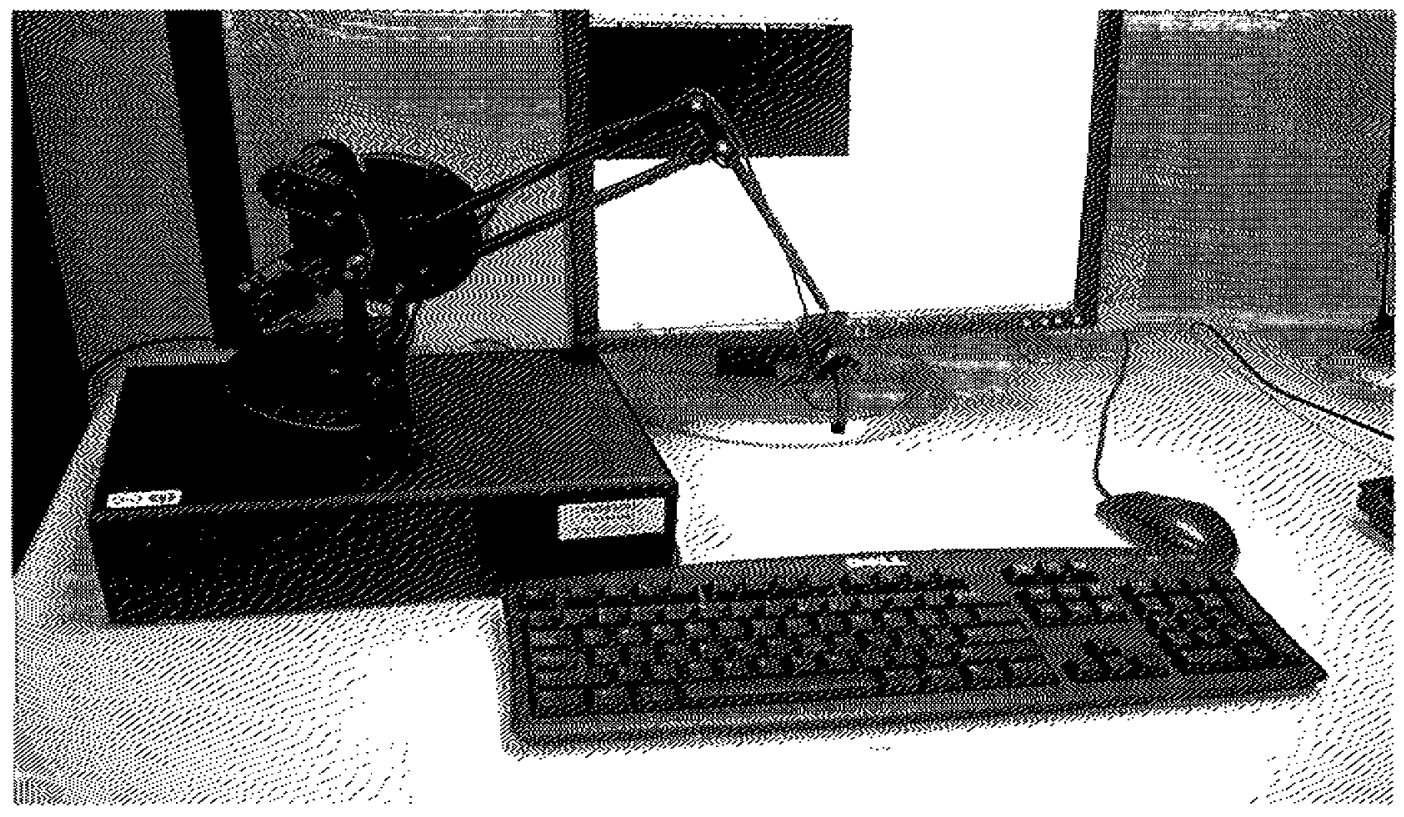

Figure 6.3: Phantom $_{2}^{\mathbf{T M}}$ robotic system.

as,

$$
\left[\begin{array}{ccc}
m_{11} & m_{12} & m_{13} \\
m_{21} & m_{22} & m_{23} \\
m_{31} & m_{32} & m_{33}
\end{array}\right]\left[\begin{array}{c}
\ddot{q}_{1} \\
\ddot{q}_{2} \\
\ddot{q}_{3}
\end{array}\right]+\left[\begin{array}{lll}
c_{11} & c_{12} & c_{13} \\
c_{21} & c_{22} & c_{23} \\
c_{31} & c_{32} & c_{33}
\end{array}\right]\left[\begin{array}{c}
\dot{q}_{1} \\
\dot{q}_{2} \\
\dot{q}_{3}
\end{array}\right]+\left[\begin{array}{c}
G_{1} \\
G_{2} \\
G_{3}
\end{array}\right]=\left[\begin{array}{c}
\tau_{1} \\
\tau_{2} \\
\tau_{3}
\end{array}\right]
$$

where $m_{j k}$ are the elements of the inertia matrix, $c_{j k}$ are the elements of the coriolis and centrifugal force matrix, $G_{j}$ are the gravity vector and $\tau_{j}$ are joint torques with $j=1,2,3$ and $k=1,2,3$. Note that we neglect the viscous and coulomb friction dynamics of the robotic system.

The components of motion dynamics of Phantom ${ }^{\mathbf{T M}}$ system are modeled as $m_{11}=\theta_{1}+\theta_{2} \cos ^{2} q_{2}+\left(\theta_{3}+\theta_{5}\right) \sin ^{2} q_{3}+2 \theta_{6} \cos q_{2} \sin q_{3}, m_{12}=0, m_{13}=$ $0, m_{21}=0, m_{22}=\theta_{4}+\theta_{5}-2 \sin \left(q_{2}-q_{3}\right), m_{31}=0, m_{23}=M_{32}=\theta_{5}-$ $\theta_{6}-2 \sin \left(q_{2}-q_{3}\right), m_{33}=\theta_{5}, c_{11}=-\left(\theta_{2} \sin q_{2} \cos q_{2}+\theta_{6} \sin q_{2} \sin q_{3}\right) \dot{q}_{2}+\left(\left(\theta_{3}+\right.\right.$ $\left.\left.\theta_{5}\right) \sin q_{3} \cos q_{3}+\theta_{6} \cos q_{2} \cos q_{3}\right) \dot{q}_{3}, c_{12}=-\left(\theta_{2} \sin q_{2} \cos q_{2}+\theta_{6} \sin q_{2} \sin q_{3}\right) \dot{q}_{1}, c_{13}=$ $\left(\left(\theta_{3}+\theta_{5}\right) \sin q_{3} \cos q_{3}+\theta_{6} \cos q_{2} \cos q_{3}\right) \dot{q}_{1}, c_{21}=\left(\theta_{2} \sin q_{2} \cos q_{2}+\theta_{6} \sin q_{2} \sin q_{3}\right) \dot{q}_{1}$ $c_{22}=\theta_{6} \cos \left(q_{2}-q_{3}\right)\left(\dot{q}_{3}-\dot{q}_{2}\right), c_{23}=\theta_{6} \cos \left(q_{2}-q_{3}\right)\left(\dot{q}_{2}-\dot{q}_{3}\right), c_{31}=-\left(\theta_{3}+\theta_{5}\right) \sin q_{3} \cos q_{3} \dot{q}_{1}-$ $\theta_{6} \cos q_{2} \cos q_{3} \dot{q}_{1}, c_{32}=0, c_{33}=0, G_{1}=0, G_{2}=\theta_{7} \cos q_{2}$ and $G_{3}=\theta_{8} \sin q_{3}$. 


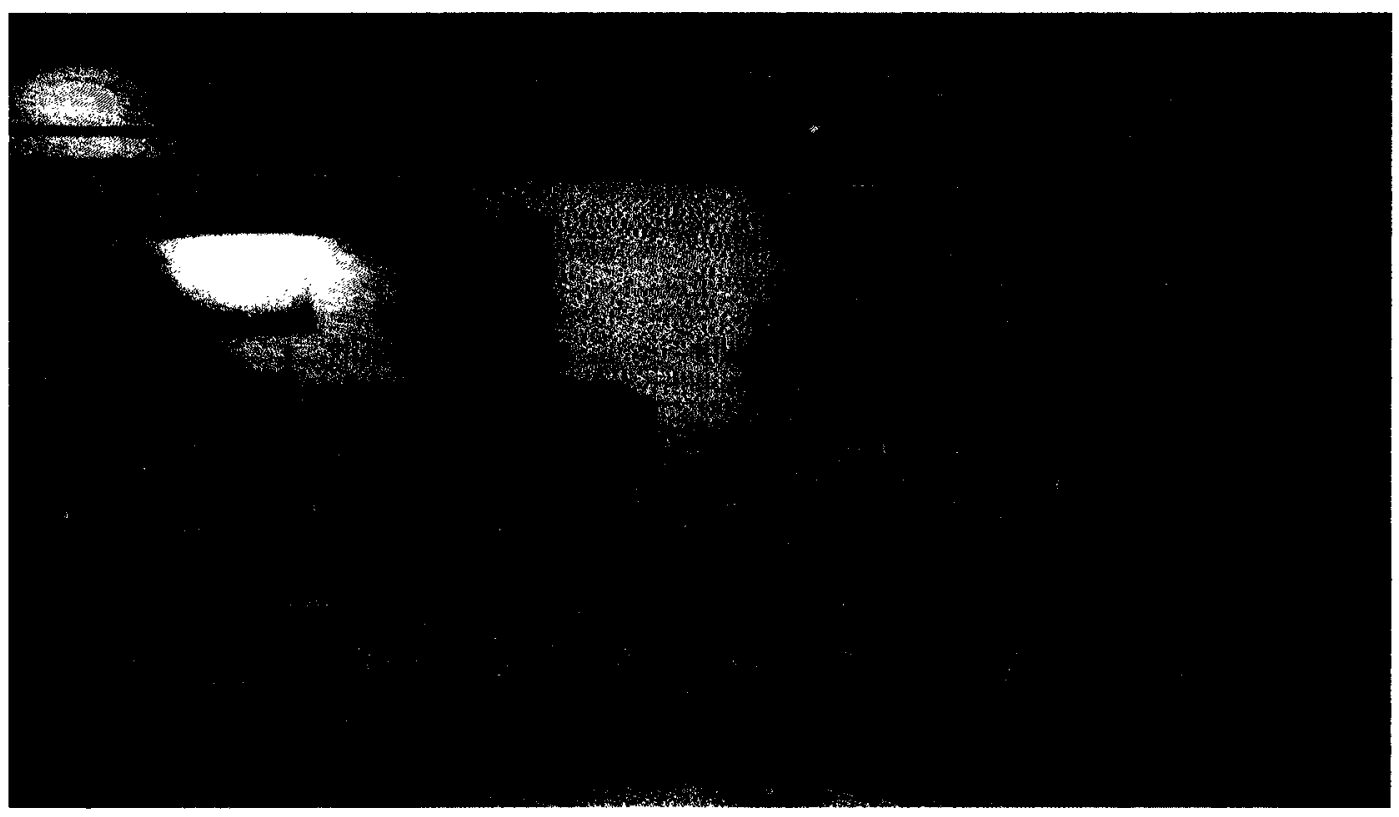

Figure 6.4: Phantom ${ }_{1}^{\mathbf{T M}}$ robotic manipulator is tracking desired trajectory with an additional $0.85 \mathrm{~kg}$. load attached to its end-effector.

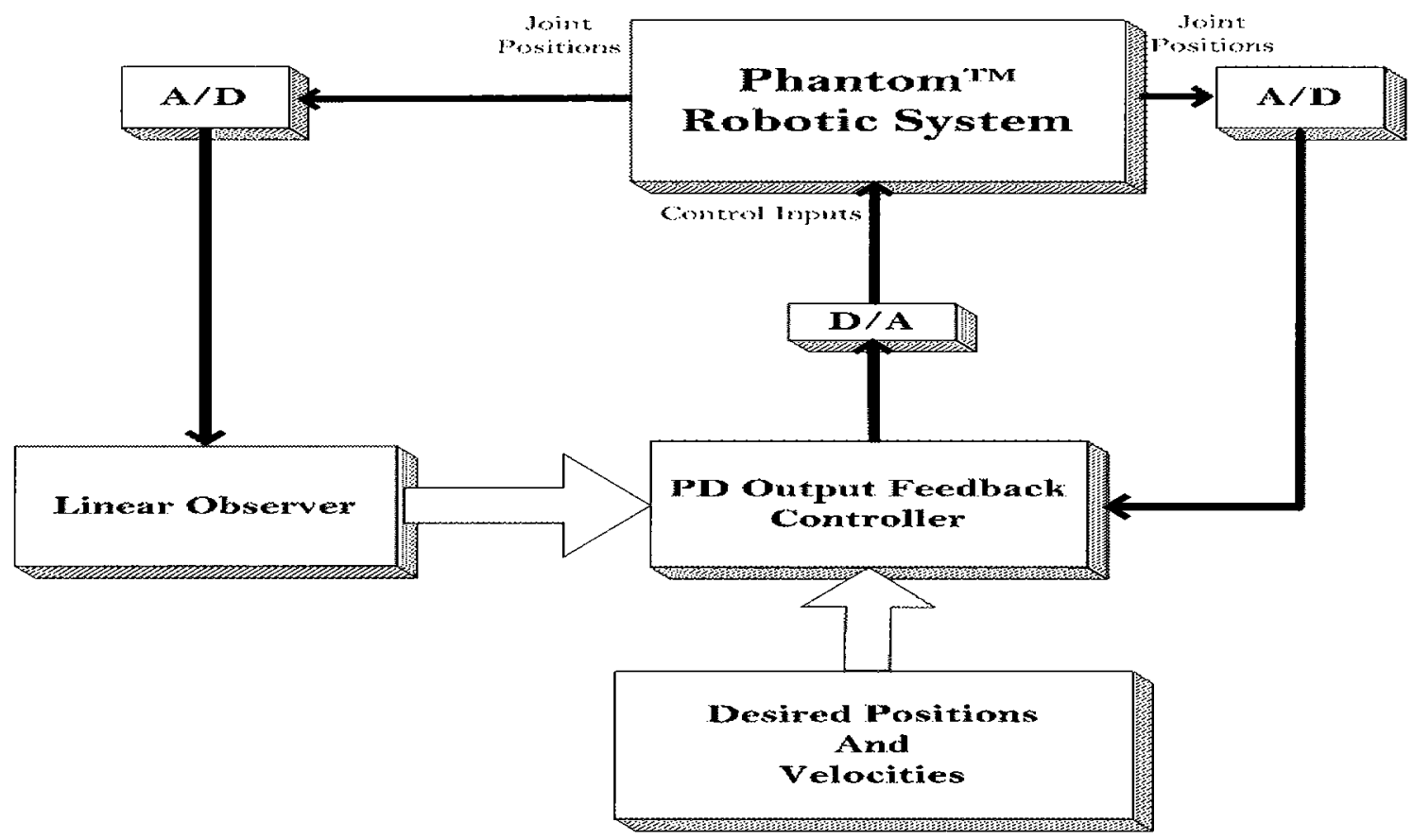

Figure 6.5: Implementation block diagram for real-time operation of PD-output feedback design with Computer/Simulink/Real-time Workshop/SensAble. 
The Phantom ${ }^{\mathrm{TM}}$ dynamical model (6.1) depends on number of physical parameters. In our experimental evaluation, the parameter sets are chosen as $\theta_{1}=$ $\left(I_{y 1}+I_{y 3}+I_{z 2}\right), \theta_{2}=\left(I_{y 2}-I_{z 2}+m_{2} r_{2}^{2}+m_{3} l_{1}^{2}\right), \theta_{3}=\left(I_{z 3}-I_{x 3}-I_{y 3}\right)$, $\theta_{4}=\left(I_{x 2}+m_{2} r_{2}^{2}+m_{3} l_{1}^{2}\right), \theta_{5}=\left(m_{3} r_{3}^{2}+I_{x 3}\right), \theta_{6}=m_{3} r_{3} l_{1}, \theta_{7}=g\left(m_{2} r_{2}+m_{3} l_{1}\right)$ and $\theta_{8}=g m_{3} r_{3}$ where $m_{j}$ with $j=1,2,3$ are the mass of the $j$-th link, $I_{x j}, I_{y j}$ and $I_{z j}$ are the moment of inertia of each link and $r_{j}$ are the center of masses for each link. It is worth noting to point out that one may choose different parameter sets resulting different nonlinear regressor model $Y(q, \dot{q}, \ddot{q})$. Using above defined parameter sets, we then construct nonlinear regressor model $Y(q, \dot{q}, \ddot{q}) \in \Re^{3 \times 8}$ as follows

$$
Y(q, \dot{q}, \ddot{q})=\left[\begin{array}{llllllll}
Y_{11} & Y_{12} & Y_{13} & Y_{14} & Y_{15} & Y_{16} & Y_{17} & Y_{18} \\
Y_{21} & Y_{22} & Y_{23} & Y_{24} & Y_{25} & Y_{26} & Y_{27} & Y_{28} \\
Y_{31} & Y_{32} & Y_{33} & Y_{34} & Y_{35} & Y_{36} & Y_{37} & Y_{38}
\end{array}\right]
$$

with $Y_{11}=\ddot{q}_{1}, Y_{21}=0, Y_{31}=0, Y_{12}=\left(\cos ^{2} q_{2} \ddot{q}_{1}-2 \cos q_{2} \sin q_{2} \dot{q}_{1} \dot{q}_{2}\right), Y_{22}=$ $\cos q_{2} \sin q_{2} \ddot{q}_{1}^{2}, Y_{32}=0, Y_{13}=\left(\sin ^{2} q_{3} \ddot{q}_{1}+2 \sin q_{3} \cos q_{3} \dot{q}_{1} \dot{q}_{3}\right), Y_{23}=0, Y_{33}=$ $-\sin q_{3} \cos q_{3} \dot{q}_{1}^{2}, Y_{14}=0, Y_{24}=\ddot{q}_{2}, Y_{34}=0, Y_{15}=\left(\sin ^{2} q_{3} \ddot{q}_{1}+2 \sin q_{3} \cos q_{3} \dot{q}_{1} \dot{q}_{3}\right)$, $Y_{25}=\left(\ddot{q}_{2}+\ddot{q}_{3}\right), Y_{16}=\left(2 \cos q_{2} \sin q_{3} \ddot{q}_{1}-2 \sin q_{2} \sin q_{3} \dot{q}_{1} \dot{q}_{2}+2 \cos q_{2} \cos q_{3} \dot{q}_{1} \dot{q}_{3}\right), Y_{26}=$ $-2 \sin \left(q_{2}-q_{3}\right) \ddot{q}_{2}-\sin \left(q_{2}-q_{3}\right) \ddot{q}_{3}+\sin q_{2} \sin q_{3} \dot{q}_{1}^{2}-\cos \left(q_{2}-q_{3}\right)\left(\dot{q}_{2}-\dot{q}_{3}\right)^{2}, Y_{35}=$ $\left(\ddot{q}_{2}+\ddot{q}_{3}-\sin q_{3} \cos q_{3} \dot{q}_{1}^{2}\right), Y_{36}=-2 \sin \left(q_{2}-q_{3}\right) \ddot{q}_{2}-\cos q_{2} \cos q_{3} \dot{q}_{1}^{2}, Y_{17}=0, Y_{27}=$ $\cos q_{2}, Y_{37}=0, Y_{18}=0, Y_{28}=0$ and $Y_{38}=\sin q_{3}$.

The model parameters for two Phantom ${ }^{\mathrm{TM}}$ devices are identified via using least square method [49]. For brevity, we removed details parameter identification procedure as they can be found in existing literatures $[36,77]$. Using the least square method, one obtains the following parameter values for Phantom ${ }_{1}^{\mathbf{T M}}$ robotic system as; $\theta_{1}=50, \theta_{2}=60, \theta_{3}=48, \theta_{4}=80, \theta_{5}=9, \theta_{6}=8, \theta_{7}=235$ and $\theta_{8}=270$.

Similarly, the least square technique gives the following parameter values for Phantom ${ }_{2}^{\mathbf{T M}}$ robotic system as $\theta_{1}=32, \theta_{2}=34, \theta_{3}=20, \theta_{4}=74, \theta_{5}=1, \theta_{6}=2$, $\theta_{7}=-926$ and $\theta_{8}=-685$. The joint position is measured in radians and the 


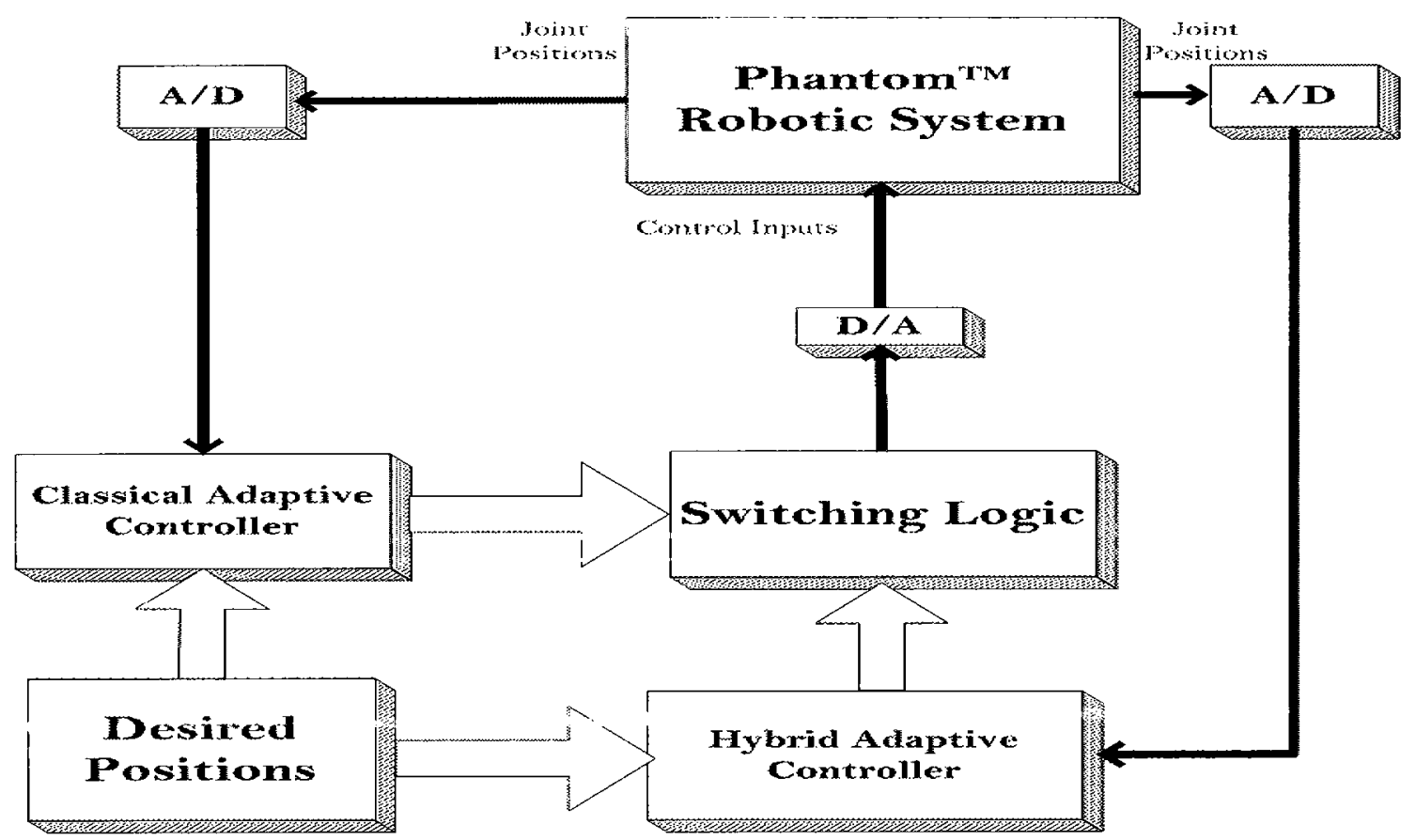

Figure 6.6: Implementation block diagram for real-time operation of hybrid adaptive design with Computer/Simulink/Real-time Workshop/SensAble.
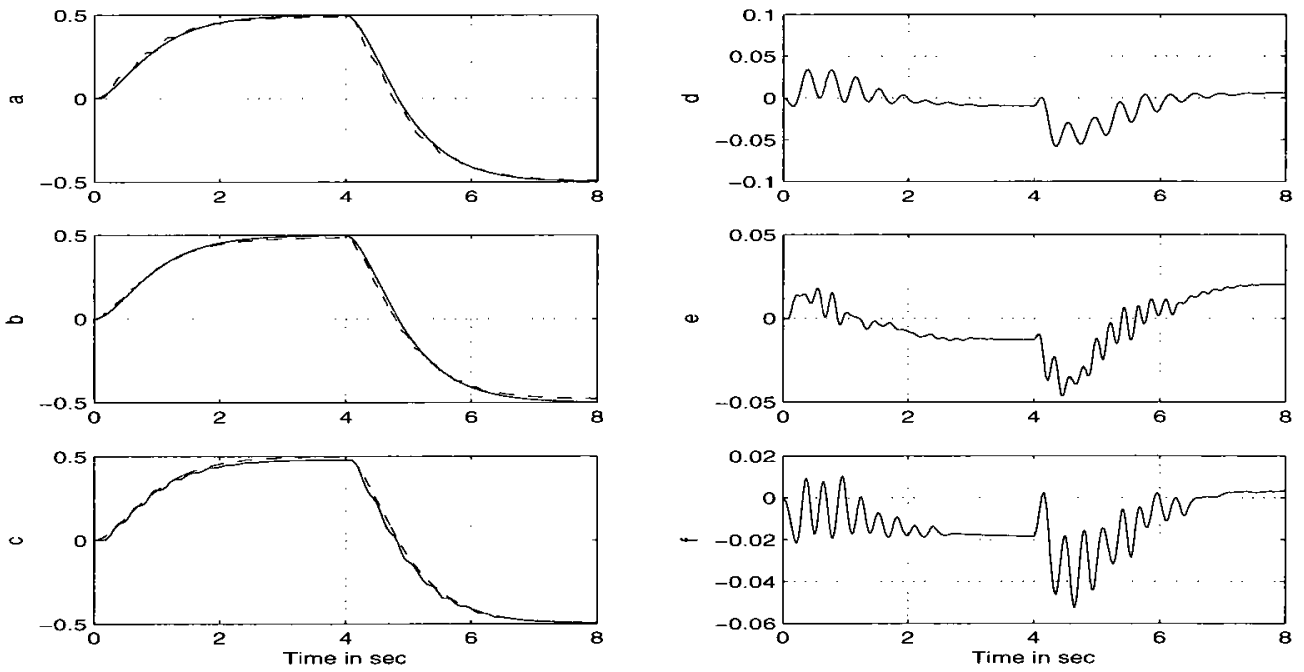

Figure 6.7: Left column: a, b \& c) The desired (solid-line) and output tracking (dash-line) for joints 1, 2 and 3, Right column: d, e \& f) The tracking errors for joints 1, 2 and 3 under PD state feedback design of Theorem 2.2. 

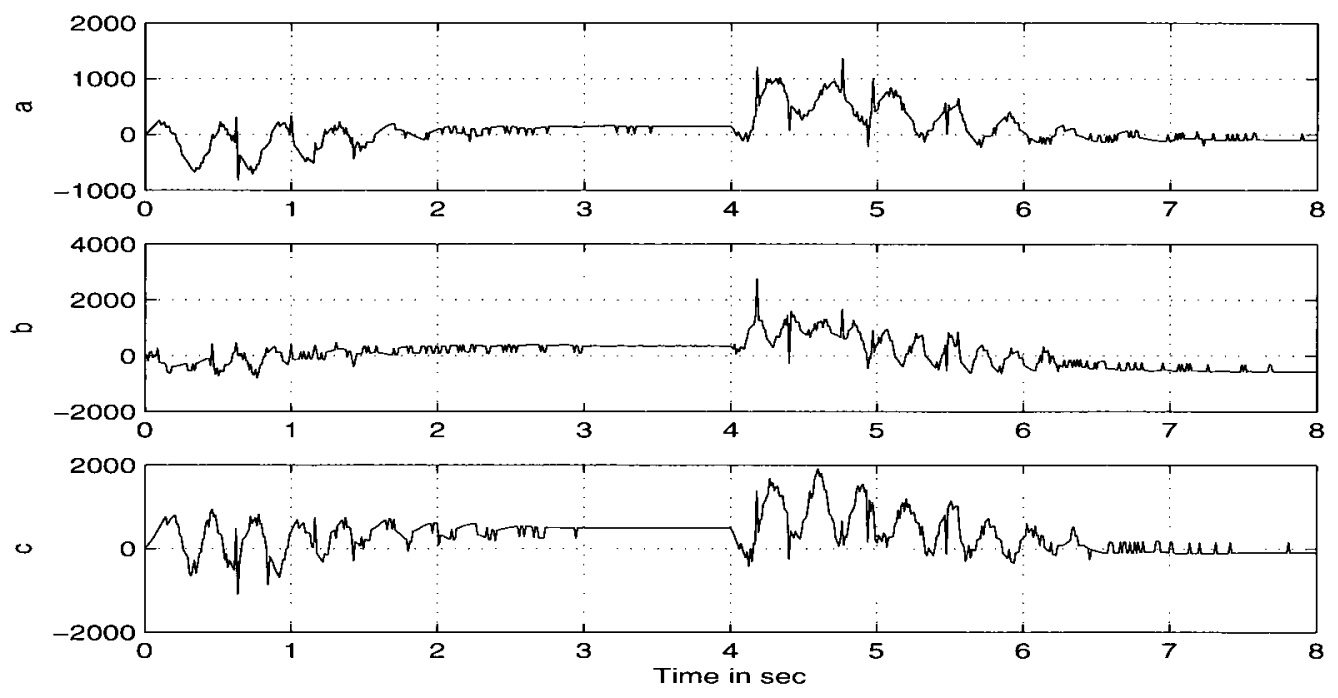

Figure 6.8: $\mathrm{a}, \mathrm{b} \& \mathrm{c}$ ) The control inputs for joints 1, 2 and 3 under PD state feedback design.

control input torque is given in numerical values between -32768 to $32767 .^{12}$

\subsection{Trajectory Generation}

To generate the desired reference trajectory for the given robot to follow, a square wave with a period of 8 seconds and an amplitude of \pm 0.5 radians is pre filtered with a critically damped 2nd-order linear filter using a bandwidth of $\omega_{n}=2.0$ $\mathrm{rad} / \mathrm{sec}$. The step reference inputs are not preferred in real-time applications as such initial jump may reduce the lifetime for the bearing. However, if one uses the step reference trajectory, then very small step sizes are required. Specifically, our main target is to use a desired trajectory that usually uses in industrial robotic systems $[4,5,6]$.

\footnotetext{
${ }^{1}$ The unit for all joint position and tracking error Figures reported in this chapter are given in radians [36].

${ }^{2}$ The unit for all control input torque Figures presented in this chapter are given in numerical values [36].
} 

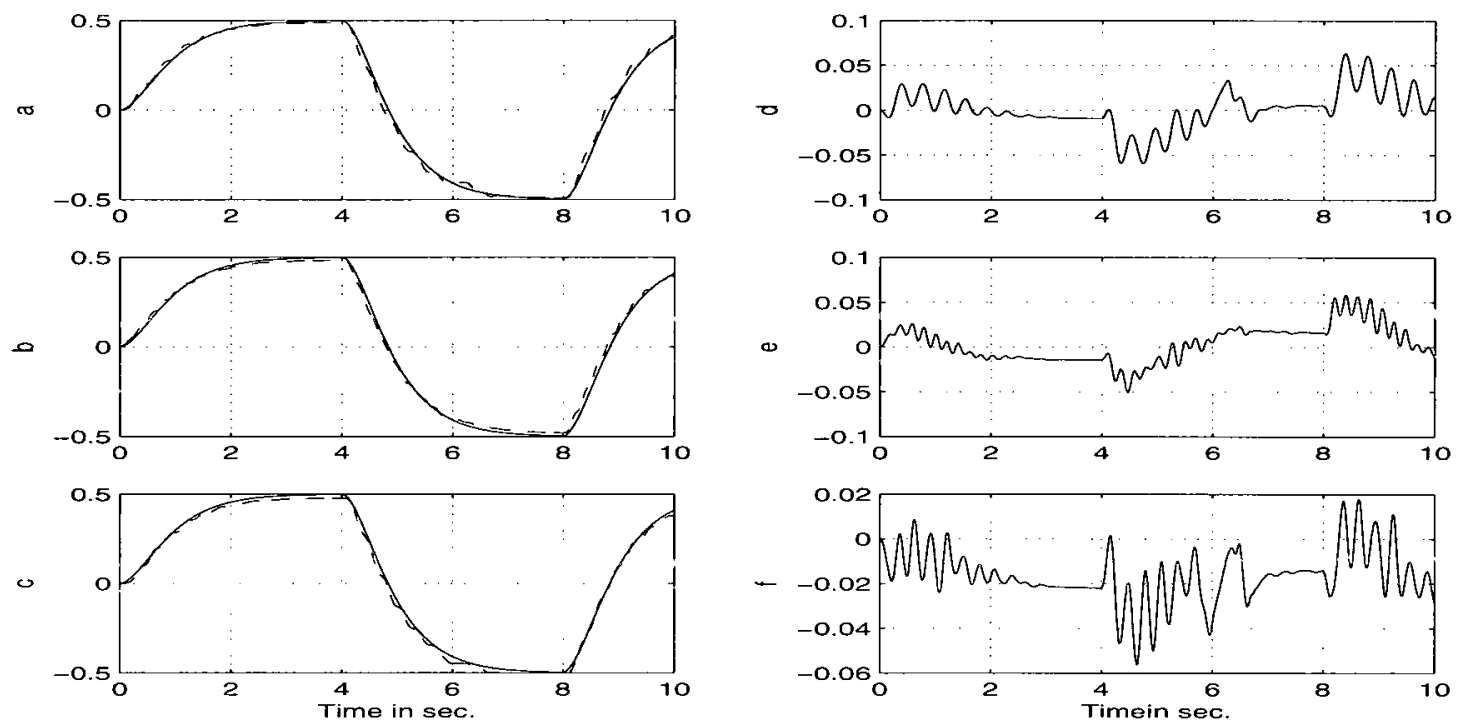

Figure 6.9: Left column: a, b \& c) The desired (solid-line) and output tracking (dash-line) for joints 1, 2 and 3, Right column: d, e \& f) The tracking errors for joints 1, 2 and 3 under PD state feedback design of Theorem 2.2 under dynamic model changes.

\subsection{Implementation Results with PD-based Lin- ear Control System Design: State and Out- put Feedback Case}

In this section, we implement model-free linear control of Theorem 2.2 and Theorem $2.3[85,94]$ on Phantom ${ }^{\text {TM }}$ systems. We first use state feedback (position-velocity) based PD type linear control design of Theorem 2.1 to track a given desired tracking trajectory. As the experimental system only provides joint position measurement, the velocity signals are estimated via differentiating the position signals.

To obtain desired control signals to follow the desired path, let us take the coefficients of the PD controller as: $\lambda=15 I_{3 \times 3}$ and $K=\operatorname{diag}(800,1200,1200)$. The values of $\mathrm{PD}$ controller are chosen to ensure acceptable transient tracking performance. With these design parameters, we then apply state feedback design 

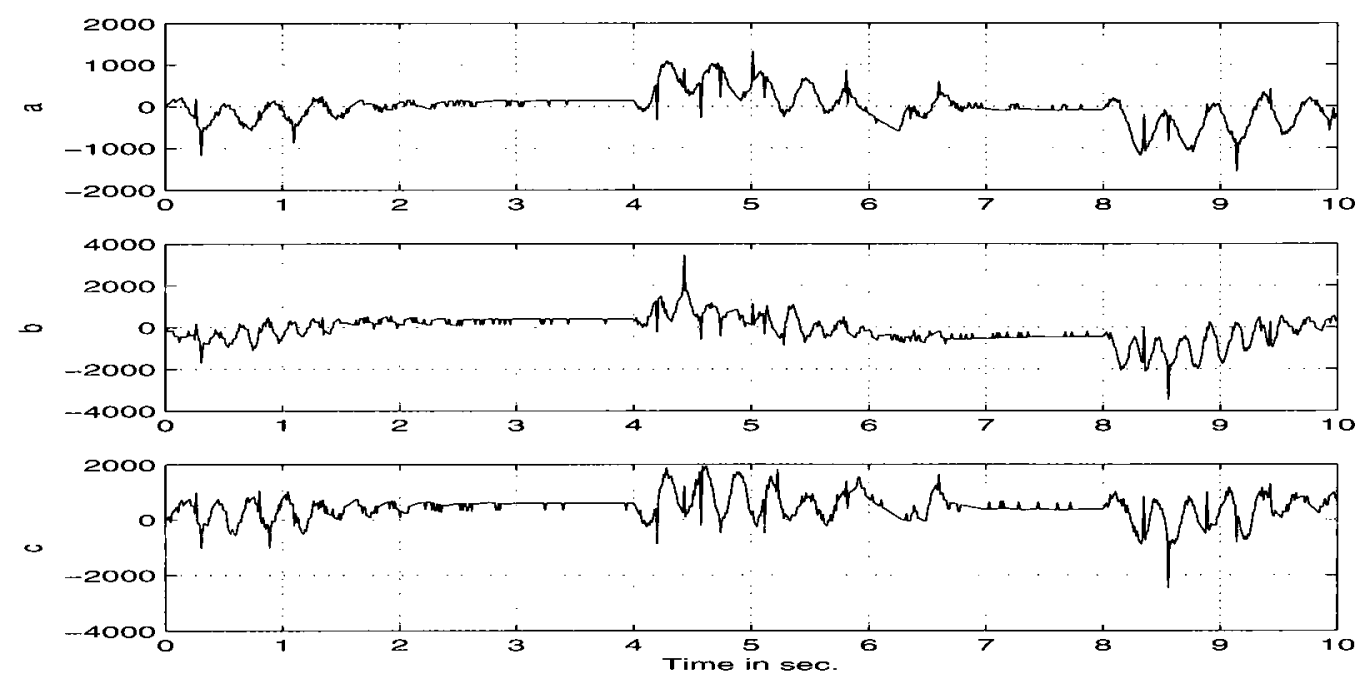

Figure 6.10: a, b \& c) The control inputs for joints 1, 2 and 3 with Theorem 2.2 under model dynamic changes.

of Theorem $2.2[85,94]$ on the given Phantom 1 TM robot system. The tested results are given in Fig 6.7 and Fig. 6.8.

To examine the robustness property of Theorem 2.2, we aim to increase the modeling error uncertainty when the manipulator is tracking the given reference trajectory. For this purpose, we first operate the plant under the same parameters that used in previous experiment. Then, we add $0.86 \mathrm{~kg}$. mass externally to the joint 2 at about 6 sec. in order to change operating dynamics. The experimental results are given in Fig. 6.9 and Fig. 6.10. One can see from these Figs. that the design is insensitive to the dynamical model parameter changes as the tracking errors slightly increase at the time of the dynamics changes. This is mainly because control algorithm is independent of the model dynamics. We can also see from Fig. 6.8 and Fig. 6.10 that the state feedback design generates relatively large control efforts due to the presence of high-frequency control chattering phenomenon as the design uses the derivative action of the position signals.

We now apply PD output feedback design of Theorem $2.3[85,94]$ on the given 
robotic system (6.1). The control objective in Theorem 2.3 is to track the same reference trajectory as used for Theorem 2.2 provided that the velocity signals are unknown. The unknown velocity signals are estimated by using linear observer. For comparison purpose, we consider the same position controller design parameters that applied in state feedback based design of Theorem 2.2. Then define observer design constants as $H_{1}=15 I_{3 \times 3}, H_{2}=5 I_{3 \times 3}$ and small value of $\epsilon$. The values of $H_{1}, H_{2}$, and $\epsilon$ are chosen such that the observer error systems are faster than that of the controller closed loop systems. As we noticed from our theoretical development that the observer speed that is the value of $\epsilon$ plays a significant rules to meet the desired control objectives. Therefore, our interest in this experiment is to analyze the influence of the observer speed on the tracking performance.

In our experimental evaluation, we consider relatively smaller (higher speed) and higher values of $\epsilon$ (smaller speed) as $\epsilon=0.1$ and $\epsilon=.01$ to examine the effect of the observer speed on the tracking performance. With these design constants, we then apply Theorem 2.3 on the Phantom ${ }_{1}^{\text {TM }}$ robotic systems. The experimental results are given in Figs. 11 to 6.14. Fig. 11 and Fig. 6.12 depict the control performance with smaller observer speed $\epsilon=0.1$ while the Fig. 6.13 and Fig. 6.14 represent the tracking convergence under higher observer speed $\epsilon=.01$.

From these results, we can notice that the model-free PD-output feedback design can achieve the desired tracking objectives as the tracking error converges closed to zero. By comparing the control inputs under Theorem 2.2 and Theorem 2.3 , we can also notice that the control efforts under state feedback based design are larger than the control input required with the output feedback design.

Let us investigate the robustness property of Theorem 2.3 with respect to dynamical model parameter changes as experimented for Theorem 2.2. In this test, we add approximately $0.56 \mathrm{~kg}$. mass to the joint 2 in order to change operating dynamics at 6 sec. The obtained experimental results are given in Fig. 6.15 and Fig 6.16. From these Figs., one can observe that the control system is insensitive to the dynamical model parameter changes as the tracking errors slightly increase 

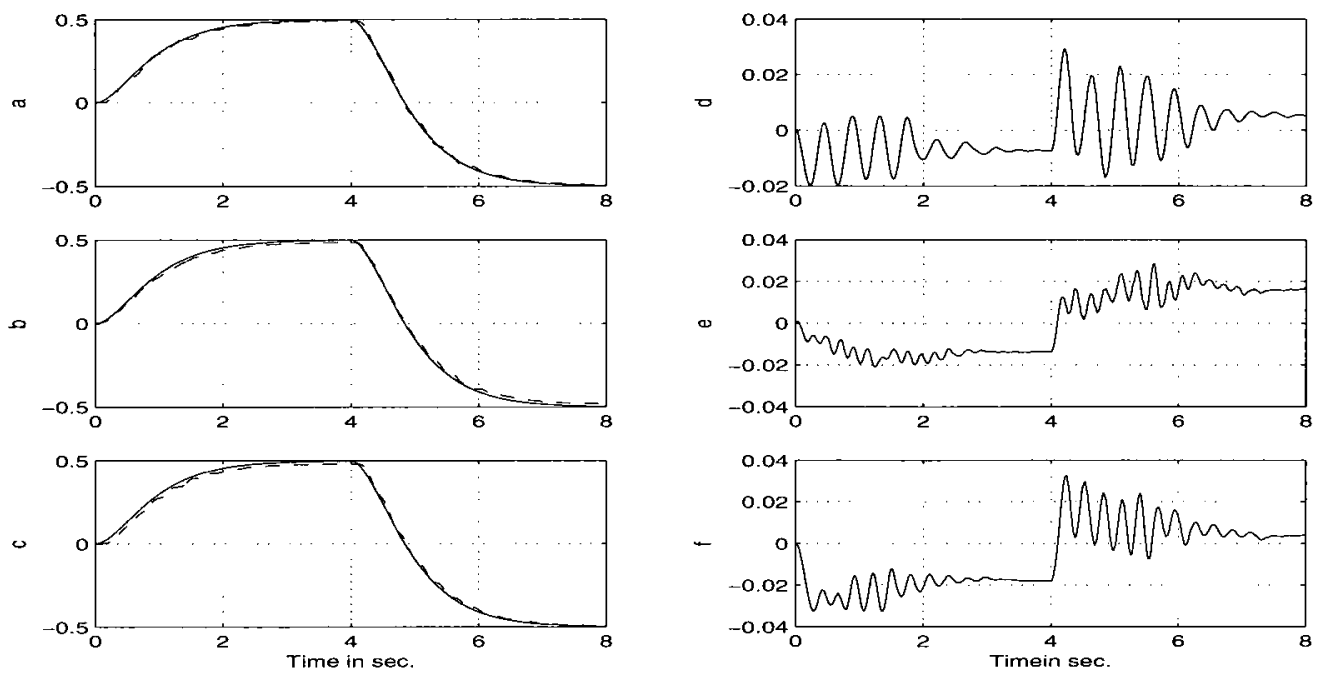

Figure 6.11: Left column: a, b \& c) The desired (solid-line) and output tracking (dash-line) for joints 1, 2 and 3, Right column: d, e \& f) The tracking errors for joints 1, 2 and 3 with PD output feedback design of Theorem 2.3 with $\epsilon=0.1$.

at the time of the model dynamic changes. This is mainly because PD control law is independent of system dynamics.

\subsection{Implementation Results with CE-based Non- linear Adaptive Control Design: State and Output Feedback Case}

We now evaluate CE principle-based nonlinear adaptive feedback control law [82] on 3-DOF Phantom ${ }^{\text {TM }}$ systems. The purpose of this evaluation is to compare the tracking performance between the model-free PD (non adaptive) linear control design $[85,94]$ and the model-based nonlinear adaptive design [82]. We first consider 

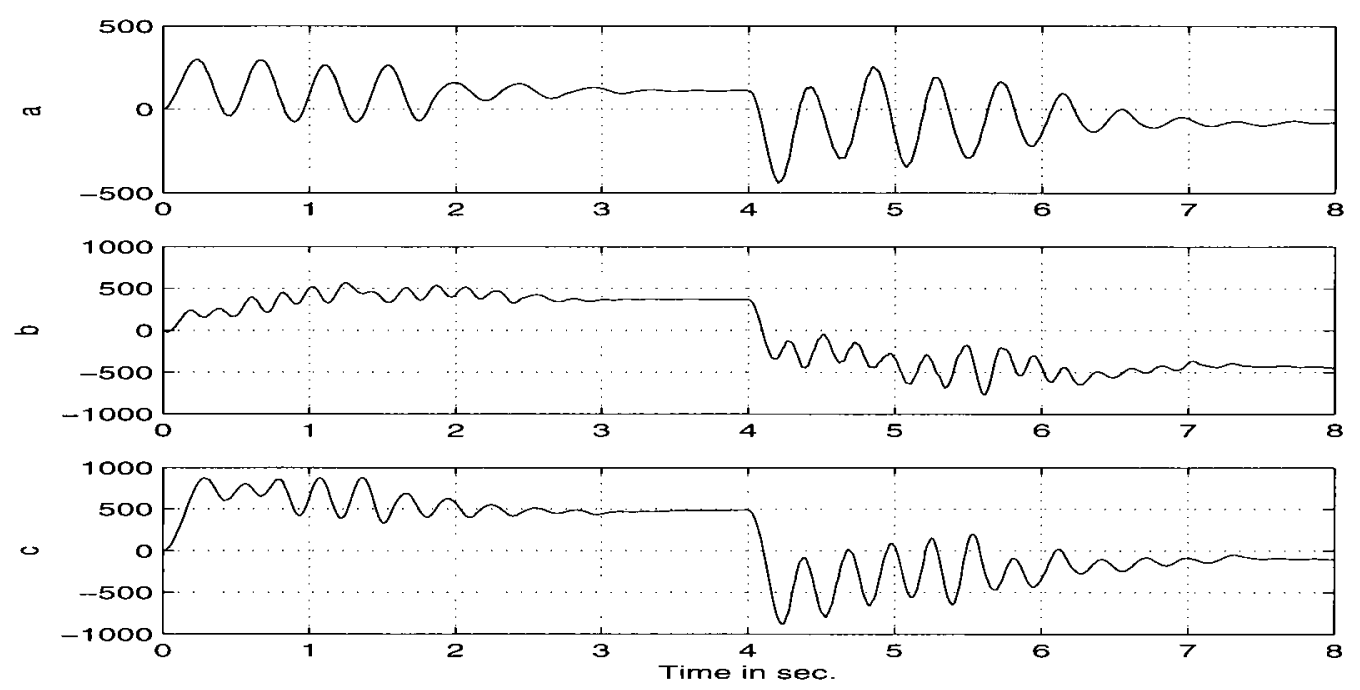

Figure 6.12: a, b \& c) The control inputs for joints 1, 2 and 3 under Theorem 2.3 using with $\epsilon=0.1$.

the following CE principle-based adaptive state feedback control algorithm [82] ${ }^{3}$

$$
\begin{aligned}
\tau\left(e, Q_{d}, \hat{\theta}\right) & =Y\left(e, \dot{q}_{d}, \ddot{q}_{d}\right) \hat{\theta}-K_{P} e_{1}-K_{D} e_{2} \\
\dot{\hat{\theta}} & =-\Gamma Y^{T}\left(e, \dot{q}_{d}, \ddot{q}_{d}\right) S
\end{aligned}
$$

where $Y\left(e, \dot{q}_{d}, \ddot{q}_{d}\right) \hat{\theta}=\hat{M}(q) \ddot{q}_{d}+\hat{C}\left(q, \dot{q}_{r}\right) \dot{q}_{d}+\hat{G}(q), K_{P} \in \Re^{n \times n}, K_{D} \in \Re^{n \times n}, S=$ $e_{2}+\lambda e_{1}, \dot{q}_{r}=\left(\dot{q}_{2}-\lambda e_{1}\right), \lambda=\frac{\lambda_{0}}{1+\left\|e_{1}\right\|}, \lambda_{0}>0$ and $\hat{M}, \hat{C}($.$) and \hat{G}($.$) define the$ estimates of the $M(),. C($.$) and G($.$) , respectively. To remove the discontinuous$ property from learning estimates, $\hat{\theta}$ can be adjusted with the smooth parameter projection scheme (5.5) as

$$
\dot{\hat{\theta}}_{i}=[\operatorname{Proj}(\hat{\theta}, \Phi)]_{i}=\left\{\begin{array}{cc}
\gamma_{i i} \Phi_{i} & \text { if } a_{i} \leq \hat{\theta}_{i} \leq b_{i} \text { or } \\
& \text { if } \hat{\theta}_{i}>b_{i} \text { and } \Phi_{i} \leq 0 \text { or } \\
& \text { if } \hat{\theta}_{i}<a_{i} \text { and } \Phi_{i} \geq 0 \\
\gamma_{i i} \bar{\Phi}_{i} & \text { if } \hat{\theta}_{i}>b_{i} \text { and } \Phi_{i}>0 \\
\gamma_{i i} \breve{\Phi}_{i} & \text { if } \hat{\theta}_{i}<a_{i} \text { and } \Phi_{i}<0
\end{array}\right.
$$

\footnotetext{
${ }^{3}$ For simplicity, we represent $Y\left(e, \dot{q}_{r}, \dot{q}_{d}, \ddot{q}_{d}\right)$ by $Y\left(e, \dot{q}_{d}, \ddot{q}_{d}\right)$
} 

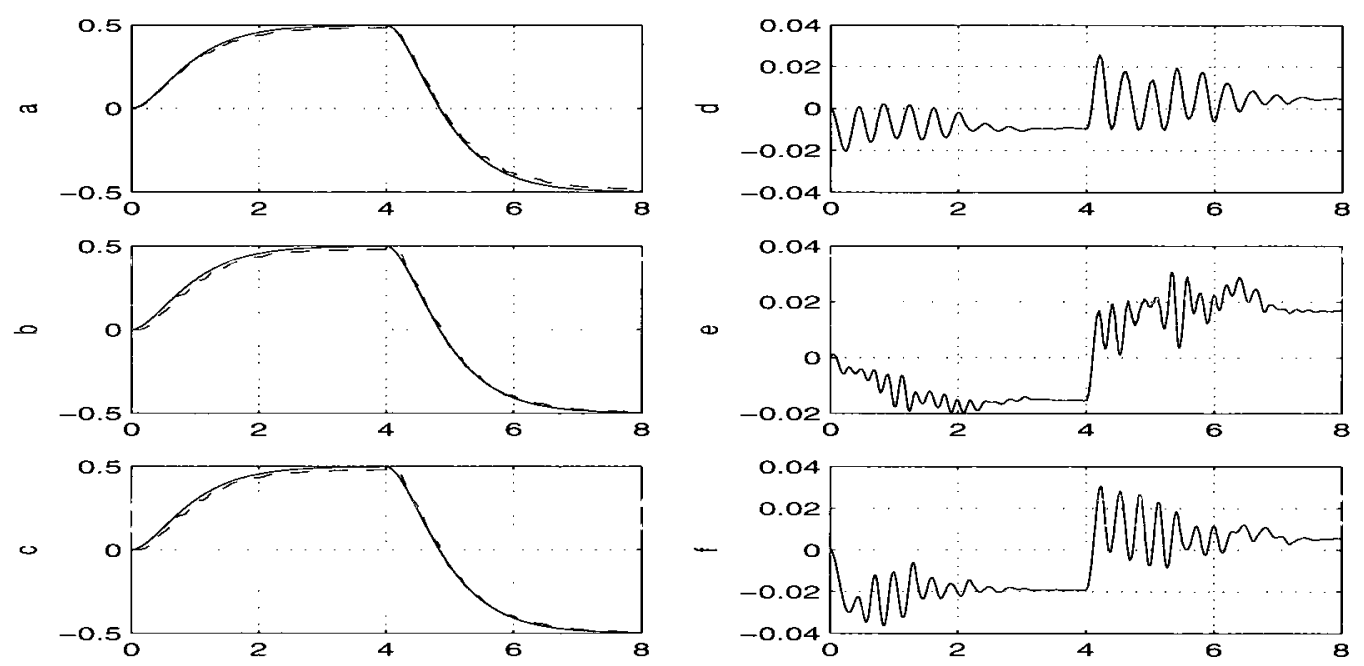

Figure 6.13: Left column: a, b \& c) The desired (solid-line) and output tracking (dash-line) for joints 1, 2 and 3, Right column: d, e \& f) The tracking errors for joints 1, 2 and 3 with PD output feedback design of Theorem 2.3 using with $\epsilon=0.01$.
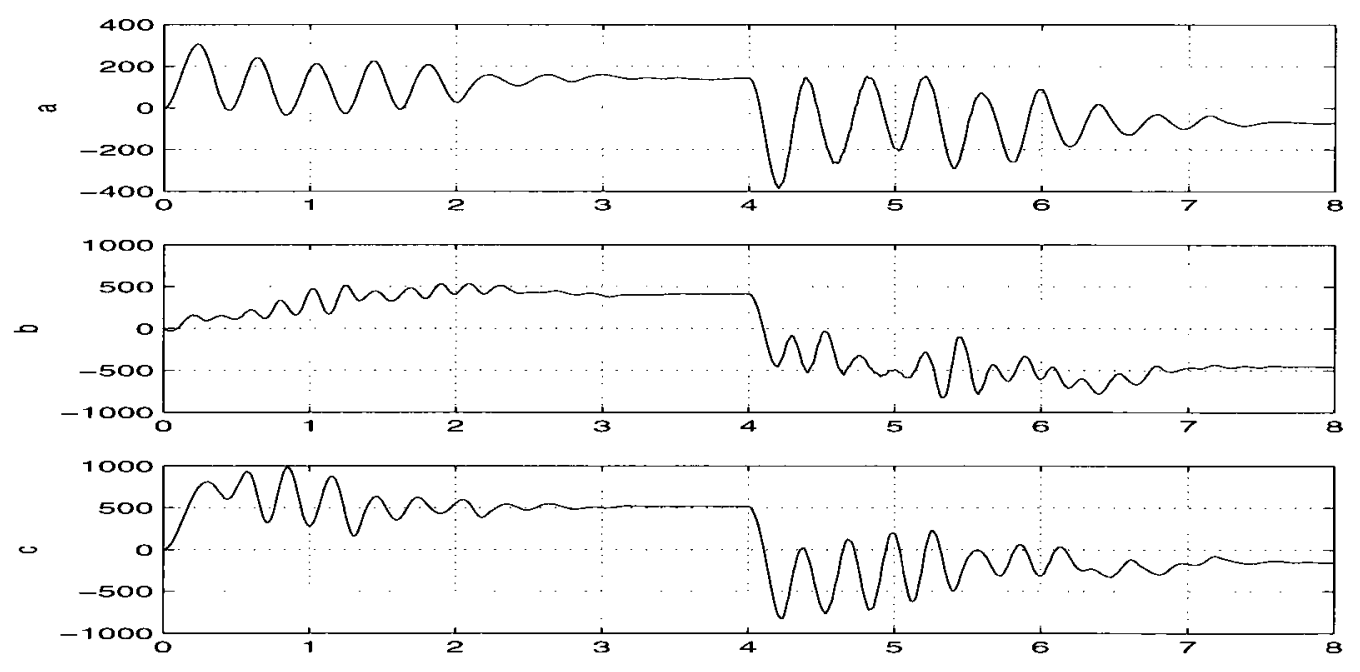

Figure 6.14: a, b \& c) The control inputs for joints 1, 2 and 3 using with Theorem 2.3 for $\epsilon=0.01$. 
$\bar{\Phi}_{i}=\left[1+\frac{b_{i}-\hat{\theta}_{i}}{\delta}\right] \Phi_{i}, \breve{\Phi}_{i}=\left[1+\frac{\hat{\theta}_{i}-a_{i}}{\delta}\right] \Phi_{i}, \Phi_{i}$ is the $i$-th element of the column vector, $-Y^{T}\left(e, \dot{q}_{d}, \ddot{q}_{d}\right) S$ and $\delta>0$ is chosen such that $\Omega \subset \Omega_{\delta}$ with $\Omega_{\delta}=\left\{\theta \mid a_{i}-\delta \leq \theta_{i} \leq\right.$ $\left.\left.b_{i}+\delta\right\}, 1 \leq i \leq p\right\}$ and $\left.\theta \in \Omega=\left\{\theta \mid a_{i} \leq \theta_{i} \leq b_{i}\right\}, 1 \leq i \leq p\right\}$. The bounds on $\theta$ are obtained by using least square identification technique.

To construct the regressor model $Y\left(e, \dot{q}_{d}, \ddot{q}_{d}\right)$, we use the model dynamics represented by equation (6.1) where we replace $\dot{q}$ in coriolis and centrifugal matrix $c(q, \dot{q})$ by $\dot{q}_{r}=(\dot{q}-\lambda e)$. The unknown velocity signals in (6.3), (6.4) are obtained via differentiating the position signals obtained from encoders that attached with the joint of the system. The value of PD controller design parameters are chosen as $\lambda_{0}=2, K_{P}=\operatorname{diag}(7500,1200,1200)$ and $K_{D}=\operatorname{diag}(500,800,800)$. The gains $\Gamma$ are chosen to achieve faster parameter learning via using trial and error search technique as $\Gamma=\operatorname{diag}(350,350,350)$. Using with these parameters sets, we obtained experimental results depicted in Fig. 6.17 and Fig. 6.18. We can notice from these results that transient tracking error under adaptive state feedback design is relatively larger than the tracking errors obtained under PD based state feedback design.

To reduce the tracking errors further, let us increase the learning gains from $\Gamma=\operatorname{diag}(350,350,350)$ to $\Gamma=\operatorname{diag}(550,550,550)$ and keep other controller design parameters similar to our last evaluation. The tested results are shown in Fig. 17. The excessive control chattering phenomenon with large control efforts can be clearly observed from Fig. 6.19.

One may further increase the learning gains to improve the tracking performance. But, the problem is that the control system may goes unbounded as a result of large control efforts. To depict this situation, we present the tested results in Fig. 6.20 and Fig. 6.21, where the learning gains are increased from $\Gamma=\operatorname{diag}(550,550,550)$ to $\Gamma=\operatorname{diag}(700,700,700)$. Note that all other control design parameters for this test are kept similar to our last experiment. However, we had to stop the experiment at about $1.56 \mathrm{sec}$. due to excessive control chattering phenomenon causing very large control action. 

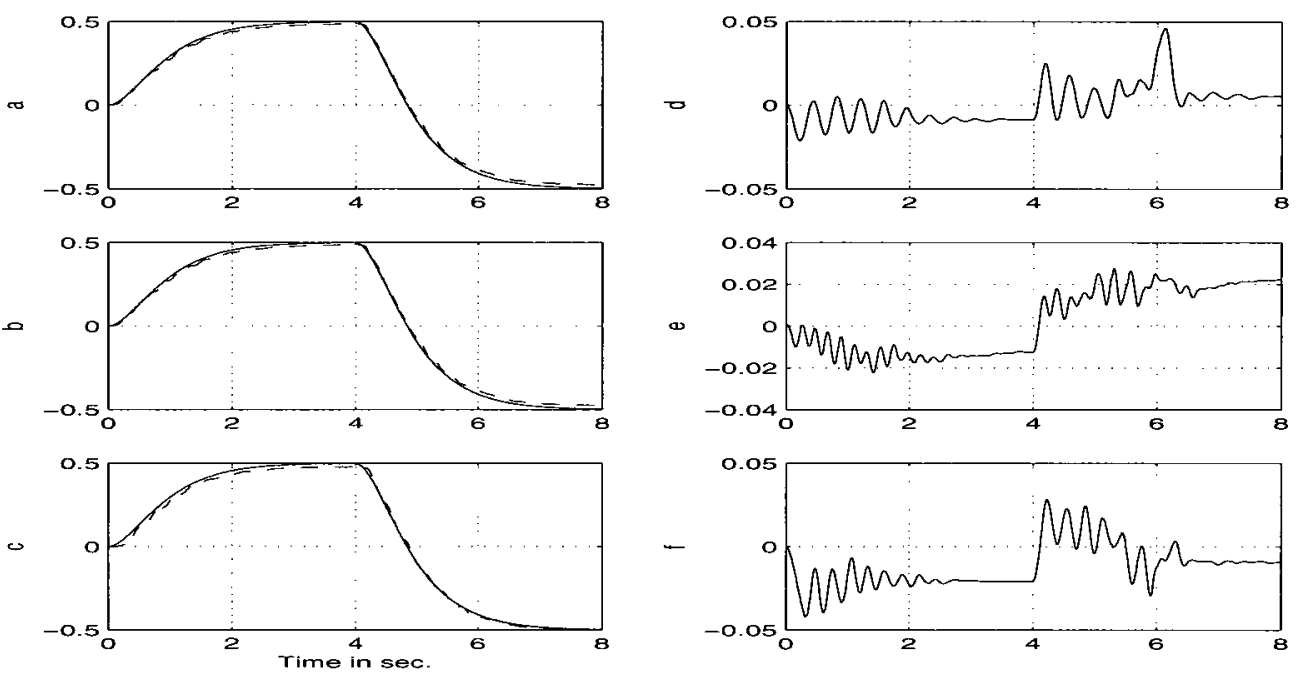

Figure 6.15: Left column: a, b \& c) The desired (solid-line) and output tracking (dash-line) for joints 1, 2 and 3, Right column: d, e \& f) The tracking errors for joints 1,2 and 3 with Theorem 2.3 under parameter changes with $\epsilon=0.1$.

Let us now replace the velocity signals in (6.3), (6.4) to develop nonlinear adaptive output feedback control form as $[82]^{4}$

$$
\begin{aligned}
\tau\left(\hat{e}, Q_{d}, \hat{\theta}\right) & =Y\left(\hat{e}, \dot{q}_{d}, \ddot{q}_{d}\right) \hat{\theta}-K_{P} e_{1}-K_{D} \hat{e}_{2} \\
\dot{\hat{\theta}} & =-\Gamma Y^{T}\left(\hat{e}, \dot{q}_{d}, \ddot{q}_{d}\right) \hat{S}
\end{aligned}
$$

where $Y\left(\hat{e}, \dot{q}_{d}, \ddot{q}_{d}\right) \hat{\theta}=\hat{M}(q) \ddot{q}_{d}+\hat{C}\left(q, \dot{\hat{q}}_{r}\right) \dot{q}_{d}+\hat{G}(q), K_{P} \in \Re^{n \times n}, K_{D} \in \Re^{n \times n}$, $\hat{S}=\hat{e}_{2}+\lambda e_{1}, \dot{\hat{q}}_{r}=\left(\dot{\hat{q}}_{2}-\lambda e_{1}\right), \lambda=\frac{\lambda_{0}}{1+\left\|e_{1}\right\|}, \lambda_{0}>0$ and $\hat{M}, \hat{C}($.$) and \hat{G}($.$) define the$ estimates of the $M(),. C($.$) and G($.$) , respectively. The unknown velocity signals$ in $(6.3),(6.4)$ is now replaced by the output of the linear observer as

$$
\dot{\hat{e}}_{1}=\hat{e}_{2}+\frac{H_{1}}{\epsilon} \tilde{e}_{1}, \dot{\hat{e}}_{2}=\frac{H_{2}}{\epsilon^{2}} \tilde{e}_{1}
$$

We also replace $\dot{\hat{q}}$ in coriolis and centrifugal matrix $c(q, \dot{\hat{q}})$ by $\dot{\hat{q}}_{r}=(\dot{\hat{q}}-\lambda e)$ to formulate the regressor model $Y\left(\hat{e}, \dot{q}_{d}, \ddot{q}_{d}\right)$. Then, we use the same controller design parameters that used in adaptive state feedback based design as $\lambda_{0}=2, K_{P}=$

\footnotetext{
${ }^{4}$ For simplicity, we represent $Y\left(\hat{e}, \dot{\hat{q}}_{T}, \dot{q}_{d}, \ddot{q}_{d}\right)$ by $Y\left(\hat{e}, \dot{q}_{d}, \ddot{q}_{d}\right)$
} 

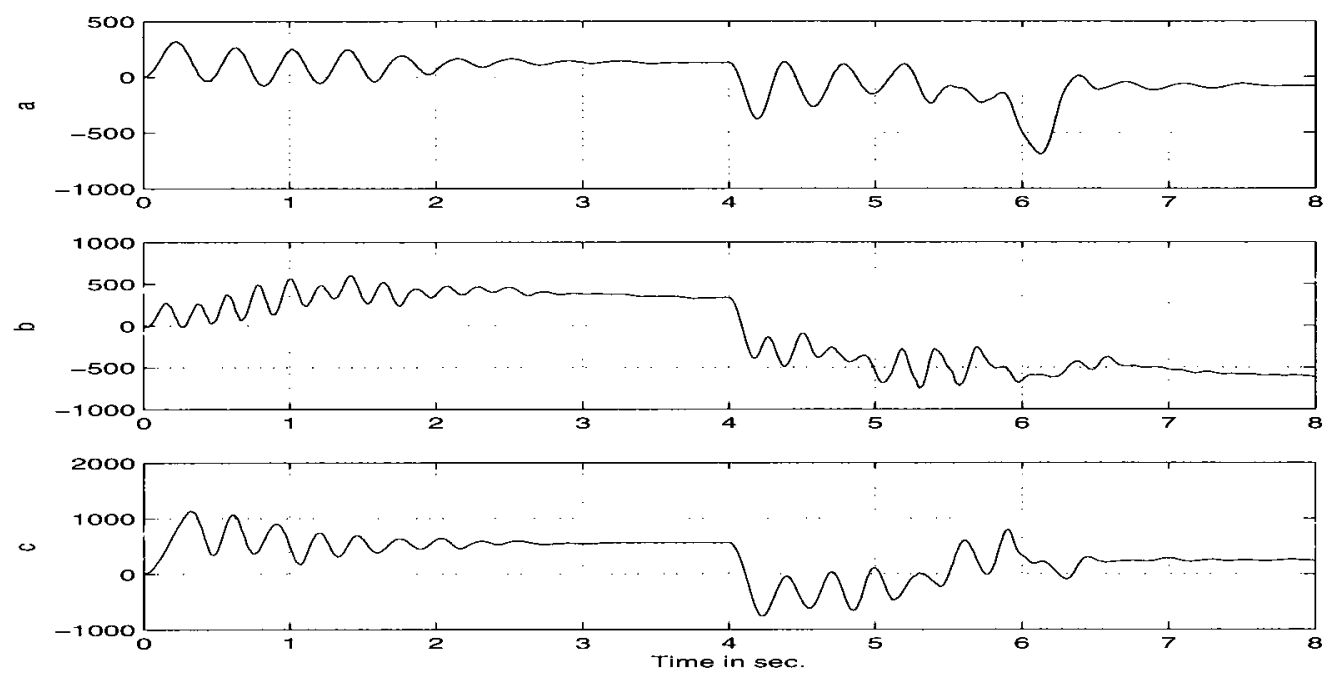

Figure 6.16: a, b \& c) The control inputs for joints 1, 2 and with Theorem 2.3 under parameter changes with $\epsilon=0.1$.

$\operatorname{diag}(7500,12000,12000), K_{D}=\operatorname{diag}(500,800,800)$ and $\Gamma=\operatorname{diag}(350,350,350)$. The observer design parameters are chosen as $H_{1}=15 I_{3 \times 3}$ and $H_{2}=5 I_{3 \times 3}$ and small value of $\epsilon$ as $\epsilon=0.03$. The tested results with nonlinear adaptive output feedback design (6.5) are given in Fig. 6.21 and Fig. 6.22.

To reduce the tracking errors, one requires to increase observer-controller gains. However, we have noticed in our earlier experimental evaluation for state feedback based design that the designer cannot increase the controller gains due to the presence of the control chattering activity. The requirement of high speed observer makes the CE-based adaptive output feedback design even more complex in real-time application as high observer speed generates high-frequency chattering and infinitely fast switching control phenomenon.

To illustrate this argument, let us increase the observer speed via decreasing the value of $\epsilon$ from $\epsilon=0.03$ to $\epsilon=0.02$, but we keep all other design parameters same as our last experimental test. The tested results are shown in Fig. 6.24 and Fig. 6.25. From these Figs., we can notice that the control efforts under nonlinear 

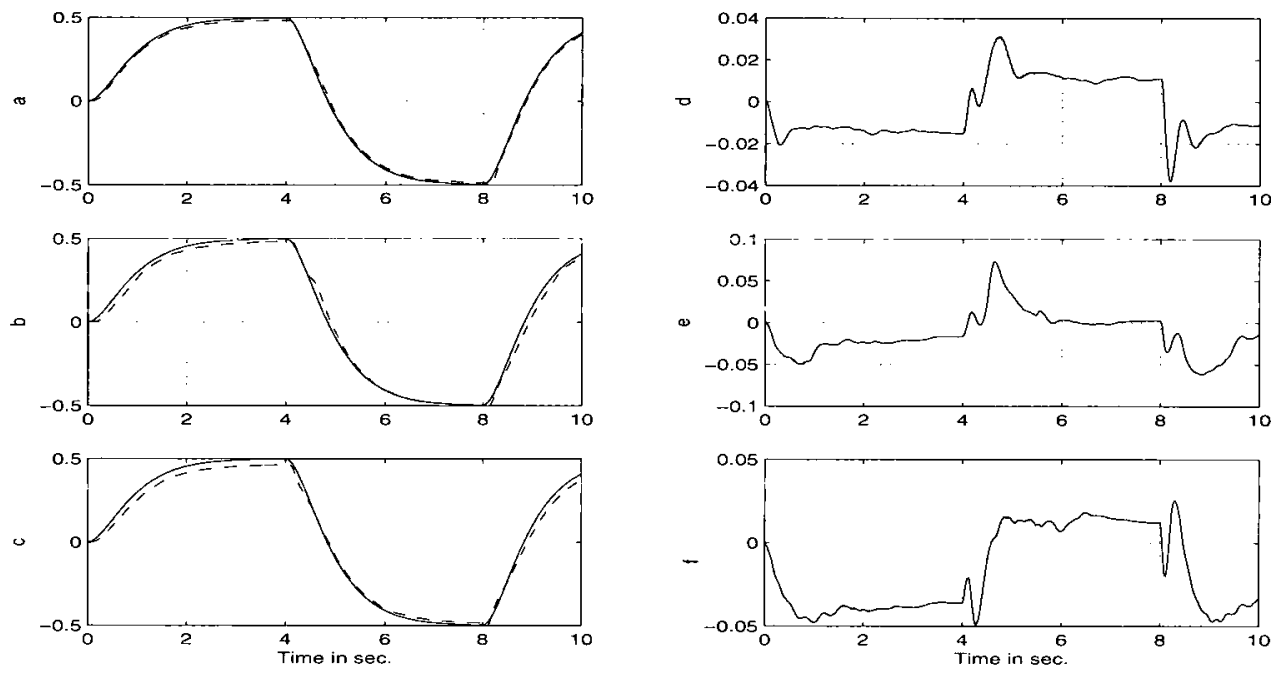

Figure 6.17: Left column: a, b \& c) The desired (solid-line) and output tracking (dash-line) for joints 1, 2 and 3, Right column: d, e \& f) The tracking errors for joints 1,2 and 3 with algorithm (6.3), (6.4).
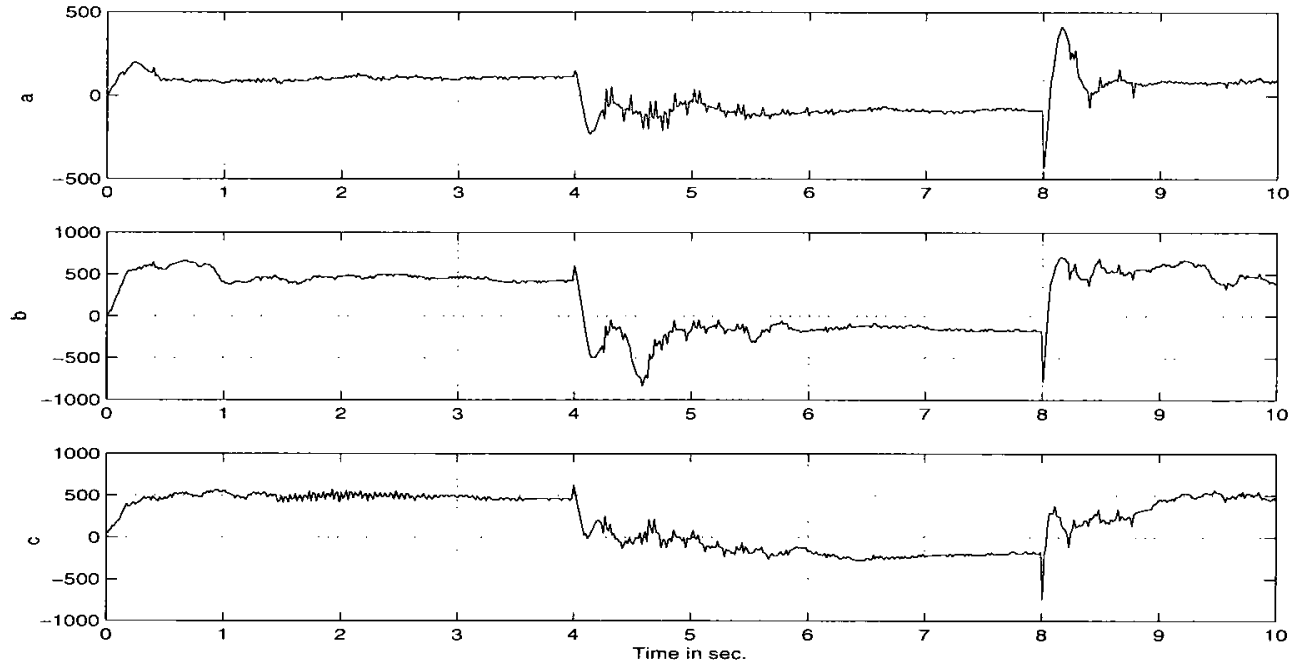

Figure 6.18: a, b \& c) The control inputs for joints 1, 2 and 3 with nonlinear adaptive state feedback control (6.3), (6.4). 

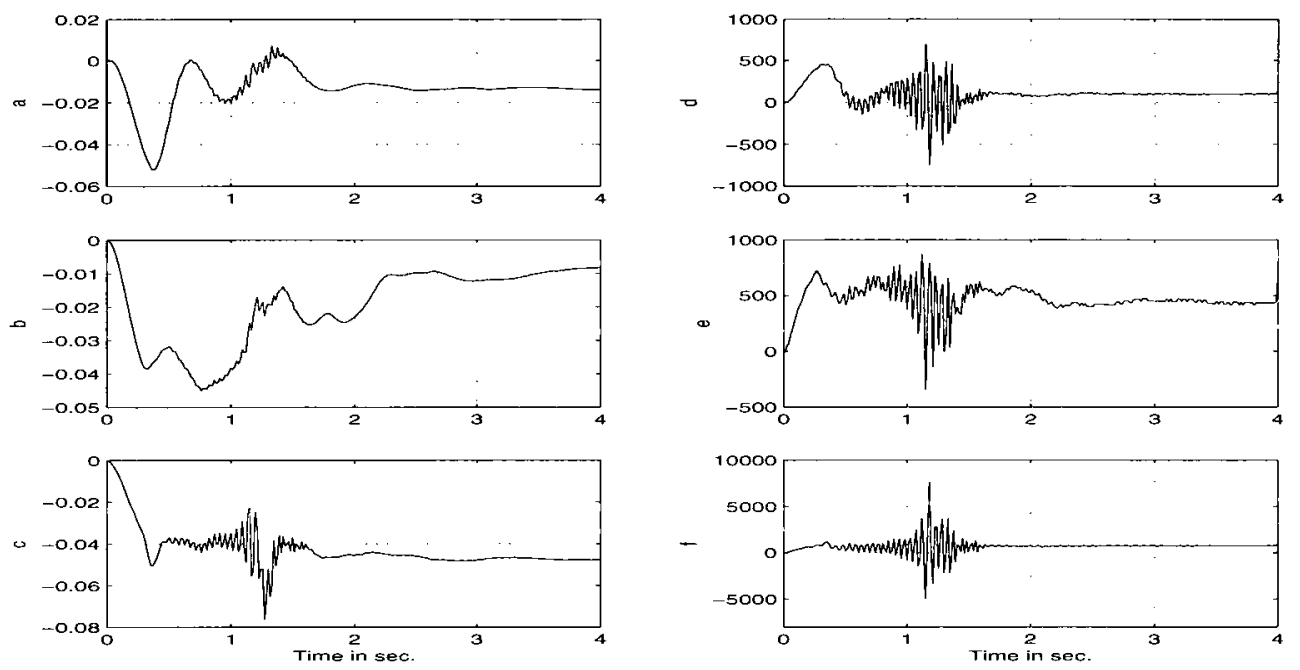

Figure 6.19: Left Column: a, b \& c) The tracking errors for joints 1, 2 and 3, Right Column: d, e \& f) The control inputs for joints 1, 2 and 3 under nonlinear adaptive controller with learning gains $\Gamma=\operatorname{diag}(550,550,550)$.
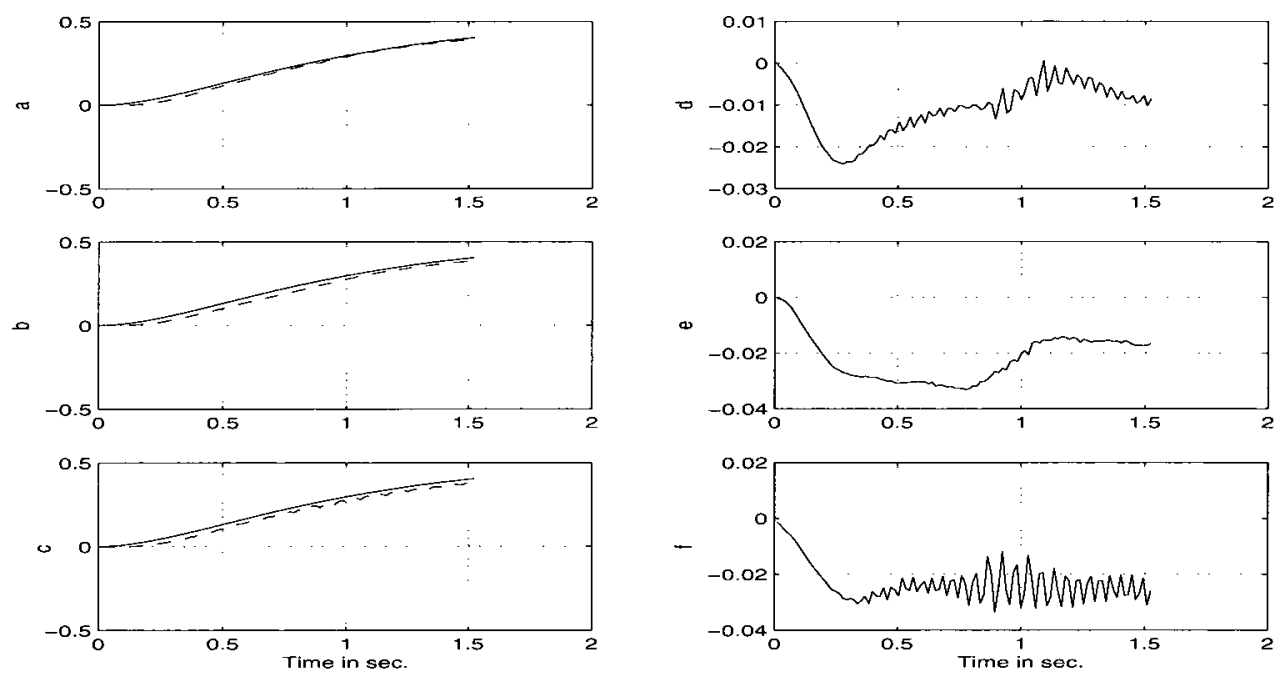

Figure 6.20: Left Column: a, b \& c) The desired (solid-line) and output tracking (dash-line) for joints 1, 2 and 3, Right Column: d, e \& f) The tracking errors for joints 1,2 and 3 with algorithm (6.3), (6.4). 

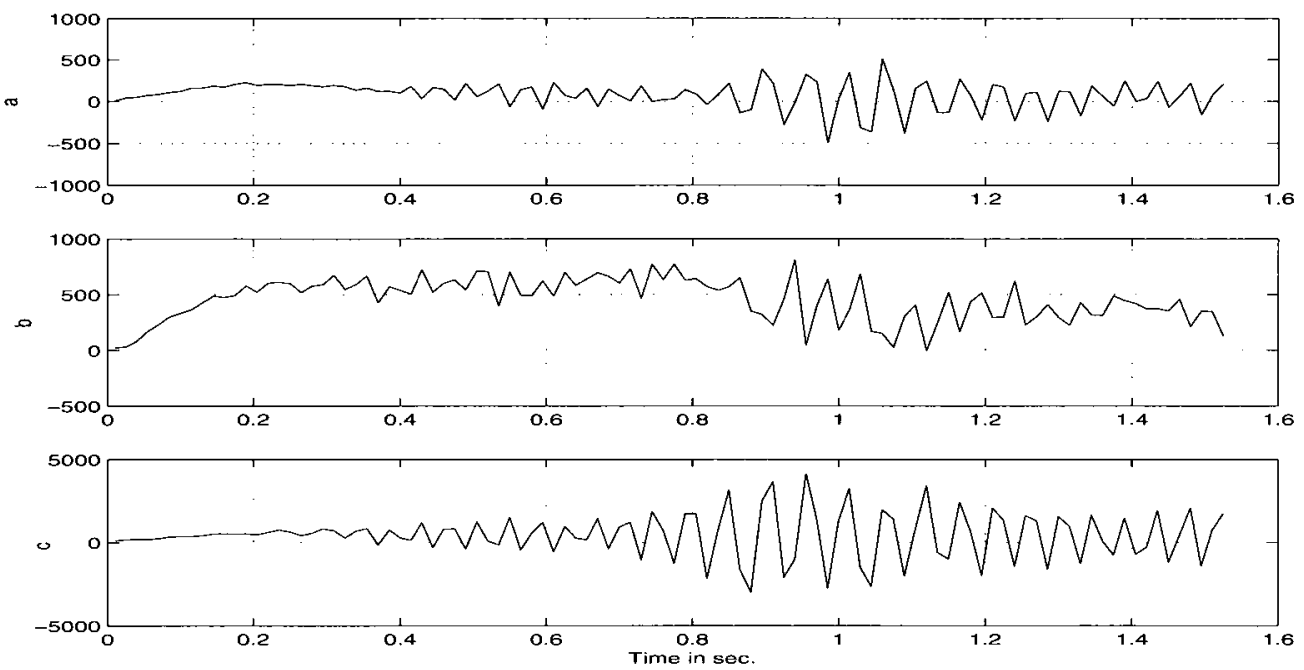

Figure 6.21: a, b \& c) The control inputs for joints 1, 2 and 3 with (6.3), (6.4) under $\Gamma=\operatorname{diag}(700,700,700)$.

adaptive output feedback becomes very large values before the nonlinear control system goes unbounded.

Remark 6.1: It is worth noting to observe from our various experimental evaluations that, in the face of the large scale parametric uncertainty, the model-free and model-based nonlinear adaptive control demands high controller-observer gain in order to achieve good tracking performance. The problem is that the designer cannot increase the gains as much required to obtain good tracking as high gains generates very large control effort saturating the control input resulting unbounded control system. To reduce the observer-controller gains and improve the tracking convergence, one may use hybrid adaptive control mechanism derived in [86, 95, $101]$. 

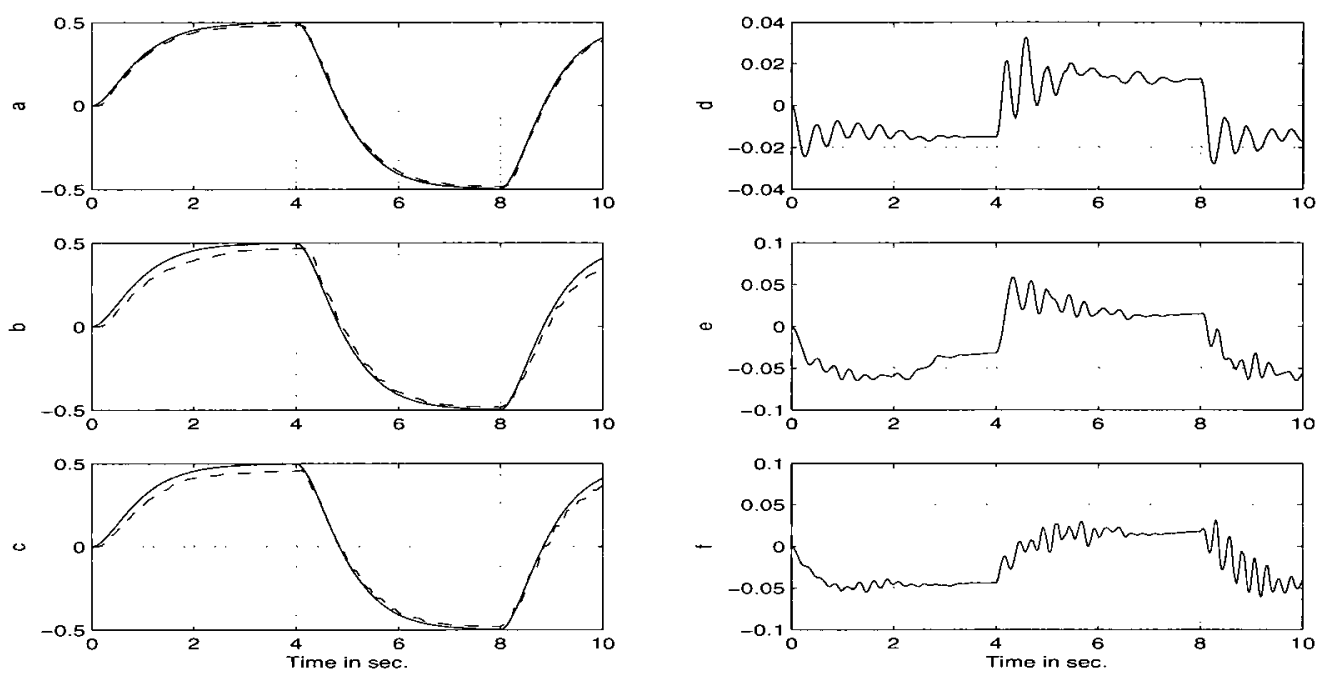

Figure 6.22: Left column: a, b \& c) The desired (solid-line) and output tracking (dash-line) for joints 1, 2 and 3, Right column: d, e \& f) The tracking errors for joints 1, 2 and 3 with nonlinear adaptive output design (6.5), (6.6) with $\epsilon=0.03$.

\subsection{Experimental Results with Multi-model Hy- brid Adaptive Design: State Feedback Ap- proach}

In this section, we experimentally verify hybrid adaptive control system [86, 95, 101] on 3-DOF Phantom ${ }^{\mathbf{T M}}$ robot systems shown in Fig. 6.2 and Fig. 6.3.

The idea of using hybrid adaptive design is to reduce observer-controller gains from classical control approach. To explore that experimentally, let us first implement multi-model hybrid adaptive design on a 3-DOF Phantom ${ }_{1}^{\text {TM }}$ robot system where the parameters $\theta=\left[\theta_{1}, \theta_{2}, \theta_{3}, \ldots ., \theta_{8}\right]^{T}$ are arbitrarily equally split into a finite numbers as $\Omega=\bigcup_{i=1}^{11}\left\{\Omega_{i}\right\}$, that is, 

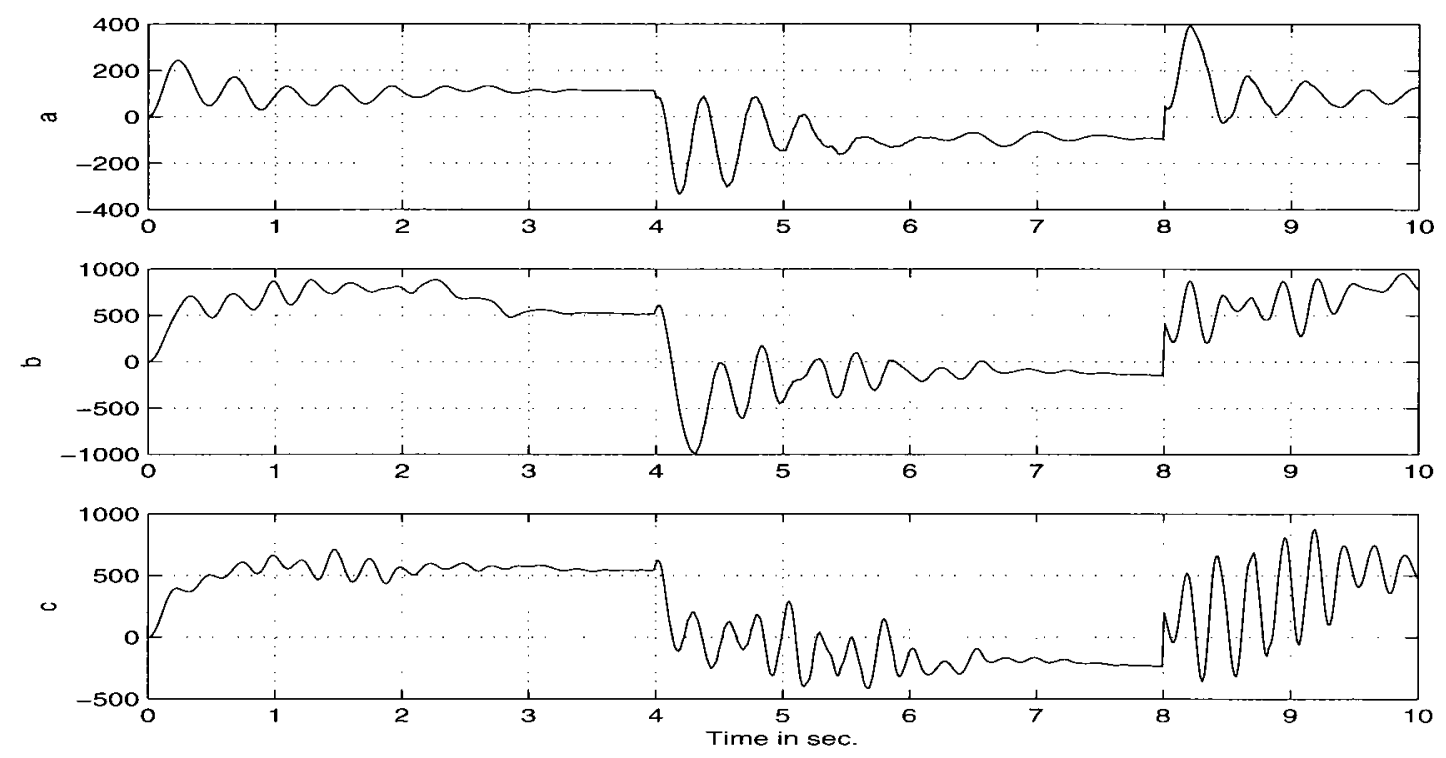

Figure 6.23: a, b \& c) The control inputs for joints 1, 2 and 3 under (6.5), (6.6) with $\epsilon=0.03$.
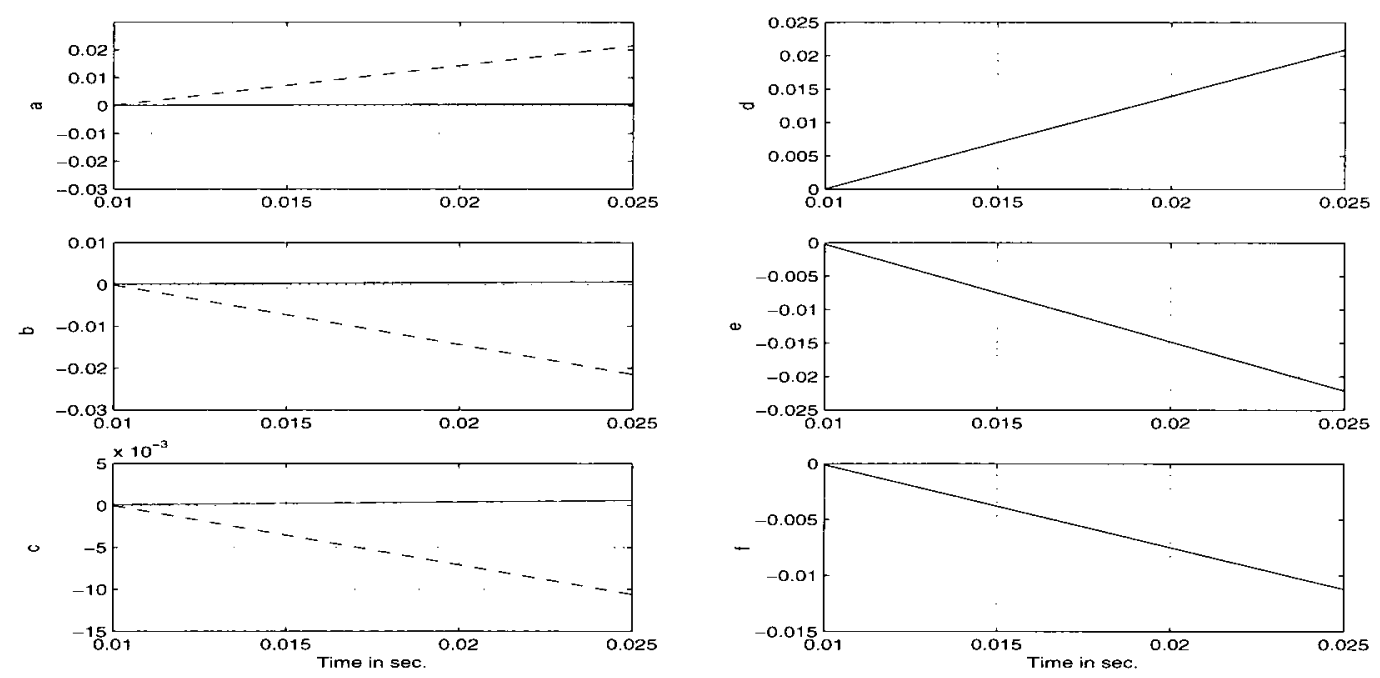

Figure 6.24: Left column: a, b \& c) The desired (solid-line) and output tracking (dash-line) for joints 1, 2 and 3, Right column: d, e \& f) The tracking errors for joints 1, 2 and 3 under nonlinear adaptive output design (6.5), (6.6) with $\epsilon=0.02$. 

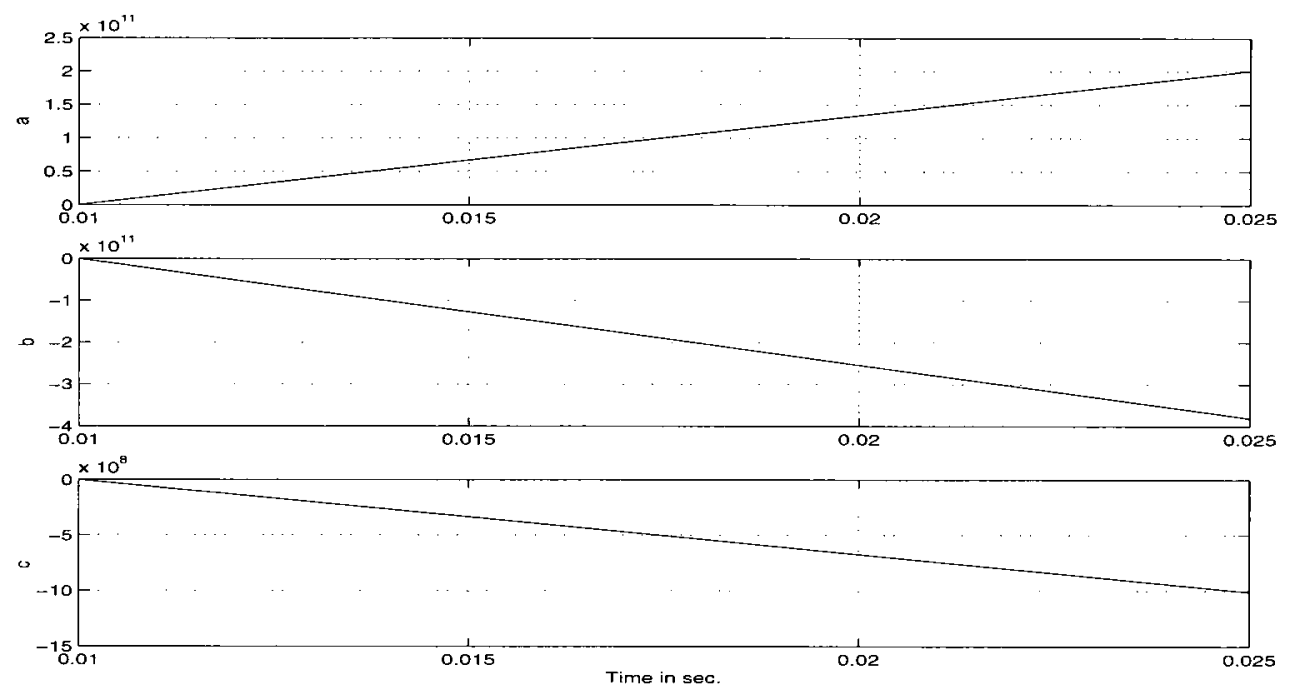

Figure 6.25: a, b \& c) The control inputs for joints 1, 2 and 3 with CAOFB design (6.5), (6.6) with $\epsilon=0.02$.

$$
\begin{aligned}
\Omega= & \bigcup_{i=1}^{11}\left\{\theta_{i}\right\} \\
= & \{0,7, \ldots ., 63,70\} \times\{0,8, \ldots ., 72,80\} \times\{0,6, \ldots ., 54,60\} \\
& \times\{0,12, \ldots . ., 108,120\} \times\{0,2, \ldots . ., 18,20\} \times\{0,2, \ldots ., 18,20\} \\
& \times\{0,32, \ldots . ., 288,320\} \times\{0,32, \ldots . ., 288,320\} \subset \Re^{8}
\end{aligned}
$$

The bounds of the parameter and the number of candidates are arbitrarily chosen to investigate overall tracking convergence property with respect to unknown parameter bounds and smaller number of candidates. We also notice from our parameter distribution that none of the candidates are exactly equal to the identified parameters $\theta$.

The control design parameters $\lambda_{0}, K_{P}$ and $K_{D}$ are common to all $i=11$ candidate controllers. The value of $\lambda_{0}, K_{P}, K_{D}$ and $\Gamma$ are chosen as $\lambda_{0}=2, K_{P}=$ $\operatorname{diag}(750,1200,1200), K_{D}=\operatorname{diag}(500,800,800)$ and $\Gamma=\operatorname{diag}(550,550,550)$. With these design constants, we now construct a set of candidate controller corresponds 

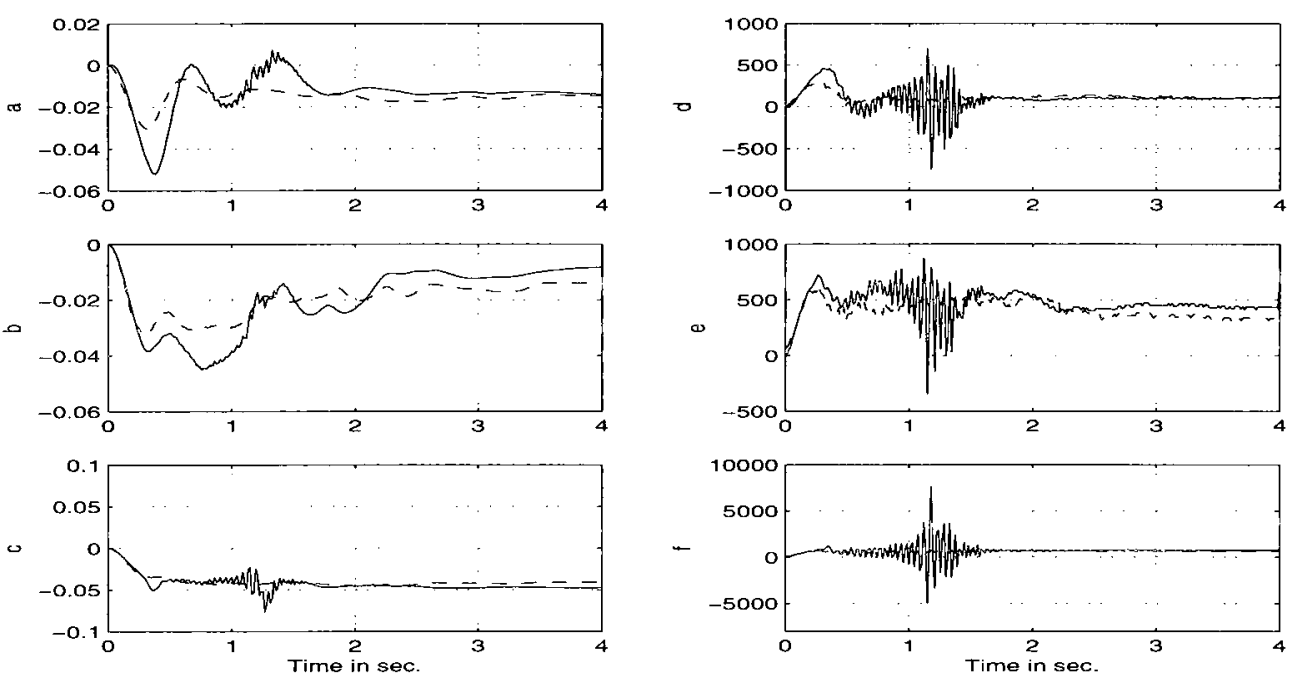

Figure 6.26: Left Column: a, b \& c) The tracking error (dash-line is for hybrid adaptive design and solid-line is for CAC algorithm (6.3)) for joints 1, 2 and 3, Right Column: d, e \& f) The control inputs (dash-line is for hybrid adaptive design and solid-line is for CAC algorithm (6.3)) for joints 1, 2 and 3.
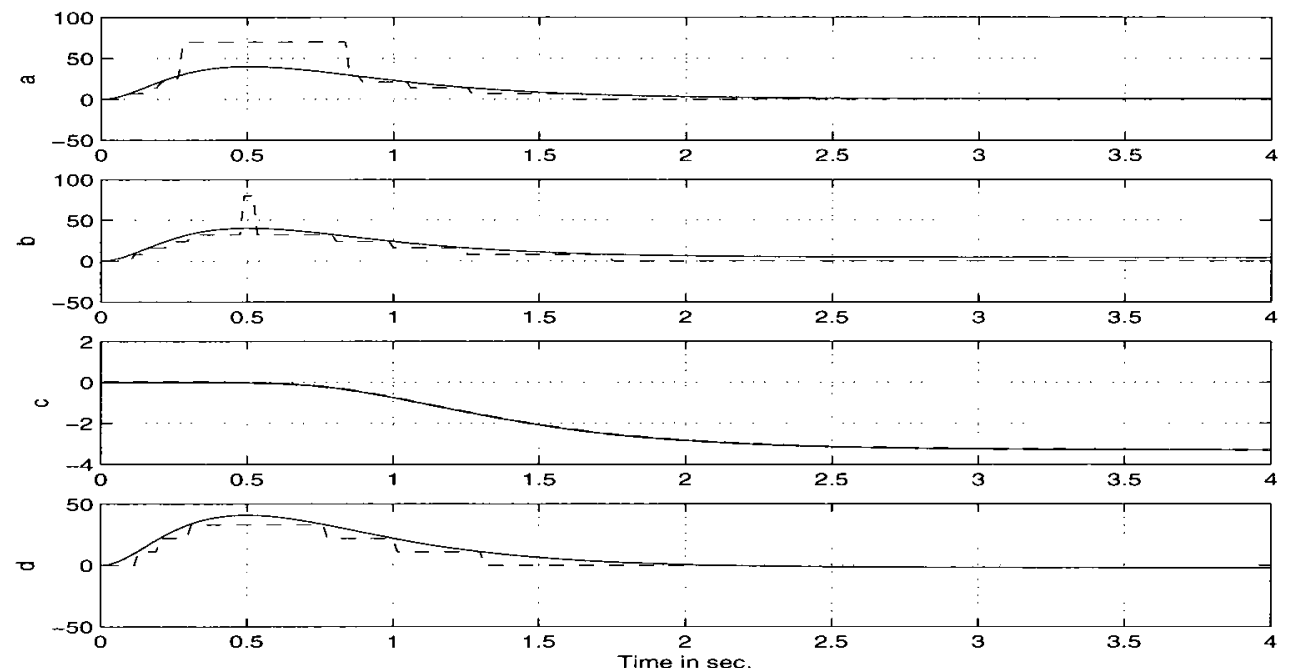

Figure 6.27: a, b, c \& d) The parameter estimate $\theta_{1}, \theta_{2}, \theta_{3}$ and $\theta_{4}$ with hybrid adaptive design where the dash-line is for hybrid adaptive design and the solid-line is for the estimate defined by (6.3). 
to candidate models $\theta_{i}$ as a state feedback

$$
\tau^{i}\left(e, Q_{d}, \theta_{i}\right)=Y\left(e, \dot{q}_{d}, \ddot{q}_{d}\right) \theta_{i}-K_{P} e_{1}-K_{D} e_{2}
$$

with $i=11$. Then, we choose $t_{d}=0.003$ and follow the steps of Algorithm 5.3 to implement Theorem $5.6[86,95,101]$ on the given Phantom ${ }_{1}^{\text {TM }}$ system. The tested results are depicted in Figs. 6.26 to 6.28 .

For comparison, we also present the implementation results of $\mathrm{CAC}$ design with the same set of controller design parameters. The dash-solid line of these Figs. represent the control performance obtained under Theorem 5.6 and the solid-line shows the tracking convergence of the model based CAC design (6.3), (6.4). In view of these Figs., we can notice that the control chattering under CAC design increase with the increase of learning gains causing very large control efforts while chattering free control action with smaller control input under hybrid design can be observed. In addition, the multi-model based hybrid design provides better tracking performance than $\mathrm{CAC}$ design with smaller learning controller gains.

Let us inspect the control performance of the hybrid design with respect to dynamical model parameter changes. To induce such model dynamic changes, we consider two cases in our next experimental evaluation.

In first case, we increase the parametric error uncertainty by adding approximate $0.85 \mathrm{~kg}$. mass externally into end effector of the Phantom ${ }_{1}^{\text {TM }}$ robotic system. We then define the following set of controller design parameters as $\lambda_{0}, K_{P}, K_{D}$ and $\Gamma$ are chosen as $\lambda_{0}=2, K_{P}=\operatorname{diag}(7500,1200,1200), K_{D}=\operatorname{diag}(500,800,1000)$ and $\Gamma=\operatorname{diag}(700,700,700)$. We noticed from our design parameters that we increase the learning gains $\Gamma$ from our last evaluation to examine the effect of the chattering phenomenon. The tested results with Theorem $5.6[86,95,101]$ under parameter changes are shown in Figs. 6.29 to 6.31.

By comparing the dash-line of Fig. 6.26 with Fig. 6.29, we can observe that the tracking errors and control efforts are slightly higher than the tracking errors obtained in our last evaluation. This is due to the fact that we increase the 

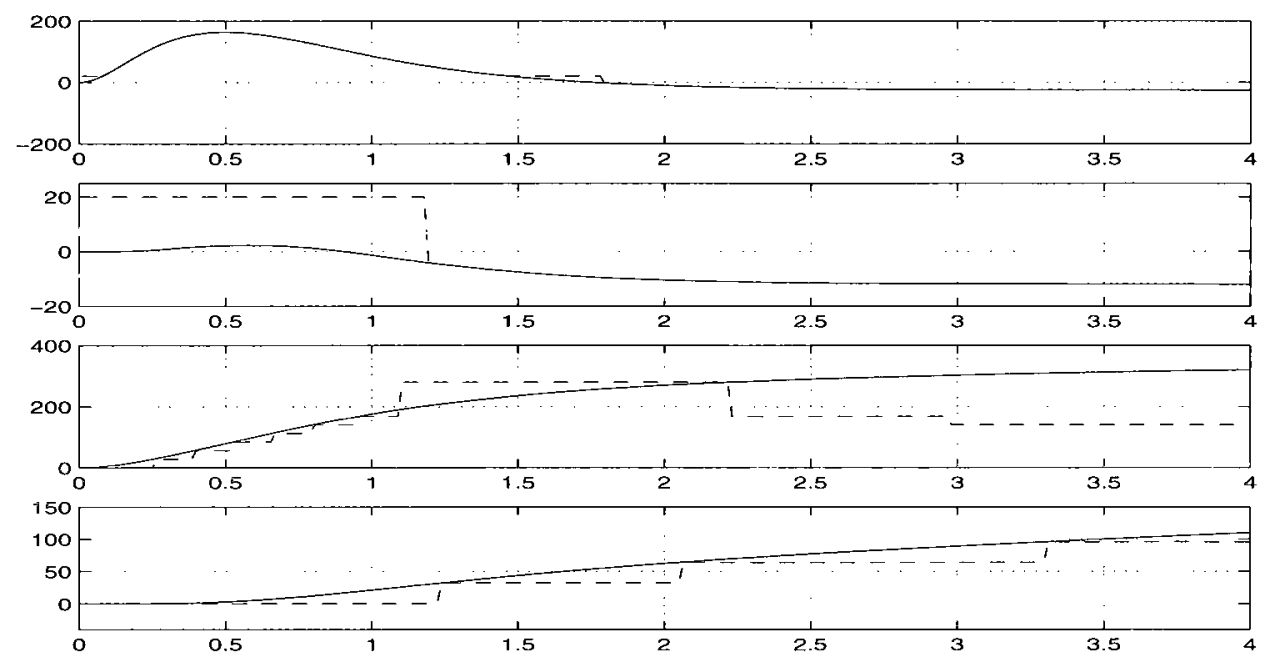

Figure 6.28: a, b, c \& d) The parameter estimate $\theta_{5}, \theta_{6}, \theta_{7}$ and $\theta_{8}$ under (6.3) (solid-line) and hybrid adaptive design (dash-line).
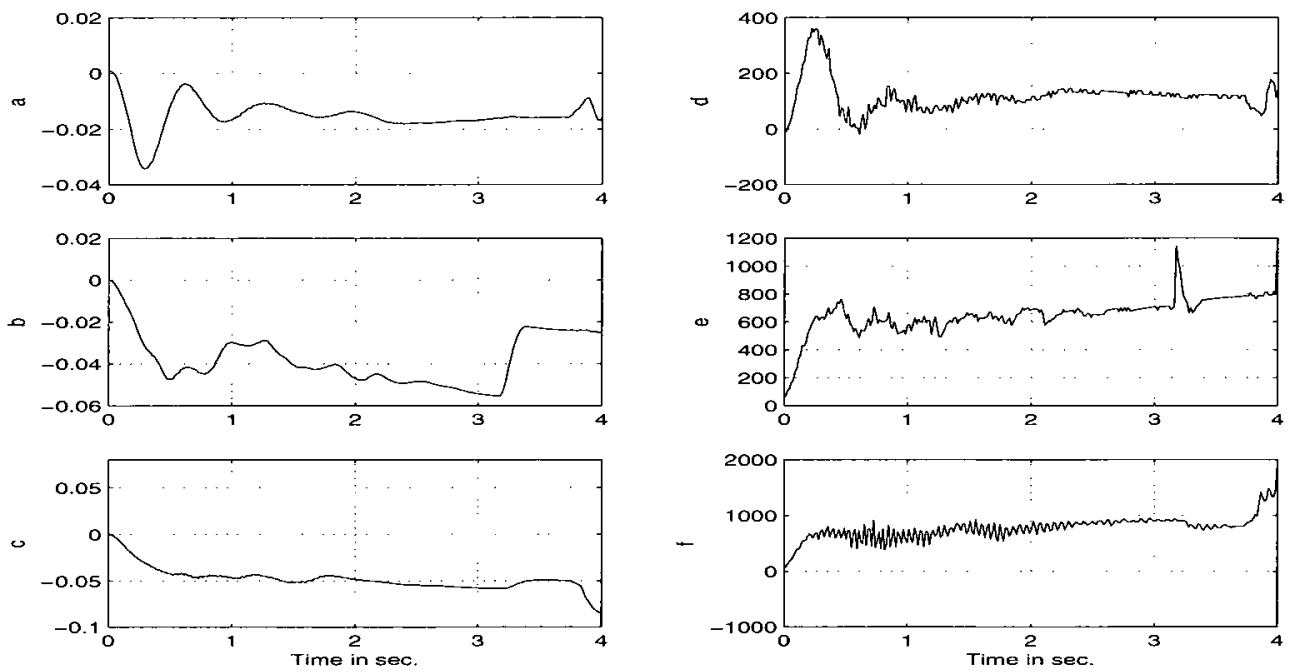

Figure 6.29: Left Column: a, b \& c) The tracking errors for joints 1, 2 and 3, Right Column: d, e \& f) The control inputs for joints 1, 2 and 3. 

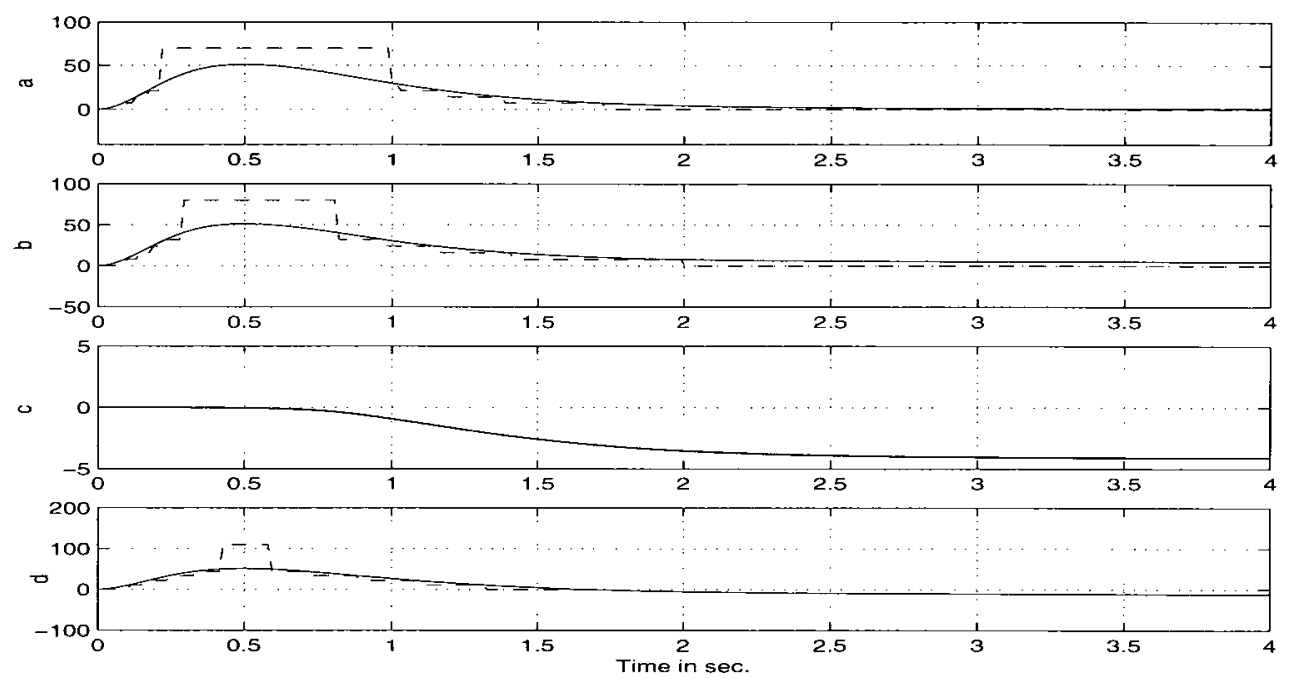

Figure 6.30: a, b, c \& d) The parameter estimate $\theta_{1}, \theta_{2}, \theta_{3}$ and $\theta_{4}$ with (6.3) and hybrid adaptive design of Theorem 5.6. The dash-line is for hybrid adaptive design and the solid-line is for algorithm (6.3).

modeling error uncertainty by adding an external loads to the system.

We noticed from our various experimental evaluations that, even with the presence of large modeling errors, the control efforts under hybrid adaptive design are relatively smaller and free from control chattering activity. For comparison, we also apply CAC design of (6.3), (6.4) under the same set of design parameters as defined above, but the control system goes unbounded as soon as we start the experiment.

In second case, we change the operating dynamics on-line while the Phantom ${ }_{1}^{\mathbf{T M}}$ robot is executing specified task. To create such environment in our experimental study, let us first operate the system with the above distributed parameter sets. Then, at approximately $4.075 \mathrm{sec}$, we externally add $0.85 \mathrm{~kg}$. mass into the second link to increase the modeling error uncertainty.

In view of the parameter sets, there are two dynamics changes in the whole process dynamics. Then, we implement Theorem 5.6 [95] on the given system with 


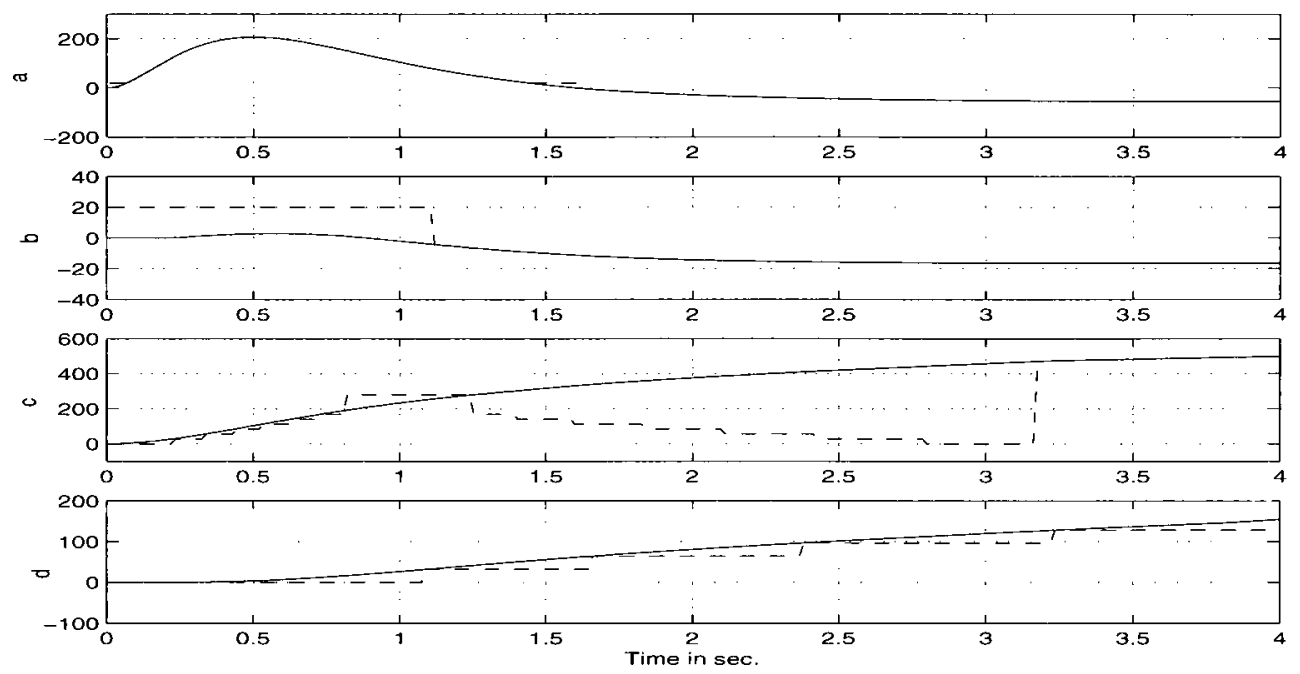

Figure 6.31: a, b, c \& d) The parameter $\theta_{5}, \theta_{6}, \theta_{7}$ and $\theta_{8}$ under classical estimate (6.3) (solid-line) and hybrid adaptive design of Theorem 5.6 (dash-line).
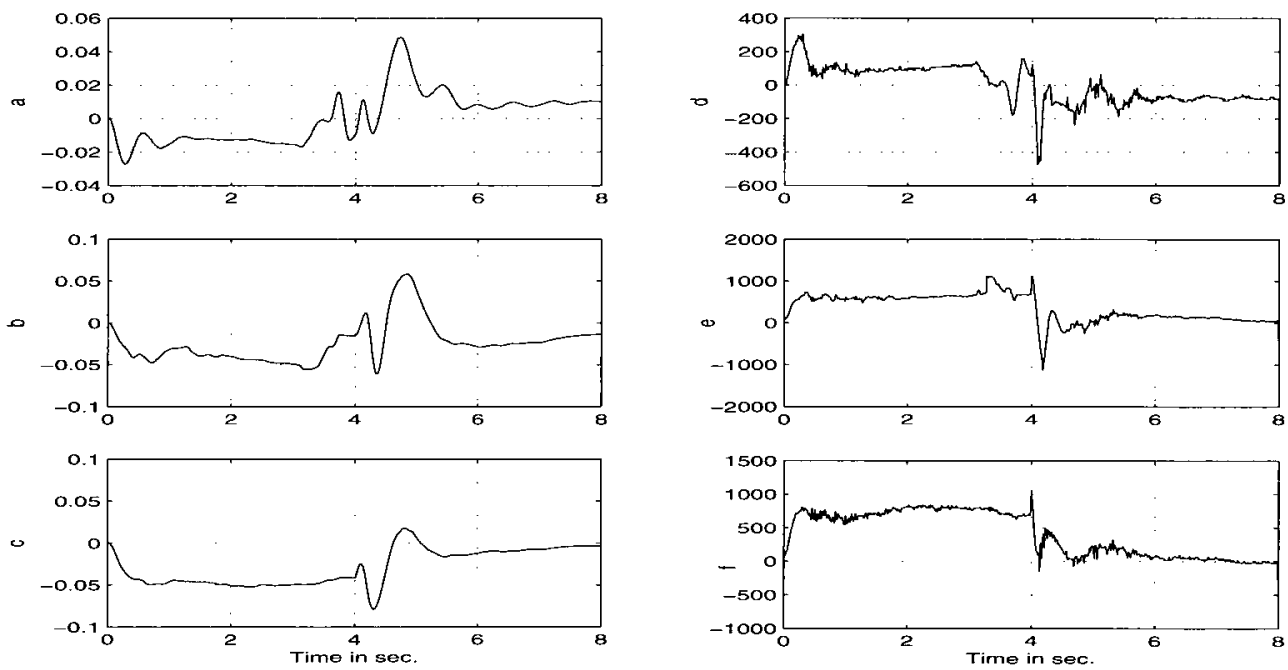

Figure 6.32: Left Column: a, b \& c) The tracking errors for joints 1, 2 and 3, Right Column: d, e \& f) The control inputs for joints 1, 2 and 3 with additional load attached to the end-effector. 


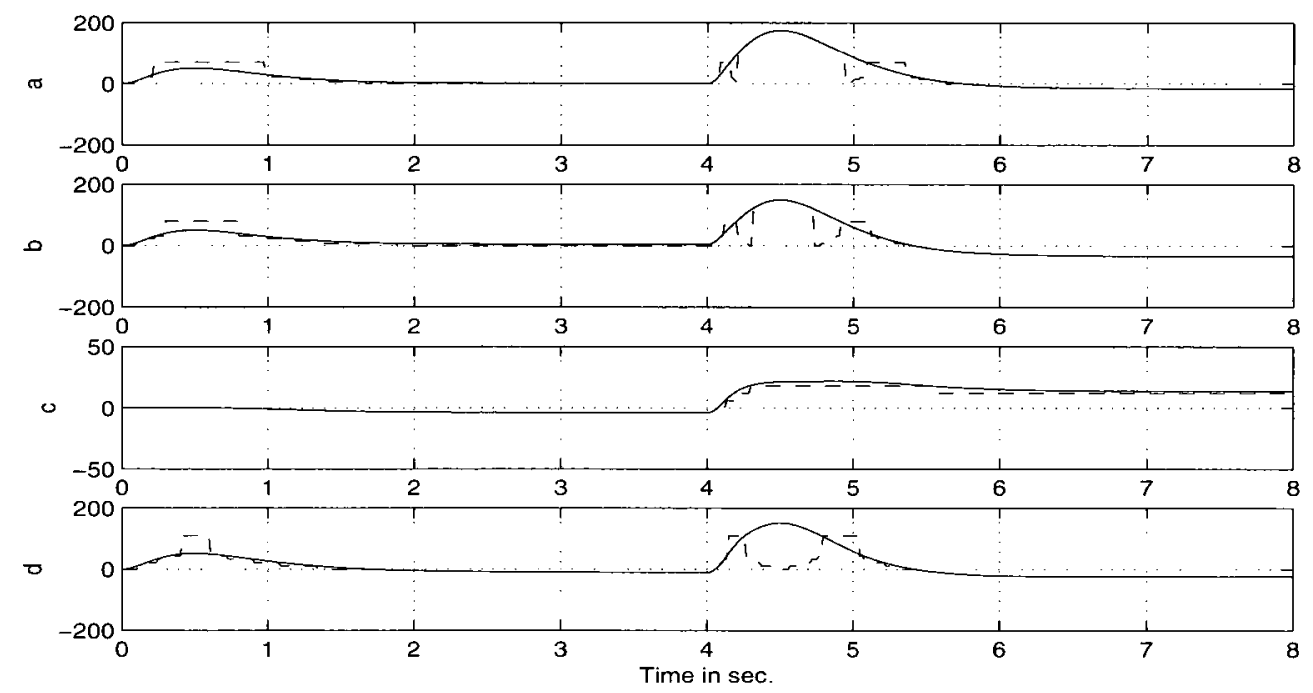

Figure 6.33: a, b, c \& d) The parameter $\theta_{1}, \theta_{2}, \theta_{3}$ and $\theta_{4}$ with classical estimate and hybrid adaptive design. The dash-line is for hybrid adaptive design and the solid-line is for algorithm (6.3) with additional load attached to the end-effector.

the same design constants that applied for our last implementation. The tested results are depicted in Figs. 6.32 to 6.34.

From these experimental results, we can observe that the tracking errors and control efforts at the time of dynamical changes are little larger than the tracking errors and control efforts obtained under fixed dynamic case. We can also view from these Figs. that the control inputs with sudden dynamic changes are free from chattering activity. It is important to note that, with the same design parameter sets, the control system with CAC algorithm (6.3), (6.4) goes unbounded due to the presence of high controller gains.

Let us consider that the parameters $\theta$ does not belong to the distributed sets. To create such experimental set-up, we now use Phantom $_{2}^{\text {TM }}$ robotic system to test hybrid and CAC algorithms. The parameter distribution, number of controllers and all other design parameters for hybrid adaptive design are remained similar to our previous experiment for Phantom ${ }_{1}^{\text {TM }}$ robotic system. 

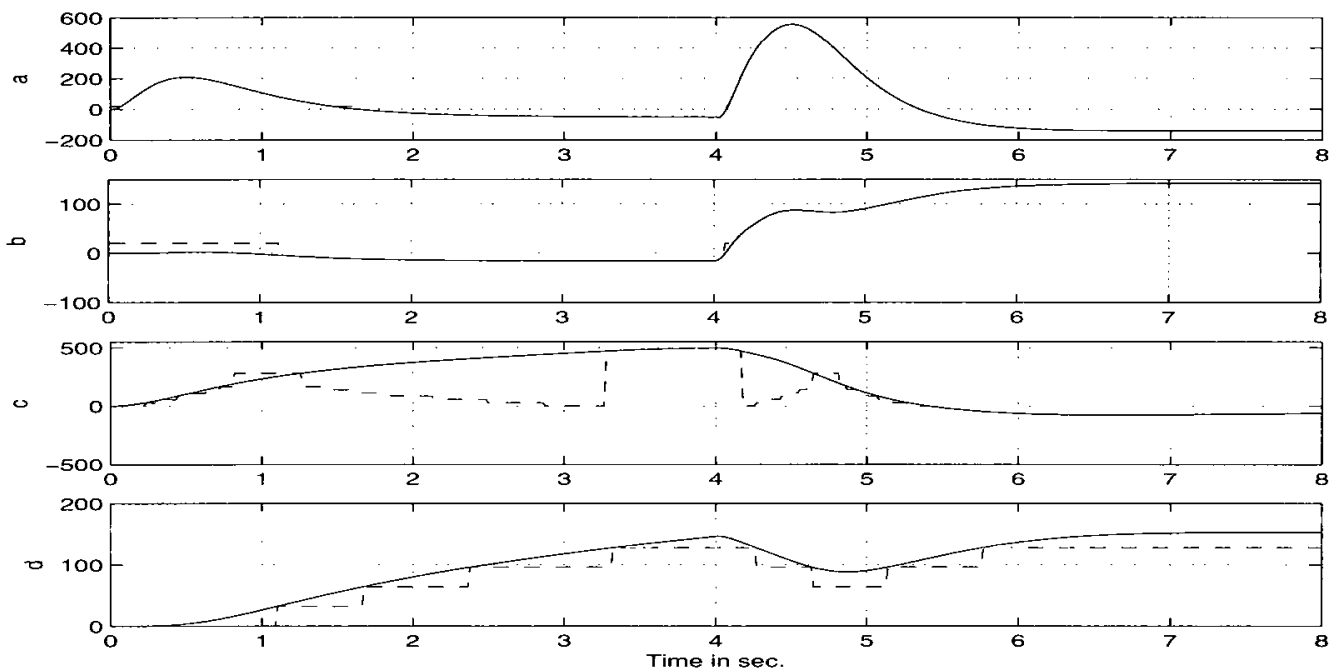

Figure 6.34: a, b, c \& d) The parameter $\theta_{5}, \theta_{6}, \theta_{7}$ and $\theta_{8}$ under CAC estimate (solid-line) and hybrid adaptive design (dash-line) with additional load attached to the end-effector.

In view of the identified and distributed parameters, we can see that the model parameters of Phantom ${ }_{2}^{\text {TM }}$ are different from Phantom ${ }_{1}^{\text {TM }}$ system. By comparing identified and distributed parameters, we can also see that the estimation errors are very large. We then apply both Theorem 5.6 and CAC algorithm on the Phantom ${ }_{2}^{\mathbf{T M}}$ robotic system with the following design parameters; $\lambda_{0}=2, K_{P}=$ $\operatorname{diag}(7500,1200,1200), K_{D}=\operatorname{diag}(500,800,800)$ and $\Gamma=\operatorname{diag}(500,500,500)$. The tested results for both algorithms are shown in Figs. 6.35 to 6.37. In view of dash and solid-line of Fig. 6.35, we can notice that the tracking errors and control efforts under hybrid adaptive design are smaller than the tracking errors and control inputs obtained with CAC approach. 

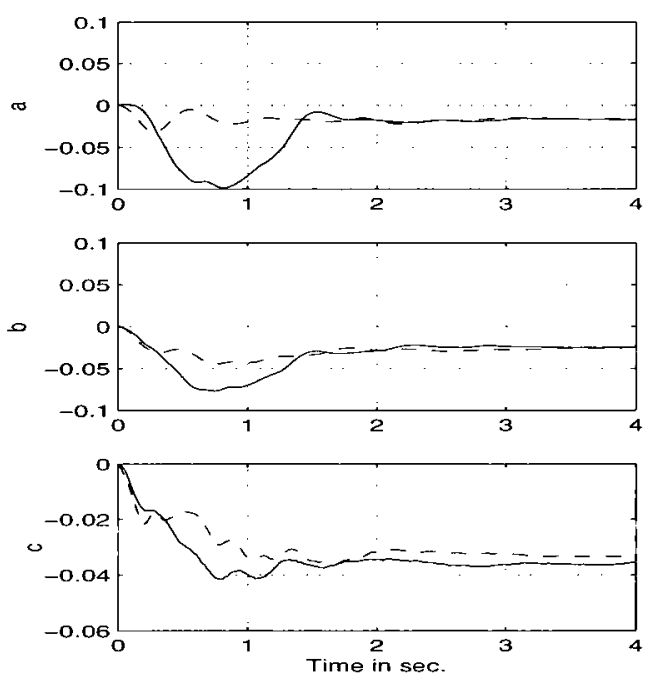
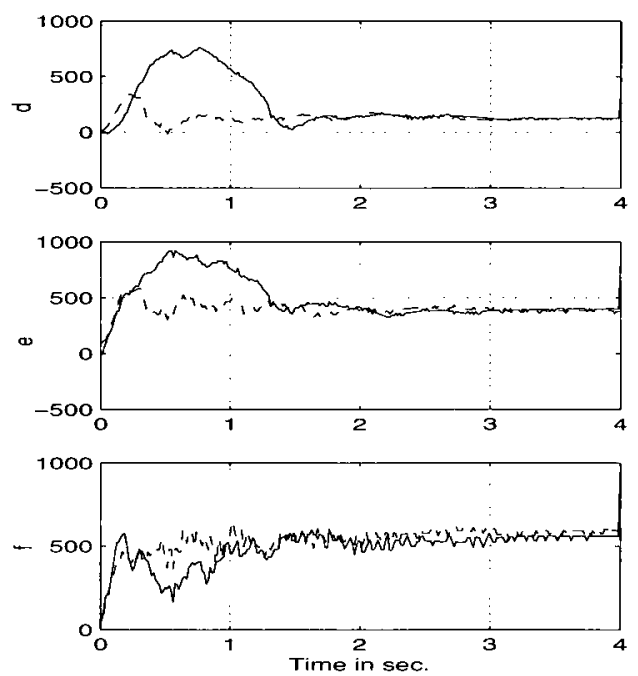

Figure 6.35: Left Column: a, b \& c) The tracking errors (dash-line is for hybrid adaptive design of Theorem 5.6 and solid-line is for CAC) for joints 1, 2 and 3, Right Column: d, e \& f) The control inputs (dash-line is for Theorem 5.6 and solid-line is for CAC) for joints 1,2 and 3 .
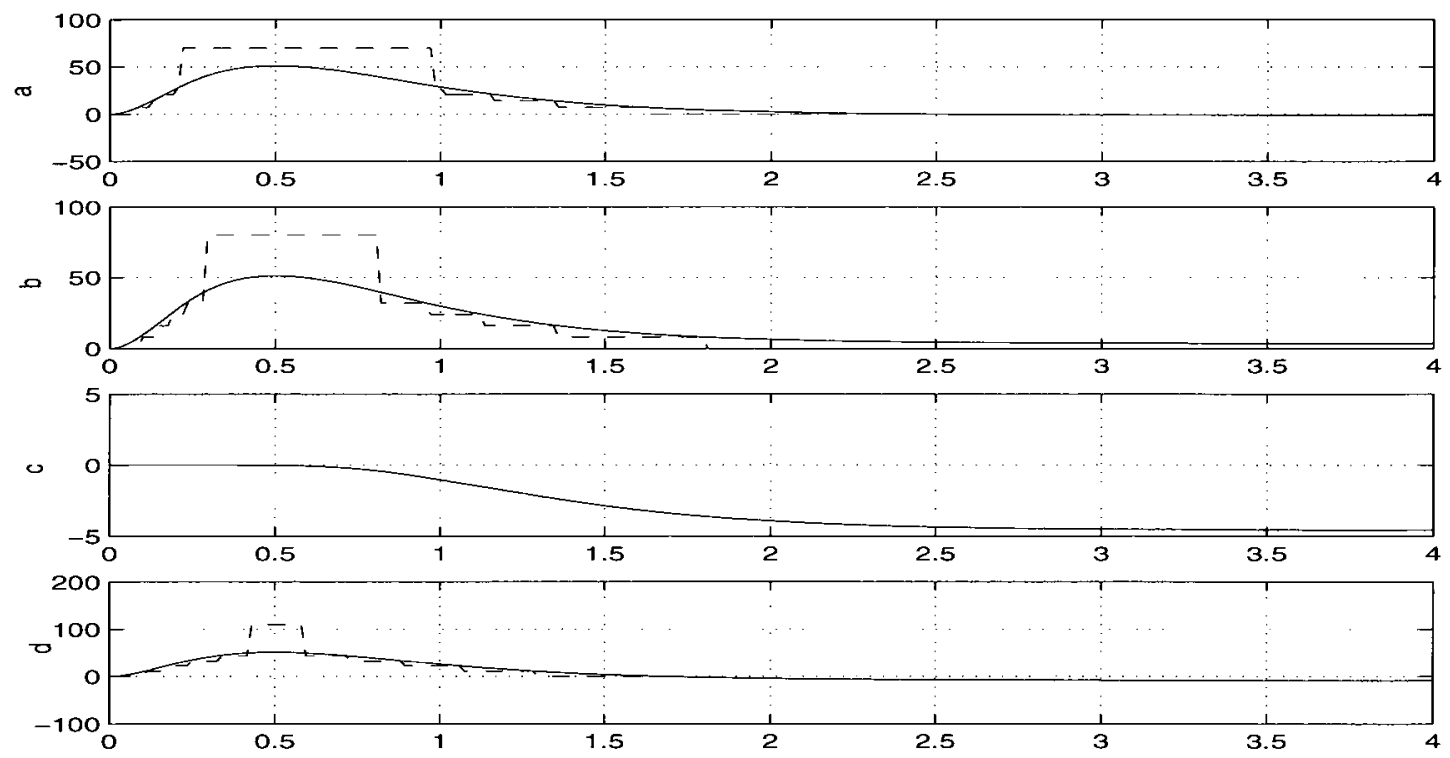

Figure 6.36: a, b, c \& d) The parameter estimate $\theta_{1}, \theta_{2}, \theta_{3}$ and $\theta_{4}$ with algorithm (6.3) (solid-line) and hybrid adaptive design (dash-line) under dynamic model parameter changes. 

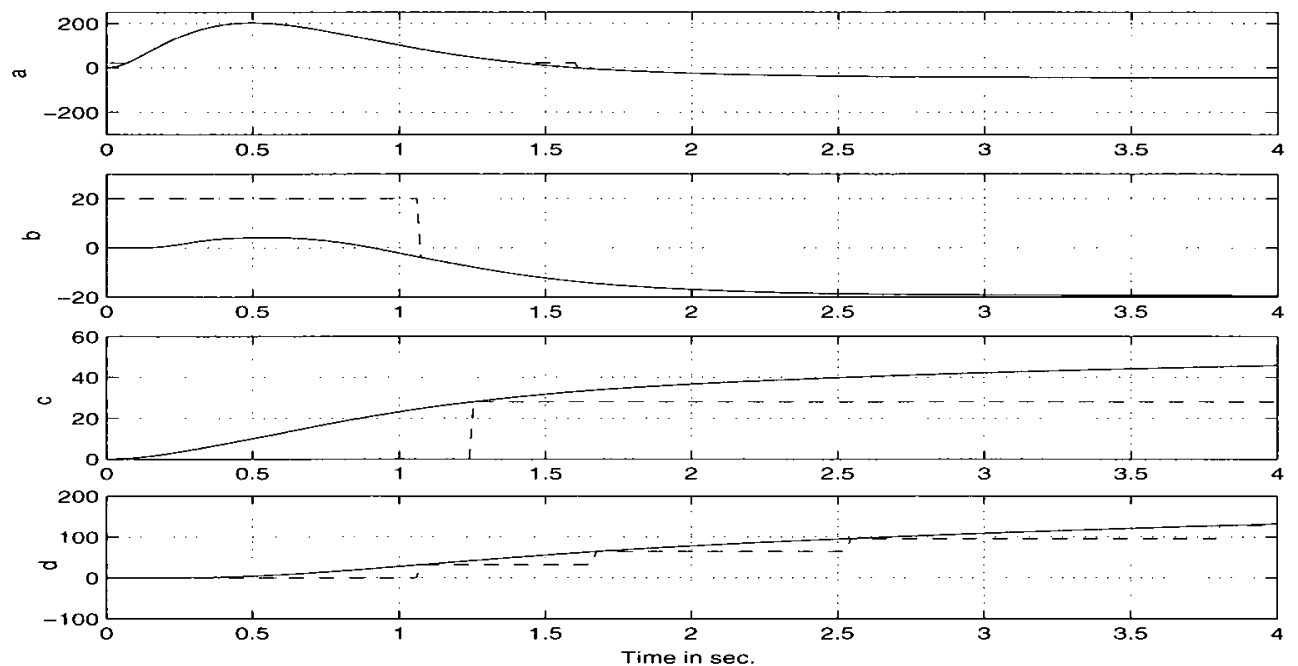

Figure 6.37: a, b, c \& d) The parameter estimate $\theta_{5}, \theta_{6}, \theta_{7}$ and $\theta_{8}$ under CE-based CAC design (6.3) (solid-line) and hybrid adaptive design (dash-line) with dynamic model parameter changes.

\subsection{Experimental Results with Multi-model Hy- brid Adaptive Design: Output Feedback Ap- proach}

We now implement and evaluate output feedback based multi-model hybrid adaptive control of Theorem $5.7[86,101]$ on the given Phantom ${ }_{2}^{\text {TM }}$ system. The controller-observer parameters are selected as $\lambda_{0}=2, K_{P}=\operatorname{diag}(750,1200,1200)$, $K_{D}=\operatorname{diag}(500,800,800), \Gamma=\operatorname{diag}(500,500,500), H_{1}=15 I_{3 \times 3}, H_{2}=5 I_{3 \times 3}$ and small value of $\epsilon$ as $\epsilon=0.05$. Notice that the controller design parameters are kept similar to our previous experiment. Then, the small value of $t_{d}$ is arbitrarily chosen as $t_{d}=0.005$. We then follow the design steps of Algorithm $5.3[86,101]$ to implement Theorem 5.7 on the Phantom ${ }_{1}^{\text {TM }}$ system. The tested results are depicted in Figs. 6.38 to 6.40 (dash line).

For comparison, we also implement the single model based CAOFB design 

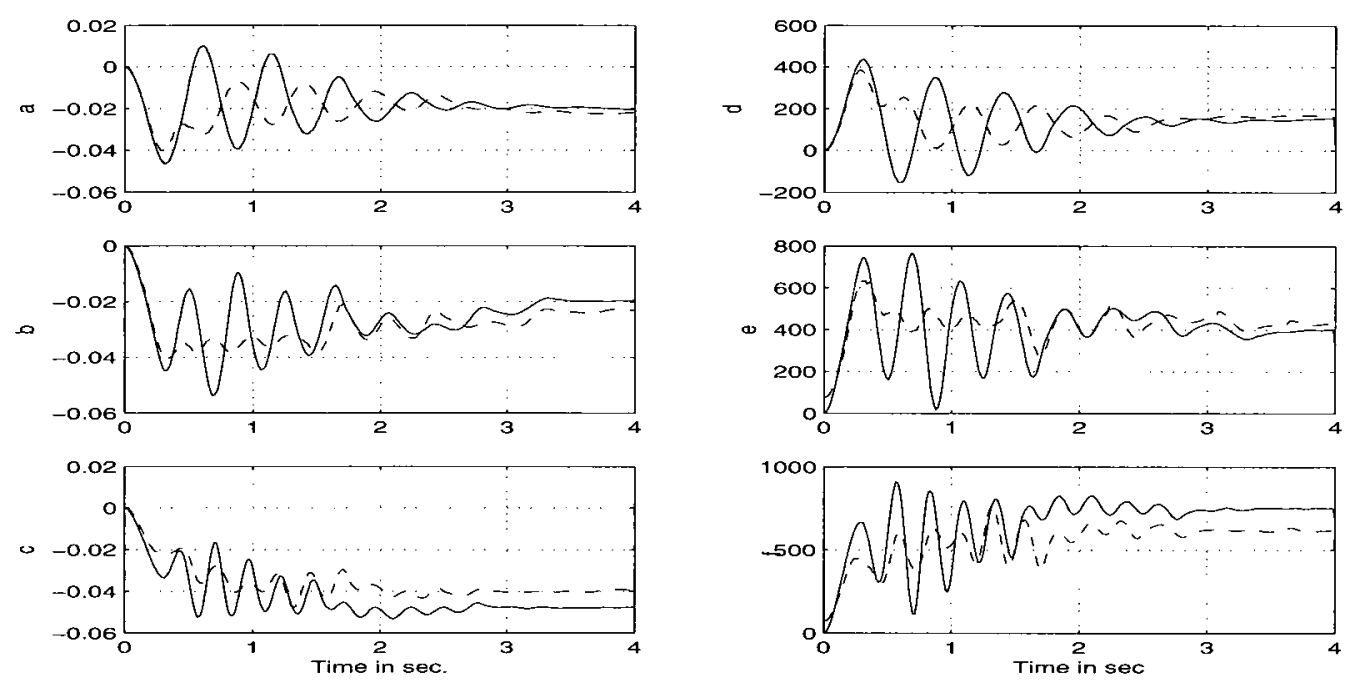

Figure 6.38: Left Column: a, b \& c) The tracking errors (dash-line is for hybrid adaptive output feedback design and solid-line is for CAOFB design (6.5)) for joints 1, 2 and 3, Right Column: d, e \& f) The control inputs (dash-line is for hybrid design of Theorem 5.7 and solid line is for (6.5)) for joints 1,2 and 3.

(6.5) on the same system with the same set of observer-controller design constants. The conducted results are depicted in the solid-line of Figs. 6.38 to 6.40.

By comparing solid and dash-line of Fig. 6.38, we can observe that the tracking errors and control efforts under Lyapunov-based multi-model hybrid adaptive design are smaller than the tracking errors and control inputs obtained with CAC design. The sampling time was set to $0.005 \mathrm{sec}$. for all the experimental results reported in this paper.

Remark 6.2: Notice from our different experimental evaluations that the parameter estimate with hybrid adaptive approach does not converge to the actual one. This is because candidate models are chosen in such away that they are not equal to actual parameters as we intentionally choose smaller number of candidate models with large modeling error uncertainty to examine the tracking performance of the hybrid design. 

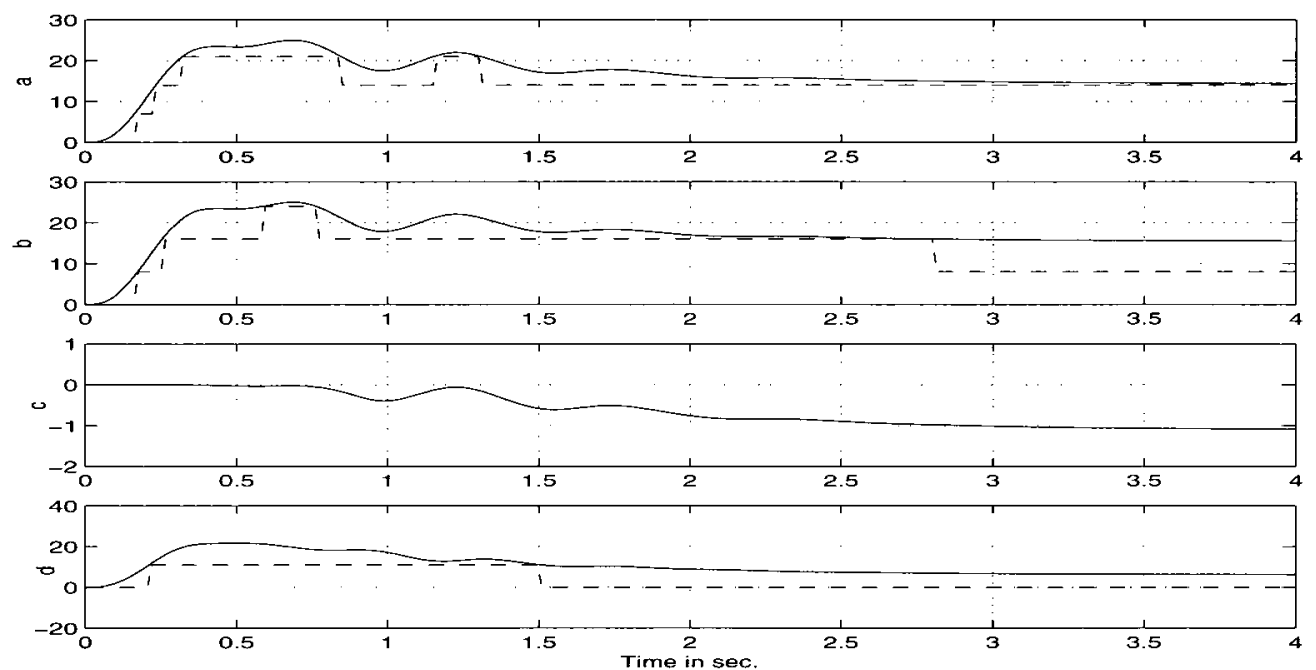

Figure 6.39: a, b, c \& d) The parameter estimate $\theta_{1}, \theta_{2}, \theta_{3}$ and $\theta_{4}$ with estimation algorithm (6.5) (solid-line) and hybrid adaptive output feedback design of Theorem 5.7 (dash-line).

\subsection{Summary}

In this chapter, we have experimentally tested some classical $[82,85,94]$ and hybrid adaptive control schemes $[86,95,101]$ on 3 -DOF Phantom ${ }^{\mathbf{T M}}$ robotic systems. Various experiments have been conducted to analyze the tracking performance of classical and multi-model based hybrid control design. These experimental results have clearly showed that the multi-model based hybrid adaptive design achieves better tracking performance than the single model-based classical control approach with relatively smaller values of observer-controller gains. The hybrid design reduced the controller-observer gains by selecting a candidate controller among the finite set of candidates through on-line estimation of the Lyapunov-function inequality. The closed-loop stability under switching has been given by using the derivative of the Lyapunov-function candidate. The hybrid design increased the convergence speed of adaptation mechanism via switching the parameter estimate of the classical controller into a family of candidate model, which best approximates 


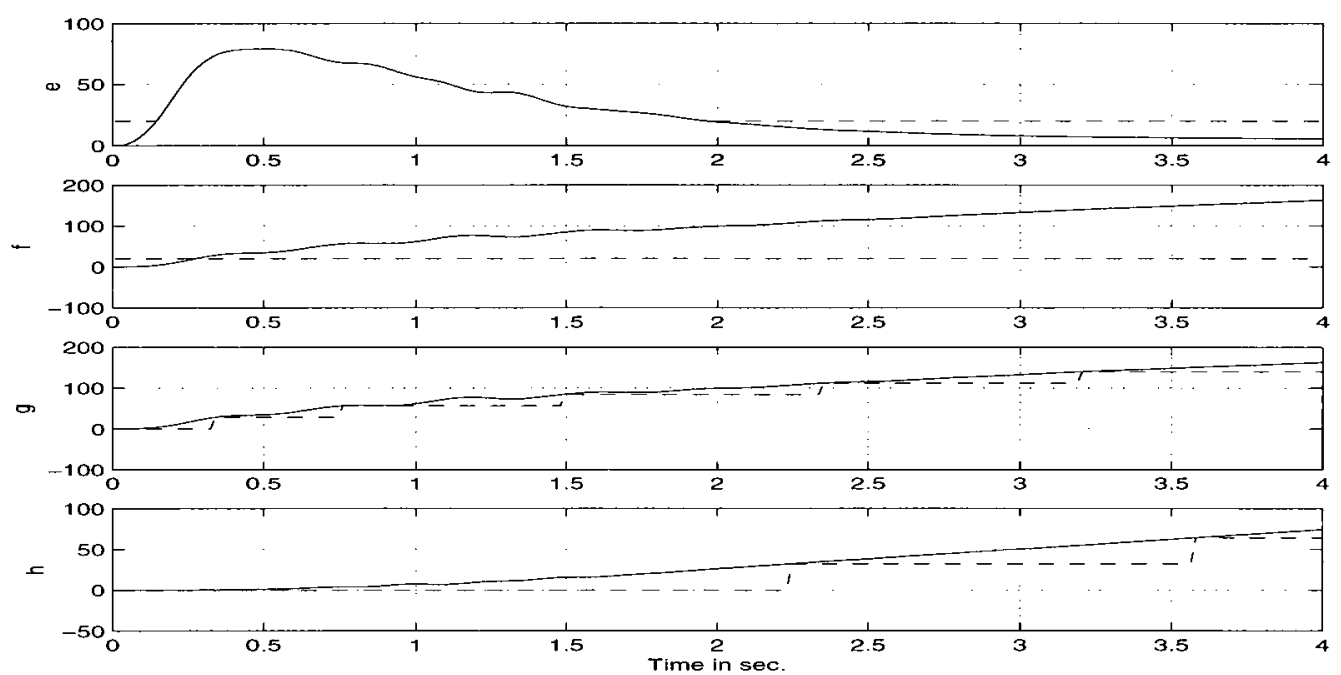

Figure 6.40: e,f, g \& h) The parameter estimate $\theta_{5}, \theta_{6}, \theta_{7}$ and $\theta_{8}$ under classical estimator (6.5) (solid-line) and hybrid adaptive output feedback design of Theorem 5.7 (dash-line).

the plant at each instant of time. 


\section{Chapter 7}

\section{Conclusions}

\subsection{Thesis Contributions}

The contributions of the thesis can be summarized as follows.

In Chapter 2, we have introduced model-free linear observer-controller strategy for uncertain robot manipulators. The design can be employed to deal with the practical problem associated with the position-velocity based PD control system for industrial robot manipulators. The method introduced in this thesis can be directly employed to industrial robot manipulators that are operating under filtered version of the PD controller via replacing the unknown velocity signals by the output of the linear observer.

In chapter 3, robust adaptive fuzzy control system has been developed for robust trajectory tracking control problem for a class of nonlinear mechanical systems. The design combined robust adaptive control with direct and indirect adaptive fuzzy system. This hybrid control paradigm used to learn and compensate the effect of the external disturbances and modeling errors that appears from fuzzy approximation errors. In the presence of the modeling errors and disturbance uncertainties, the designed method provided a smooth controller-observer algorithm for highly uncertain nonlinear mechanical systems. 
The contributions of the Chapter 4 can be divided into two parts. We first introduced single model based output feedback sliding mode control technique for nonlinear systems. This method used to deal with the problem associated with state-feedback based sliding mode control design reported in the literature. Then, various distributed sliding mode control algorithms have been designed to improve the tracking performance with smaller values of the observer, discontinuous and learning control design parameters in the presence of large scale modeling/parametric uncertainty. The distributed robust control method can be deployed to avoid of using chattering and infinitely fast switching control problem from single model robust control strategy.

Chapter 5 introduced novel multi-model based hybrid adaptive control technique for trajectory tracking control problem for robot manipulators. First, single model CE principle-based adaptive output feedback control strategy has been introduced. The stability condition has been used to find the lower bound on the observer speed that reflects the uncertainties in initial conditions and initial parameters. Second, we have proposed multi-model based hybrid adaptive control system strategy to obtain robust tracking performance with respect to large scale modeling errors. The method extended the design derived in the first part of this chapter. One of the main goals of the multi-model based hybrid control system was to achieve robust tracking performance with smaller values of observer-controller gains in the presence of large scale modeling errors. The design used multi-models of the system dynamics and operating loads in order to identify different operating environments and generate an appropriate candidate control input vector. The method started with a single model classical controller. Then, for every control input, the candidate waits in the feedback loop for very short periods of time and checks the pre-specified switching inequality to see whether or not there was a change in the operating conditions (for example, the system manipulates or picks up a new load). If there is a change in the environment, then the switching criterion will identify and switch the acting controller to a candidate that satisfies 
the switching condition before a control action is taken. Therefore, the proposed technique can be viewed as an extension of the classical design which is feasible for real-world applications for large-scale MIMO nonlinear mechanical systems.

Chapter 6 presented experimental results of some single model based classical and multi-model based adaptive designs on 3-DOF Phantom ${ }^{\text {TM }}$ robot manipulators. These experiments have studied to compare the tracking performance of classical and multi-model control approach. These experimental results have clearly demonstrated that the multi-model based design achieves better tracking performance than the classical control design with relatively smaller values of observercontroller gains.

\subsection{Manuscripts Published, Accepted, Under Re- view and Submitted}

The work presented in this thesis resulted in a number of refereed journals and conference proceedings.

[1] S. Islam and P. X. Liu, Output feedback sliding mode control for robot manipulators, Robotica, In Press, 2009.

[2] S. Islam and P. X. Liu, PD output feedback control design for industrial robotic systems, IEEE/ASME Transactions on Mechatronics, Vol. 15, no. 3, 2010.

[3] S. Islam and P. X. Liu, A hybrid adaptive control for robust trajectory tracking for robot manipulators:Theory and Experiment, Robotica, In Press, 2010.

[4] S. Islam and P. X. Liu, Robust adaptive fuzzy output feedback for robotic manipulators, IEEE/ASME Transactions on Mechatronics, In Press, 2010.

[5] S. Islam and P. X. Liu, Hybrid control system for a class of nonlinear mechanical systems: Theory and experiment, Accepted for publication in Mechatronics, 2010. [6] S. Islam and P. X. Liu, Robust sliding mode control for robot manipulators, IEEE Transactions on Industrial Electronics, In Press, 2010. 
[7] S. Islam and P. X. Liu, Adaptive iterative learning control for robot manipulators without using velocity signals, Robotica, Under Review.

[8] S. Islam and P. X. Liu, Hybrid adaptive sliding mode control for robot manipulators, Robotics and Autonomous Systems, Under Review.

[9] S. Islam and P. X. Liu, Robust adaptive output feedback control of robotic systems, IEEE Transactions on Control Systems Technology, Under Review.

[10] S. Islam and P. X. Liu, Intelligent control for a class of nonlinear systems: theory and experiment, IEEE/ASME Transactions on Mechatronics, Under Review. [11] S. Islam and P. X. Liu, Experimental comparison of model-free and model based output feedback control design for robotic systems, IEEE/ASME Transactions on Mechatronics, Under Review.

[12] S. Islam, Adaptive output feedback for robot manipulators using linear observer, Proceedings of the International Conference on Intelligent System and Control, November 16-18, Orlando, Florida, 2008, pp. 38-43.

[13] S. Islam and P. X. Liu, Adaptive sliding mode control for robotic systems using multiple parameter models, 2009 IEEE/ASME International Conference on Advanced Intelligent Mechatronics (AIM), Suntec International Convention and Exhibition Centre, Singapore, July 14-17, 2009, pp. 1775-1780.

[14] H. M. Schwartz and S. Islam, An evaluation of adaptive robot control via velocity estimated feedback, In Proceedings of the International Conference on Control and Applications, Montreal, Quebec, May 30-June 1, 2007, pp. 325-333.

[15] S. Islam and P. X. Liu, PD output feedback control design for robotic manipulators, 2009 IEEE/ASME International Conference on Advanced Intelligent Mechatronics (AIM), Suntec International Convention and Exhibition Centre, Singapore, July 14-17, 2009, pp. 1539-1544.

[16] S. Islam and P. X. Liu, Adaptive output feedback control for robot manipulators using Lyapunov-based switching, 2009 IEEE/RSJ International Conference on Intelligent Robots and Systems (IROS), St. Louis, USA, October 11-15, 2009, 
pp. 899-905.

[17] S. Islam and P. X. Liu, Robust tracking using hybrid control system, Proceeding of the 2009 IEEE International Conference on Systems, Man, and Cybernetics (SMC), San Antonio, TX, USA, October 11-14, 2009, pp. 4571-4576.

[18] S. Islam and P. X. Liu, Adaptive fuzzy output feedback control for robotic manipulators, Proceeding of the 2009 IEEE International Conference on Systems, Man, and Cybernetics (SMC), San Antonio, TX, USA, October 11-14, 2009, pp. 2704-2709.

[19] S. Islam and P. X. Liu, Robust control for robot manipulators by using only join position measurements, Proceeding of the 2009 International Conference on Systems, Man, and Cybernetics (SMC), San Antonio, TX, USA, October 11-14, 2009, pp. 4113-4118.

[20] S. Islam and P. X. Liu, Disturbance rejection in adaptive control for robotic systems, Presented in 2010 IEEE/ASME International Conference on Advanced Intelligent Mechatronics (AIM), July 6-9, Montreal, Canada.

[21] S. Islam and P. X. Liu, Adaptive iterative learning control for robot manipulators without using velocity signals, Presented in 2010 IEEE/ASME International Conference on Advanced Intelligent Mechatronics (AIM), July 6-9, Montreal, Canada.

[22] S. Islam and P. X. Liu, A distributed hybrid sliding mode control system for robot manipulators, Presented in 6th IEEE International Conference on Automation Science and Engineering (CASE), August 21-24, Toronto, Canada.

\subsection{Future Research}

The following are possible topics for the future research.

1. The fuzzy based model-free control methods developed in Chapter 3 is not experimentally verified on real systems. Therefore, it would be interesting to in- 
vestigate the tracking performance of these algorithms on real-world applications. 2. The hybrid adaptive control strategy proposed in Chapter 5 and Chapter 6 is based on using a priori known structure of the system dynamics. Though, the theoretical developments are verified through simulation and experimental evaluations, the requirement of a priori known system structure may influence the tracking performance in real-world applications as the perfect model structure in many real-world control applications may be unrealistic. To relax the requirement of the system dynamics from multi-model based hybrid adaptive design, it would be interesting to introduce multiple-neuro and multiple-parameters model based hybrid adaptive fuzzy approach. This approach will be employed to remove the demand of the model structure represented by the nonlinear regressor and developed a model-free hybrid control strategy for large-scale nonlinear systems. The convergence property will be shown along the line of the results proposed in Chapter 3 and Chapter 5 .

3. In our future work, the results presented in this thesis will be applied in haptic and Internet based teleoperation for biomedical mechatronic applications. More specifically, the hybrid adaptive and adaptive fuzzy control system will be employed to improve the system performance in delay-dependent robot-assisted telesurgical tracking tasks. 


\section{Bibliography}

[1] A. S. Morse, Supervisory control of families of linear set-point controllers-Part I, IEEE Transactions on Automatic Control, Vol. 41, pp. 1413-1431, 1996.

[2] A. Rojko and Karel Jezernik, Sliding-mode motion controller with adaptive fuzzy disturbance estimation, IEEE Transactions on Industrial Electronics, Vol. 51, no. 5, pp. 963-971, 2004.

[3] A. Tayebi, Adaptive iterative learning control for robot manipulators, Automatica, Vol. 40, pp. 1195-1203, 2004.

[4] A. Tayebi, S. Abdul, M. B. Zarembi and Y. Ye, Robust iterative learning control design: application to a robot manipulator, IEEE/ASME Transactions on Mechatronics, Vol. 13, no. 5, pp. 608-613, 2008.

[5] A. Tayebi and S. Islam, Adaptive iterative learning control for robot manipulators: Experimental results, Control Engineering Practice, vol. 14, pp. 43-851, 2008.

[6] A. Tayebi and S. Islam, Experimental evaluation of an adaptive iterative learning control scheme on a 5-DOF robot manipulators, In Proceeding of IEEE International Conference on Control Applications, Taipei, Taiwan, September 2-4, pp. 1007-1011, 2004.

[7] A. Teel and L. Praly, Tools for semi-global stabilization by partial state and output feedback, SIAM journal of Control and Optimization, Vol. 33, 1995. 
[8] A. V. Topalov and O. Kaynak, On line learning in adaptive neurocontrol for compliance tasks of robotic manipulators, IEEE Transactions on Systems, Man, and Cybernetics, Vol. 31, pp. 445-450, 2001.

[9] B. S. Chen, H. J. Uang and C. S. Tseng, Robust tracking enhancement of robot systems including motor dynamics: a fuzzy-based dynamic game approach, IEEE Transactions on Fuzzy Systems, Vol. 6, pp. 538-552, 1998.

[10] C. Canudas De Wit and N. Fixot, Adaptive control of robot manipulators via velocity estimated feedback, IEEE Transactions on Automatic Control, Vol. 37, no. 8, 1992, pp. 1234-1237.

[11] C. Canudas De Wit and N. Fixot, Robot control via robust estimated state feedback, IEEE Transactions on Automatic Control, Vol. 36, pp. 1497-1501, 1991.

[12] C.-J. Lin, Variable structure model following control of robot manipulators with high-gain observer, JSME, International journal Series C, Vol. 47, no. 2, pp. 591-600, 2004.

[13] C.-Ho Choi and Nojun Kwak, Robust control of robot manipulator by modelbased disturbance attenuation, IEEE/ASME Transactions on Mechatronics, Vol. 8, no. 4, pp. 511-513, 2003.

[14] C. Hua, X. Guan and G. Duan, Variable structure adaptive fuzzy control for a class of nonlinear time delay systems, Fuzzy Sets and Systems, Vol. 148, no. 3, pp. 453-468, 2004.

[15] C. Hua and P. X. Liu, Convergence analysis of teleoperation systems with unsymmetric time-varying delays, IEEE Transactions on Circuits and SystemsII, Vol. 56, no. 3, 2009, 240-244. 
[16] C. Hua, P. X. Liu and X. Guan, Backstepping control for nonlinear systems with time delays and applications to chemical reactor systems, IEEE Transactions on Industrial Electronics, Vol. 56, no. 9, pp. 3723-3732, 2009

[17] C. Mnasri and M. Gasmi, Robust output feedback full-order sliding mode control for uncertain MIMO systems, IEEE International Symposium on Industrial Electronics, pp. 1144-1149, 2008.

[18] C. Y. Su, Q. Wang, X. Chen, S. Rakheja, Adaptive Variable Structure Control of a class of Nonlinear Systems with Unknown Prandtl-shlinskii Hysteresis, IEEE Transactions on Automatic Control, 50, no. 12, pp. 2069-2074, 2005.

[19] David G. Wilson, Gordon G. Parker, Gregory P. Starr and Rush D. Robinett, Output feedback sliding mode control for a planar flexible manipulator, Proceedings of the ASCE Specialty Conference on Robotics for Challenging Environments, 8-14, 1998.

[20] D. Angeli and E. Mosca, Lyapunov-based switching supervisory control of nonlinear uncertain systems, IEEE Transactions on Automatic Control, Vol. 47, no. 3, pp. 500-505, 2002.

[21] E. S. Shin and K. W. Lee, Robust output feedback control of robot manipulators using high-gain observer, Proceeding of IEEE International Conference on Control Applications, pp. 881-886, 1999.

[22] E. Kim, Output feedback tracking control of robot manipulators with model uncertainty via adaptive fuzzy logic, IEEE Transactions on Fuzzy Systems, Vol. 12, no. 3, 2004.

[23] F. L. Lewis, C. T. Abdallah, and D. M. Dawson, Control of Robot Manipulators. New York: MacMillan, 1993.

[24] G. Bartolini and I. Zolezzi, Variable structure systems nonlinear in the control law, IEEE Transactions on Automatic Control, Vol. AC-30, pp. 681-684, 1985. 
[25] G. Liu, Control of robotic manipulators with consideration of actuator performance degradation and failures, Proceeding of the IEEE International Conference on Robotics and Automation, Seoul, Korea, May 21-26, 2001.

[26] G. Liu and A. A. Goldenberg, Comparative study of robust saturation-based control of robot manipulators: analysis and experiments, The international Journal of Robotics Research, Vol. 15, no. 5, pp. 473-491, 1996.

[27] G. Liu and A. A. Goldenberg, Robust control of robot manipulators based on dynamics decomposition, IEEE Transactions on Robotics and Automation, Vol. 13, no. 5, pp. 783-789, 1997.

[28] G. Liu and A. A. Goldenberg, Experiments on robust control of robot manipulators, Proceeding of the IEEE International Conference on Robotics and Automation, Nice, France, May, 1992.

[29] H. Asada and J. J. Slotine, Robot Analysis and Control. New York:Wiley, 1986.

[30] H. Berghuis, R. Ortega, and H. Nijmeijer, A robust adaptive robot controller, IEEE Transactions on Robotics and Automation, Vol. 9, pp. 825-830, 1993.

[31] M. Hamerlain, The new robust control using the theory of generalized variable structure, IEEE International Symposium on Industrial Electronics, July 1014, Athens, Greece, 1995.

[32] H. Hashimoto, K. Maruyama, and F. Harashima, A microprocessor-based robot manipulator control with sliding mode, IEEE Transactions on Industrial Electronics, Vol. 34, no. 1, 1987.

[33] H. Medhaffar, N. Derbel, and T. Damak, A decoupled fuzzy indirect adaptive sliding mode control with application to robot manipulator, International Journal of Modeling, Identification and Control, Vol. 1, no. 1, 2006. 
[34] H. M. Schwartz, Model reference adaptive control for robotic manipulators without velocity measurements, International Journal of Adaptive Control and Signal Processing, Vol. 8, pp. 279-285, 1994.

[35] I. G. Polouchine and A. Tayebi, Anticipating iterative learning control of robot manipulators, Proceeding of American Control Conference, Vol. 14, 2006, pp. 843-851.

[36] I. G. Polouchine, Force-reflecting tele operation over wide-area networks, $P h$. D. Thesis, Carleton University, Ottawa, Canada, 2009.

[37] J.-B. Pomet and L. Praly, Adaptive nonlinear regulation estimation from the Lyapunov equation, IEEE Transactions Automatic Control, Vol. 37, pp.729$740,1992$.

[38] J. M. Daly and David W. L. Wang, Output feedback sliding mode control in the presence of unknown disturbances, Systems and Control Letters, Vol. 58, no. 3, pp. 188-193, March 2009.

[39] J. Daly and H. M. Schwartz, Experimental results for output feedback adaptive robot control, Robotica, Vol. 24, pp. 727-738, 2006.

[40] J. Daly and H. M. Schwartz, Nonlinear adaptive output feedback control of robotic manipulators, Proceeding of the International Conference on Robotics and Automation, Spain, April, 2005.

[41] J. J. E. Slotine and W. Li, Adaptive manipulator control: a case study, IEEE Transactions on Automatic Control, Vol. 33, pp. 995-1003, 1988.

[42] J. J. E. Slotine and W. Li, Applied nonlinear control, Upper Saddle River, NJ: Prentice Hall, 1991.

[43] J. J. E. Slotine and S. S. Sastry, Tracking control of nonlinear system using sliding surface, with application to robot manipulators, International Journal of Control, Vol. 38, pp. 465-492, 1983. 
[44] J. Y. Hung, W. Gao and J. C Hung, Variable structure control: A survey, IEEE Transactions on Industrial Electronics, Vol. 40, pp. 2-22, 1993.

[45] J. T. Wen and S. Murphy, PID control for robot manipulators, Rensselaer Polytechnic Institute, CIRSSE Document no. 54, 1990.

[46] K. D. Young, Controller design for robot a manipulator using theory of variable structure control, IEEE Transactions on Systems, Man, and Cybernectics, Vol. 8, pp. 101-109, 1978.

[47] K. Erbatur and O. Kaynak, Use of adaptive fuzzy systems in parameter tuning of sliding mode controllers, IEEE/ASME Transactions on Mechatronics, Vol. 6, no. 4, 2001, pp. 474-482.

[48] K. Erbatur, O. Kaynak and A. Sabanovic, Robust control of a direct drive manipulator, Proceeding of IEEE ISIC/CIRA/ASAS Joint Conference, Gaithersburg, MD, September 14-17, 1998.

[49] K. J. Astrom and B. Wittenmark, Adaptive control, Addition-Wesley, 2nd edition, 1995.

[50] K. S. Narendra and A. M. Annaswamy, Stable Adaptive Systems, Prentice Hall, New Jersy, 1988.

[51] K. W. Lee and H. K. Khalil, Adaptive output feedback control of robot manipulators using high-gain observer, International Journal of Control, Vol. 67, no. 6, 1997, pp. 869-886.

[52] K. Y. Lim and M. Eslami, Robust adaptive controller designs for robot manipulator systems, IEEE Transactions on Robotics and Automation, Vol RA-3, pp. pp. 54-66, 1987.

[53] L. C. Fu and T. L. Liao, Globally stable robust tracking of nonlinear system using variable structure control and application to a robot manipulator, IEEE Transactions on Automatic Control, Vol. 34, pp. 345-1350, 1990. 
[54] L. X. Wang, A Course in Fuzzy Systems and Control, Englewood Cliffs, Nj: Prentice-Hall, 1997.

[55] M. Akar, Robust tracking for a class of nonlinear systems using multiple models and switching, Proceeding of the American Control Conference, Portland, OR, pp. 4056-4061, 2005.

[56] M. Erlic and W. Lu, A reduced-order adaptive velocity observer for manipulator control, IEEE Transactions on Robotics and Automation, Vol. 11, pp. 293-303, 1995.

[57] M. Llama, R. Kelly, V. Santibanez and H. Centeno. An Adaptive Fuzzy Controller for Robot Manipulators: Theory and Experimentation, International Journal of Factory Automation, Robotics and Soft Computing. Issue 1, January 2009, pp. 122-131.

[58] M. L. Corradini, T. Leo, and G. Orlando, Robust stabilization of a class of nonlinear systems via multiple model sliding mode control, Proceeding of the American Control Conference, Chicago, IL, pp. 631-635, June 2000.

[59] M. L. Corradini and G. Orlando, Transient improvement of variable structure controlled systems via multimodel switching control, Journal of Dynamic Systems, Measurement and Control, Vol. 124, pp. 321326, June 2002.

[60] M. Q. H. Meng, Application of an efficient neural network to identification and adaptive control of unknown robot dynamics, International Journal of Intelligent Control and Systems, Vol. 1, no. 4, pp. 459-468, 1996.

[61] M. Q. H. Meng, Comparison study of model-based and non-model-based robot controllers," In Proceeding of IEEE International Conference on Systems, Man and Cybernetics, Vancouver, Canada, 1995, pp. 61-66. 
[62] M. Q. H. Meng and W.-S. Lu, A unified approach to stable adaptive force/position control of robot manipulators, In Proceeding of the American Control Conference, 1994.

[63] M. R. Akbarzadeh and T. R. Shahnazi, Direct adaptive fuzzy PI sliding mode control for a class of uncertain nonlinear systems, IEEE international conference on Systems, Man and Cybernetics, Vol. 3, 2005.

[64] M. S. Branicky, Multiple Lyapunov functions and other analysis tools for switched and hybrid systems, IEEE Transaction on Automatic Control, Vol. 43, pp. 475-482, 1998.

[65] M. S. De Queiroz, J. Hu, D. Dawson, T. Burg, and S. Donepudi, Adaptive position/force control of robot manipulators without velocity measurements: Theory and experimentation, IEEE Transactions on systems, man, and $c y$ bernetics, Vol. 27, 1997, pp. 796-809.

[66] M. Takegaki and S. Arimoto, A new feedback method for dynamic control of manipulators, Transactions on ASME Journal of Dynamic Systems, Measurement, and Control, Vol. 103, pp. 119-125, 1981.

[67] M. W. Spong and M. Vidyasagar, Robot dynamics and control, New York: Wiley.

[68] M. W. Spong, On the robust control of robot manipulators, IEEE Transactions on Automatic Control, Vol. 37, no. 11, pp. 1782-1786.

[69] P. Ioannou and J. Sun, Robust adaptive control, Prentice Hall, 1996.

[70] P. R. Pagilla and M. Tomizuka, An adaptive output feedback controller for robot arms: stability and experiments, Automatica, Vol. 37, 2001, pp. 983-995.

[71] P. Rocco, Stability of PID control for industrial robot arms, IEEE Transactions on Robotics and Automation, Vol. 12, no. 4, 1996. 
[72] P. Tomei, Adaptive PD controller for robot manipulators, IEEE Transaction on Robotics Automation, Vol. 7, pp. 565-570, 1991.

[73] R. Kelly, Comments on Adaptive PD controller for robot manipulator, IEEE Transaction on Robotics Automation, Vol. 9, pp. 117-119, 1993.

[74] R. Kelly, Global positioning of robot manipulators via PD control plus a class of nonlinear integral actions, IEEE Transactions on Automatic Control, Vol. 43, no. 7, pp. 934-938, 1998.

[75] R. Kelly, A tuning procedure for stable PID control of robot manipulators, Robotica, Vol. 13, pp. 141-148, 1995.

[76] R. Matinez and J. Alvarez, Hybrid sliding-mode-based control of under actuated system with dry friction, IEEE Transactions on Industrial Electronics, Vol. 55, no. 11, 2008.

[77] R. M. Murray, Z.Li and S. S. Sastry, A mathematical introduction to robotic manipulation, CRC press, 1994.

[78] R. Ortega, A. Loria, and R. Kelly, A semiglobally stable output feedback $P I^{2} D$ regulator for robot manipulators, IEEE Transactions on Robotics Automation, Vol. 40, pp. 1432-1436, 1995.

[79] S. Arimoto and F. Miazaki, Stability and robustness of PID feedback control for robot manipulators of sensory capability, Robotics Research; First International Symposium, Cambridge, MA: MIT Press, pp. 783-799, 1984.

[80] S. A. Morris and S. Khemaissia, A neural network based adaptive robot controller, Journal of Intelligent and Robotic Systems, Vol. 15, pp. 3-10, 1996.

[81] S. Arimoto, T. Naniwa, V. Parra-Vega, and L. Whitcomb, A quasi natural potential and its role in design of hyper-stable PID servo-loop for robotic systems, In Proceeding CAI Pacific Symposium Control and Industrial Automation Application, pp. 110-117, Hong Kong, 1994. 
[82] S. Islam, Adaptive output feedback for robot manipulators using linear observer, Proceedings of the International Conference on Intelligent System and Control, November 16-18, Orlando, Florida, pp. 38-43, 2008.

[83] H. M. Schwartz and S. Islam, An evaluation of adaptive robot control via velocity estimated feedback, In Proceedings of the International Conference on Control and Applications, Montreal, Quebec, May 30-June 1, pp. 325-333, 2007.

[84] S. Islam and P. X. Liu, Adaptive sliding mode control for robotic systems using multiple parameter models, 2009 IEEE/ASME International Conference on Advanced Intelligent Mechatronics (AIM), pp. 1775-1780, Suntec International Convention and Exhibition Centre, Singapore, July 14-17, 2009.

[85] S. Islam and P. X. Liu, PD output feedback control design for robotic manipulators, 2009 IEEE/ASME International Conference on Advanced Intelligent Mechatronics (AIM), pp. 1539-1544, Suntec International Convention and Exhibition Centre, Singapore, July 14-17, 2009.

[86] S. Islam and P. X. Liu, Adaptive output feedback control for robot manipulators using Lyapunov-based switching, 2009 IEEE/RSJ International Conference on Intelligent Robots and Systems (IROS), St. Louis, USA, pp. 899-905, October 11-15, 2009.

[87] S. Islam and P. X. Liu, Robust tracking using hybrid control system, Proceeding of the 2009 IEEE International Conference on Systems, Man, and Cybernetics (SMC), San Antonio, TX, USA, October 11-14, pp. 4571-4576, 2009.

[88] S. Islam and P. X. Liu, Adaptive fuzzy output feedback control for robotic manipulators, Proceeding of the 2009 IEEE International Conference on Systems, Man, and Cybernetics (SMC), San Antonio, TX, USA, pp. 2704-2709, October 11-14, 2009. 
[89] S. Islam and P. X. Liu, Robust control for robot manipulators by using only join position measurements, Proceeding of the 2009 International Conference on Systems, Man, and Cybernetics (SMC), San Antonio, TX, USA, October 11-14, pp. 4113-4118, 2009.

[90] S. Islam and P. X. Liu, Disturbance rejection in adaptive control for robotic systems, Presented in 2010 IEEE/ASME International Conference on Advanced Intelligent Mechatronics (AIM), July 6-9, Montreal, Canada.

[91] S. Islam and P. X. Liu, Adaptive iterative learning control for robot manipulators without using velocity signals, Presented in 2010 IEEE/ASME International Conference on Advanced Intelligent Mechatronics (AIM), July 6-9, Montreal, Canada.

[92] S. Islam and P. X. Liu, A distributed hybrid sliding mode control system for robot manipulators, Presented in 6th IEEE International Conference on Automation Science and Engineering (CASE), August 21-24, Toronto, Canada.

[93] S. Islam and P. X. Liu, Output feedback sliding mode control for robot manipulators, Robotica, In Press, 2009.

[94] S. Islam and P. X. Liu, PD output feedback control design for industrial robotic systems, IEEE/ASME Transactions on Mechatronics, Vol. 15, no. 3, 2010.

[95] S. Islam and P. X. Liu, A hybrid adaptive control for robust trajectory tracking for robot manipulators:Theory and Experiment, Robotica, In Press, 2010.

[96] S. Islam and P. X. Liu, Robust adaptive fuzzy output feedback for robotic manipulators, IEEE/ASME Transactions on Mechatronics, In Press, 2010.

[97] S. Islam and P. X. Liu, Hybrid control system for a class of nonlinear mechanical systems: Theory and experiment, Accepted for publication in Mechatronics, 2010. 
[98] S. Islam and P. X. Liu, Robust sliding mode control for robot manipulators, IEEE Transactions on Industrial Electronics, In Press, 2010.

[99] S. Islam and P. X. Liu, Adaptive iterative learning control for robot manipulators without using velocity signals, Robotica, Under Review.

[100] S. Islam and P. X. Liu, Hybrid adaptive sliding mode control for robot manipulators, Robotics and Autonomous Systems, Under Review.

[101] S. Islam and P. X. Liu, Robust adaptive output feedback control of robotic systems, IEEE Transactions on Control Systems Technology, Under Review.

[102] S. Lim, D. Dawson, and K. Anderson, Re-examining the Nicosia-Tomei robot observer-controller from a back-stepping perspective, IEEE Transactions on Control Systems Technology, Vol. 4, 1996, pp. 304-310.

[103] W.-S. Lu and M. Q. H. Meng, Regressor formulation of robot dynamics: computation and applications, IEEE Transactions on Robotics Automation, vol. 9, no. 3, pp. 323-333, 1993.

[104] S. Lim, D. Dawson, and K. Anderson, Re-examining the Nicosia-Tomei robot observer-controller from a back-stepping perspective, IEEE Transaction on Control Systems Technology, Vol. 4, 1996, pp. 304-310.

[105] S. Nicosia and P. Tomei, Robot control by using only joint position measurements, IEEE Transactions Robotics Automation, Vol. 35, no. 9, 1990, pp. 1058-1061.

[106] S. Sastry and M. Bosdon, Adaptive control: Stability, Convergence and Robustness, Printice-Hall, 1989.

[107] S. Y. Yi and M. J. Chung, A robust fuzzy logic controller for robot manipulators with uncertainities, IEEE Transaction on Systems, Man, and Cybernetics $B$, vol. 27, pp. 706-713, 1997. 
[108] V. I. Utkin, Variable structure systems with sliding mode, IEEE Transaction on Automatic Control, Vol. 22, pp. 212-222, 1977.

[109] W. Chen and M. Saif, Output feedback controller design for a class of MIMO nonlinear systems using high-0rder sliding-mode differentiators with application to a laboratory 3-D Crane, IEEE Transaction on Industrial Electronics, Vol. 55, no. 11, 2008.

[110] W. E. Dixon, M. S. Queiroz, F. Zhang and D. M. Dawson, Tracking control of robot manipulators with bounded torque inputs, Robotica, Vol. 17, 1999, pp. 121-129.

[111] W. Lu and M. Q. H. Meng, Regressor formulation of robot dynamics: computation and applications, IEEE Transactions on Robotics Automation, Vol. 9, no. 3, 1993, pp. 323-333.

[112] W. Pedrycz, Fuzzy control and fuzzy systems, Willey, Toronto, 1993.

[113] X. Yang and Q.-H. Meng, Real-time tracking of robot manipulators with online learning based approach, In Proceedings of IEEE Canadian Conference on Electrical and Computer Engineering, Shaw Conference Center, Edmonton, Alberta, Canada, May 9-12, 1999.

[114] Y. C. Chang and B. S. Chen, Robust tracking design for both holonomic and nonholonic constrained mechanical systems: adaptive fuzzy approach, IEEE transaction on Fuzzy Systems, Vol. 8, pp. 46-66, 2009.

[115] Y. D. Meddah and A. Benallegue, A stable neuro-adaptive controller for rigid robot manipulators, Journal of Intelligent and Robotic Systems, Vol. 20, pp. 181-193, 1997.

[116] Y. Guo and P. - Y. Woo, An Adaptive fuzzy sliding mode controller for robotic manipulators, IEEE Transaction on systems, Man, and Cybernetics, Vol. 33, no. 2, pp. 149-159, 2003. 
[117] Y. H. Kim and F. L. Lewis, Neural network output feedback control of robot manipulators, IEEE Transaction on Robotics and Automation, Vol. 15, pp. 301-309, 1999.

[118] X.-G. Yan and C. Edwards, Adaptive sliding-mode-observer-based fault reconstruction for nonlinear systems with parametric uncertainties, IEEE Transaction on Industrial Electronics, Vol. 55, no. 11, 2008.

[119] X. Lu, An investigation of adaptive fuzzy sliding mode control for robotic manipulators, Master Thesis, Carleton University, 2007. 